\title{
Memory formation, neurodegeneration and drugs : behavioral, neurochemical and neuroanatomical investigations in the rat
}

Citation for published version (APA):

Prickaerts, J. H. H. J. (1998). Memory formation, neurodegeneration and drugs : behavioral, neurochemical and neuroanatomical investigations in the rat. [Doctoral Thesis, Maastricht University]. NeuroPsych Publishers. https://doi.org/10.26481/dis.19981029jp

Document status and date:

Published: 01/01/1998

DOI:

10.26481/dis.19981029jp

Document Version:

Publisher's PDF, also known as Version of record

Please check the document version of this publication:

- A submitted manuscript is the version of the article upon submission and before peer-review. There can be important differences between the submitted version and the official published version of record.

People interested in the research are advised to contact the author for the final version of the publication, or visit the DOI to the publisher's website.

- The final author version and the galley proof are versions of the publication after peer review.

- The final published version features the final layout of the paper including the volume, issue and page numbers.

Link to publication

\footnotetext{
General rights rights.

- You may freely distribute the URL identifying the publication in the public portal. please follow below link for the End User Agreement:

www.umlib.nl/taverne-license

Take down policy

If you believe that this document breaches copyright please contact us at:

repository@maastrichtuniversity.nl

providing details and we will investigate your claim.
}

Copyright and moral rights for the publications made accessible in the public portal are retained by the authors and/or other copyright owners and it is a condition of accessing publications that users recognise and abide by the legal requirements associated with these

- Users may download and print one copy of any publication from the public portal for the purpose of private study or research.

- You may not further distribute the material or use it for any profit-making activity or commercial gain

If the publication is distributed under the terms of Article 25fa of the Dutch Copyright Act, indicated by the "Taverne" license above, 


\section{Memory Formation, Neurodegeneration and Drugs}

Behavioral, Neurochemical and Neuroanatomical Investigations in the Rat 
Memory formation, neurodegeneration and drugs: Behaviorat, neurochemical and neuroanatomical investigations in the rat / Jozef Hendrik Hubertus Joannes Prickaeris. - Maastricht:

Neuropsych Publishers Maastricht. - Ill.

Thesis Manatricht University. - With ref. -

With summary in Duich.

ISBN 90-75579-08-X

NUGI 743746

Subject headings: neurodegeneration, cognition, nootropics

\section{Production: Datawyse I University Press Maastricht}

Neuropsych Publishers is a nonprofit organization which airrs at promoting the science of 'Brain and Behavior' and improwing the application of the products of this science in health care and education. Neuropsych Publisher accomplishes these aims by publishing books, dissertations and other products of scientific activity, by disseminating educational materials and publication of tests, assessment scales and other psychonteric instruments in the field of Neuropsychology, Neuropsychiatry and ofher areas within the domain of Brain and Behavion.

Postal addrew:

Neuropsych Publisthers

Department of Psychiatry and Neuropsychology

Section Neuropsychology, Neuropsychiatry and Psychobiology

Maastricht Uniwersity

P.O. Box 616

$6200 \mathrm{MD}$ Maastricht

The Netherlands

Cover illestration: The hippocampal formation and septal area of the rat (adapted fron a photograph by J.P. Broad in 'The hippocampus as an interface between cognition and emotion' by J A. Gray (1984) in Animal Cognirion (eds. Roitblat H.L., Bever T.G. and Terrace H.S.), pp. 607-626. Lawrence Erlbaum, Hillsdale, NJ.) 


\section{Memory Formation, Neurodegeneration and Drugs}

Behavioral, Neurochemical and Neuroanatomical Investigations in the Rat

\section{PROEFSCHRIFT}

ter verkrijging van de graad van doctor an de Universiteil Masstricht, op gezag van de Rector Magnificus, Prof. Dr. A.C. Nieuwenthuijzen Kruseman, volgens het besluit van het College van Decanen, in het openbaar te verdedigen op donderdag 29 oktober 1998 om 14.00 uur

door

Jozef Hendrik Hubertus Joannes Prickaerts

geboren op 31 maart. 1966 te Berg en Terblijt 
Promoloren:

Co-promotor:

Beoondelingsconmissie:
Prof. Dr. J. Jolles

Prof. Dr. H.W.M. Steinbusch

Dr. A. Blokland

Prof, Dr. J. Troost (voorzitter)

Prof. Dr. C.E. Blanco

Prof. Dr. J.F.M. Smits

Prof. Dr. P.R. Băr (Universiteit Utrecht)

Prof. Dr. P.G.M. Luiten (Rijksuniversiteit Groningen)

The studies described in this thesis were carried out at the European Graduate School of Neuroscience "EURON" (Department of Psychiatry and Neuropsychology, Maastricht University, Maastricht, The Netherlands) and BAYER AG (CNS-Research, Cologne, Germany).

Publication of this thesis was financially supported by: BAYER AG. Hoechst Marion Roussel B.V., SERVIER R\&D Benelux N.V., Sigma-Tau Ethifarma B.V., and UCB Pharma B..... Furthermore, additional financial support for publication by the "Stichting Alzheimer Fonds' and 'Intemationale Stichting Alzheimer Onderzoek' is gratefully acknowledged. 
Paranimfen:

F.M.D.G. Stikvoort

Drs. M.L.J.H. Prickaerts

Voor iedereen die heeft bijgedragen... 
"Is het de mens wel mogelijk om van de volle warheid kennis te nemen?".

Xenofanes van Kolofoon

(Griekse natuurfilosoof en dichter, wijfde eeuw w. Chr.) 


\section{Table of Contents}

Prologue. . . . . . . . . . . . . . . . . . . . 9

1 Introduction ............................ 11

Plasticity. . . . . . . . . . . . . . . . . . . . . . . 11

Operational definitions of memory formation . . . . . . . . . . II

Brain structures, neurotransmitters and neuromodulators. . . . . . . . . 14

Neurodegeneration . . . . . . . . . . . . . . . . . 47

Mechanisms of neurodegeneration in aging and Alzheimer's disease. . . . 47

Animal models of neurodegeneration . . . . . . . . . . . . . . 58

Aims . . . . . . . . . . . . . . . . . 77

Plasticity . . . . . . . . . . . . . . . 77

Neurodegeneration . . . . . . . . . . . . . . 79

2. Spatial discrimination learning and choline aceryltransferase activity

in streptozotocin-treated rats: Effects of chronic treatment with acetyl-L-carnitine . . 93

Introduction. . . . . . . . . . . . . . . . . 93

Materials and Methods . . . . . . . . . . . . . . . . . . 94

Results. . . . . . . . . . . . . . . . . . . 9 95

Discussion . . . . . . . . . . . . . . . . . 96

3 Brain enzyme activities after i.c.v. injection of streptozotocin

in rats receiving acetyl-L-carnitine . . . . . . . . . . . . . . . . 10I

Introduction. . . . . . . . . . . . . . . 10I

Materials and Methods . . . . . . . . . . . . . . . . . . 102

Results. . . . . . . . . . . . . . . . . . 103

Discussion ....................... 105

4 Cognitive performance and neurodegenerative markers in hippocampus, septum and striatum after an i.c.v. injection of streptozotocin: A correlation analysis . . . M

Introduction. . . . . . . . . . . . . . . . M 11

Materials and Methods . . . . . . . . . . . . . . . . . . . 1/2

Results . . . . . . . . . . . . . . . . 116

Discussion ...................... . . . 125

5 Nitric oxide synthase does not mediate neurotoxicity after an i.c.v.

injection of streptozotocin in the rat. . . . . . . . . . . . . . . 133

Introduction. . . . . . . . . . . . . . . . . . 134

Materials and Methods . . . . . . . . . . . . . . . . . 134

Results . . . . . . . . . . . . . . . . 137

Discussion ...................... 142 
6 Acute effects of acetyl-L-carnitine on sodium cyanide-induced behavioral and biochemical deficits. . . . . . . . . . . . . . . . 155

Introduction. . . . . . . . . . . . . . . . . 155

Materials and Methods . . . . . . . . . . . . . . . . 156

Results . . . . . . . . . . . . . . . . . . 158

Discusstion ......................... 161

7 Possible role of nitric oxide-cyclic GMP pathway in object recognition:

Effects of 7-nitroindazole and zaprinast . . . . . . . . . . . . . 167

Jntroduction, . . . . . . . . . . . . . . . . . . . 167

Materials and Methods . . . . . . . . . . . . . . . . 168

Results .. . . . . . . . . . . . . . . . . . 171

Discussion . . . . . . . . . . . . . . . . . . . 176

8 Behavioral, neurochemical and neuroanatomical effects of chronic postnatal L-NAME treatment in meonatal and adult rats . . . . . . . . . . . . 185

Introduction. . . . . . . . . . . . . . . . 186

Materials and Methods . . . . . . . . . . . . . . . . . . 186

Results . . . . . . . . . . . . . . . . . . . . . 190

Discussion . . . . . . . . . . . . . . . . . . 198

9 Concluding remarks. ....................... 211

Summary . . . . . . . . . . . . . . . . . . 223

Samenvatting. . . . . . . . . . . . . . . . . . 229

Dankwoord . . . . . . . . . . . . . . . . . . . 235

Curriculum Vitae . . . . . . . . . . . . . . . . . . . 237

Publications . . . . . . . . . . . . . . . . . . . . . . . 238 


\section{Prologue}

\section{Memory formation, neurodegeneration and drugs: Behavioral, neurochemical and neuroanatomical investigations in the rat}

The experiments described in this thesis were part of the research program of the division of neurodegeneration and plasticity (European Graduate School of Neuroscience "EURON', Department of Psychiatry, Maastricht University). Neurodegeneration can be best delined as a decline in function and dying of neuronal tissue. This thesis focusses on Alzheimer's disease as a neurodegenerative disorder which is characterized by a progressive deterioration of memory function [8], In 1995 it was estimated that about 20 million people were affected worldwide by this memory loss disorder [1]. A number that is expected to increase in the near future because of the "graying" of the population in the western world. The other aspect of the division's research line is neuronal plasticity, which is the ability of neuronal tissue to change or adapt. In this thesis the term plasticity is mainly used for physiological aspects of learning and memory processes: also referred to as synaptic plasticity [e.g. 2]. But plasticity is also applicable to developmental aspects of neuronal tissue [e.g.6] and neurochemical/neuroanatomical recovery after a decline in function due to, for instance, neurodegeneration le.g. 91.

In this thesis, neurodegeneration and plasticity were studied on three levels: 1) behavior, 2) neurochemistry, and 3) neuroanatomy. Behavior was mainly assessed in several memory tasks (e.g. spatial discrimination leaming). After behavioral testing, the brains of the rats were used for either biochemical measurements (e.g. enzyme activities) or anatomical studies (histology, immunocytochemistry). The behavioral and biochemical measures were correlated and the results about possible relationships, together with the morphological data, were used to gain insight into functioning of several brain structures during physiological (i.e. plasticity) or pathological (i.e. neurodegeneration) conditions.

\section{Aims and outline of the thesis}

The cause(s) and the mechanism(s) of neuronal cell death of neurodegenerative diseases are still largely unknown. Because of this limited understanding of neurodegenerative mechanisms, it is difficult to develop a suitable therapy. Unfortunately, there is no animal species which naturally develops a meurodegenerative disorder. Consequently, a good animal model of neurodegeneration is in great demand, and preferably one that has an interface with the possible cause(s). With a good model one can gain a better understanding of neuropathological processes underlying neurodegenerative disorders and one also has to possibility to tes: therapies which enhance neuronal function or prevent neuronal dysfunction. Furthermore a better insight in neurophysiological (or 'healthy') processes is also very useful in developing a therapy based on enhancement of neuronal function. This thesis focusses on Alzheimer's disease and learning and memory (or "cognitive") processes, and as a consequence the main themes of this thesis are:

\footnotetext{
- Characterization of an animal model of neurodegeneration

-Gain insight in neuropathological processes leading to neurodegenerative disorders

-Testing of nootropics (cognition enhancing/neuroprotective substances)

-Gain insight in neurophysiological processes involved in learning and memory
} 
In the first chapter an overview is given of plasticity, neurodegeneration, and aims of the thesis, respectively. With regard to plasticity, operational definitions of, and behavioral tasks measuring learning and memory are presented. Thereafter, brain structures and neurotransmitter/neuromodulator systems inwolved in memory formation are summarized. Neurodegenerative processes are mainly discussed by taking Alzheimer's disease as an example. In addition, models of neurodegeneration and therapies for neurodegenerative disorders (as mainly based on the previously discussed mechanisms) are presented.

The following chapters describe the experiments with two animal models of an impaired neuronal function. One model, i.c.v. injected streptozotocin (Chapter 2-5), is based on a chronic inhibition of the energy metabolism, and can be regarded as a model of neurodegeneration $[4,5]$. The other model, i.c.v. injected sodium cyanide (Chapter 6), is based on a short period of acute energy depletion, and can be used to study the acute effects of drugs that are presumed to preserve neuronal function during low energy states [3]. Some experiments were done in collaboration with pharmaceutical companies, especially the testing of a putative nootropic effect of acetyl-L-carnitine in our models (Sigma-Tau Company. Pomezia-Rome, Italy; Chapter 2, 3 and 6). To gain more insight into the neurodegenerative mechanism of streptozotocin, this model was further characterized behaviorally and both biochemically (Chapter 4) and anatomically (Chapter 5).

The other chapters describe the experiments with regard to neuronal plasticity, both memory formation (Chapter 7) and developmental processes (Chapter 8 ). Since nitric oxide has been ascribed an important role in neuronal plasticity $[\mathrm{e} . \mathrm{g} .7,10]$, the role of nitric oxide was studied in depth in these contexts. Finally, the main findings and possible implications of the above-mentioned studies are discussed in the concluding remarks (Chapter 9).

\section{REFERENCES}

1. Brarinaga M. (1995) Missing Alzheimer's gene found. Science 269, 917-918.

2. Bliss T. V. P. and Collingridge G. L. (1993) A synaptic model of memory: Long-tem potentiation in the hippocampus, Nature $361,31-39$.

3. Blok]and A Bothoner J., Honig W. and Jolles J. (1993) Behavioral and biochemical effects of acute central motabolic inhibition: Effects of acetyl-L-carnitue. Eur. A. Prannacol. 235, 275-281.

4. Blokland A. and Jolles I. (1993) Spatial learing deficit and reduced hippocampal ChA activity in rats after an ic unject hon of streptozotocin. Pharmacol. Biochew. Behav. 44,491-494.

5. Blokiand A. and Jolles J. (1994) Behavioral and biochentical effects of an icw injection of streptorotocin in old Lewis rats. Pharmacol. Biachem. Behav, 47, 833-837.

6. Braty R. J. Gorfer J. A., Monroe M. T. and Swann I. W. (1994) Developmentall allerations in the serusinivily of hppocampal WMDA receptors to AP5. Brain Res. Dew. Brain Res. 83, 190-196.

7. Fin C., Cunha C. A., Bromberg E., Schmitz. P. K. Bianchin M. Medina J. H. and lizquierdo I. (1995) Exporiments suggesting a role for nitric oxide in the hippocampus in memory processes. Newrobiot. Leamr. Men. 63, $113-115$.

8. Friedland $\mathbb{R}$. P. (1993) Alzeimer's disease: Clinical leatures and differential diagnosis. Menrology 43 (Siuppl. 4), S4.5-\$51.

9. Horwitz B. (1987) Neuroplasticity and the progression of Alzheimer"s disease. Intern. J. Neuroscience 4 , I. 14.

10. Kalb R. G. and Agostini J, (1993) Molecula evidence for niflic oxidemediated motor neuron dewelopment. Nerusosit. 57, $\|-8$. 


\section{Chapter 1}

\section{Introduction: Plasticity}

Neuronal plasticity is the ability of neuronal tissue to change or to adapt. Plasticity applies to neurochemical and neuroanatomical recovery after a decline in function due to several factors such as lesions, aging and meurodegenerarion [s.g. 14.3$]$. But it is also applicable to developmental aspects of neuronal tissue [e. 42]. Physiological aspects of learning and memory processes are also considered as a form of neuronal plasticity [e.g. 33]. This part of the introduction deals with the latter aspect: learning and memory. With regard to learning and memory processes I prefet to use the term mentory formation. This because on the one hand, one first has to learn something before it comes to memory, but at the other hand without some memory there can be no learning. In the following overview several aspects of memory itself will be presented, that is how is memory defined (operationally), and how is it measured (in behavioral tasks)? Thereafter, brain structures and neurotransmitter/ neuromodulator systems involved in memory formation are summarized. At the same time the decline in memory formation in aging and neurodegeneration is discnssed to give additional insight into functioning of memory. Alzheimer"s disease is taken as an example of a neurodegenerative disorder, since this disease is characterized by a progressive deterioration of memory function and its incidence increases more and more because of 'graying' in the western world. Possible cause(s) of neurodegeneration are discussed in the following part Neuradegeneration of this chapter.

\section{Operational Definitions of Memory Formation}

\section{MEMORY DEFINITIONS}

Memory has been defined with several operational definitions and the mechanisms of memory formation can be subdivided into several steps. First an organism has to encode information before that information can be stored into memory. The efficiency of learnimg and memory processes depends on non-mnemonic factors such as attention, motivation and emotion (71). Figure $\mathbb{i}$ illustrates the processes thought to be involved in learning. In luman studies nemory is moslly subdivided into short-term (less then minutes) and longuterm (hours or greater) memory. while the short-term component is also often referred to as working memory [2t]. The long-term component is further subdivided into procedural (e.g. classical conditioning, skills/habits) and declarative (events and facts) memory [301. In animal studies the declarative memory is often subdivided into working and reference memory [251]. Moreover, working and reference memory may be regarded as synonymous with episodic (events) and semantic (facts) menory in human studies.

When working with experimental animals it is useful to make a hierarchic subdivision of memory. Firstly, one determines the modality such as spatial memory or object memory. I will give no opinion on whether short-term or long-term memory is involved, although there are researchers who make this distinction 931 or even a further one into declarative and procedural memory [3301. Secondly, one determines whether it is acquisition (learning) or retention of information which is being measured. During acquisition, processes come up such as encoding of information and its storage into memory, which together can also be referred to as consolidation 
of information. During retention, after acquisition, retrieval of information out of memory is being tested. In one-trial learning it is more useful to talk of consolidation and retrieval of information in stead of acquisition and retention [155]. During acquisition of more than one trial learning, retrieval of information is of course also very important. Thus, there is no learning without retrieval of information from memory "while at the same time there is no memory without leaming first. Hence, memory formation is an appropriate term to refer to both learming and memory processes, as has already been mentioned. Thirdly, one can make possible use of the operational definitions as formulated by Olton [251]: working and reference memory. Working memory contains trial-depend information such as the number of places that have been visited during a trial. Reference memory contains trial-independent information such as the fixed location of a food reward in subsequent trials.

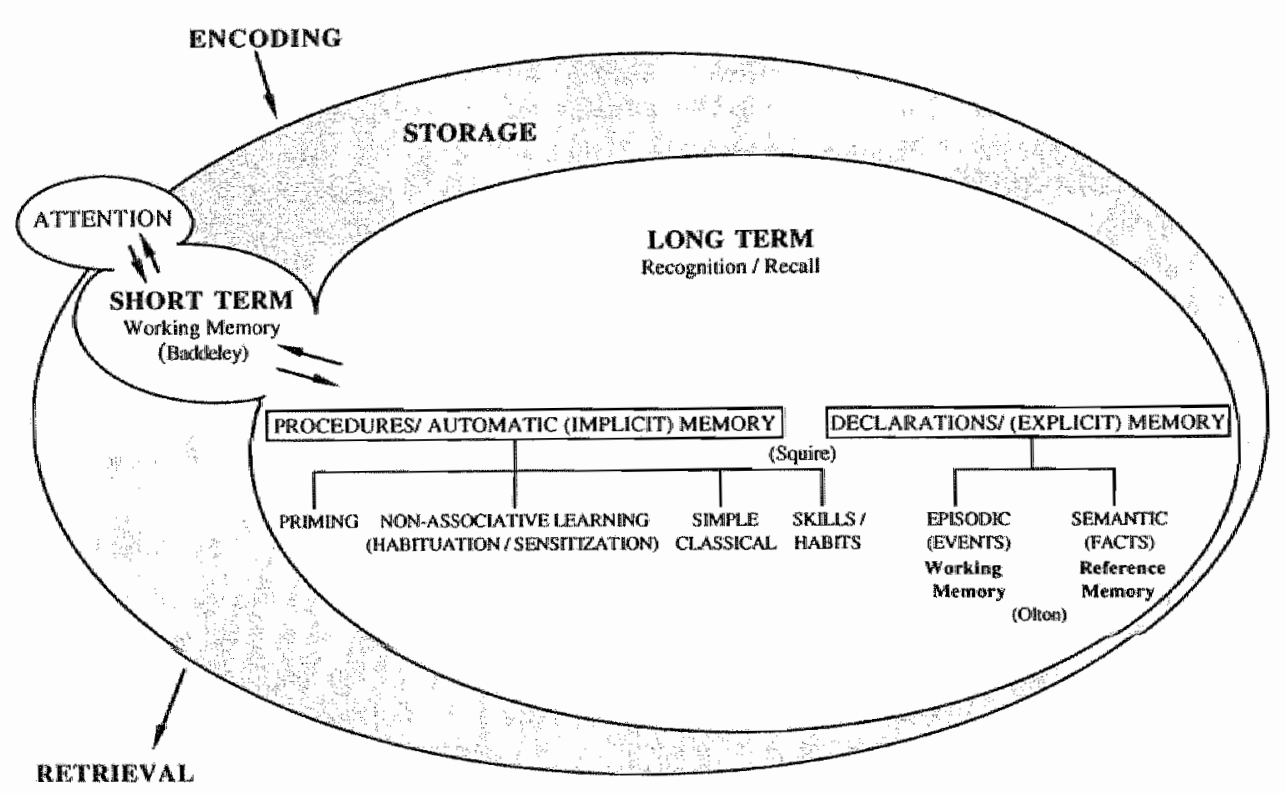

FIG. I. Schematic diagram showing the mnemonic processes thought to be involved in learning. Based upon concepts postulated by Baddeley $[21]$, Oton [251] and Squire [330] (adapted from D'Metlo and Steckler [71]).

\section{MEMORY TASKS}

There are many behavioral tasks used for measuring memory-related behavior (for a short summary see [94]). Of great importance for this thesis is the acquisition and retention of spatial information as measured in the Morris water escape task [2291. In the spatial version (hidden water escape platform at a fixed position) of this task, "place" learning and memory can be assessed, i.e. rats have to make a 'spatial' map by using cues/objects from the environment (allocentric representation), in onder to orientate themselves spatially and thus being able to learn the task. In addition, next to the use of this allocentric representation of the environment ("place" learning), 
rats may use exteroceptive 'cues' (visual/smel/tactile) which mark a certain point of orientation (cue" learning, e.g. a cue version of the Morris water sscape task with a visible platform at a variable position). Thirdly, rats can make use of an effective sequence of responses, such as turning left or right ('response" learning or egocentric orientation).

For this thesis also working memory is of relevance as measured in both a delayed non-matching to position (DNMTP) procedure in a Skimer box and an object recognition rask (ORT). However, it has to noted that both tasks differ wn the stimulus modality used: DNMTP or its counterpart, DMTP tasks assess spatial working memory, whereas the ORT assesses object or non-spatial memory 11031. The place version of the Morris water escape task can also be regarded as a task measuring spatial reference menory since a rat has to remember the (trial-independent) platform position over subsequent trials.

Besides that spatial information in the Morris water escape task and D(N)MTP tasks has to be memorized, also the use of procedurall learning and memory is necessary such as learning to search for an escape platform or to press a lever. However, this is not likely to be the case in one-trial learning tasks such as the ORT, which has, therefore, been suggested to assess purely working memory [103]. But whether the ORT measures working memory is questionable because the intertrial interval is rather long (greater than hours). Therefore, it is more appropriate to talk about the ORT as a task measuring object recognition memory only, but allowing to investigate consolidation and retrieval of information depending on the time of possible treatments [155]. This is also applicable to measuring memory performance in the passive avoidance task, which is also a one-trial learning task. Another one-trial learning task is the $\mathrm{T}$ (or $\mathrm{Y}$-)maze. This alternating task assesses specifically working memory. In a spatial configuration, spatial working menory is assessed as comparable with the D(N)MTP procedure in a Skinner box. In addition, object or non-spatial working memory can be assessed in the T-maze [4]. The latter is comparable with the delayed (non-)matching to sample (D(N)MTS) procedures in a Skinner box which also assess some sort of object working memory [139]. It has to be noted that the D(N)MTS procedure is one of the most used methods to measure memory performance in humans and monkeys. Taken together, delayed (non-)matching procedures in de skinner box as well as alternation procedures in the T-maze allow the assessment of memory, both spatial (position) and object (sample) memory.

When performing behavioral studies one has to keep in mind that memory performances may be influenced by non-mnemonic processes such as attention, motivation and emotion. These memory-independent processes can be detected in the delayed (non-)matching procedures as an overall effect on the rate of forgetting. Especially in aversive tasks using electrical shocks such as two-way active avoidance learning in the shuttle box and passive avoidance learning, which are both widely used, non-mnemonic factors like emotional/motivational processes maty interfere with memory performance. This greatly complicates the interpretation of the behavioral data in these tasks. Therefore, it is useful to use also noncognitive tasks such as the open-field test which measures anxiety-related behaviors. Moreover, the open field test offers the possibility to investigate whether there are treatment effects on locomotor activity, and to monitor whether these or other possible effects on anxiety influence the performance in memory tasks [ses $38.39,55.270$.

Finally, there are many more factors which influence test data significantly such as strain, gender, task, operational definitions, housing conditions, cliet, and biological rhythms [12.350], which is especially the case for the rather subtle effects in aging studies $\{24 \mid$. For instance, especially the strain of rats used is a decisive factor for the outcome of aging studies 137,327$]$, and because of learning effects aged rats are not well suited for aging studies with a longitudinal set-up $[28,31,351,365]$. 


\section{Brain structures, neurotransmitters and neuromodulators}

\section{BASAL FOREBRAIN AND ACETHYLCHOLINE}

\section{Cholinergic hypothesis}

During aging there is, in general, a decline in memory performance, as has been detected for spatial memory leg. 315 . and working memory [e. 86 . However, some subjects are more sensitive for in age-related decline in cognitive (memory-related processesy function than others. Concomitantly, it is possible to make an arbitrary subdivision of aged individuals based on their level of cognitive dysfunction. It has been suggested that the degree of age-related cognitive dysfunction of subjects may be associated with experiences of so-called health-related factors, such as organic solvents, head trauma or alcohol for example [144. 166]. In animals studies a comparable arbitrary subdivision of aged animals can be made, e.g. cognitive impaired versus non-impaired aged rats [e.g. 1, 34, 118, 262].

Alzheimer"s disease (AD) is a neurodegenerative disorder which is characterized with a very severe disnuption of cognitive functioning. Compared to age-matched controls, AD patients have an impaired spatial discrimination learming [e,g, 3]. Working memory as measured in the DMTS procedures is delay-independently affected in AD patients 225,2931 . This suggests that other non-mnemonic processes are involved, like for instance attention which has indeed been demonstrated 1621. Furthermore, the deterioration of working memory (as short-term memory or as episodic component of declarative memory) occurs at the early stages of $\mathrm{AD}$, while at a later stage the reference memory (as semantic component of declarative memory) deteriorates, whereas the procedural memory is still relatively preserved $[22,226,3291$.

In 1982 the cholinergic hypothesis was formulated by Bartus et al. 225] suggesting, based on a review of animal and human studies thus far, that there exists a relationship between the cholinergic dysfunction in the aged/demented brain and cognitive impairments [see also 78, 172, 2691, The degeneration of the basal forebrain cholinergic system is the most consistent and significant change in a specific neural system in the brain of patients with $A D$ [312]. According to the cholinergic hypothesis the extensive loss of cholinergic neurons within the medial septum, diagonal band of Broca, and the nueleus basalis of Meynert in AD patients may underlie some componens of the memory impairments associated with dementia. As a consequence, the metrotransmitter acelylcholine (ACh) and the cholinergic structures of the basal forebrain have primarily received (and still receive) interest in cognitive research. The basal forebrain cholinergic system includes the septum and diagonal band of Broca which both project to the hippocampal formation, and the nuclets basalis magnocellularis (NBM) which projects to the frontal and parietal cortex. The NBM in the rodent is thought to be homologous to the nucleus basalis of Meynert in humans. The amygdala is also associated with the basal forebrain and receives (though little) cholinergic input from the NBM [222, 269]. The basal forebrain (cholinergic) system is depicted in Figure 2 which illustrates the major connections between brain structures plus neturotransmitters/neuromodulators involved in memnonic processes.

\section{Hippocampal formation}

The hippocanpal formation is a component of the basal forebrain cholinergic system that in early years already attracted the attention of researchers interested in its role in cognitive function [245]. Based on the early findings some hypotheses were fornulated with regard to the hippocampus" function in learning and memory. It is of importance to have an idea about the functioning of the hippocampal formation, also with respect to the basal forebrain cholinergic system, in order to 


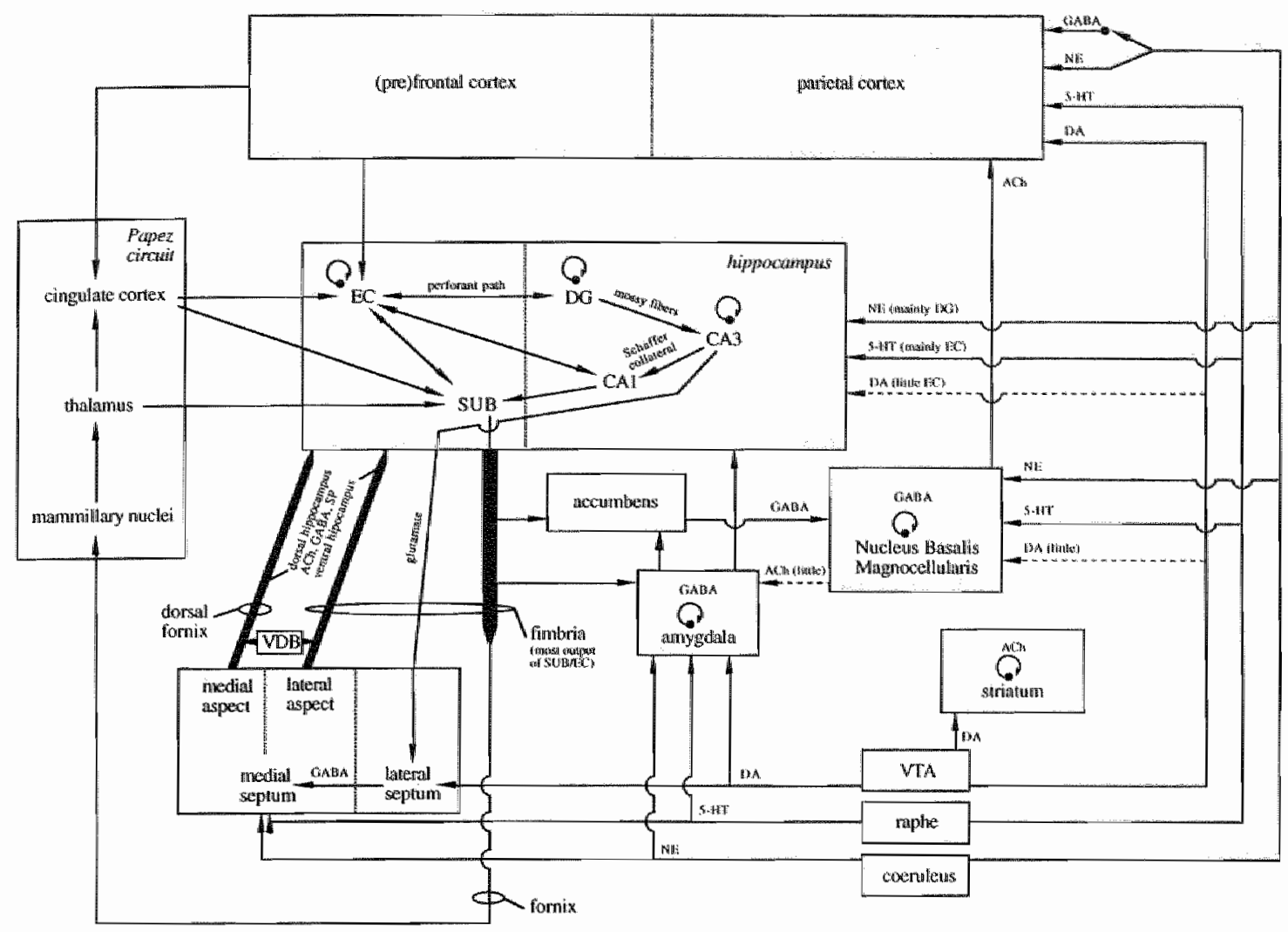

FIO. 2. Diagrara showing the major connections between hippocampus, nucleus basalis matgnocelularis, contex and amygdala plus neurotransmitters/neuromodulators involved in mnemonic processes. Based upon concepts from Decker [80], Gray [130], Jarrard [160], Jones [167] and Paxinos [265]. The gray areas represcent the basal forebrain system. Abbreviations: ACh, acetylcholine; DA, dojamine; DG, dentate gyrus; EC, entorhinal contex; GABA. $\gamma$ Arninobutyric acid: 5-HT, 5-hydroxytrypamine (serotonin); NE, norepinephrine; SP, substunce B; SUB, subiculum; VDB, wental diagonal band of Broca; VTA ventrat tegmentat area.

understand the results of cognitive studies and thus gain tnsight into learning and memory processes. Acconding to the various hypotheses the hippocampus is involved in: 1) the formation of a cognitive/spatial map [2451,2) working memory $250-2531,3$ ) a temporal memory buffer 12811 , or 4) declarative memory [229.330!

The cognitive/spatiall mapping theory proposes that the hippocampus is especially involved in an allocentric (spatial/place) memory system establishing a cognitive map of the enwiromment. An egocentric (response) memory system is ascribed to other parts of the brain. In both the working memory and temporary memory buffer theory the spatial or non-spatial nature of information being processed is not important, but the hippocampus is thought be involved in memory system that emphasizes the temporal/personal context that separates one instance from another. 
According to the working memory theory, the hippocampus is selectively concemed with holding information that is persistent only within a short period of time (working memory containing trial-dependent information), but not information that remains constant over time (reference memory containing trial-independent information). The predictions of the temporal memory buffer are similar to those of the working memory theory, except that for short-term working memory (i.e. with temporal gaps on the order of a few seconds), it should be possible for a limited-capacity, short-term memory buffer to support effective performance in the face of hippocampal damage. The declarative memory theory makes a distinction between a declarative (events and facts) memory system that is thought to be dependent on the hippocampus and a hippocampal-independent procedural (skills/habits) memory system. Most animal studies testing these various bypotheses support the cognitive/spatial map theory and the hypothesis has been extended in so far that the hippocampal formation plays an especially important role in processing and remembering spatial and contexnal information (for a rewiew see [160]). However, it should be noted that the hippocampus can still play a role in the other assumed functions, but not exclusively as was stated by the various hypotheses separately. For instance, the hippocampus plays a role in spatial working memory, but is also involved in spatial reference memory [353]. Currently, also other parts of the hippocampal formation besides the hippocampus itself, such as the entorhinal cortex. or subiculum, are receiving more and more interest with regard to their role(s) in memory runctions, of which the contribution has already been found to be of an essential nature $128,160,167,230,236,280]$.

As far as the acquisition and rettention of spatial information is concerned, it has been suggested in animal studies that the spatial map is stored mainly in the parietal cortex which is accomplished via the hippocampus $[85,174,219]$. Hence, the hippocampus is neccesary for the consolidation of spatial information. Accordingly, human studies reported that the hippocampus is involved in the acquisition of new factual knowledge, that is anterograde declarative memory (e.g. the acquisition of spatial information) [22].

As far as working (or episodic) and reference (or semantic) memory is concemed, it has been suggested that, according to the working memory theory, the hippocampal formation is the neural substrate that subserves working memory $185,171,174,203]$. The neocortex and in particular the parietal cortex, could represent the neural substrate associated with reference memory $[85.171,174]$. However, one might expect that both memory components cannot operate completely independently from each other. For instance, it can be suggested that without working memory it will be difficull to add new information to the reference memory. Accordingly, human studies have demonstrated that without episodic (working) memory, subjects have great difficulty adding new information 10 semantic (reference) memory [22]. Likewise, rodent studies showed that hippocampal lesions do not exclusively impair working memory, but also reference memory le.g. $161,171,35.31$. Therefore, it can be argued that there is no strict dissociation between these two memory components, while there is also ewidence that both memory components do not represent the same arpect of spatial menory [17!. 352!

Based on the above mentioned findings the role of the basal forebrain cholinergic system in spatial memory formation can be described as follows. The septohippocampal system would be mainly responsible for the formation of the spatial map and its storage in the parietal cortex (cf. working memory). The NBM/parietal cortex system would be mainly responsible for the retrieval of the information stored in the spatial cortex (cf. reference memory). It is noteworthy to mention that hnman studies demonstrated that also other portions of the temporal lobe (besides the thippocampus) are also involved in the retrieval of previously learned knowledge, that is retrograde declarative memory (e.g. the retention of spatial information) [22]. 
The spatial version of the Monts water escape tasks involves mainly reference nemory, that is the position of the water escape platform. In other spatial tasks stch as the cone field $[352.353)$ and the radial maze [161.252.253] both spatial working and spatial reference memory can be measured. Spatial leaming/memory can also be assessed in D(N)MTP procedures in the skimer box and alternation experiments in spatial T-mazes, which in both cases is likely to involve a working memory component mainly. It was found that damage to the (cholinergic) septohippocampal system impaired the spatial memory performance in both a T-maze $[210,248,255,28 \%]$ and a D(N)MTP lask $[7,93]$. Non-spatial or object working memory performance can be assessed in a non-spatial or object T-nnaze as well as a D(N)MTS task. It was found that the object memory as measured in the Twaze was not, or only mildy affected by septohippocampal (cholinergic) lesions $[146,233,248,289,316]$. The D(N)MTS task, which is mainly used to rest nonhuman primates, demonstrated that only after extensive damage to the septohippocampal system object memory performance was clamaged 81 . In addition, the ORT, which can be regarded as an object (working) memory task, rewealed no behavioral effects after septohippocampal damage [104. 210]. All these object memory related findings are in agreement with those of the cue (colo object) learning/memory experiments in Morris water escape tasks and radial maze tasks which did not demonstrate any effect, or only a small one when compared to effects on spatial learning/memory $[18,209,228,229.338]$. To summarize, object (recognition) memory is dependent on the integrity of the hippocampus. However, probably to a lesser extent when compared to the dependency of spatial memory on hippocampal (cholinergic) function.

In the following overview, the findings of spatial memory tasks, predoninantly the Morris water escape task and radial maze task, are discussed mainly. Furthermore, working memory is discussed predominantly by D(N)MTP findings, possibly complenented with findings of the spatial T-maze. Especially studies using rats are taken into consideration, but when none existed, studies with mice and/or monkeys are used. Only when no spatiall studies existed at all, then findings of the non-spatial I-maze, D(N)MTS task or ORT were discussed. It can ahready be stated that aging studies only found small, or even no effects of aging on the performances in these object-related tasks as compared with the findings on memory performance in the spatial counterparts of these tasks $15,57,93,208,3077$. These anging effects are in correspondence with the above mentioned findings concerning the (cholinergic) septohippocampal system, which is likely to be of more importance for spatial than for object memory. Finally, shock motivated tasks such as passive and active avoidance learning are discussed sporadically; only when no other data is awailable or when a very important finding has been done using these tisks.

\section{Acetylcholine}

With the cholinergic hypothesis in mind, still many animal, mainly rodent, studies are performed investigating the cholinergic basal forebrain system by measuring cholinergic markers such as acetylcholinesterase (AChE) activity and levels [221.224], choline acetyltransferase (ChAT) activity and levels 1224,321 , high-affinity choline uptake (HACU) 181, 110, 206.357, and acety/cholline (ACh) synthesis and release [223, 368]. Moreover, correlation of the behavional spatial data with the biochemicalanatomical data in many aging studies with rodents in combination with transplant experiments (e.g. fetal cholinergic septum tissue in hippocampus of aged subjects 160. 114, 115, 2391) still confirm the relationship between cholinergic basal forebrain dysfunction and cognitive function in aging/dementia (e.g. AChE [108], ChAT [91. 107, 152, 207), HACU 1120, 121, 1871, and ACh 1147). In addition, in D(N)MTP lasks it has been found that aging and treatment with cholinergic grafts had at delay-dependent, i.e. specific (spatial working) memory effect $193,95,961$.

Also a lot of rodent studies have been done in which the cholinergic basal forebrain was 
manipulated with physical, toxic or pharmacological (using anticholinergic agents) lesions of the septohippocampal system or NBM. With respect to these studies it is already noteworthy to mention that one should dissoctate $\mathrm{ACh}$ receptors in nicotinic and muscarinic receptors (see also below). Mostly, it was intended to develop a model of (cognitive) aging or AD. Hence, it was checked whether the spatial behavioral data could be correlated with the biochemical/netroanatomical data. In many cases indeed a relationship between a behavioral dysfunction and chollnergic basal forebrain dysfunction was demonstrated (e.g. muscarine blockade $[199,204,363$ and nicotine blockade [195, 199.). Likewise, in D(N)MTP tasks it was found that lesions of the septohippocampal system resulted in delay-dependent defects, that is (spatial) memory specific impairments 17.93,96, 981. Many of these putative models of AD have been used to test cognitive enhancing therapies, mostly based on cholinergic transmission. An example is Tacrine, which is an acetylcholinesterase inhibitor, of which the first results in human [186] and animal [109] studies were promising. But eventually it tumed out to be rather disappointing since Tacrine had only a modest effects in human DNMTS tests [272] and a delay-independent improvement, i.e. a nonspecific memory effect, in animal D(N)MTP tests [93. 295]. Both findings indicate that in a cognitive dysfunction and dementia there is more at hand than only a cholinergic dysfunction. Another putative treatment for memory disorders showed the same disappoining results ultimately (nicotine, see below) which together with an accumulating body of further evidence question the cholinergic hypothesis in general and the role of ACh in memory formation in particular.

Initially, many rodlent models of AD, which were based on the cholinergic hypothesis, used a MBM lesion or an administration (mostly peripherally) of scopolamine, a nonselective muscarinic antagonist (see 3201. To lesion the NBM (or septum) ibotenic (IBO) acid a $N$-methy] D -aspartate (NMDA) antagonist, has been widely used $[102,137,176,180,234,273,298,358]$. It was found that cholinergic markers (e.g. AChE and ChAT) were decreased in the projection areas of the cortex (or hippocampus) while at the same time the cognitive (spatial) performance was impaired, which is conform the cholinergic hypothesis. Surprisingly, both IBO and quisqualic (QUIS) acid (also a glutamate receptor antagonist, but as later turned out not of the NMDA receptor but of the amino-3-hydroxy-5-methyl-4-isoazolepropionate (AMPA) receptor) lesion of the NBM resulted in a decreased cortical ChAT metivity $[144,97.99 .334]$, whereas only $\amalg B O$ acid lesion resulted in cognitive (spatial) impairments $[97,3341$. In D(N)MTP tasks it was found that IBO acid had a delay-independent effect [931, and QU1S acid had no [951, or a mild delay-independent effect [294]. Moreover, it has been reported that cognitive function can recover after an IBO acid lesion of the NBM, while cortical ChAT activity is still low [14]. Furthermore, it has been found that ChAT activity can allso recover after such a lesion [360]. All these findings have also been reported for nonhuman primates tested in D(N)MTS tasks [see 359]. Therefore, it is apparent that NBM lesions do not provide a simple animal model of all the cognitive and cholinergic deficits in aging and AD fsee also $97,231,2321$, although this was initially suggested by the cholinergic hypothesis of dementia. Recently, it has been suggested that the NBM model is better a model of attentional deficits $231.359 \|$

Another animal model which still receives a considerable amount of interest as a possible model of $\mathrm{AD}$, is the scopolamine model in which scopolamine is administered to subjects which indeed seemed to disrupt the (spatial) cognitive function, according to the cholinergic hypothesis [0. 36. 1991, although some results pointed into the direction of a nonspecific drug effect [e.g. 326]. In addition, it was found that scopolamine administration (and peripherally with certainty) affects cognitive functioning of rodents nonspecifically since delay-independent effects were found in D(N)MTP tasks $[93,99]$ and other re]ated cognitive tasks $[13,181]$. Hence, deficits in attention may 
coexist with, or be responsible for the cognirive impaiments associated wh the scopolamine treatment Isee also 44,501 . Based on these findings the relevance of the scopolamine model for $\mathrm{AD}$ can be questioned [see also 27, 291].

Based on the above mentioned results with D(N)MTP tasks, whether or not involving scopolamine and basal forebrain excitatory amino acid (IBO and QUIS) lesions as models of the (cholinergic) cognitive deficit in $\mathrm{AD}$, it can be argued that the cholinergic system of the basal forebrain and especially the NBM, is involved in attention, rather than memory [35. 74.231. 232. 359], Thus, age-related changes in attentional processes could explain the changes in cognitive functioning [331]. Furthermore, enhancement of attentional functions, which can be seen as early-step information processes, represents a cognitively and clincally relevant goal for treatment of memory disorders $[35,302,30 \mathrm{z}]$.

\section{Nicotine and Muscarine}

To have a better understanding of the biochemical and behavioral effects of cholinergic interventions one should dissociate $\mathrm{ACh}$ receptors in nicotinic and muscarinic receptors. Human studies (nicotine [e.g.69] and musarine [e.6. 241]) as well as animal studies (nicotine [e.g. 310] and muscarine (e.g. 187. 205. 266. 2741) revealed that there is an age-related decline in the nicotinic and muscarinic receptor function, probably due to a decrease in its receptor numbers. Administration of nicotinic antagonists [195, 199, 246] or muscarinic antagonists [36, 47,61, 199, 204, 126, 361, 369.3] impaired (spatial) memory performance. However, the relevance of the results for memory when obtained after administration, especially systemically, of a nonselective muscarinic receptor (e.g. attopine or scopolamine) has been questioned [326, 361]. This can be further corroborated by the nonspecific memory data of the D(N)MTP tasks [93, 99], which led to the already' mentioned questioning of the relevance of the scopolamine model for memory disorders. The muscarinic receptors can be further divided in $M_{1}$ and $M_{2}$ receptors, which is an additional reason to use selective muscarinic antagonists (or agonists) instead of nonselective ones when studying muscarinic receptor function. In the cortex of the AD brain a major decrease in nicotinic receptors has been found $241,270,286$, 337). To a lesser degree there seems to be a decrease in $\mathrm{M}_{2}$ receptors (when affected), whereas the $\mathrm{M}_{\mathrm{1}}$ receptors seem to be preserved (or even increased) in number in the cortex of the $\mathrm{AD}$ brain [241, 270, 339, 362].

The nicotine receptor is associated with presynaptic cholinergic nerwe terminals and it has

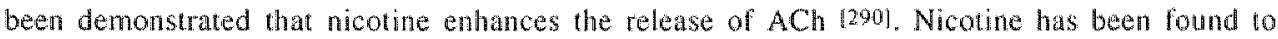
improve memory performance in humans 1356], nonhuman primates [3468, adult rats [194. 3231. septohippocampal lesioned rats 179, 196] and aged rats [17, 323]. However, also no, or nonspecific memory effect of nicotine have been found in DNMTP tasks with nonhuman primates 11454 and adult rats $[98.295]$. Correspondingly, nicotine has been shown to be effective in enhancing attention next to, or together with its cognitive enhancing function in nomal humans and AD [.356. Furthermore, it has been suggested that nicotine enhances cognitive performance due 10 its interactions, either directly or through $\mathrm{ACh}$ release, with the muscarinic receptor or release of norepinephrine, dopamine, serotonin and GABA 1346].

In the hippocampus of the rat the $\mathrm{M}_{1}$ receptor is when compared to the $\mathrm{M}_{2}$ receptor, the most abundant muscarinic receptor and is thought to be responsible for the postsynaptic action of ACh in this structure [328]. The majority of the thippocampal $\mathrm{M}_{2}$ receptors are localized presynaptically on cholinergic terminals and are thought to regulate $\mathrm{ACh}$ release by functioning as inhibitory autoreceptors 13281. These assumptions have been corroborated by a study which demonstrated that the (working) memory performance of rats was ameliorated by a $M_{1}$, receptor agonist as well as a $M_{2}$ receptor antagonist (after their local administration $(2471)$. In addition, $M_{2}$ receptor antagonists 
have recently been reported to facilitate spatial memory in aged impaired rats (after systemic administration $\left[39.2771\right.$ ). Moreover, it was found that the $\mathrm{M}_{2}$ receptors may be increased in the hippocampus of spatially impaired aged rats $\left[20.277\right.$. Hence, administration of selective $\mathrm{M}_{1}$ receptor agonists and $M_{2}$ receptor antagonists deserve consideration as a useful therapy for memory disorders, like for instance occurring in $\mathrm{AD}$ [ses also $19 \mathrm{l}$.

Finally, the role of the cholinergic deficit in cognitive disorders mist not be overemphasized since it has been found that, apart from a decrease in cholinergic neurotransmission, different monoaminergic neurotransmitter systems are changed in AD [e.g. 129, 138]. Furthermore, animal studies have demonstrated that next to ACh more neurotransmitters/neuromodulators are involved in (spatial) cognition (eg. 2181. Likewise, next to the basal forebrain cholinergic system more brain structures/systems are involved in cognitive function, and the interactions between these systems contribute to the cognitive deficits associated with aging and $\mathrm{AD}[0 . \mathrm{g} .80 .189]$ as will be further discussed below.

\section{GLUTAMATE}

\section{N-merhyl-D-asparrate receptors}

N-methyl-D-aspartate (NMDA) receptors have gained a lot of interest because of the assumption that activation of these receptors appears to be essential for the induction of hippocampal longterm potentiation (LTP), which is thought to be the physiological substrate for learning and memory [c.g. 33] (see section Synaptic plasticity). The role of the excitatory amino acid transmitter glutamate, which binds to the NMDA receptor, in learning and memory processes has been mainly investigated with competitive (e.g. D-2-amino-5-phosphonopentanoic acid (AP5)) and non-competitive antagonists (e.g. dizolcipine (MK-801)) of the NMDA receptor. In rodent studies with spatial aasks it was fround that administration of NMDA receptor antagonists impaired spatial learning and/or blocked hippocampal LTP induction in wivo $[2,73,227,287,313]$. However, the NMDA-receptor-dependent LTP as the mechanism of learning and memory has also been questioned $[173\}$. On the other hand it has recently been demonstrated that spatial learning induced an increase in hippocampal glutamate release ex vivo as did LTP induction in vitro [284]. Also, it has recently been demonstrated that in vivo LTP coincided well with the advance of spatial learning [1511.

It has been demonstrated that there is an age-related decrease in the glutamate receptor binding capacity of the nat contex and hippocampus 1188 . 324. In addition, this reduction in hippocampall ghtamatergic neurotransmission is likely to be related to the spatial learning deficit [188. 267], but see [238], To summarize the role of the glutamate receptor, reviewing the spatial Viterature, it can be argued that nomal functioning of the hippocampal glutamatergic system is necessary for place learning, that is encoding and storage of spatial information, but not for retention of place lesning, nor for cue leaning [218]. Additionally, it can be argued that the glutamate receptor function is imbolved in (spatial) working memory as measured in the DMTP task [3691. although it function in this respect is lather complicated because it has been found that a non-competitive NMDA anlagonist had a delay-independent impairment, while a competitive NMDA antagonist had a specific memory performance defect [03]. All these findings are in agrement with the previously mentioned findings for the hippocampal cholinergic system (see previous sections)

It has been found that next to NMDA receptors, AMPA receptors in the hippocampus are involved in memory performance of the spatial Morris water escape task 12001 and passive 
avoidance task [5]. More in detail, learning the spatial task involved hippocampal NMDA and AMPA receptors, while consolidation of spatial infomation (inmediately after each daily learning session) involves NMDA receptors and retrieving such information (just prior to the final resting trial) involves AMPA receptors [200]. The latter is in line with findings in passiwe avoidance tasks suggesting that NMDA receptor blockade hinders performance due to consolidation inhibition. whereas AMPA receptor blockade hinders performance due to retrieval intibition [463]. Whether these findings have implications for the previous findings with $I B O$ and QUIS is interesting, i.e. do the behavioral effects of IBO lesions correspond with AMPA receptor blockade and QUIS lesions with NMDA blockade? However, it should be noted that the effects of $1 B O$ and QUIS lesions, which have mainly been investigated with respect to the NBM, are rather large and crude, and thus nonspecific. Nevertheless, some parallels can be drawn since QUTS lesions compared to IBO, caused milder, on in general no impairments in several behavioral tests [0.g. 93, 97]. In addition, the largest QUIS-induced imparment, that is when an impairment was found, seems to be especially related to retrieval of information as found in the passive avoidance task [97].

The assumption that NMDA receptors, through their involvement in thippocampal LTP, may be required for spatial and some other forms of learning, is also referred to as the NMDA-LTP-memory hypothesis [cf. 48]. However, recently this hypothesis is more and more questioned. For instance, it has been reported that NMDA antagonists did not prevent place learning of rats if they were first non-spatially pretrained, which led to the suggestion that place learning is independent of NMDA transmission, i.e. NMDA receptors may not be required for encoding of the spatial map [23,305]. Furthermore, in contrast with earlier studies, NMDA antagonists affected cue learning which led to the suggestion that impaired place learning of naive rats trained under NMDA antagonism might be due to drug-induced sensorimotor disturbances (e.g. hyperactivity) $[49,3061$. In addition, it was found that although place learning could still be normal, hippocampal LTP in vivo was blocked under NMDA antagonism [23, 48, 30.5]. Thus, the NMDA-LTP relationship remains intact $\llbracket c \hat{f} .65]$, although it is questioned whether it is involved in memory formation [48. 177].

\section{Giutanatergic hyporhesis}

In addition to the cholinergic hypothesis of $A D$, an glutamatergic hypothesis of $A D$ has been formulated (212). It was suggested that many of the cognitive deficits of $\mathrm{AD}$ are related to a glutamatergic dysfunction, i.e. dementia is caused by the loss of ghtamatergic receptors from the neocortex and hippocampus. Several animals model have been developed based on this hypothesis by denervation of glutamatergic transmission [111, 23\%]. However, the glutamatergic hypothesis has, just as the cholinergic hypothesis, shortcomings. A major aspect in this respect is that a glutamatergic overactivity is neurotoxic (the so*called excitotoxic cell death $[26,348]$ ). This has implications for possible therapies with glutamatergic agonists. Although glutamatergic agonists (e.g. NMDA, cycloserine) facilitate and improve cognitive behavior and induce LTP in rodent models (for a review of animal models and drugs related to the glutamatergic hypothesis see (111), their potentially neurotoxic actions must also be taken into account which therefore limits its usefulness for treating the cognitive complaints of $\mathrm{AD}$ patients.

\section{VENTRAL TEGMENTAL AREA AND DOPAMINE}

\section{Parkinson's disease}

The neuromodulator dopamine (DA) is involved in another neurodegenerative disorder, viz. 
Parkinson's disease (PD). PD is anatomically characterized by a loss of striatal DA which is caused mainly by degeneration of dopaminergic neurons in both substantia nigra pars compacta (A9 cell group) and ventral tegmental area (A10 cell group). PD is behaviorally characterized by locomotor impairments (bradykinesia in combination with rigidity, tremor, and postural imbalance) which have been related to the DA depletion in the striatum [142]. Further, cognitive dysfunctions, which appears to be selectively for procedural learning and not for declarative memory 12963 , can be observed in about $25 \%$ of $\mathrm{PD}$ patients $[263,292.345$. A lendency to persevere and difficulties in shifting conceptual sets can be observed in PD patients [190], and an impaired attention in PD patients has also been found [62]. Hence, it has been argued that a frontal dysfunction underlies the cognitive defucits in PD patients $[72,292,345]$. Finally, the occurrence of depression can be observed in PD patients and recently it has been suggested that the raphe nucleus is, consequently, involved in the pathogenesis of PD [336],

The striatum can be subdivided into the dorsal striatum or caudate nucleus-putamen and the ventral striatum or nucleus accumbens, and is a part of the basal ganglia. The basal ganglia consist further of the pallidum (external and internal segment of the globus pallidus and ventral pallidum), the subthalamic nucleus, and the substantia nigra (pars compacta and pars reticulata). The caudate-putamen (CPu) is the input structure of the basall ganglia receiving glutamatergic and dopaminergic input. The output structure of the basal ganglia consists of the internal segment of the globus pallidus (GPi) and the substantia nigra pars reticulata (SNr) which project to thalamic nuclei controlling motor outpul. The input (CPU) and output (GPi/SNr) structures of the basal ganglia are connected with each other by means of two pathways, i.e. a "direct' and an "indirect" pathway. The direct pathway consists of the GABA/substance P projections from the CPu to the GPi/SNr. The indirect pathway is respectively constituted by GABA/enkephalin projections from the CPu to the externat segment of the globus pallidus (GPe), which in turn has GABAergic projections to the subthalamic nucleus (STN). The STN provides an excitatory glutamatergic input to the SNr/GPi. The output neurons of the SNr and GPi use GABA to inhibit thalamic neurons projecting to the cortex. A 'balance' in the two striatal pathways seems to be essential for the normal regulation of movement. DA acting via $D_{1}$ receptors would have a stimulating effect on the direct pathway, whereas via $D_{2}$ receptors $D A$ is thought to have an inhibitory effect on the indirect pathway.

The consequence of the loss of DA in PD is thought to be an increase in the inhibitory output from the GPi/SNr [82]. Firstly, because the dopaminergic inhibition of the indirect pathway is reduced and hence the glutamatergic excitation predominates in the CPu. In turn the glutamatergic neutons of the STN becomes desinhibited, which leads to an increased stimulation of the GABAergic neurons in the GPiSNr, and thus to an increased inhibition of the neurons in the thalamus controlling the motor output of the cortex. Secondly, the dopaminergic stimulation of the direct pathway is reduced and in turn the GABAergic neurons of the GPi/SNr become desinhibited. thus also resulting in an increased inhibition of thalamic neurons. Ultimately, supposedly at a loss of about $75 \%$ of striatal DA, the inhibition of cortical activity accounts for (most of) the motor impaiments in PD.

The cause of the degeneration of dopaminergic neurons in the substantia nigra pars compacta (SNC) and the accompanying loss of striatal DA is still unknown. Recently it is thought that degeneration of serotonergic neurons in the dorsal raphe nucleus, which are known to project to the CPu, might contribute to the reductions of striatal DA levels and thus explain the occurring of depression as well 1336]. In general, PD patients are treated pharmacologically, by supplement and or substiution of $\mathrm{DA}$ with the $\mathrm{DA}$ precursor and $\mathrm{D}_{1} / \mathrm{D}_{2}$ agonist L-DOPA or with $\mathrm{D}_{2}$ receptor agonists [366]. However, the clinical efficacy of $D_{1}$ receptor stimulation is still under debate [355], 
which is probably due to the dellicate ballance between the direct and indirect pathways. Recently, even a possible therapeutic value of a $D_{1}$ antagonist is being exaluated [15]. Because the glutanatergic system is thought to interact with the dopaminergic system -with the CPu receiwing glutamatergic input from the cortex as a principal site, but also in the GPi/SNr region-to control the motor output from the basal ganglia, it has been hypothesized that an overactivity of the glutamatergic system of the basal ganglia underlies the pathophysiology of PD [309.331]. Hence, it has been proposed that glutamate antagonists may be useful adjuvants 10 L-DOPA in antiparkinson therapy, as had been demonstrated in rodent models of PD (DA-depleted innimals) $[182,333]$.

Finally, it is noteworthy to mention that a 6-hydroxydopamine (6-OHDA) lesion of the SNC. which provides the dopaminergic input to the direct and indirect psthways $\left(D_{1}\right.$ and $D_{2}$, respectively?[309,332]) starting in the $\mathrm{CPu}$, is regarded as one of the most appropriate rat and monkey models of PD [32]. In addition, an excitotoxic lesion (IBO [32] as well as QUIS [1131) of the $\mathrm{CPu}$, mainly the putamen, may be regarded as a model of Huntington's disease.

\section{Dopamine}

Reviewing de spatial literature with 6-OHDA lesions of the striatal system and peripheral administration of DA agonists (e.g. apomorphine) or antagonists (e.g. haloperidol), indicates that all these interventions impair place leaming [192,218], but also place retention and cue learning [218]. The latter two findings indicate that alterations of DA activity produce sensorimotor or motivational impairments. Despite these findings suggesting that DA is not by itself an important modulator of learning and memory, but may be more related to ancillary processes, it has been demonstrated in aging studies with rodents that there is a DA hypofunction in the basal forebrain and striatum which may be related to the age-induced cognitive deficits 180.2181 . However, an interference of the cholinergic system cannot be ruled out since there is also a cholinergic hypofunction in the basal forebrain and striatum of aged rats [43, 108, 110, 152, 207, 310].

It has already been established that $\mathrm{ACh}$ and DA may play complementary roles in motor function since the hypokinesia of $\mathrm{PD}$ can also be attenuated with treatment $\mathrm{ACh}$ antagonists next to the DA agonists [68]. The striatum appeared to be the morphological substrate for the complementary roles of these two transmitter systems in motor function 146]. Recently, it has been demonstrated more in detail how the cholinergic neurons play a central role in the control of striatal activity via muscarinic receptors, viz. $M_{1}$ receptors might facilitate striatal DA release and $\mathrm{M}_{2}$ receptors might inhibit striatal DA release [322]. At the moment it has become clear that, like motor behavior, cognitive function may be influenced by the balance between $A C h$ and $D A$, as demonstrated in several, mainly spatial learning experiments with lesions and/or peripheral administration of drugs $[193,197,198,216,217,335]$. Thus, DA seems to be involved in memory processes, though in relationship with $\mathrm{ACh}$. But it was demonstrated that it is a very complex and somehow antagonistic interaction between DA and $\mathrm{ACh}$ in which the muscarinic and nicotinic interactions with DA appear to involve different subpopulations of dopaminergic receptors. The $D_{\|}$receptor system has interactive effects with muscarinic blockade effects, though surprisingly both a $D_{1}$ agonist and $D_{1}$ antagonist reverse the cognitive deficit from muscarinic receptor blockade. In addition, a $D_{2}$ agonist appears to have an ameliorating effect on the cognitive impairments after nicotinic blockade. The interactions of the $D_{1}$ and $D_{2}$ system with cholinergic mechanisms may be important for the development of treatments of $A D$ (e.g. administration of a $\mathrm{D}_{1}$ or $\mathrm{D}_{2}$ agonist).

Compared to the serotonergic raphe nucleus and noradrenergic locus coeruleus, the dopaminergic ventral tegmental area (VTA) has only few projections to the cholinergic NBM and 
hippocampus 1265 (see Fig. 2). However, a likely site for dopaminergic/cholinergic interactions important for cognitive functioning may be the lateral septal area in which GABAergic neurons may mediate the inhibition of hippocampal cholinergic activity by dopaminergic neurons from the VTA since it has been demonstrated that VTA or lateral septum lesions increased hippocampal cholinergic activily $[116,3701$ and cognitive performance $[101,16,117,340]$ (see Fig. 2). Based on these finding it can be assumed that positive action of a $\mathrm{D}_{1}$ antagonist on cognition might be exerted wia the latleral septum. Another candidate for the site of memory processes may be the striatum itself because striatal cholinergic manipulations are known to influence cognitive performances 130 . 84, 325]. In addition, the striatum may be the site for the dopaminergic/cholinergic interaction in cognition because the $A C h$ level increases in the striatum after systemic administration of a $D_{1}$ agonist [335. Another indication of striatal involvement is the positive effect on cognition after striatal $\mathrm{D}_{1}$ or $\mathrm{D}_{2}$ agonist administration $[260]$. However, this was only with respect to striatumdependent aspects of the cognitive task used, i.e. che learning (see below). In addition, it was found that hippocampal administration of a $D_{\|}$or $D_{2}$ agonist has a positive effect on cognitive performance also, though again only on the hippocampus-dependent aspects of the task, i.e. place learning $[260)$ (see below). Nevertheless, these findings may be of relevance for a memory disorder therapy based on DA, as has already been mentioned above. Finally, it has recently been found that next to the dopaminergic/cholinergic interaction, the inhibitory neurotransmitter GABA in the striatum appears to be of significance for cognitive functions $158 \% 29 \%$.

The different parts of the dorsal striatum or CPuin are related to distinct functions. Thus, whereas the dorsolateral $\mathrm{CPu}$ (non-limbic striatum) controls sensorimotor function, the ventromedial part (limbic striatum) is implicated with affective (emotion and attention) behavior [125]. In PD, it is mainly the dorsal part of the CPu which is affected [131]. How the dorsal striatum affects the cognitive performance in PD patients is not clear yet, but it is likely that the memory imparments may be attributed to the caudate nucleus but not the putamen nucleus [141]. Further it might be possible that its limbic part affects learning and memory via enotional, motivational or attentional processes. Another possibillity might be that the dopaminergic/cholinergic relationship in the non-limbic striatum is disturbed, but in how far this would influence cognition is not completely clear yet (see above), let alone in $\mathrm{PD}$. Some have argued that the cognitive dysfunction in PD patients may be due to a fromtal cortex dysfunction of which the cause is unknown 1263.292. 3451. To have an indication of the role of the dorsal striatum in cognition, the effects of lesions (electrolytic or excitotoxic) of the CPu, nainly the caudate nucleus, have been investigated in several spatial tasks. It was found that the caudate nucleus was not involved in place learning 166. $177.213,249,257-259 \mid$, while it appeared necessary for cue learning $183,213,257,258$ and response (egocentric) hearning $160.477,25 \%$. The behavioral effects of lesions of the CPu have also been inwestigated in DNMTP tasks and because of the nonspecific memory defects after excitotoxic lesions, it may be argued that the CPu has probably attentionall functions as well [87. 92).

The findings about an inwolvement in response learming and attentional processes of the $\mathrm{CPu}$ striatum in rats resemble the findings with regard to the medial prefrontal cortex in rats (see section Prefrontal cortex). Concomitantly, the medial prefrontal cortex has projections to the dorsal striatum, especially to the medial portion of the caudate nucleus [349]. Compared with the hippocampus there is a functional dissociation between the dorsal striatum, especially its (dorso)lateral portion, and the dorsal part of the hippocampus for piace learning on one hand (dorsall hippocampus), and response and cue learning on the other hand (dorsal striatum) [213, 257. 258. 2601. With respect to response learning it has recently been suggested that striatal involvement is mediated by a dorsal striatum subregion other than the one involved in cue learning [249]. Future studies using local administration of DA antagonist including also other structures next to the 
striatum, e.g. hippocampus, prefrontal cortex or nucleus accumbens, will give more insight into the putative cognitive functions of the striatum [e.g. 29 .

The nucleus accumbens or ventral striatum is also involved in cogntive function as was demonstrated by (excitotoxic, 6-OHDA or electrolytic) lesion experiments. For instance, lesions of the nucleus accumbens resulted in delay-dependent impaiments in DNMTP tasks [9z.232\}. Further, lesions of the nucleus accumbens affected memory formation as was found in spatial tasks mainly [308, 311, 338, 341]. Cognitive function is thought to be influenced by the accumbens through its output via the ventral pallidum (and then eventually wia the mucleus medialis dorsalis) to areas of the medial prefrontal cortex necessary for cognitive function [288]. The specific and spatial memory deficits after accumbens lesions are in correspondence with effects of hippocampal lesions. This may indicate an influence of the dorsal part of the hippocampus on accumbens-related cognitive processes, although direct morphological evidence is lacking. Further, both the amygdala and medial prefrontal cortex project to the accumbens and with respect to the latter's cognitive processes only the anygdala may be assumed to be involved also Iff. 4.5I.

The accumbens has been found to be also involved in locomotor bethavior for which there seems to be a functional relationship between the ventral part of the hippocampus and the accumbens which can be atributed to a direct connection from the hippocampus to the accumbens (or indirectly from the VTA). The medial prefrontal cortex is probably not involved in a locomotor effect although it projects also to the accumbens and receives input from the ventral hippocampus [45, 206]. The amygdala, which projects also to the accumbens, appears neither to be involved in locomotor behavior [45]. Finally, the accumbens has been found to play a role in emotional reactivity as well. This bas to do with its connections with the anygdalin and hippocampus, which are both part of the limbic system (see Fig. 2 and section Limbic system). The nucleus accumbens is, therefore, even considered to be an interface between limbic and striatal sensorimotor structures/functions [see 341].

To summarize, the dorsal striatum (CPu) receives input from different areas of the cerebral corlex (including prefrontal, parietal and temporal association areas as well as sensorimotor cortex) and allows these, via projections to the globus pallidus and substantia nigra, and then to the thalamis to influence (pre)motor cortex [288]. With regard to cognition it may be suggested that at sort of a 'motor' plan is stored in the striatum, and to a certain evolving sequence of input information the appropriate motor output pattern would be looked-up. The ventral striatun (accumbens) projects via the ventral pallidum to the medial prefrontal cortex. Thus, the prefrontal cortex which is involved in cognitive functions can be provided with information about which response has been selected or has just been performed (28B). While the striatum may play a role only in specific forms of (motor) memory, it is likely that DA in relationship with other neurotransmitters systems infuences cognirive functioning in general. However, which interactions between DA and other neurotransmitters exist between the VTA, striatum, hippocampus and prefrontal cortex, and its consequences for cognition and locomotor behavior still needs further clarification.

\section{RAPHE NUCLEI AND SEROTONIN}

The raphe nucleus has serotonin (5-hydroxyryptamine; 5-HT) projections to the hippocampal formation (preferentially from the medial raphe mucleus to the entorhinal cortex) and NBM (4l. 265] (see Fig. 2). Dysfunction of this serotonergic system has been found in aging and in $\mathrm{AD}$. For 
instance in $A D$ brain the number of $5-\mathrm{HT}_{2}$ receptor decreases while the number of $5-\mathrm{HT}_{1}$ receptors remains relatively constant $\{\epsilon$. 215\}. Whether the serotonergic projections are involved in cognition is not completely clear and further research is necessary, moreover to have prospects for development of a serotonergic therapy for $A D$ 19. Alas, a first impression was rather not promising as it was found that aged rats that were impaired in place learning or spatial working memory tasks showed little or no change in 5-HT levels of several brain structures, although 5. HT"'s metabolites showed some changes [e.g. 18\%. 344]. Likewise, 5-HT depletions due to 5,7-dihydroxytryptamine (5,7-DHT) infusion, either i.c.4. 1240) or into the dorsal raphe [285], did not impan place learning or had only a modest effect on spatial working memory [358]. However, these 5-HT lesions combined with medial septum [240] or NBM [285] lesions producied marked deficits in place learning when compared with the basal forebrain lesions alone. Hence, despite the relatively modest effects of 5-HT depletion on learning and memory, interactions between 5-HT and $\mathrm{ACh}$ may well play an important role in cognitive function.

However, in a DNMTP task it was found that a 5,7-DHT lesion of the raphe (both medial and dorsal) did not affect performance as might be expected, while it did not worsen the memory, though nonspecific, defect of a NBM lesion [2941. Likewise, in more recent spatial studies it has been found that selectiwe deafferentation of hippocampal 5-HT terminals by infusion of 5,7-DHT into the fimbria/fornix had no effect by itself on spatial task performance (but see [10]), while again it did not substantially contribute to the disruptive effects of lesioning the medial septum [235]. Also, othen contrasting results have been obtained with administration of 5-HT ligands into the hippocampus, which showed that 5-HT itself was yet involved in place learning [52], and into the periphery, which showed that 5-HT and ACh may play complementary roles in passive avoidance learning [11.242]. Possible explanations are that the type of 5-HT receptor should be taken into consideration (there are indications that especially the postsynaptic 5-HT IA $_{\text {receptor }}$ may be of relevance $[53,54,220]$ ), and whether nicotine or muscarinic receptors play a role in possible serotonergic interactions. To summarize, not all memory impairments induced by concurrent manipulations of the serotonergic and cholinergic system can be attributed to a serotonergic modification of the cholinergic system, while also influences on memory via other general functions (e.g. attention, anxiety) should not be neglected [56].

Interventions that reduce 5-HT function such as administration of 5,7-DHT or 8hydroxy-2-(di-n-propylamino) tetralin (8-OH-DPAT: a 5-HT 1 A receptor agonist) have been demonstrated to produce in general anxiolytic (i.e. 'anti-conflict') effects [324. 3471. This is consistent with the hypothesis that 5 - HT, which level may increase as a result of 'stress' [367], is anxiogenic and a reduced 5-HT function leads to a reduced anxiecy [153]. At the moment benzodiazepines, GABA receptor facilitation and 5-HT $\mathrm{H}_{\mathrm{A}}$ agonist are widely used as anxiolytics $[243,268]$. It has been suggested that benzodiazepines (e.g. Diazeparn) as a result of facilitating GABA receptors, exert some of their anxiolytic effects indirectly through an inhibition of 5-HT neurotransmission [324, 3671. In addition, based on recent findings it should not be ignored that the cholinergic, dopaminergic and NMDA system are probably also involved in anxiety, although its mechanisms of action or its interaction with the serotonergic system are not completely clear [106]. For instance, it has been found that muscarine antagonists are anxiogenic, a $D_{2}$ agonist is anxiolytic and NMDA antagonists are anxiolytic. Anxiety can interfere or influence cognition, i.e. anxiety reductions produce an impairment in cognition as formulated in the so-called reduced motivation hypothesis. Thus, anxiolytic effects of a reduced 5-HT function due to 8-OH-DPAT might explain the affected performances in place learning [52] and passive avoidance learning [220]. It has been suggested though, that the impaired place learning in a Morris water escape task, which is also an aversive task, was not influenced by an anxiolytic effect of a reduced 5-HT function [53. 
541. This is in agreement with the assumption that the anxiolytic effects of benzodiazepines, GABA and NMDA antagonists do not influence place learning or even learning in general (see section $G A B A)$. But in passive avoidance learning, which is aversive because of the electric shocks, the anxiolytic effect of the reduced 5-HT function probably interfered with memory processes [55],

Besides that the raphe nucleus projects to the hippocampus, it also projects to another limbic structure, viz. the anygdala l265] (see Fig. 2). At the moment it is thought that the anxiolytic action of the 5-HT 1 a agonists (like 8-OH-DPAT) arise primarily from their actions on the presynaptic inhibitory $5-\mathrm{HT}_{1 \mathrm{~A}}$ autoreceptors in the dorsal raphe leading to a reduced 5 -HT release in the amygdala 1106, 132]. The memory deficits of 5.HT $1 \mathrm{~A}$ agonists are thought to be a resull of their action on postsynaptic 5-HT , receptors in the hippocampus $[5.54 .54$. It is interesting to note that there is evidence that the postsynaptic 5-HT ${ }_{1 \mathrm{~A}}$ receptors in the hippocampus which receive $5-\mathrm{HT}$ projections from the medial raphe, are also associated with an antidepressant action [106. 12:]. This corroborates the use of $5-\mathrm{HT}_{1 \mathrm{~A}}$ agonists as antidepressant next to its use as anxiolytics [243].

Recently, the dorsal raphe has been connected with PD [see 336]. The cause of PD is still not known but it is characterized by a decrease in striatal DA levels and a degeneration of DA neurons in the SN, which leads to motor disturbances. Serotonergic efferents of the dorsal raphe are known to innervate both the striatum and $\mathrm{SN}$ and may therefore have a modulatory influence on the nigrostriatal dopaminergic pattway. 5-HT levels decrease in PD and thus remowal of the 5-HT input might result in a reduction of striatal DA levels and/or loss of inhibition by dopaminergic $\mathrm{SN}$ neurons. Hence, it has been hypothesized that degeneration of the raphe serotonergic neurons could be one of the major events in the onset of $\mathrm{PD}$. Hence, it is interesting to study whether the occurrence of depression which is often observed in $\mathrm{PD}$ patients, can be related to this hypothesis.

\section{LOCUS COERULEUS AND NOREPINEPHRINE}

The dorsal noradrenergic bundle is the noradrenergic pathway from the locus coeruleus to the septum, hippocampus (mainly dentate gyrus) and cortex [265] (see Fig. 2). The locus coeruleus and other neuronal systems containing norepinephrine (NE) are danaged in $A D$ [27l]. In addition, $\alpha_{2}$-receptors in the projection areas of the locus coeruleus seem to be decreased in AD brain [170]. Furthermore, abnomalities of brain NE function are implicated in age-related memory loss in both humans $[214]$ and nonhuman primates $[19]$. However, aged rats showing spatial learning deficits actually had no 1399 or even increased $\mathrm{NE}$ levels in the brain 1119$]$. In thdition, manipulations of the noradrenergic system by causing lesions ( $6-\mathrm{OHDA}$ or $N-(2-$ chloroethyl)-N-ethyl-2-bromobenzylamine (DSP-4)), or treatment with B-adrenergic antagonists (e.g. propanolol) had no, or only little effect on spatial learning and working memory [80. 135. 218.319 .358 . This suggests that NE itself is not necessary for cognitive function per se (cf. 5-HT). But there is evidence that NE and ACh interact in influencing cognitive processes. Synergistic interactions between NE manipulations (e,g. locus coeruleus lesion or B-antagonist propanolol) and cholinergic manipulations (e.g. NBM or septum lesion) have been reported for spatial learning and working memory, though sometimes an interaction is best described as only additive, or even no interaction at all has been found 180$]$.

Interestingly, it has been found that administration of an $\alpha_{2}$-antagonist (indaxoly improved spatial retention, probably dene to its action on the noradrenergic imhibitory autoreceptor [300]. At the same time, administration of another $\alpha_{2}$-antagonist (phentolamine) ameliorated the effects of a septohippocampal lesion on spatial learning [1351. Especially the latter finding is in contrast with at proposed synergistic interaction between the cholinergic and noradrenergic system. Thus, it has 
been suggested that a cholnergic dysfunction can be allewiated with a reduced noradrenergic function $30 \mathrm{n}$. Biochemical and electrophysiological findings have already indicated that NE is indeed able to exert an inhibitory action on cortical neurons both directly via ca-adrenergic receptors, and indirectly via NE modulation of GABA. At hippocampal level this precise mechanism of action of the interaction wan $\mathrm{NE}$ met $\mathrm{ACh}$ is not fully clear, but for the cholinergic system a dissociation has to be made in this respect between $M_{1}$ and $M_{2}$ receptors [see 80 . In addition, a dissociation between $\alpha$ and 8 receptors has to be made since a 3 -adrenergic antagonist (propanolol) did not attenuate, like an $\alpha_{2}$-antagonist, but did potentiate the effects of a septohippocampal lesion on spatial learning [135].

Therefore, until now there is no detailed understanding of the extent and importance of chotinergic/noradrenergic interactions in leaming and memory. However, this understanding is essential if one wants to apply adrenergic intervention in cognitive deficits. An example in this context is that an $\alpha_{2}$ wagonist (clonidine) improved cognitive performance of both aged nonhuman primates [19] and rats with combined cholinergichoradrenergic lesions [134]. However, treatment of AD patients with another $\alpha_{2}$-agonist (guanfacine) did not improve the impaired cognitive performance [701. This finding further underlines that treatment of memory deficits requires intervention of more than one neurotransmitter/nelaromodulator.

\section{GABA}

\% Aminobutyric acid (GABA) is the major inhibitory meurotransmitter in the mammalian central nervous system. Pharmacological manipulations with GABA agonists have revealed that GABA is negatively involved in cognition $180,156,159,218]$. The G.ABA receptor is positively modulated by benzodiazepines which have also its own receptors in the mammalian central nerwous system and are typically associated with anxiery reduction [243]. It is generally known that benzodiazepines have amnestic effects which may be due to the activation of its receptors, although it can also be assumed that the amnestic effects of benzodiazepines can be attributed to GABAergic influences $180,157,218,243]$. Interestingly. NMDA receptors antagonists, which impair cognition, have also anxiolytic effects similar to those of benzodiazepines $[67.105,275,3147$. At the same time the effects of NMDA antagonists, GABA agonists and benzodiazepines on cognition appear to be similar since, for instance, all these drugs impair place learning, but mot place retention and che learning 12181. Hence, there may be a relationship between the anxiety state of a rat and cognition as suggested by the motivation reduction hypothesis. However, present evidence suggests that the anxiolytic properties of benzodiazepines, GABA agonists and NMDA antagonists are not sufficient to affect learning and memory in general $[105.218]$. Though more specifically the latter is in contrast with the previously mentioned anxiolytic influences of serotonergic 5-HT $1 \mathrm{~A}$ receptor agonists on memory performance in shock motivated tasks [55].

GABA input to both the NBM (mainlly from the nucleus accumbens) and the medial septum (mainly from the lateral septum; see Fig. 2) may play an important role in the regulation of cholinergic neurons in the basal forebrain and may, thus, mediate some of the previously mentioned $N E / A C h$ and DA/ACh interactions. Although the effects of GABA agonists and benzodiazepines on memory are not completely compatible to the cholinergic drugs effects on memory, there is considerable evidence to state that the interactions with ACh underlie some of GABA's action on memory, which was for instance already apparent from age-related spatial leaming impaiments 11.801. It has been suggested that during spatial learning, glutamatergic lateral septum synapses participatte, presumably through the septall GABAergic interneurans, in the 
(inhibitory) control of cholinergic septohippocampal neurons [122. 162] (see Fig. 2). Whether such a control system in the NBM exits, has still not been directly ellucidated, though GABAergic manipulations of the NBM also affect performances in tasks which are sensitive to cholinergic manipulations [see 80]. Because the interactions of GABA and other neurotransmitter/neuronodulator systems are presumably so complex, future researeh assessing the effects of GABA and benzodiazepines on learning and memory, should check for other features like dissociating between the two subiypes of each receptor, respectively GABA and $G A B A_{B}$, and $\omega_{1}$ and $\omega_{2}$. Likewise, the interactions of GABA and benzodiazepines with $A C h$, especially in the septum (and NBM) need further study in order to gain more insight in the role of the GABAergic system in cognition. Hopefully, the results of future research into the role of GABA in cognition can make an attribution to useful treatments, which may be based on GABAergic antagonism, of cognitive impairments [112, 302].

\section{PREFRONTAL CORTEX}

The prefrontal cortex (PFC) is thought to have a function in: response inhibition (perseverance), temporal structuring of information, orientation (mainly egocentric), social and affective behavior, spontaneity of behavior, and finally, odor perception [183]. Based on the first two functions the PFC is mainly of importance for temporal structuring of information in temporal learning tasks [e.g. 254]. Recently, it has been demonstrated that during temporal learning there is an inverse dopaminergic relationship between the PFC and the striatum, that is dopaminergic activity in the PFC and striatum is enhanced and reduced, respectively [154].

With regard to spatial orientation it has been demonstrated that the medial PFC (MPFC), an area that presumably is homologous to the dorsolateral PFC in monkeys and primates, is necessary for egocentric (response) learning $177,179,184]$. However, the involvement of the mPFC in the expression of allocentric (place) learning is equivocal since no effects of PFC lesions have been found in several spatial tasks $176,179,184,2991$. The effects on allocentric learning that had been observed in earlier studies [75, 185] may be explained by an impaired temporal structuring of spatial information [178], or by effects on perseverance (e.g. repeating of incorrect responses [127]) or structuring of information (e.g. wrong learning and applying of rulles [364). In DMTP tasks the effects of pharmacological lesions of the PFC or mPFC were studied and it was suggested that because of the nonspecific effects, the (m)PFC plays a role in attentional processes $192,100,1401$ Concomitantly, it has recently been demonstrated in humans with prefrontal damage that their attention was impaired $1126 !$.

The findings with regard to orientation indicate a dissociation between the mPFC and hippocampus/parietal cortex in their involvement in the expression of egocentric and allocentric learning. It might even be argued that the mPFC mediates the construction of an egocentric cognitive map (compare this with the role of the hippocampus and parietal cortex with regard to an allocentric cognitive map). However, future research is necessary to elucidate the role of the mPFC in (response) learning and memory. This implies studying other structures and neurotransmitters/modulators also. For instance, the caudate nucleus is also involved in the expression of egocentric learning $[2591$ and not allocentric learring $1213,25 \%, 258]$ 
The amygdala is part of the limbic system which is a group of structures including also the hippocampus, septum and cingulate cortex. The latter three sitructures form the Papez circuit. which receives projections from and returns them to the hippocampus (subicular area) via the mammillary bodies, the anterior thallamus and the cingulate cortex $[130,261]$ (see Fig. 2). The limbic system is proposed to be the strictural basis for emotion and motivation in which the amygdala plays a key role 1334 . As previously already mentioned the emotional status of a subject can influence or interfere with its cognition [130]. It has been found that amygdala lesions produced impaiments in an aversive task such as active avoidance learning [201,304]. On the other hand, amygdala lesions did not produce deficits in spatial learning [174] and spatial working memory [6].

The amygdala is also part of the basal forebrain cholinergic system which receives input from the NBM which predominantly projects to the parietal cortex [136, 222]. Thus based on the above mentioned findings there appears to be a dissociation of function for memory between the NBM and its cholinergic targets, i.e. the parietal contex and the amygdala. In general, the NBM chollinergic projections to the parietal cortex are involved in spatial memory, whereas the NBM-mediated cholinergic input into the amygdala might be of critical importance for memory associated with amygdala function, e.g. avoidance learning [175]. Besides that the amygdala receives cholinergic input from the NBM, it also receives noradrenergic, dopaminergic, and serotonergic input from, respectively, the locus coeruleus, VTA and raphe [265] (see Fig. 2).

Recently, inwestigations of the participation of interactions in the amygdala between acetylcholine and other neuotransmitters/neuromodulators in avoidance learning have been attempted. Post-training infusion of the NMDA antagonist AP5 or the muscarinic antagonist scopolamine into the amygdala, medial septum or hippocampus blocked the consolidation of passive avoidance learning, which was counteracted by infusion of a $G A B A_{A}$ receptor antagonist into these structures [156, 159]. Post-training infusion of the AMPA antagonist 6-cyano-7-nitroquinoxaline-2,3-dione (CNQX) into any of the three structures impaired two-way active avoidance learning, but probably by blocking retrieval processes [163]. Based on these finding and assuming that LTP is involved in learning and memory (see section Synaptic plasticity), it has been suggested that LTP activated by the learning experiences in the hippocampus and/or amygdala and medial septum is the crucial event (for a review see [158]). Thus, NMDA receptor blockade is thought to block LTP induction and hinder retention due to consolidation inhibition, whereas AMPA receptor blockade at the time of testing hinders LTP expression and retention due to retrieval inhibition [163]. In addition, it is suggested that GABA receptors (nodulated by benzodiazepines [157) inhibit post-4raining memory processes [156, 159]. The entorhinal cortex is Whought to intervene in post-training memory processing after the other limbic regions and integrates consecutively acquired memories [1.58]. Recently, it has been found that an amygdala lesion resulted in an impaired induction of LTP in the hippocampus [148], which therefore adds evidence to the assumption that LTP in the hippocampus and/or amygdala and medial septum is the crucial event $\| 158 \rrbracket$, It seems that noradrenergic transmission in the amygdala is also inwolved in avoidance learning $[156,202]$. In addition, indirect evidence exists that there is an interaction between the noradrenergic and cholinergic system in the amygdala in awoidance learming [1501. Finally, although anygdala lesions did not result in spatial learning deficits per se, it has been reported that spatial retention may depend on dopaminergic activity of the amygdala [256]. However, this was not a specific spatial deficit, as also cue learning was impaired.

Another structure which is commonly associated with the limbic system and thus emotion and motivation, is the nucleus accumbens [354]. Concomitantly, it has been found that the nucleus accumbens appears to mediate anxiety [28.3 and depression [149], probably through an interaction with its dopaminergic transmission. Normally, the nucleus accumbens is suggested to play a 
specific role in cognitive and locomotor behavior (see section Wentral tegmental area and dopamine).

\section{PHOSPHONNOSITIDE SIGNAL TRANSDUCTION SYSTEM}

It may be assumed that for cholinergic activity, postsynaptic processes (e.g. the muscarinic second messenger system) are as important as presynaptic processes (e.g. the synthesis and release of ACh). This is corroborated by the finding that the age-related decrease in the hippocampal phosphoinositide signal transduction system correlated with the spatial learning deficit in the Morris water escape task [59]. In the brains of AD patients it has been found that the phosphoinositide signal transduction system was impaired [168. 169]. Thus, it nay be suggested that a future goal for treatment of cognitive impairments is the development of substances which activate the muscarinic receptor-coupled phosphoinositide signal transduction (e.g. by activating the phosphoinositide metabolism).

\section{SYNAPTIC PLASTICITY}

During normal synaptic transmission in the hippocampus, glutamate mediates the so-called evoked excitatory potential (EPSP) after single shock stimulation of the presynaptic neuron via the AMPA receptor on the postsynaptic neuron. This receptor has an ion channel through which the $\mathrm{Na}$ t ions flow into the neuron causing the depolarization. Glutamate also binds to the NMDA receptor. However, this receptor contributes little to the EPSP because of the voltage dependent $\mathrm{Mg}^{2+}$ block of the to this receptor coupled ion channel, through which $\mathrm{Ca}^{2+}$ ions can fllow into the neuron. Nevertheless, the activation of the NMDA receptors is lileky to be essential for the induction of LTP, as has been demonstrated in in vitro studies [c.g. 64]. LTP can be defined as a stable and long-lasting increase (lasting even up to several weeks) in the efficiency of symaptic transmission after electrical conditioning of the pathway, that is stimulating the presynaptic neurons with high frequency pulses, e.g. $50 \mathrm{~Hz}$. During the prolonged depolarization the $\mathrm{Mg} 2$ block is removed and $\mathrm{Ca}^{2}+$ can enter the cell through the NMDA receptor channel. This entry of Ca2t is the probable trigger for LTP induction. After LTP induction the efficiency of symaptic transmission is increased, i.e, the same electrical stimulus, hence the same symaptic input, produces an increased postsynaptic potential when compared with the postsynaptic EPSP response before LTP.

The increase in the efficiency of synaptic transmission in the hippocampus is a form of synaptic plasticity which is believed to be the physiological substrate for learning and memory leg. 331. This is supported by several studies with regard to the hippocampus, which is especially essential for spatial learning and memory processes [229.245]. For example, administration of NMDA receptor antagonists impair spatial learning and block hippocampal LTP induction in vivo 12. 73. 287. In addition, it has recently been demonstrated that spatial learning induces changes in the septohippocampal system of rodents, like for example an increase in hippocampal glutamate release ex wivo [284] or an increased synaptic efficacy in vivo [122, 151. 162].

After induction of LTP, the NMDA receptor plays mo more role in mainiaining the LTP and LTP cannot be influenced anymore [133]. Hence the question arizes what mechanisms maintain the increase in synaptic transmission. Or formulated in another way: what is the locus of LTP mantenance? At the moment research is concentrating at changes in glutamatergic transmission 
occuring at the postsynaptic site (e.g. an increase in the amount or sensitivity of AMPA andor NMDA receptors to glutamate) or at the presynaptic site (e.g. an increase in the synthesis and/or release of glutamate). There are several studies indicating that postsynaptic changes occur (e.g. NMDA receptor regulation; which are probably mediated by protein kinase $\mathbb{C}$ mediated protein phophorylations $1264,278,2791$. On the other hand there are also indications that presynaptic changes occur because it has been found that the increase in synaptic transmission is maintained probably by an increase in presynaptic glutamate release 1881 . This presynaptic modification of neurotransmitter release after the postsynaptic induction of LTP by activation of NMDA receptors, suggests that there must be an intercellular (retrograde) messenger which is released from the postsynaptic cell after induction of LTP (or signal that LTP induction is occuring) (see 331.

It should be noted that there is also another form of synaptic plasticity, viz. long-term depression (LTD). However, it is not clear whether LTD contributes to learning and memory. LTD is induced by a low frequency of stimulation, e.g. $1-3 \mathrm{~Hz}$. It has been proposed that the magnitude of the rise in intracellular $\mathrm{Ca}^{2}+$ might determine whether LTP or LTD is induced [211], A large rise in intracellutar $C^{2}{ }^{2+}$ concentrations is thought to result in the induction of LTP by activating calcium/calmodulin-dependent protein kinase II (Ca/CaMKII), whereas a modest rise in intracellubar $\mathrm{Ca}^{2+}$ induces LTD, presumably by activating Cat2-dependent protein phosphatases. In principle, it is proposed that the balance between the activities of the protein kinases and protein phosphatases influences synaptic strength by controlling the phosphorylarion state of certain (though unidentified) proteins, thus suggesting postsynaptic changes. In addition, the production of a retrograde messenger may also be involved in LTD induction, thus suggesting presynaptic changes also [see 211].

There are several candidates suggested to act as retrograde messengers. Arachidonic acid and platelet-derived factor, which are phospholipase $\mathrm{A} 2$ derived lipids, were initially considered as possibilities [se 33]. Recently, nitric oxide (NO) has received considerable amount of interest as a possible retrograde messenger. This because $\mathrm{NO}$ is produced by a calcium/calmodulin-dependent enzyme NO synthase which can be stimulated by the intracellular $\mathrm{Ca}^{2+}$ influx as a result of NMDA receptor activation [123]. Furthermore, NO is short-lived and freely diffusable, which is regarded as ideal properties for a retrograde messenger [33, 24$]$. Several experiments have indeed demonstrated that NO synthase inhibition blocked induction of hippocampal LTP both in vitro $116,40.244$ and in vivo 1901 .

Finally, it is interesting to mention that there is also experimental evidence supporting a role of CarCaMKII in LTP and memory processes. For example, mutant mice that do not express Ca/CaMKIl are deficient in their abillty to produce LTP and exhibit spatial learning deficits 1347 . 3181. In addition, it has recently been found that spatial learning alters hippocampal Ca/CaMKII activity in the at [343]. Furthermore, indirect evidence shows that this is probably mediated via NO after NMDA receptor stimulation [164, 655 ]. Al] these findings with respect to Ca/CaMKII suggest postsynaptic changes in stend of presynaptic changes for LTP maintenace, but nevertheless it seems that NO is still involved in LTP and memory processes.

\section{REFERENCES}

1. Abdullah F.A., Abu-Bakia M.A.J., Culaminici M. R., Stephenson J.D. and Siden J.D. (1995) Importance of lorebrin cholinergio and $\mathrm{GABAergic}$ systems to the age-related deticits in water maze performance in rats. Newrobiol. Aging 16,41-52.

2. Abrahan W.C. and Mason S.E (1988) Effects of the NMDA receptorchannel antagonists CPP and MK. 801 on the hippocampal field potentials and long-term potentiation in anesthezised rats. Brain Res. 462 , 4046 . 
3. Adelstein T.B., Kesner R.P. and Stassherg D.S. (1992) Spatah recognition and spatiat order methory hin patients with dementia of the Alzheime's type. Neuropsychologica $30,59-67$.

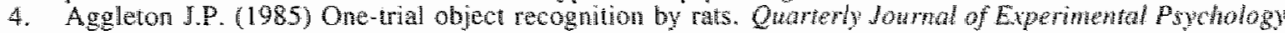
Comparanine and Physiological Psychalogy. 37B, 279.294

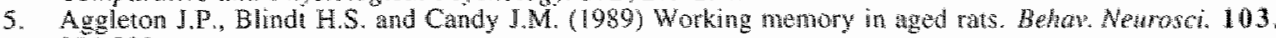
$975-983$.

6. Aggleton J.P., Blind H.S. and Rawins J.N. (1989) Effects of anygdaloid and anygdaloid-hippocampal lesions on object recognition and spatial working memory in ras. Befhat Neurosed. 103, 962-974.

7. Aggleton J.P. Kein A.B., Rawlins J.N., Hunt P.R. and Sabgal A. (1992) Removal of the happosingus and transection of the fomik produce comparable doficits on detayed non-matching to position by rats. Behav. Bram Rer. 52, $61-71$.

8. Aigner T.G., Mitchell S.J., Aggleton J.P., DeLong M.R., Struble R.G., Price D.L., Wenk O.L., Petigrew K.D. and Mishkin M. (1991) Transient impaiment of recognition memory following ibotemio acid lesions of the basal forebrain in macaques, Exp. Brain Res. 86, 18-26.

9. Alman H.J. and Wormile H.J. (1988) What is the nature of the rold of the serolonergic merwous system an learning and memory: Prospects for development of an erfective meatment strategy for senile dententim Neurobiol. Aging $\$$ "627-638.

10. Allman H.J., Nomile H.J., Galloway M.P., Ramirez A. and Azmitia E.C. (1990) Enhaced spanal discrimination learning in rats following 5,7-DHT-induced serotonergio deafferentation of the hippocanpus. Brain Res. 518, 61-66.

11. Altman H.J., Stone W.S. and Ogren S.O. (1987) Evidence for a possible functional interaction between serotonergic and cholinergic mechanisms in memory retrieval. Behow. Newal Biol. 48, 49-62.

12. Andrews J.S. (1996) Possible confounding influence of strain, age and gender on cognitive perlormance in rats. Cogninive Brain Res. 3, 251-267.

13. Andrews J.S., Gratzmer M and Stephens D.N. (1992) Etfects of cholinergic and non-cholinerio drugs on visual discrimination and delayed visual discrimination performance in rats. Psychopharnicanotog: 106, 523530 .

14. Andrews J.S. Grutzner M. and Stephens D.N. (1994) The effects of ibotenic ancid lesions of uhe basal forebrain on visual discrimination performance in rats. Brain Res. Butlesin 34, 407-412.

15. Andringa G., Lubbers L.J. Drukarch B., Sto of J.C. and Cools A.R., The DI antagonist SKF 83959 exerts mild antiparkinsian effects in MPTP-treated rhesus monkeys. First Duth Endo-Newro Meeting, Doonwonth. 1997, pp. 33, P3.

16. Arancio O. Levram V. "Tsien R.Y., Kandel E.R. and Hawkins R.D. (1996) Nitric oxide acts as a rarograde messenger during long-tem potentiation in cultured hippocampal neurons. $J$. Physiol. (Paris) $10,321-122$

17. Arendash G. W. Sanberg, P.R. and Sengstock $G$ J. (1995) Nicotine entunces the learning and memory of aged rats. Pharmacol. Biochern. Behav. $\$ 1,517-523$

18. Arendt T. Allen Y., Marchbanks R.M., Schtgens M.M., Sinden J., Lantos P.L. and Cray J.A. (1989) Cholinergic system and memory in the rat: Effects of chronic ethanol, embryonic basal forebrain brain transplants and excitotoxic lesions of cholinergic basal forebraimi projection system. Neuroscience 33, 435462 .

19. Arnsten A.F.T., Cai J.X. and Goldman-Rakic P.S. (1988) The alphat adrenergic agonist guanfacine improve memory in aged monkeys whout sedative or hypertensive side-effects: Evidonce lor alphaz stulitypes. A. Nernosci. $8,4287-4298$.

20. Aubert 1. Rowe W. Meaney M.J., Gether S. and Quition R. (1995) Cholinorgic matkers in aged Cognitively impaired Long-Evans rats. Neuroscience 67, 277-292

21. Baddeley A. (1986) Working Memory. Oxford University Press.

22. Baddeley C.A. (1995) Mandbook of nemomy disorders. Whitey.

23. Bannerman D.M., Good M.A. Butcher S.P. Ramsay M. and Morris R.G.M. (1995) Distunct contiponents of spatial leaming revealed by prior traning and NMDA receptor blockade. Nature $378.182-186$.

24. Bames C A. (1990) Animal models of age-related cogntive dechine. In Handbook of Neuropsychology. (ed. Boller F. and Gratman 1.), Vol. 4, pp. 169-196. Elsevier Science Publishers.

25. Bartus R.T., Dean II. R.L. Beer B. and Lippa A.S. (1982) The cholinergie hypothesis of geriatric memory dysfunction. Science 217 " $408-417$

26. Beal M.F., Hyman B.T, and Koroshetz W. (1993) Do defects in mitochondrial energy metabol wism undertie the pathology of neurodegenerative cliseases? Trefols Nenosch. 16, 125-131.

27. Beatly W. Butters $N$, and Janowsky D. (1986) Pattems of memory fature after scopolamine treatmant Bmplications for cholinergic by pothesis of dementia. Behav Newrot Biol. 45, 196-211.

23. Beatly W.W. (1988) Preserwation and loss of spatial memory in aged rats and humans: Implications for the analysits of memory dystunction in dementia. Nevrobiol. Aging 9,557-561

29. Beninger $R_{\text {m }}$. and Ranaldi R. (1993) Microinjections of filpenthixol into the caudate-putament but not the rucleus accurnbens, amygdala or frontal cortex of ras produce iniresession declines in food rewarded operant responding. Behav. Brain Res. 55, 203-212.

30. Bemudez Ration F. Mujica. Gonzalez M. and Prado Alcala R.A. (1986) Is cholinergic antivity of the striatum inwolwed in the acquisition of positively-motivated betawiors? Phamacol Biochen. Behaw. 24. $715-719$. 
31. Bierley R.A. Rixen G.J., Troster A.I. and Beatty W.W. (1986) Preserved spatial memory in old rats survives 10 months without lraining. Behow. Neural Biol, $45,223-229$.

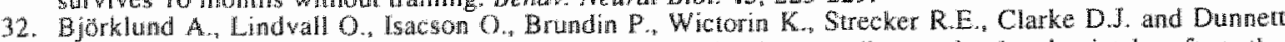
S.B. (1987) Mechanisms of achion of intracerebral neural mplants studies on nigral and stratal grafts to the IEsioned stratum. Trends Meurase: 10,509-516

33. Blis T.V.P. and Collingridge G.L. 1993) A synaptic model of memory: Long-tern potentiation in the hippocsmpus. Nature $361,31-39$.

34. Bloktand A. (1993) Age-related changes in comethion between behavioral and biochemical parameters in Lewis rats. Behav. Nenfal Brol. 60, 52-61.

35. Blokland A. (1996) Acetylcholine: A neurounansmitler for learning and memory? Brain Res. Rev. 21.285300.

36. Blokland A. Honig W. and Raajmakers W. (1992) Effect of intra-hippocalmpal scopolamine injections in at repeated spatial acquisition lask in the rat. Psychopharmacology 109, 373-376.

37. Blokland $A$. Honig $W$, and Rauijmakers W. (1.994) Age related changes in spatial discrimination learning performance in Lewis rats. Psychobiology 22, 149-155.

38. Blokiand A and Jolles 3 . (1994) Behavioral and biochemical effects of an ic injection of streplozotocin in old Lewis rats. Pharmacol. Biochent. Buthaw, 47, 833-837.

39. Bloklarid A. Prickuerts I. and Ravimakers W. (1992) Reduced level of ankiety in adult Lewis rats after chronic ethand consumption. Physiol. Behov. 51, 245-248.

40. Bon C., Bothme G.A., Doble A., Stutzmann J.-M. and Blanchard J.-C. (1992) A role for nitric oxide in longuterm potentiation. Eur d. Pharmacol 4, $420-424$.

41. Bonwento G., Seatlon B., Claustre $Y$. and Rouquier $L$. (1992) Effect of local injection of 8-OH-DPAT into the dorsal or median raphe nuclei on extracellula levels of serotonin in serotonergic projection areas in the rat brain. NewrosciLest. 137, $101-104$.

42. Brady R.J., Gonter J.A., Monroe M.T. and Swann J.W. (1994) Developmental alterations in the sensitivity of hippocampal MMDA receptors to ADS. Brom Res. Dev Brain Res. 83. 190-196.

43. Bratt A.M., Kelly M.E, Domeney A.M., Naylor R.J. and Costall B. (1994) Aged Stone maze-imparied rats exhibit reduced striatal muscarinic receptor densities. NeuroReport $\mathbf{5}, 2678-2682$.

44. Broks P., Presion G.C., Traub M., Poppleton F., Ward C. and Stahl S.M. (1988) Modelling dementia: Effects of scopolamine on memory and attention. Neuropsychologian 26, 685-700.

45. Burns L.H., Robbins "T.W and Everitt B.J. (1993) Differential effects of excitotoxic lesions of the basolateral amygdata, ventral subiculum and medial prefrontal cortex on responding with conditioned reinforcement and locomotor activity potentiated by intra-accumbens infisions of $D$-amphetamine. Behav, Brain Res. 55, 167-183.

46. Butcher L.L.. Wool N.J., Albanese A. and Butcher S.H. (1981) Cholnergic-monoaminergic interactions in selected regions of the brain: Histochemical and pharmacologic analyses. In Cholmergic mechanisms: phylogenetic aspects, centrat and peripheral synapses, and chical significance. (ed. Pepeu $\mathrm{O}$. and Ladinsky H.), pp. 723-738. Plenum. New York.

47. Bymaster F.P., Heath 1. Hendrix J.C. and Shannon H.E. (1993) Comparative behavional and neurochemicat aclivilies of aholinergic antagonists in rats. J. Phamacol. Exp. Ther, 267, 16.24.

48. Cain D.P., Saucier D. and Boon F. (1997) Testing hypotheses of spatial laarning: The role of NMDA receptors and NMDA-mediated long-term potentiation. Behav, Brain Res. 84, 179-193.

49. Can D.P, Saueier D., Hall J. Hargreaves. E. L. and Boon F. (1996) Detalled behavioral analysis of wate maze ancisition under APV or CNQX: Contribution of sensorimotor alstubances to drug-induced acquisilion deficis. Behav. Nerosci, 110, 86-102.

50. Cillahan M.J. Kinsori J.I. Harhatigh R. F., Reeder T.M. and Davis R.E. (1993) Continnous ICV infision of scopolamine impairs sustained atiention of Rhesus Monkeys. Newobiol. Aging $14,147-151$.

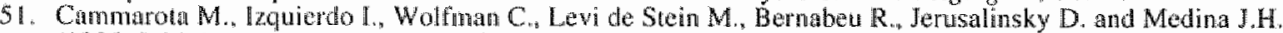
(1995) lnhibutory aroidance traning induces rapid and selective changes in 3[H]AMPA receptor binding in the rat hippowampal formation. Netrobiol. Leam. Mem, 64, 257-264.

52. Carli M., Ladarova M., Temezynska E. and Samanh R. (1992) Stimulation of 5-HTIA receptors in the dorsal hippocanpus impars acguistion and pertormance of a spatial task in a water maze. Brom Res., 50 50.

53. Carli M. Luschi R. Garofalo P. and Samanin R. (1995) 8-OH.DPAT impairs spatial but not visual learing in water maze by stimalang 5-HTL Aeceptors in the hippocampus. Behan. Brain Res. 67, 6774.

54. Carli M and Samanin R. ( 1992$)$ 8.Hydroxy-2-(di-n-propylamino)tetralin impairs spatial learning in a water maze: Role of postsymaplic 5-HTIA iecepiors. Br. N. Pharmacol. 105, 720-726.

55. Carli M., Tatarezynska E. Cerva L. and Samanin R. (1993) Stimulation of hippocampal 5m HT 1 receptors causes amnesin and anxiolytic-like bu not antidepressant-like effects in the rat. Eur. J. Pharmacol. 234. $2|5-22|$

56. Cassel J.C. and Jelsch H. (1995) Serotonergic modulation of chollinergic function in the cantral nervous system: Cognitive implications. Nearoscience 69. 1 -4 1 .

57. Cavoy A. and Delacout J. (1993) Spatial but not object recogmition is impared by aging in rats. Physiol. Behay $53,527-530$ 
58. Chavez M.E., Salado Castillo R., Sanchez Alavez M. Quirarte C.L. and Prado Aleala R.A. (1995) Postraining injection of GABAergic antagoniss into the striatun produces retrograde amnesia. Newrobiot. Lean. Mem. 63, $296-300$.

59. Chouinard M.L. Gallagher M. Yasuda R.P., Wolfe B.B. and Mckinney M. (1995) Hippocampal

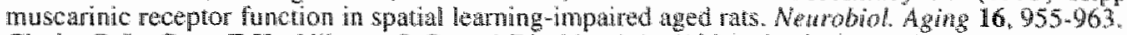

60. Ciake D.J. Cage F.H., Nilsson O.G. and Bjorklund A. (1986) Crated septal nemons from cholineraic symaptic connections in the dentate gyrus of behaviorally impained aged rats. 4. Comp. Menol. 252, 483. 492.

61. Clarke P.B.S. and Fibiger H.C. (1990) Reinforced alternation performance is unpatred by museminic but not by nicounic receptor blockade in rats. Beflaw Brain Rer. $36,207-207$.

62. Claus J.J. and Mohr E. (1996) Attentional deficits in Alzheimer's, Parkinson's, and Huntington's diseases. Acta Newrol Scand $93,346-351$.

63. Cole B.J., Klewer M., Jones G.H. and Stephens D.N. (1993) Contasting effects of the compouive NMDA antagonist CPP and the non-competitive NMDA antagonist MK 801 on performance of an operan delayed matchig to postion task in rats. Psychopharnacology Ber. 111, $465-471$

64. Collingridge G.L. and Bliss T.V. (1987) NMDA receptors: Their wole in long-lem potentiation. Spacial Issue: Excitatory amino acids in the brain: focus on NMDA receptors. Trends Nenrosici. $10,238-293$.

65. Collingridge G.M. and Bliss 17. W.P. (1995) Memories of NMDA recepors and L.TP. Themds Nemosci. 18 . $54-56$.

66. Cook D. and Kesner R.P. (1988) Caudate rucleus and memory for egocentric localization. Behan. Newal Biol. 49, 332-343.

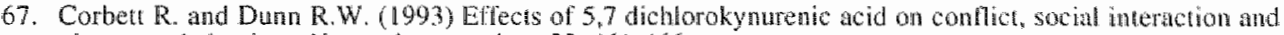
pllus maze behavions. Nenropharmacology $32,461-466$.

68. Colzias G.C., Papawasiliow P.S. and Ginos I.Z. (1976) Therapentic approaches in Parkinson"s disease: Possible roles of growih hormone and somatostatin. In The basal ganglia (ed. Yahr M. D.). pp. 305 .313. Rawen Presis Mew York.

69. Court J.A., Piggon M.A. Pery E.K., Balow R.B. and Pery R.H. (1992) Age associated decline in high affinity micotine binding in buman brain frontal cortex does not correlate with the changes in cholino acetyltranferase activity. Newroscience Research Conmunication. 10,125-1133.

70. Crook T. Winer E., Rothwell A., Winterling D. and McEntree W. (1992) Noradrenergic intervention in Alzheiner's disease. Psychophamacology Bullerin 28, $67-70$.

71. DMello G.D. and Steckler T". (1996) Animal models in cognive behavioural pharmacology: An overview. Cogn. Brain Res. 3, 1996.

72. Daum I. Schugens M.M., Spieker S., Poser U., Schonle R.W. and Birbumer N. (1995) Menory and skill acquistion in Parkinson's disease and frontal lobe dysfunction. Cortex 31, 413-432.

73. Davis S., Butcher S.P. and Morris R.G.M. (1992) The NMDA recptor antagonist D-2-amino-5 phosphopentanoate (D-APV) impairs spatial learning and LTP in vito at intracerebral concenuations comparable to those that block LTP in vitro. J. Neurosci. 12, 21-34, 1992.

74. Dawson G.R., Heyes C.M. and Iversen S.D. (1992) Pharmacological mechanisms and animal models of cognition. Behaw. Pharmacol 3, 285-297.

75. De Brabander J.M., De Bruin J.P.C. and Van Eden C.G. (1991) Comparisory of neonatal and adult medial

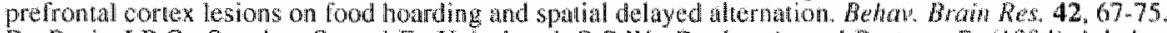

76. De Bruin J.P.C., Sanchez-Santed F., Hensbroek R.P.W., Donker A and Postmes P. (1994) A bohavioral analysis of rats with damage to the umedial prefrontal cortex using the Morris water mate: Evidence lor bethavoral flexibility, but rot for impanted spalial navigation. Brain Res. 652, 323-333.

77. De Bruin J.P.C., Swinkels W.A.M and De Brabander J.M. (1997) Response leanong of rats in a Morris water raze: Involvement ol the medial prefrontal contex. Behav Bram Res. 85, 47.55.

78. Decker M.W. (1987) The eflects of aging on hippocatmpat and cortical projections of the foncbrain cholinergic system. Braw Res. Rew. 12,423-438.

79. Decker M. W. Majchrzak M.J. and Anderson D.J. (1992) Effects of nicotine on spatial metnory deticis in rats with spatial lesions. Bran Res. 572, 28 1.285.

80. Decker M.W. and McGaugh J.L. (1991) The role of interactions betwen the cholinergic system and other neuromodulatory systems in learning and nemory. Symapse $7,151-168$.

81. Decker M. W. Pelleymounter M.A. and Gallagher M. (1988) Effects of traning on a spatial muenory tusk on high affinily chollme uptake in hippocampus and contex in young adull and aged rats. J. Neurosci. 8,90 m 99.

82. DeLong M.R. (1990) Primate models of mowement disorders of basal ganglia ongin. Trends Nerosci. 13. $281-285$

83. Devan B.D., Goad E.H. and Petri H.L. (1996) Dissociatom of hippocampal and stratal contributions to spatial navigation in the water maze. Newrobiol Leam. Mem. 66, 305-323.

84. Diaz del Guante M.A., Carbonell Hernandez C. Quirante G., Cruz Morales. 5.E., Rivas Arancibia S. and Prado Alcala R.A. (1993) lintrastratal injection of choline accelecates the acquisition of positively rewarded behators. Broin Ries. Bull. 30.671-675.

85. DiMautia B.D. and Kesmer R.P. (1988) Spatial cognitive maps: Differential role of partat conex and hippocampal fomation. Behav. Neurosct. 102,47 - 480. 
86. Dobbs A.R. and Rule B.C. (1989) Aduh age diferences in working memory. Psychol. Agng 4, 500.503.

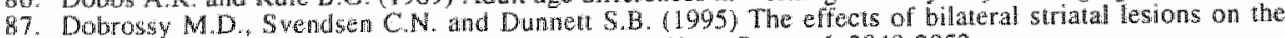
acquistion of an operant lest of short term memory. Newropeport 6. 2049-2053.

88. Dolphin A.C. Errintion M.L. and Bliss T.V.P. (1982) Long-term potanition of the perforant path it vivo is associated wth increased glutamate release. Nature $297,496-498$.

89. Doods H.W. Quirion R. Mihm G., Enge W., Rudolf K. Entzeroth M, Schiav G.B., Ladinsky H. Bechell W.D., Eustrger H.A. Mandla K.D. and Eberlein W. (1993) Therapeutic potentials of CNS-active M2 andagonists: Novel structures and pharmacollogy. Life Sici. $52,497.507$.

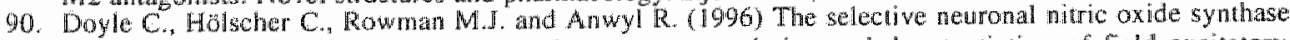
antaponst 7-ntro indazole biocks both long-term potentation and fepotentiation of fleld exchatory postsynaplic potentials in tal hippocampal $C A$ in wivo. J. Neatoset. $16.418-424$.

91. Dunbar O.E. Rylett R.J. Schmidi B.M., Sindair R.C. ant Willams L.R. (1993) Hippocampal choline acelyluansferase activily correlates with spatal hearning in aged rats. Brain Res. 604, $266-272$.

92. Dunnet \$.D. (1900) Role of prefrontal Cortex and stratal outpul systems in short-term memory deficits associated wilh ageing, basal forebrain lesions, and cholinergic rich grafts. Can. J. Psychol, 44, $210-232$

93. Dumet S. B. (1993) The role and repair of forebrain cholinergic systems in shormerm memory. Sudies using the delayed matching-to-gosinion lask in tats. Adw. Newol 59,53-65.

94. Dunnet1 S.B. (1994) Strategies for tesing learning and memory abílities in transplanted rats. In Functional newal ransplantahon. (ed. Dunneth S. B. and Bjorklund A.) pP. 217-251. Raven Press, New York.

95. Dunnet S.D., Badman F. Rogers D.C. Evenden J.L. and Wersen S.D. (1988) Cholinergic grafts in the necontex or hippocampus of aged rats: Reduclion of delay dependent deficis in the delayed non-matching to position task. Exp. Nethol. 102, $57-64$.

96. Dumetr S.B., Evienden J L. and lwersen S.D. (1988) Delay-apendent short-tern memory deficits in aged rans. Pyychophawnacology $96,174-180$.

97. Dumet S.F., Everit B.J. and Robbins T.W. (1991) The basal forebrain-cortical cholinergic system: Interpreting the functional conseguences of excitotoxic lestons. Trends Neurosci, 14, 494-501.

98. Dunnet S.B. Mantel F.L. and Iversen S.D. (1900) Proactive interference effects on short-term memory in rats: 11. Eltects in young and aged rats. Wehav. Newroser 104, 666-670.

99. Dunnet 5.B. Rogers D.C. and Jones G.H. (1989) Effects of nucleus basalis magnocellularis lestions in rats on delayed matching and nonmatching to position tasks. Eur. Wo Newosci. 1, 395-406.

100. Dunneu S.B. Wareham A. T. and Torres E.M. (1990) Chollnergic blockade in prefrontal cortex and hippocampus disrupts short-ferm memory in rats. Newnokeport $1.61-64$.

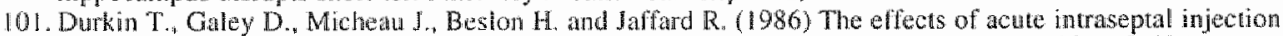
of haloperidol in wivo on hippocampal chollingric function in the mouse. Brain Res. 376, 420-424.

102. El-Defawy S.R., Coloma In., Thamandas K., Boegman R.J., Benninger R.J.and Wirsching B.A. (1985) Functional and neurochemical cortical cholinergic impaiments followitg neurotoxic leston of the nucleus basalis magnocellularis in the rat. Neurobiol. Aging 6, 325-330.

103. Ennaceur A. and Delacour J. (1988) A new one-trial test for nearobiological studies of memory in tats. 1: Behavioral dala. Behat. Brain Res. 31,47.59.

104. Ennacer $A$. and Meliani $K$. (1992) A new one-trial test for neurobiological studies of nemory in rats. III. Spatial ws. non-spatinl working memory. Behaw Brak. Res. 51. 83-92.

105. Faiman C.P. Viu E., Skathick P. and Trullas $\mathbb{R}$. (1994) Diferental affects of compotmds that act an

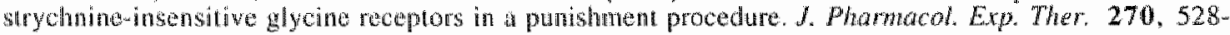
$53 x^{3}$

106. File S.E. (1996, Recentevelopments in anxiely, stress, and depression. Pharmacot. Brocherw. Behaw. 54. $2 \cdot 12$

107. Fischer W. Chen K.S., Gage R.H. and Bjorklund A (1992) Progressive decline in spatial learning and

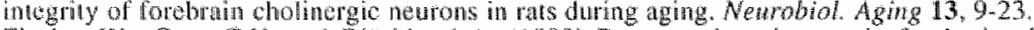

108. Fischer W. Gage ${ }^{*}$ H. and Biokklund A. (1989) Degenerative changes in forebrain cholinerge nuclei conelute wh cogntiva impaiments in aged rats. Eur. J. Newrosci. 1, 34-45.

109. Flod J.F (1988) Elfect of acute arecoline, taxine, and arecoline + tacrine post-training administation on retention in tate middle-aged rais. J. Gerontol. BIOL SCl, 2, B54-B56.

110. Forloni $\mathrm{O}$. and Angaren $N$, (1992) Dectased [3H Jhemicholinhm binding to high-affinity cholne uptake siles in aged rat brin. Broin Res, $570,354-357$.

111. Fraticis P.T. Pangalos M.N. and Bowen D.M. (1992) Animal and drug nodelling for Alzheiner symaptic pathology. Prog. Newobiot 39,5 57.545

112. Froest! W. Mickel S.J., Von Sprecher G. Diel P.J., Hall R. G. Maer L., Strub D., Melillo V. Baumann P.A. and Bernisconi R. (1995) Phosphinic acid analogues of GABA. 2. Selective, orally active GABAB antagonists. I. Med. Chem. 38, 3313,3331 .

113. Futido J.C. and Mazuck M.F. (1996) Behatioral characterization of quinolinate-induced lesions or the medial stritum: Relewance tor Huntington's disease. Exp. Mewrol 138. 158-168.

14. Gage FH. and Bjönklund A (1986) Cholinergic septal grafts into the hippocampal formation improve spatiall leatning and memory in aged rats by an atropine-sensitive mechanism.d. Nererosci. 6, $2837-2847$.

115. Gage F.H., Biorklund A., Steveni U., Dunnet S.B. and Kelly P.A.T. (1984) Intranippocampal septal grafts andionate learning impatments in aged rats. Science 225, 533-536. 


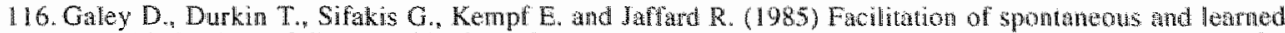

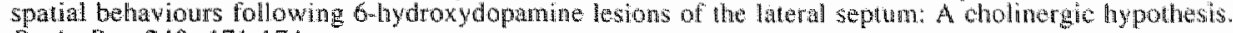
Brain Res. 340, 171-174.

117. Galey D. Toumane A., Durkin T. and Iaffard $\mathbb{R}$. (1989) In vivo modulation of septo hippocampat cholinergic activity in mice. Relationships wh spatial reference and working momory performance. Be wat Brain Res. 32, 163-172.

113. Gallagher M. and Burwell R.D. (1989) Relationship of age-related decline across several behavioral domans. Neurobial. Aging 10.69l-708.

119. Gallagher M., Burwell R.D. Kod5i M.H. McKinney M., Southartand S. Vella-Rountec L. and Lewis M.F. (1990) Markers for biogenic amines in the Aged rat brain: Retationship to decline in spatial farming ability. Nerrobiol. Aging 11, 507-514.

120. Gallagher M. and Pelleymounter M.A. (1988) An dge-related leaming delicit: Choling uptake distuguishas "impaired" and "unimpaired" rats. Newnobiol. Aging 9, 363-369.

121. Gallagher M. and Pelleymounter M.A. (1988) Spatial learning deficits in old rats: A model for menory decline in the aged. Newrobiol. Aging 9, 549-556.

122. Garcia $\mathbb{R}$., Voumba R.M. and Jafard R. (1993) Spatial discriminaion learning induces L TP-like changes in the lateral septum of mice. NeuroRepont $5,329-332$.

123. Garthwate d. (1991) Glutamate, nitric oxide and cell-cell signalling in the nerwous system. Thends Neurosci. 14,60-67.

124. Garthwate J. and Boulton C.L. (1995) Nütric oxide signaling in the central nervous system. Amm. Rew. Physiol. 57, 683-706.

125. Gerfen G.R and Sawchenko P.E. (1984) An anterograte neuroanatomical tracing method that shows the detated morphology of neurons, their axons and terminals: Immunohistochemical localization of an axonally transported plant lectit, Phaseolus vulgaris-leuconglutinin (PHA-L). Bran Res. 290, $219-238$.

126. Godefroy O. and Rousseaux M. (1996) Divided and locused antention in patienas with lesion of the prefronial cortex. Brain Cogn. 30, 155-174.

127. Goldman-Rakic P.S. (1984) Modular organization of prefirontal contex. Trends Newosci. 7.419-424.

128. Goodlet C.R., Wuchols J.M., Halloran R.W. tand West J.R. (1989) Long-term deficils in water maze spatial condisional altermation performance following remohippocampal lesions in rats. Behan. Brain Res. 32, 63. 67 .

129. Gottries C.G., Andolfsson R., Acquilonius S.M., Carlsson A., Eckernas S.A., Nordberg A., Oreland L., Svennerholn L., Wiberg A. and Wimblad B. (1983) Biochemicat changes un dementa disorders of the Alzheiner type (AD/SDAT). Newrobiol. Aging 4, 261-271.

130. Gray J.A. (1984) The hippocampus as an interface between cognition and enotion. In Amimal Cogninion (ed. Roitblat H. L. Bever T. G and Terrace H. S.). pp. 607-626. Lawrence Erlbaun associanes Ine, Hillsdale, New Jersey.

131. Graybiel A.M. (1993) Functions of the nigro-striatal system. Clin. Nerrosit. 1, 12-17.

132. Greaff F.G. Guimarães F.S., De Andrade T.G.C.S and Deakin J.F.W. (1996) Role of 5-HT in stress, anxiety, and depression. Pharmacol. Biochem. Behaw, 54, $129-141$.

133. Gustafsson B. and Wigström H. (1990) Basic features of long-term potentiation in the hippocampus. Sew. Nearosci. $2,321-333$

134. Haroutunian V.. Kanof P.D., Tsuboyama G. and Davis K.L. (1990) Restoration of cholinomimetio ancivity by clonidine in cholinergic plus noradrenergic lestoned rats. Brain Res. $507,261-266$.

135. Harell L.E. Peagler $A$ and Parsons D.S. (1990) Adrenoreceptor andagonist tratment influchces recovery of learning followng medial septal lestons and hippocampal sympathetic ingrowith. Pharmacol. Brochern. Behow. 35, $21-28$.

136. Hellendall R.P., Godfrey D.A., Ross C.D., Armstrong D.M. and Price J.L. (1986) "fhe distribution of chotine acetyluansferase in the rat amygdaloid complex and adjacent cortical areas, as determined by quantative micro-assay and immunohistochemistry. J. Comp. Nearol. 249, 486 498.

137. Hepler D.J., Olton D.S., Wenk G.L. and Coyle J.T. (1985) Legions in nucleus magnocellulanis and mediat septal area of rats produce qualitatively similar menory impanments. 8 . Neurose. 5, 866-873.

138. Herregodts P. Bruyland M.. DeKeyser J., Solhedd C., Michotte Y, and Ebinger G. (1989) Monouminergic meurotransmither in Alzhemer's disease. J. Neurol. Sci. 92, $101-116$.

139. Herremans A.H., Hijen T.H. and Slangen J.L. (1994) Validity of at delayed conditional discrimination lask as a model for working memory. Physiol, Behaw. 56, 869-875.

1.40. Hememans A.F.J. Hijzen T.H. Weborn P.F.E. Olivier B. and Slangen J.L. (1996) Eltects of infusion of cholinergic drugs into the prefrontat contex area on delayed matching 10 position performance in the rat. Brain Res. $711,102-111$.

141. Holthoff Deno V.A., Kessler J., Hertolz K., Borner H. Piotrzyk U. Wurker M. Ghaemi M., Wisonthand K., Wagner R. and Heiss W.D. (1997) Functional effects of striatal dysfunction in Parkinson disease. Aroh. Newrol 54, 145-150.

142. Hornykiewicz O. (1973) Dopamine in the basal gangliat Is role and therapeutic implicalions. Br. Med. Bull. 29, $171-178$.

143. Horwitz B. (1987) Neuroplasticity and the progression of Alzhemer's disease. Mnewn. J. Newrowerence 41 , 1.14. 


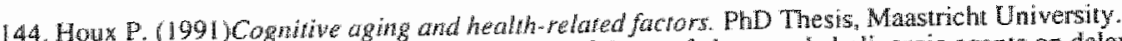

145. Hudzik T. and Wenger G.R. (1993) Effects of drugs of abuse and cholinergic agents on delayed machingto-sample responding in the squirrel monkey. I. Pharmacol. Exp. Ther. 265, $120-127$.

146. Huston A.E. and Aggleton J.P. (1987) The aflects of eholinergic drugs upon recognition memory in rats. Q. J. Exp. Psychol 39B, 297,314

147. Wegam $\$$. (1994) Behavioral impairment in radial-am maze leaning and acetyloholine content of the hippocampus and cerebral cortex in aged mice. Behav. Brain Res. 65, 103-111.

148. Kegaya $\Psi$. Siato $H$ and Athe $\mathrm{K}$. (1995) Requirment of basolateral amygdala neuron activity for the induction of long-term potentiation in the dentate gyrus in vivo. Brath Res. 671. 351-354.

149. Lipperato A. Cabib S. and Puglisi-Allegra S. (1993) Repeated stressful experiences differently affect the lime-dependent responses of the mesolimbic dopamine system to the stressor. Brain Res. 601, 333 336.

150. Introiti Collison I.B. Arai Y. and McGaugh J.L. (1989) Strin terminalis lesiong attentate the effects of postraining oxotremotine and atropine on retention. Psychobiology 17, 397-401.

151. Shihara $K$. Missuno $K . .$, Ishikawa M. and Sasa M. (1997) Behavioral LTP during learning in rat inppowanpal CA3. Behav. Brain Res. 83, 235-238.

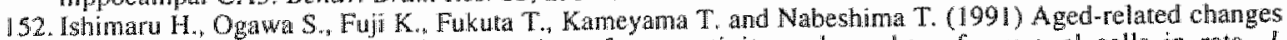
in learning and memory, cholune acetyleranferase activity and number of neuronal cells in rats. $J$. Phamolobiodyn. 14, 321-325.

153. Tversen S.D. (1984) 5.HT and anxiety. Neuropharmacology 23, 1553-1560.

I54. Iwano H. Yamamuro $Y$. Hori $K$, Yamochi " $T$ ". and Nomura M. (1997) Effects of discrimination learning on the rat striatal dopaninergic activity: A microdialysis sidy. NewReport 8, $1095-1098$.

155. Jzquierdo L. (1989) Different forms of post-training processing. Behaw. Neural Brol. $\mathbf{5 1 , 1 7 2 - 2 0 2 .}$

156. Trquerdo 1., Da Cumba C., Rosar R., Jerusalinsky D., Ferreira M.B.C. and Medina M.H. (1992) Neurotransmitter receptors involved in memory processing by the amygdala, medial septum and hippocampus of rats. Behor. Newral Biol. $58,16026$.

157. Izquierdo I. and Medina J.H. (1991) GABA A receptor modulation of memory. The role of endogenous benzodiazepines. Trends Pharmacol. Sci. $12,260-265$.

158. lzquierdo I. Medina J.H. Bianchin M., Walz R. and et al. (1993) Memory processing by the limbic system: Role of specillic neurotransmitter systems. Behaw. Brain Res. 58, $91-98$.

159. Izquierdo 1., Medina J.H., Jerusalinsky D. and Da Cunha C. (1992) Post-training memory processing in mygdata, septum and hippocampus: Role of benzodiazepine/GABA-A receptors and theit interaction with othen neurotransmitter systems. Rew. Netrasci. 3, il :-23.

160. Jarrand L.E. (1993) On the role of the hippocampus in learning and memory in the rat. Behaw. Mevral Bhol. 60. 926 .

161. Jamard L.E., Okatichi H., Steward O. and Goldschmidt R.B. (1984) On the role af hippocampal connections in the performance of place and cue tasks: Comparison with damage to hippocampus. Behaw. Neurosci. 98, $946-954$.

162. Jarrard R., Voumba R.M., Marighetto A. and Garcia R. (1997) Long-term patentiation and long-term depression in the lateral septum in spatiall working and reference memory. I. Physiology (Paris) 90, 339341.

163. Jerusalinsky D., Ferreira M.B.C., Walz R., Da Silva R.C., Bianchin M., Ruschel A., Median J.H. and Laquierdo I. (1992) Amnesia by initsion of glutamate receptor blockers into the amygdala, hippocampus and tenthorhinat cortex. Bellaw. Netral Biol, 58,76-80.

164. Johnston H.M. and Morits B.J. (1994) NMDA and nitric oxide increase microtubule associated protein 2 gene expression in hippocampal granue cells. N. Newrochem. 63, 379-382.

165. Jhmston H.M. and Morris B.J. (1995) N-methyl-D-aspartate and mitric oxide regulate the expression of

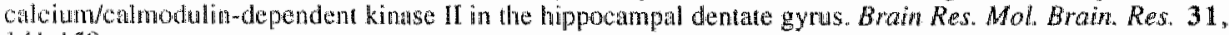
$141-150$.

166. Jolles I., Vethy F.R.I. Riedel W.I. and Houx P.J. (1995) Cognitive impanment in elderly people. Prefisposing factors and implications for experinental drug studies. Drugs and Aging 7.450.479.

167. Jomes R.S.G. (1993) Enthorhinal-hippocampal connections: A speculative view of their function. Trends Neurosci. 16. $58-64$.

168. Jope R.S. Song L., Li X. and Powers R. (1994) Impaired phosphoinositide hydrolysis in Aluheimer's distase brain. Neurobiol. Aging 15.221-226.

169. Jope R.S. Song L. and Powers R.E. (1997) Cholinergic activation of phosphoinositide signaling is impaired in Alzheinar's disease brain. Newrobiol. Aging 18, $111-120$.

170. Kalana R.N. and Andon A.C. (1991) Adrenergic receptors in aging and Alzheimer's disease: Decreased apha 2 receptors demonstrated by [3H]p-aminoclonicine binding in prefrontal cortex. Neurobiol. Aging 12, $131-136$

171. Kamctani H. and Kesner R.P. (1989) Retrospective and prospective coding of information: Dissociation of purictal cortex and hippocampal formation, Behow. Nearosci. 103,84-89.

172. Kutuman R. (1986) Alzheimer"s disease. N. Eng. N, Mod. 314, 964-973.

173. Keith J,R. and Rudy J.W. (1990) Why NMDA-receptor-dependent long-lerm potentiation may not be a mechanisty of lowning and memory: Reappraisal of the NMDA-receptor blockade strategy. Psychobiology
18, 251-257. 
174. Kesner R.P. (1986) Newnoblogical wiews of memory. In Learnug and menom: A biological wow, (ed. Martinez. Jr. J L. and Kesmer R. P. M p. 399-438. Acadenic Press, Or tando.

175. Kesner R.P. (1988) Reevaluation of the contribution of the basal forebrain cholinergic systen 10 momory. Newobiol. Aging 9, $609-616$.

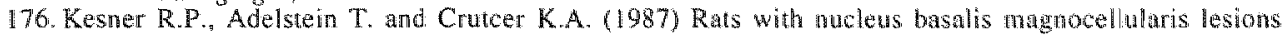
mimic mononic symptomatology observed in patrents wh dementia of the Alzhemer's ype. Behas. Nerrosci. 101, $451-456$.

177. Kesner IR.P., Bolland B.L. and Dakis M. (1993) Menory for spatiallocations, motor responsas, and objects: Triple dissociation anong the thippocampus, caudate nucleus, and extrastriate visual cortex. Exp. Brain Res. 93, 462.470 .

178. Kesner R.P. and Holbrook T. (1987) Dissociation of item and order spatial memory in rats following medial prefronal cortex lesions. Neuropsychologia 25, 653-664.

179. Kesner R.P., Rarnsworth G. and DiMatia B.V. (1989) Double dissociation of egocentric and allocentric space following medial prefromtal and parietal cortex lesions in the rat. Behan. Newnosel. 103, 956-961.

180. Kessler J., Markowitsch H.J. and Sigg G. (1986). Memory related role of the posterior cholinergic system. Int. J. Neurosei. 30, $101-119$.

18H. Kirk. R.C., White K.G. and McNaughton N. (1988) Low dose scopolamine affects diseriminability but not rate of forgeting in delayed conditional discrimination. Psychopharmacology $96,541-546$.

182. Klockgether $T$. and Turski L. (1990) NMDA anagonists potentiate antiparkinsonian actions of L-dopa in monoamine-depleted rats. Amn. Newrol. 28,539-546.

183. Kolb B. (1984) Functions of fromal cortex of the rat: A comparative revew. Brain Res. Reu. 8.65.98.

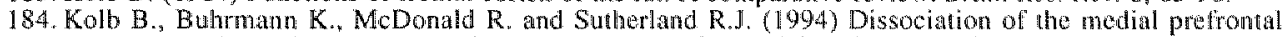
cortex, posterior parietst, and posterior temponal cortex for spatial navigation and recognition memory in the rat. Cereb. Cortex 4, 664-80.

185. Kolb B., Sutherland R.J. and Whishaw I.Q. (1983) A comparison of the contributions of the frontal and parietal association cortex 10 spatial localization in rats. Behav. Newrosi. 97, 13-27.

186. Koopmans Summers W., Majovski L.V., March G.M., Tachiki K. and Kling A. (1986) Oral tetrabydroaminoseridine in long-tern treatment of senile chementia, Alzheimer type. N. Eng. J. Med. 315, 1241.1245.

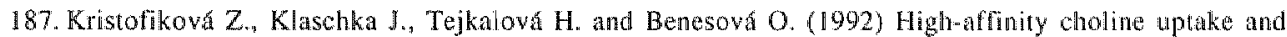
muscarinic receptors in rat brain during aging. Arch. gerontol. Geriat, 15, 87-97.

188. Le Jeune H., Cecyre D..., Rowe W., Meaney M.J. and Quirion R. (1996) Jonotropic glutanate receptor subtypes in the aged memory-impaired and unimpaired Long-Exans rat. Neuroscience 74, 349-363.

189. Lee J.M., Ross E.R., Gower A., Paris J.M., Marterissom R. and Lorens S.A. (1994) Spatial leantuing deficis in the aged rat: Newrosnatomical and newrochemical corfelates. Brain Res. Bull. 33, 489-500.

190. Lees A.J. and Smitt E. (1983) Cognitive deficits in the early stages of Parkinson's disease. Brican 106. $257-270$.

191. Levey A.L. (1996) Muscarinic acetylcholine receptor expression in memory circuits: Implications tor treatment of Alzheimer disease. proc. Natl Acad. Sci. U.S.A. 93. $13541-13546$.

192. Levin E.D. (1988) Psychopharmalogical effects in the radial-arm maze. Neurosci. Biobehas" Res. 12, 169 175.

193. Levin E. D. (1988) Scopolamine interactions with DI and D2 antagonists on radial maze performance in rats Behau. Newral Biot. 50, $240-245$.

194. Levin E. D. Briggs. S.J., Christopher N.C. and Rose J.E. (1992) Prersistence of chronic nicotine induced cogritive faciliation. Behat, Nearal Biol, 58, 152-158.

195. Levin E.D., Castongut M. and Ellison G.D. (1987) Erfects of the nicotinic receptor blocken mecamylamine on radialmarm maze perfomance in rats. Behaw. Neural Blol. 48, 206-212.

196. Levin E... Christopher N.C. Briggs S and Rose J.E. (1993) Chronidu nicoline rewerses working menory deficits caused by lesions of the fimbria or medial basaloconicall projection. Cogn. Brain Res. 1, 137-143.

197. Levin E.D., MeGurk S.R., Rase J.E. and Butcher L.L. (1990) Chohnugic-dopanincergic interactions in cognitive perfomance. Behav. Nearal Biol. 54, 271-299.

198. Levin E D. and Rose J.E. (1991) Interactiwe effects of DI and D2 agonists with scopolamine on radial arm mave performance. Phamacol. Biochen. Behaw. 38, 243-246.

199. Levin E.D. and Rose $\mathbb{~}$.E (1991) Nicotinic and muscarinic interactions and choice accuracy int the radial-arm maze. Brain Res. Bull. 27, 125-128.

200. Larag K.C.., Hon W. Tyan Y.M. and Liao W.L. (1994) Involvenent of hippocampal NMDA and AMPA receptors in acquistion, formation and retrieval of spatial memoty in the Morris water maze. Chin. $J$, Physiol. 37, 201-212

201. Liang K.C., McGaugh J.L. Martinez J.L.,., Jersen R.A., Vasquez B.J. and Messing R.B. (1982) Postraining amygdaloid lesions impair retention of an inbibitory avoidace response. Behav. Brain Res. 4,237 249.

202. Liang K.C., McGaugh J.L. and Yao H.-Y. (1990) Involwernent of amygdala pathways in the influence of post-training intramygdala norepinephrine and perpheral epimephrine in memory storage. Bran Res. 508 . $225-233$

203. Lindner M.D. Balch A.H. and VanderMaelen C.P. (1992) Short forms of "raference-" and "working- 
memory" Morris water naze for assessing age-related deficits. Behav. Newral Bol. 58, 94-102.

204. Lindner M.D. and Schallert T. (1988) Aging and atropine effects on spatial navigation in the Morris water lask. Behav. Neurssck. 102,621-634.

205. Lippa A.S., Loultis C.C., Rorrosen I. Cordasco D.M., Critchent D.I. and Joseph J A. (1985) Confomational changes in muscarinic receptors may produce diminished cholinergic neurotransmission and memory deficits in aged rats. Neurobiol. Aging 6, 317-323.

206. Lipska B.K. Jaskiw G.E. and Weinberger D.R. (1994) The effects of combined prefrontal cortical and hippocampal damage on dopamine-related behaviors in rats. Pharmacol. Biochem. Behav. 48, 1053-1057.

207. Luine $V$. and Hearns M. (1990) Spatial memory deficuls in aged rats: Contributions of the cholinergic system assessed by ChAT. Brain Res. 523, 321-324.

208. Lukaszewska I. and Radulska A. (1994) Object recognition is not impaired in old rats. Acta Neurobiol. Exp. 54. 143.150 .

209. MHarzi M, and Jarrard L.E. (1992) Strategy selection in a task with spatial and nonspatial components: Eiffects of frmbria-fomix lesions in rats. Behow. Neural Biol. $58,171-179$.

210. M'Harzi M. Jarrard L.E., Willig F., Palacios A, and Delacour J. (1991) Selective fumbria and thalamic lesions differentially impair forms of working memory in rats. Behov. Neural Biol. 56, 221-239.

211. Malenka R.C. (1995) LTP and LDP: Dynamic and interactive processes of synaptic plasticity. Neuroscientist 1, 35-42.

212. Maragos W.F., Greenamyre J.T. Penney J.B. and Young A.B. (1987) Glutamate dysfunction Azheimer's disease: An thypothesis. Trends Neurosci. $10,65-68$.

213. McDonald R.J. and White N.M. (1994) Parallel information processing in the Water Maze: Evidence for independent memory systems in wolving dorsal stratum and hippocampus. Behov. Neural Biol. 61, 260270.

214. McEntre W.J. and Crook T.H. (1990) Age-assceiated memory impairment: A role for catecholamimes. Neurology $40,526,530$.

215. McEntree WJ. and Crook T,H. (1991) Serotonin, memory, and the aging brain. Psychopharmacology (Berlin) 103, 143-149.

216. MoGurk S.R., Levin E.D. and Butcher L.L. (1988) Cholinergic-dopaminergic interactions in radial-arm maze performance. Behav. Neural Biol. 49, 234-239.

217. MeGurk S.R., Levin E.D. and Butcher L.L. (1992) Dopaminergic drugs reverse the impairment of radial maze perfonnance caused by lesions involving the cholinergic medial pathway. Neuroscience 50, 129-135.

218. McNamara R.K. and Skelton R.W. (1993) The neuropharmacological and neurochemical basis of place learning in the Morris water maze. Brain Res. Rev. 18, 33-49.

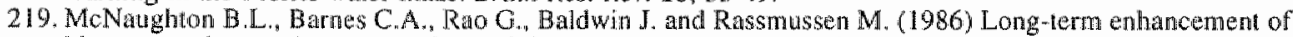
hippocampal synaptic transmission and the acquisition of spatial information. J. Neurosci. 6, 563-571.

220. Mendelson S.D., Quartermain D., Francisco T. and Shemer A. (1993) 5-HT/A receptor agonists induce anterograde amnesia in mice through a postsynaptic mechanism. Eur. J. Pharmacol. 236, 177-182.

221. Menegur A., Bisso G.M. and Michalek H. (1992) Age-related changes in acetylcholinesterase and its molecular forms in various brain areas of rats. Neurochem. Res. 17. 785-790.

222. Mesularm M.M., Mufson E.J., Wainer B.H. and Levey A.I. (1983) Central cholinergic pathways in the rat: An overview based on an alternative nomenclature (Ch. 1-Ch.6). Nenroscience 10, 1185-1201.

223. Meyer E.M., Onge E.S.T, and Crews F.T. (1984) Effeces of aging on rat cortical presynaptic cholinergic processes, Netrobiol. Aging 5, 315-317.

224. Michatek. H., Fontune S. and Pintor A. (1989) Age-related differences in brain choline acetyltransterase, cholinesterases and muscarinic receptor sites in two strains of rats. Neurobiol. Aging 10, 143-148.

225. Monoy E.A. Kirk R.C. and McNaughton N. (1992) Alzheimer's dementia produces a loss of discrimination but no increase in rate of memory decay in delayed matching to sample. Neturopsychologia 30, 133-143.

226. Morris R.G. and Baddeley A.D. (1988) Primary and working memory functioning in Alaheimer-type dementia. J. Clin. Exp. Netropsychol 10, 279-296.

227. Morris R.G.M. (1989), Synaptic plasticity and learning: Selective impaiment of learning in rans and blockade of long-term potentiation in viwo by the NMDA receptor antagonist AP5. J. Neurosei. 9, 3049. 3057.

228. Morris R.G.M., Anderson E., Lynch G.S. and Baudry M. (1986) Selective impaiment of learning and blockacle of long-term potentiation by an $N$-methyl- D-aspartate receptor antagonist, AP5, Nante 319,774 . 776.

229. Morris R.G.M., Garrard P., Rawlins J.N.P. and O"Keefe J. (1982) Place nawigation is impaired in rats with hippocampal lesions. Nature 297,6812683.

230. Morris R.G.M., Schenk F. Tweedie F. and Jarrard L.E. (1990) Ibotenate lesions of hippocampus and/or subiculum: Dissociating components of allocentric spatial learning. Eur. J. Neurosci. 2, 1016-1028.

231. Muir J.L. (1997) Acetylcholine, aging, and Alzheimer's disease. Pharmacol. Btochem. Behav. 56, 687-696.

232. Muir J.L., Page K.J.. Sirinathsinghij D.J.S., Robbins T.W. and Everitu B.J. (1993) Excitotoxic lesions of basal forbrain cholinergic neurons: Effects on learning, memory and attention. Behav. Brain Res. 57, 123 . 131.

233. Mumby D.G, and Pinel J.P.J. (1994) Rhinal cortex lesions and object recognition in rats. Behav. Neurosci. $1108,11-18$ 
234. Muray C.L. and Fibiger H.C. (1986) Plocarpine and psychostigmine artenuate spanal memory

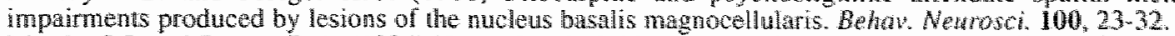

235. Murtha S.J. and Pappas B.A. (1994) Newochemica, histopathological and mnemonic effects of conbined lesions of the medial septall and serotomin afferents to the hippocampus. Bram Res. 651, 16-26.

236. Myher T. (1992) Selective lesions in the temporal-hippocampal region of the rat: Fifion on acquisition tand retention of a wisual discrimination lask. Behaw. Neural Biol. $58,8-15$.

237. Mybrer T. (1993) Animal models of Alzhener's disease: Gluamalergic denervation as an aldormative approach to cholinergic denervation. Neurosci. Biobehav. Rev. 17, $195-202$.

238. Nicolle M.M. Bizon J.L and Gallagher M. (1996) In vitro autoradiography of ionotropic glutamate receptors in hippocampus and sitriatum of aged Long-Evans rats: Relationship to spatial learning. Neuroscience 74, 741-756.

239. Nilsson O.O., Shapiro M.L., Gage F.H., Oton D.S. and Björkhund A. (1987) Spatal leaming and menory following fimbria-fornix transection and grafting of fetal septal neurons to the hippocumpus. Fxp. Brain $\operatorname{Res}, 67,195-215$.

240. Nilssion O.G., Strecher R.E., Daszuta A. and Björklund A. (1988) Combined cholinergic and serotonergic denervation of the forebrain produces severe defteits in a spatial leaning ask in the rat. Brain Mes. 453 , $235-246$.

241. Nordberg A., Alafuzoff 1 , and Winblad B. (1992) Nicotinic and muscarinic subtypes in the human brain: Changes with aging and dementia. J. Nenrosct. Res. 31, 103-111.

242. Normile H.J. and Alman H.J. (1992) Effects of combined acetylchohinesterase inhibition and serotonergic receptor blockade on age-associated memory impaiments in ras, Nemobiol Aging 13, 735-740.

243. Nut D. I. 1993) The approach to new anxiolylics. J. Pharm. Phamacol. 45, 352-354.

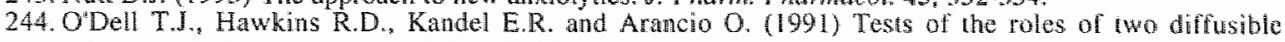
substances in long-term potentiation: Evidence for nitric oxide as a possible ealy retrograde messenge Proc. Nan Acad. Sci. U.S.A. 88, $11285-\llbracket 1289$.

245. O'Keefe J. and Nadel L. (1978) The hippocanpus as a cognime map. Clarendon Press, Oxford.

246. Ohno M. Yamamoto T. and Shigenori W. (1993) Blockade of hippocampal nicotinic receptors umptirs working memory but not reference memory in rats. Pharmacol. Biochen. Behan. 45, 89.93.

247. Onno M. Yamamoto T. and Watanabe S. (1994) Blockade of hippocampal ML muscalinic receptors impairs working memory performance of tats. Bran Res. $650,260-226$.

248. Okatih H. (1987) Performance and dominanil strategies on place and cue tasks following hippocampal lesions in rats. Pychobiology 15, 58-63.

249. Oliveira M.G.M., Bueno O.F.A., Pomarico A.C. and Gugliano E.B. (1997) Strategies used. by hippocampal and caudate-putamen-lesioned rats jn a leaming task. Neurobiol learn. mem, 68, 32.40.

250. Olion D.S. (1983) Memory functions and the hippocampus. In Nerabrobiog of he hippocampus. (ed. Seifert W.), pp. 335-373. Acadernic Press, London.

251. Ottom D.S., Becker J.T. and G.E. H. (1980) Hippocampal function: Working memory or cogntive mapping. Physiol. Psychol. 8, 239-246.

252. Olion D.5. Becker J.T. and Handelman O.E. (1979) Hippocampus, space, and memory. Behav. Brian Selences $2,313-365$.

25.7. Olton D.S. and Papas B.C. (1979) Spatial memory and hippocampal function. Neuropsychologia 17.669. 682 .

254. Olion D.S., Wenk G.L., Church R.M. and Meck W.M. (1988) Altention and the fromtal contex as examined by simultaneous temporal processing. Nesropsychologia, $307-315$.

255. Ondy J.M. Thomas G.J., Volpe B.T., Dunlap W.P. and Colombo P.M. (1988) An animat modal of: hunan-lype memory loss based on agimg, lesion, forbran ischeman, and drug studies wilh the rat. Neturobiol. Aging, 9,667-683.

256. Packard M. G. Calill L. and McGaugh J.L. (1994) Amygdata moduation of hippocampal-dependent and caudate nucleus dependent memory processes. Proc. Natl Acad. Sei. U.S.A. 91,8477-8481.

257. Packard M.G., Hirsch R. and White N.M. (1989) Differential elfecis of fornix and caudate nucleus lesions on two radial maze lasks: Evidance for mulaple memory systems. J Nenrosci. 9, 1465-1472.

258. Packard M.G. and McGaugh J.L. (1992) Double dissiociation of fornix and caudate aucleus lesions un acquisition of two water maze tasks: Funther evidence for multiple memory systems, Behaw. Nerrasei. 106 439.446.

259. Packard M.G. and McGaugh J.L. (1996) Inwctivation of hippocampus or caudate nucleus with lidocaine diferentially affects expression of place and response learning. Neurobial. Lean. Menory 65.65-72.

260. Packind M.C. and White N.M. (1991) Dissociation of hippocampus and caudate nucleus memory systems by postraning intracerebral injection of dopamine agonists. Behav. Newrosci. 105, 295-306.

261. Papez J.W. (1937) A proposed mechanism of emotion. Archives of Nelurological Psychiatry 38, 725 743.

262. Pappas B.A., De la Torre J.C. Davidson C.M., Keyes M.T. and Fortin T. (1996) Chronic reduction of cerebral blood thow in the adult rat: Late-emerging CA 1 cell loss and memory dystumetion. Brain Res. 708 . 50.58 .

263. Partiot A., Yerin M., Pillon B., Teixeira Ferreira C., Agid Y. and Dubois B. (1996) Delayed response tasks in basal ginglia lesions in man. Further ewdence for a strialom frontal cooperation in behavioural adaptation. Neuropsychologia $34,709.721$. 
264. Pasinelli P., Ramakers G.MJ, Urban IJ.A., Hens J.JH. Oestreicher A.B., Graan PN.E. and Gispen W.H. (1995) Long-lerm potentation and synaptic protein phosphorylation. Behaw. Brain Res: 66, 53-59.

265. Paxinos $\mathrm{G}$ (1994) The rat herwous system. 2 nd edn. Academic Press, San Diego.

266. Pedigo N.W.J. Minor L.D. and Kumtei T.N. (1984) Cholinergic drug effects and brain muscarinic receptor binding in aged rats. Newrobiol A ging $5,227-233$.

267. Pelleymounter M.A., Beaty $G$ and Gallagher M. (1990) Hippocampal 3H.CPP binding and spatial

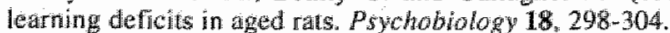

268. Perourka S.J. (1995) 5-HT receptors: Past, present and funne Trends Neurosei. 18, 68-69.

269. Perry $\mathbb{E} . K$. (1986) The cholinergic hypothesis - Ten years on. Br. Med. Bull. 42,63*69.

270. Perry E.K., Smith C.J. Coun J.A. and Perry R.H. (1990) Cholinergic nicotinic and muscarinic receptors in dementia of Alzlime Parkinson and Lewy body types. J. Neural. Transm. Park. Dis. Dement. Sect. 2 , $149-158$.

271. Perry E.K., Tomlinson B.E., Blessed G., Perry R.H., Cros A.J. and Crow T.J. (1981) Nenropathological biological observations on the noradrenergic system in Alzheimer"s disesse. N. Neurol Sci. 51, $279-287$.

272. Perryman K.M. and Fituen L.J. (1993) Delayed matching-10 sample performance during a double-blind trial of tactine (THA) and lecthin in patients with Alahelmer's disease. Life Sci. 53, 479-486.

273. Petcmel A., Hughey D., Wenk $O$ and Ollon $D$. (1988) Basal forbrain and memory: Neurotoxic lesions impain serial reversals of apatial discrimination. Psychobiology 16,54-58.

274. Pietrak E.R., Wilce P.A. and Shanley B.C. (1989) Plasticity of brain receptors in aging rats: The adaptive response to scopolamine and ethanol treatment. Newosci. Lett. 104, 331-335.

275. Plaznik A. Palejko W. Nazar M. and Jessa M. (1994) Effects of antagonists at the NMDA receptor complex in iwo models of anxiety. Eun. Neuropsychopharmacol 4, 503-512.

276. Prickaerts J., Ratijmakers W. and Blokland A. (1996) Effects of myocardial infarction and captopril therapy on anxiety-related behaviors in the rat. Physiol. Behav, 58,43-50.

277. Quirion R., Wilson A.. Rowe W . Aubert I., Richard J., Doods H., Parent A., White N. and Meaney M.J. (1995) Facilitation of acelylitholine relase and cognitive performance by an $M_{2}$-muscarinic receptor antagonist in aged nemory-impaired rats. J. Neurosci. 15, 1455-1462.

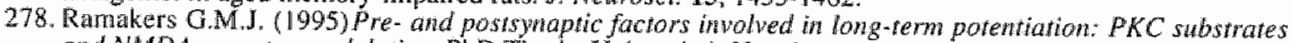
and NMDA receptor modulation. PhD Thesis, Universiteit Urectht.

279. Ramakers G.M.J., De Graan P.E.N., Urban I.J.A., Kraay D., Tang T., Pasinelli P. Oetreicher A.B. and Gispen W.H. (1995) Temporal differences in the phosphorylation state of pre- and postsynaptic protein kinase C substrates B50-10AP-43 and neurogranin during long-term potentiation. The J. Biol. Chem. 270. $13892 \cdot 13898$.

280. Rasmussen M. Barnes A. and McNaughton B.L. (1989) A systematic tesf of cognitive mapping, workingmemory, and temporal discontiguity itheories of hippocampal function. Psychobiology 17, 335-348.

281. Rawlins J.N.P. (1985) Associations across time: The hippocampus as a temporary memory store. Behaw. Brain Sci. 8, $479-496$.

282. Reading P.J. and Dunneti S.B. (1991) The affects of excitotoxic lesions of the nucleus accumbens on a matching to position task. Behav. Brain Res. 46, 17-29

283. Reinstein D.K., Hanugan I.H. and Isacson R.L. (1982) Time course of certain behavioral changes after hippocampal damage and their alteration by dopaminergic intervention into mucleus accumbers. Phambcol. Brochem. Behaw. 17, 193-202.

284. Richer-Levin G. Camerari L. and Bliss T.V. (1995) Long-term potentiation and glutamate rellease in the dentate gytus. Links to spatial learing. Behav. Brain. Res. 66, 37-40.

285. Rickkinen P. Sirvio d. and Riekkinen P. (1990) Interaction between raphe dorsalis and nucle us basalis magnocellularis in spatial lesuing. Brain Res. $527,342-345$.

286. Rinne J.O., Myllykyla T., Lomberg P. and Marjamaki P. (1991) A postmortem study of brain micotimic receptors in Patkinson's and Alzheimer's disease. Brain Res. $\mathbf{5 4 7 , 1 6 7 - 1 7 0 .}$

287. Robinson G.S., Crooks G., Shinkman P.O. and Gallagher M. (1989) Behavional effects of MK-801 mimic deficits associated with hippocampal damage. Psychobiology $17,156-164$.

288. Rols E.T. (1994) Netrophysiology and cognitive functions of the striatum. Rev. Neurol. Paris 150, 648660 .

289. Rothblat L.A. and Kromer L.F. (1991) Object recognition memory in the rat: The role of the hippocampus. Behaw. Brain Res. 42, 25-32.

290. Rowell P.P. and Winkler D.L. (1984) Nicotinic stimulation of [3H]acetyloholine rellease from mouse cercbral cortical symaptosomes. If Nenrochem. 43, 1595-1598.

291. Rupniak N.M.J., Samson N.A., Tye S.J., Field M.J. and Iversen S.D. (1991) Evidence against a specifie effect of cholnergic drugs on spatial memory in primates. Behav. Brain Res. 43, 1-6.

292. Sagar H.J., Sullivan E.V., Gabrieli J.D., Corkin S. and Growdon J.H. (1988) Temporal ordering and shortterm memory deficits in Parkinson's disease. Brain $111,525-539$.

293. Sahgal A., Galloway P.H. McKeith 1.G., Lloyd S., Cook J.H., Ferrier N. and Edwardson J.A. (1992) Mathing-to-sample deficis in patients with sente dementias of the Alzhemer and Lewy body types. Arch.
Nenol. 49, 1043-1046. 294. Salugal A. and Keith A.B. (1993), Combined serotonergic-cholinergic lesions do mot disrupt memory ür rats.
Pharmacol. Brochen. Behov. 45, 995-1001. 
295. Sahgal A., Keith A.B. and Lloyd S. (1990) Effers of nicotine, oxutenorine and 9-amino $1,2,3,4$ tetrahydroacridine (acrilae) on matching and main-matching 10 postaton in rats: No evidence lor memonic enhancement. J. Psychophamacol. 4,210-218.

296. Saint Cyr I.A., Taylor A.E. and Lang A.E. (1988) Procedural learning and neostriatal dystunction in máti. Brain 111. $941-959$

297. Salado Castillo R, Diaz da Guante M.A., Alvarndo, R. Quirarte G.L. and Prado Alcala R.A. (1996) Effects of regional GABAergic blockade of the striatum on memory consolidaton. Newrobiol. Leary. Wem. 66 . $102-108$

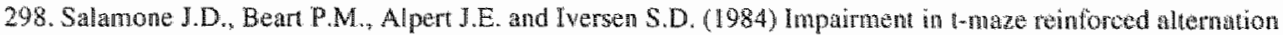
pertomance following nucleus basalis magnocellunaris lesions in rats. Behaw. Brain hes, 13,63.70.

299. Sanchez-Sanced F. De Bruin J.P.C., Heinsbroek R.P.W. and Verwer R.W.H. (1997) Spatiall delayed alternation of rats in a T-maze: Effects of neurotoxic lesions of the medial profromal cortex and of T-maze rotations. Behav. Brain Res, 84,73-79.

300. Sara J.J. and Devauges $V$. (1989) Idazoxan, an $\alpha-2$ antagonist, facilitates memory retrieval in the mat. Behav. Neural Biol. 51, $401-411$.

301. Sara S.f. (1989) Noradrenergic-interaction: Its possible nole in memory dystunction associnted with senile dementia. Arch. Gerontol. Gerion. Suppl. $1,99-108$.

302. Sarter M. and Bruno J.P. (1997) Trans-synaptie stimulation of contical acetylcholine and enhancement of attentional functions: A. rational approach for the development of cognition enhancers. Behove. Broin Res. $83,7-14$.

303. Sarter M., Bruno J.P., Givens B., Moore H., MoGatugh J. and MoMahon K. (1996) Neuronal meshanisms mediating drug-induced cognition enhancement: Cognitive activily as a necessary intervening variable. Cogn. Brain Res. 3, 329-343.

304. Sarter M. and Markowitsch H.J. (1985) lnvolvement of the amygdala in learning and menory: A critical review, whth emphasis on anatomical relations. Behav. Neurosci. $99,342.380$.

305. Saucier D. and Cain D.P. (1995) Spatial learning without NMDA receptosdependent long-term potentiation. Nature 378, 186-189.

306. Saucier D., Hargreaves E. B., Boon F. Vanderwolf C.H. and Cain D.P. (1996) Detailed behavional analysis of water maze acquisition under systentic NMDA or muscarinic antagonism: Nonspatial pretraining climinates spatial hearning deficits. Behav. Neurosci. 110, 103* 16.

307. Scali C., Casamenti F. Pazzagli M. Bartolini L. and Pepeu G. (1994) Nerve growth factor increases extracelluhar acetylcholine levels in the parietal cortex and hippocampus of aged rats and testores object recognition. Newrosci. Lett. 170, 117.120.

308. Schacter G.B. Yang C.R. Innis N.K. and Mogenson G.J. (1989) The role of the hippocampal-nucleus accumbens pathway in radial-arm maze pertormance. Brain Res. 494, 339-349.

309. Schmidt W.J., Bubser M. and Haber W. (1992) Behavioural phamacology of glutamate in the basal ganglia. J. Neural. Transm. Suppl. 38,65-89.

310. Schulz D.W. Kuchel G.A. and Zigmond R.E. (1993) Decline in response to nicotine in aged rat striatum:

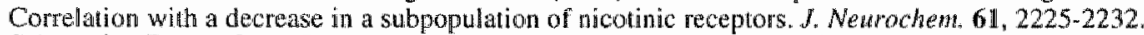

31 I. Schwarting R. and Carey R.J. (1985) Deficits in inhibitory avoidance after newrotoxic lesions of the ventral stratum are neurochemically and behaviorally selective. Behav. Brain Res. 18, 279-283.

12. Selkor D.J. (1991) The nolecular pathology of Alzhemer"s disease. Neuron 6, 487-498.

313. Shapho M.L. and Caramanos 2. (1990) NMDA artagonist MK-801 impairs acquisition but not perfomance of spatial wonking and peference memory. Psychobiology 18, 231-243.

314. Sharma A.C. and Kulkarni S.K. (1993) Evidence for benzodiazepine receptor intoraction with MKK 801 in ankiety related behaviour an rats. Indian J. Exp. Brol. 31. 191-193.

315. Sharps M.J. and Gollin E.S. (1987) Memory for object loc ation in young and elderly adullis. $J$. Geronol. $42,336-341$.

316. Straw C. and Aggleton J.P. (1993) The effects of formix and medial prefromal lesions on delayed non matching-to-sample by rats. Behav. Brain. Res. 54, $91-102$.

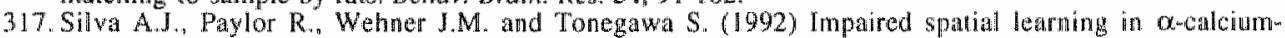
calmodulin kinase 11 mutant mice. Science $257,206-211$.

3.18. Silva A.J., Stevens C.F. Tonegawa S. and Wang Y. (1992) Deficient hippocampal long term potentiation in $\alpha$-calciun-calmodulin kinase II mulant mice. Scrence 257, 201-206.

319. Sirvio J., Lahtinen H. Riekkinen P.J. and Riekkinen P.J. (1994) Spatal learning and noratrendine content in the brain and periphery of young and aged rats. Exp. Netrol. 125, 312-315.

320. Snith G. (1988) Animal models of Alzheimer's disease: Experimental cholinergic denervation. Brain Ren Rev, 13, 103-118.

321. Smith M.L., Deadwyler S.A. and Booze R.M. (1993) 3. D reconstruction of the cholinergic bagal fortbrair system in young and aged rats. Neurobiol. Aging 14, 389-392.

322. Smolders 1., Bogaert L., Ebinger G. and Michote Y. (1997) Muscatinic modulation of striatad dopamine, glutamate, and GABA release, as measured with in vivo microdidysis. f. Newrochem $68,1942-1948$.

323. Socci D.J., Sanberg. P.R. and Arendash G.W. (1995) Nicotine enhances morris water maze performance of young and aged rats. Neurobiol. Aging 16,857-860.

324. Söderpalm B. and Engel J.A. (1991) lawolvement of the GABAAbenzodiazepine choride ionophore 


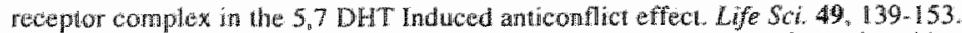

325. Solana Figueroa R, and Prado Alcala R.A. (1990) Retrograde amnesia produced by intrastriatal atropine and its reversal by cholne. Lfo $\$$ ch, $46,679-86$.

326. Spangler E.L Clachich M.E., Cuntis N.J. and Ingram D.K. (1989) Age-related impairment ir complex maze learning in rats. Relutionship to neophobia and cholnergic anagonism. Newobiol. Aging 10 , 133 [4.

327. Spungler E.L. Waggie K.S., Hengemilyle J., Roberts D. Hess B. ard Ingran D. K. (1994) Behavioral assessment of aging male figher 344 and brown norway rat strains and their fil hybrid. Weurobiol. Aging $15,319.328$.

328. Spencer D.G., Horyath E. and Traber $\$$. (1986) Direct antoradiographic determination of M1 and M2 muscarinic acetylcholime receptor distribution in the rat brain: Relation to cholinergic nuclei and projections. Bratin Res. 380, 59-68.

329. Squire J.R. (1986) Machanisms of meraty. Sulence $232,1612-1619$.

330. Squire L.R (1992) Decharave and nonwedaraive memory: Mulliple bran systems supponting learning and memory. J. Cogn. Neurosici, 4, 232-243.

33. Stankov L. (1988) Aging, attention, and intelligence. Psychol. Aging 3, 59-74.

332. Starr M.S. (1995) Glutamate/dopamine D1/D2 balance in the basal ganglia and its relevance to Parkinson's disease. Symapse 19, 264-293.

333. Star M.S. and Star B.S. (1994) Comparison of the effects of MMDA and AMPA antagonists on the locomotor activily induced by selective D1 and D2 dopamine agonists in reserpine-rreated mice. Psychopharmacology (Berlin) 114, 469-476.

334. Steckler T. Andrews J.S. Marten P. and Turroer J.D. (1993) Effects of NBM lesions with two neurotoxins on spatial mentory and atoshaping. Pharmacol Biochem. Behav. 44, 877-889.

335. Steele T.D., Hodges D.B., Jr., Levesque T.R., Locke K.W. and Sandage B.W., Jr. (1996) The Dl agonist dihydrexidine releases acerylcholune and improves cognition in rats. Ann. $N$. Y. Acad. Sci 777, 427-30.

336. Steinbusch HI.W.M. and De Vente J. (1997) New wistas on the neurobiology of depression. Co-localization of serotonin-, dopamine- and nitric oxjide synthase-containing neurons in the dorsat raphe nucleus. In Depression (ed. Honig A and Van Prag H. M. . Pp 179-196. Wiley and Sons.

337. Sugaya K. Giacobin E. and Chimppialli V.A. (1990) Nicotinic acetylloline receptor subtypes in human frontal contex: Changes in Alzheimer's disease. J. Newrosci. Res. 27, 349-359.

338. Sutherland R.J. and Rodriguez A.J. (1989) The role of the fornix/fimbria and some related subcortical structures lin place learning and memory. Behaw. Brain Res. 32, 265-277.

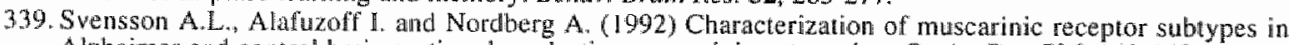
Alsheimer and control brain cortices by selective muscarinic antagonists. Brain. Res. 596, $142-148$

340. Taghzouti K., Le Moal M. and Simon H. (199i) Suppression of noradtenergic innervation compensates for behavioral deficits induced by lesion of dopaminergic terminals in the lateral septum. Brain Res. 552,124 . 128.

341. Taghzouti K. Simon H. Louilot A., Herman J.P. and Le Moal M. (1985) Behavioral study after local injection of 6-hydroxydopamine into the nucleus accumbens in the rat Brain Res. 344, 9-20.

342. Tamaru $\mathrm{M}_{\text {, }}$ Yoneda $\mathrm{Y}$. Ogita $\mathrm{K}$. Shimizu J. and et al. (1991) Age-related decreases of the N-methyl-Daspartate receptor complex in the rat carebral cortex and hippocampus. Brain Res. 542, $83-90$

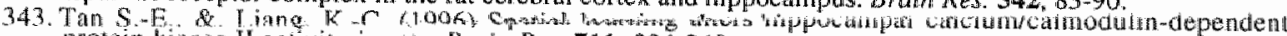
Prowin kndse II activity in rats. Bram Res. 711. 234.240.

344. "Tanda H. Taira T. Picpponen T" P. and Honkanen A. (1994) Effect of sex and age on brain monoamines and spatiall learning in rats. Newobial. Aging $15,733-741$,

345. Thylor A.E., Stint Cyr J.A. and Lagg A.E. (1990) Memory and leaming in early Parkinson"s disease: Evidence for a "frontal lobe syndrome". Brain. Cogk. 13, $211 \times 232$.

346. Tery A.V. (199) Scoplamine reversal of nicotine enhanced delayed matching-lo-sample performance in monkeys, Pharmacol. Biochem. Beham, 45, 925-929.

347. Treit D., Robinson A., Ronzinger S. and Pesold C. (1993) Anxiolytic effects of serotonergio interventions in the shock-probe burying test and the elevated plus-maze. Behav. Brain Res. 54, 23 - 34.

348. Turski L. and Turski W.A. (1993) Towards on whdersianding of the role of glutamia in neunodegenerative disopders: Dnergy metabolism and neuropathology. Experimentia 49, 1064-1072.

349. Uylings H.B.M. and Var Ecien C.G. (1990) Qualitative and quantiative comparison of the prefromtal cortex in rat and primates, including humans. In The prefrowal contex" its stracture, function and pathology, progess in Brain Res. (ed. Uylings H. B. M., Van Eden C. G. De Bruin I. P. C., Cormer M. $A$. and Feenstra M. G. P.), Vol. 85 , pp. 31.62 . Elsevier, Amsterdam.

350. Van der Stay E...) and Blokinnd A. (1996) Behavional difference between outbred Wistar, inbred Fischer 344, Brown Norway, and hybrid Fuscher 344x Brown Norway rats. Physiol Behav. 60, 97-109.

351. Van der Staty F.J, and Blokland A. (1996) Repeated assessment of spatial discrimination performance of aged rats in the Motris water escape tasik. Neurobiol. Learm. Memn 65, 99-102.

352. Van der Stany F.J., Blokland A. and Raaimakers W.G.M. (1990) Different time course for age-related changes of behavio in a complex spatial cone-field discimination task in Lewis rats. Psyohobiology 18.
$305-311$.

353. Van der Stay F.J., Raajhnakers W.G.M., Lamners A.J.C. and Tonnaer J.A.D.M. (1989) Selective 
fimbria lesions impair acquision of working and reforence memory of was in a complex spanal discrimination task. Betaw. Brain Res. 32, 151-161.

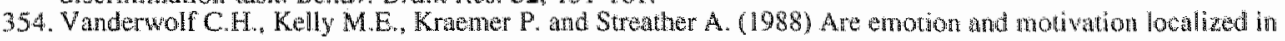
the limbic system and nucleus accumbens? Behav, Brow Res. $27,45-58$.

355. Wachel H. (1991) Antiparkinsonian dopamine agonists: A review of the pharmookinetics and meuropharmacology in anmals and humans. J. Newrot. Transm. 3, $151-201$.

356. Warburton D.M. (1992) Nicotine as a cognitive enhancer. Bol Psychiatro 16, 181-191.

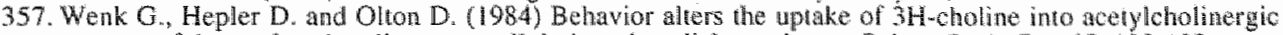
meurons of the nucleus basalis magnocelluharis and medial septal area. Behav. Broin Res. 13, 129-138.

358. Wenk G., Hughey D., Boundy V., Kim A., Walker L. and Olton D. (19g7) Neurotransmitters ard menory: Role of chalinergic, serotonergic, and noradrenergic systerns. Behav. Nentasci. 101, 325-332,

359. Wen G.L. (1993) A primate model of Alzheimer's disease. Behav. Brang Res. 57, $117-122$.

360. Wenk O.L. and Olton D.S. (1984) Recowery of neocortical choline acelylransferase activity lollowing ibotenic acid injection into the nucleus basalis of Meynert in rats. Bram Res. 293, $184,186$.

361. Whishaw I.Q. and Tomie J.-A. (1987) Cholinergic receptor blockade produces imparments in in sensormotor subsystem for place nawigation in the rat: Evidence from serisory, motor, and acquistion tests in a swimming pool. Behaw. Neurosict 101, 603-616.

362. Whitehouse P.J. and Au K.S. (1986) Cholinergic receptors in aging and Alzhemer's disense. Prog. Newol. Prychopharmacol. Biol. Psychiat 10,656.

363. Willner P.. Wise D. and Elis T. (1986) Specific dismuption of spatial behavior in mats by central muscarinic receptor blackade. Psychopharmacology (Berl) $90,229.235$.

364. Winocur G. (1991) Functional dissociation of the hippocampus and prefontal conter in leaming and memory. Psychobiology 19, 11-20.

365. Wimter J.C. (1997) The effects of age on continuous learning in the radial maze. Phystod Behav. $61,609$. 612.

366. Wolters E.C. (1992) Basic concepts in the treatment of Parkinson's disease. In Curmetronds in the treatrrent of Parkinson's disease. (ed. Agid $\mathrm{Y}$.), pp. 39.48. John Libbey and Comp., Londion.

367. Wright I.K. Upton $N$ and Marsden C.A. (1992) Effect of estabilished and putative anxiolytics on extracellular 5-HT ind 5-HIAA in the ventral hippocampus of rats during belawiour on the elevated $X$-maze. Psychopharmacology 109, 338-346.

368. Wu C.F., Bertorelli R., Sacconi M., Pepeu G. and Consolo S. (1988) Decrease of brain acetylcholine release in aging freely-nnoving rats detected by microdialysis. Neurobiot. Aging 9, 357-361.

369. Yamamoto T., Hironalka K. and Ueki S. (1990) Leaming and menory in rats with delayed matching to lever location procedures. Eut. J. Pharmacol. 183 , 2359-2360.

370. Yanai I., Rogel-Fuclas Y., Pick. C.G., Sloikin T., Siedler F.J., Zahalka E.A. and Newnan M.E. (1993) Septohippocampal cholinergic changes after destruction of the Al0-septal dopaminergic pahways. Nerrophamacology $32,113-117$. 


An




\section{Introduction: Neurodegeneration}

Neurodegeneration can be best defined as a decline in function and dying of neuronal tissue. The cause(s) and precise mechanism(s) of neuronal cell death of neurodegenerative diseases are still largely unknown. An overview is given of the processes which are thought to be involved in neurodegeneration thus far. Neurodegenerative processes are mainly discussed by taking Alzheimer's disease as an example. Similar processes involved in aging are also discussed. Additionally, models of neurodegeneration (and therapies for neurodegenerative diseases) based on several mechanisms are presented with an emphasis on those with relevance for the experiments described in this thesis.

\section{Mechanisms of neurodegeneration in aging and Alzheimer's disease}

\section{ALZHEIMER'S DISEASE}

Alzheimer's disease (AD) is the most common neurodegenerative disease next to Parkinson"s disease (PD) and Huntington"s disease (HD). About 55-75\% of the cases of adult-onset dementia are of the Alzheimer type and in 1995 it was estimated that about 20 million people are affected worldwide by this memory-loss disorder [4]. AD is characterized clinically by a progressive deterioration of memory, attention, personality, intellect, and language function [78]. In addition to the clinical syndrome, AD is characterized histochemically by the occurrence of (diffuse and meuritic) senile plaques and neurofibrillary tangles 42131 . The only certain diagnosis is by means of brain biopsy. Neuritic senile plaques are 'mature' plaques which are found extracellulat and consist of a central core of amyloid fibrils surrounded by dystrophic neurites together with reactive microglia and astrocytes $98^{3}$. Amyloid $(A B)$, which is the major protein in plaques, is a 39.43 amino acid long fraction of the amyloid precursor protein (APP). Normal APP is a membrane spanning glycoprotein and is thought to have a function in regeneration and repair of cell membranes 214 . Neurofibrillary tangles, which are found within brain cells, appear as bundles of paired helical filaments of tau [96]. Tau, which is the major protein found in neurofibrillary tangles, is a microtubule-associated protein of the cytoskeleton and, nomally, is thought to promote microtubule assembly and to stabilize microtubules in axons and thus helps to maintain axonal transport.

A key question in amyloiclosis is: how does APP generate AB? The major pathway of APP metabolism results in the release of secreted forms of APP (SAPP) which probably have a neuroprotective action [159], and a soluble form of $A B$ (SAB) which is released during normal metabolism [257. Modifications in APP processing could lead to the formation of AB and some researchers think that in this case membrane damage is necessary; alterations in membrane properties affect the membrane insertion and cleavage of APP resulting in the formation and accumulation of $A B$ [189.257]. It is also possible that $S A B$ is the precursor of $A B$ and some researchers think that in this case radicalization of $S A B$ is necessary so it can cross-link and form anyloid fibrils $[158,257]$. But as will be discussed hereafter, there are more putative factors causing or contributing to amyloidosis and $\mathrm{AD}$ pathology in general such as a decrease in metabolism, oxidative stress, and/or genetic factors. Whether $A B$ is neurotoxic is a controversial issue at present 


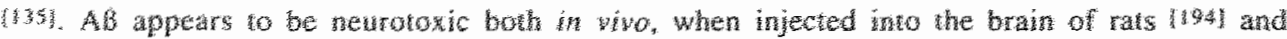

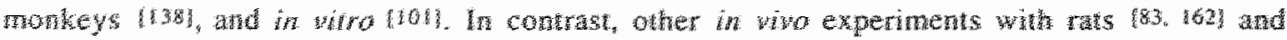

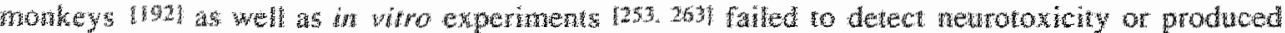

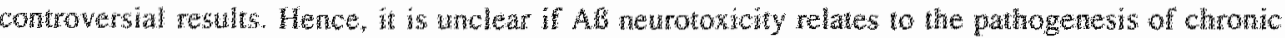
neuromal dogeneration in AD.

Furtwermor, it is not clear wheher here is a divect relatonshp between anyloid deposthon and tangles formation and wheh prowess preceles he other one. Seweral studies have shown that netrofibrillary tangles and semile plaques are correlated wh each other and severit explanations have been offered to link the wo processes with each other. For instance, it is possible that both plaques and tangles arise as a result of a common process 3 ach as an abuormal protein

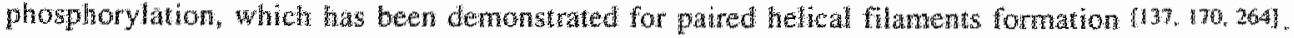
Another possibility is, for instance, that soluble Af in drofuse plaques may come into contact wh paired helcal fitaments atul other proteins reledsed from dying newons, and thes diffuse plagues are conwerted in newritic piaques (241). However, these are all recent assumptons which need futher inwestigation. Naturally, there ane more possible explanatons, but thus is is iltustrated that it is sill unclear whether plaques and tangles are primary causes or secondary events of $\mathrm{AD}$.

Morphological studies of the bratu of $\mathrm{AD}$ patents tevealed symaptic and neuronal loss anext the netrofibrihary changes and anyloid deposition in plaques [28\}. Additionaly, divease severity was associated with a hierarchy of pathological changes in the entominat cortex, hippocampal fomation and the cortex (2a). Likewise, the cholinergic activity (e.g. choline acetytransferase (ChAT) and acetyloholnesterase (AChE) activity) was most affected in the entorhinal cortex and

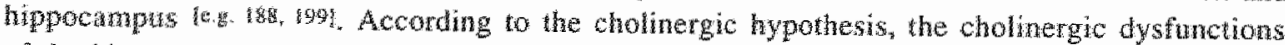
of the angocampal formation and the basal forebran cholinergic system in general, may underlie the cognitive imparments of $A D$ 6. 5I. In addition, it has been found that the morphological features (senile plaques, nextohbrilaty tangles, symaptic and cell loss) in the AD brain not only correlate with each other $10.22,45,62]_{*}$ but can also correlate with the cholinergic deficiencies (e.g.

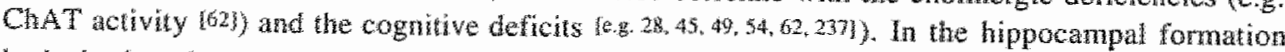
both the loss in synapses [34] and the loss in cells [22] seems to be related to the neurofibrillary tatigles. Further, it was found that in the entorhinal cortex the level of amyloid (AB load), level of paared helicat filaments (PHF load), number of senile plaques and number of newrofibrillary tangles correlated with each other and the cognitive dysfunction [45]. It is generally thought that neurofibrillary tangles correlate better than senile plaques [28, 6.2], but especially the AB load seems to be the strongest predictor of premortem cognitive dystunction 45 .

Based on the above findings it can be argued that plaques related variables (AB load and senile plaques) and tangles related variables (number of neurofibrillary tangles, paired helical fituments load or tau hyperphosphorylation) in the hippocampal formation and entorhinal cortex underlie the cognitive deficits displayed by AD patient. However, ather established neuropathologies like synaptic loss and cell death may also be rellated to cognitive dysfunction in AD, though no correlative study can demonstrate causality. Hence, the exact cause or causes of the etiopathology of $\mathrm{AD}$ remain unclear. It is possible that there is an underlying common denominator, or multiple fundamenal defects, which give rise to the related neuropathologies, as will discussed hereafter. 


\section{NEURODEGENERATION IN AGING}

\section{Metabolism andor oxidative stress}

It is assumed that there is an age-related decrease in activity of the mitochondrial electron transport chain. Animal (rodents and nonhuman primates) studies showed that in the aging brain there is a decrease in activity of enzyme complex I (NADH dehydrogenase [27, 57]) and enzyme complex IV (cytochrome $c$ oxidase $[17,27,46.154]$ ). More recently, an indication for a defect in complex. IV has also been found in human brain [I19]. Several processes have been suggested to underlie the age-related decrease in enzyme activities of the electron transport chain such as oxidative stress, mutations in nuclear DNA (nDNA) and mitochondrial DNA (mDNA), changes in mitochondrial membranes, and/or inpaired enzyme activities of the electron transport chain 117, 27. 57. 2191. All these processes can be related to each other and can interact in affecting the respiratory chain, as will be illustrated below. It should be noted that it has been found that mtDNA mutations can also be genetically inherited, thus resulting in energy deficits and compromised tissue function [251].

Oxidative stress is caused by the accumulation of oxidants which include hydrogen peroxide $\left(\mathrm{H}_{2} \mathrm{O}_{2}\right)$, and the superoxide anion $\left(\mathrm{O}^{2}\right)$ and hydroxyl $(\mathrm{OH})$ radicals. These oxidants are produced continuously at a high rate as a by-product of aerobic metabolism and damage macromolecules, including DNA, proteins, and lipids [219]. During aging there is an increase in oxidative stress, probably due to an incompetent anti-oxidant system as reflected in decreased levels of glutathione, which is necessary to detoxify peroxides $\| 17$. The oxidation of membrane proteins, including plasma membrame ATPases and mitochondrial inner membrane proteins constituting to the electron transport chain, can alter the efficiency of electron transport [219. 265]. The peroxidation of lipid components in plasma and mitochondrial membranes may contribute to the decrease in fluidity (or increase in rigidity) of cellular membranes [2.19]. A consequence of the mitochondrial membrane damage might include loss of mitochondrial respiratory control, although it has been demonstrated that these two aspects appear to be unrelated 265. Finally, oxidative damage can cause mutations in mDNA and nDNA, which also encodes for many proteins of the mitochondria. This is confirmed by a progressive age-related accumulation in oxidative damage to NDNA and, preferentially. mtDNA in human brain [165]. Such mutations can result in lowered efficiencies of electron transport components $|219|$. The above mentioned detrimental processes are depicted in Figure 3.

To summarize, oxidative damage, in particular oxidative damage to mitochondria (e.g. enzymes, membranes and DNA) causes the decline in mitochondrial function during aging (21y) Subsequently, this mitochondrial dysfunction causes the cellular energy deficits and thus can impair cellular functioning. However, it can also be suggested that the oxidative stress, which causes the oxidative damage, is a result of mitochondriall dysfunction, because the respiratory chain and especially an impaired one, is the major source of free radicals in a cell [17, 154, 219.265] (see Fig. 3). This indicates that there is a chicken and egg problem regarding the primary cause or wltimate event resulting in a decline in mitochondrial and cellular function.

\section{Atrophy}

The above mentioned energy metabolism deficits and oxidative damage might cause the neuronal dysfunction occurring during aging and this could subsequently lead to neuronal loss. It should be noted that there still is some controversy whether global neuronal loss or only global atrophic changes (as depicted in Fig. 3) takes place in the aging brain (and also in the AD brain as will be discussed later). At least it can be argued that there is a regionally specific atrophy in the brain, 


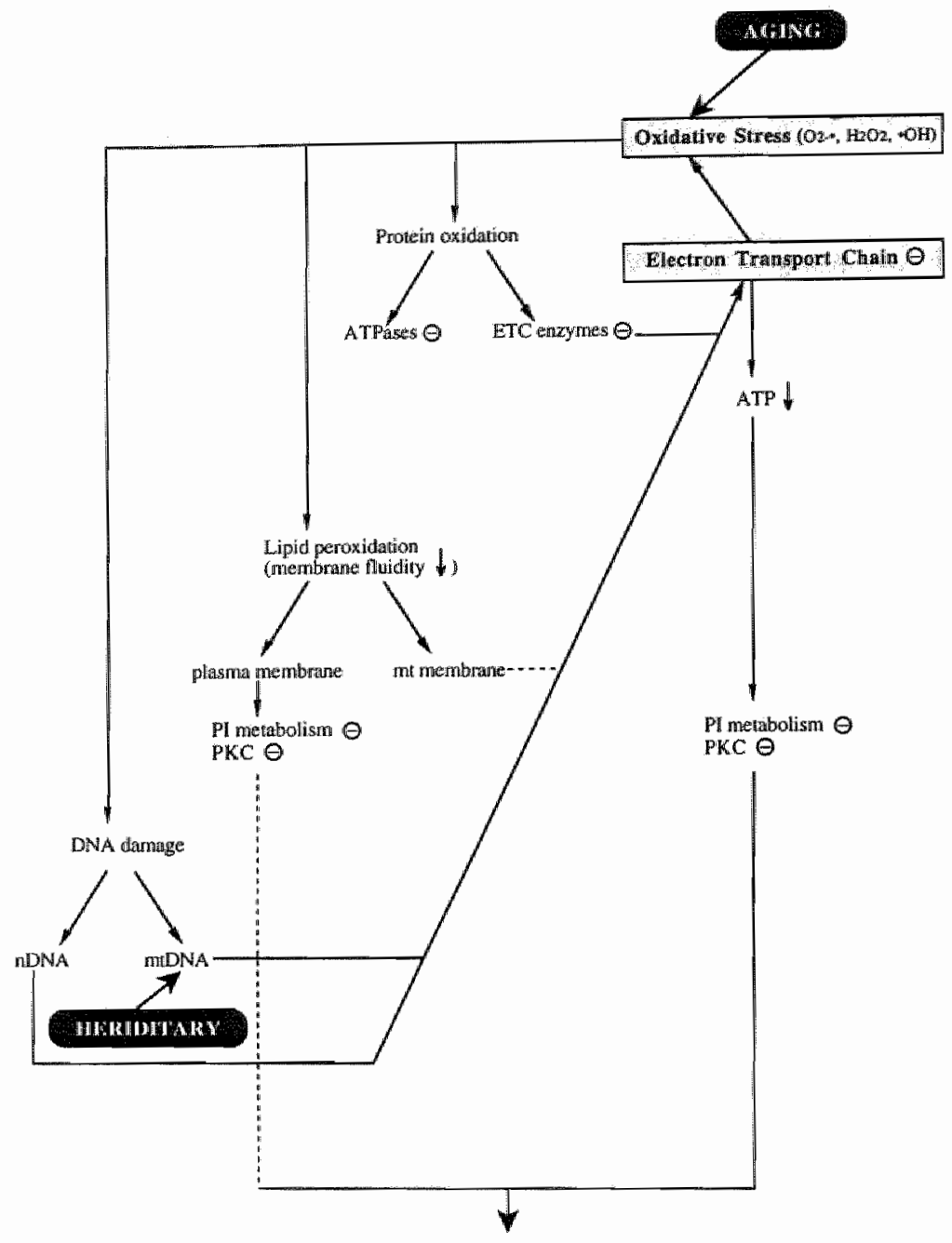

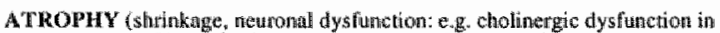
ACll synthesis'relenge and Muzcarunic second messenger system)

FIG. 3. Schernatic diagram showing the processes thought to be involved in neuronal dysfunction due to aging. Abbreviations: ACh, acetyleboline; ETC, electron transport chain; mt, mitochondral; n, nuclear; PI, phosphoinositide; PKC, protein kinase C. Symbols: $\downarrow$, decrease: $(-)$, inhibition.

e.g. the hippocampus [49], though even this is also contradicted, i.e. no (regional) atrophy in the hippocampus [23:) or no (global) atrophy in the brain at all [oul].

With respect to the cholinergic function it has been found that there is a cholinergic cell loss (in size and number) in the basal forebrain (e.g. medial septum, but not nucleus basalis magnocellularis) of cognitively impaired aged rats $[70]$, though this has not been corroborated in 
another study [197]. Howewer, it is already known that when comparing age-rellated changes in neuron size and number, the data are often conflicting which ma be attributed to differences in brain region, species, strain and age [73]. In addition, another candidate, next to the neuronal loss, for structural correlates of age-related cognitive impairment is the symaptic loss (in size and numbers) as has been found in aged rat hippocampus [88], although again this has also been contradicted [205].

\section{Phosphoinositide signal iransduction}

Rodent studies demonstrated that the age-related decrease in cholinergic function, that is acetylcholine synthesis and release $[91.260]$, could be due to an age-related decrease in oxidative metabolism, that is a decrease in glucose utilization 1911. Moreover, the decrease in glucose utilization could be related to an age-related decrease in cognitive functioning 181.2591. Hence, it can be suggested that the age-related decreases in cholinergic and/or cognitive function may be due to alterations in energy metabolisn. Furthermore, an affected energy metabolism may result in oxidative stress. Both a decreased energy metabolism and oxidative stress might affect the metabolism of phosphoinositides and protein kinase $\mathrm{C}$ activity and it has been observed that during aging the phosphoinositide nnetabolism in the brain is affected 124, 150.2021.

The metabolism of phosphoinositides plays an important role in the receptor-mediated signal transduction, e.g. the postsynaptic muscarinic receptor response. For a detailed description see [24]. Briefly, an extracellular signal, such as a neurotransmitter or growth factor, initiates signal transduction through the membrane by binding to a specific receptor which is coupled to at "second messenger-producing system" at the cytosolic side of the membrane. Receptors coupled to the phosphoinositide signall transduction mechanism are coupled to phospholipase $C$ (PLC). This coupling could be achieved via an intermediate G-protein or protein tyrosine kinase. PLC hydrolyses phosphatidylinositol 4,5 -biphosphate $\left(\mathrm{PIP}_{2}\right)$ which is formed by the consecutive phosphorylation of phosphatidylinositol (PI) to phosphatidylinositol 4mphosphate (PIP) by PI 4-kinase and of PIP tot PIP 2 by PIP 5-kinase. The receptor-stimulated hydrolysis of PIP results in the release of the second messengers inositol- $1,4,5$, trisphosphate (IP,) and diacylglycerol (DAG). DAG remains in the plasma membrane and activates protein kimase $C$ (PKC) in the presence of $\mathrm{Ca}^{2}+$ which is mobilized by $\mathrm{IP}_{3}$.

Aging reduces the membrane tratnslocation and activation of PKC activity $18,80,245]$. Thus, aging may be associated with changes in the phosphoinositide signal transduction mechanism that is linked to the muscarinic receptor. This is corroborated by a recent study in which an age-related dysfunction of the postsynaptic muscarinic receptor second messenger system was found, while the muscarinic receptors themselves seem to be intact in the aged rat brain $[40]$. Furthermore, the dysfunction of the postsynaptic muscarinic receptor system, as indicated by a decrease in metabolisn of phosphoinositides, was correlated with the spatial learning deficil of these old rats. "The impaired phosphoinositide signal transduction system may allso explain other observed age-related alteration in cholinergic function in rat brain like the reductions in acetylcholine synthesis and release during aging [245], because the activation of PKC appears to modulate the release of a number of neurotransmitters, though its precise role in the release-process remains unknown $[80,157]$. Taken together, the age-related alterations in cholinergic function may be due to alterations in energy metabolism which affects the metabolism of phosphoinositides [6. 244]. In Figure 3 the possible contribution of an affected phosphoinositide metabolism and PKC activity to an age-rellated (chollinergic) neuronal dysfunction is incorporated.

Finally, it has been found that oxidative stress is also involved because membrane 
peroxidation in young thts mimicked aging with respect to the translocation of $\mathrm{PKC}$ which could account for a decrease in acetyleholine release in the brain of aged rats 1168 . Howewer, in the brain of aged rats no increase in lipid peroxidation was found (although there was a small increase in protein oxidation) [4:], thus suggesting only a minor or partial role of oxidative stress in age-related cholinergic dysfunction (see Fig. 3).

\section{NEURODEGENERATION IN ALZHEIMER'S DISEASE}

\section{Merabolison}

There appears to be a relation between the age-related decrease in function of the electron transfer chain and meurodegenterative disorders as it has been found that the activity of the electron transfer chain is decreased in the brains of patients with Parkinson's disease, which appears to be selective for enzyme complex 1 activity [215.2161. Likewise, the brains of patients with Alzheimer's disease showed a decrease in gene expression and activity of enzyme complex IV [37, 133, 258]. Genetic deficits, i.e. mutations in m.DNA, may underlie these impaiments in oxidative metabolism of AD patients [118, 223], as is shown in Figure 4 which illustrates neurodegenerative processes in general while putative sources of plaques and tangles in the AD brain are also depicted.

The involvement of the energy metabolism in neurodegenerative processes is also apparent from the obserwation that brain tissue of $\mathrm{AD}$ pattents shows marked reductions in pyruvate dehydrogenase complex activity [32] and $\alpha$-ketoglutarate dehydrogenase complex activity [156, 238], although the cause the enzymes' deficiency is yet unknown. Furthermore, the brains of $\mathrm{AD}$ patients showed also a major decrease in glucose utilization [50. 115, 1151. Hence, based on the observations of deficits in respiratory chain, decreases in metabolic enzymes and glucose utilization, it can be argued that oxidative energy metabolism is decreased in AD brain.

Based on these observations it has been suggested that ghcose transport may be rate limiting for glucose utilization in AD brain 12241, because in the brain of AD patients decreased concentrations of the two glucose transporters GLUTI (microvessels and glial cells) and GLUT3 (neurons) have been found [99, 224]. However, there is no evidence that brain intracellular glucose is zero in $\mathrm{AD}$ or that glucose utilization in this condition is limited by glucose transport [184]. When glucose transport is rate limiting for utilization, intracellular glucose is essentially zero. Conversely, when glucose utilization is limited by glucose phosphorylation (i.e. glycolysis), intracellular glucose is mensurable. Therefore, the importance of the decreases of the glucose transporters in the pathogenesis of $\mathrm{AD}$ remains to be determined.

It is possible that the defects in energy metabolism due to neurodegenerative processes add to, or potentiate the deficits in energy metabolism of nomal aging and subsequently result in an energy production of a neuron that is below a critical threshold. Concomitantly, it has been hypothesized that a (primary) impaiment of energy metabolism leads to a (secondary) neuronal death and thus may play a role in neurodegenerative disorders $[10,12,243]$. Briefly, impaiment of oxidative energy metabolism, as can be measured in AD and PD, resulls in reduced levels of ATP. ATP is produced in the mitochondrion and most of the ATP in the brain is used by plasma membrane ion-motive. ATPuses ( $\mathrm{N}^{+1} / \mathrm{K}^{+}$and Ca2 + -ATPases) to restore the ionic gradients that have been run down by synaptic transmission. Initially, interference with the $\mathrm{Na}+\mathrm{K}^{*}-\mathrm{ATP}$ ase retards repolarization of the plasma membrane. This disturbance will cause increases in $\mathrm{Nat}$ concentration in the cytosol, lessening the voltage-dependent $\mathrm{Mg} 2+$ block of $N$-methyl-D-aspartate (NMDA) channels, which as a result will be activated by endogenous levels of glutamate. Thus, the 
flux of $\mathrm{Na}^{+}$and $\mathrm{Ca}^{2}+$ through these NMDA channels will be enhanced. The removal of $\mathrm{Ca}^{2}+$ is inhibited because the effectiveness of the $\mathrm{Na}+\mathrm{Ca}^{2}+$ exchange sy stem is decreased by the increased intracellular $\mathrm{Na}^{+}$concentration, and because the Ca2+-ATPase is inhibited by the energy impairment. The enhanced flux of $\mathrm{Ca}^{2+}$ and $\mathrm{Na}{ }^{+}$through NMDA channels in ATP-deficient neurons creates an even greater demand for ATP by the ATPases which soon depletes the cytosolic ATP. At high concentrations of intracellular Cat*, mitochondria take up $\mathrm{Ca}^{2 *}$ from the cytosol. The consequence of $\mathrm{Ca}^{2}+$ overload of mitochondria is irreversible blockade of the respiratory chain and energy metabolism leading to activation of mitochondrial phospholipases and mitochondrial damage (see Fig. 4). This sequence of events leads rapidly to additional elevation of $\mathrm{Ca}^{2}+$ in the cytosol of energy-deprived neurons, and consequently to self-destructive processes such as activation of phospholipases, endonucleases, and proteases (see Fig. 4). The above described mechanism is referred to as a NMDA-mediated neurotoxicity or excitotoxic mechanism of cell death [12, 243]. Furthermore, the importance of a $\mathrm{Ca}^{2}+$ homeostasis has led to the formulation the 'calcium hypothesis' of $\mathrm{AD}$ and brain aging [132]. In this view the intracellular $\mathrm{Ca}^{2}+$ concentrations play a central role in modulating the direction of the equilibrium governing regeneration and degeneration. In addition, the cause(s) of $A D$ can be anything, thus not energy deficits only, but it will eventually disrupt the cellular mechanisms for maintaining cytosolic $\mathrm{Ca}^{2+}$ concentrations homeostasis which thus provides a final common pathway for cell dysfunction and death.

Because of the decrease in glucose utilization in the brain of AD patients, it has been hypothesized that the degree of cognitive dysfunction of $\mathrm{AD}$ is related to the level of oxidative energy metabolism in the brain [see 116]. In addition, it recently has been hypothesized that the cholinergic deficit and amyloid accumulation in the brain are secondary phenomena caused by the major decline of glucose metabolism in AD [166]. The key substrate of acetylcholine synthesis is acetylcoenzyme $A(A c e t y l C O A)$ which in the brain is exclusively synthesized in the glycolytic pathway, because the brain is unable to synthesize acetylcholine from fatty acids or in the lipid metabolism [242]. Glucose in the AD brain is so low that acetylcholine synthesis is no longer possible because of the need to preserve electrolytic/ionic homeostasis and life by a sufficient ATP production by degradation of AcetylCoA in the citric cycle. Because the whole amount of ATP is so low in the AD brain. APP cannot be correctly inserted in the plasma membranes and, consequently, the proteolytic splitting of APP is incomplete and results in AB fragments (see Fig. 4). Nomal APP has a membrane repair function $[2141$ and the absence of this function causes atrophic/necrotic changes, which finally results in a loss of symapses and neurons. It has also been suggested that the altered membrane insertion and spliting of APP is a result of alterations in plasma membrane properties [ct. 189, 257] (see Fig. 4).

\section{Oxidative stress}

Oxidative stress may also play a role in the pathogenesis of neurodegenerative diseases [cr. 183]. For instance, it has been suggested that free radicals could contribute to the pathogenesis of AD by impacting on amyloidosis [79]. Vice Versa, $A B$ in plaques appears to increase or contribute to the production of free radicals thus causing oxidative stress. 114). Therefore, mechanisms should and have been proposed combining these aspects. In this view accumulation of $A B$, which is also found in diffuse plaques of normal aged brain, is a result of and contribution to both a reduced energy availability and increased oxidative processes [158]. Furthermore, it was suggested that, ultimately, these events might lead, or contribute to neurofibrillary tangles formation.

The matter becomes even more complicated when it is realized that oxidative stress and energy depletion are, as has been previously mentioned, a chicken and egg problem. Free radicals 
damage protein, lipids and DNA, and thus can influence the energy metabolism (see Fig. 3 and 4). For unstance, two important classes of enzymes whose activity has been found to be impaired by free radicals are the plasma membrane ATPages and the mitochondrial inner membrane electron transport complexes [265]. Both impaiments can contribute to the excitotoxic/metabolic insult $1 \mathrm{ch} .12 .2431$ and thus may play a role in neurodegenerative process of $A D$ [1581. An increased oxidative damage has also been found in $\mathrm{NDNA}$ and particularly in mDNA in AD brain tissue $\left\{164 \|_{\text {and }}\right.$ thus contributes to mitochondrial dysfunction. However, an increased radical formation (oxidative stress) is also the result of a mitochondrial dysfunction, which alternatively (as just mentioned) may be a consequence of increased oxidative damage. Therefore, it is very difficult to determine in how far oxidative stress plays primary and/or secondary role( $(5)$ in the pathogenesis of $\mathrm{AD}$, which applies also for the role(s) of the energy metabolism in this respect.

It is interesting ro note that in the brain of $A D$ patients the paired helical filaments of tau in tangles and the $A B$ fibrils in plaques can form advanced glycation end product (AGEPS), which are peptides cross-linked to sugars [103, 262]. An increased accumulation of AGEPS can also be observed in de aging human brain [133). These AGEPs can aggregate and induce oxidative stress. Hence it has been suggested that the glycation of $A B$ and tau could contribute to or be causally involved in the netrodegenerative damage in AD 1103,262,2641. However, it has been proposed that the glycation of $A B$ in $A D$ results from the free radical generation by $A B$ itself [160]. In this view, a reduced energy metabolism and oxidative stress cause the radicalization of $A B$ (see Fig. 4). $A B$ radicals promote cross-linking of the peptide to itself and to sugars (glycation). Therefore glycation of $A B$ may be causally not involved in the neurodegeneration associated with $A D$, though the initial radicalization is of importance in the pathogenesis of $A D$ and for cross-linking of $A B$ with itself forming the amyloid fibril 1581. Recently, it has been suggested that apolipoprotein $\mathrm{E}$ (see below) which was found in senile plaques and neurofibrillary tangles, or a fragment of apolipoprotein E, may also be glycated [59].

\section{Generics}

About $75 \%$ of the cases of $\mathrm{AD}$ occurs at ages greater than 65 years, without a known family history of disease occurrence [122]. This form of AD is called sporadic or late-onset AD. In the remaining $25 \%$ of $\mathrm{AD}$ cases seem to be inherited and are referred to as familial or early-onset AD. A mutation on chromosome 14 in gene $S / 82$ is responsible for $80 \%$ of he cases of familial $\mathrm{AD}[4,207,218]$. APP is encoded on chromosome 21 , thus mutations of the APP genes could lead to an altered APP processing resulting in the deposition of AB. But mutant APP genes turned out to account for only $2 \%$ to $3 \%$ of fumiliat AD cases [4, 22, 208]. The remaining AD familial cases are neither related to mutations of chromosome 14 nor chromosome 21 . Recently, on chromosome 1 a candidate gene (STM2) has been found which is wery similar to gene $S / 82$ on chromosome 14 1421. Possibly, mutations in this gene account for the remaining cases of familial AD [4] (see Fig. 4). The normal cellular functions of $S T M 2$ and $S 182$ are unknown, but mutations in these genes could alter intracellular protein trafficking of APP and ultimately lead to altered APP processing and increased production of $A B 1142\}$.

Recently, it has been found that for late-onset $A D$, the apolipoprotein $E$ E4 (ApoE-E4) allele elevates risk for $\mathrm{AD}$ (see Fig. 4), possibly by reducing the age of onset [43], whereas the $\varepsilon 2$ allele may be protective $[421$, ApoE is a protein that ferries cholesterol through the blood stream and the ApoE locus on chromosome 19 has three alleles: ApoE-E2, ApoE-E3, and ApoE-E4; the latter two allelles are most common. It is not completely clear how ApoE is linked or contributing to the pathogenesis of $\mathrm{AD}, \mathrm{e}, \mathrm{g}$. plaques and tangles formation. But it has been found that the isoform apolipoprotein E4 (ApoE4) binds much more rapidly and tightly to AB than does the 


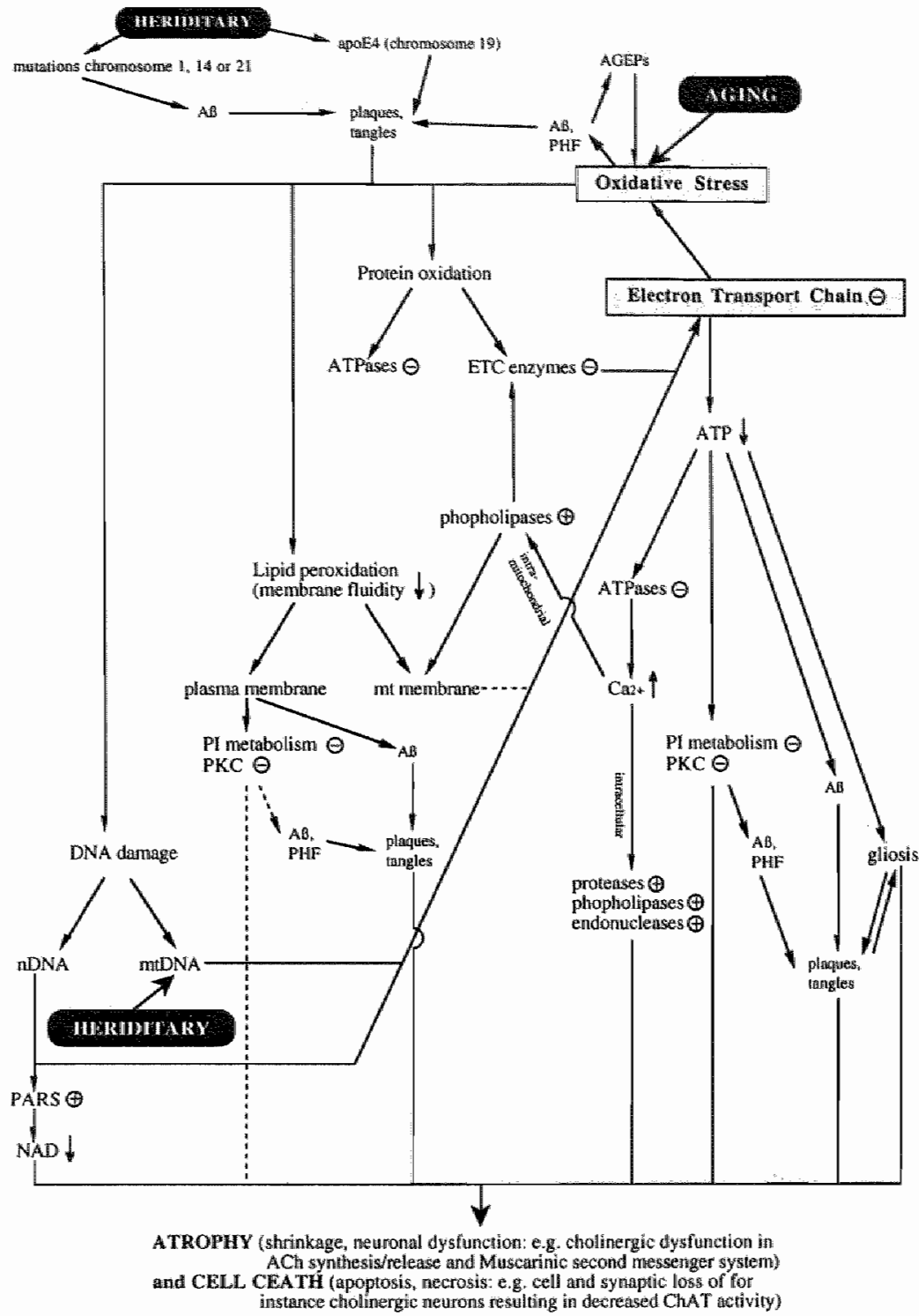

FIG. 4. Schematic diagram showing the processes thought to be inwolved in neuronal dysfunction and cell death due to meurodegeneration in general, i.e. the metabolic/excitotoxic insult and oxidative damage. In particular the putative sources of plaques ( $A B$ deposits) and tangles (paired helical filaments) in Alzheimer's disease are depicted. Additionally , the possible role of the enzyme poly (ADP-ribose) synthetase (PARS) in cell death is shown. DNA damage due to free radicals resul in the activation of the enzyme PA.RS which is involved in repair of DNA damage. However, activation of this enzyme reduces cellular NAD and is associated with a decreased cellular survival [2:10]. Abbrewiations: $A B$, amyloid; $A C h$, acetylcholine; $A G E P s$, advanced glycation end products; apoE apolipoprotein $\mathrm{E}$ : ChAT, choline acetyltansferase; ETC, electron transport chain; mt, mitochondrial; n, nuclear; PHF, paired helical filaments; PI, phosphoinositide; PKC. protein kinase C. Symbols: $\downarrow$, decrease; $\uparrow$, increase; $(-)$, inhibition; $(+)$, stimulation. 
protein isoform ApoE3 2301 . More recently, it has been shown that ApoEA induces AB fibril formation, and that ApoE2 antagonizes this process [147]. ApoE4 does not bind to kau, whereas ApoE3 binds strongly to tau, possibly slowing down the initial rate of tau phosphorylation (211). Hyperphosphorylated tau does not bind to microtubules and may self-assemble into paired helical filaments [170]. Thus, it can be hypothesized that ApoE3 may slow down tangles formation [cr. 211].

\section{Cell death}

The whole brain as well as its separate structures (mainly medial temporal structures like the hippocampusy of AD patients show a decline in weight and volume $[22,49,61,1981$. Howewer, it is not clear whether a global neuronal loss in the brain of $A D$ patients underlies these morphological changes [233), although there is clear evidence that in some specific regions neural loss occurs like in the hippocampus $[22,252]$ and locus coeruleus [233]. Whether this neural loss occurs via a necrotic or an apoptotic mechanism is not known, but in vitro experiments revealed that $A B$ induces a necrosis rather than an apoptosis mechanism [13. However, it has been suggested that no cell loss takes place in the neocontex and nucleus basalis magnocellutaris of $\mathrm{AD}$ brain implicating that neuronal atrophy (cell shrinkage) rather than cell death is the major phenomenon involved in $\mathrm{AD}$ $[198,233]$. In Figure 4 both atrophy and cell death are depicted.

When cells are thought not to be lost but only inactive, this makes a difference for therapeutic strategies preventing A.D as formulated in the "use it or loose it concept, namely, that activation of neuronal systems might prevent their degeneration and restore their function during aging and AD [232]. This concept is contrary to the oxidative stress theory of neurodegenerative diseases, which predicts that the most active neurons are also the ones that are most affected, which has been paraphrased as the "wear and tear" concept [232]. On the other hand, the "use it or loose it" concept is in agreement with the decline in brain metabolism, and thus suggests that global stimulation of the brain (e.g. by charging with cognitive tasks or by stimulating central metabolism) has a positive effect on neuronal activity.

In the hippocampus 11144 , nucleus basalis magnocellularis [2.37] and cortex [206] of AD patients also synaptic loss has been found. The assumption that neuronal atrophy and not neuronal loss is the main hallmark of $\mathrm{AD}$ [233] increases the relevance of the loss of synapses as a structural correlate of the cognition deficits of AD patients.

\section{Gliosis}

The expression of glial fibrillary acidic protein (GFAP), the major intermediate filament protein of astrocytes, has been demonstrated to increase with age in human brain (and rodent brain) le.g. 174!. Although the signats which are leading to upregulation of GFAP are unclear, it seems that the functioning of the energy metabolism is involved in the regulation of astrocytes during aging lof. 175). The process 'reactive astrogliosis' is a stereotypic reaction of astrocytes to virtually any severe damage to brain tissue caused by physical (e.g. ischemia [6.5]) and chemical (e.g. excitoxic [139]) insults and by several pathological states associated with for instance aging [175], AD [77] or multiple sclerosis [201]. The reactive changes are characterized by astrocyte proliferation and/or extensive hypertrophy. Concomitantly, the expression of GFAP increases during the development of reactive astrogliosis. Hence, it can be suggested that GFAP upregulation and astrogliosis are markers of brain aging and neurodegeneration. Astrocytes are found in senile plaques 198. 248?. The major question is whether the astrocytes are involved in AD. For instance, astrocytes may be merely reacting to the lesions, i.e. they are affected by the (toxic) plaques and tangles, or the pathology occurring in AD (see Fig. 4). On the other hand astrocytes may be causal or contributory to $A D$; astrocytes might cause the deposition of $A B$ or act in concert with $A B$ to 
produce the neurodegenerative pathology of $\mathrm{AD} 77,209$ ) (see Fig. 4). This is corroborated by a sudy suggesting that a compromised brain energy metabolism (due to an impared brain microcirculation) causes neurons to release astroglial mitogens signalling reactive astrocytes to proliferate and become hypertrophic (see Fig. 4). This astroglial reaction ostensibly contributes to the formation of senile plaques and neurofibrillary tanglas [239].

Microglia are the resident immune competent (macrophage-type) cells in the central nervous system and become activated in response to injury, proliferate, change morphology and participate in the inflammatory process [48]. Senile plaques contain next to activated astrocytes also activated microglia and diverse inflammatory proteins inchuding cytokines and complement factors (for a summary see [67]). As for astrogliosis, the question arises what role microgliosis play in the pathogenesis of $\mathrm{AD}$. Microgliosis may be merely a reaction to the pathology of $\mathrm{AD}$. This is in agreement with findings that $A B$ activates complement which is further postulated to stmulate microglia (and astroglia) [38) and that AGEPs (of AB fibrils, paired hellical fillaments, and Apo-E) may serve as a signal for activation of microglia [59]. On the other hand, microglia nay play a specific or causal role in the formation of senile plaques, that is the conversion of diffuse plaques unto neuritic plaques [148], In addition, growth factors and stimuli from microglia affect astrocytes, and wise versa $[581$. To summarize the role of astrogliosis and microgliosis, it is likely that the gliosis reaction is reinforced by the neuropathology of $\mathrm{AD}$ as well as that gliosis has a contribution in causing the neuropathology.

\section{Phosphoinositide signal transduction}

Like during aging, the metabolism of phosphoinositides as well as the activity of $\mathrm{PKC}$ is affected in the brain of $A D$ patients [se 24]. This may have implications with regard to the pathogenesis of $A D$ as is partly shown in Figure 4 which illustrates the possible contribution of an affected phosphoinositide metabolism and PKC activity to plaques and tangles formation in the AD brain.

Firstly, like during aging, the alterations in phosphoinositides metabolism and PKC activity may lead to a disturbed phosphoinositide signal transduction in the brain 124,202$]$ which is corroborated by recent findings that the postsynaptic muscarinic receptor responses are impaired in $A D[125,126]$. In addition, becanse the phosphoinositides signal transduction modulates APP processing, it is believed that the reduced signaling activity increases $A B$ [126].

Secondly, it has been suggested that abnomal phosphorylation or hyperphosphorylation of tau is involved in the formation of paired helical filaments $[96,120,137,470,264]$, although it has also been reported that phosphorylation may be of secondary importance in this respect 1141. 151. PKC is able to phosphorylate tau [9] and an altered PKC function can, therefore, be connected with the formation of neurofibrillary tamgles. Protein phosphorylation and the occurrence of senile plaques seems also to be related, i.e. APP may be phosphorylated by PKC [84]. However" still no direct evidence has been provided that APP phosphorylation by PKC is responsible for the altered processing of APP [see 202].

Thirdly, a possible function of PKC is the regulation of protein tyrosine phosphorylation [178). Because tyrosine kinase activity probably regulates PI 3-kinase activity in the AD brain, these findings could be directly related to the decreased PI 3-kinase activity in the AD brain 125]. The possible involwement of PI 3-kinase and protein tyrosine kinase in the regulation of cyloskeletal rearrangements in addition to the decline in PI kinase activity [25. 123] and tyrosine kinase activity [217] suggest that these enzyme activities may be involved in the pathology of $\mathrm{AD}$ [4].25]. This is because neurofibrillary tangles are composed of wrongly phosphorylated cytoskeletal components.

Finally, it can be noted that as a result of changes in membrane integrity/fluidity the activities 
of the enzymes involved in the metabolism of phosphoinositides are probably not (PIP kinase [26, 124] and PLC [125. 221]) or only minor (PI 4-kinase [25. 123]) affected in AD (for a detailed review see [241), and probably neither during aging [124].

\section{Animal models of neurodegeneration}

Unfortunately, there is no animal species which naturally dewelops a neurodegenerative disorder. Consequently, a good animal model of neurodegeneration is in great demand, and preferably one that has an interface with the possible cause(s). In addition, since the understanding of neurodegenerative mechanisms is still limited, it is difficult to develop a suitable therapy. Especially a therapy directed at prevention of, or slowing down the neurodegenerative disorder, in stead of a therapy directed at its symptoms anly. Therefore, models of neurodegeneration are needed: on the one hand to gain a better understanding of neuropathological processes underlying neurodegenerative disorders, and on the other hand to have the possibility to test therapies which enhance neuronal function or prevent neuronal dysfunction.

In several studies it has been found that toxins which impair energy metabolism by inhibiting the electron transpont chain, cause selective neurodegeneration which was comparable with that of humans [see 12.243\}. The cell death occurring after administration of these toxins is due to a so-called excitotoxic/metabolic insult which involves overstimulation of the NMDA receptor (see section Metabolism). Thus, blockade or inhibition of the central energy metabolism may provide a model of neurodegeneration. Oxidative stress may also play a role in causing cell death associated with neurodegeneration [ch. 183] (see section Oxidative stress). Thus, models of neurodegeneration can also be developed with toxins causing oxiclative stress. But there are a lot of other possibilities. For example, many models are based on a specific cholinergic lesion, but there is sufficient evidence to dismiss such an unitary transmitter concept model. Moreover, other aspects are also involved, e.g. other neurotransmitters/neuromodulators and/or nerve growth factor. This should be kept in mind when developing a model of neurodegenerative diseases, like for instance $\mathrm{AD}$ [113]. $\mathrm{AD}$ is characterized by a heterogeneity of behavioral and cognitive features and cannot be definitively diagnosed. This heterogeneous disorder is probably caused by genetic mutations or properties, metabolic deficits and/or oxidative damage. Besides cognitive deficiencies, $\mathrm{AD}$ is characterized by amyloid plaques, neurofibrillary tangles, symapse loss, neuron loss, cholinergic deficits and inflammatory processes/gliosis (see section Alzheimer's disease). An animal model displaying one or all of these features would provide a powerful tool in elucidating the ctiopathology of the disease. However, aged rodents do not accumulate AB, while at least some of the new world monkey species preferentially accumulate $A B$ in blood vessels. Old world monkeys and humans deposit $A B$ in plaques and blood vessels and have thus been used as models for studying amyloid deposition in aging and AD [193]. However, there are some factors that should be taken into consideration when evaluating experiments of nonhuman primates. Especially the finding that the distribution pattern of amyloid deposits is different among species, i.e. from vascular anyloid with occasional diffuse plaques to neuritic plaques with varying levels of vascular amyloid [86]. In addition, the length of the AB peptide mainly present in senile plaques is different from humans, viz. $A B_{40}$ for nomhuman primates wersus $A B_{42}$ for humans [87]. Because rodents do not accumulate $A B$, a lot of effort is recently being done trying to create transgenic rodents which yet develop this feature of AD. Moreover, the development of transgenic organisms is one of the main focuses of scientific research at the moment.

Not until one has a good model of neurodegeneration, it remains difficult to develop and test 
therapies for cognitive impairments accompanying the neurodegenerative disorder. The development of these therapies is since almost twenty years mainly based on the neurotransmitter acetylcholine (according to the cholinergic hypothesis), while since almost ten years the NMDA receptor (according to the glutamatergic hypothesis), and even more recently the enzyme No synthase, is used as point of interest [see 1211. However, therapies based on acetylcholine itself have only achieved little clinical progress to date. More recent investigation have suggested that a more specific therapy directed at the cholinergic receptors, that is one which results in a specific stimulation of the cholinergic system, probably has more therapeutic potential (see previous part Plasticity, section Basal forebrain and acetylcholine). Although interference with the NMDA receptor or NO synthase has resulted in interesting findings, like a memory enhancement after stimulation of both (see previous part Plasticity, sections Glutamate and Synaptic plasticity), there are also enough conflicting data complicating its application. This is probably due to the potential neurotoxic effects of overstimulating both systems, which conld result in an excitotoxic/metabolic insult (see section Metabolism). But this property offers possibilities to develop models of neurodegeneration based on the NMDA or NO system, like administration of NMDA agonists or NO donors. Furthermore, there are indications that administration of NMDA antagonists and NO synthase inthibitors are effective in treating neurodegenerative disorders $[12.39 .243]$. Hence, stimulation of the NMDA receptor or NO synthase within some sort of physiological range can be used for treatment of neuronal dysfunction under relatively normal or mild pathological conditions, whereas under severe pathological condition it is perhaps better to inhibit these systems to treat neurological impairments.

Firstly, a summary of some animall models of neurodegeneration is given hereafter. Secondly, some therapies against neurodegenerative processes are discussed. Only models and therapies relevant for this thesis, and with an accent on $\mathrm{AD}$, are summarized.

\section{ANIMAL MODELS}

\section{Lesions}

Physical (e.g. electrically, knife cuts) and pharmacological (e.g. ibotenic acid, quisqualic acid, 6-thydroxydopamine) lesions in several brain areas of experimental animals are widely used as models of human neurodegenerative disorders. Well-known examples are lesions of the hippocampal system as a model of $\mathrm{AD}$, and lesions of the substantia nigra as a model of PD. For more information see the previous part of this chapter (Plasticity) in which these models have already been discussed.

\section{Inhibition of electron transport chain}

Complex I (NADH dehydrogenase). Systemic 1-methyl-4-phenyl-1,2,3,6-tetrahydropyridine (MPTP) administration results in the development of a PD-like syndrome in humans and several animal species [225]. MPTP is assumed to induce selective neurotoxicity in nigrostriatal dopaminergic neurons wa its metabolite 1-methyl-4-phenylpyridinium ion (MPPi) which irrewersibly inhibits the electron transport chain at complex I [107, 196]. There is evidence that an excitotoxic mechanism is involved in causing MPP* neurotoxicity 12031 . In addition, there are several indications that free radicals may also play a role in the neurotoxic process $[7.411,236]$. However, it is not fully clear which is the major or primary mechanism of MPTP/MPP+-induced neurotoxicity. This is of course partly due to the fact that energy depletion and oxidative stress are a chicken and egg problem (see section Metabolism andlor oxidative stress). 
Complex I/ (succinate detydragenase). 3-Nitropropionic acid (3-NP) is an irreversible inhibitor of complex II and it probably causes neuronal damage via a NMDA-dependent, that is an excitotoxic mechanism, although a non-NMDA-dependent mechanism appears to be also involved [1. 2431. Systemic administration of 3-NP caused bilateral striatal damage, impaiments in motor functions, and a slightly affected memory (passive avoidance) performance, and thus would be a useful model of Huntington's disease [220]. In comparison with systemic administration, intrastriatal injections of 3-NP resulted in more profound hypoactivity, greater loss of memory function, and more sewere striatal damage [136). Thus, this prowes to be an even more useful model of Huntington's disease.

Complex IV (cyrachrome oxidasel. Cyanide (CN) is an inhibitor of complex IV and systemic administration (i.w.) causes a period of 'histotoxic' hypoxia in the brain [149]. Cyanide does not cause neuronal damage per se [29], and low doses of cyanide are converted within minutes by mitochondrial sulphur transferase into non-toxic sulphur cyanide [104]. There are indications that allso an excitotoxic mechanism may be involved in cyanide-induced neurotoxicity [186], which thus may eventually cause neuronal damage. In our laboratory we used cyanide, that is sodium cyanide $(\mathrm{NaCN})$, to dewelop a model which allows the assessment of cognitive behavior during a period of acute central "histotoxic" hypoxia [ci. 149]

It has been found that systemic administration (s.c.) of azide, which is another inhibitor of complex IV, impaired (spatial) learning of rats $[15,16]$. Likewise, systemic administration (s.c.) of NaCN impaired (spatial) memory performance of guinea-pigs [47]. It has to be kept in mind that a systemic administration can complicate the interpretation of the behavioral data in terms of central effects because of possible peripheral effects (e.g. motor effects). In our model we, therefore, injected $\mathrm{NaCN}$ i.c.v to avoid peripheral systems being affected. We have found that i.c.v. administration of $\mathrm{NaCN}$ impaired (spatial) memory performanee of rats [19]. Furthermore the hippocampal phosphoinositide metabolism, which is ATP-dependent, was affected, indicating that energy metabolism was indeed impaired after NaCN administration [19].

\section{Inhibition of glucose metabolism}

We have suggested that treatment of middle-aged rats with i.c.v. injected streptozotocin (STREP, one single injection) may provide a relewant model of neurodegeneration. This assumption was based on previous findings of a cognitive (passive avoidance) deficit [161, and a decrease in hippocampal choline acetyltransferase activity [109]. Besides that i.c.v. injected STREP affected the cholinergic system, it had also been found to affect other neurotransmitter systems as was evident from a reduction in serotonin and noradrenaline levels in several brain areas [601. Especially, our assumption was based on the findings that middle-aged rats treated with STREP show deficits in several learning and memory tasks, which could be related to a hippocampal dysfunction [20, 21 . More in detail, there was a correlation between a cognitive (spatial learning) deficit and a decline in hippocampal choline acetyltransferase activity in STREP-treated rats.

Initially, STREP has been and still is being used with systemic administration to expertimentally induce diabetes mellitus in rats [e.g. 31. 69]. STREP is thought to act via several mechanisms, such as inhibition of the insulin synthesis and secretion in pancreas islet cells [23]. This could be due to oxidative damage since nuclear DNA breaks have been found together with an activation of poly (ADP-ribose) synthetase [23]. Moreover, it has been found that STREP stimulated $\mathrm{H}_{2} \mathrm{O}_{2}$ generation, which induced nuclear DNA fragmentation in pancreatic B-cells, thus leading to their destruction eventually [235]. Further, it has been found that STREP decreased the autophosphorylation of the insulin receptor tyrosine kinase [127). This indicates that STREP probably binds/interacts with the tyrosine kinase domain of the insulin receptor [0. 30]. Recently it 
has been demonstrated that STREP-induced diabetes in the rat is associated with decreased sensitivity and responsiveness to insulin, but unchanged insulin clearance (on which together with insulin secretion plasma insulin levels depend) 131. This finding may be interpreted as that an affected insulin receptor is of more importance than an affected plasma insulin concentration for STREP-induced diabetes.

Normally, insulin is thought to regulate glucose transport into the cell by stimulating the synthesis of glucose transporters and their translocation to, and activation at the plasma membrane [130]. The autophosphorylation of the insulin receptor may be the initial event in insulin action, but how it is, or might lead to a signalling event is uncertain (128). Hence, both an inhibited insuln synthesis/secretion and a decreased insulin receptor signalling in the target cells may impair the transport of glucose, and lead to a decrease in glucose utilization. Accordingly, it has been found that STREP treatment resulted in the suppression of an insulin responsive glucose transporter [85]. After insulin receptor activation, endocytosis of the insulin receptor takes place, for which a part of the insulin receptor is of importance that is also of relevance for autophosphorylation 155 , and to which STREP probably binds [30]. Taken together, STREP may impair the energy metabolism. in the cell and long-term effects of STREP can be explained by the impaired insulin receptor endocytosis. In contrast, it can also be suggested that STREP generates oxidants (e.g. $\mathrm{H}_{2} \mathrm{O}_{2}[2351$ ), which cause DNA damage and thus eventually leads to a slow cell death. For both mechanisms of action of STREP it is necessary that STREP enters the cell, although it is nor clear how. Because of the resemblance of STREP with glucose it could be possible that STREP is transported into the cell by a glucose transporter. Especially pancreatic B-cells have many glucose transporters [IA4], which could explain why STREP affects these cells specifically.

It is (still) largely unknown what the working mechanism of i.c.v. injected STREP is, but it has been suggested that STREP impairs the brain glucose metabolism $[161,176.17 \%]$. This is in agreement with the basic assumption for this model that STEP would have the same effect in the central nervous system as in the periphery, i.e inhibition of a brain insulin producing system and/or a decreased insulin receptor function (also referred to as an impairment in the brain insulin/insulin receptor system). It has been found that i.c.v. injected STREP indeed decreased glucose utilization [63] and inhibited energy metabolism [176, 177] in the cortex and hippocampus. Furthermore, the reduction in central glucose utilization appears to be caused by a decrease in the activities of enzymes of glycolysis (whille there were no changes in concentrations of its intermediates) [191].

In this context it is important to note that it has been found that both short and long-term i.c.v. administration of insulin had almost no effect on energy metabolism and no effect at all on insulin receptor density 1 Hol. These findings may suggest that possible influences of i.c.v injected STREP on central insulin concentrations have no consequences, while it is more likely that the central effects of STREP are mainly mediated via the insulin receptor, i.e. inhibition of its autophosphorylation. This assumption is in agreement with what can be thought to be the working mechanism of STREP in the periphery fof. 3I, and is in agreement with some previous suggestions pointing in this direction [see 63. 191]. Hence, more studies regarding the phosphorylation of the yrosine kinase of the insulin receptor in the brain should be helpful ill deciphering the working mechanism of STREP. However, neurodegenerative effects due to oxidative damage cannot be excluded yet, since a probable mechanism of action of STREP could be that it induces primarily oxidative stress, which results in for instance DNA damage [ct. 23, 64. 235].

It is remarkable that the limbic system and mainly its hippocampal formation is one of the brain structures which have the largest number of insulin receptors on neurons 1112,2461 and the highest glucose need [005]. This suggests that this brain system is probably one of the most 
sensitive to STREP treament. Furthermore, some neurons in the hippocampal formation may synthesize and secrete insulin [56], although it is thought that most, if not all brain insulin originates from the periphery [2465]. This further reduces the relevance of the putative effect of STREP on the insulin production in the brain.

\section{Cholinergic and nerve growth factor specific toxins}

Many models of neurodegeneration and in particular of $A D$ are based on disraption of the cholinergic system. For the development of these models several compounds have been used which were thought to be selectively toxic for cholinergic neurons. A well known example is AF64A, which is structurally similar to choline and has been used as a model of AD [226]. Although AF64A is still frequently being used, its cholinergic selectivity and its usefuiness as a model of $A D$ can be questioned. This because both local and i.c.v. administration of AF64A in the brain appeared to cause rather widespread and non-specific neuronal damage.

Because all cholinergic neurons are responsive for nerve growith factor (NGF), many attempts at the specific destruction of cholinergic neurons in the basal forebrain have been made using NGF. For example, a NGF-diphtheria toxin conjugate (NGFT) has been used [222]. Another example is a immunotoxin (saporin, an irreversible inhibitor of protein synthesis) coupled to an antibody directed against NGF receptor (1.92-IgG-saporin) which produced near to total loss of cholineacetyl transferase-positive neurons of the medial septum and nucleus of the diagonal band, as well of those in the mucleus basalis magnocellularis [2541. The effectiveness of 192-IgG-saporin is currently under investigation, but it seems to have a rather selective effect on both cholinergic neurons and specific behaviors [e.g. 240]. Furthermore, another antibody against NGF that has recently been tested, appears to have similar sellective effects [247]. These findings seem to encourage further development of models of neurodegeneration based on NGF function.

\section{Nitric oxide}

NO may be involved in neurodegenerative processes which offers possibilities to develop models of neurodegeneration. However, this line of research has just begun and thus far it did not yield any useful models of neurodegeneration. For instance, in vivo administration of a NO donor did not have any clear results and mainly it is not clear in how far NO mediates NMDA neurotoxicity [95, 102, 145]. However, there is some evidence that NO does mediate NMDA neurotoxicity as was found in studies after an in vivo administration of a NMDA agonist [97, 229]. But fhis finding is probably more of relevance for models of ischemia than for models of neurodegeneration [cf. 33 . $36,39,129,179,1801$.

\section{Transgenic aminals}

No rodent develops a neurodegenerative disorder !ike for instance $\mathrm{AD}$, but at the moment much work is done trying to create transgene rodents which can develop one or more features of $A D$ Especially transgenic animals which can develop extracellular anyloid deposits have the interest (for a review see $(94 \mid)$ ). In this respect, there were two studies which examined the possibility of AB-based transgenes. However, in one study no effects at all were found [see 256], and in the other one neurodlegeneration was present, but no amyloid deposits were observed [140]. Other studies used the APP as angle. For instance transgenic mice that express full-length APP [195]. Variable extracelluhar amyloid deposits could be observed in these transgenes, and spatial learning was affected [se 94 . Another model is transgenes with mutant APP (Val7 17->Phe) overexpressing APP and having extracellular amyloid deposits 1821. However, this model still needs behavioral validation. Another transgenic mouse with mutant APP (Lys670->Asn: Met671->Leu) also. 
showed extracellular amyloid deposits and has been behaviorally rested, although it showed rather nonspecific spatial deficiss [1:17]. Very recently, transgenes have been devaloped expressing the carboxy terminus of APP which also showed the amyloid deposits [173. Furthermore these ransgenes demonstrated specific deficits in spatial learning and in maintenance of long-tem potentation. Hence, this transgenic model looks rather promising.

Next to APP transgenic mice, also transgenes are being developed based on the expression of the E4 isoform of ApoE [146]. But the results of these experiments are still very preliminary. At a later stage it may be interesting to interbreed the APP and ApoE4 transgenic animals.

As mentioned above, transgenic mice for $A B$ itself showed no clear neurodegenerative effects. In addition, an infusion of $A B$ in the rodent brain affects memory performance in awoidance tasks and spatial tasks, but only the consolidation and not the retrieval of information appears to be affected 174, 75, 162, 1721. In addition, indications for neurodegenerative affects of in vivo administration of $A B$ were found $[138,172,194]$. However, some studies do not report a neurotoxio effect after acute or chronic administration of $A B$ [8 83.162$]$. The above mentioned studies mainly differ with regard to the length of the $A B$ peptide. Hence, all these findings raise questions abou: the possible roles of non-AB APP domains and/or factors associated with plaque formation. So there is a need for an approach which elevates $A B$ without elevating APP. In an recent study AB. infusion had only a minor effect (as could be expected), that is few plaque-like deposits [76]. Howewer, large deposits could be found after a combined infusion of $A B$ and the growth factor TGFB. So now there is a model which allows one to introduce $A B$ and other plaque-associated factors without overexpression of potentially confounding APP domains.

It has been reported that transplantated cells from the brain of mice irisomic for chronosome 16 (portions of this chromosome are genetically homologous with human chromosome 21) induced AD-like pathology [200]. This led to the assumption that the trisomy 16 mouse could be a useful model of AD. However, in another study, trisomy 16 brain transplants did not develop AD-like pathologies (e.g. plaques) [228]. This finding questions the usefulness of this model for AD. Another possibility of developing an animal model of AD using grafts could be the transplantation of $A B$-secreting cells in the brain. However, the usefulness of such a model is also limited as it has been found that transplants of human $A B$-secreting neurons neither induced $A B$ deposition nor induced other $A D$ lesions in the rat brain [153].

\section{THERAPIES}

\section{Noomopics}

Nootropics is a term used to describe compounds that have neuroprotective and/or cognition enhancing effects. In general every drug that has such properties, irrespective of its mechanism of action, can thus be named. Mostly, the term nootropics is used for pirazetam-like compounds that have cognition enthancing properties, but for which no commonlly accepted mechanism of action has been established (for a review see [93]). It is important to note that there can be a difference between an effect defined as neuroprotective and one defined as cognition enhancing. For instance, a drug that acutely enhances cognition does not need to be neuroprotective against neuronal damage which is caused later on.

The mechanisms of action by which compounds exert their nootropic effects and, therefore, can be used as a therapy for a neurodegenerative disorder, can be very diverse. There are more or less 'global' therapies which can be used for the treatment of several neurodegenerative disorders, like for instance calcium channel blockers (e.g. Nimodipine) and free radical scavengers (e.g. 
vitamin C). Moreover, there are "specific" therapies which are directed at a specific aspect of one neurodegenerative disorder (e.g. a strategy directed against/preventing amyloidosis in AD). Hence, there are many possibilities to develop therapies (and nootropics), but only the most relevant ones for this thesis are discussed hereafter.

\section{Neurotransmitters/neuromodulators and neural iransplans}

There are many neurotransmitter/neuromodulator therapies in which ligands or degradationintibitors are administered. For instance, $A D$ patients are treated with Tacrine, an acetylcholinesterase inhibitor, and PD patients are treated with L-DOPA, a dopamine ligand. In addition, fetal transplants offer a possibility for treatment, which is certainly the case in PD by transplanting dopaminergic-rich fetal tissue in the degenerated substantia nigra. For a more detailed view see the previous part Plasricity in this chapter where all these aspects have already beeri described.

\section{Stimularion of energy metabolism}

Dementia of the Alzheimer type is accompanied by disruption in glucose utilization that has been suggested to contribute to its characteristic memory impaiment [116]. According to the cholinergic hypothesis, this cognitive impaiment is related to a decline in cholinergic markers in the $\mathrm{AD}$ brain 16.131 .1871 . In addition, it has been suggested that a cholinergic deficit. that is a decline in acetylcholine synthesis, is caused by a decreased glucose utilization in the AD brain [106]. Hence, it may be assumed that glucose administration, or global stimulation of energy metabolism, could diminish cholinergic deficits and/or cognitive impairments in AD patients.

Sevaral studies have reported that glucose administration improved memory performance in AD patients [152] and aged subjects [185]. However, it has to be noted that a cognitive enhancing effect in young adult can be disputed [9. The precise mechanisms underlying the memory improvement are speculative. Based on findings in rodent studies it has been suggested that the memory enhancement induced by glucose administration could result from an enhancement of brain acetylcholine synthesis and/or release [134, see also 91]. On the other hand it has been found that insulin may also play a role in aneliorating the cholinergic and/or memory deficits [167]. Recently, it has been reported that insulin administration is indeed able to improve cognitive performance in AD patients $[44]$.

Stimulation of the central energy metabolism is in agreement with the 'use it or loose it' concept, which states that activation of neuronal systems might prevent their degeneration and restore their function during neurodegeneration [212]. Thus, stimulating the central energy metabolism directly, but also cognitive enhancement therapy itself, would have a positive effect on neuronal function. However, this implicates a higher metabolism which is in contradiction with the "wear and tear" concept predicting that the most active neurons are also the most affected ones [232]. The 'wear and tear' concept is, therefore, in agreement with the oxidative stress theory suggesting that a food restriction, or better referred to as caloric restriction, would result in lless neuronal damage, since a low metabolism causes less oxidative stress. There is strong evidence from a number of animal studies that food restriction retards the aging process (e.g. it increases life span and prevents age-associated diseases [155]), which is in agreement with the oxidative stress theory. However, there are several indications that active cells and even active humans function longer at a better level 1232], which is, therelore, not in agreement with the oxidative stress theory, but in line with the "use it or loose it' concept. Also, there are several indications that compounds which stimulate central energy metabolism can prevent neurodegenerative damage (e.g. acetyl-L-carnitine; see below). All these findings together suggest, once more, that the cause of 
neurodegeneration has to do with both oxidative stress and energy metabolism deficits.

Acetyl-L-camitine (ALCAR) is regarded as a compound which attenuates neurodegenerative processes by having a beneficial effect on the energy metabolism, viz. vila a stimulation of the use of alternative energy sources, such as lipid substrates or ketone bodies $11,2,18,182,249,2501$. Concomitantly, it has been found that chronic ALCAR treament attenuated the cognitive decline in aged rats $15,35,89,90 \%$. Furthermore, it was found that the progression of $A D$ was reduced in chronically ALCAR-treated patients [190, 227]. However, it has to be mentioned that the putative nootropic effect of ALCAR might be attributed also to other mechanisms of action (e.g. an indirect enhancement of cholinergic function via stimulation of the NGF receptor [23at]).

\section{Neurotrophic factors}

Neurotrophic factors (NTF) from astrocytes and neurons influence the expression of gene products known to stabilize $\mathrm{Ca}^{2+}$ homeostasis and suppress free radical accumulation: NTFs bind to receptors with tyrosine kinase activity and, through several intermediate kinase steps, mitogen-activated protein kinases (MAPKs) are activated. MAPKs mediate the strvival-promoting actions of NTFs (and also phosphorylate tau in a manner similar to that in AD) 1158 .

One of the earliest and therefore much investigated neurotropic factors is NGF. It had been found that infusion of NGF into the brain (i.c.v.) reversed lesion-induced cholinergic degeneration in the basal forebrain of rats $[100,106]$. This improvement in cholinergic function may be due to an effect of NGF on the residual cholinergic neurons 153, 691. Cognitive impaiments in basal forebrain lesioned animals were also reversed by NGF treatment 1661 . Furthermore, in aged rats the NGF partly reversed the cholinergic degeneration in basal forebrain (and striatum), and improved learning and memory deficits in behaviorally impaired aged animals $[68,69,71,72,255]$,

It has been demonstrated that NGF levels are rather constant in the brain during anging or only a slight increase was found $[108,212]$. This finding supports the view that degenerative changes in the basal forebrain cholinergic system that are know to occur in the behaviorally impaired aged rat are not caused by reduced availability of NGF in the cholinergic target areas. Correspondingly, NGF levels in the brain of AD patients are not decreased 11711. However, it should be noted that these findings do not exclude the possibility of disturbances in the NGF transduction pathway.

Basal forebrain lesion experiments with both young and aged animals showed that NGF treament which is adequate for promoting recovery in young adult rats, does not apply fully 10 aged rats as NGF did not reverse the cholinergic deficits in the lesioned aged rats 1204. Moreower, in basal forebrain lesioned aged animals there appeared to be an attenuated increase in NGF levels when compared to the NGF response in lesioned young rats [2121. These findings suggest that the therapeutic potential of NGF may be limited by the amount of neuronal damage.

Another neurotrophin is brain-derived NGF (BDGF). However, in contrast to NGF BDGF was, even when administered together with NGF, without substantial effect in lesioned basal forebrain rats (71) as well as in aged rats [52]. Thus, its therapeutic potential can be questioned. At the moment NGF is further evaluated as a therapeutic approach to cholinergic dysfuncrion, perhaps even cognitive dysfunction. However, NGF does not cross the blood-brain bartier and hence orally active NGF synthesis stimulators are being developed at the moment $1261 \mathrm{j}$.

\section{Inhibition of NO sywhose}

Some therapies are directed at diminishing the neurotoxicty of NO by inhibiting NO synthase. However, direct applications would mainly involve treatment of ischemia (stroke [39]), although they appear to offer also some future strategies for treatment of neurodegenerative (cognitive) impaiments $[121]$. 


\section{Activation of phosphoinosinide signal transduction}

According to recent studies, therapies should be based on substances "activating muscarinic receptor-coupled phosphoinositide hydrolysis" [40, 125, 126], since in $A D$ (and aging) mosily no changes in number (and intactness) of muscarinic receptors was found, whereas its functionatlity was affected. This could explain the limited therapeutic responses achieved by administration of cholinomimetrics to patient with $\mathrm{AD}$.

\section{Glia-derived-growth factors and antiinflammatory factors}

Glial cells produce many of the neuropeptides, transmitters and growth factors that are produced also by neurons (for a review see [163]). The roles that these glia-derived factors play in injury are not well understood, but potentially these factors could contribute to observed pathological changes, or alternatively might be important in recovery from brain injury $\llbracket 77,163]$. Glia-derived-growth factors have attracted interest as potential treatments for a number of neurodegenerative conditions including $\mathrm{AD}$ and $\mathrm{PD}$ [see 163]. At the moment it is unclear which receptors and how many second and third-messenger pathways are involved in the activation of astrocytes. The more pathways involved in activation of reactive glia-derived factor production, the better the chance for eventual therapeutic interventions that will inhibit deleterious effects associated with brain injury and reactive gliosis while sparing or angmenting neuroprotective effects. Gliosis in AD brain is accompanied with the presence of activated astrocytes and microglia and many inflammatory components like cytokines and complement factors in senile plaques [67]. As a matter of fact, microglia are the macrophage cells of the brain [48]. Thus, evidence is accumulating that antiinflammatory therapies can delay the onset and may even slow the progression of AD [180]. Preliminary findings have suggested some possible benefit, though more investigations and clear evidence are needed into the role of inflammation and its related therapeutic interventions in AD.

\section{REFERENCES}

1. Auneli T., Micchell A., Di Cocco M.E.L. Ghirandi O , Giulinni A. Ramacci M.T. and Conti F. (1994) Elfect of actyl- Learmitine on recovery of brain phosphorus metabolites and lactic acid level during reperfusion after cerebral ischemia in the rat - study by 31P-and IH-NMR spectroscopy. Broin Res.643, 92.99 .

2. Aurdi T. Micthel A. Ricciolini R. Di Cocco M.-A., Rantacei M.T., Angelucci L. Ghirgrdi O. and Cont $\mathrm{F}$. (1990) Aging bram: Elfect of acetyl-L-carnitine teatment on wat brain energy and phospholipid metabolism. A study by $31 \mathrm{P}$ and IH NMR spectroscopy. Brain Res" 526, 108-112.

3. Azari N.P. (1991) Efrect of glucose on memory processes in young aduls. Phychopharmacology 105. $521-524$

4. Barinaga M. (1995) Missing Alzheimen's gene found. Science 269, $917-918$.

5. Banes C.A., Markowska A.L., Ingran D.K. Kannetani H., Spangler E.L., Lemken V.J. and Ohton D.S (1990) Acetyl-L-cantine 2: Effects on learning and memory performance of aged rats in silmple and complex mases Neurobiol. Aging 11, 499-506.

6. Bartus R.T. Dear III R.L.. Beer B. and Lippa A.S. (1982) The cholinergic hypothesis of geviatric memory dysfunction. Srience 217, 408-417.

7. Bates T.E. Heales S.J.R. Davies S.E.C., Boakye P. and Clark J.B. (1994) Effects of 1-methyl-4. phenylpyridinitum on isolated rat brän mitochondria: Evidence for a primary involvement of energy depletion. J. Newrochem. 63,640 648.

8. Banaini F. Elkabes S., Bergamaschi S., Ladisa V., Luchi L., De Graan P.N.E., Sehumrnan T., Wetsel W.C. Trabucchi M. and Govoni $\$$. (1995) Protein kinase $C$ activity, translocation and convertional isoforms in aging rat brain. Neurobiol. Aging $16,137-148$.

9. Bandier J, Lee S.H. and Cole R.D. (1987) Separation of the different microtubule-associated tau protein species from bovane brain and the mode II phosphorylation by CA2+/phospholipid dependent protein kinase C. J. Biol. Chem. 262, 17584-17590.

10. Beal M.F. (1992) Does impaiment of energy metabolism result in excitotoxic neuronal death in 
neurodegenerative illnesses? Am. Neurol. 31. 119-130.

11. Beal M.F., Brouiller E., Jenkins B., Ferrarne R.J., Kowall N.W., Miller J.M., Storey E., Srivasava R., Rosen B, and Hyman B.T. (1993) Neurochemical and histologic characerization of striatal excitotoxic lesions produced by the nutochondrial toxin 3-nitroproprionic acid. $J$. Neurosci. $13,4181-4192$.

12. Beal M.F., Hyman B.T. and Koroshetz W. (1993) Do defects in mitochondriat energy merabolism underlic the pathology of neurodegenerative diseases? Trends Neurosci. 16, 125-131.

13. Behl C., Davis J.B., Klier F.G. and Schubert D. (1994) Anyloid beta peptide induces necrosis rather than apoptosis. Brain Res. 645, 254-264.

14. Behl C., Davis J.B., Lesley R. and Schubert D. (1994) Hydrogen peroxide mediates anyloid B protein toxicity. Cell 77, 817-827.

15. Bennett M.C., Diannond D.M., Stryker S.L., Parks J.K. and Parker W.D.J. (1992) Cytochrome oxidase inhibition: A novel animal model of Alzheimer's disease. Geriatr. Psychiatry Neurol 5, 93-101.

16. Bennet M.C. and Rose G.M. (1992) Chronic sodium azide treatment impairs learning of the morris water maze task. Behav. Neural Biol. 58, 72-75.

17. Benzi G., Pastoris O., Marzanico F., Villa R.F., Dagani F. and Curti D. (1992) The mitochondrial alectron transfer alteration as a factor involwed in the brain aging. Neurobiol. Aging 13, 361-368.

18. Benzi G., Villa R.F., Dossena M., Vercesi L. Gorini A and Pastoris O. (1984) Cerebral endogenous substrate utilization during the recovery period after profound hypoglycemia. J. Neurosci. Res. 11, 4.37450 .

19. Blokland A., Bothmer J., Honig W. and Jolles J. (1993) Behawional and biochemical efrects of acute central metabolic inhibition: Effects of acetyl-L-carnitine. Eur. J. Pharmacol. 235, 275;281.

20. Blokland A. and Jolles J. (1993) Spatial learning deficit and reduced hippocampal ChAT activity in rats after an icw injection of streptozotocin. Pharmacol. Biochem. Behat. 44, 49 1-494.

21. Blokland A. and Jolles J. (1994) Behavioral and biochemical effects of an icv injection of streptozoloein in old Lewis rats. Phamacol. Biochem. Behay, $47,833 \mathrm{~m} 837$.

22. Bobinski M., Wegiel J., Wisniewski H.M., Tarnawski M., Bobinski M., Reisberg B., De Leon M.J. and Miller D.C. (1996) Neurofibrillary pathology-correllation with hippocampal formation atrophy in Alzheimer disease Neurobiol. Aging 17,909-919.

23. Bolaffi J.L., Nagamatsu S., Harris J. and Grodsky G.M. (1987) Protection by thymidine, an inhibitor of polyadenosine diphosphate ribosylation, of streptozotocin inhibition of insulin secretion. Endocrinology 120. 2117.2122 .

24. Bothmer J, and Jolles J. (1994) Phosphoinositides, aging and Alzheimer's disease. Biachim. Biophys. Acta $1225,111-124$

25. Bothmer J., Markerink M. and Jolles J. (1994) Evidence for a selecrive decrease in type 1 phosphatidylinositol kinase activity in brains of patients with Alzheimer's disease. Dementia 5, 6-11.

26. Bothmer J., Markerink M. and Jolles J. (1994) Phosphoinositide kinase activities in synaptosomes prepared from brains of patients with Alzheimer's disease and controls. Neurosci. Letr. 176, 169-172.

27. Bowling A.C., Mutisya E.M., Walker L.C., Price D.L., Cork L.C. and Beal M.F. (1993) Age-dependent impairment of mitochondrial function in primate brain. J. Neurochen. 60, 1964-1967.

28. Braak H. and Braak E. (1991) Neuropathological stageing of Alzheimer-related changes. Acta Neusropathol. $82,239-259$

29. BrierIy J.B. Brown A.W. and Calverley J. (1976) Cyanide intoxication in the rat: Physiological and neuropathological aspects. J. Neurol. Neurostarg. Psychiary 39, $129-140$.

30. Burant C.F., Tretselaar M.K. and Base M.G. (1986) Diabetes-induced functional and structural changes in insulin receptors from rat skeletal muscle. J. Clin. Invest. 77, 260-270.

31. Burvin R., Armoni M. and Karnieli E. (1994) In vivo insulin action in normal and streptozotocin-induced diabehic rats. Physiol Behav $56,1-6$.

32. Bulterworth R.F. and Besnard A.M.M. (1990) Thiamine-dependent enzyme clisanges in temporal cortex of patients with Alzheimer's disease. Metab. Brain Dis. 5, 179-184.

33. Caldwell M., ONeill M., Earley B. and Leonard B. (1994) NG-nitro-L-arginine protecis against ischemiainduced increases in nitric oxide and hippocampal neuro-degeneration in the gerbil. Eur. J Phamacol. 260, $191-200$.

34. Callahan L.M. and Colernan P.D. (1995) Neurons bearing neurolibrillary tangles are responsible for salected synaptic deficits in Alzhemer's disease. Neurobiol. Aging 16, 311-314.

35. Caprioli A., Ghirardi O., Ramacci M.T. and Angelucci L. (1990) Age-dependent deficits in radial maze performance in the rat: Effect of chronic treatment with acetyl-L-Carnitine. Prog. Neuxopsychophthomacol. Biol. Psychiar. 14, 359-369.

36. Chal moniuk M. and Strosznajder J. (1995) Brain ischemia-reperfusion injury activates neuronal, constitutive form of nitric oxide synthase. Mollecular action of nitric oxide. Eur. J. Neurosci. Suppl. 6, $26,15.28$.

37. Chandrasekaran K., Giordano T. Brady D.R., Stoll J., Martin L.J. and Rapoport S.L. (1994) Impairment in mitochondrial cytochrome oxidase gene expression in Aztheimer's disease. Mol. Bran Res. 24, $336-340$.

38. Chen S., Frederickson R.C.A. and Brunden K.R. (1996) Neuglial-mediated immunoinflammatory responses in Alzheimer's disease: Complement activation and therapcutic approaches. Neurobiol. Aging 17, 781-788.

39. Choi D.W. (1995) Calcium: Still center-stage in hypoxic-ischemic neuronal death. Tresds Neurosci. 18, 
$58-60$

40. Chounard M.L. Gallagher M. Yasuda R.P., Wolfe B.B. and McKinney M. (1995) Hippocampal muscarinic receptor funcion in spatid learning-impaired aged rats Neurobiol. Aging 16.955-963.

41. Cin M. and Moretti, A. (1995) Studies on fipid peroxidation and protein oxidation in the aging brain. Nourobial Aging $15,53-57$.

42. Corder E.H., Satudern A.M., Rusch N.J., Schritmater W.J., Schmechel D.E., Gaskall P.C., Rimmler I.H., Locke P.A. Conneally P.M. Schmader K.E. Small G.W., Roses A.D., Haines I.L. and PericakVance M.A. (1994) Protective efcet of apolipoprotein E type 2 allele for late onser Alzheimer Disease. Nat. Gener. $7,180-184$.

43. Corder E.H. Saunders A.M., Stritmatter W.J., Schmechel D.E., Gaskell P.C., Smal G.W., Roses A.D., Haines J.Lu. and Pericak.Vance M.A. (1993) Gene dose of Apolipoproten E type 4 allele and the risk of Altheiner's disease in late onset families. Science $261,921 \times 923$.

44. Craft S., Newcome J., Kanne S. Dagogo.Jack S., Cryer P., Sheline Y., Luby J., Dagogowack A. and Alderson A. (1996) Memory improwement following induced thyperinsuhinemia in Alzhemers disease Neurobiol. Aging 17, 123.130.

45. Cummings B.J. Pike C.J. Shankle R. and Cotman C.W. (1997) B-amyloid deposition and other measures of neuropathology predict cognitiwe stadus in Alzhemer's disease. Neurobiol. Aging 17, $921-933$.

46. Curti D., Giangare M.C. Redolfi M.E., Fugaccia I and Benzi G. (1990) Age-related modifications of cytochrome coxidase activity in discrete brain regions. Mech. Ageing Develop. 55, 171-180.

47. DMello G.D. (1986) Eflects of sodium cyanide upon swimming performance in guinea-pigs and the confermant of protection by pretreatment with paminopropriophenone. Neurobehav. Toxicol. Teratol. 8 . 171.178.

48. Divis E.J., Foster T.D. and Thomas W.E. (1994) Cellular forms and functions of brain mictoglia. Brain Rer., Bull. 34, 73-78.

49. De Leon M.J., George A.E.,Golomb J. Tarshish C., Convit A., Kluger A., De Santi S. Mc Rae T. Ferrus S.H., Reisberg B. Ince C., Rusinek H., Bobinski M.., Quinu B., Miller D.C and Wisniewski H.M. (1997) Frequency of hippocampal formation atrophy in normal agng and Alzheimer's disease. Newrobiol. Aging 18,1.11

50. De Leon M.J. Harris S.H., George A.E., Reisberg B., Christman D.R. Kricheff I.L. and Wolf A.P. (1983) Computed tomograply and positron emission transaxial tomograply evaluation of normal aging and Alzheimer's Disease. J Ceneb. Blood Flow Metab. 3, 391-394.

51. Decker M.W. (1987) The eftects of aging on hippocampal and cortical projections of the foretrain cholinergic system. Bran Res. Rev. 12,423-438.

52. Dekker A.D., Fagan, A.M., Gage, F.H. and Thal, L.I. (1994) Effects of brain-derived neurotrophic factor and nerwe growth factor on remaining neurons in the lesioned nueleus basalis magnocellularis. Brain Res. 639, $149-155$.

53. Dekkel. A.J. and Thal L.J. (1993) Nerwe growth factor increases cortical choline acetyltransferasempositive fiber staining without affecting cortical cholinergic neurons. Brain Res. 601, 329-332.

54. DeKosky S. and Scheff $S$. (1990) Symapse loss in frontal coutex biopsies in Alzheimer's disease: Correlation with cogritiwe severity. Ank. Neurol. 27, 457-464,

55. Desbois C.. Capeau J., Hainalt J. Wiak P., Reynet C., Weissiere D. Caron M., Picard J., Guerte-Milla M. and Cherqui $G$. (199!) Differental role of insulin receptor autophosphorylation sites $1162-1163$ in the longtem insulim stmulation of glucosemanspont, glycogenesis and protein synthesis. J. Biol. Chem. 267 , $13488-13497$.

56. Dovaskaur S.U., Giddings S.d., Rajakumar P.A. Camaghi L.R., Menon R.K. and Zahn D.S. (1994) Insulin gene expression and insulin synthes in mammalian neuronal cells. J. Biof. Chem. 269.8445-8454.

57. Di Monte D.A., Sandy, M. S., DeLanney, L. E., Jewell, S. A. Chan, P., Irwin, I. and Langston, W, (1993) Age-dependerat changes in mitochondrial energy production in striatum and cerebellum of the monkey brain. Nenodegeneration $2,93-99$.

58. Dickson D.W. and Lee S.C. (1996) Microglia. In Texbook of uerropathology. (ed. Davis R. L. and Robertson D. M.). Williams and Wilkins, Ballimore.

59. Dickson D.W., Sintcropi S. Yen S.-H. Ko L.W. Mathiace L.A. Bucala R. and Vlassara H. (1996) Glycation and microglial reaction in lesions of Alzheimer's disease. Newrobiol. Aging 17, 733.743.

60. Ding A. Nitsch R. and Hoyer $S$. (1992) Changes in brain monoaminargic neurotransmitter concentrations in rat aller intracerebroventricular injection of streptozotocin. $J$. Cereb. Blood Flow . Metab. 12, 103-109.

61. Donble K.L. Halliday G.M., Kril J.J., Harasly I.A., Cullen K., Brooks W.S., Creasey H. and Broe G.A. (1996) Topography of brain arophy during nomal aging and Alzheimer"s disease. Neurobiol. Aging 17 ,
$513-521$.

62. Dournaud P., Deleare P.. Hauw J.I. and Epelbann J. (1995) Differental comelation between neurochemical deficits, neuropathology, and cogninive staus in Alzhemer"s disease. Neurobiol. Aging 16,817-823.

63. Duelli R., Schöck H., Kuschinsky W. and Hoyer S. (1994) Intracerebrowentricular injection of streptozotocin induces discrete local changes in cerebral glucose wilization in rats. Int. J. Devi Mewrosc: $12,737-743$. 64. Duhin W.E. and Wyse B.M. (1969) Studies on the ability of compounds to block diabetogenic activity of
streptozotocin. Diabetes 18, 459 . 
65. Fahrig T. (1994) Changes in solubiliry of ghat fibillary acidic proten after ischemic brain drame in the mouse. Netorochem.63,1796-1801.

66. Fernandez C.1, Gonzalez O., Soto J., Alvarez L. and Lores T. (1996) Effects of chronic infusion of nerve growh factor (NGF) in rats with nucleus basallis magnocellularis lesion. Mol. Chem. Nenropathol 28 . $181-184$.

67. Fiach C.E. and Marchalonis J J. (1996) Evolutionary perspectives on amyloid and inflammatory features of Alzheimer's discase. Newrobial. Aging 17,809-816.

68. Fischer W. (1994) Nerve growth factor reverses spatial memory imparments in aged ruts. New rochew. Int. $25,47.52$

69. Fischer W., Bjorklund A. Chen K. and Gage FH. (1991) NGF improves spaial memory in aged rodants. as a function of age. J. Newosci. $11,1889-1906$.

70. Fischer W., Gage F.H. and Björklund A. (1989) Degenerative chäges in forebran cholnerghe nuclei comelate with cognitive impaiments in aged rats. Eur. J. Newrosci. 1. 34-45.

7 I. Fischer W., Surevaag A., Wiegand S.J. Lindsay R.M. and Bjorklund A. (1994) Reversal of spatial menory impaiments in aged rats by nerve growth factor and neurotrophims 3 and $4 / 5$ but not by bran-deriwed neurotrophic factor. Proc: Nal Acad. Sci. U.S.A. 91, 8607-8611.

72. Fischer W. Wictorin K., Bjorklund A. Willams L.R. Varon S. and Gage F.H. (1987) Ameliomation of cholinergic meuron atrophy and spatial menory impairment in aged rats by nerve growth factor. Natume $329,65-68$.

73. Flood D.G., Coleman, P. D. (1988) Neuron numbers and sizes in aging brain: Comparisons of human; monkey, and rodent data. Neurobiol. Aging. 9, 453-463.

74. Flood J.F., Morley J.E. and Roberts E. (1991) Amnestic effects of mice of four syluhetic peptides homologous to amyloid beth protein from patients with Alzheimar's disease. Proc. Nall Acad. Sci. U.S.A. $88,3363-3366$

75. Flood J.F., Morley J.E. and Roberts E. (1994) An amyloid beta-protein frament, A beta[12-281, equipotently impars post-training memory processing when injected into different limbic system siructures. Brain Res, $663,271-276$.

76. Frautschy S.A., Yang F., Calderón L. and Cloe G.M. (1996) Rodent models of Alzheimer's disease: Rat $A B$ infusion approaches to amyloid deposits. Newrobiol Aging 17, 311-321.

77. Frederickson R.C.A. (1992) Astroglia in Alzheimer"s disease. Newrobiol. Aging 13, 239-253.

78. Friedland R.P. (1993) Alzheimer's disease: Clinical features and differential diagnosis. Neurology 43 (Suppl. 4), S45-\$51.

79. Friedich A.L. and Butcher L.L. (1994) Involwenent of free oxygen radicals in Bramyloidosis: An hypothesis. Newrobiol Agwg 15, 443-455.

80. Friedman $\mathbb{E}$. and $W$ ang $\mathrm{H}$. $-\mathrm{Y}$. (1989) Affects of age on brain cortical protein kinase $\mathrm{C}$ and its mediation of 5uydroxytryptamine release. J. Neurochem. 52, 187-192.

81. Gage F.H., Kelly P.A.T. and Björklund A. (1984) Regional changes in brain glacose metabolism reflect cognitive impaiments in aged rats. $J$. Neurosci. 11, 2856-2865.

82. Games D., Adams D., Alessandrini R , Barbour R.. Berthelette P. Blackwell C., Carr T., Chemens J., Donaldson T., Gillespie F., Guido T., Hagopian S., Johnson-Wood K. Khan K., Lee M., Leibowilz P., Lieberburg 1., Lutule S., Masliah E. McConlogue L., Montoya-Zavala M. Mucke L., Paganini L., penniman E., Power M., Schenk D., Seubert P., Snyder B, Soriano F., Tan H. Vitale d., Wadsworth S.

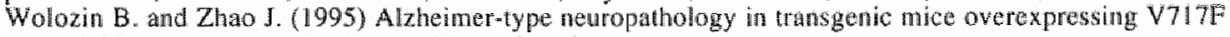
B-amyloid precursor protein. Narwe 373, $323,527$.

83. Games D., Khan K.M. Soriano F.G. Davis D.L.K.B. and Lieberbung 1. (1992) Lack of Alzheiner pathology after B-amyloid protein injections in rat brain. Neurobiol. Aging 13.569-576.

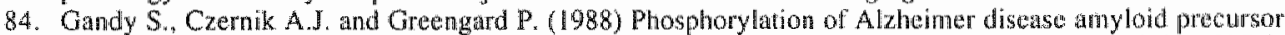
peptide by protein kinase $\mathrm{C}$ and Ca2+/calmodulin-dependent protein kinase II. Proc Nat Acad. Sci. U.S.A. $85,6218-6221$.

85. Garvey W.T., Huecksiead T.P. and Birnbaum M.J. (1989) Pretranslational suppression of an insulinresponsive glucose transporter in rats with diabetos mellitus, Scrence $245,60-63$.

86. Gearing M., Rebeck G.W. Hyman B.T.. Tigges J, and Mirra S.S. (1994) Neuropathology and apolipoprotein E profile of aged chimpanzees: Impilications for Alatimer disease. Proc. Nath Acad. Sci. U.S.A. 91, 9382.9386 .

87. Gearing M., Tigges J., Mori H. and Mura S.S. (1996) AB40 is a major form of B-amyloid in nonthuman primates. Neurobiol. Aging 17,903.908.

88. Geinisman Y., de Toledo-Morrell L. and Mornell F. (1988) Aged rats need a preserved complement of perforated axospinous synapses per hippocampal neuron to maintain good spatial memory. Brain Res. 398, $266-275$

89. Ghirardi O. Caprioli A. Milano S. Giulani A., Ramacci M.T. and Angeluce L. (1992) Aclive Avoidance Learning in old rats chronically treated will levocamilime acelyl. Physiol. Behav. $52,185-187$.

90. Ghirardi O. Milano S., Ramacci M.T. and Angelucei L. (1988) Effect of acetyl-L-carnitine chronic treatment on discrimination models in aged rats. Physial. Behaw. 44, 769.773.

91. Gibson G.E and Peterson C. (1981) Aging decreases oxidative metabolism atrod the release and synthesis of acetylcholine. Newrochens. 37, 978-984. 


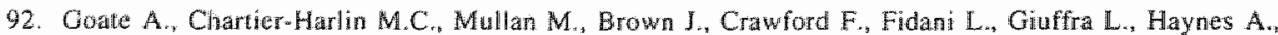
Irving $\mathbb{N}$, James $\mathrm{L}$., Mant R., Mewion P., Rooke K. Roques P. Tallool C. Pricak-Vance M., Roses A., Willianson R. Owen M. and Hardy $\mathbf{~} .(1991)$ Segregation of a missense mutation in the amyloid precursor protein gene with familial Alzheimer's disease. Nature 349, 704-706.

93. Couliaev A.H. and Scming A (1994) Piracetam and other structurally rekted nootropics. Bram Res. Rev. $19,180-222$

94. Grenberg B.D., Savage M.J. Howland D.S. Al S.M. Siedlak S.L. Perry G., Siman R. and Soot R. W. (1996) APP transenes: Approaches towards the development of animal models for Alzheimer disease nuturopathology. Newrobiol. Aging 17, 153 171

95. Gross P.M., Weater D.F. Bowers R.J., Wag S. Ho L.T., Pang JJ. and Espinosa F.J. (1994) Neurotoxicily in conscious rats following intraventricular SNAP, a nitric oxide donor. Neurophamacology $33,915-927$

96. Grundke-Jqbal I., Iqbal K.,Tung Y.C. Quinlan M., Wisniewski H.M. and Binder L.I. (1986) Abnornat phosphorylation of the microtubule-associated proten tau (tau) in Alzheimer cyloskeletal pathology. Proc. Natl Acad. Scí. U.S.A. 83, 49134917

97. Hammer B. Davis Parker Jr. W. and Bennet J.P. (1993) NMDA receptors increase OH radicals in vivo by using nitric oxide synthas and protein kinase C. NeuroReport 5, 72-74.

98. Hardy $\mathrm{J}$ and Alsop D. (1991) Amyloid deposition as the central event in the aetiology of Alzheimer's disease. Trends Pharmacol. Set 12, 383-388.

99. Harik S.L. and Kalaria R.N. (1991) Blood-brain bareier abnomalities in Alzheimer's disease. Anm. N. Y. Acad. Sici $640,47-52$

100. Haroutumian V., Kanof P.D. and Davis K.L. (1986) Partial rewersal of lesion-induced deficits in cortical cholinergic markers by nerwe growth factor Brain Res. 386, 397-399.

H01. Hartigan M.R. Kunkel D.D., Nguyen L.B. and Malout A.". (1995) Bata amyloid is neutoxic in hippocampal shice chltures. Neurobiof. Agimg 16, 779-789.

102. Harrington C.A. Mobley S.L. wnd Wenk G.L. (1994) Nitric oxide formation does not underlie the mernory deficits produced by iboterate injections into the nucleus basalis of rats. Behow. Neurosci. 108, $277,283$.

1.03. Harrington C.R and Colaco C.A.L.S. (1994) Alzheiner's disease. A glycation connection. Nature 370, $24.7-248$

104. Hatlway D.E. (1984) Molecalar aspects of toxicology pp. 84. The Royal Society of Chemistry, London.

105. Hawkins R.A. Mans A.M. and Davis D.W. (1983) Glucose avallability to individual cerebral structures as correlated to glucose metabolism. N. Neurochem. 40, 1013-1018.

106. Hefti F. (1986) Nerve growth factor promotes surwival of septal cholinergic neurons after fimbrial transections. J. Netrosci. 6, 2155-2162.

107. Heikkila R.E. Nicklas W.J. and Duwoisin R.C. (1985) Dopaminergic toxicity after the stereotaxio administration of the 1-methyl-4-phenylpyridinum ion (MPP+) to rats. Neurosci. Let. 59. 135-140.

108. Hellweg R., Fischer W., Hock. C., Gage F.H. Bjorklund A. and Thoenen H. (1990) Nerve growth factor levels and choline acelyltransferase activity in the brain of aged rats with spatial memory impairments. Brain Res. 537, 123-130

109. Hellweg R., Nitsch R., Hock C., Jaksch M. and Hoyer S. (1992) Nerwe growth factor and choline acetyltransfetase activity levels in the rat brain following experimental impairment of cerebral glucose and ancrigy metabolism. I. Neurosoi. Res. $31,479-486$.

110. Hennebeng N. and Hoyed S. (1994) Shortuterm or long-tem infracerebroventricular (i,c.v.) injection of Insulin exhibits discrete anabolic effect on cerebral energy metabolism in the rat. Newrosci. Lett. 175
153-156.

111. Heo J.H. Kim D.Q. Bahng H.R. and Kim J.-S. (1995) Dimethylthiourea prevents MPTP-induced decrease in 13 H dopamine uptake in rat striatal slices. Brain Res $671,32[-324$.

112. Hill J.M., Lesniak M.A. Pert C.B. and Roth J. (1986) Autoradiographic localization of insullin receptors in ran bran: Promineme in olfactory and limbic areas. Neurosience 17, $1127-1138$.

113. Holtum J.R. and Gertion $\mathrm{S}$ (1992) The cholinergic model of dementa, Azheimer type" Progression trom the unitary transmitter concept. Dememia 3, 174.185.

1 14. Honer W.G., Dickson D.W., Gleson J. and Davies P. (1992) Regional synaptic pathology in Alzheimer's clisease Nenrobior. Aging 13, 375-382.

IIS. Hoyer S. (1991) Abnormalities of. glucose metabolism in Alaheiner's disease. Ann. N. Y. Acad. Sci. 640, $53-57$

116. Hoyer S. (1992) Oxidative energy metabolism in Alzheimer brain. Mol. Chem. Newropathol. 16, $207-224$.

17. Hsaio K. Chapmán P., Nilsen S, Eckman C., Harigaya Y. Younkin S., Yang F. and Cole G. (1996) Correlative memory deficits, AB elevation and anyloid plaques in transenic mice. Science 274, 99-103.

118. Hutchin $T$, and Cortopassi $G$. (1995) A mitochondrial DNA clone is associated with increased risk for Alaheimer disense. Proc. Narl Acad. Sci, U.S.A. 92, 6892-6895.

19. ladecola C. Pelligrino D.A., Moskovitz M.A. and Lassen N.A. (1994) Nitric oxide synthase inhibition and cenbbrovascular regulation. J. Cereb. Blood Flow Merab. 14, 175-192.

120. Thara $Y$. Nukina $N$. Miura $\mathbb{R}$ and Ogawara $M$. (1986) Phosphorylated tau protein is integrated inco pared helical filaments in Alzheinmers disease. J. Biochem. 99, 1807-1810

121. Ingram D.K., Shimada A., Spangler E.L., Kari H., Hengemihle J., Kuo H. and Greig N. (1996) Cognitive 
enthancement. New strategies for stmulating cholinergic, glutamatergic, and mitric oxide systems. Anm. N.Y. Acod. Sci. $786,348-361$.

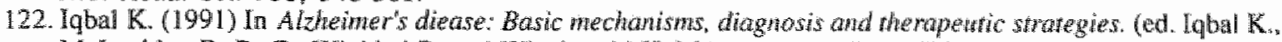
Mclachlan D. R. C., Winblad B. and Wisniewski H. M.), pp. L-5. Wiley, Chichester.

123. Jolles J., Bothmer J., Markerink M. and Ravid R. (1992) Phosphatidylinositol kinase actuiry is reduced in Alzhemer's disease. J. Neurachern. $2326-2329$.

124. Jolles J., Bothmer J., Markerink M. and Ravid R. (1993) Reduced phospladitylinositol kinase activity in Althemer's disease: Effects of age and onset. Dementia $4,81-86$.

125. Jope R.S., Song L., Li X. and Powers R. (1994) Impaired phosphoinostide hydrolysis in Alzheimars disease brain. Neurobiol. Aging $15,221-226$.

126. Jope R.S., Song L. and Powers R.E. (1997) Cholinergic activation of phosphoinositide signaling is impaired in Alzheimer's disease brain. Neurobiol. Aging 18, 111-120.

127. Kadowaki T., Kasuga M. Akanuma Y., Ezaki O. and Takaku F. (1984) Decreased autophosphorylation of the insulin receptor-kinase in streptozotocin-treated rats. $J$. Biol. Chem. $259,14208-14216$.

128. Kahn C.R. (1985) The molecular mechanism of insulin action. Amm Rev. Med. $36,429.451$.

129. Kahn R.A., Panah M. and Weinberger J. (1997) Modulation of ischemic excitatory neurotransmitter and gamma aninobutyric acid release during global temporary cerebrall ischemia by selective metronal niteic oxide synthase inhibition. Anesthesia Analgesia 84, 997-1003.

130. Karmieli E. and Armoni M. (1990) Regulation of glucose transporters in diabetes. Horm. Res. 33, 99. \04.

131. Katzman R. (1986) Alzheimer's disease. N. Eng. J. Med 314, $964-973$.

132. Khachaturian Z.S. (1994) Calcium hypothesis of Alzheimer's disease and brain aging. Ann. N.Y. Acad. Sci. $747,1-11$.

133. Kish S.J., Bergeron, C., Rajput, A., Dozic, S., Mastrogiacono, F., Chang, L. J., Wilson, J. M., DiStefano, L. M., Nobrega, N. (1992) Brain cytochrome oxidase in Alzheimer's disease. d. Newrochem. 59, $776-779$.

134. Kopf S.R. and Baratti C.M. (1994) Memory-improving actions of glucose: Involvenent of a central cholinergic muscarinic mechanism. Behav. Newal Biol. 62, 237-243.

135. Kosik K. and Coleman P. (1992) is B-amyloid neurotoxic? Neurobiol. Aging 13, 535-627.

136. Koutowzis T.K. Borlongan C.V., Freeman T.B., Cahill D.W. and Sanberg P.R. (1994) Intrastratal 3w mitropropionic acid: A behavioral assessment. NeuraReport 5, 2241-2245.

137. Kowall N., Beal M.F., McKee A.C. and Kosik K.S. (199li) Okadaic acid produces dose-dependent meurotoxic lesions and increased phosphorylation of neurofilament and tat proteins in vivo. $\mathrm{J}$. Cell. Biol. $115,385 \mathrm{a}$.

138. Kowall N.W. MeKee A.C., Yanker B.A. and Beal M.F. (1992) In vivo neurotoxicity of beta amyloid [18(140)] and the [B(25-35)] fragment. Neurobiol Aging 13,537-542.

139. Kunkler P.E. and Kraig R.P. (1997) Reactive astracytosis from excitotoxic injury in happocampal organ culture parallels that seen in viwo. $J$. Cereb. Blood Flow Merab. 17, 26-43.

140. LaFerla. F.M. Tinkle B.T., Bieberich C.J., Haudenschild C. and Jay G. (1995) The Alzheimer's AB peptide. induces netrodegemeration and apoptotic cell death in trangenic mice. Nat. Genet. 9, 21-30"

141. Lai R.Y.K., Gertz H.N.-J., Wischik D.J., Xuereb J.H., Mukaetowa-Ladinska E.B., Harrington C.R., Edwards P.C., Mena R., Paykel E.S., Brayne C., Huppert F.A., Roll M. and Wischik C.M. (1995) Exarnination of phophorylated tau protein as a PHF-precursor at early stage Alzheimer's disease. Nethrobiol. Aging $16,433-445$.

142. Lewy-Lahad E., Wasco W. Poorkaj P., Romano D.M., Oshima J., Petugell W.H., Yu C.E., Jondo P.D., Schnidt S.D., Wang K., Crowley A.C.. Fu Y.H., Guenette S.Y., Galas D., Nemens E., Wijaman E.M., Bird T.D., Schellenberg G.D. and Tamzi R.E. (1995) Candidate gene for the chromosome I famillal Alzheimeris disease locus. Science 269, 973-977.

443. Li J.J., Surini M. Catsicas S. Kawashima $\mathbb{E}_{\text {and }}$ Constatin B. (1995) Agemependent accumulation of advanced glycosylation products in human newrons. Newrobiol. Aging 16, 69-76.

144. Lienhard GE., Slot $\mathbb{J}$. W. James D.E. and Mueckler M.M. (1992) How cells absorb glacose. Sci. Am. $266,86-91$

145. Loiacono R.E. and Beart P.M. (1992) Hippocampal lesiors induced by microinjection of uitric oxide donor nitroprusside. Eur. J. Phamacol. 216, 331-333.

146. Loring J.F., Paszty C., Rose A. MeIntosh T.K., Murai H., Pierce J.E.S., Schiani S.R., Wymore K., Lee W.M.- Y., Trojamowski J.Q and Peterson K.R. (1996) Rational degign of an animal model for Alaheimer's disease: Introduction of multiple human genomic transgenes to reproduce $A D$ pathology in a rodent. Nenrobiol Aging 17, 173-182.

147. Ma J. Lee A., Brewer H.B., Das S. and Potter H. (1994) Amyloid associated proteins a antichymotrypsin and apolipoproten E promote assembly of Aldheimer B-protein into filaments. Nature 372, 92-94.

148. Mackenzie I.R.A. Hao, C. and Munoz, D.G. (1995) Role of mioroglia in senile plaque formation. Newrobiol. Aging 16, 797-804.

149. MacMillan V.H. (1989) Cerebral energy metabolism in cyanide encephalopathy. J. Cereb. Blood Flow Merab. $9,156-162$.

150. Magnoni M.S., Govoni S., Batami F. and Trabucchi M. (1991) The aging brain: Protem phosphorylation as a target of changes in neuronal function. Life Sci. $48,373.385$. 
151. Mandelkow E.-M., Biernat J, Gustke N., Trinczek B. and Mandelkow E. (1995) Tau domains, phosphorylation, and interactions with microtubules. Neurabiol Aging 16, 355-363.

152. Manning C.A. Ragozzino M.E and Gold P.E. (1993) Glucose enhancement of memory in patients with probably senile dementia of the Altheimer's type. Neurobiol. Aging 14, 523-528.

153. Mantione J.R., Kleppner S.R.. Miyazono M. Werikin A.M., Lee V.M.-Y. and Trojanowski J.Q. (1995) Human neurons that constitutively secrete $A B$ do not induce Alzheimer's disease pathology following transplankation and long-term survival in the rodent brain. Brain Res. 671, 333-337.

154. Martinez M., Ferrandiz M.L., De Juan E. and Miquel J. (1994) Age-related changes in gfutathione and lipid peroxide content in mouse synaptic mitochondria: Relationship to cy tochrome coxidase decline. Neurosci. Leti. $170,121-124$.

155. Masoro $\mathrm{E}$. (1988) Food restriction in rodents: An evaluation of ats role in the study of aging. J. Gerontol. BIOL $5 \mathrm{Cl}, 43, \mathrm{~B} 59-\mathrm{B} 64$.

156. Mastrogiacomo F., Bergeron C, and Kish S.J. (1993) Brain $\alpha$-ketoglutarate dehydrogenase complex activity in Alzhemer"s disease. J. Newrochemu. 61, 2007-2014.

157. Mathies H.J.G.. Palfrey H.C. Hirning L.D. and Miller R.J. (1987) Down regulation of protein kinase C in netronal cells: Effects on neurotransmiter release. J. Neurosci. 7, 1198-1206.

158. Mattson M.P. (1995) Degencrative and protective signalling mechanisms in the neurofibrillary pathology of AD. Nevrobiol. Aging 16, 447-463.

159. Mattson M.P. Barger S.W., Cheng B. Lieberburg I., Smith-Swintosky V.L. and Rydel R.E. (1993) BAmyloid precursor protein metabolites and loss of neuronal $\mathrm{Ca}^{2}+$ homeostasis in Alzheimer's disease. Trends Neztosci. 16, 409.414.

160. Matson M.P., Carne J.W. and Butterfield D.A. (1995) A tombstone in A.lzheimer's? Nature 373, 48I

161. Mayer $G$., Nisch R. and Hoyer S. (1990) Effects of changes in peripheral and cerebral glucose metabolism on locomotor activity, learning and memory in addult male rats. Brain Res. 532, 95-100.

162. McDonald M.P., Dahl E.E. and Overmier J.B. (1994) Effects of exogenous f5-amyloid peptide on retention for spatial learning. Behov. Neurat Biol. 62, 60-67.

163. McMillian M.K., Thai L., Hong J.-.S., O'Callaghan J.P. and Penneypacker K.R. (1994) Brain injury in a dish: A model for reactive gliosis. Trends Neurosci. 17, 138-142.

164. Mecocci P., MacGarvey U. and Beal M.F. (1994) Oxidative damage to mitochondrial DNA is increased in Alzheimer's disease. Ann. Neurol. 36, 747-751.

165. Mecocci P. MacGarvey U, Kaufman A.E., Kooniz D., Shoffner J.M., Wallace D.C. and Beal M.F. (1993) Oxidative damage to mitochondrial DNA shows marked age-dependent increases in human brain. Anm. Neurol. 34, 609-616.

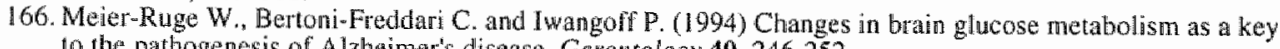
to the pathogenesis of Alzheimer"s disease. Ge rontology 40, $246-252$. 167. Messier C. and Destrade C. (1994) Insulin attenuates scopolamine-induced memory deficits. Psychobiology
22, 16-21.

168. Meyer E.M., Judkins J.H., Momol A.E. and Hardwick E.O. (1994) Effects of peroxidation and aging on rat neocortical ACh-release and protein kinase C. Newrobiol. Aging. 15, 63-67.

169. Mordes J.P. and Rossini R.A. (1981) Animal models of diaberics. Am. J. Med. 70, 353-360.

170. Morishima-Kawashima M., Hasegawa M., Takio K., Suzuki M., Yoshida H., Watanabe A., Titani K. and Ihara Y. (1995) Hyperphosphoryllation of tau in PHF. Neurobiol. Aging 16, 365-380.

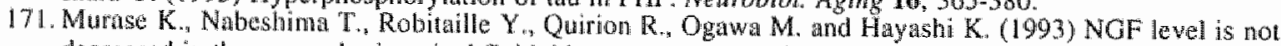
decreased in the serum, brain-spinal fluid, hippocampus, or parietal contex of individuals with Alzheimer"s discase. Biochem. Bioplys. Res. Conm 193, 198-203.

172. Nabeshima T. and Nitta A. (1994) Memory impairment and neuronal dysfunction induced by beta-amyloid protein in rats. Tohoku. J. Exp. Med. 174, $241-249$.

173. Nalbantoglu J., Tirado-Santiago G., Lahnsaïni A., Poirier J, Goncalves O., Verge G., Momoli F., Weiner S.A. Massicote G., Julien J.-P and Shapiro M.L. (1997) Imparred learning and LTP in mice expressing the carboxy terminus of the Alzheimar amyloid precursor protein. Nature 38\%, 500-505.

174. Nichols N.R. Day J.R. Laping N.J., Johnson S.A. and Finch C.E. (1993) GF.AP mRNA increases with age in rat and human brain. Neurobiol. Aging 14, 421-429.

175. Nictols N.R. Finch C.E. and Nelson J.F. (1995) Food restriction delays the age-related increase in GFAP in mRNA in rat hippocampus. Neurobiol. Aging 16, 105-1.10.

176. Nitsch R. and Hoyer $\mathrm{S}$. (1991) Local action of the diabetogenic drug, streptozotocin, on glucose and energy metabolisn in the brain cortex. Neurosci. Lett. 128, 199-202.

177. Nitsch R., Mayer G. and Hoyer S. (1989) The intracerbroventriculary streptozotocin-treated rat: Impairment of cerebral glucose metabolism resembles the alterations of carbohydrate metabolism of the brain in Alzheimer's disease. J. Neural Trans. 1, 109-110.

178. Northwood J.C. and Davis R.J. (1989) Protein kinase C inhibition of the epidermal growt factor receptor tyrosine protain kinase activity is independent of the oligomeric state of the receptor. J. Biof. Chem. 264 .
$5746-5750$.

179. Nowicki J.P. Duval D. Poignet H. and Scatton B. (1991) Nitric oxide mediates neuronal death after focal cerebral ischemia in the mouse. Eur. J. Pharmacol 204, 339-340.

180. O'Barion M.K. and Finch C.E. (1996) Inflammatory mechanisms and anti-inflammatory therapy in 
Althemer's disease. Neurobiol. Agtng 17,669-671.

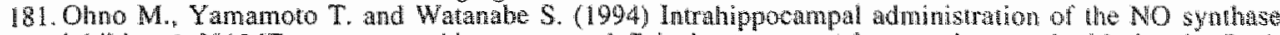
inhbitor L-NAME prevents working memory deficis in rats exposed to mansient cercbral ischemia. Bfan Res. 634, 173-177.

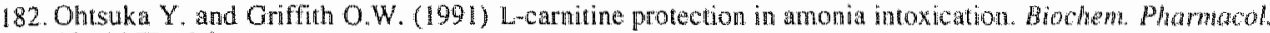
12. $1957-1961$.

183. Olanow C.W. (1993) A nadical hypothesis for newrodegeneration. Trevds Nestosc. 16,439.44.4.

184. Pardridge W.M. (1994) Glucose transport and phosphorylation: Which is rate limitig br brain glticose Wilization? Anm. Wewor, 35, 511-512.

185. Parsons M.W. and Gold P.E. (1992) Glucose enhancement of memory in elderly humans: An inverted-U dose-response curve Newrobiol. Aging 13, $401-404$.

186. Patel M.N., Yim G.K.W. and Isom G.E. (1992) Blockade of N-Methyl-D.Aspentate receptors preyents cyande-induced netronal injury in primary hippocampal cultures. Toxicol. Appl. Pharmacol, 115, 124. 129.

187. Perry E.K. (1980) The cholinergic system in old age and Alzhentmer's disease. Age Ageing 9, 1-8.

188. Perry E.K., Johnson M. Kerwin J.M., Piggot M.A., Coun J.A., Shaw P.J., Ince P.G., Brown A. and Perry R.H. (1992) Convergent cholinergic aclivities in aging and Alzhemer's disease. Neurobiol. Aging $13,393-400$

189. Pettegrew J.W., Panchalingam, K., Klunk, W. E., Kanter, J. N., McClure, R. J. and Muenz, L. R. (1994) Alterations of cerebral metabolism in probable alzheimar's disexse: A prelinamary study. Neurobiol. Agimg $15,117 \% 132$.

190. Pettegrew J.W., Klunk W.E., Panchalingam K., Kanfer J.N. and McClure R.J. (1995) Clinicnl and neurochemical effects of acetyl-L-camine in Alzlyeimer's disease. Neurobiot. Aging 16, 1.4.

191. Plaschke $\mathrm{K}$. and Hoyer S. (1993) Action of the diabetonic drug streptozotocin on glycolytic and glycogenolytic metabolism in adult rat brain cortex and hippocanpus. Int. J. Devl Neurosci, 11, 477 483.

192. Podlisny M.B. Stephanson D.T., Frosch M.P., Lieberburg I., Clemens J.A. and Selkoe D.J. (1992) Synthetic amyloid B-protein fails to produce specific neurotoxicity in monkey cerebral contex. Newrobiol. Aging 13, 561-568.

193. Price D.L., Martin L.J., Sisodia S.S., Wagster M.V., Koo E., H., Walker L.C., Koliatsos V.E. ard Cork L.C. (1991) Aged nonhuman primates: An animal model of age associated nearodegenerative disease. Brain Pathol. II., $287-296$.

194. Proper E.A., de Jong G.L, Luiten P.G.M. and Korf J. (1995) Local administration ol B-amyloid (B25-35) peptide in the nucleus basalis of meynert affects cholinergic neurons. Ewr. J. Neurosici. Stuppl. 8, 171 , 68.07.

195. Quon D., Wang Y., Catalano R, Scardina I.M., Murakami K. and Cordell B. (1991) Formation of betaamyloid protein deposits in brains of transgenic mice. Nature 352, 239-241.

196. Ramsay R.R., Krueger M.J., Youngster S.K., Gluck M.R., Casida J.E and Singer T.P. (199l) Ineraction of 1-methyl-4-phenylpyridinium lom (MPP+) and its analogs with the rotenone/piericidin binding site of NADH detyydrogenase. J. Neurochem. 56, 1184-1190.

197. Rasmussen T., Schliemanen T. Sprensen J.C., Zimmer J. and West M.J. (1996) Memory impared aged rats: No loss of principal hippocampal and subicular neurons. Neurobiol. Aging 17, 143-147.

198. Regeur L., Badsberg Jensen G. Pakkenberg H. Evans S.M. and Pakkenberg B. (1994) No global neocortical nerve cell loss in brans from patients with senile dementia of Alaheimer's lype. Newrobiol. Aging 15, 347-352.

199. Renikainen K.J., Riekkinen P.J., Paljaryi L.., Souninen H., Helkala E.L.. Jolkkonen J. and Lakkso M. (1988) Cholinergic deficit in Alzhemer's disease: A study based on CSF and anopsy ata. Neurodhem. Res. 13, 135-146.

200. Richards S.I. Waters I.J. Beyreuher K., Masters C.L. Whishik C.M. Sparkman D.R., White C.L., Abraham C.R. and Dunnett S.B. (1991) Transplants of mouse trisomy 16 hippocimpus provide a model of Alzheimer is disease neuropathology, EMBO i. 10, 297-303.

201. Rosengren L.E. Lycke I. and Anderson 0. (1995), Gliallibrillary acidic protein in CSF of ritulipte sclerosis patients: Relation to neurological deficit. J. Neurol. Sci. 133, 61-65.

202. Sainon T., Masliah E. Jin L..W., Cole G.M., Wieloch T. and Shapiro I.P. (1991) Biology of disease: Protein kinases and phosphorylation in meurologic disorders and cell death. Lab. Tnvest. 64, 596-61.6.

203. Santago M., Venero J.L.. Machado A and Cano J. (1992) In vivo protection of striatum from MPPt meurotoxicity by N-methyl-D-aspartate antagonists. Brain Res. 586, 203-207.

204. Santucci A.C., Kanof P.D. and Haroutunian V. (1993) Cholinetgic marker deficits induced by lesions of the nueleus basal is of Meynert are altenuated by nerwe growth factor in youmg, but not aged. F344 rats. Brain Res. 609, 327.332.

205. Scheff S.W., Anderson, K.J. and DeKosky. S.T. (1985), Strain comparison of synaptic density in hippocampal CA 1 of aged rats. Neurobiol Aging 6, 29-34.

206. Scheff S.W. and Price D.A. (1993) Synapse loss in the temporal lobe in Alzheimer's disease. Amm. Newnol. $33,190-199$.

207. Schellenberg G.D., Bird T.D. Wijsman E.M., Orr H.T., Anderson L., Nemens E., White J.A., Bonnycastle L. Weber J.L., Alonson M.E., Poter H., Heston L.L. and Maruir G.M. (1992) Genetic 


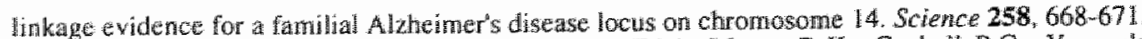

2018. sethellemberg G.D., Pericak-Vance M.A., Wijman E.M., Moore D.K., Gaskell P.C. Yamaoka L.A., Bebout IL Anderson L., Welsh K.A., Clark C.M. Marin G.M., Roses A.D. and Bird T.D. (1991) Linikage andysis of familial Alzheimers disease, using chromosome 21 markers. Am. J. Hum. Gener. 48. 563.583 .

209. Schipper H.M. (1996) Astrocytes, brain aging, and newrodegemeration. Neurobiol Aging 17, $467-480$.

210. Schraufintter I.U., Hyslop P.A. Hinshew D B. Spragg R.G., Sklar L.A. and Cochrane C.G. (1986) Hydrogen peroxide-induced injury of cells and ils prevention by intuibiors of poly(ADP-rbose) polymerase. Proc. Noil Acad. Sci. U.S.A. 83, 4908-4912.

211. Schritmater W.J. Weisgraber K.H., Goedert M. Satuders. A.M., Huarg D. Corder E.H., Dong L.M., Jakes R., Albertis M.J. and Gibert J.R. (1994) Hypothesis: Microwbule instability and paired hedical flament formation in the Altheimer disease brain are related to apolipoprotein E genotype. Exp. Neurol. $125,163-171$.

212. Scou S.A., Liang S. Wentertner J.A. and Cnutcher K.A. (1994) Increased NGF-like activity in young but not aged rat hippocampus after septal lesions. Neurobiol. Aging 15, 337-346.

213. Sulkoe D J. (1991) The molecular pathology of Alzeimer's disease. Neuron 6,487-498.

214. Sukoe D.J. Podinsny M.D.,Joachim C. Wikers E.A., Les G., Fritz L.C. and Oltersdorf T. (1988) Betaamyloid precursor protein of Alzheimer"s disease occurs at $110 \mathrm{~m}$ 10 135 -kilodalton nembrane-associated proteins in neural and nomeural ussue. Proc. Nanl Acad. Sci. U.S.A. 85, 734 1-7345.

215. Shapir: A.H.V. Cooper J.M., Dexter D., Clark J.B., Jenner P. and Marsden C.D. (1990) Mitochondrial complex 1 defliciency in Parkinson"s disease. $J$ Newrochem. 54, 823-827.

216. Shapira A.H.V., Marn V.M. Cooper J.M. Dexter D., Daniel S.E., Jenner P.. Clark J.B. and Marsden C.D. (1990) Anatomic and discase specificity of $\mathrm{NADH} C \mathrm{CQ}$ reductase (Complex I) deficiency in Parkinson's disease. Nenrochem. 55, 2142-2145.

217. Shapiro 1.P. Maslah E. and Saitoh T. (1991) Altered protein byrosine phosphorylation in Alzheimer's disease. J. Neurochem. 56, $1154-1162$.

218. Sherrington R., Rogaev E. I. Liang Y., Rogateva E.T., Levesque G., Ikeda M., Ci H. Lin G., Holman K. Tsuda T. Mar L., Foncin J.-F. Bruni A.C., Montesi M.P., Sorbi S., Rainero I., Pinessi L.. Lee I... Chumakow I., Pollen D., Brookes A., Sanseau P., Polinsky R.J., Wasco W., Da Silva H.A.R., Haines J.L. Pericak-Vance M.A. Tanzi R.E., Roses A.D., Fraser P.E., Rommens J.M. and St. Georges-Hyslop P.H. (1995) Cloning of gene bearing missense mutations in early onset familial Aldheimer's disease. Nature $375,754-760$.

219. Shigenaga M.K. Hagen, T.M. and Ames, B.N. (1994) Oxidative damage and mitochondrial decay in aging. Proc. Nont Acad Sci. U.S.A. 91, 10771-10778.

220. Shimano Y., Kumazaki M.x Sakurai T., Hida H., Fujimoto 1., Fukuda A. and Nishino H. (1995) Chronically administered 3 -nitropropionic acid produces selective lesions in the striatum and reduces muscle Ionus. Obes. Res. 3, $1071-7323$.

221. Shimohama S., Fujimoto S. Taniguchi T. and Kimura I. (1992) Phosphatidylinositol-specific phospholipase $\mathrm{C}$ activĭty in the postmortem human brain: No alterations in Alzheimer's disease. Brain Res. $579,347-349$

222. Shiosakn S. (1992) Attempts to make models for Alzheimer's disease. Neurosci. Res. 13. $237-255$.

223. Shofiner J.M., Brown M.D., Tononi A., Lon M.T. Cabell M.F., Mirra S.S., Beal M.F., Yang C.-C., Gearing M., Salvo R., Wats R.L., Juncos J.L., Hansen L.A. Crain B.J., Fayad M. Reckord C.L. and Wallace D.C. (1993) Mitochondrial DNA mutations associated with Alzheimer's and Parkinson's disease. Genomics 17, $171-184$

224. Sinpxson 1.A., Chundu K.R., Dänes-Hill T., Honer W.G. and Davies P. (1994) Decreased concentrations of CLLTI and GLUT3 glucose transponers in the brans of patien is with Alzheimer's disease. Amm. Neurol $35,546.551$

225. Singet T.P. and Rumsany R.R. (1990) Mechantsm of the neurotoxicity of MPTP. Fed. Eur. Biochem. Soc. $274,1-8$

226. Sunili G. (1988) Animal models of Alzeimer"s disease: Experimental cholinergic denerwation. Brain Res. Rev, 13, 103-118.

227. Spagnoli A. Lucca U., Menasce G. Bandera L., Cizza G., Forloni G., Tentamanti M., Franura L.* Tiruboschi P. Comelli M., Senin U., Longo A., Petrini A., Brambilla G. Belloni A., Negr C..

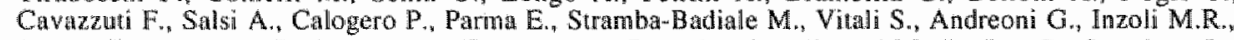
Sentus G. Caregnato R., Peruzza M., Favaretto M., Bozeglav C. Alberoni M., De Leo D., Serraiotto L. Baiocchi A. Scoccia $S_{. .}$Chlotta P. and leractano D. (1991) Long-term acetyl-L-carnitine treatment in Alaheimer's diseatse. Newrotogy 41, 1726-1732.

228. Stoll J., Babo A. Aull B., Rapoporn S.I. and Fine A. (1993) Long term transplants of mouse trisomy 16 hippocampal neurons, a model for Down's syndrome, do not develop Alzheimer's disease newropathology. Brain Res: 610, 295-304.

229. Strijbos P.L.M. Knowles. R. and Rotwwell N.J. (1993) Nitric oxide mediates glutamate neurotoxicity in the rat striatum. Brain Res. Assac. Abstr. 10, 46, 8.6.

230. Stritmatce W.J., Saunders A.M., Schmechel D., Pericak-Vance M., Enghild I., Salvesen G.S. and Roses A.D. (1993) Apolipoprotein E: High-avidity binding to B-anyloid and increased frequency of type 4 allele 
in late-onset familial Alzhemer's disease. Proc. Nol Acad Sol U.S.A. 90, $1977-1981$.

231. Sullvar E.V. Marsh L., Mathalon D.H. Lim K. O. and Ptefferbiam A. (1995) Age-relatid decline un MRI volumes of temporal lobe gray mater but not hippocampus. Neurabiol Aging 16, 591-606.

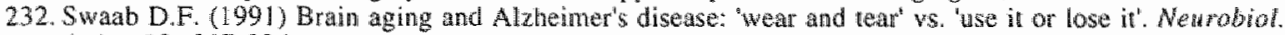
Aging $12,317-324$

233. Swatb D.F., Holman M.A., Lucassem P.J. Salehi A. and Uylings H.B.M. (1994) Neuronal atrophy, not cell death, is the main hallmark of Alzheimer's disease. Nowrobiol. Aging 15, 369-371.

234. Taglialatela G., Angelucei L . Ramacci M.T. Werbach Perez K., Jackson G.R and Penez-Polo IR. (1991) Acetyl-L-carmitine enhances the response of PC 12 cells to nerve growth factor. Dev. Bratu. Res. 59. 22I230 .

235. Takasu N., Komiya I. Takayuki A., Nagasawa $Y$ and $Y$ amada $T$. (1991) Streptozotocin-and alloxaninduced 1202 generation and DNA fragmentation in pancreatio islets. Diabetes 40, $1141 \% 1145$.

236. Ter Horsi G.J. Knigge M.F. and Van der Wal A. (1992) Newrochemical losioning in the rat brain with

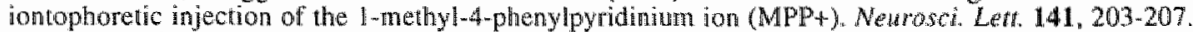

237. Terry R.D., Masliah E., Salmon D.P., Buters N.. DeTeresa R., Hill R. Hanse L.A. and Katzman R. (1991) Physical basis of cognitive alterations in Alzheimer's disease: Synapse loss is the muajor correlate of cognitive impaiments. Ann. Neurol. 30, 572-580.

233. Terwel D., Bothmer F., Meng E., Woll E., Markerink M. and Jolles J. (1994) Reduced enzyme activities in Alzheimer"s disease are sensitive to hypoxia. Nearobiot. Aging 15 (Suppl.1), $\$ 120$.

239. Torre de la J.C. (1994) Impaired brain microcirculation may trigger Alzheimers disease. Neurosci. Biobehav. Rev. 18, 397-401.

240. Torres E., Pery T.A., Blokland A., Wilkinson L.W. and Dunnet S.B. (1994) Behavioural. histocluemuch and biochenical consequences of selective immunolesions in discrete regions of the basal forebrain cholinergic system. Neuroscience $63,95-122$

241. Trojanowski J.Q. Shin R.-W., Schmidt M.L. and Lee V.M.-Y. (1995) Relationship between plaques, langles, and dystrophic processes in Alzheimer"s disease. Newrobiol. Aging 16, 335-345.

242. Tucek S. Ricny J. and Dolezal V. (1990) Advances of biology of cholinergic neurons. Anw. Newrot. 51 , $109-115$.

243. Turski L. and Turski W.A. (1993) Towards an understanding of the role of gluamate in neurodegenerative disorders: Energy metabolism and neuropathology. Experimewtia 49, $1064-1072$.

244. Undie A.S. and Friedman E. (1993) Diet restriction prevents aging-induced defucts in brain phosphoinositide metabolism. J. Gerontol. 48, B62-B67.

245. Undie A.S., Wang H.-Y. and Freidman E. (1995) Decreased phospholipase C-B inmunoreactivity, phosphoinositide metabolism, and protein kinase $C$ activation in senescent $F-344$ rat braun. Newrobiol. Aging 16, 19-28.

246. Unger J.W., Livingsion J.N. and Moss A.M. (1991) Insulin receptors in the central nervous system: Localization, signaling mechanisms and functional aspects. Prog. Neurobiol, 36, 343-262.

247. Van der Zee C.E., Lounenssen S., Stanisz J. and Diamond J. (1995) NGE deprivation of adult rat brain results in cholinergic hypofunction and selective impatrments in spatial learning. Eur, J. Neurosct. 7, 160 168.

248. Vijayan V.K., Geddes J., Anderson K., Chang-Chui H. Ellis W. and Colmen C.W. (1991) Astrocyte hypertrophy in Alzheimer's disease hippocampal formation. Exp. Newrol. 112, 72*78.

249. Villa R.F. and Gorini A. (1991) Action of L-acetylcamitime on different cerebral mitochond rial populations from hippocampus and stratum during aging. Newrochem. Res. 16, $1125-1132$.

250. Villa R.F., Gorini A. Zanadi F. and Benzi G. (1986) Action of L-acetylearnitine on different ceretonal mitochondrial populations tram hippocampus. Aroh. Int. Pharptacodyo. 279, $195-211$.

251. Wallace D. (1992) Mitochondrial genetics: A paradigm for ageing and newodegenerative diseases? Science $256,628-632$

252. West M.J. (1994) Differences in the pattern of hippocampal neuron loss in normal aging and Alzheinger's disease. Lancer 344, 287-293.

253. Whitson J.S. and Appel S.H. (1995) Neurotoxicity of AB amyloid protein in witro is not altered by calcium channel blockade. Newrobiol. Aging 16, $5-10$,

254. Wiley R.G. Oetumann T.N. and Lappi D.A. (1991) Immunolesioning: Selective destruction of centrial and peripheral neurones in vivo usitig an immunotoxin to the rat NGF receptor. Soc. Neurosc. Absrr. 17, 222,

255. Williams L.R. Rylett R.J... Moises H.C. and Tang A.H. (1991) Exogenous NGF affects cholinergic transmitter function and $Y$-maze behavior in aged Fischer 344 male rats. Cas!. J. Neurol. Sci. 18, $403-407$.

256. Wirak D. Bayner R. Ramabhadran T.C. Fracasso R.P. Hart J.T., Hauer P.E., Hsiau P., Pekar S.K.. Scangos G.A., Trapp B.D. and Unterbeck A.J. (1992) Science 255, 1445 .

257. Wisniewski T., Ghiso J. and Frangione B. (1994) Alzheimer's disease and soluble AB. Newrobiol. Aging 15, $1.43-152$.

258. Wong-Riley M.T.T. (1989) Cytochrome oxidase: An endogenous melabolic marker for newronal actútity. Trends Nerrosici. 12, 94-101.

259. Wree A., Kaever C., Birgel B., Schleicher A., Horvath E. and Zilles K. (1991) Locul cerebral glucose utilization in the brain of old, learning impained rats. Histochemistry $95,591-603$,

260. Wu C.F., Bertorelli R. Sacconi M. Peper G. and Consolo S. (1988) Decrease of brain wcelylcholine 
relcage in aging freefy-moving rats detected by microdialysis. Neurobiol. Aging 9, 357-361.

261. Yamada K., Nitta A., Hasegawa $T$., Fuji $K$, Hiramalsu M., Kameyama $T$, Furukawa $Y$., Hayashi $K$. and Nabeshima T. (1997) Orally active NGF synthesis stimulators: Potential therapeutic agents in Alzheimer"s disease. Bethav Broin Res. 83, 117-122.

262. Yan S.-D., Chen X., Schmidt A.-M., Brett J., Godman G., Zou Y.-S., Scot C.W. Caputo C. Frappier T. Snith M.A., Perry G., Yen S.H. and Stern D. (1994) Glycated tau protein in Alhemer disease: A mechanism for induction of oxidan stress. Proc. Natl Acad. Sci. U.S.A. 91, 7787-7791.

263. Yanker B.A. Duffy L. protein: Reversal by tachykinin neuropeptides. Science 250, 279-282.

264. Yen S.-H. (1995) Alzheimer neurofibrillary lesions: Molecular nature and potential roles of different components. Neurobiol. Aging 16, 381-387.

265. Zhang Y.. Marcillat O.* Givivi C. Ernster L. and Davies K.J. (1990) The oxidative inactivation of mitochondrial deerron transport chain componenis and ATPase. J. Biol. Chem. 265, 16330-16336. 


\section{Introduction: Aims}

\section{Plasticity}

Nitric oxide (NO) is suggested to be involved in memory formation as a retrograde messenger necessary for the induction of long-term potentation (LTP). This because NO is produced by a calcium/calmodulin-dependent enzyme NO synthase which can be stimulated by the intracellular $\mathrm{Ca}^{2+}$ influx as a result of $\mathrm{N}$-methyl-D-aspartate (NMDA) receptor activation 1601 . Furthemore, NO is short-lived and freely diffusible, which is regarded as ideal properties for a retrograde messenger $\{21.611$. Several experiments have indeed demonstrated that NO synthase inhibition blocked induction of hippocampal LTP both in vitro $15,29,1011$ and in wivo [50] and thus supported the hypothesis that NO acts as a retrograde messenger. Systemic administration of NO synthase inhibitors in rats impaired their acquisition of spatial discrimination learning $226.37,53,72,92.94,94$. 15. 152]. The retention of spatial information, as measured in a probe trial, was nor impaired when NO synthase was inhibited after initial learning $137,94,99,152$. Furthermore, NO synthase inhibition did not impair cue learning $[37,53,72]$, whereas spatial reversal learning appeared affected [72].

All these above mentioned findings are in correspondence with the effects of NMDA antagonists on spatial leaming and/or hippocampal LTP $[1,43,90,95,120,128]$. However, it has also been found that systemic administration of a NO synthase inhibitor does not impair spatial learning per se, as reversal learning and one trial learning was not affected in the Morris water task [10]. Likewise, NO synthase inhibition did not affect spatial leaming in another spatial task [142]. These findings, therefore, question a pivotal role of $\mathrm{NO}$ in spatial discrimination learning. Concomitantly, it has been found that although hippocampal LTP could still be induced after NO synthase inhibition under certain conditions, it was not so strong anymore (in height and length) both in vitro $[31,39,41,64,66,67]$ and in vivo $[9,11 \%$. Based on these finding it can be concluded that $\mathrm{NO}$-dependent and $\mathrm{NO}$-independent forms of hippocampal LTP exist [6. 61].

In contrast to the above mentioned findings which were obtained with systemic administration of substances, it is perhaps better to use local or i.c.v. administration when studying whether No has a direct effect on learning, like has been done in some studies in which was found that NO is involved in spatial learning [117], passive avoldance leaming [58, 117], and working memory 1103], More in detail, indications were found that $\mathrm{NO}$ regulates hippocampal processes generated during or shortly after acquisition of a task 167,38, 581. Thus it can be argued that NO affects mechanisms in the hippocampus that are important for memory consolidation, that is encoding and storage of information.

The diffusible NO is a powerful activator of the cyclic GMP (cGMP)-synthesizing enzyme, soluble guanylyl cyclase (SGC) [96. It has been demonstrated that the distribution of 5 GC appears complementary with that of NO synthase, that is NO synthase was found in postsynaptic structures and CGMP accumulation in presynaptic elements 1311 . This suggests that activation of $5 G C$ is therefore likely to be a major mechanism of NO signal transduction in the brain 1 see also 471. Severall mechanisms of action of the presynaptically formed OGMP have been offered to explain the maintenance of LTP [see 61]. For example cGMP regulates cGMP-gated channels and thus regulates ion flux directly which depolarizes the presynaptic terminal and can influence glutamate release $[155]$. It is also known that CGMP activates cGMP-dependent protein kinases (PKG) which phosphorylate certain proteins, and cOMP has an influence on CAMP-phosphodiesterases (PDEs) 
which regulate the AMP concentrations [12al. It may be suggested that both these effects of cGMP can infuence the synthesis and/or release of glutamate. The participation of $C A M P$ in the cGMP response is probably vial CAMP-dependent protein kinases [ct. 124$]$ and cAMP-dependent ion channels [cl. 155],

It was found that cGMP is inwolved in the induction of hippocampal LTP in viro $[39,661$. Recent in vitros studies using $\$$ GC inhibitors $[4,30,31,154]$ or PKG inhibitors $[4,154]$ have shown that $\triangle G C$ and PKG indeed contribute 10 LTP. But comparable with NO, there is a hippocampal form of LTP that is independent of CGMP and one that is CGMP-dependent 31\%. Future research with behavioral experiments is needed to elucidate the role of cGMP in learning and memory processes. Very recently, indications have been found that cGMP regulates hippocampal processes generated immediately after acquisition of a passive avoidamce task 118. 19]. This is in agreement with earlier findings regarding NO and thus it can be argued that CGMP, just as has already been done for NO [17, 38, 58], affects mechanisms in the hippocampus that are important for memory consolidation.

At least two isoforms of the NO synthase exist which are stimulated by $\mathrm{Ca}^{2+}$ : neuronal $(\mathrm{n})$ and endothelial (e) NO synthase. Next to these two so-called constitutive isoforms, an inducible (i) isoform of NO synthase has been identified, furst isolated in macrophages (for a review see [45] and (761). The iNO synthase, which is also found in astrocytes, is induced by endotoxins and cytokines, such as $\gamma$-interferon and lipopolysaccharide. Once iNO synthase is expressed it produces NO continuously. nNO synthase, found in neurons, and eNO synthase, found not only in endothelium but also in neurons, are constituive in the sense that their activation does not require enzyme protein synthesis. At the moment it not clear which constitutive isoform of NO synthase is involved in the induction of LTP. It is assumed that nNO synthase is involved, although it has been found that in mutant mice lacking nNO synthase, hippocampal LTP can still be induced [102]. In addition, it has been found that a functional (membrane-targeted) eNO synthase is required for LTP [84]. These findings suggested that eNO synthase, rather than $\mathrm{nNO}$ synthase, generates NO within the postsynaptic cell during LTP. This is supported by anatomical sudies which show that nNO synthase is probably absent in hippocampal pyramidal neurons and is only concentrated in interneurons of the hippocampus [49]. In contrast, eNO synthase is highly concentrated in both pyranidal cells and granule cells of the dentate gyrus. Future research using inhibitors of NO synthase which are thought to be specilic for eNO synthase or nNO synthase might give more informution about the role of constifutive NO synthase in synaptic plasticity and memory formation. Recently, it has been reported in a study using knock-out mice for eNO synthase and/or nNO synthase, that only LTP was reduced in double knock-out mice 1130 . This indicates that both isoforms of constitutive NO syathase can compensate for each other in the single knock-onts. Moreover, it suggests that both isoforms can be involved in memory processes, though it has very recenty been argued that the contribution of nNO synthase to LTP would be minimal IIS!!

Finally. it should be kept in mind that stimalation of ADP ribosyltransferase by NO, which leads to the ADP-ribosylation of certain proteins, may explain LTP induction in the hippocampus in addition to LTP induction due to 5 GC activation 174, 126, 127]. Interestingly, long-term depression (LTD) which is another form of synaptic plasticity and is present in the cerebellum, appears to involve also $\mathrm{NO}$ and $\mathrm{SGC}$, although the expression of $\mathbb{L T D}$ appears to be postsynaptic, e.g. a decreased sensitivity of amino-3-hydroxy-5-methyl-4-isoazolepropionate (AMPA) receptors 161. 70. 1261. Moreover, LTD has apparently, just like LTP, a NO-independent component «see 611.

Besides a function of $\mathrm{NO}$ in the central (and peripheral) nervous system as a putative retrograde messenger in long-term potentiation (LTP), suggesting a role for NO in memory 
formation, NO has more (putative) functions. In macrophages it mediates cytotoxicity. NO also plays role in wascular regulation by acting as a wasodilator in endothelial cells. In frot NO was first called endothelium-derived relaxing factor until in 1987 it was discovered that these two substances were one and the same (107). NO may mediate vasodilatation also via perivascular natves (for a review see [76]). In situations of excessive production NO may function as a neuroloxin, suggesting a role for $\mathrm{NO}$ in neurodegenerative disorders (for a review see $[4,5]$ ). At the moment the vascular, memory and neurodegenerative (e.g. during ischemia) potentials of NO have the main interest of research. But other putative functions of NO are quickly increasing in number. For instance, some studies suggest a role of NO in locomotor $1116,123,133.1521$ and emotional 1409.1491 processes. Finally, most interesting is the finding that NO besides playing a tole in LTP/memory formation, is also involved in another form of neuronal plasticity, viz. the development of the nervous system $[71.83,122]$.

A part of this thesis focusses on neuronal plasticity and in this context the results of experiments have been described which try to elucidate the possible role of NO in both memory and developmental processes. In a first experiment the role of the NO-cGMP signal transduction pathway in memory formation processes was studied by manipulating NO and cGMP concentrations (Chapter 7). In a second experiment we tested the role of NO in neuronal development by chronic postnatal inhibition of NO synthase and its possible functional consequences on memory performance during later life (Chapter 8).

\section{Neurodegeneration}

Blockade or inhibition of the central energy metabolism could provide a model of neurodegeneration (see previous part Neurodegenenation, section Animal models). Based on this assumption the streptozotocin (STREP), i.c.v. injected, model has been developed in our laboratory [23, 24]. The STREP model is based on a chronic inhibition of the energy metabolism. Another model was developed to study the acute effects of drugs that are presumed to preserve neuronal function during low energy states. This model is based on al short period of acute energy depletion by i.c.N. injected sodum cyanide (NaCN) 122]. In this thesis the STREP model has been further characterized behaviorally, biochemically and anatomically. In addition, the therapeutic potential of acetyl-Lacarnitine was tested in both models. Figure 6 gives an overview of the possible role(s) of STREP and $\mathrm{CN}$ in degenerative mechanisms in relation with the putative regenerative mechanisms of acetyl-L-carnitine.

\section{ACETYL-L-CARNITINE}

Acetyll-carnitine (ALCAR) is regarded as a compound which attenuates neurodegenerative processes by having a beneficial effect on the energy metabolism, viz. via a stimulation of the use of alternative energy sources, although its putative nootropic effect can be attributed also to other mechanisms of action (see below). It is of importance to keep in mind that ALCAR is the acetylated form of L-carnitine, both of which occur naturally in the brain and play an important role in the transfer of acyl groups across the mitochondrial membrane for subsequent ismoxidation 201. However, this role of $\mathrm{L}-\mathrm{carnitine}$ is of minor importance in the brain, since the brain almost exchsively depends on the oxidation of glucose to meet its energy demands. 
In animal studies it has been found that chronic treatment with ALCAR prevented an impaired cognitive performance in aged rats $[12,35,62.63]$. In human studies it has been found that the progression of AD was significantly reduced in patients chronically treated with ALCAR [112. 1321. Several possible mechanisms of action of ALCAR in the brain have been hypothesized to explain Ihese neuroprotective effects on cognitive function. Many explanations are based on $\mathbb{L}$-carnitine. However, ALCAR treatment is thought to be more effective than L-camitine treatment because it is quite possible that the hydrophobic acetyl moiety of ALCAR improves the ability of the drug to cross the blood-brain barrier and to be taken up by neuronal tissue [33]. To gain more insight in the possible mechanism(s) of action by which ALCAR may exert a nootropic effect in the models of neurodegeneration (NaCN and STREP) as described in this thesis, only the relevant hypotheses are described hereafter.

\section{Stimulation of the use of alternative energy sourcesi}

In a number of studies the catabolic effect of acute administration of ALCAR and L-camitine have been evaluated. It was found that ALCAR and L-carnitine stimulate the use of allernative energy sources, such as lipid subsitrates or ketone boclies, but on the other hand it inhibits the "glycolytic flow" $[7,8,16,105,147,148]$. ALCAR administration could, by enhancing the utilization of alternative energy sources, therefore, compensate for the decrease in glucose utilization during AD (and aging) and, consequently, preserve cholinergic/cognitive function $[73,91]$. To test this assumption we evaluated behavioral (Chapter 2) and biochemical (Chapter 3) effects of chronic treatment with ALCAR ( $v$ ua drinking water) in the STREP model, since STREP reduces the central metabolism of glucose (and thus ATP; see Fig Fig. 6) and can, therefore, be seen as a chronic energy deficiency model of neurodegeneration $[51,97,98,114]$.

Formation of ATP-independent acyl-L-camitines reserwoir and/or detoxification of acylCoAs

The composition of neuronal membranes undergoes age-associated changes $\mid 125 \rrbracket$ and conconitantly it has been found that in aged rat brain there is a decrease in activity of the enzyme Iysophospholipid acyltransferase which is necessary for the reacylation of plasma membrane phospholipids out of lysophospholipids and acylCoAs [8, 9:3]. These acylCoAs are produced out of free fatty acids (orignating from deacylation of membrane phospholipids) by the enzyme acylCoA synthetase which requires energy (ATP). Arduini ex al. 160 proposed a working mechanism for L-carnitine which suggested that L-carnitine is involved un brain phospholipid metabolism via the enzyme carnitine acyltransferase (probably a plasma membrane associated protein). According to Arduini's model L-carnitine provides an ATP-independent reservoir of acyl groups for reacylation, by shifting the equilibrium for carnitine acyltansferase toward acyl-L-camitines. This could be of main importance when the ATP-dependent reacylation route via acylCoA synthetase is inhibited by a low energy metabolism in the brain, like during hypoxia or ischemia (see below). In addition, this might also explain the observed increase in acyl-L-carnitines during aging [87], i.e. thus a removal of a possible excess of acylCoAs, which might arise because reacylation is reduced $[8,93]$, takes place.

The above mentioned mechanism is probably not per se of importance in normal healthy adult rats, unless membranes have been damaged due to for instance hypoxia, ischemia or hypoglycemia and thus racylation/deacylation processes become involved. When energy metabolism is impaired due to ischemia [78], hypoxia or hypoglycemia [2] there is an increase in deacylation of membrane phospholipids and a decrease in B-oxidation. This results in an increase in free faty acids and acylCoAs, respectively, of which the latter are toxic for the mitochondrion 
both via an inhibition of the electron chain enzyme complexes [105] and va mitochondial membrane damage [110], and thus can contribute to cell death (see Fig. 6). It seems likely that ALCAR via L-carnitine diminishes the accumulation of toxic acylCOAs by shifting the equilibrium for carnitine acyltransferase towards acyl-L-carnitines [0f. 105]. However, this may be of minom relevance in the brain becanse of the low carnitine acyltransferase activity in the brain due to its preference for glucose. But a detoxification function (and also the formation of a ATP-independent acyl reservoir) may still play a role, since it appears that next 10 an increase in. acylCoAs, there is an increase in acyl-L-carnitines in the brain during ischemia [see 25]. Taken together, it is likely that an increase in ALCAR and/or L-carnitine levels prevents, by formation of acyl-L-carnitines, a too high level of acylCoAs both inside and outside the mitachondrion. A mitochondrion membrane associated carnitine acyltransferase may be responsible for this inside the mitochondrion, while at its outside a mitochondrion and/or plasma membrane associated enzyme may be involved [a. 61 .

Next to an increase in acylCoA levels, there is an increase of acetylCoA levels in the mitochondrion during high energy demands, which leads to an allosteric inhibition of pyruvate dehydrogenase. In this respect it is thought that L-carnitine has a positive effect in the periphery, that is an increase in both muscle metabolism and physical performance during exercise at maximal intensity [146]. This effect was attributed to L-carmitine acting as an acceptor of the acetyl groups, that is L-carnitine lowers the acetylCoA level and thereby releases the inhibition of van de pyruwate dehydrogenase. However, this mechanism is questioned [75] and in animal studies it has been found that ALCAR had no effect in sensorimotor tasks [44.89].

We have already demonstrated that chronic treatment with ALCAR had a protective effect on spatial memory performance after acute cyanide intoxication in our NaCN model [22]. This preservation of neuronal function could be explained by the formation of an acyl-L-carnitines reservoir during ALCAR treatment, so reacylation processes can contimue during the short-lasting energy deficit caused by cyanide (an inhibitor of the enzyme complex IV of the electron transport chain [88]; see Fig. 6). Furthermore, a detoxification function of ALCAR could be also imvolved, i.e. removal of toxic acylCoAs. However, chronic ALCAR treatment had no effect on the affected hippocampal energy metabolism of cyanide-treated rats (as measured by the energy-dependent phosphoinositide metabolism) [221. This indicates that ALCAR did not enhance the utilization of alternative energy sources during the acute cyanide-induced energy depletion. Moreover, this supports the notion that the protective effect of chronic ALCAR treatment in the $\mathrm{NaCN}$ model can be attributed to the formation of a reservoir of activated atcyl groups, i.e. acyl-L.-camitines. Based on an earlier study of Rosenthal et at. [121], it was decided to evaluate also the behavioral effects of acute ALCAR administration (intravenous) in the NaCN model. We assumed that an acute administration of ALCAR could possibly be more effective than a chronic ALCAR treatment (Chapter 6).

\section{Direct enhancenent of cholinergic funciton}

Acute administration of ALCAR may enhance cholinergic function directly by providing acetylCOA for acetylcholine synthesis 179.1501 . This is in agreement with a prewious finding that (chronic) ALCAR administration resulted in an increased 'high affinity choline uptake' in aged rat brain [42], because choline is next to acetylCoA, the other important precursor for acelylcholine synthesis [143]. It has to be noted that glucose, independently of ALCAR, keeps providing acetylCoA for the synthesis of acetylcholine U150).

A cholinergic enhancement effect of ALCAR can be also attributed to L-carnitine [1:9], that is 
ALCAR may just serve as a source of L-carnitine. L-carnitine would be expected to facilitate the iransfer of acety/COA actoss the mitochondrial membrane in the cytosol, by shifting the equilibrium of the normal transfer of acetylCOA (in the constitution of ALCAR) across the mitochondrion membrane via canitine acetyltransferase towards the cytosol, where the synthesis of acetylcholine occurs [119]. Thus, mitochondrial acetylCoA could be used for anabolic processes, like acetylcholine synthesis.

\section{Stimulation of nerve growth factor receptor}

A large number of aging studies and lesion experiments have demonstrated that nerve growth factor (NGF) provides trophic support to the cholinergic basal forebrain system [e.g. 57.59]. An important step to this trophic effect of NGF is its binding to membrane receptors (NGFR) and the subsequent internalization of the NGF-NGFR complex at the cholinergic terminal [106]. It has been demonstrated that ALCAR retards the age-associated loss in NGFR in the basal forebrain, as increases in NGF binding to its receptor were found in the aged rat brain after ALCAR treatment 10. This tropic effect of ALCAR on the cholinergic system of aged rats might be explained by an improwed binding and internalization of the NGFR-NGF complex after ALCAR treatment [137]. In addition. it seems likely that ALCAR increased the synthesis of NGF [138], although an increase in expressiton and anterograde transport of NGFR from the soma to terminals cannot be ruled out either $[1361$. Correspondingly, it has been found that ALCAR increases NGF levels (and choline acety/transferase activity) in the basal forebrain of fimbria-fornix lesioned rats [113] and aged rats [1.39\%.

\section{Increase in glutathione.}

Chronic treatment with ALCAR has been found to elevate the level of the oxidant scavenger glutathione in the rodent substantia nigra [55, 36]. This suggests that ALCAR offers protection against oxidative stress. In addition, ALCAR may offer protection against oxidative stress via the above mentioned trophic effect on NGF, since NGF is also known to play a role in oxidant homeostasis [108] (see Fig. 6).

\section{STREPTOZOTOCIN}

\section{Sireptozotocin as a model of nearodegeneration}

Although several studies have provided evidence that an i.c.Y. injection of STREP inhibits the utilization of glucose (and thus ATP; see Fig. 6) in the brain and consequently leads to an inhibition of the metabolic capacities of neurons $[5 \mid, 97,98,114]$, it has to be noted that the exact mechanism by which STREP induces neurodegenerative effects remains unclear, thus far. For instance, oxidative damage may be (also) involved in causing an impaired neuronal function [ef. 27 , 52], since STREP has been found 10 generate $\mathrm{H}_{2} \mathrm{O}_{2}[140)$ (see Fig. 6). To gain more insight into the possible mechanism of action of STREP, we further characterized the STREP model behavionally and both biochemically (Chapter 4) and anatomically (Chapter 5),

\section{Streptozotocin and nitric oxide}

Figure 5 shows that there are several conversion reactions of NO after its synthesis has been stimulated by NMDA receptor stimulation initially. Overstimulation of NMDA receptors may result in NMDA-mediated neurotoxicity, also referred to as an excitotoxic mechanism which is regarded as a process which may result in neurodegeneration [13, 14, 145]. Experiments with cell 


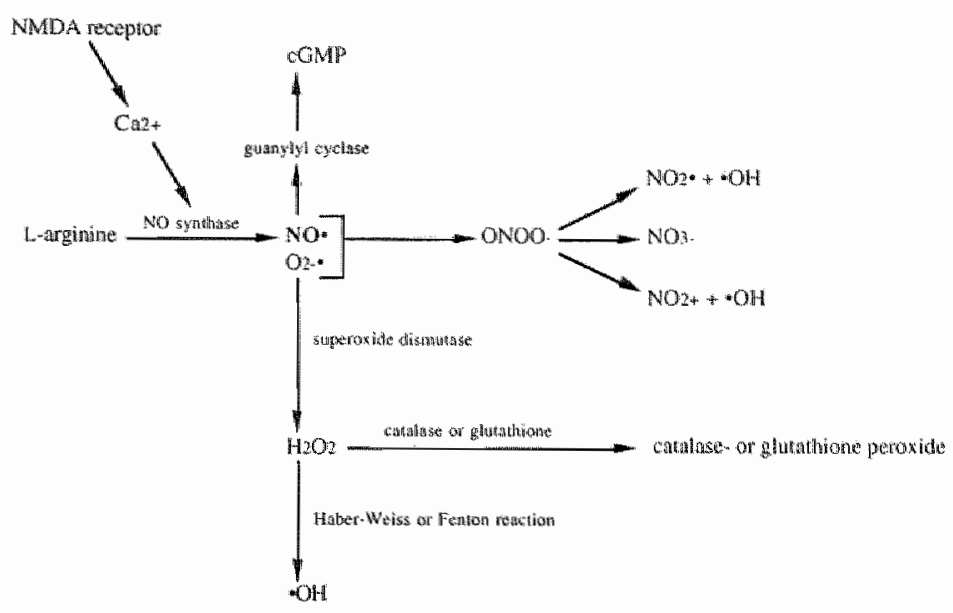

FIG. 5. Conversion reactions of nitric oxide (NO). Influx of calcium cations into the cell after NMDA receptor stimulation activates NO synthase which uses L-arginine as a precursor for NO formation. NO binds to guanylyl cyclase and thus stimulates the synhesis of cGMP. Normally, in air $\mathrm{NO}$ is oxidized $10 \mathrm{NO}_{2}$ (nitrogen dioxide) followed by dimerization to $\mathrm{N}_{2} \mathrm{O}_{4}$ which then reacts with water to form $\mathrm{NO}_{2}{ }^{-}$(nitrite) and $\mathrm{NO}_{3}^{-}$(nitrate). In aqueous solution $\mathrm{NO}$ is oxidized to $\mathrm{NO}_{2-}$ which subsequently may be further oxidized to $\mathrm{NO}_{3-}$ [77]. However, NO may react with the $\mathrm{O}_{2}^{-}$(superoxide anion) to form the $\mathrm{ONOO}^{-}$(peroxynitrite anion), which is an extremely potent oxidizing agent. Under physiological conditions peroxymitrite protonates to form ONOOH (peroxynitrious acid), which spontaneously decomposes to the $\mathrm{NO}_{2}$ radical and the hydroxyl (OH) radical; but most of ONOOH (about $70-80 \%$ ) isomerizes to $\mathrm{NO}_{3}$. Sometimes metal tons can catalyze the cleavage of peroxynitrite to $\mathrm{NO}_{2}{ }^{+}$ (nitronium) and $\mathrm{OH}^{-}[15]$. Nitronium is also very damaging by its nitration of tyrosine in proteins. ONOO. formation can be prewented by the conversion of $\mathrm{O}_{2}$ - by superoxide dismutase to hydrogen peroxide $\left(\mathrm{H}_{2} \mathrm{O}_{2}\right)$. The reactive $\mathrm{H}_{2} \mathrm{O}_{2}$ can eventually be scavenged with catalase or glutathione or still form the $\mathrm{OH}$ radical, mainly vis the Fenton reaction (catalyzed by metal ions) but also via the Halber-Weiss reaction (catalyzed by the superoxide anion).

cultures and slices of brain tissue showed that NMDA-mediated neurotoxicity can be attenuated with $\mathrm{NO}$ synthase inhibitors $[* 6,809$. In similar experiments it was also found that administration of a NO donor (sodium nitroprusside) was neurotoxic 146.811. In addition, NO synthase inhibitors blocked an augmented NO synthase activity and/or cGMP concentration due to NMDA receptor stimulation $[32,46]$. All these in vitro findings indicate that NO (and perhaps CGMP) play a role in NMDA-mediated neurotoxicity. However, some experiments with cell cultures have provided evidence against a (primary) toxic action of NO (and CGMP) after NMDA receptor stimulation, because NO synthase inhibitors did not protect against NMDA-mediated neurotoxicity [48, 111]. In contrast, a NMDA antagonist, MK-801, was nenroprotective after NMDA receptor stimulation.

In wivo animal models of central ischemia (carotid occlusions), in which NMDA receptor stimulation is probably responsible for the neurotoxic insults, revealed that NO synthase inlibition, just as NMDA antagonism, had a neuroprotective effect $134,36,40,82,100$. However, experiments with direct in wivo administration of a NO donor or a NMDA agonist did not clarify whether NO formation after NMDA agonist administration is directly responsible for the neurotoxicity $[65,69,36]$. But recently it has been found that $\mathrm{NO}$ synthase inhibition was. 
neuroprotechive after NMDA agonist administration in vivo 134]. In addition, another in wivo brain experiment demonstrated that a NO synthase inhibiter (just as MK-801) blocked the production of the toxic $\mathrm{OH}$ radical after $\mathrm{NMDA}$ administration [68]. Behavioral experiments might make further substantial contributions in gaining insight how NO is involved in NMDA-medated neurotoxicity. For instance, it has already been found that hippocampal intibition of NO synthase attenuared an ischemia-induced cognitive deficit [104].

Based on the in witro and in wiwo findings it may be assumed that NO plays a role in NMDA-mediated netrotoxicity, while cCMP is probably not involved [f. 69l. The contribution of NO to neurodegenerative processes must be seen as oxidative damage caused by the NO radical itself, although other explanations for cellular damage are possible, for instance as a result form nitrosylation of proteins by NO or ONOO- ${ }^{4.5]}$ (e.g. NO inhibits tyrosine kinases activity by nitrosylation of the receptor proteins $[\$ 4,129]$ ). However, it is difficult to assess which compounds like for instance $\mathrm{ONOO}, \mathrm{NO}_{2}$ and $\mathrm{OH}$ are involved in mediating neuronal cell death (see Fig. 5). With regard to oxidative damage it has been demonstrated that damage to DNA, and subsequently poly A.DP ribose synthetase (PARS) activation, was caused by NO [153] and ONOO- [135]. For lipidperoxidation it has already been found that this may be caused by $\mathrm{ONOO}$ and $\mathrm{NO}_{2}[15,118]$. Finally, regarding possible protein oxidation, it has been found that NO inhibited electron transport chain enzymes (e.g. complex [V [28]).

NO might play a role in STREP-induced neurodegeneration. Firstly, because inhibition of the energy metabolism by STREP could start an excitotoxic metabolism, in which NO may be inwolved via NO synthase (see Fug. 6). Secondly, it has been demonstrated that STREP, because of its nitroso molety, may liberate the reactive NO [85, 141, 144] (see Fig. 6). NO might be associated with the results of some former studies with STREP in which oxidative DNA damage was found. However, it was found that the DNA damage was caused by $\mathrm{H}_{2} \mathrm{O}_{2}$ whose production was stimulated by STREP, whereas on the production of its $\mathrm{O}_{2}$ - intermediate no effect was found [140]. Therefore, how STREP stimulates $\mathrm{H}_{2} \mathrm{O}_{2}$ generation remains unknown. To evaluate the possible role of $\mathrm{NO}$ in STREP induced neurodegeneration we performed a behavioral and anatomical study in which one STREP-treated group of rats was also treated with the NO synthase inhibitor L-NAME to investigate whether NO formation is involved in a possible excitotoxic mechanism after STREP treatment (Chapter 5). In addition, to investigate whether NO liberated from STREP may be responsible for the neurotoxic effect, one group of rats was treated with the NO donor sodium nitroprusside.

\section{REFERENCES}

1. Abraham W.C. and Mason S.E. (1988) Elfects ol the WMDA receptor/channel antagonists CPP and MK801 on the hippocampal field potentials and long-term potentiation in anesthezised rats. Braw Res. 462 , 40.46 .

2. Ando $S$. Waki H. and Kon K. (1993) Diferemial laty acid release from CA ll and CA3 regions of rat hippocampal slices under hypoxia and hypoglycemia. Newrosci. Lett. 151,48-50.

3. Angehcei L. Ramacei M.T. Taglialatela C. Hulsebosh C., Morgan B., Wertbach-Perez K. and Perez-Polo IR. (1988) Nerve growh lactor binding in aged rat central nervous system: Effect of acelyl-L-carnitine. $J$. Newrosci. Res. 20, $491-496$.

4. Arancio O., Kandel E.R. and Hawkims R.D. (1995) Aclivity-dependent long-term enhancement of transmitter release by presynaptic 3",5"cyclic GMP in cultured hippocampal neurons. Nature 376, 7.4-80.

5. Arancio O., Levram V., Tsien R. Y. Kandel E.R. and Hawkins R.D. (1996) Nitric oxide acts as a retrograde messenger during long-tarm potentiation in cullured hippocampal neurons. $J$. Physiol. (Paris) 90, $321-322$.

6. Arduni A., Denisowa N., Virmani A., Avrova N., Eederici G. and Arrigoni-Martell E. (1994) Evidence for the involvement of carnitine-dependent long-chain acyltransferases in neuronal wiglyceride and phospholipid acid turnover. N. Nearochem. 62, $1530-1538$. 


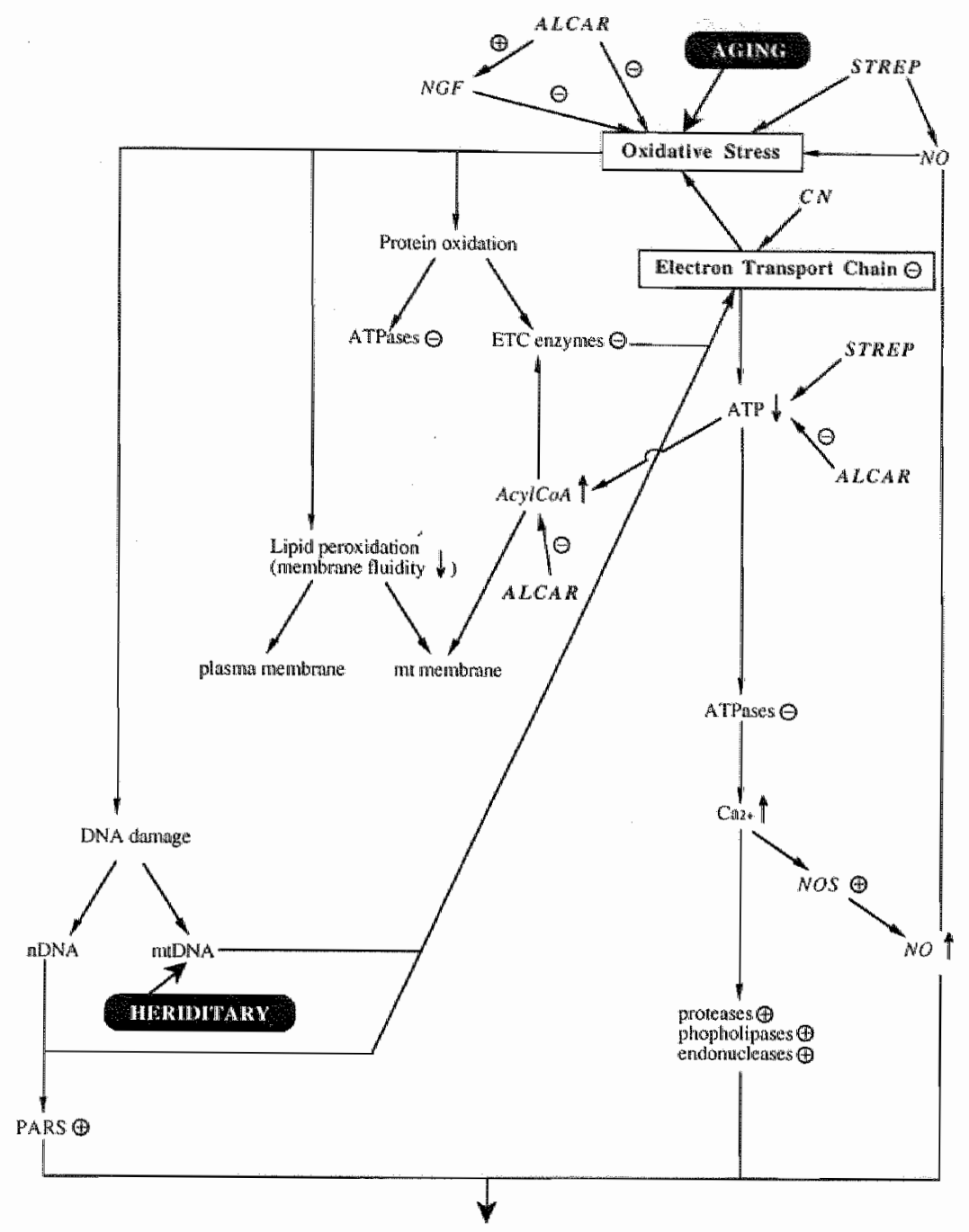

ATROPHY (shrinthage, neuromal dysfunction) and CELL DEATH (apoptos is, necrosis)

FIG. 6. Neurodegenerative mechanisms (see previous part Neurodegeneration, Fig. 4) in which the possible role of NO and the possible (chronically) working mechanism(s) of STREP are shown. It is still not completely clear which of the reactive groups related to NO lead to which type of specific neuronal damage (see Fig. S). STRREP is known to result in (chronic) inhibition of the glucose metabolism. Further, STREP can cause oxidative stress by gemeration of $\mathrm{NO}$ and $\mathrm{H}_{2} \mathrm{O}_{2}$. In addition, the angle of the (acute) effect of $\mathrm{CN}$ is depicted in connection with that of the possible role of acylCoA in cell death. Putative protective effects of ALCAR (and NGF) are also shown. Abbreviations: AcylCOA, acetylcoenzyme A; ALCAR, acelyl-L-carnitine; CN, cyanide; ETC, electron unsport chain; mt, mitochondrial; NGF, nerve growth factor; NO, nitric oxide; NOS, nitric oxide synthase; n, nuclear; PARS, poly (ADP-ribose) synthetase; STREP, streptozotocin. Symbols: $\downarrow$, decrease; $\uparrow$, increase; (- $)$, inhibition; $(+)$, stimulation. 
7. Aureli T., Micheli A. Di Coceo M.E.L. Ghirardi O.. Giumani A., Ramacci M.T. and Cont F. (1994) Effect of acetyl-L-carnitine on recovery of brain phosphorts metabolites and lactic acid level during repertusion afier cerchral ischemia in the rat - sudy by $31 \mathrm{P}$ and $1 \mathrm{H}$-NMR spectroscopy. Brain Res. 643 . $92-99$.

8. Aureli T. Miccheli A., Ricciolini R., Di Coceo M.,A, Ranacei M.T. Angelucci L., Ghirardi O. and

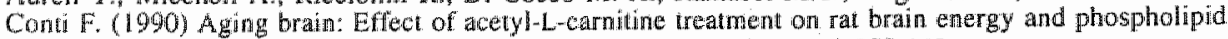
metalofism, study by $31 \mathrm{P}$ and IH NMR spectroscopy. Brain Res. 526, 108.112.

9. Bannerman D.M. Bucher S.P. and Morr.s R.G. 1995) Intracerebroventricular injection of nitric oxide synhase inhibitor does not affect long-term slope potentiation in wivo. Neurophanmacology 33 , 1387 1397.

10. Bamerman D.M. Chapman P.F., Kelly P.A.T., Butcher S.P. and Morris R.G.M. (1994) Inthibition of ritric oxide synthase does not imparir spatial lleaining. N. Netrosci. 14, 7404-7414.

11. Binneman D.M. Chapman P.F., Kelly P.A.T., Butcher S.P. and Moris R.G.M. (1994) Inhibition of nitric oxide synthase does not prevert the induction of long-term potentiation in vivo. J, Neurosci. 14 , $7415-7425$.

12. Barnes C.A., Markowska A.L. Ingram D.K. Kametani H. Spangler E.L., Lemken V.J. and Olton D.S. (1990) Acetyl-L.carmitime 2: Effects on learring and menory performance of aged rats in simple and complat matues. Newrobiol. Aging $11,499-506$.

13. Beal M.F. (1992) Does impentment of energy metabolism result in excutotoxic newronall death in neurodegenerative illnesses? Anm. Neurot. 31, ॥19-130

14. Beal M.F. Hyman B. $\mathrm{T}$. and Korostretz. W. 1993) Do defects in mitochondrial energy metabolism underlie the pathology of neurodegenerative diseases? Trends Neurosci. 16, 125-131.

15. Beckman J.S. Ischiropoulos H., Zhu L., Van der Woerd M., Smith C. Chen J., Harrison J., Martin J.C. and Tsai M. (1992) Kinetics of superoxide dismutase-and iron-catalyzed nitration of phenolics by peroxymitule. Areh. Biochen. Biophys. 298, 438-445

16. Benzi G. Villa R.F., Dossena M., Vercesi L. Gorini A. and Pastoris O. (1984) Cerebral endogenous substrate ulilization during the recovery period after profound hypoglycemia. $J$. Newrosci. Res. 11 , 437450 .

17. Bermabeu R. de Stein M.A., Fin C., Izquierdo I. and Medina J.H. (1995) Role of hippocampal NO in the acquisition and consolidation of inhibitory avoldance learning. NearoReport 6, 1498-1500.

18. Bermabeu R. Schmitz P., Fallace M.P., Izquierdo I, and Medina J.H. (1996) Hippocampal cGMP and cAMP are differentially involved in memory processing of inhibitory avoidance learning. NeuroReport 7 , $585-588$.

119. Bernabeu R., Schroder N., Quevedo J. Cammarota M. Lequierdo I. and Medina J.H. (1997) Further evidence for the involvement of a hippocampal cGMP/cGMP-dependent protein kinase cascade in memory consolickation. NenroRport 8,2221.2224.

20. Hieber L.L. (1988) Carnitine. Amn. Rev. Biochem. 57, 261-283.

21. Bliss T.V.P. and Collingridge G.L. (1993) A synaptic model of memory: Long-term potentiation in the hippocampus. Nature 361.31-39.

22. Blokland A., Bothmer J., Honig W, and Jolles J. (1993) Behtavioral and biochemical effects of acute central metabolic inhibition: Effects of acetyl-L-carnitine. Eur. J. Pharmacol, 235, 275-281.

23. Blokland A and Jolles I. (1993) Spatial learning deficin and reduced hippocampal ChA activity in rats after ath hew injection of streptozotocin. Phammacol Biochem, Behaw 44, 491-494.

24. Blokland A and Jolles J. (1994) Behavioral and biochemical effects of an icv injection of streptomotocin in old Lew is rets. Pharmacol. Brochem. Behov. 47, 833-837.

25. Bohan T.P. (1992) Edilorial comment. Siroke 23, 1317-1318.

26. Bohme G.A., Bon C., Lemane M., Reibaud M., Piot O., Stutzmann J.M., Doble A. and Blanchard J.-C (1993) Altered synaptic plasticily and memory formation in nitric oxide symhase inhbitor treated rats. Proc. Nall Acod. Sci. U.S.A. $90,9191-9194$.

27. Bolaff J.L., Nagamatsu S., Harris J. and Grodsky G.M. (1987) Protection by thymidine, an inhibutor of polyadenosine diphosphate abosylation, of streptozotocin inbibition of insulin secretion. Endocrinology $120,2117.2 \| 22$.

28. Bolah̆os M.P., Peuchen S., Heales S.J.R., Land J.M. and Clark J.B. (1994) Nitric oxide-mediated inhibition of the mitochondrial respiratoly chain in cultured astrocytes. N. Neurochem. 63, 910-916.

29. Bon C., Botme G.A., Doble A., Stumann J.M. and Blanchard J.-C. (1992) A role for witric oxide in long-term potentiation. Eur: J. Phamacol. 4, 420-424.

30. Boulton C.1. Irving A.J., Sotham E., Potier B., Gathwaite J. and Collingridge G.L. (1994) The nitric

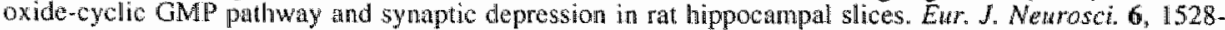
1535 .

31. Boultow C.L., Southam E, and Garthwaite J. (1995) Nitric oxide-dependent long-term potentiation is blocked by a specific inhibitor of soluble guanylyl cyclase. Neuroscience 69, 699-703.

32. Bredi D.S. and Snyder S.H. (1989) Nitric oxide mediates glutamate linked entancement of cGMP levels in tho cerabellum. Proc. Not Acad. Sci. U.S.A. 86, 9030-9033.

33. Burlina A.P., Sershen H., Debler E.A. and Lajtha A. (1989) Uptake of acetyl-Lwcarnitine in the brain. Newrochen. Res. 14, 489-493. 
34. Caldwell M. ONenl M. Earley B. and Leonard B. (1994) NG.nitro-L-arginine protects aganst ischentam

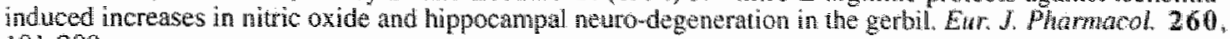
$191-200$

35. Caprioli A. Ghirardi O., Ramacei M.T. and Angeluoci L. (1990) Age dependent defoits in radial maze performance in the rat: Effect of chronic treatment with acety-Licarmine. Prog. Neuropsychopharmacol. Biol Psychiar. 14, 359-369.

36. Chalimoniuk. M. and Strosztajder I. (1995) Bratn ischema reperfusion injury acruates neuronal, constitutive form of mitric oxide syrthase. Molecular action of nutric oxide. Eur. J. Metrosci. Suppll. 6. 26, 15.28.

37. Chapman P.F. Atkins C. Allen M.T. Haley J.E. and Stemmetz J.E. (1992) Inhibition of nitric oxide symthesis impairs two different forms of learning. Newrokepont 3,567-570.

38. Chem I., Zhang S., Zuo P. and Tang L. (1997) Memory related changes of nitric oxide synthase activity and nitrite level in rat brain. NewroReporr $8,1771-1774$.

39. Chetkowich D.M. Klann, E. and Sweatt, J. D. (1993) Nitric oxide synthase independent long-lernt potentiation in area CAI of hippocampus. Neurokepont $4,919.922$

40. Choi D W. (1995) Calcium: Sill center-stage in hypoxic-ischemic neuronal death. Trends Nenoscis. $58-60$

41. Cummings J.A., Nicola, S. M. and Malenka, R. C. (1994) Induction in rat hipponampus of long lerm potentiacion (LTP) and long-term depression (LTD) in the presence of a nitric oxide synuase hnbibitor. Newrosi. Lent. $176,110-114$.

42. Curti D., Dagani F., Marzalico F. and Benzi G. (1986) Aging and acelyl-L-carnitine treatment: Eftects on some cerebral biochemical parameters. In Biological Psychiony. Developments in Psychiaty (ed. Shagass C. et al.). Vol. 7, pp. 1343-1345. Elseviers Science Publishing, Naw York.

43. Davis $S$., Butcher S.P. and Morris R.G.M. (1992) The NMDA receptor antagonist D-2mamino-5 phosphopentanoate (D-APV) impairs spatiat learning and LTP in wivo at intacerebral concentrations comparable to those that block LTP in vitro. J. Nenrosci. 12, 21-34, 1992.

44. Davis S., Markowska A.L., Wenk G.L. and Bames A. (1993) Acetyl-L carnitine: Behavioral, electrophysiologicat, and neurochemical effects. Neurobiol Aging 14, 107-1 is.

45. Dawson T.M. and Dawson V.L. (1995) Nitric oxide: Actions and pathological roles. Newrosctentist 1. 718 .

46. Dawson V.L., Dawson T.M., London E.D., Bredt D.S. and Snyder S.H. (1991) Nitric oxide mediates glatamate neurotoxicity in primary cortical cultures. Proc. Natl Acad. Sci. U.S.A. 88, 6368-6371.

47. De Vente J. and Steinbusch H.W.M. (1992) On the stimulation of soluble and particulate guanylate cyclase in the rat brain and the inwolvement of nitric oxide as studied by CGMP immunocytochemistry. Acta Histochem. 92, 13.38.

48. Demerlé-Pallardy C., Lonchampt M-O., Chabrier P.E. and Braquet P. (1991) Absence of inplication of Larginine/nitric oxide pathway on netronal cell injury induced by $\mathbb{L}$ iglutamate or hypoxia. Brochen. Biophys. Res. Comm. 181, $456-464$.

49. Dinerman J.L., Dawson T.M., Schell MJ., Snowman A. and Snycler S.H. (1994) Endothealial nitric oxide synthase localized to hippocampal pyramidal cells: Implications for synaptic plasticity. Proc. Narl Acad. Sci. U.S.A. 91, 42!4-4218.

50. Doyle C., Hobscher C., Rowruan MJ. and Anwy) R. (1996) The selective neturonal nitric oxide synuhase antagonist 7-mitro indazole blocks boh long-lerm potentiation and depotentiation of field excitatoty posisynaptic potentials in rat hippocampal CAl in wivo. N. Newmosci. 16, 418-424.

51. Dueli. R. Schröck H. Kuschinsky W. and Hoyer S. (1994) Intracerebrowentricular injection of streptozotocin induces discrete local changes in cerebral glucose utilization in rats. Int. D. Dew Newnosc. $12,737-743$.

52. Dulin W.E. and Wyse B.M. (1969) Srudies on the ability of compounds to block diabetogenic fuctivity of streptozotocin. Diaberes $18,459$.

53. Estall L.B., Grant $S_{3} J$ and Cicala G.A. (1993) Inhibilion of nitric oxide (NO) production selectively impars learning and memory in the rat. Pharnacol. Bochem. Behaw 46, 959-962.

54. Estrada C., Comez C. Martin-Nieto J., De Frutos T, Jirnenez A. and Villalobo A. (1997) Nirric oxide reversibly inhibits the epidemal growth factor receptor tynosine kinase. Biochem. J. 326, 369.376.

55. Fariello R.G., Ferraro T.M., Golder G.T., DeMattei M., Phillips T. and Shug A.L. (1986) Hypotheses on the role of the carnitine system in normal and aging brain. In Biological Psychiony. Dewelopments in Psychiary (ed. Shagass C. al.) Yol. 7, pp. 1377-1339. Elsevier Science Publishing, New York.

56. Fariello R.G. Ferraro T.N. Golden G.T. and DeMatia M. (1988) Systemic acetyl-tharnitine elevates nigral levels of glutathione and GABA. Life Sci. 43, 289-292.

57. Femandez C.l. Gonzalez O., Soto J., Alvarez L. and Lores T. (1996) Elfects of chronic infusion of nerve growh factor (NGF) in rats with nucleus basalls magnocellularis lesion. Mol. Chem. Newropathol. 28. $181-184$

58. Fin C., Cunha C.A. Bromberg E., Schmiz P.K. Bianchin M., Medna I.H. and lzquierdo I. (1995) Experiments suggesting a role for nitric oxide in the hippocampus un memory processes. Netrobiol. Leam. Mewn. 63. 113-115.

59. Fischer W., Bjorklund $A$, Chen $K$, and Gage F.H. (1991) NGF improves spatial memory in aged rodents as 


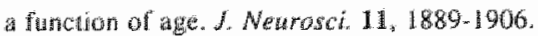

60. Garhwate 1. (1991) Gulatiate, nitic oxide and cellwell signalling in the nerwous system. Trends Neurased $14,60-67$

61. Garthorite J. and Boulton C.L. (1995) Nitric oxide signaling in the central nerwous system. Anma. Rew. Physiot. $57,683-706$.

62. Ghrardi O. Caprioli A. Milano S., Gulani A., Rarnacci MT and Angelucei L. (1992) Active Awoidance Learning in old rats chronically trealled with levocamitine acelyl. Physiol Behov. 52, 185-187.

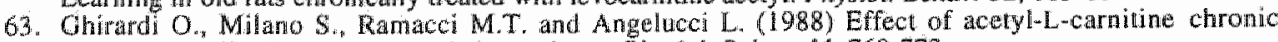
treatment on discrimination models in aged rats. Physiol. Beho*, 44,769-773.

64. Gribkotf V.K. and Lum-Ragan, J. T. (1992) Evidence for nitric oxide synthase inhibior-senstive arad insernsitive hippocampal synaptie potentiation. Joumal of Neurophysiology $68,639-642$.

65. Gross P.M. Weaver D.F., Bowers R.J. Nag S., Ho L.T. Pang IJ. and Espinosa E.J. (1994) Neurotoxicily in comscious rats following intrawentricular SWAP, a nitric oxide donor. Newrophantacology $33,915,927$

66. Hatey J.F. Wilcox, C. L. and Chapman, P. F. (1992) The role of nitric oxide in hippocampal long-term potentichton. Neuron 8, 211-216.

67. Haley J.E. Malen, P. L. and Chapman, P. F. (1993) Niric oxide synthase inhibitors block long-term potentiation induced by weak but not strong tetanic stimulation at physiological brain temperatures in rat hippocampal slictes. Newrasci. Leti. 160,85-88.

68. Hammor B., Davis Parker W. Ir and Bennet J.P. (1993) NMDA receptors increase OH radicals in vivo by using nitric oxide synthase and protein kinase C. NeuroReport 5, 72-74.

69. Harrington C.A., Mobley S.L. and Wenk G.L. (1994) Nitric oxide formation does not umderlie the memory deficits produced by ibotenate injections into the nucleus basalis of rats. Behav. Neurosci. $108,277-283$.

70. Hartell N.A. (1996) Inhibition of coMP breakdown promotes the induction of cerebellar long-tern deptession. The J. Meurosci. 16, 2881-2890.

71. Hindley 5. Juturlin B.H.J., Gysters J.W. Middlemiss P.J., Herman M.A.R. and Rathbone M.P. (1997) Nitric oxide donors ahance neurotrophin induced nearite outgrowth through a cMM dependent mechanism. d. Neuroset Res, 47, 427-439.

72. Hölscher C., McGlinchey L., Anwyl R. and Rowan M.J. (1996) 7-Mitro indazole, a selective neuronal nitric oxide synthase inhibitor in vivo, impairs spatiall learning in the rat. Learn. Mem. 2, 267-278.

73. Hoyer S. (1992) Oxidativenergy meiabolism in Alztweimer brin. Mol. Chent. Neuropathol. 16, $207-224$.

74. Huang A.M. and Lee E.H. (1995) Role of hippociampal nitric oxide in memory retention in rats. Pharmacol. Blochew Behav. 50, 327-332.

75. Hultman E, Cederblad G. and Harper P. (1991) Carnitine administration as a lool of modify energy metabolism during exercise. Eur. J. Appl. Physiol. 62, 450.

76. Iaclecola C. Pelligrino D.A., Moskoviz M.A. and Lassen N.A. 1994) Nitric oxide synthase inhibition and cerebrovascular regulation. J. Cereb. Blood Flow Metab. 14, 175-192.

77. Ignarro L.J., Fukuto J.M., Griscavage J.M., Rogers N.E and Byrns $\mathbb{R}$.E. (1993) Oxidation of nitrie oxide in aqueous solution 10 nitrite but not nitrate: Comparison with enzymatically formed nitric axide from $L$ arginine. Proc. Natl Acad. Sci. U.S.A. 90,8103-8107.

78. Ikeda M. Yoshida S., Busto R., Santiso M. and Ginsberg M.D. (1986) Polyphosphoinositides as a probable source of brain free faty acids accumulated at the insel of ischemia. $d$. Neurochem. 47, 123-132.

79. Inyperato A. Ranacei M.T. and Angelucci L. (1989) Acetyl-L.camntine enhances acetylcholime ralease in

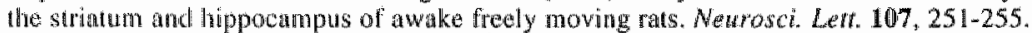

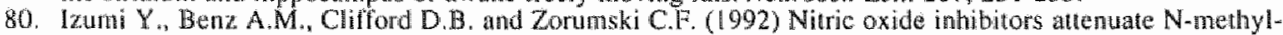

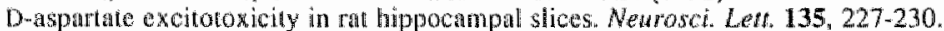

81. Lami Y., Benz A.M. Clifrond D.B. and Zonumski C.F. (1993) Newrotoxic effects of sodium nitroprusside in rat hippocampal slices. Exp. Neurol. 121, 14-23,

82. Kahr R.A. Pand M. and Weinberger J. 1997) Modulation of ischenuic excitatory neurotrarasmutter and gamma amiabbityric ncid releas during global iemporary cerebral ischemia by selective neuronal nitric oxide synthase inhabition. Anesthesia Analgesia 84, 997-1003.

83. Kalb R.G. and Agostini d. (1993) Molecular evidence for nitric oxide-mediated motor neuron development. Neurosed. 57, 1-8.

84. Kantor D.B. Lanzein M., Stary J., Sandoval G.M., Smith B., Sullvan B.M., Dawidson N. and Schuman E.M. (1906) A role for endothetial NO synthase in LTP revealed by adenowirus-raediated inlibition and reseute, Science $274,1744.1748$.

85. Kwon N.S., Lee S.H. Choi C.S. Kho T. and Lee H. S. (1994) Nitric oxide generation from Stroptozatocin. The FASEB Jommal 8.529-533.

86. Loiacono R.E. and Beart P.M. (1992) Hippocampal lesions induced by microinjection of nitric oxide donor nitroprusside. Em. J. Phamacol, 216,331-333.

87. Mancenri F. Arseni A., Chiodi P., Ramacei T. and Angelucci L. (1990) Levels of carnitines in brain and ohar tissues of rats of different ages: Effect of acetyl-L-caminine administration. Exp. Gerontol. 25, $127-$ 134.

88. MacMillan V.H. (1989) Cerebral energy metabolism in cyanide encephalopathy. J. Cereb. Blood Flow Nerab. $9,456.162$ 
89. Markowska A.L. Ingram D.K., Bames C.A., Spangler E.L., Lemken V.J., Kamerani H., Yee W. and Olton D.S. (1990) Acety/-L-carnitine I: Etfects on mortality, pathology and sensory-moror pertomanoe in aging rats. Neurobiol. Aging 11, 491-498.

90. McNamara R.K. and Skelion R.W. (1993) The neurophamacologional and nemochemicial basis of place learning in the Morris water maze. Brain Res. Rev. 18, 33-49.

91. Me ier-Ruge W., Bertoni-Freddari C. and Iwangof P. (1994) Changes in brain glucose nutwbolism as a key to the pathogenesis of Alzheimer's disease. Gerontology $40,246-252$.

92. Meyer R.C., Spanglier E.L., Patel N. and Ingram D.K. (1996) Inhibition of newronal niaric axide synthase mpairs learning of rats in a 14 -unit T-maze. Soc. Nerrasci. 22, 139.62.3.

93. Miccheli A., Delfini M., Aureli T. Di Cocco M.E. Tomasini A., Calderini G. and Conti F. (1988) Aging brain: H-1 and P-3I NMR spectroscopy study on energy and phospholipid metabolism. Magm. Res. Med. Bial. 1, 157.168.

94. Magensen J., Wornwein G., Gustafson B. and Ermens P. (1995) L-Nitroarginine reduces hippocampal mediation of place learning in the rat. Neurobiol. Leam. Mem, 64, 17-24.

95. Morris R.G.M. (1989) Synaptic plasticity and learning: Selective impaiment of learning fin rats and blockade of long term potentiation in vivo by the NMDA receptor antagonist APS. J. Newroci. 9, 3049. 3057 .

96. Murad F., Mittal C.K. Arnold W.P., Katsuki S. and Kinura H. (1978) Guanylate cyclase: Activation by azide, nitro compounds, nitric oxide, and hydroxyl ratical and inhibition by hemoglobin and myoglobin. Adn. Cyclic Nucl. Res. 9, 145-158.

97. Nitsch R. and Hoyer S. (1991) Local action of the diabetogenic drug streptozotocin, on glucose and energy meiabolism in the brain cortex. Neurosci. Lett. 128, 199-202.

98. Nitsch $\mathbb{R}$, Mayer $G$, and Hoyer $S$. (1989) The intracerebroventriculary streptozotocin-treated nat Impaiment of cerebral glucose metabolism resembles the alterations of carbohydrate notabolism of the bran an Alzheimer's disease. J. Neural Trans. 1, 109-110.

99. Noda Y., Yamada K. and Nabeshima 'T. (1997) Role of nitric oxide in the effect of aging on spatial menory in rats. Behav. Brain Res. 83, 153-158.

100. Nowicki J.P., Duwal D., Poignet H. and Scatton: B. (1991) Nitric oxide mediates neuronal death after focal cerebral ischemia in the mouse. Eur. J. Pharmacol. 204, 339-340.

101. O'Dell T.J., Hawkins R.D., Kandel E.R. and Arancio O. (1991) Tests of the roles of lwo diffusible substances in long-term potentiation: Evidence for nitric oxide as a possible early retrograde messenger. Proc. Nat Acad. Sci. U.S.A. 88, 11285-11289.

102. O'Dell T.J., Huang P.L., Dawson T.M., Dineman J.L., Snyder S.H., Kamdal E.R. and Fishman M.C. (1994) Erudothelial NOS and the blockade of LTP by NOS inthibitors in mice lacking nemromal NOS. Science $265,542-546$.

103. Ohno M., Yamamoto T. and Watamabe S. (1993) Deficits in working memory following inhibition of hippocampal nitric oxide symthesis in the rat. Brian Res. 632, 36-40.

104. Ohno M., Yamamoto T. and Watanabe S. (1994) Intrahippocanpal administration of the NO synthase inhibitor L-NAME prevents working memory deficits in rats exposed to transient cerebral ischemia. Brain Res. 634, 173.177.

105. Ohtsuka $\mathrm{Y}$. and Griffith O.W. (1991) L-carnitine protection in amonia intoxication. Bhochem. Phamencol. 12. $1957-1961$.

106. Otender E.J and Such R.W. (1980) Sequestration of 125 \%labelled B nerve growth fachor by sympathetic neurons. A. Biol Chem. 255,9338-9343.

107. Palmer R.M.J., Ferrige A.G. and Mancada S, (1987), Nilric oxide release accounts for the biological activity of endothelium-derived relaxing factor. Nature 327, $524-526$.

108. Pan 2. and Perez-Polo R. (1993) Role of nerve growth factor in oxidant homeostasis: glutathone metabolism. . Newrochem.61, 1713-1712.

109. Papa M. Pellicano M.P, and Sadile A.G. (1994) Nitric oxide and long-tern habituation to novelty in the rat. Ann. N. Y. Acad. Sct. 738, 316-234.

110. Pastorino J.G., Snyder J.W., Serroni A., Hoek J.B. and Farber J.L. (1993) Cyclosporin and carnitine prevent the anoxic death of cultered hepatocytes by imbibiting the mitochondrial permeability transition. $J$. Biol. Biochen. 268, 1379:-13798.

111. Pauwels P.J. and Leyson J.E. (1992) Blockade of nitric oxide formation does not prevent glutamate-induced neurotoxicity in neuronal cultures from rat hippocempus. Neurosci. LeHt 143, 27-30.

112. Pategrew J.W., Klunk W.E., Panchalingam K. Kanfer J.N. and McClure R.J. (1995) Clinical and neurochemical effects of acety-L-carnitine in Alzheimer's disease. Newrobiol Aging 16, 1-4.

113. Piowesan P., Pacifici L., Taglialaela G. Ramaci M.T. and Angelucci L. (1994) Acetyl- L warnitine treatment increases choline acetylumsterase activity and NGF levels in the CNS of adull pats following total fimbriafornix transection. Brain Res. 633,77-82.

114. Plaschke K. and Hoyer S. (1993) Action of the diabetonic drug streptozotocin on glysolytic and glycogenolytic metabolism in adult rat brain cortex and hippocanpus. Int. S. Dew Nourosch. 11.477-483.

115. Prendengast M.A., Buccafuseo JJ. and Terry J., A.V. (1997) Nitric oxide symhase inhibition impairs spatial mavigaton learning and induces conditioned taste aversion. Pharmacol Biochem. Behat. 57. 347. 352 . 
116. Pudiak C.M. and Bozarh M.A. (1993) LNAME and MK.801 attenualle sensitization to the locomotor

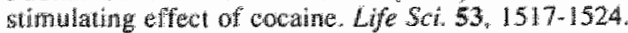

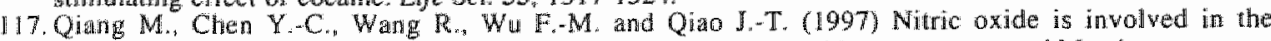
formation of learning and memory in rats: Sudiss using passive avoidance response and Morris water maxe taskik. Behav. Phammacol. 8, 183-187.

118. Radi R. Beckmai J.S. Bush K.M. and Freman B.A. (1991) Peroxymite induced membrane lipid peroxintation: The cylosolic potential of superoxide and nitric oxide. Arch Biochem. Biophys. 288, 481 . $487 x$

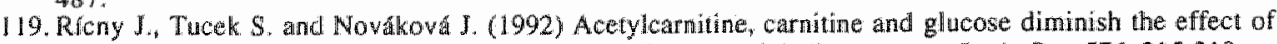
muscarinic antagonist quinuclidinyl benzilate on striatal acetyleholine content Brain. Res. 576, 215-219.

120. Robinson G.8., Crooks $\mathrm{G}$. Shirkman $\mathrm{P}$. $\mathrm{s}$. and Gallagher M. (1989) Behavioral effects of MIK-801 mimic deficits essociated with hippocampal damage. Psychobiology 17, 156-164.

121. Rosenthal R.E. Williams R., Bogat Y.E. Gixtson P.R. and Fiskum G. (1992) Prevention of postischemic neurologic injury through potemiation of bran energy metabolism by acetyl-L-carnitine. Stroke $23,1312 \times 1317$

122. Roskams A.J."Bredt D.S., Dawson T.M. and Romet: G.V. (1994) Nitric oxide mediates the formation of symaptic connections in developing and regenerating olfactory receptor metrons. Newron 13, 289.299.

123. Sandi $C$, Venero $C$. and Guara $C$. (1995) Decreased spontaneous motor activity and startle response in nitric oxide synthase inhibitor-1reated rats Eur, J Pharmacol 277, 89-97.

124. Schmidt H.H.H.W., Lohmann S.M. and Walter U. (1993) The nitric oxide and coMP signal transduction system: Regulation and mechanism of action. Biochim. Biophys. Acro $1178,153-175$.

125. Schoeder $F$. (1984) Role of membrane lipid asymmetry in aging. Newrobiot. Aging $5,323-333$.

126. Schuman E.M. and Madison D.V. (1994) Nitric oxide and synaptic function. Amnu. Rev. Neurosci. 17. $153-183$.

127. Schuman E.M., Meffert M.K. Seluuman H. and Madison D.V. (1994) Ar A.DP-ribosyltransferase as at potential target for nitric oxide action in hippocampal long-term potentiation. Proc. Nath Acad. Sci. U.S.A. 91, 1958-11962.

128. Shapira M.L. and Caramanos Z. (1990) NMDA antagonist MK-801 mpairs acquisition but not pertomance of spatial working and reference memory. Psychobiology 18, $231-243$.

129. Simon D.I., Mullins M.E. Jia L., Gaston B., Singel D.J. and Stamller J.S. (1996) Polynitrosylated proteins: Characterization, bionclivity, and functonal consequences. Proc. Nat Acad. Sci. U.S.A. 93, $4736-4741$.

130. Son H., Hawkins R.D., Martin K., Kiebler M., Huang P.L., Fishman M.C. and Kandell E.R. (1996) Long-tern potentiation is reduced in mice that are doubly mutant in endathelial and metronal nitric oxide symulase. Cell 87, 1015-1023.

13H. Southam E. and Garthwate J. (1993) The nitric oxde-cyclic GMP signalling pathway in rat brain. Nearopharmacology $32,1267-1277$.

132. Spagnoli A., Lucca U., Menasce G., Bandera L., Ciza G., Forloni G., Tetramanti M., Frattura L., Tiraboschi P., Comelli M., Senin U, Longo A., Petrini A., Brambilla G., Belloni A., Negri C., Cavazzuti F., Salsi A, Calogero P., Parma E." Stramba-Badiale M., Vitali S., Andreoni G., Inzoli M.R., Santus G., Caregnato R., Peruzza M., Favarelto M., Bozeglav C., Alberoni M. De Leo D.. Serrainto I. Baincetvi A., Scouch S., Culoth P. and leracitano D. (1991) Long-term acetyl-L-carmitine treatment in Alzheimer's disease. Newrology $41, \| 726-1732$.

133. Star M.S. and Star B.S. (1995) Do MMDA receptor-mediated changes in motor behawiour involve nitric oxide? Eur. Pharmacol. 272, $211-217$.

134. Strijbos P.J.L.M., Knowles R. and Rothwell N.J. (1993) Nitric oxide mediates gitanate neurotoxicity in the lat striatum. Brain Res. Assoc. Abstr. 10, 46, 8.6.

135. Szabó C. Zingareli B., O'Conmo M. and Sulzman A.L. (1996) DNA sirand breakage activation of poly (ADP*ribose) synthetase and cellular energy depletion are inwolved in the cy totoxicity in macrophages and smooth musclo cells exposed to peroxyminite. Proc. Nat Acad. Ses. U.S.A. 93, 1753-1758.

136. Tagiliatatela O. Angelucci L., Ramacci M.T., Foreman P.J. and Perez-Polo J R. (1990) 1251-B-Nerve growth factor binding is reduced in rat brain after stress exposare. N. Neurasci. Res. 25, 331 1 335.

137. Tagliatatela G. Angelucei L., Ranacei M.T., Werbach Berez K., Jackson G.R. and Perez-Polo J.R. (1991) Aceryl-L-carnitine enhances the response of PC12 cells to nerve growth factar. Dev, Brann. Res. 59, 221230 .

138. Taglialanela G. Amgelucci $L$. Ramacci M.I., Werbach-Perez K., Jackson G.R. and Perez-Polo J.R. (1992) Stimulation of nerve growth factor receptors in PC 12 by aceyl-L-carmitine. Biochem. Pharmacol. 44, 577585.

139. Taglialatela G., Navarra D., Cruciani R. Ramacci M.T. Alema G.S. and Angeilucci L. (1994) Acetyl-Lcarnutine treathent increases nerve growh factor levels and cholne acetyltumsferase activity in the central newous system of aged rats. Exp. Gerontol. 29,55-66.

140. T"akasu N., Komya L. Takayuk A., Nagasawa Y. and Yamada T. (1991) Streptozotocin- and alloxan. induced $\mathrm{H} 2 \mathrm{O2}$ generation and DNA fragmentation in pancreatic istets. Diabetes 40,1141-1145.

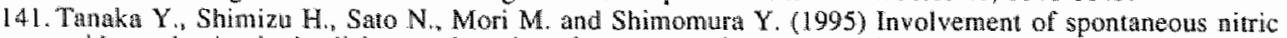
oxide production tn the diabetogenic action of streptozolocin. Phamacology $50,69-73$. 
142. Tobin J.R. Gorman L.K., Baxter M.G. and Traystman R.J. (1995) Nitric oxide synthase intribition does nor impair visual or spatall discrimination learning. Brain Res. $694,177-182$.

143. Tucek S., Ricny I. and Dolezal V. (1990) Advances of biology of cholinergic neurons. Adw. Meurol. 51, $109-115$.

144. Turk J. Corbett J.A., Ramanadham S., Bohrer A. and McDaniel M.L. (1993) Biochemical evidence for nitric oxide formation from streptozolocin in isolated pancreatic islets. Biochem. Biophys. Res. Conm. $197,1458-1464$.

145. Turski L. and Turski W.A. (1993) Towards an understanding of the rolle of gluamate in neurodegeneratiwe disorders: Energy metabolisn and neuropathology, Experimentia 49, 1064-1072.

146. Veechiet L., Dilisa F., Pieralisi G., Ripari P., Menabo R., Giamberardino M.A. and Siliprandi N. (1990) Influence of $L$-carnitine administration on maximal physical exercise. Eur. J. Appl. Physiol. 61, 486-490.

147. Villa R.F. and Gorini A. (1991) Action of L-acetylcarnitine on different cerebral mitochondrial populations from hippocampus and striatum during aging. Neurochem. Res. 16, 1125-1132.

148. Villa R.F., Gorini A., Zanada F, and Bienzi $G$. (1986) Action of L-acetylcarnitine on different cerebral mitochondrial populations from hippocampus. Arch. Int. Pharmacadyn. 279, 195-2l1.

149. Volke V., Koks S., Vasar E., Bourin M., Bradwejn J. and Mannisto P.T. (1995) Inthibition of nitric oxide synthase causes anxiolytic-like behaviour in an elevated plus-maze. Neuroreport 6, 1413-1416.

150. White H.L. and Scates P.W. (1990) Acetyl L-carnitine as a precursor of acetylcholine. Neurochem. Res. 15, $603+607$.

151. Wilson R.I., Yanovsky J., Godecke A., Stevens D.R., Schrader J, and Haas H.L. (1997) Endothelial nitric oxide synthase and LTP. Nature $386,338$.

152. Yamada K., Noda Y., Nakayama S., Komori Y., Sugihara H., Hasegawa T. and Nabeshima T. (1995) Role of mitric oxide in learning and memory and in monoamine metabolism in the rat brain. Br. J. Pharmacol. $115,852-858$.

153. Zhang J., Dawson V.L., Dawson T.M. and Snyder S.H. (1994) Nitric oxide activation of poly(ADP-ribose) synthetase in neurotoxicity. Science $263,687-689$.

154. Zhuo M., Hu Y., Schultz C., Kandel E.R. and Hawkins R.D. (1994) Role of guanylyl cyclase and cGMP. dependent protein kinase in long-term potentiation. Nature 368, 635-639.

155. Zufall F. (1995) Cyclic nucleotide-gated channels, nitric oxide, and neural function. Neuroscientist 2, 2432. 


\title{
Spatial discrimination learning and choline acetyltransferase activity in streptozotocin-treated rats: Effects of chronic treatment with acetyl-L-carnitine*
}

\author{
Jos Prickaerts, Arjan Blokland2, Wiel Honigl, Fanping Meng 3 and Jelle Jolles
}

European Graduate School of Neuroscience' 'EURON', 'Deparment of Psychiamy and' Neuropsychology, and 2Department of Psychology, Maastrich University. The Netherlands ${ }^{3}$ Deparment of Microbiology and Immunology, Yanbian Universiry College of Medicine, China

\section{ABSTRACT}

Treatment of rats with i.c.\%. injected streptozotocin may provide a relevant model of neurodegeneration that is induced by a decrease in the central metabolism of glucose. Acetyl-L-carnitine enhances the utilization of alternative energy sources and by such a mechanism of action acetyl-L-carnitine could antagonize the effects of streptozotocin treatment. In this siludy the effects of chronic treatment with acetyl-L-carnitine were evaluated on spatial discrimination learning in the Morris water escape task and choline acetyltransferase activity of middle-aged streptozotocin-treated rats. Chronic treatment with acetyl-L-carnitine attenuated both the streptozotocin-induced impairment in spatial bias and the decrease in hippocampal choline acetyllansferase activity. These findings indicate that acetyl-L-carnitine treatment has a neuroprotective effect, although further studies are needed to characterize the mechanism of action of acetyll-L-carnitine in this model.

\section{INTRODUCTION}

Recently, an animal model for metabolic dysfunction has been developed in which the centmal metabolism of glucose is decreased by i.c. v. injection of streptozotocin (STREP) [19.24, 25. 281. Besides a reduction in variables of energy metabolism after STREP treatment, the concentrations of different neurotransmitters, including the cholinergic transmission, were also reduced 112. 17]. This decrease in neurotransmitter concentrations suggests that the STREP-induced metabolic inhibition leads to a global neurodegeneration. Accordingly, cognitive deficits have been observed in middle-aged and old rats treated with i.c.v. injected STREP $[8$, 列.

Aceryl-L-camitine (ALCAR) is the acetylated form of L-carnitine, both of which occur naturally in the brain and play an important role in the transport of acyl-groups across the mitochondrial membrane for subsequent B-oxidation [6]. It has been suggested that ALCAR is involved in brain energy metabolism by stimulating the use of altemative energy sources, such as lipid substrates or ketone bodies $[2,3,5]$, Thus, it could be argued that a STREP-induced decrease in central glucose energy metabolism could be attenuated by ALCAR through an enhancement of

\footnotetext{
* This siudy has been published in Brain Res. 674, 142-146 (1995).
} 
the utilization of alternative energy sources.

In the present study we examined the effects of chronic treatment with ALCAR on spatial discrimination learning in middle-aged STREP-treated rats. In addition, we measured hippocampal ChAT activily and evaluated the relation between this biochemical marker and the behaviorat variables.

\section{MATERIALS AND METHODS}

\section{Awhals and treament}

We used twenly-four 18-months old male Lewis rats, randomly assigned to a control group $(n=7)$, a STREP-treated group $(n=9)$, or an ALCAR/STREP-Ireated growp $(n=8)$. No ALCAR control group was used because spatial discrimination learning of the middle-aged Lewis rat is not yet impaired when compared with the performance of young Lewis rats and in a previous study we did not find an effect of chronic ALCAR treatment on spatial discrimination learming of young Lewis rats [7]. All animals were housed individually in standard Makrolon cages on sawdust bedding in an air-condinoned room (about $20^{\circ} \mathrm{C}$. They were kept under a $12 / 12 \mathrm{~h}$ light-dark cycte (lights on from 09,00 to $21.00 \mathrm{~h}$ ). ALCAR treatment was started two weeks before surgery. The ALCAR/STREP group wats given ALCAR $75 \mathrm{mg} / \mathrm{kg}$ per day; the drug was dissolved in a $0.1 \%$ solution of saccharine (approximately $10 \mathrm{ml}$ ). The control and STREP group received a comparable amount (10 mly of saccharine solution. The solution was given in the morning in drinking tubes next to the standard drinking water. All arts drank the solution within 10 min. One week before behavioral testing, the rats were anesthetized with pentobarbital $(60 \mathrm{mg} / \mathrm{kg}$, i.p.) and placed in a stereotaxic frame. The ALCAR/STREP and STREP group were given a bilatcral i.c.\%. injection of STREP $(1,5 \mathrm{mg} / \mathrm{kg}$ body weight dissolwed in $4 \mu$ salline, $2 \mu l /$ injection site $)$. The stereotaxic coordinates were $-0.8 \mathrm{~mm}$ anterior, $1.55 \mathrm{~mm}$ lateral and $-3.8 \mathrm{~mm}$ ventral from the bregna [26]. The control group underwent the same surgical procedures but saline was injected i.c.v. instead of STREP. One ALCAR/STREP rat died shortly after the surgery for unknown reasons.

\section{Behcovioral procedures}

Ten days after surgery, the rats were trained on a standard spatial Morris water escape task [22] in a black water tank (diameter $1.22 \mathrm{~m}$ ). The rats were started from four different, randomly chosen, start positions and trained to find an invisible platorm (diameter $11 \mathrm{~cm}$ ) at a fixed position in the water tank, $1 \mathrm{~cm}$ below the surface of the watter. The water temperature was about $22^{\circ} \mathrm{C}$. A trial lasted until a rat had found the platform or until $60 \mathrm{~s}$ had clapsed. If a rat did not find the platform widhin $60 \mathrm{~s}$, it was placed on the platform for $3 \mathrm{~s}$ and then removed from the water tank. On the first dayy, the rats were given four trials. On the three subsequent days, the rats were givon eight trials a day. The time between subsequent rials was about 10 min. A probe trial lasting $60 \mathrm{~s}$, during which the platlorm was removed from the water tank, was given on the last day after the last trial.

\section{Chat achiviry}

Three weoks after surgery the rats were killed. The brain was taken out of the skull and the frontal cortex, hippocampus septum and the striam were dissected bilaterally. ChAT actiwity was measured as described in our previous study [8], using Fomum's method [13].

\section{Statistical analysis}

During acquisition of the Morris task, the mean escape latency was calculated per block of four triats. In the probe trials, the time spent in each quadrant was measured. Treatment effects during acquisition training were evaluated with a two-factorial (Treatment and Session) analysis of variance (ANOVA) with repeated measures over Session. For the probe trial, treatment effects on the time spent in the training quadrant were analyzed with a one-factorial (Treatment) ANOVA. Additional t-statistics were performed to analyze whether the time spent in the training quadrant differed from the chance level (15 s). Treatment effects were evaluated in more detail by Duncan"s post hoc multiple range test. Interdependence between behavioral masures and ChAT activity was analyzed by using Pearson's correlation coefricient. 


\section{RESULTS}

All rats reduced their escape latencies during acquisition of the Morris water escape task (Session effect: $F_{6,120}=24.76, p<0.01$; see $\mathbb{F i g}$. 1 A). There was no statistical reliable difference between groups when analyzed over the total training period (general mean: $F_{2.20}=2.05$, n.s.). In the probe trial, the groups showed a difference in the times spent in the training guadrant (Treatment effect: $F_{2,20}=4.71, p<0.05$; see Fig. IB). Both the control group and the ALCAR/STREP group spent more than $15 \mathrm{~s}$ in the training quadrant $(r \mathrm{~s}>2.98, p<0.05)$, whereas the time that the STREP-treated rats spent in the training quadrant did not differ from chance level $\left(t_{8}=0.85, n . s\right)$. Post hoc analysis revealed that the control group and the ALCARSTREP group spent more time in the training quadrant than the STREP group. Furthermore, it was found that the time spent in the training quadrant was the same for the ALCAR/STREP group and the control group.

The three experimental groups differed in hippocampal ChAT activity $\left(F_{220}=9.59, p<0.00\right.$; see Table 1). Post hoc analysis revealed that hippocampal ChAT activity was decreased in STREP-treated rats (both STREP and ALCAR/STREP) as compared with control rats. Additionally, it was found that the hippocampal ChAT activity of the ALCAR/STREP group was higher than that of the STREP group. There were no differences between the groups in ChAT activity in the frontal cortex and striatum $\left(F_{\mathrm{S}}<\mathbb{1} .17\right.$, n.s.). Septal ChAT activity tended to be increased after STREP treatment $\left(F_{2,20}=3.23,0.05<p<0.1\right)$. Post hoc analysis showed that there was no difference in septal ChAT activity between the STREP-treated and the control rats. However, the septal ChAT activity of the ALCAR/STREP group was higher than that of the control group (see Table 1).
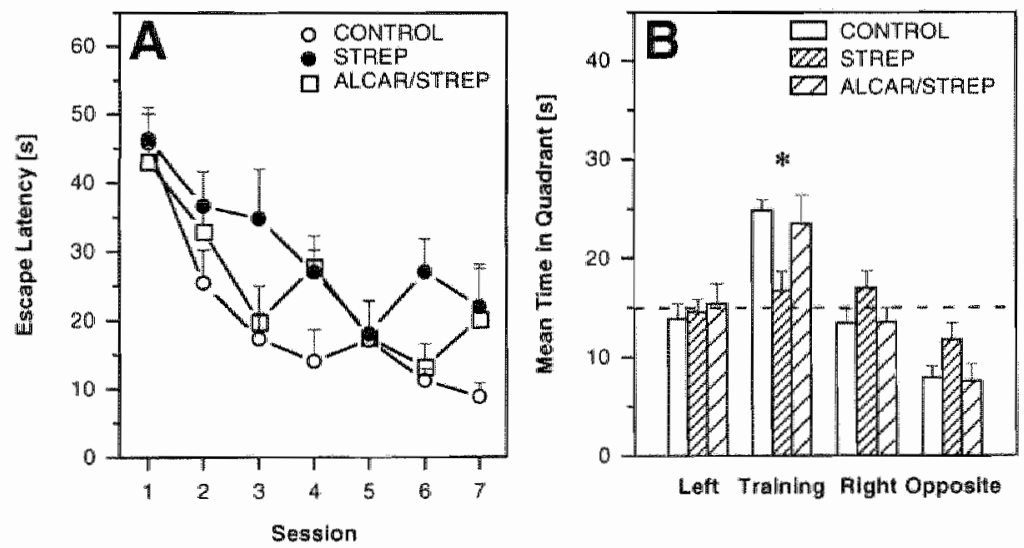

FIG. 1. Performance of 18-month-old control, streptozotocin-treated (STREP), and acetyl-Lcarnitine/streptozotocin-treated (ALCAR/STREP) Lewis rats in the Morris water escape task. (A) Mean escape latency $(+$ S.E.M.) during acquisition of the task. (B) Time (+S.E.M.) spent in each quadrant in the probe trial. TRAINING: quadrant where the platform was located during training. ADJ.-R/ADJ.-L: quadrant located immediately to the righ/left of the training quadrant. OPPOSITE: quadrant located 180 degrees from the training quadrant. Treatment effect: * $p<0.05$. 
TABLE 1, Choline acetyltransferase (ChAT) activity of different brain regions from 18-month-old control, streptozotocin-treated (STREP), and acetyl-Lcarnitine/streptozotocin-reated (ALCAR/STREP) Lewis rats.

\begin{tabular}{lrrr} 
Brain region & \multicolumn{3}{c}{ ChAT activity (nmol/mg protein/htS.EM.) } \\
& CONTROL & \multicolumn{1}{c}{ STREP } & ALCAR/STREP \\
\hline Hippoctampus & $90.7 \pm 2.40 \mathrm{a}$ & $33.6 \pm 10.04 \mathrm{~b}$ & $62.5 \pm 11.95 \mathrm{c}$ \\
Frontal cortex & $81.3 \pm 1.50^{\mathrm{a}}$ & $84.9 \pm 2.69 \mathrm{a}$ & $86.1 \pm 1.97 \mathrm{a}$ \\
Striatum & $203.9 \pm 7.17 \mathrm{a}$ & $192.5 \pm 5.30^{\mathrm{a}}$ & $203.5 \pm 6.46 \mathrm{a}$ \\
Sephum & $99.5 \pm 8.80^{\mathrm{a}}$ & $110.4 \pm 7.15 \mathrm{ab}$ & $134.8 \pm 13.23^{\mathrm{b}}$ \\
\hline
\end{tabular}

Means with the same superscript are not different (Duncan's multiple range test, $p<0.05$ ).

Hippocampal ChAT activity correlated negatively with the mean escape latency of the STREP group during the last five acquisition sessions in the Morris water escape task, where the mean escape latency of the control and $\mathrm{S}\rceil \mathrm{REP}$ groups diverged $\left(r_{\mathrm{p}}=-0.75, p<0.05\right.$; see Fig. 2$)$. There was no correlation between the two measures in the CON group and the ALCAR/STREP group ( $r$ respectively, -0.12 and -0.07 , n.s.; see Fig. 2). In none of the experimental groups there was a correlation between septal ChAT activity and escape latency $(-0.21<r s<0.66$, n.s. $)$.

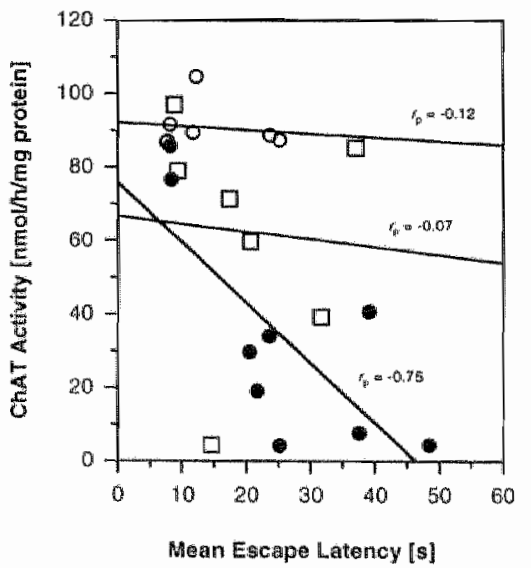

Mean Escape Latency [s]
FIG. 2, Relation berween hippocampal choline acetyluransferase activity and the average escape latency during the last five sessions of traning in the Morris task for 18-month-old control (open circles), streptozotocinutreated (closed circles), and acetyl-L-carnitine/streptozotocintreated (open squares) Lewis rats. Pararson's correlation coefficient $\left(y_{p}\right)$ : control rats: $r_{\mathrm{p}}=-0.12, \mathrm{n} . \mathrm{s}$; streptozotocin-treated rats; $r p=-0.75$, $p<0.05$; acetyl-L-carnitinel streptozotocin-treated rats: $r_{p}=-0.07$, n. S.

\section{DISCUSSION}

The present study replicated our previous findings that spatial discrimination learning in the Morris task is affected in middle-aged rats by STREP treatment and that this cognitive impairment is related to hippocampal ChAT activity [8]. Chronic treatment with ALCAR attenuated the STREP-induced impaiment in the spatial bias during the probe trial and attenuated the 
STREP-induced decline in hippocampal ChAT activity. This indicates that, in this animal model,chronic treatment with ALCAR has a beneficial effect at a behavioral and biochemical level.

ALCAR treatment did not affect the escape latency during acquisition of the Morris spatial mavigation task although the ALCAR/STREP group displayed a clear spatial bias towards the training quadrant during the probe trial that was indistinguishable from that of the control rats. It could be argued that escape latency is not a sensitive enough measure of spatial discrimination learning performance [see 30], and that other measures (swim path; learming index 15 et 151 ) might have detected a beneficial effect of ALCAR treatment on the acquisition of the Morris task. During the probe trial, the STREP-treated rats had no spatial bias towards the previous position of the platform. This indicates that these rats had not learned the spatial position of the escape platform during the acquisition of the task. Because spatial discrimination learning is highly dependent on the integrity of the hippocampus [23], these data suggest that hippocampal function was intact in the control and ALCAR/STREP-treated rats but not in the STREP-treated rats.

In line with previous studies, STREP treatment decreased hippocimpal ChAT activity 18.99 .171 In contrast, we found that ChAT activity in the frontal cortex, striatum, and septum was not affected three weeks after STREP treatment. This night be due to masking effects exerted by compensatory nerve growth factor (NGF) mediated stimulation of ChAT activity in the remaining functional neurons, as suggested by Hellweg et al. [17]. However, it could also be argued that i.c.y. injected STREP did not affect cholinergic cell bodies that project to the frontal cortex, striatum and septum, and that this is why no changes in ChAT activity, which is a marker for stmotural changes in cholinergic nerve endings, were observed in these areas.

Chronic treatment with ALCAR attenuated the decrease in hippocampal ChAT activity after STREP treatment. This suggests that ALCAR thad a neuroprotective effect on (septal) cholinergic neurons after a STREP-induced decrease in central energy metabolism. This neuroprotective action could be mediated by the supposed energy enhancing mechanism of action of ALCAR, i.e. the stimulation of the use of alternative energy sources $[2,3,5]$. However, it has been suggested that ALCAR has a positive effect on the neurotrophic action of NGF on the septohippocampal cholinergic system [1, 29]. Thus, a positive effect of ALCAR on NGF could also explain the increase in ChAT activity in the septum and hippocampus in the ALCAR/STREP group. This is consistent with the results of a recent study by Piovesan et al. [27], in which it was found that ChAT aetivity in the septum was increased in septo-hippocampal-lesioned rat as compared to sham-operated untreated rats after chronic treatment with ALCAR. The increase in septal ChAT activity of the ALCAR/STREP group as compared to the control group was remarkable. Although speculative, it could be argued that the increased septal ChAT activity is a synergistic effect of a cholinergic lesion, which leads to increased ChAT levels in the septum [16], and the positive effects of ALCAR on the neurotrophic action of NGF.

It was found that hippocampal ChAT activity was correlated with the STREP-induced spatial discrimination deficit. This is in line with the notion that the hippocampal cholinergic system is highly involved in spatial discrimination learning leg. 11 . However, the results for wo rats in the ALCAR/STREP group do not support this notion. As can be seen in Figure 2, one rat had a very low hippocampal ChAT activity and a good spatial discrimination performance, and the other had a high hippocampal ChAT activity and a poor spatial discrimination performance. Thus, hippocampal ChAT activity does not necessarily predict spatial discrimination performance. Moreover, these results suggest that the ALCAR-evoked erhancement of performance cannot be explained solely in terms of enhanced hippocampal ChAT activity. Recent studies suggest a role for interactions between the cholinergic system and other neuromodulatory systems in larang. and memory [se 10,20l. Further studies are needed to examine the possible effect of ALCAR on 
different neurotranimitter systems in the middle-aged STREP-treated rat and their possible relevance for learning and memory.

After dissection of the brain, a macroscopically inspection revealed that the septum had degenerated after STREP treatment, i.e. a reduction in tissue weight and a somewhat watery morphological appearance. These findings suggest that more neurotransmitter systems are affected in this model [er. 12). This effect was less dramatic in the ALCAR/STREP group, suggesting that ALCAR attenuates the STREP-induced septal degeneration. Although these qualitative observations could not be evaluated adequately, they support the observations of a higher level of hippocampal ChAT activity in the ALCARISTREP group than in the STREP group. However, these observations need to be quantified in further studies.

A dramatic decrease in glucose utilization can be obserwed in the brains of Alzheimer"s disease patients and led to the suggestion that the cognitive dysfunction of Alsheimer"s disease is related to a reduction in central glucose metabolism [18]. Similarly, a reduced brain glucose utilization is related to the age-related decline in cognitive function in rats $[14,31]$. It has been found that i.c.v. injected STREP impairs the energy metabolism of the rat brain by reducing the central metabolism of glucose $[24,25,28]$ and affects spatial learning in rats [4. 8]. The change in central energy metabolism as an underlying process may explain the similarity between the biochemical and cognitive changes in Alzheimer's disease and middle-aged STREP-treated rats. Recently it has been suggested that the decrease in central glucose metabolism that can be observed in Alzlieimer's disease is of importance in the development of the cholinergic deficit and the Bamyloid accumulation in the brain [21]. Therefore, it could be hypothesized that the middle-aged STREP-treated rat could be regarded as an animal model of Alzheimer's disease.

To summarize, in this study we found that chronic treatment with ALCAR attenuated both the STREP-induced impairment in spatial bias during the probe trial and the decrease in hippocampal ChAT actiwity. Therefore, it can be suggested that ALCAR attenuates a STREP-induced decrease in central glucose metabolism by stimulating the use of alternative energy sources. However "the present sudy gave no direct eyidence for this conclusion and therefore this remains speculative. Further histological, neurochemical, and behawioral tesearch is needed to examine these effects of ALCAR and STREP.

\section{ACKNOWLEDGEMENTS}

We thank Sigma-Tau Company (Pomezia-Rone, Italy) for the partial financial support and for providing us with acetyl-L-carnitine.

\section{REFERENCES}

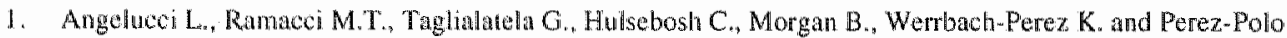
J.R. (1988) Nerve growth factor binding in aged tat central nervous system: Effect of acetyl-L-carnitine. $J$. Newrosed. Res. $20,4911-496$.

2. Aureli T., Miccheli A. Dil Cocco M.E.L., Ghirardi O., Giuliani A., Ramacci M.T. and Conti F. (1994) Eflect of acey-L-carnitine on recovery of brain phosphorus metabolites and lactic acid level during reperfusion alter cerebral ischemia in the rat - study by $31 \mathrm{P}$-and $1 \mathrm{H}-\mathrm{NMR}$ spectroscopy. Brain Res. 643 , 92.99.

3. Aureli T., Miccheli A., Ricciolini R., Di Cocco M.A., Ramacci M.T. Angelucci L., Ghirandi O. and Conti F. (1990) Agirg brain. Fillect of aceryl-L carnitine treatment on rat bran energy and phospholipid metabolism. A sudy by $31 \mathrm{P}$ and $1 \mathrm{H}$ NMR spectroscopy. Brain Res, $\mathbf{5 2 6}, 108-112$.

4. Babbedge R.C., Bland Ward P.A., Hart S.L. and Moore P.K. (1993) Inhibition of rat cerebellar nitric oxide symthase by 7 -ritro indazole and related substituted indazoles. Br. J. phapracol. 110, 225-228. 
5. Benzi G., Villa R.F. Dossena M. Veresi L. Gorini A. and Pastoris O. (1984) Cenebral endogenous substrate utilization during the recovery period after profound bypoglycernia. 4 . Newrosct. Res. 11, 437. 450 .

6. Bieber L.L. (1988) Camitine. Anm Rew. Bhochen. 57, 261-283.

7. Blokland A. Bothmer J. Hong W, and Jolles J. (1993) Behavioral and biochemical affects of acule cantral metabolic inhibition: Effects of aceyl-L-carnitine. Eur. J. Phannacol. 235, 275-281.

8. Blok land A and Jolles J. (1993) Spatial learning deficit and reduced hippocampal Cha I schity in lats after

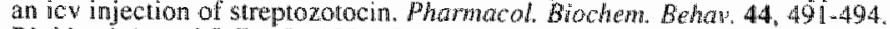

9. Blokland A. and Jolles $\mathfrak{I}$. (1994) Behavioral and biochemical atfect of an icv injection of streptozotocin in old Lewis the Phomacol Biochem. Behat, 47,833.837.

10. Decker M.W. and McGangh J.L. (1991) The role of interactions between the cholinergie system and other neuromodulatory systems in learning, and menory, Symapse 7, 151-168.

11. Decken M.W. Pelleymounter M.A. and Gallagher M. (1988) Enfects of raining on a spanial menory tusk

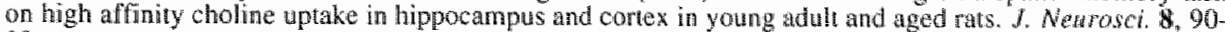
99.

12. Ding A. Nitsch R, and Hoyer S. (1992) Changes in brain monoaminergic neurotransmilter concentrations in rat after intracerebroventricular injection of streptozotocin J. Ceveb. Blood Flow Metab. 12, 103-109.

13. Fonnum F. (1975) A rapid radiochenical method for the detemanation of choline acelylumsferase. $d$. Neurochem. 24, $407-409$.

14. Gage F.H. Kelly P.A.T. and Bjothlund A. (1984) Regional changes in brain glacose unetabolism reflect cognitive amparments in aged rats. Nenrosci. 11, 2856.2865:

15. Gallagher M., Burwell R. and Burchinal M. (1993) Severity of spatiat learning impairment in aging: Development of a learning index for performance in the Morris water maze. Behav. Nemrosci. 107, 618 . 626.

16. Gasser U.E., Weskamp G., Otten U. and Dravid A.R. (1986) Time counse of the atevation of nerve growth factor (NGF) content in the hippocampus and septurn following lesions of the septohippocampal pathway in rats. Brain Res. $376,351-356$.

17. Hellweg R., Nitsch R., Hock C., Jaksch M. and Hoyer S. (1992) Nerve growh factor and choline acetyltransferase activity lewels in the rat brain following experimental impaiment of cerebral glucose and energy melabolism. J. Newrosci. Res. 31, 479-486.

18. Hoyer S. (1992) Oxidative energy metabolism in Aldneiner brain. Mol. Chent. Neuraponol. 16.,207-224.

19. Mayer G. Witseh R. and Hoyer S. (1990) Effects of changes, in peripheral and cerebral glucose metabolisum on locomoton activity, learing and menory in adult male rats. Brain Res. $\mathbf{5 2}, 95-100$.

20. McNamara R.K. and Skelon R.W. (1993) The neuropharmacological and neurochemical basis of place learning in the Morris water maze. Brain Res. Rev. 18,33-49.

21. Meier-Ruge W. Bertoni-Freddari $C$. and Wwangoff $P$. (1994) Changes in brain glucose meabolism as a key to the pathogenesis of Alzheimer's disease, Gerontology $40,246-252$.

22. Morris R.G.M. (1981) Spatial localization does not requite the presence of local cues. Learn. Moriv. 12, $239-261$.

23. Morris R.G.M. Garard P. Rawlins J.N.P. and O'Keefe J. (1982) Place navigation is unpatred in rats with hippocampal lesions. Natane 297,681-683.

24. Wisch R. and Hoyer S. (1991) Local action of the diabetogenic drug. streptozolocin, on glucose and encrgy metabolism in the brain cortex, Neurosci. Letr. 128, 199-202.

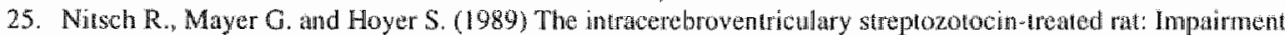
of cerebral glucose metabolism resenbles the alorations of cabohydate metabolism of the bitin in Alzheimer's disease. J. Neurat Trans. 1, 109-110.

26. Paxinos $\mathrm{G}$, and Wasson $\mathrm{C}$ (1986) The rat brain in srereotoric coodinates. 2nd edn. Academic Press. San Diceno.

27. Plovesan P., Pacifici L. Taghialaela G., Ramaci M.T. and Angelucei L. (1994) Acetyl-L-carnitime treatment increases choline acelytransterase activily and NGF levels in the CNS of adult fats following ton finbria fornix transection. Brain Res..633,77-82.

28. Plaschke K. and Hoyer $\$$. (1993) Action of the diabetonic drag streptozotocin on glycolytio and glycogenolytic metabolism in aduli rat brain contex and hippocampus. Int. Dev/ Newroser. 11, 477-483.

29. Taglialatela G., Angelucci L., Ramacci M.T., Werbach Perez K., Jackson G.R. and Peraz-Polo J.R. (1992) Simulation of nerwe growth factor receptons in PC 12 by acetyl-L-carnitine Bhochen. Pharmacol. 44,577 585 .

30. Van der Staay F.J. and de Jonge M. (1993) Effects of age on water escape bohavior and on repeated acquisition in rats. Behav. Neurat Biol. 60, 33-41

31. Wree A., Kaever C, Birgel B., Schleicher A., Horvath E. and Zilles K. (199l) Locall cerebral glucose utilization in the brain of old, learning impared rats. Histochemistry $95,591-603$. 



\title{
Brain enzyme activities after i.c.v. injection of streptozotocin in rats receiving acetyl-L-carnitine*
}

\author{
Dirk Terwel1, Jos Prickaerts 1, Fanping Meng2 and Jelle Jolles1 \\ IEuropean Graduate School of Neuroscience 'EURON', Department of Psychiatry and \\ Newropsychology, Maastrich University, The Netherlands \\ 2Deparment of Microbiology and Immunology, Yambian University College of Medicine, China
}

\begin{abstract}
Intracerebroventricular injection of streptozotocin has been introduced as a means to inhibit glucose utilization in the rat brain, and to induce changes in neurotransmitter systems and behavior which led to the assumption that streptozotocin-treatment might be of relevance to inwestigate pathogenetic aspects of Alzheimer's disease. In this study, enzyme activities previously investigated in Alzheimer's disease (peptidases, dehydrogenases and acetyltransferases) were measured in the septum and hippocampus of control and streptozotocin-treated rats. Streptozotocin-treated rats receiving acetyl $\mathrm{L}$-carnitine were also included in the experiments in order to assess possible neuroprotective effects of this substance. All enzyme activities in the septum were affected by streptozotocin, with the exception of choline acetyltransferase activity. By contrast, choline acetyltransferase activity was the only enzyme activity affected in the hippocampus. The weight of the septum was reduced in streptozotocin-treated animals. These findings indicate that i.c.v. injection of streptozotocin causes septal damage and, consequently, changes in enzyme acitivities. These enzymatic changes do not closely resemble the more specific changes in enzyme activities as observed in Alzheimer's disease. Acetyl-L-carnitine partly preverted this damage, as reflected by an attenuation of the streptozotocin-induced dectease in hippocampal choline acetyltransferase activity. This finding indicates that streptozotocin-treated rats may be valuable to test neuroprotective effects of substances.
\end{abstract}

\section{INTRODUCTION}

Recently, Nitsch and Hoyer [24] introduced i.c.v. injection of streptozotocin (STREP) as a means to reduce brain glucose utilization. Besides a reduced glucose utilization after i.c.v. injection of STREP, cholinergic and monoaminergic deficits and behavioral impairments have been observed $17,8,12,483$. These deficits have been suggested to resemble those of Alzheimer's disease and, consequently, STREP-treatment is assumed to be of relevance to investigate pathogenetic changes involved in Alzheimer's disease 17,25, 321. The mechanism through which STREP causes its effects on the brain has not been inwestigated as yet. Hoyer and collaborators speculated that its action on the central nervous system might be similar to its peripheral action, i.e. the destruction of insulin-secreting cel]s or interference with the insulin receptor system $124,25,321$. Systemic

\footnotetext{
* This sudy has been published in Eur. J. Pharmacol. 287, 65-7I (1995).
} 
administration of STREP is used to induce diabetes mellitus in experimental animals [3t. Pancreatic B-cells are selectively destroyed by STREP [34], although the selective vulnerability of these cells to STREP is not entirely understood. Several studies have demonstrated that inhibitors of poly-ADP ribosylation, which is stimulated by single-strand DNA breaks, decrease the effect of STREP on paricreatic B-cells (e.g.9. 13). This suggests that STREP causes DNA demage, which is in accordance with the finding that STREP generates. DNA-darmaging free radicals in cultures of pancreatic islets $[40]$.

The present siudy addresses the question whether changes in enzyme activities as observed in Alzheimer's disease are also observed after i.c.y. injection of STREP. Thus, the possible relevance of the STREP.treated rat as a model for Alzheimer's disease was studied. Several enzyme activities previously studied in Alzheimer's disease $[11,19,21,41,42]$ were measured in the septum and hippocampus three weeks after i.c.y. injection of STREP or saline. The enzyme activities measured in the studies were those of prolyl endopeptidase, a-ketoglutarate dehydrogenase, carnitine acetyltransferase and choline acetyltransferase, which are affected in Alzheimer"s disease, and those of aminopeptidase and glutamate dehydrogenase, which are not affected in Alzheimer's disease. Cholime acelyltransferase activity was used as a marker of cholinergic neurons, which die or shrink in Alzheimer's disease. The other enzyme activities are of a ubiquitous nature. The present study also addresses the question whether in the brain $\alpha$-ketoglutarate dehydrogenase is selectively affected by STREP as compared to glutamate dehydrogenase, which has been shown to be the case in pancreatic B-cells [35].

L-carnitine and its acetylated form, acetyl-L-carnitine (ALCAR), are endogenous substances in various organs, including the brain [5]. L-carnitine has a role in the B-oxidation by mitochondria of organs such as liver and muscle. This role of $\mathrm{L}$-camitine is of minor importance in the brain, since the brain almost exclusively depends on the oxidation of glucose to meet its energy demands. However, L-carnitine and ALCAR may be involved in the regulation of metabolic pathways in the brain (and other organs) by having an influence on the regeneration of COA and modulation of the level of acetylCOA in the mitochondria. In addition, ALCAR may have a role as a precursor of acetyllcholine, by providing the acetyl moiety for acetylcholine synthesis [43].

Chronic treatment with ALCAR has been shown to prevent impaired neuronal functioning in aged rats, both at the netrochemical level $[23,29,39]$ and at the behavioral level $[3,10,15,161$. Moreover, it has been found that chronis treatment with ALCAR increases cholinergic functioning after fimbria-formix transection [31!, and attenuates behavioral deficits induced by i.c.*. treatment with cyanide [6] or STREP [33]. However, no uniform explanation has been offered to explain these neuroprotective effects of ALCAR. As neuronal funcrioning may be affected after STREP treatment, the effects of chronic treatment with ALCAR on the enzyme activities mentioned were also evaluated in the STREP-treated rats.

\section{MATERIALS AND METHODS}

\section{Animals}

For the first experiment 23 male Lewis rats of 18 months of age were used. The rats were divided over three groups of $7-9$ animals. One group of rats was given ALCAR $(75 \mathrm{mg} / \mathrm{kg} / \mathrm{day})$ in the drinking fluid $(0.1 \%$ saccharine). After wo weeks on ALCAR the rats were anesthetized with pentobarbital (60 mg/kg. i.p.) and placed in st stereotaxic frame. A skin incision was made to free the skull and two holes were drilled above the tateral ventricles. The amimals were given an injection of $2 \mu l$ of STREP solution in each lateral ventricle (total 
dose $1.5 \mathrm{mg} / \mathrm{kg}$ body weight). The stereotaxic coordinates were $-0.8 \mathrm{~mm}$ anterior, $1.55 \mathrm{~mm}$ lateral and $-3.8 \mathrm{~mm}$ ventral from bregma [30], STREP was dissolved in saline just prior to injection. Another group of rats was not given ALCAR in the drinking fluid, but received injections of STREP only. A third group of animals received injections with saline. Ten days after injection, the rats were used for behavioral experiments of which the results have been published previousiy ( $[33$ ) and see Chapter 2). Three weeks after injection the animals were decapitated and the brains were removed from the skull in a cold room for dissection of the septum and hippocampus. For the second experiment 16 rats were used, divided over two groups of 8 animalls. The rats were treated in the same manner as in the first experiment, except that none of the rats was treated with ALCAR.

\section{Enzyme assays}

Brain tissues were homogenized in 19 volumes of $50 \mathrm{mM}$ sodium phosphate (pH 7.4). Part of the howogenates was centrifuged at high speed to obtain particle-free fractions. In both experiments prolyl endopeptidase, aminopeptidase and choline acetyltransferase activities were determined. In addition, carnitine acetyltransferase activity was determined in the first experiment and $\alpha$-ketoglutarate delaydrogenase, and glutamate dehydrogenase activities in the second experiment. All enzyme activities were not determined in a single experiment because of insufficient material. Additionally, one STREP-treated rat of the second experiment could not be wsed for measurements of prolyl endopeptidase and glutamate dehydrogenase activities because of insufficient material.

Prolyl endopeptidase activiry. Prolyl endopeptidase activity was determined spectrophotometrically with $\mathrm{N}$-succiryl-Gly-Pro-Leu-Gly-Pro-7-amido-4-methylcoumarin as substrate. The substrate $(0.1 \mathrm{mM})$ was incubated with $10 \mu \mathrm{l}$ cytosol in $200 \mu$ of a buffer consisting of $50 \mathrm{mM}$ Tris (pH 7.4), $1 \mathrm{mM}$ DTT and $1 \mathrm{mM}$ EDTA, at $37^{\circ} \mathrm{C}$. After $1 \mathrm{~h}$ of incubation the reaction was stopped by the addition of $0.3 \mathrm{ml} / \mathrm{M}$ acetic acid. Extinction was measured at a wavelength of $373 \mathrm{~nm}$.

Antinopeptidase activity. Aminopeptidase activity was measured with alamine p-nitroanilide as substrate. The substrate $(0.5 \mathrm{mM})$ was incubated with $10 \mu \mathrm{l}$ cytosol in $200 \mu 150 \mathrm{mM}$ Tris $(\mathrm{pH} 7.4)$ at $37^{\circ} \mathrm{C}$. After 30 min of incubation the reaction was stopped by the addition of $0.3 \mathrm{ml}$ ethanol. Extinction was merasured at a wavelength of $410 \mathrm{~nm}$.

$\alpha$-Ketoglutarate dehydrogenase acrivity. $\alpha$-Keloglutarate dehydrogenase activity wals determined in the homogenates according to the procedure of [20].

Glutamate dehydrogenase activity. Glutamate dehydrogenase activity was determined spectrophotometrically. The reaction mixture consisted of $50 \mathrm{mM}$ Tris $(\mathrm{pH} 8.0), 2.5 \mathrm{mM}$ EDTA, $100 \mathrm{mM}$ ammonium acetate, $0.2 \mathrm{mM}$ NADH, $1 \mathrm{mM} \mathrm{ADP}, 7 \mathrm{mM} \alpha$-ketoglutarate, and $20 \mu 12 \%$ homogenate in a final wolume of $1 \mathrm{ml}$. The reaction, at ambient temperature, was started by the addition of $\alpha$-ketoglutarate. The disappearance of NADH was monitored at a wavelength of $340 \mathrm{~nm}$. To calculate enzyne activity a correction was mado for the change in extinction in the absence of $\alpha$-ketoglutarate.

Choline aceryltransferase and carnitine acetyltransferase. Clnoline acetyltransferase ncliwity was measured in the homogenates according to the method of [14], The method for the determination of carnitine acetyltransferase activity was identical to the method for the determination of choline acetyltransferase activity, except that L-carnitine hydrochloride was used as substrate instead of choline chloride and the reaction was stopped with 10 mM phosphate ( $\mathrm{pH} 3.0)$ instead of $14 \%$ trichloroacetic acid.

\section{Statistical analysis}

Data of the first experiment were subjected to a one-way analysis of variance with treatment as the dlependent variable. Treatment effects between the three experimental groups were cvaluated in more detail by at Student-Newman-Keuls test. The effect of treatment in the second experiment was analyzed by comparing the two experimental groups with a i-test. Interdependencies between measures were analyzed using Pearson's correlation coefficient $\left(r_{\mathrm{p}}\right)$.

\section{RESULTS}


TABLE 1. Enzyme actiwites in the sepun of saline-injected, streptozolocin-injected and acetyl-L-carnitine-irsaled, streptozotocin-injected rats of amerment 1 .

\begin{tabular}{llll}
$\begin{array}{l}\text { Enzyme accivity } \\
\text { (nmol/h/mg protein) }\end{array}$ & Control & Streptozotocin & $\begin{array}{l}\text { Acetyl-L-carnitine, } \\
\text { streptozotocin }\end{array}$ \\
\hline Prolyl endopeptidase & $72 \pm 5^{\mathrm{a}}$ & $46 \pm 5^{\mathrm{b}}$ & $56 \pm 6^{\mathrm{b}} \mathrm{b}$ \\
Aminopeptidlase & $568 \pm 24 \mathrm{a}$ & $486 \pm 17 \mathrm{~b}$ & $564 \pm 24 \mathrm{ab}$ \\
Camitine acelyltransferase & $1219 \pm 31^{\mathrm{a}}$ & $858 \pm 70^{\mathrm{b}}$ & $949 \pm 71^{\mathrm{b}}$ \\
Choline acetyltransferase & $100 \pm 9^{\mathrm{a}}$ & $110 \pm 7 \mathrm{ab}$ & $135 \pm 13^{\mathrm{b}}$ \\
\hline
\end{tabular}

Dallat represent means \pm S.E.M. Means of enzyme activities with the same superseript are not different (Student-Newman-Keuls test, $p<0.05$ ).

TABLE 2. Enzyme activities in the hippocampus of saline-injected, sitreprozotocin-injected and acetyi-L-canitine-treated, streptozotocin-injected rais of experiment 1.

\begin{tabular}{llll}
$\begin{array}{l}\text { Enzyme activity } \\
\text { (nmol/h/mg protein) }\end{array}$ & Control & Streptozotocin & $\begin{array}{l}\text { Acetyl-L-carnitine, } \\
\text { streptozotocin }\end{array}$ \\
\hline Prolyl endopeptidase & $97 \pm 4^{\mathrm{a}}$ & $93 \pm 4 \mathrm{a}$ & $95 \pm 4 \mathrm{a}$ \\
Aminopeptidase & $439 \pm 14^{\mathrm{a}}$ & $406 \pm 20^{\mathrm{a}}$ & $467 \pm 37 \mathrm{a}$ \\
Carnitine acetyltransferase & $654 \pm 11^{\mathrm{a}}$ & $592 \pm 27^{\mathrm{a}}$ & $634 \pm 21^{\mathrm{a}}$ \\
Choline acetyltransferase & $91 \pm 2^{\mathrm{a}}$ & $34 \pm 10^{\mathrm{b}}$ & $63 \pm 12^{\mathrm{c}}$ \\
\hline
\end{tabular}

Data represent means \pm S.E.M. Means of enzyme activities with the same superscript are not different (Student-Newman-Keuls test, $p<0.05$ ).

TABLE 3. Pearson's correlation coefficients for data from experiment 1 on sepum weight and enzyme activities.

\begin{tabular}{lll} 
Eernzyme activity & $r_{\mathrm{p}}$ & $\beta$ \\
\hline Prolyl endopeptidase & 0.838 & $<0.001$ \\
Aminopeptidase & 0.594 & $<0.01$ \\
Carnitine acetyltransferase & 0.754 & $<0.001$ \\
Clodine acenyltransferase & -0.272 & n.s. \\
\hline
\end{tabular}

\section{Experinent 1}

Septum weights \pm S.E.M. (mg) were $17.0 \pm 1.0,9.2 \pm 1.3$, and $11.9 \pm 1.9$ in the control, the STREP-ingected and the ALCAR-treated, STREP-injected rats, respectively. The weight of the septum was reduced after injection of STREP in both the animals treated with ALCAR and the untreated animals $(p<0.01)$. The weight of the hippocampus was unchanged (data not shown).

The enzyme activities in the septum of the saline-injected, the STREP-injected and the ALCAR-treated, STREP-injected rats are given in Table I. The three experinental groups differed in septal prolyl endopeptidase activity $(p<0.05)$, aminopeptidase activity $(p<0.05)$ and carnitine acetyltransferase activity $(p<0.01)$. Septal choline acetyltransferase activity only tended to be different between the groups $(0.05<p<0.1)$. Post hoc analysis revealed that prolyl endopeptidase, aminopeptidase and carnitine acetyltransferase actiwities were reduced in the septum of the STREP-injected animals, whereas choline acetyltransferalse activity was not changed. Treatment with ALCAR did not prevent prolyl endopeptidase and carnitine acetyltramsferase activities from being reduced. Aminopeptidase activity was spared by treatment with ALCAR. 
However, septal choline acetyltransferase activity of the ALCAR-treated, STREP-injected rats was even higher than that of the saline-injected rats. In the hippocampus of all experimental groups prolyl endopeptidase, aminopeptidase, and camitine aceryltransferase activities were unaftected (see Table 2, $p s>0.05$ ), whereas choline acetyltransferase activity was affected $(p<0.01)$. Post hoc analysis showed that treatment with ALCAR attenuated the decrease in choline acetyltransferase activity in the hippocampus elicited by STREP.

High correlations were found between septwm weight and enzyme activities, except choline aceryltransferase activity (see Table 3). The correlation between choline aceryltransferase activity in the septum and that in the hippocampus was poor $\left(r_{p}=-0.085\right.$, n.s.), but the correlation between septum weight and choline acetyltransferase activity in the hippocampus was high ( $r_{\mathrm{p}}=$ $0.865, p<0.001\rangle$.

\section{Experiment 2}

Septum weights \pm S.E.M. (mg) were $17.2 \pm 0.5$ and $7.9 \pm 1.5$ in the control and the STREPinjected rats, respectively. The weight of the septum was reduced after injection of STREP ( $p<$ 0.001). The weight of the hippocampus was unaffected (data not shown).

Prolyl endopeptidase, aminopeptidase, $\alpha$-ketoglutarate dehydrogenase, and glutamate dehydrogenase activities in the septum were reduced after injection of STREP (see Table 4), whereas in the hippocampus these enzyme activities were not affected by STREP. However, choline acetyltransferase activity in the hippocampus of the STREP-injected rats was reduced compared with that of the control animals (see Table 5).

High correlations were found between septum weight and enzyme activities (see Table 6). The correlation between septum weight and choline acetyltransferase activity in the hippocampus was high $\left(r_{p}=0.917, p<0.001\right)$.

\section{DISCUSSION}

In the present study the effects of STREP on enzyme activities in the septum and hippocampus of control rats and of rats treated with ALCAR were studied. STREP affected enzyme activities in the septum rather indiscriminately, except choline acetyltransferase activity, whereas it affected only choline acetyltransferase activity in the hippocampus. ALCAR did not spare all the anzyme activities in the septum, but seemed to preserve the cholinergic function in the hippocampus to some extent. The gemeral effect of STREP on enzyme activities in the sepium contrasts with its reportedly selective effects on enzyme activities in pancreatic B-cells $[3.5]$. This difference might be explained by the possibility that STREP may have caused cell death in the septum, wheresis enzyme activities in pancreatic 6-cells have been measured in cells surviving the insult by STREP.

The above findings suggest that cholinergic neurons in the septum were relatively spared from the degenerative effects of STREP. This does not necessarily mean that cholinergic neurons are less vulnerable to STREP than other cells, since many of the non-cholinergic cells in the septum are closer to the injection site than the cholinergic neurons. The relative sparing of the cholinergic neurons may have been enhanced by ALCAR. Histological and immunocytochemical studies are in progress to assess which brain structures are affected by STREP.

Specific choline acetyltransferase activity in the septum was unaffected by STREP, whereas specific choline acetyltransferase activity in the hippocampus was reduced. This seems surprising, since the hippocampus receives a cholinergic input from the septum. However, the septurn weight was reduced, whereas the hippocampus weight was unaltered, so that in both structures total 
TAELE 4. Enzyme activities in the sepumm of saline-injected and streptozotocm-injected rats from experiment 2 .

\begin{tabular}{lrr}
\hline Enzyme activity (nmol/h/mg protein) & Control & Streptozotocin \\
\hline Frolyl endopeptidase & $66 \pm 3$ & $35 \pm 6^{* *}$ \\
Aminopeptidase & $567 \pm 12$ & $418 \pm 29^{* *}$ \\
a-Ketoglutarate delydrogenase & $545 \pm 33$ & $306 \pm 27^{* *}$ \\
Glutamalte delinddrogenase & $5938 \pm 218$ & $2602 \pm 149^{* *}$ \\
\hline
\end{tabular}

Data represent means \pm S.E.M. Asterisks indicate differences with respect to control ( $t$-test, $p<0,0011)$.

TABLE 5. Enzyme activities in the hippocampus of saline-injected and streptozotocininjected rats from experiment 2 .

\begin{tabular}{lrr}
\hline Enzyme activity (nmel/h/mg protein) & Control & Streptozotocin \\
\hline Prolyl endopepticlase & $137 \pm 10$ & $128 \pm 8$ \\
Aminopeptidase & $682 \pm 48$ & $607 \pm 26$ \\
C.Ketoglutarate dehydrogenase & $615 \pm 45$ & $650 \pm 49$ \\
Glutanate debydrogenase & $8106 \pm 308$ & $8065 \pm 279$ \\
Choline acetyltransferase & $92 \pm 2$ & $39 \pm 13^{*}$
\end{tabular}

Data represent means \pm S.E.M. Asterisk indicate differences with respect to control $(t$-test, $p<0.011$.

TABLE 6. Pearson's corralation coefficients for data from experiment 2 on septum weight and enzyme activities.

\begin{tabular}{lll} 
Enzyme acivity & $r_{\mathrm{p}}$ & $p$ \\
\hline Prolyl endopeptidase & 0.977 & $<0.001$ \\
Aminopeptidase & 0.901 & $<0.001$ \\
anKewoglutarate dehydrogenase & 0.691 & $<0.01$ \\
Glutamate dehydrogerase & 0.845 & $<0.001$ \\
\hline
\end{tabular}

choline acetyltransferase activity was reduced. In addition, total choline acetyltransferase activities in these two structures were strongly correlated $\left(r_{\mathrm{p}}=0.751, p<0.001\right)$. Therefore, the reduced cholline acetyltransferase activity in the hippocampus can be explained by an effect of STREP at the level of the septum.

Interestingly, choline acetyltransferase activity correlated highly with carnitine acetyltransferase activity in the hippocampus $\left(r_{p}=0.749, p<0.001\right)$, although this latter activity was not significantly reduced after injection of STREP. In addition, hippocampal carnitine acetyltransferase activity correlated both with septal carnitine acetyliransferase activity $\left(r_{\mathrm{p}}=0.611\right.$, $p<0.01)$ and with septum weight $\left(r_{\mathrm{p}}=0.768, p<0.001\right)$. These correlations suggest that carnitine acetyltransferase may be linked to the septohippocampal pathway. This may have to do with the possibility that the enzyme is preferentially located in cholinergic nerve endings. This possibility is in accordance with some previous findings. First, lesion of the habenulointerpeduncular tract has been showin to result in reduced activity of both carnitine acetyltransferase and choline acetyltransferase in the interpeduncular nucleus [36]. Second, it has been reported that nerve growth factor (NGF) increases both carnitine acetyltransferase and 
choline acetyltransferase activities in PC12 cells [44]. Moreover, these results suggest that preferential localization of carnitine acetyltransferase in chollinergic terminals is a general phenomenor.

The fact that enzyme activities were affected rather indiscriminately in the septum together with a reduced weight of the septum suggests that STREP acts as a non-selective neurotoxin near the site of injection. Hoyer and collaborators, who used the same dose of STREP as used in this study, found rather widespread effects of STREP on energy metabolism and neurotransmitter systems and ascribed these effects to a possible interference of STREP on the action of insulin. However, our finding of septal degeneration is not in line with such a specific effect of STREP on the action of insullin in the brain. The destruction of septal-hippocampal and sepial-cortical comections may have contributed to the effects of STREP in locations distant from the septum. Therefore, with respect to the mechanism of action of STREP an alternative hypothesis may be formulated. Since it has been shown that STREP causes its effects on pancreatic B-cells by the generation of hydrogen peroxide [40], it can be hypothesized that STREP does so in the central nervous system as well.

There is evidence that oxidative stress is important in the pathogenesis of Alzheimer's disease and other neurodegenerative diseases (reviewed by [26]). This evidence is for the most part indirect, consisting of alterations in the accumulation of peroxidation products in tisstes 11,271 and changes in the activity of enzymes sensitive to changes in redox status 122. 421. Interestingly, it has recently been shown that $\mathbb{B}$-amyloid peptide increases the production of oxygen free radicals by stimulating a NADPH oxidase-like activity or by an auto-oxidative process [4, 17]. However, i.c.v. injection of STREP caused a general decline in the enzyme activities determined, whereas in Alzheimer"s disease some of these enzyme activities are reduced and others are not [21, 421. The effect of STREP on the septum may have been too strong to mimic what is going on in Alzheimer's disease. In addition, the septum may not be the most appropriate area to induce Alzheimer's disease-like features, since brain areas other than the septum are affected by Alzheimer"s disease. Future studies should investigate the effects of low doses of STREP in brain areas that are affected in Alzheimer"s disease.

Two effects of ALCAR reported in the literature may be of relevance to explain the positive effects of ALCAR on the septohippocampal cholinergic system. Firstly, ALCAR increases the binding of NGF to its receptor in aged rat central nervous system |2|. Secondly, in PCI2 cells ALCAR increases the synthesis of the NGF" receptor " 381 and the subsequent internalization of NGF [37], which is important in the action of NGF. Correspondingly, it has been found that ALCAR increases NGF levels and choline acetyltransferase activity in the central nervous system of fimbria-formix transectioned rats [31] and aged rats [39]. Finally, the fact that NGF plays a role in oxidant homeostasis $28 \rrbracket$ strengthens the possibility that NGF is involved in the newroprotective effect of ALCAR against the damaging effects of STREP.

In conclusion, i.c.v. injection of STREP causes structural damage in the septum. This damage causes enzymatic changes that do not closely resemble the more specific changes in enzyme activities as observed in Alzheimer"s disease. ALCAR partly prevents this dumage, as reflected by an attenuation of the STREP-induced decrease in hippocampall choline acetyltransferase activity. This finding indicates that STREP-treated rats may be valuable to test neuroprotective effects of substances. Furthermore, it demonstrates that ALCAR has a neuroprotective action on cholinergic neurons. However, little is known about the neuroprotective effect of ALCAR on non-cholinergic neurons. Injection of low doses of STREP in brain sites other than the septum in combination with assessment of markers of specific neuronal populations may be a way to study the neuroprotective effects of ALCAR on non-cholinergic neurotransmitter systems. 


\section{ACKNOWLEDGEMENTS}

We thank Signid Tau Company (Pomazia-Rome, Italy) for financial support and for providing us with acelyl-Luarnitine.

\section{REFERENCES}

1. Andorn A.C., Britton R.S. and Bacon B.R. (1990) Evidence that lipid peroxidation and total iron are increased in Alzheimer"s brain. Neurobiol. Aging 11, 316.

2. Angelucci L., Ramacci M.T., Taglialatela G., Hulsebosh C., Morgan B., Werbach-Perez K. and PerezPolo J.R. (1988) Nerwe growth factor binding in aged rat central nerwous system: Effect of acetyl- $\mathrm{L}$ carnitine J. Meurosci. Res. 20,491-496.

3. Barmes C.A., Markowska A.L., Ingram D.K., Kamelani. H., Spangler E.L., Lemken V.J. and Olton D.S (1990) Acetyl-L-carnitine 2: Effects on learning and memory performance of aged rats in simple and complex mazes. Neurobial Aging 11, 499-506.

4. Behl C., Davis J.B., Lesley R, and Schubert D. (1994) Hydrogen peroxide mediates arnyloid ts protein toxicity. Cell 77, 817.827.

5. Bieber L.L. (1988) Carnitine. Ann. Rev. Biechem. 57, 261-283.

6. Blokland A., Bohmer J., Honig W. and Jolles J. (1993) Behavioral and biochemical effects of acute central metabolic inhibition: Effects of acetyl-L-carnitine. Eur. J. Phamacol. 235, 275-281.

7. Blokland $\mathrm{A}$ and Jolles J. (1993) Spatial learning deficit and reduced hippocampal ChAT activity in rats after an icv injection of streptozotocin. Pharmacol. Biochem. Behav. 44, 491-494.

8. Blokland A. and Jolles. J. (1994) Behavioral and biochemical effects of an icv injection of streptozotocin in oldl Lewis rats. Phawmacol. Biochem. Behav, 47, 833-837.

9. Bolaffi J.L.. Nagamatsu S., Harris J, and Grodsky G.M. (1987) Protection by thymidine, an inhibitor of polyadenosine diphosphate ribosylation, of streptozotocin inhibition of insulin secretion. Endocrinology 1.20. $2 \rrbracket 17-2122$.

10. Caproli A., Ghirardi O., Ramacci M.T. and Angelucci L. (1990) Age-dependent deficits in radial maze performance in the rat: Effect of chronic treatment with acetyl-L-carnitine. Prog. Neuropsychophamacol. Biol. Psychiat. 14, 359-369.

11. Davies P. (1979), Neurotransminter-related enzymes in senile dementia of the Alzheimer type. Brain Res. 171, 319-327.

12. Ding A. Nitsch R. and Hoyer S. (1992) Changes in brain monoaminergic neurotransmitter concentrations in rat after intracerebroventricular injection of streptozotocin. J. Cereb. Blood Flaw Metab. 12, 103-109.

13. Dulin W.E. and Wyse B.M. (1969) Studies on the ability of compounds to block diabetogenic activity of streptozotocin. Diabetes $18,459$.

14. Fonnum F. (1975) A rapid radiochemical method for the determination of choline acetyltransferase. $J$. Neurochem. 24, 407-409.

15. Ghirardi O., Caprioli A., Milano S., Giwiani A., Ramacci M.T. and Angelucci L. (1992) Active avoidance learning in old rats chronically treated with levocarnitine acetyl. Physiol. Behav. 52, 185-187.

16. Ghirardi O. Milano S., Ramacci M.T. and Angelucci L. (1988) Effect of acetyl-L-carnitine chronic treatment on discrimination models in aged rats. Physiol. Behaw, 44, 769-773.

17. Goodman Y., Steiner M.R., Steiner S.M. and Mattson M.P. (1994) Nordihydroguaiaretic acid protects hippocampa! neurons against amyloid is-peptide toxicity, and attenuates free radical and calcium acoumulation. Brain Res. 654, 171-176.

18. Hellweg R.. Nitsch R., Hock C., Jaksch M. and Hoyer S. (1992) Nerve growth factor and chine acetyltransferase activity levels in the rat brain following experimental impairment of cerebral glucose and energy metabolism. J. Neurosci. Res. 31, 479-486.

19. Kalaria R.N. and Harik S.I. (1992) Carnitine acetyltransferase activity in the human brain and its microvessels is decreased in Alzheimer's disease. Amm. Neurol. 32, 583-586.

20. Lai J.C.K. and Cooper A.J.L. (1986) Brain $\alpha$-ketoglutarate dehydrogenase complex: kinetic properties, regional distribution, and effects of inhibitors. J. Neurochem. $47,1376-1386$.

21. Mastrogiacomo F., Bergeron C. and Kish S.J. (1993) Brain c-ketoglutarate dehydrogenase complex activity in Alzheimer"s disease. J. Neurochem. 61, 2007-2014.

22. Morrison L.D. Bergeron C. and Kish S.J. (1993) Brain S-adenolsyl methionine decarboxylase activity is increased in Alzheimer's disease. Neurosci. Leth 154, 141-144.

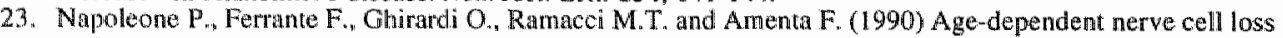
in the brain of Sprague-Dawley rats: Effects of a long-term acetyl-L-carnitine treatment. Anch. Geronol. Geriatr. 10, 173-185.

24. Nitsch $R$ and Hoyer S. (199 L) Local action of the diabetogenic drug, streptozotocin, on glucose and energy metabolism in the brain cortex. Neurosci. Letr. 128, 199-202.

25. Nitsch R., Mayer G. and Hoyer S. (1989) The intracerebroventriculary streptozotocin-treated rat: 
Impaiment of cerebral glucose metabolism resembles the alterations of carbohydratle metabolism of the brain in Alzheimer's disease. $\lambda$. Neural Trans. $1,109-110$.

26. Olanow C.W. (1993) A radical hypothesis for neurodegeneration. Trends Neurosci. 16, 439-444.

27. Palmer A.M. and Burns M.A. (1994) Selective imerease in lipid peroxidation in the inferior temporal corfex in Alzheimer's disease. Brain Res, 645, 338-342.

28. Pan Z. and Perez-Polo R. (1993) Role of nerve growth factor in oxidant homeostasis: glutahtone metabolism. J. Neurochem. 61, 1713-1712.

29. Patacchioli F.R., Amenta F., Ramacci M.T., Tagialatiela G., Maccari S. and Angelucci L. (1989) Acetyl. L-carnitine reduces the age-dependent loss of glucocorticoid receptors in the rat hippocampus: An autoradiographic study. J. Nemrosci. Research $23,462-466$.

30. Paxinos G. and Watson C. (1986) The rar brain in stereotaxic coordinates. 2nd edn., Academic Press, San Diego.

31. Piovesan P., Pacifici L., Taglialaela G., Ramaci M.T. and Angelucci L. (1994) Acetyl-L-carnitine treatment increases choline aceiyltransferase activity and NGF levels in the CNS of adult rats following total fimbriafornix transection. Brain Res. 633, 77-82.

32. Plascinke $\mathrm{K}$. and Hoyer $\mathrm{S}$, (1993) Action of the diabetomic drug streptozotocin on glycolybic and glycogenolytic metabolism in adult rat brain cortex and hippocampus. Int. Devl Neurosci. 11, 477-483.

33. Prickaerts J., Blokland A., Honig W., Meng F. and Jolles J. (1995) Spatial discrimination learning and choline acetyltransferase activiny in streptozotocin-treated rats; Effects of chronic treatment with aceiyl-Lcarnitine. Brain Res. 674, 142mit6.

34. Rakieten N., Rakisten M.L. and Nadkarni M.V. (1963) Studies on the diabetogenic action of streptozotocin. Cancer Chentother. Rep. 29,91.

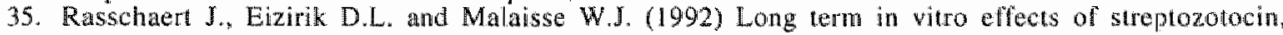
interleukin-1, and high glucose concentration on the activity of mitochondrial dehydrogenases and the secretion of insulin in pancreatic islets. Endocrinology 130, 3522-2528.

36. Sterri S.H. and Fonnum F. (1980) Acetyl-CoA synthesizing enzymes in cholinergic nerve terminals. $J$. Neurochem. 35, 249-254.

37. Taglialatela G., Angelucci L. Ramacci M.T., Werbach-Perez K. Jackson G.R. and Perez-Polo J.R. (1991) Acetyl-L-carnitine enhances the response of PC12 cells to nerve growth factor. Dev. Brain. Res. 59, 221 . 230.

38. Taglialatela G., Angelucci L., Ramacci M.T., Wertbach-Perez K., Jackson G.R. and Perez-Polo J.R. (1992) Stimulation of nerve growth factor receptors in PC12 by acetyl-L.carnitine. Biochem. Pharmacol. 44, 577 . 585.

39. Taglialatela G., Navarra D., Cruciani R., Ramacci M.T., Alema G.S. and Angelueci L. (1994) Acetyl-Lcarnitine treatnent increases nerve growth factor levels and choline aceiyltransferase activity in the central nervous system of aged rats. Exp. Gerontol. 29, 55-66.

40. Takasu N., Komiya I., Takayuki A., Nagasawa Y. and Yamada T. (1991) Streptozotocin- and alloxaninduced $\mathrm{H}_{2} \mathrm{O} 2$ generation and DNA fragnentation in pancreatic islets. Diabetes $40,1141-1145$.

41. Terwel D., Bothmer F. Meng E., Wolf E., Markerink M. and Jolles. J. (1994) Reduced enzyme activities in Alzheimer's disease are sensitive to hypoxia. Neurobiol. Aging 15 (Suppl.11), S120.

42. Terwel D. Markerink M. and Jolles. I. (1992) Peptidases are affected differently in neocontical regions of brains from patients with Alzheimer's disease. Dementia 3, 108-113.

43. White H.L. and Scates P.W. (1990) Acetyl-L-carnitine as a precursor of acetylcholine. Nemrochem. Res. 15, 603-607.

44. White H.L. and Scates P.W. (1991) Stimulation of carnitive acetyliransferase in PC12 calls by nerve growth factor: relationship to choline acetyltransferase. Neurochen. Res. 16, 63*66. 


\title{
Cognitive performance and neurodegenerative markers in septum, hippocampus and striatum after an i.c.v. injection of streptozotocin: A correlation analysis*
}

\author{
Jos Prickaerts? Thomas Fahrig2 and Arjan Blokland3 \\ European Graduate School of Neuroscience "EURON", Department of Psychiary and \\ Newropsychology, and 3 Departwent of Psychology, Mastrich University, The Netherlands \\ 2 Bayer AG, CNS-Research, Cologne, Germany
}

\begin{abstract}
In the present study we evaluated the effects of an intracerebroventricular injection of sireptozotocin on cognitive behavior and biochemical markers in the brain of middle-aged Wistar rats. Intracerebroventricular injected streptozotocin has previously been reported to decrease the central metabolism of glucose. We found that streptozolocinmtreated rats showed an impaired cognitive performance in the delayed non-matching to position task and the Morris water escape task. The streptozotocin-treated rats could be subdivided into a non-impaired and an impaired group on the basis of the performance in the delayed non-matching to position task. This subdivision also applied to the performance in the Morris water escape task which indicates that streptozotocin induced an individual cognitive decline across several behavioral domains. Glial fibrillary acidic protein, an indicator of reactive astroglial changes, was measured in three different (soluble, Triton X-100 soluble and crude cytoskeletal) protein fractions and its content in the fractions of the septum, hippocampus and striatum of streptozolocin-treated rats was increased. Furthermore, the glial fibrillary acidic protein response of each protein fraction to streptozotocin treatment appeared to be differently regulated. In streptozotocin-treated tats the choline acetyltransferase activity was decreased in the hippocampus only, which was correlated with the hippocampal glial fibrillary acidic protein contents of all three hippocampal protein fractions, thus suggesting that the cholinergic deficit is a consequence of direct damage to the hippocampus. The cognitive deficits in both tasks were related to the increased glial fibrillery acidic protein contents, especially of the soluble and cytoskeletal fraction, and the decreased choline acetyltransferase activity in the hippocampus. Taken together, these findings indicate that it is important to take into account which protein fraction has been used for measuring the glial fibrillary acidic protein response to a stressor. Furthemore, intracerebroventricular injected streptozotocin may provide a relevant model for studying neurodegenerative changes due to a metabolic insufficiency and testing neuroprotective effects of substances.
\end{abstract}

\section{INTRODUCTION}

A dramatic decrease in glucose utilization can be observed in the brains of Alzheimer's Disease

\footnotetext{
* This study has been submitted to Behav Brain Res.
} 
(AD) patients and led to the hypothesis that the cognitive dysfunction of $A D$ is related to a reduction in central glucose metabolism 1301, More recently, it has been hypothesized that the cholinergic deficil and anyloid accumulation in the brain are caused by the major decrease in glucose metabolism in the AD brain [41]. Several studies have prowided evidence that a single intracerebroventricular (i.c.v.) injection of streptozotocin (STREP) reduced the central metabolism of glucose in middle-aged rats $[13,46,47,50]$. Concomitantly, a decrease in cognitive function $[3,4,37$. 5.1] and a decrease in, among others cholinergic neurotransmission [3. $4,11.27$. 51] were observed in STREP-treated rats. Therefore, it has been suggested that the STREP-treated rat could be regarded ass relevant animal model of sporadic $\mathrm{AD}[3,13,46,47,50]$.

In prewious experiments we have shown that rats that received an i.c.v. injection of STREP were impaired in learning a spatial Morris water escape task 19.41. Interestingly, this impaiment in spatial discrimination performance correlated with a reduction of hippocampal choline acetyltransferase (ChAT) activity and suggested that these two parameters were related. Therefore, it can be proposed that a single i.c.v. injection of STREP provided an animal model for studying the relation between the hippocampal cholinergic system and spatial discrimination performance.

The finding that the impaired spatial discrimination performance correlated with a decreased hippocampal ChAT activity corroborated the notion that the cholinergic neurotransmission is involved in cognitive processes. However, two observations did not support this apparent conclusion. First, a single i.c.v. injection of STREP also affected different monoaminergic neurotransmitters in the brain [11]. Thus, the impaired behavioral performance could also be related to a functional decline of other neurotransmitters. Another interesting observation was made in a study in which we tested the effects of acetyl-L-carnitine on the STREP-induced deficit [51]. In that study we replicated the correlation between hippocampal ChAT activity and spatial discrimination performance in STREP-treated animals. However, this correlation was not found in animals treated with STREP and acetyl-L-carnitine. This suggests that hippocampal ChAT activity does not necessarily predict cognitive performance in a spatial Morris task.

Although it has been suggested that STREP inhibits the utilization of glucose in the brain and consequently leads to an inhibition of the metabolic capacities of neurons $[13,46,47,50]$, it has to be noted that the exact mechanism by which an i.c.v. injection of STREP induces neurodegenerative effects remains unclear, thus far. For instance, oxidative damage may be involved in causing an impaired neuronal function since STREP generates reactive compounds (e.g. $\mathrm{H}_{2} \mathrm{O}_{2}[60]$ ).

The present study extends our previous efforts to understand the effects of STREP treatment on behavior and the neurodegenerative effects of this neurotoxin. Rats were tested in the Morris water escape task, in order to replicate our previous findings. Behavioral effects of STREP treament were also tested on the performance in the delayed non-matching to position (DNMTP) lask, a task that is assumed to assess (spatial) working memory $[$ see 1, 16]. Thereafter, ChAT activity and glial fibrillary acidic protein (GFAP) content in the septum, hippocampus and striatum were determined. ChAT activity, which is decreased in AD brain le.g. 3\%, was chosen to monitor neurodegenerative changes of the cholinergic system, whereas GFAP was used as an indirect marker for neuronal damage in general, as has also been observed in AD brain [21]. Finally, the biochemical markers were correlated with the behavional data to evaluate putative relationships between parameters $[3,51]$.

\section{MATERIALS AND METHODS}




\section{Animals}

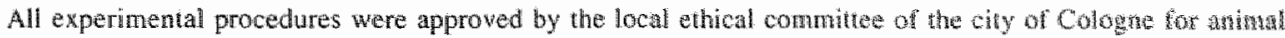
experiments and met German governmental guidelimes. We used twenty-sis 14-month-nh mate Wistar gats (Winkelmann, Borchen, Germany), which were already experienced in the DNMTP tak from presious angr

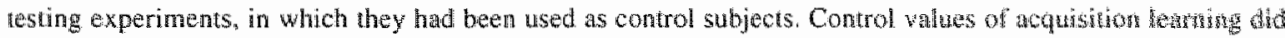
not change over a period of 6 months. Acquisition training in this experiment was stared after a wash-oul pertod of 8 weeks and 2 weeks before surgery. The animals were housed in groups of two rats in standard Mathoton? cages on sawdust bedding in an air-conditioned room (about $20^{\circ} \mathrm{C}$. They were kept under at $12 / 12-$ hour bightegark cycle (lights on from 08.001020 .00 h). Normally, rats had free access to food and water, but during the DNWTP task they were subjected to a food-deprivation regime, in which $10-12 \mathrm{~g}$ dry laboratory chow was given at we ond of each day of testing.

\section{Singery}

After two weeks of reacquisition training in the DNMTP task, the rats were assigned to a control group and a STREP group. The groups were matched for performance during the delays of the second week of training. Two weeks before retesting in the DNMTP task, the rats were anesthetized with chloral hydrate (400 mg/kg body weight in a $20 \%$ solution, i.p.) and placed in a stereotaxic frame. STREP was dissolved in a citrate buffer (s $\mathrm{mM}, \mathrm{pH}=5.5$ ). The STREP group was given a bilateral i.c.v. injection of STREP ( $1.5 \mathrm{mg} / \mathrm{kg}$ body weight dissolved in $4 \mu$ l citrate buffer, $2 \mu l /$ injection site). The stereotaxic coordinates were $-0.8 \mathrm{~mm}$ anterior, $1.5 \mathrm{~mm}$ lateral, and $-4.0 \mathrm{~mm}$ ventral from the bregma [49]. The control group underwent the same surgical procedures, but citrate buffer was injected i.c. $v$. instead of STREP. Three rats died during surgery, i.c. before being treated, for reasons unknown. After surgery the rats were housed individually and had access to food and water ad lib. During the experiment body weights and water consumption were monitored. One control rat and one STREPatreated rat died shortly after surgery. Further, one control rat and one STREP-treated rat died later on, during behavioral testing, for reasons unknown. Therefore, the numbers of rats per group used for analysis were: control, $n=7$ and STREP, $n=12$.

\section{Delayed non-matching to position task.}

Two weeks after surgery, the rats were again subjected to a food-depriwation program and tested in the DNMTP task for a further two weeks. A bank of ten identical Skinner boxes were used and a more detailed description of the task has been given by Dunnett $[15]$.

Procedures: At the start of a trial one of two levers was inserted into the operant chamber during the 'sample' phase. After the rat had pressed the sample lever, the lever was retracted and the rat had to poke its nose against a hinged panel that gave access to the food well. The lood well was positioned equidistantly between the wo levers. After the rat had pusthed the panel of the food well, both levers were presented to the rat (choice' phase) and the rat could choose which lever to press. In the NMTP paradigm, food reward is contingent on a lever-press in the 'choice" phase which is in the opposite position of the lever which was presented in the 'sample' phase. There was a 5-second time-out period (and no food reward) when the lever-press in the 'choice" phase was the same one as in the 'sample' phase (i.e. when the response was incorrect). Aftur the rats had acquired this task, delay interwals were introduced between the 'sample' and 'choice' phases. The delay interval wats randomly chosen from the following six altematives: $1,2,4,8,16$, or 32 seconds. During the delay interval the rat was required to press a hinged panel, which prevented the rats from developing a position or mediating strategy. The rats were given five daly sessions per week with a session duration of $45 \mathrm{~min}$. All behavioral lesting was always done between 10.00 and $16.00 \mathrm{~h}$.

Statistical analysis: The number of correct responses was calculated for each delay. The mean correct responses per five sessions was taken as the percentage correer responses per delay. This measure was analyzed per group (within-subject design) in a two-way ANOVA (Surgery and Delay) with repeated measures over Surgery and Delay. For comparisons between the wo groups (between subject design) a threc-way ANOVA (Treatment, Surgery and Delay) with repeated measures over Surgery and Delay was used. 1-statistics were used for comparisons per delay. The latency to press the choice lever was measured. Response bias was assessed by calculating Index $Y$ as formulated by Sahgal [54]; Index $Y=$ (percentage correct left responses minus percentage correct right responses) / (sum of the percentages left and right correct responses). The analysis of response bias 
was performed on absolute values of Index $Y$. Both latencies and Indices $Y$ were averaged over all delays and subsequently the mean walues per five sessions were calculated, which were analyzed with a two-way ANOVA. (Treatnent and Surgery) with repeated measures over Surgery.

\section{Morris water escape task}

After the DNMTP task, that is four weeks after surgery, the rats were fed ad lib again and their Morris water escape performanee was assessed in a black water tank (diameter $1.53 \mathrm{~m}$ ). The escape platorm (diameter $11 \mathrm{~cm}$ ) was submerged $(1.5 \mathrm{~cm})$ below the surface of the water. The water temperature was about $22^{\circ} \mathrm{C}$. The water was not made opacue because the escape platform was black and hence virfually invisible in the black tank. The water tank was divided into four quadrants which were designated training, right-adjacent, left-adjacent and opposite relative to the position of the platform. Abundant spatial cues were provided by the furniture in the room, including desks and computer equipment, and by the presence of the experimenter. A radio, which was playing softly, provided background noise during testing in the water task.

A wideo camera, mounted in the center above the circular pool, provided a picture of the pool on a TV monitor. The movements of a rat were regisfered automatically (Ethowision, Noldus equipment) and stored in a personal computer.

Procedures: The rats were started facing the wall of the tank from four different, randomly chosen, start positions, and trained to find the invisible platform at a fixed invisible position in the water tank. A trial lasted until a rat had found the platform or until $60 \mathrm{~s}$ had elapsed. If a rat did not find the platiorm within $60 \mathrm{~s}$, it was placed on the platform for $3 \mathrm{~s}$ and then removed from the water tank. On the first day, the rats were given four trials. On the three subsequent days the rats were given eight trials a day. The time between subsequent trials was about 10 min. A probe trial lasting $60 \mathrm{~s}$, during which the platform was removed from the water tank, was given alter the last trial. In the probe trial all rats were started, facing the wall of the tank, from the position opposite to the position of the renioved platform.

Statistical analysis: Four measures of the acquisition of the Morris water escape task were analyzed: the escape latency, the distance swum, swimming speed (distancellatency), and mean distance to platform [22]. The measures were averaged per rat per session of four trials. Treatment effects during the acquisition were evaluated with a two-factoriall (Treatment and Session) ANOVA with repeated measures over Session. For the probe trial, preferences for quadrants were evaluated per group by analyzing the times spent in each quadrant with a repeated measures ANOVA over Quadrant, and additional $t$-statisties which analyzed whether the time spent in a quadrant differed from the chance level (15s).

\section{CMT alcrivity and GFAP content}

Five weoks after surgery, the rals were decapituted and the heads were immediately immersed in liquid nitrogen for 5. s. Brains were taken out of the skull and the septum, hippocampus, and striatum were dissected out at $4^{\circ} \mathrm{C}$. Tissue samples were stored at $-80^{\circ} \mathrm{C}$ until used 10 mesasure ChAT activity and GFAP content. A pilot experiment. had show that there was no difference in both ChAT activity and GFAP content between frozen and fresh tissue. Brain satuples were bomogenized (10\%) in $10 \mathrm{mM} \mathrm{Na} 2 \mathrm{HPO}_{4} / \mathrm{NaH}_{2} \mathrm{PO}_{4}$ buffer ( $\mathrm{pH} 7.4$ ) containing I mM EDTA, $1 \mathrm{mM}$ EGTA and warious protease inhibitors $(10 \mu \mathrm{g} / \mathrm{ml}$ soybean trypsin inhibitor, 1 MM leupeptin, ImM phenylmethyisulfonyl thoride). One fraction of the homogenate was diluted 5 times, resulting in a $2 \%$ homogenate in $10 \mathrm{mM} \mathrm{Na} 2 \mathrm{HPO} / \mathrm{NaH}_{2} \mathrm{PO}_{4}$ buffer, whereas a second fraction was diluted 2 times, resulting in a $5 \%$ homogenate in $50 \mathrm{mM} \mathrm{Na} \mathrm{HPO}_{4} / \mathrm{NaH}_{2} \mathrm{PO}_{4}$ buffer. Protein determinations were performed according to a moditication of the Lowry procedure [25].

The 5\% homogenate fraction was used for ChAT activily measurement according to Fonrum's method [20], using [14C]acelyl coenzyme-A (Amershann, $50-60 \mathrm{mCi} / \mathrm{mM}$ ). The $2 \%$ homogenate fraction was used for mensurements of GFAP content according to the semiquantitative method as described by Fahrig [19]. Briefly, the $2 \%$ homogenates were centrifuged at $30000 \mathrm{~g}$ for $45 \mathrm{~min}$ at $4^{\circ} \mathrm{C}$ and the supernatant was atiquoted and stored at $-80^{\circ} \mathrm{C}$ (soluble protein fraction). The resulting pellet was rehomogenized at $4^{\circ} \mathrm{C}$ in $10 \mathrm{mM}$ Tris-HCl (pH 7.4), $1.5 \mathrm{M} \mathrm{NaCl}, \mathbb{m M}$ EDTA, I mM EDTA, $1 \mathrm{mM}$ EGTA, 0.5\% Triton X-100, containing protease inhibitors. After another $30000 \mathrm{~g}$ centrifugation step for $45 \mathrm{~min}$ at $4^{\circ} \mathrm{C}$, the supernatant was collected, aliquoted, and stored at $-80^{\circ} \mathrm{C}$ until used (Triton $\mathrm{X}-100$ soiuble fraction). The remaining insoluble material was incubated for 1 min at $4^{\circ} \mathrm{C}$ with $10 \mathrm{mM}$ Tris-HCl $(\mathrm{pH} 7.4), 150 \mathrm{mM}$ EDTA, $5 \mathrm{mM} \mathrm{MgCl}_{2}$, containing protease inhibitors and 100 
Hg/ml DNase I, and centrifuged at $4^{\circ} \mathrm{C}$ for $45 \mathrm{~mm}$ at $30000 \mathrm{~g}$. The supername was discarded, and the resuling pellet was resuspended in $10 \mathrm{mM}$ Tris-HCl (pH 7.4), $150 \mathrm{mM} \mathrm{NaCl}, 1 \mathrm{mM}$ EDTA, and protesse inhibitors. The resulting suspension was stored at $-80^{\circ} \mathrm{C}$ (crude cyoskeletal fracion).

Sodium dodecyl sulfate-polyacrylanide gel electrophoresis (SDS-PACE) was performed with $10 \%$ acrylamide slab gels. Proteins were transferred to polyvinylidene difluoride membranes (Miltipore) in is senidry botting apparatus and immunostaining was performed as described eartier [18!

GFAP protein, monoclonal antibodies to GFAP (clone G-A-5), and peroxidase-conjugated Fab fragments to mouse IgGs were obtained from Boebringer. Imnunoplates were purchased from Nunc. For the ELISA anti-GFAP antibodies were diluted 1:200 and the secondary, peroxidase-coupled antibodies were used at a dilution of 1:1000. For determination of the GFAP content of each protein fraction, the $O \mathrm{D}_{40}$ s values obtained from the single determinations (protein coating concentration $25 \mu \mathrm{g} / \mathrm{ml}$ per well) were related to a GFAP standard curve. GFAP standard curves were fitted by a four-parameter equation and GEAP values were calculated from this fit (Kineti Calc, Bio-Tek Instruments).

Statistical analysis: The soluble GFAP fraction and Triton X-100 GFAP fraction of one STREP animal were lost. Treatment effects on ChAT aenivity and GFAP content were analyzed using a $t$-test, Correlations between the different parameters were analyzed using Pearson's correlation coefficient $\left(r_{\mathrm{p}}\right)$. For behavioral parameters we calculated the general mean (i.e. height of curve) and the linear trend component (i.e. slope of curve) of the forgetting (DNMTP task) and learning (Morris water task) curves. The linear trend of the behavional data was used for correlation analysis since it was the most pronounced trend component, i.e. it explained more than $50 \%$ of the variation of the behavioral data of both the control group and the STREP-treated group.

\section{STREP non-impaired and STREP impairea}

Oniy the data of the second week of postoperative testing was used for further analysis, since the behavior of the rats in the inst week of postoperative testing was likely to be influenced by a non-specific effect of the surgery (see section Resulis).

As was evident from the large variability of the DNMTP data, the STREP group was particularly heterogeneous, which is in accordance with our previous behavioral findings that the susceptibijity to STREP treatment was not the same for all rats $[3,4,51]$. Therefore, we divided the STREP group into at subgroup that showed no effect of STREP treatment, chosen as no change in mean percentage correct in the postoperative session of the second week (STREP non-impaired, $n=6$ ), and a subgroup whose perfornanee was impaired after STREP treatment (STREP impaired, $n=6$ ).

Although all STREP-impaired rats had a worse mean postoperative performance when compared with the ir own preoperative performance, one STREP impaired rat had a postoperative performance of which the absollute value was higher than that of one rat of the STREP non-irnpaired group and some control rats. There was weak statistical support that this could be due to the already high preoperative perfornance of the STREP rats when compared with the control rats (Treatment effect: $F_{1,17}=4.10,0.05<p<0.10$ ). This marginat difference in preoperative performance was due to the dying of some rats after they had been assigned to a treatment group, while initially the groups of rats had been matched for their preoperative performance. Therefore, for correlation analysis we calculated the difference between the mean preoperative and postoperative performance, i.e. difference in general mean percentage correct $=$ (general mean percentage contect postoperative - general mean pefcentage correct preoperative). Since there was no indication for a difference in the linear tremd component of the preoperative performance in the DNMTP task (Treatment $\times$ Delay effect: $F_{5.85}=1.19$, n.s.), whe postoperative linear trend component was used for correlation analysis. Likewise, the postoperative lndex $Y$ was taken since there was no preoperailve difference between the control and STREP grots $\left(t_{17}=0.47\right.$, n. . s.). The subdivision of the STREP group, which was based on the DNMTP performance, was also used for the Morris water escape task. However, this does not exclude the possibility that the performance in the latter tastit of STREP impaired and non-impaired rats overlaps. 


\section{RESULTS}

\section{Delayed non-matching to position lask}

During the complete study there were no differences in body weight and water consumption between control and STREP-treated rats (data not shown). The behavioral data of the rats in the first week of postoperative testing was likely to be influenced by a non-specific effect of the surgery as was indicated by the Surgery effect on the percentage correct responses $\left(F_{1.17}=4.90, p\right.$ $<0.05)$ and latency $\left(F_{1,17}=13.55_{*} p<0.10\right)$, but without a Treatment or Surgery $\times$ Treatment interaction effect (all $F_{S}<4.06, n . s$.). Therefore, only the data of the second week of postoperative testing were used for further analysis.

Figure 1 shows the pre- and postoperative performance of the CON and STREP groups at each delay interval. During postoperative testing the CON group showed a rate of forgetting over the delay intervals that was not different from that in the week prior to surgery (see Fig. $\mathbb{A}$ ). The performance of the STREP group during postoperative testing was impaired compared with its preoperative testing (Surgery effect: $F_{1,11}=7.32, p<0.05$; see Fig. 1B). This impairment was nonspecific, i.e. the performance at all delay intervals was disrupted (Surgery $\times$ Delay effect: $F_{5,55}$ $=1.94$, n.s.). Likewise, when comparing the performance of the STREP group with the CON group, the STREP group showed an impaired performance (Treatment $\times$ Surgery effect: $F_{1,17}=$ $5.62, p<0.05$ ), which was not specific delay dependent (Treatment $\times$ Surgery $\times$ Delay effect: $F_{5,85}$ $=0.79, \mathrm{nis} . \mathrm{s}$.

It was found that the STREP impaired group showed a specific delay dependent impairment, i.e. the impairment was progressively greater as the delay interval increased (Surgery $\times$ Delay effect: $F_{5,25}=4.53, p<0.01$; see Fig. ID). A comparison per delay showed that the postoperative performance of the STREP impuired group was only different from its preoperative performance at all delays greater than $4 \mathrm{~s}$ (all is $>3.44 ; p<0.05$ ). In addition, the performance of the STREP impaired group was specifically impaired when compared to the STREP non-impaired group (Treatment $\times$ Surgery $\times$ Delay effect: $F_{5.50}=3.14, p<0.05$ ). When comparing the postoperative performance per delay it was found that the STREP impaired group only differed from the STREP non-impaired group on the delays greater than $4 \mathrm{~s}$ (all $t \mathrm{~s}>2.68 ; p<0.05$ ).

The response bias (Index $Y$ ) of the STREP group was not different from that of the control group (Treatment $\times$ surgery effect: $F_{1,17}=0.78$, n.s.; see Fig. 1E). Although inspection of Figure IE suggested that the response bias of the STEP impaired group was affected compared to the STREP non-impaired group, there whs only weak statistical evidence for this (Treatment effect: $F_{1.10}=4.14,0.05<p<0.10$ ). The latency to press the choice lever was not affected in any of the four experimental groups (Treatment $\times$ Surgery effect: $F_{1,17}=0.17$, n.s.), and had an average value of $\mathrm{I} .08 \pm 0.04 \mathrm{~s}$ (mean \pm S.E.M.).

\section{Morris water escape lask}

The measures of the acquisition of the water escape task and the performances in the probe trial me shown in Figure 2. The overall session escape latency of the STREP group was worse than that of the control group (general mean Treatment: $F_{1,17}=5.88, p<0.05$; see Fig. $2 \mathrm{~A}$ ). Both control and STREP-treated rats reduced their escape latencies during acquisition of the spatial discrimination task (Session effect: $F_{6,102}=48.81, p<0.01$ ), but the STREP group had a slower rate of improvement over sessions when compared to the control group (Treatment $\times$ Session effect: $F_{6,102}=2.25, p<0.05$ ). There was no difference in overall escape latency and rate of improvement over sessions between the STREP non-impaired and STREP impaired group (both $\left.F_{s}<2.69, n, s.\right)$. The distance swum to the platform revealed similar results as the escape latency. 

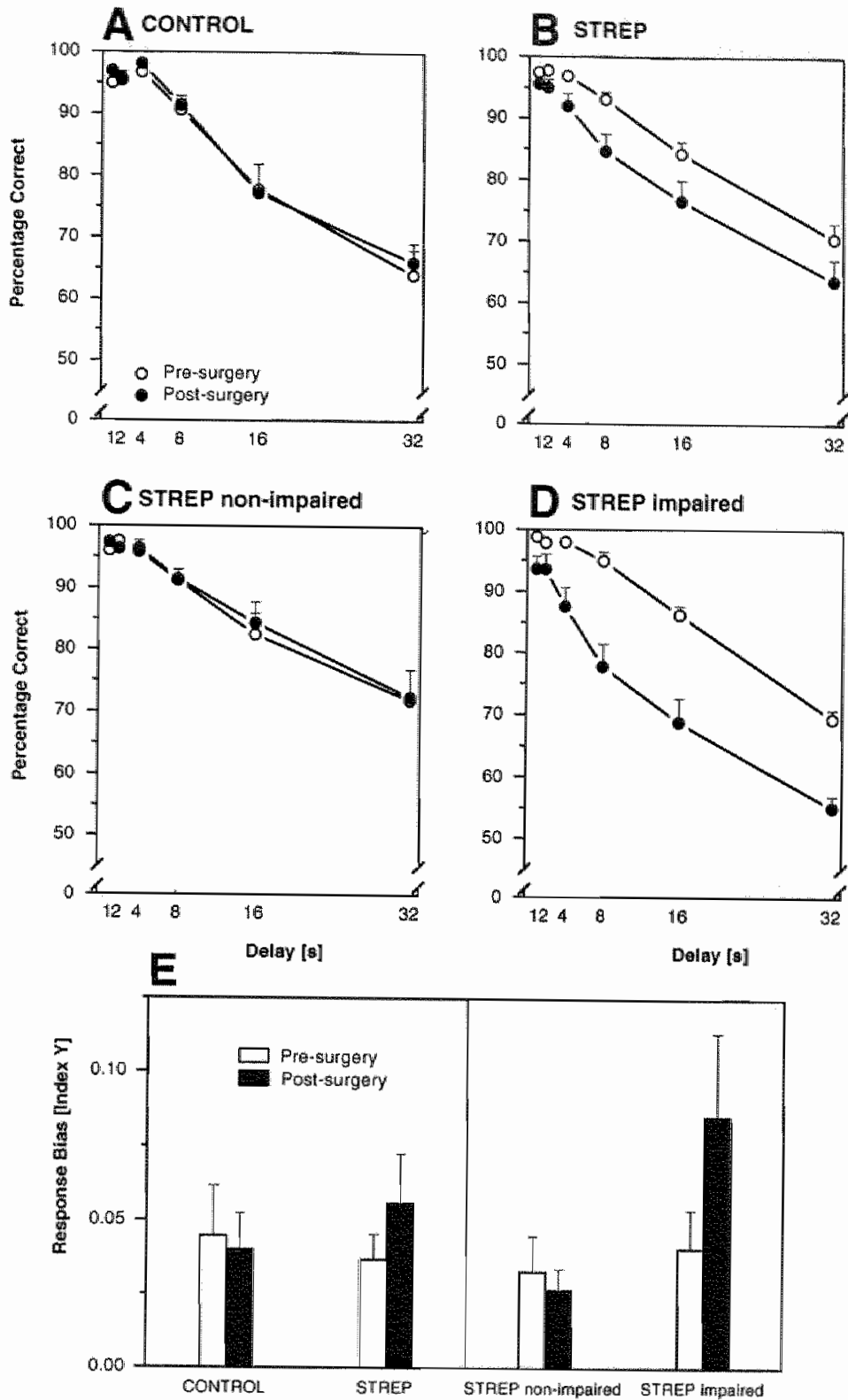

FIG. 1. Performance of 14-month-old control and streptozotocin-treated rats in a delayed non-matching to position task. The twelve animals in the streptozotocin-treated group were subdivided into two subgroups of 6 animals each on the basis of whether they sthowed no deficit at any delay interval or a decline in performance during postoperative testing. Percentage correct at each delay (1-32 s) for the control (A), streptozotocin-treated (B), streptozotocin non-impaired (C), and streptozotocin impaired (D) group. Response bias (Index $Y$ ) for each experimental group (E). Values are means ( $+S . E M$. ) of 5 days of testing during baseline training in the week prior to surgery (pre-surgery) and during postoperative testing (post-surgery). 

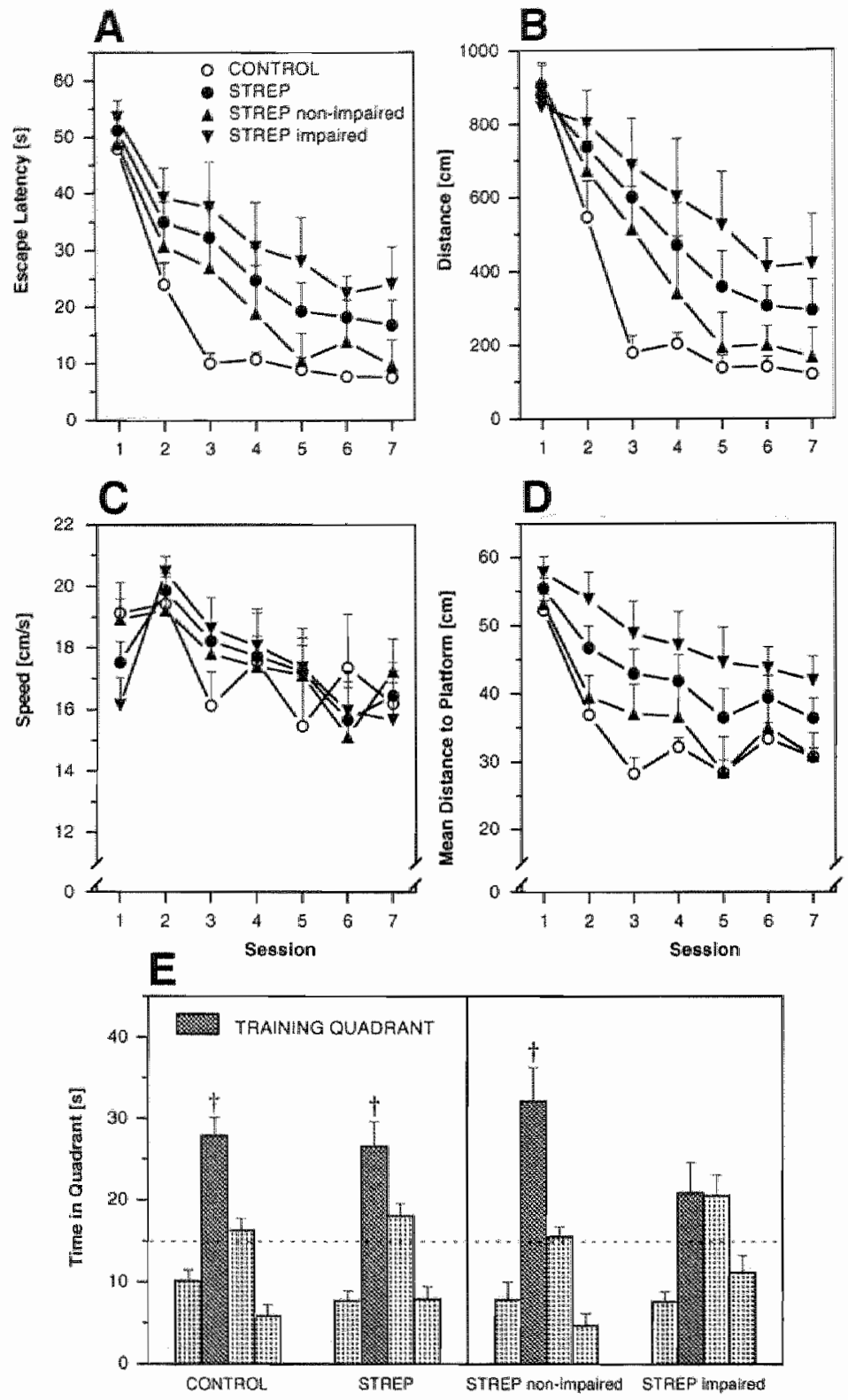

FIG. 2. Performance of 14 -month-old control and streptozotocin-treated rats in the Mornis water escape task. Streptozotocin-treated rats were subdivided into a non-impaired and inpaired group, respectively , based on their performance in the delayed non-matching to position task. Escape latency (A), distance swum (B), swimming speed (C), and average distance to platform (D) for each session of each experimental group during spatial training. Times spent in the four quadrants in the probe trial after spatial training (E). The quadrants are organized with respect to the trahing quadrant, i.e. Ieft-adjacent, rightwadjacent and opposite. Values are means (+S.E.M.). $t p<0.01$ for time spent in quadrant above chance level $(15 \mathrm{~s})$. 
On average, the STREP group swum the longest distance before finding the platform during the acquisition (general mean Treatment: $F_{11,1}=6.11, p<0.05$; sew Fig. 2 B). Both the control and STREP group reduced their distance swum during the acquisition (Session affect: $F_{6,102}=38.68, p$ $<0.011$, but the STREP group had a slower rate of improvement (Treatment $\times$ Session effect: $\left.F_{6.102}=2.61, p<0.05\right)$. Again, no differences were found between the STREP non-impaired and STREP impaired group (both $F_{\mathrm{S}}<2.73$, n.s.). During acquisition, there was a decrease in swimming speed (Session effect: $F_{6.102}=5.57, p<0.01$; see Fig. $2 \mathrm{C}$ ), but there were no differences between the control and STREP group (both $F S<1.95$, n.s), and likewise, no differences between the STREP non-impaired and STREP impaired group either (both $F \mathrm{~S}<1.35$, n.s.). The mean distance to the platform decreased over sessions during acquisition (Session effect: $F_{6.102}=31.69, p<0.01$; see Fig. $2 D$ ). However, there was only a weak statistical evidence for a difference in overall performance and rate of improvement between the control and STREP group (both $F_{S}<4.29,0.05<p<0.10$ ). But for this measure only, there was also weak statistical support for a difference in overall performance between the STREP non-impaired and STREP impaired group (general mean Treatment: $E_{1,10}=4.65,0.05<p<0.10$ ).

In the probe trial, both the control and STREP group showed a quadrant preference (Quadrant effect: both $F S>15.19, p<0.01$ ). In addition, it was found that both groups spent most of their time in the training quadrant (both $t \mathrm{~s}>3.67, p<0.01$; see Fig. 2E). The STREP non-impaired group showed a quadrant preference in the probe trial (Quadrant effect: $F_{3,18}=$ $17.73, p<0.01$ ), as was also found for the STREP impaired group (Quadrant effect: $F_{3,33}=5.14$, $p<0.05$ ). However, only the STREP non-impaired group spent most of its time in the training quadrant $\left(t_{5}=4.15, p<0.01\right)$, whereas the time spent in the training quadrant by the STREP impaired group was not different from chance level $\left(t_{5}=1.61\right.$, n.s.; see Fig. 2E).

\section{ChAT activity and GFAP content}

No differences in weight of the septum, hippocampus and striatum between control and STREP-treated rats were found (data not shown; all is $<1.44$, n.s.). Table 1 summarizes the ChAT activities and GFAP contents in the three brain structures of both experimental groups. Only hippocampal ChAT activity was decreased in STREP-treated rats as compared with control rats ( $f_{17}$ $=2.13, p<0.05$ ). In both the septum and hippocampus of STREP-treated rats mainly the soluble and Triton $\mathrm{X}-100$ fraction showed an increase in GFAP content (all $t \mathrm{~s}>3.21, p<0.01$ ), whereas in the striatum mainly the GFAP content of the Triton X-100 and cytoskeletal fraction were. increased (both $t s>4.1, p<0.01$ ). Correlation analysis revealed that only in the hippocampus of STREP-treated rats, the shree different GFAP protein fractions were related with each other (Table 2). Furthermore, in STREP-treated rats the increased hippocampal GFAP content of all three protein fractions correlated with the decreased ChAT activity (Table 2). In both cases the correlations were highest for the soluble and cytoskeletal fraction. This attaches more importance to the solluble and cytoskeletal fraction as compared with the Triton $\mathrm{X}$-100 fraction, which may be viewed more or less as an intermediate fraction between the former two.

Western blotting was performed with the anti-GFAP monodonal antibody to verify its specificity (Fig. 3). Because the Triton X-100 soluble protein fraction is intermediate between the soluble and cytoskeletal protein fraction, only the latter two fractions were assayed by immunoblotting. In both fractions, the anti-GFAP antibody reacted with one protein species migrating with a relative molecular mass of about $53 \mathrm{kDa}$ which is equivalent to that of native GFAP monomers. No major degradation products were observed in any protein fraction.

The soluble protein fraction of the control hippocampus had the highest GFAP immunoreactivity compared with that of the striatum and septam. This is consistent with the data 
TABE 1. Choline acelyltransferase (ChAT) activity (nmol/mg protein/h) and glial fibrillary axidic protein (GFAP) conteni of soluble. Triton X-100 soluble and cytoskeletal protein fractions prepared from the septum, hippociampus and striatum from 14-month-old control and streptozotocin-treated. (STREP) Wistar rats. For GFA.P measurements wells were coated at a protein concentration of $25 \mu \mathrm{g} / \mathrm{ml}$ and the resulting $O D_{405}$ was converted lo ngíml by using a GFA gtandard curve.

\begin{tabular}{lccl}
\hline & CONTROL & \multicolumn{2}{c}{ STREP } \\
\hline Septum & $71.3 \pm 7.4$ & $64.4 \pm 3.5$ & $(-10 \%)$ \\
ChAT & $10.6 \pm 1.0$ & $19.5 \pm 1.4 \dagger$ & $(+83 \%)$ \\
GFAP Soluble & $14.7 \pm 5.1$ & $42.5 \pm 6.4 \dagger$ & $(+18 \%)$ \\
GFAP Triton X-100 soluble & $12.8 \pm 2.3$ & $15.9 \pm 1.1$ & $(+25 \%)$ \\
GFAP Cyloskeletal & & & \\
Hippocaimpus & $76.0 \pm 1.7$ & $58.7 \pm 6.1^{*}$ & $(-23 \%)$ \\
ChAT & $40.8 \pm 2.1$ & $65.4 \pm 5.4 \dagger$ & $(+60 \%)$ \\
GFAP Soluble & $38.0 \pm 2.0$ & $62.1 \pm 5.3 \dagger$ & $(+63 \%)$ \\
GFAP Triton X-100 soluble & $34.9 \pm 7.7$ & $43.0 \pm 2.3 *$ & $(+23 \%)$ \\
GFAP Cytoskeletal & & & \\
Striatum & $153.7 \pm 4.1$ & $149.7 \pm 4.4$ & $(-3 \%)$ \\
ChAT & $16.8 \pm 0.8$ & $20.8 \pm 1.0 *$ & $(+24 \%)$ \\
GFAP Soluble & $3.5 .6 \pm 2.3$ & $56.0 \pm 3.5 \dagger$ & $(+57 \%)$ \\
GFAP Triton X-100 soluble & $14.9 \pm 0.8$ & $21.9 \pm 1.1 \dagger$ & $(+47 \%)$ \\
GFAP Cyloskeletal &
\end{tabular}

Vallues are shown as means \pm S.E.M. (\% change of control). $* p<0.05 ; \dagger p<0.01$ ( $t$-test).

TABLE 2. Correlation coefficients $\left(r_{p}\right)$ for glial fibrillary acidic protein (GFAP) contents of soluble (Sol), Triton X-100 (TX) and cyloskeletal (Cyt) protein fractions and choline acetyltransferase (ChAT) activities prepared from the septum, hippocanpus and striatum from 14 -month old control and streptozotocin-treated (STREP) Wistar rats.

\begin{tabular}{|c|c|c|c|c|c|c|c|c|}
\hline & \multicolumn{4}{|c|}{ CONTROL } & \multicolumn{4}{|c|}{ STREP } \\
\hline & \multicolumn{3}{|c|}{$\mathrm{GFAP}$} & \multirow[t]{2}{*}{ ChAT } & \multicolumn{3}{|c|}{ GFAP } & \multirow[t]{2}{*}{ ChAT } \\
\hline & $\overline{\mathrm{Sol}}$ & $\mathrm{TX}$ & $\overline{\mathrm{Cyt}}$ & & Sol & $\mathrm{TX}$ & $\overline{C y}$ & \\
\hline \multicolumn{9}{|l|}{ Septum } \\
\hline GPAP Sol & - & . & . & 0.05 & . & - & - & 0.41 \\
\hline GEAP TX & -0.67 & . & - & -0.18 & -0.20 & - & $=$ & 0.01 \\
\hline GPAP Cyt & 0.28 & -0.23 & - & -0.55 & 0.14 & 0.48 & . & -0.17 \\
\hline \multicolumn{9}{|l|}{ Hippocampus } \\
\hline GFAP SOL & - & - & - & -0.50 & . & - & - & $.0 .84 \dagger$ \\
\hline GFAP TX & $-0.2 \rrbracket$ & . & - & -0.29 & $0.61 *$ & - & - & $-0.7 \mathbb{1} *$ \\
\hline GFAP Cyt & 0.73 & -0.60 & . & -0.14 & $0.85 \dagger$ & $0.63^{*}$ & $\propto$ & $-0.83+$ \\
\hline \multicolumn{9}{|l|}{ Siriatum } \\
\hline GFAP SOI & . & - & - & 0.31 & - & - & - & 0.45 \\
\hline GFAP TX & .0 .24 & - & * & 0.38 & 0.46 & - & - & 0.26 \\
\hline GFAP Cyt & .0 .26 & 0.42 & - & .0 .18 & 0.52 & 0.43 & - & .0 .34 \\
\hline
\end{tabular}

* $p<0.05 ; p<0.01$

obtained from the ELISA showing the highest amount of soluble GFAP in the hippocampus of control rats. Funthermore, the immunoreactivity of the soluble fraction was increased in all three brain structures of the STREP-treated rat, just as was also found in the ELISA. The 


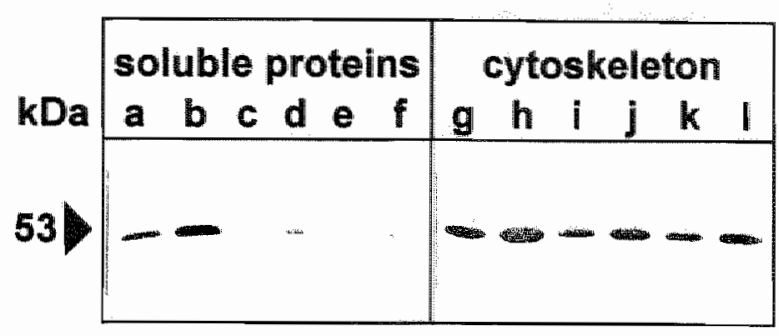

FIG. 3. Westem blot detection of GFAP in soluble (a-1) and cytoskeletal ( $g$-l) protein fractions prepared trom hippocampal $(a, b, g, h)$, striatal $(c, d, i, j)$, and septal $(e, f, k, l)$ tissue homogenates of control $(a, c, e, g, i, k)$ and streptozotocin-treated $(b, \mathrm{~d}, \mathrm{f}, \mathrm{h}, \mathrm{j}, \mathrm{i})$ rats. For soluble fractions $10 \mu \mathrm{g}$ and for cytoskeletal fractions $5 \mu \mathrm{g}$ of total protein were applied per lane. The molecular mass is indicated at the left margin ( $k D a)$.

immunoreactivity of the control cytoskeletal fraction was highest in the hippocampus and was increased in all three brain structures of the STREP-treated rats. These findings are also in agreement with the dlata from the EJISA, although the latter showed only a weak statistical increase in septal cytoskeletal GFAP $\left(t_{17}=1.98,0.05<p<0.10\right)$.

The GFAP content of the soluble and cytoskeletal fractions as determined by ELISA were approximately equal in all three brain structures of the controll rats. However, the immunoblot staining intensity of the cytoskeletal GFAP fractions was much more prominent than that of the soluble fractions. This indicated that the GFAP content of the cytoskeletal fractions as dletermined by ELISA appeared to be underestimated with respect to the soluble fraction, which is in accordance with prewious reported findings [19].

\section{Biochemical and behavioral correlations}

The results of the correlation analysis of the relationship between the DNMTP measures and both the ChAT activity and GFAP content are summarized in Table 3 and 4, respectively. No correlations were found between the behavional and biochemical measures of the control rats. Hippocampal ChAT activity correlated positively with both the difference in general mean (postoperative minus preoperative) and the linear trend component of the forgeting curve of STREP-treated rats (Table 3). An impaired performance in general has a negative value for the difference in general mean percentage correct responses. A linear trend component represents the slope of the curve, or in this case the rate of forgetting, and has always a negative value. Hence, the positive correlations indicate that a lower hippocampal ChAT activity predicts less correct responses in general as well as a higher rate of forgetting over the delays. Hippocampal ChAT actiwity of the STREP group was also found to have a negative relationship with the response bias as formulated by Index $\mathrm{Y}$. Furthermore, the ChAT activity in the striatum had a positive correlation with the linear trend component of the STREP group (Table 3).

The GFAP content of all three hippocampal protein fractions of STREP-treated rats correlated negatively with the difference in mean correct responses over all delays (Table 4), whereas their correlations with the response bias were positive. The GFAP content of the cytoskeletal protein fraction of the striatum of STREP-treated rats also had a negative correlation with the difference in general mean correct responses and a positive correlation with the response bias. For the 
TABLE 3 . Cormelation coefficients $\left(r_{p}\right)$ for measures of the de ayed non-matching to position task and choline acetyliansferase (ChAT) activities of the septum, hippocampus and striatum from 14 month-old control and streptozotocin-treated (STREP) Wistar rats during post-operative testimg.

\begin{tabular}{|c|c|c|c|c|c|c|}
\hline & \multicolumn{6}{|c|}{ ChAT } \\
\hline & \multicolumn{3}{|c|}{ CONTROL } & \multicolumn{3}{|c|}{ STREP } \\
\hline & Sept & $\mathrm{Hpp}$ & Striatum & Sept. & Hipp. & Striatum \\
\hline \multicolumn{7}{|l|}{ \% correct: } \\
\hline diff. generat mean & 0.17 & -0.09 & 0.27 & -0.57 & 0.85 & 0.09 \\
\hline linear trend & -0.06 & 0.39 & 0.43 & -0.12 & $0.81 \div$ & $0.61 *$ \\
\hline Index $\mathrm{Y}$ & -0.18 & 0.39 & -0.09 & 0.09 & $-0.74 t$ & -0.30 \\
\hline
\end{tabular}

$p<0.05 ; 1 p<0.01$

TABLE 4. Cortelation coefficients $\left(r_{p}\right)$ for measures of the delayed non-matching to position task and glial fibrillary acidic protein (GFAP) contents of soluble (Sol), Triton X-100 (TX) and cytoskeletal (Cyt) protein fractions prepared from different brain regions from 14 -month old control (A) and streptozotocin-treated (STREP; B) Wistar rats during posit-operative testing.

GFAP

\begin{tabular}{|c|c|c|c|c|c|c|c|c|}
\hline \multicolumn{3}{|c|}{ Septum } & \multicolumn{3}{|c|}{ Hippocampus } & \multicolumn{3}{|c|}{ Striatum } \\
\hline $\mathrm{Sol}$ & $\mathrm{TX}$ & $\overline{C y t}$ & Sol & $\overline{T X}$ & $\overline{\text { Cyt }}$ & $\overline{\mathrm{Sol}}$ & $\overline{T X}$ & $\overline{\text { Cylt }}$ \\
\hline
\end{tabular}

A. CONTROL

o conrect:

$\begin{array}{lrrrrrrrrr}\text { diff. general mean } & -0.41 & 0.67 & -0.49 & -0.19 & -0.24 & -0.37 & -0.20 & -0.45 & -0.04 \\ \text { linear trend } & 0.54 & -0.03 & 0.35 & -0.09 & 0.43 & -0.12 & -0.31 & 0.61 & -0.37 \\ \text { lex } \mathrm{Y} & -0.10 & -0.28 & 0.73 & -0.16 & -0.25 & 0.06 & -0.04 & 0.49 & 0.68\end{array}$

B. STREP

o correct"

$\begin{array}{lllllllll}\text { diff. general meam } \quad-0.16 & -0.16 & 0.40 & -0.89+ & -0.81 \div & -0.86 \% & -0.47 & -0.36 & -0.747\end{array}$

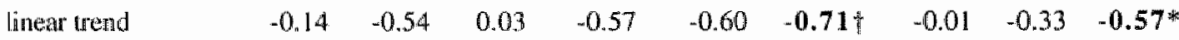

$\begin{array}{llllllllll}\text { Index } \mathrm{Y} & 0.21 & 0.22 & -0.32 & 0.75+ & 0.68 * & 0.64 * & 0.12 & 0.36 & 0.65 *\end{array}$

$p<0.05 ; t p<0.01$

cytoskeletall GFAP fraction of both the hippocampus and striatum of STREP-treated rats, a negative correlation with the linear trend component was found.

The results of the correlation analysis of the relationship between the measures of the Morris water task and both the ChAT activity and GFAP content are summarized in Table 5 and 6 , respectively. No correlations were found between the behavioral and biochemical measures of the control rats, although sometimes rather high correlation coefficients were found. Correlation coefficients of STREP-treated rats reached statistical significance already at lower values, since a higher number of subjects was involved. Hippocampal ChAT actiwity of STREP-treated rats correlated negatively with both the general mean and the linear trend component of the measures escape latency and distance swum (Table 5). This indicates that a lower ChAT activity predicts higher escape latencies and swimming distances (i.e. a worse cognitive performance) in general, and a slower rate of improvement during training. Hippocampal CHAT activity also correlated negatively with the general mean of the mean distance to platform of STREP-treated rats. Furthermore, the ChAT activity in the striatum had a negative correlation with the linear trend of the measure escape latency (Table 5). 
TABLE 5. Correlation coefficients ( $r_{p}$ ) for measures of the Morris water tass and choline acetyltransferase (ChAT) activities of the septem, hippocampus and striatum from $\| 4$-month-old control and streptozotocin-treated (STREP) Wistar rats.

\begin{tabular}{|c|c|c|c|c|c|c|}
\hline & \multicolumn{6}{|c|}{ ClnAT } \\
\hline & \multicolumn{3}{|c|}{ CONTROL } & \multicolumn{3}{|c|}{ STREP } \\
\hline & Sept. & Hipp. & Striatum & Sept. & Hipp. & $\overline{\text { Striatum }}$ \\
\hline \multicolumn{7}{|l|}{ Escape latency: } \\
\hline general mean & .0 .24 & -0.49 & -0.75 & 0.21 & -0.721 & -0.09 \\
\hline linear trend & .0 .02 & 0.16 & 0.22 & -0.38 & $-0.59 *$ & $0.59 *$ \\
\hline \multicolumn{7}{|l|}{ Distance swum: } \\
\hline general mean & -0.05 & -0.17 & -0.19 & 0.22 & $-0.72 \dagger$ & -0.02 \\
\hline linear trend & 0.00 & -0.07 & -0.06 & -0.20 & $-0.79 \dagger$ & -0.52 \\
\hline \multicolumn{7}{|c|}{ Mean distance to platform: } \\
\hline general mear & -0.26 & -0.03 & -0.23 & 0.26 & $-0.74 \dagger$ & .0 .22 \\
\hline linear trend & -0.62 & -0.14 & .0 .22 & -0.32 & .0 .48 & -0.46 \\
\hline
\end{tabular}

TABLE 6. Correlation coefficients $\left(r_{\mathrm{p}}\right)$ for measures of the Morris water task and glial librillary acidic protein (GFAP) contents of soluble (Sol), Triton $\mathrm{X}-100$ (TX) and cytoskeletal (Cyt) protein fractions prepared from different brain regions from 17-month-old control (A) and streptozotocin-treated (STREP; B) Wistar rats.

GFAP

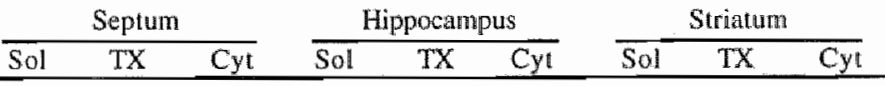

A. CONTROL

Escape latency:

$\begin{array}{lrrrrrrrrr}\text { general mean } & 0.09 & -0.62 & 0.33 & 0.31 & -0.01 & 0.29 & 0.17 & -0.21 & 0.36 \\ \text { linear trend } & 0.18 & 0.58 & -0.15 & -0.20 & 0.02 & 0.00 & -0.57 & 0.03 & -0.44\end{array}$

Distance swum:

$\begin{array}{lrrrrrrrrr}\text { general mean } & 0.43 & -0.74 & 0.40 & -0.26 & 0.47 & -0.47 & 0.26 & -0.08 & 0.02 \\ \text { linear trend } & 0.07 & 0.50 & -0.28 & 0.14 & -0.09 & 0.36 & -0.44 & -0.08 & -0.38 \\ \text { stance to platform: } & & & & & & & & \\ \text { general meart } & -0.35 & -0.36 & 0.35 & 0.51 & -0.28 & 0.36 & 0.32 & 0.29 & 0.70 \\ \text { linear trend } & -0.46 & 0.75 & -0.14 & 0.48 & -0.47 & 0.36 & -0.54 & -0.01 & 0.26\end{array}$

\section{B. STREP}

Mean distance to platform:

Escape latency:

$\begin{array}{lrrrlllllll}\text { general mean } & 0.37 & -0.17 & -0.04 & \mathbf{0 . 7 3} * & 0.40 & 0.57 & 0.10 & 0.37 & 0.53 \\ \text { linear trend } & -0.35 & 0.38 & 0.05 & 0.20 & 0.18 & 0.39 & -0.57 & -0.10 & 0.12 \\ \text { general mean } & 0.43 & -0.20 & -0.07 & 0.79 & 0.43 & \mathbf{0 . 6 1 *} & 0.18 & 0.37 & 0.55 \\ \text { linear trend } & -0.27 & 0.33 & -0.08 & 0.45 & 0.34 & 0.54 & -0.37 & 0.05 & 0.34\end{array}$

Mean distance to platform:

\begin{tabular}{lccccccccc} 
general mean & 0.31 & -0.19 & -0.17 & $\mathbf{0 . 7 5}$ & 0.44 & $\mathbf{0 . 7 0}$ & 0.12 & 0.27 & $\mathbf{0 . 6 5}$ \\
linear trend & 0.10 & -0.12 & 0.10 & 0.26 & 0.00 & 0.32 & 0.53 & -0.06 & 0.12 \\
\hline
\end{tabular}

$*_{p}<0.05 ; \dagger p<0.01$ 
The GFAP content of the soluble fraction of the hippocampus of STREP-treated rais correlated positively with the general mean of the three Morris task measures (Table 6). In addition, the GFAP content of the hippocampal cytoskelletal fraction of STREP-treated rats correlated positively with the general mean of the measures distance swum and mean distance to platform. Finally, the cytoskeletal GFAP fraction of the striatum had a positive correlation with the general mean of the measure mean distance to platform.

The relationship between the biochemical markers and the cognitive performances is depicted in Fig. 4 in order to illustrate the correlation between the measures. For the DNMTP task the difference between the postoperative and preoperative performance in mean percentage correct
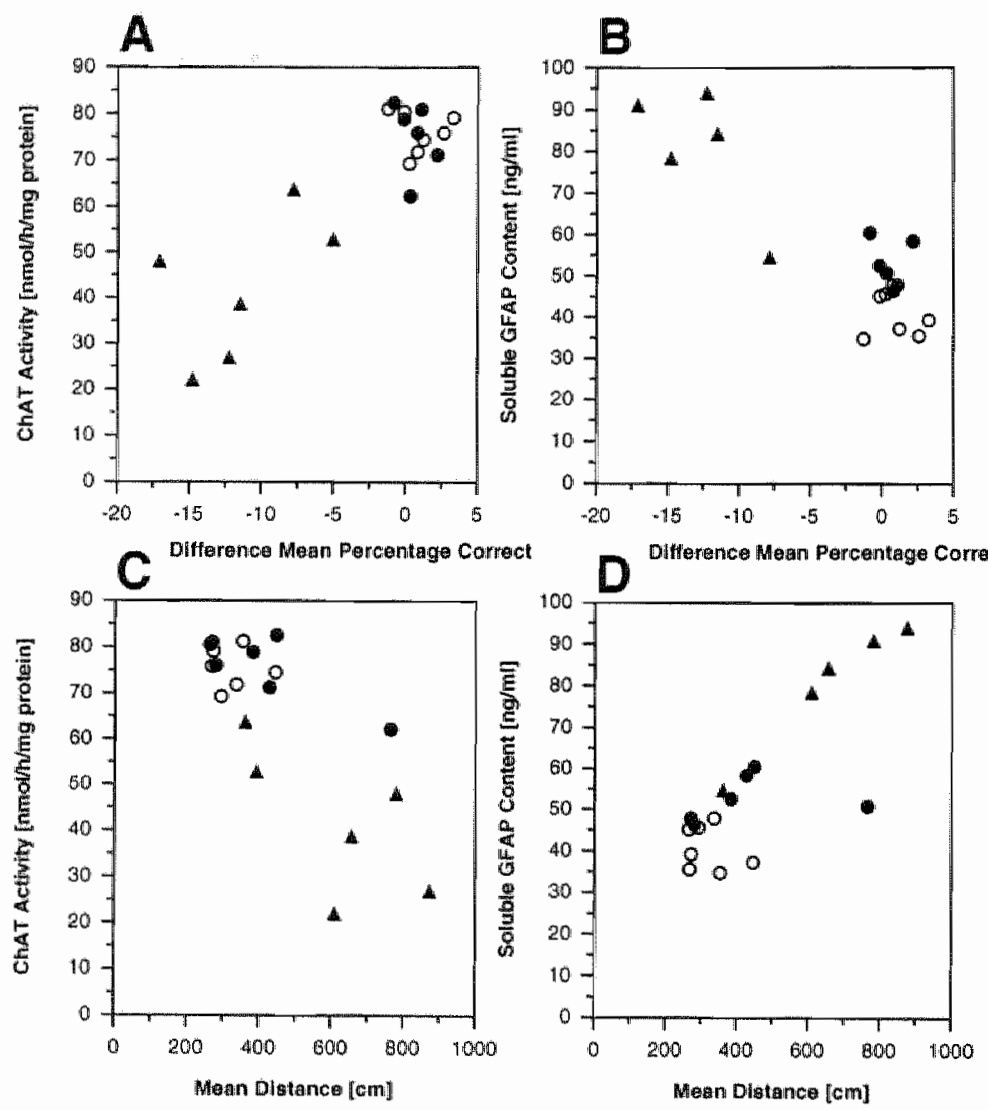

FIG. 4. Hippocampal choline acetyltransferase (ChAT) activity versus the difference in nean percentage correct responses between postoperative and preoperative performance in the delayed non-matching to position task (A), and the moan distance swum in the Morris water escape task (C) of 14-month-old control rats (open circles) and streptozotocin-treated rats. The streptozotocin-treated rats were further subdivided into non-impaired (closed circles) and inmpaired (triangles) rats based on the delayed non-matching performance. Hippocampal glial fibrillary acidic protein (GFAP) content versus the mean percentage correct in the delayed non-matching to position task (B), and the mean distance swum in the Morris water escape task (D). 
responses was taken (Fig. 4A and B). From the measures of the Morris water escape lask the mean distance swum during training was taken as example (Fig. 4C and D). The soluble GFAP protein fraction was taken as example from the GFAP fractions (Fig. $4 \mathrm{~B}$ and D).

\section{DISCUSSION}

\section{Behavioral and biochemical correlations}

I.C.v. injection of STREP affected cognitive performance in the DNMTP task and Morris water escape task. It was found that there was a large behavioral wariability in the individual susceptibility to STREP. The GFAP response to STREP of different pools of GFAP, i.e. protein fractions, was qualitatively different between septum, hippocampus and striatum. Only in the hippocampus of STREP-treated rats a decrease in ChAT activity was found. Correlation analysis of the behavioral and biochemical data revealed that the ChAT activity in the hippocampus of STREP-treated rats correlated with the cognitive performance both the general mean performance and its linear trend component) in both tasks. For the spatial Morris water escape task, in which the cognitive performance is highly dependent on the integrity of the hippocampus

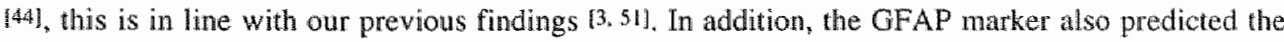
cognitive performance. Especially the hippocampal GFAP content of the soluble protein fraction, but also of the cytoskeletal fraction, was related to the cognitive performance (but only the general mean) in both tasks.

Lesions of the hippocampal system (itself or of the fimbria-fornix) of rats have been found to result in delay-dependent impairments in the DNMTP task [1. 161. The STREP-treated rats as one group had a delay-independent impairment, but a subdivision was possible which revealed in more detail that our STREP-treated rats were either not affected by STREP or showed a delaydependent impairment. The latter indicates that when memory processes were influenced, they were affected specifically. This may be related to hippocampal damage since, as already mentioned, the GFAP content of all hippocampal protein fractions was correlated with the cognitive imparment in the DNMTP task. In addition, this cognitive impairment was, as already mentioned, also related to a decrease in hippocampal ChAT activity. However, there are several findings that argue against a specific cholinergic involvement in this cognitive impairment. This can already be assumed because cognitive performances in the tasks we used, but also in general, have been suggested to be dependent on an interaction between (hippocampal) cholinergic transmission and other neurotransmitter systems and brain structures $[7,16,17,39]$. But there are also direct indications since we thave found that the response bias could be related to GFA.P content and ChAT activity in the hippocampus. This suggests that, although there was only minor statistical evidence for an effect on response bias, non-cognitive aspects were also influenced by STREP. Secondly, i.c.v. injected STREP has been found to affect also monoaminergic neurotransmission [1]]. Thirdly, in a previous study we have found indications that the STREP-induced cognitive impairment (in the Morris water escape task) appeared to be not only dependent on a decrease of hippocampal ChAT activity [5:].

Interestingly, correlations were found between the GFAP content in the cytoskeletal protein fraction of the striatum and the GFAP contents in all protein fractions of the hippocampus as well as the ChAT activity in the hippocampus of STREP-treated rats (data not shown). This does not necessarily mean that there is also an anatomical or functional relationship between the hippocampus and striatum, but it corroborates the finding that the GFAP content in the striatal cytoskeletal protein fraction correlated with the mean cognitive impairment in both the DNMTP 
task and Morris water escape lask, just as has been found for the hippocampal GFAP protein fractions. Also interestingly was the finding that, although striatal ChAT activity was not affected after i.c.y. STREF injection, its activity correlated with the linear trend component in both tasks. Therefore, it may be suggested that an i,c.v. injection of STREP caused striatal damage which had consequences For cognitive performances. Accordingly, it has been demonstrated in several studies that the striatum is involved in (some aspects of) cognition [e.g. 9. 12. 38. 5\%],

In recent studies the GFAP marker has been correlated with behavioral measures $18,35,48,56,591$. An immunocytochemical study of hippocampal excitotoxic lesions demonstrated a correlation between GFAP immunoreactivity at several hippocampal lesion sites and the cognitive performance deficit in a fixed ratio task 1561. Which GFAP fraction is being measured immunocytochemically is largely unknown, but it might be suggested to be the cytoskeletal fraction, since the other fractions may be lost due to the immunostaining protocol. In a rat model of cerebral ischemia it was found that the increased density of GFAP immunoreactivity in the hippocampus correlated with radial arm maze but not Morris water escape impairment [48]. However, it should be noted that in this ischemic model ChAT activity was unaffected in the hippocampus. In an aging study, in which a soluble protein fraction was used, no correlation was found between the hippocampal GFAP content and a cognitive measure in the Morris water escape task $15 \%$. This is in line with the results of other aging studies providing indications that hippocampall GFAP in a soluble protein fraction [35] as well as ummunocytochemically measured $[\mathbb{1}]$, is probably not related with the cognitiwe performance deficit in the Morris water task. However, there is some contradiction between these studies in whether or not the amount of GFAP, as measured with these two methods, is affected by aging. But at the level of messenger RNA for GFAP, it has been found in the former aging study that there was an increase in its amount in the hippocampus which was correlated with the age-related cognitive impairment. In addition, a correlation was found between striatal GFAP, both soluble GFAP and messenger RNA for GFAP, and the cognitive behavior [59]. Once more this underlines the importance of an intact striatum for cognitive functioning. But even more importantly, it indicates that the GFAP response of brain structures is specifically dependent on the type of stressor (e.g. STREP, ischemia, aging).

\section{GFAP}

The process "reactive astrogliosis" is a stereotypic reaction of astrocytes to virtually any severe neuronal damage and, probably, neuronal cell death caused by physical (e.g. ischemia [19]) and chemical (e.g. excitoxic (34)) insults and by several pathological states associated with for instance aging [45], AD [2]] or multiple sclerosis [53]. The reactive changes are characterized by astrocyte proliferation and/or extensive hypertrophy. Concomitantly, the expression of GFAP increases during the development of reactive astrogliosis. Hence, it can be suggested that GFAP upregulation (and astrogliosis) is a marker of neurodegeneration. The GFAP content in the hippocampus was increased in all three protein fractions, which were also related with each other. However, neither in the septum nor in the striatum there was a correlation between the GFAP contents in the three protein fractions. The soluble and cytoskeletal GFAP protein fraction of the hippocampus appeared to have the most physiological relevance, since they were strongly correlated with each other, with hippocampal ChAT activity, and with behaviorall measures. The Triton X-100 soluble fraction may be viewed as an intermediate fraction between the soluble and cytoskeletal fraction. Immunoblotting showed that in both the soluble and cytoskeletal protein fraction of the three brain structures only GEAP monomers were measured. But more importantly, it was found that brain structures reacted differently to STREP, i.e. there was a difference between the brain structures in the GFAP increase in the separate protein fractions. This might be due to 
differences in distribution of STREP throughout the brain or differences betwed structures in susceptibility to STREP. But the finding is in agreement with previous study of ischemia, in which it was found that the most pronounced increase in GFAP in the correx. was in ins soluble protein fraction [19]. Therefore, when studying the GFAP response to a stressor one has to rathe that different pools of GFAP exist, which are differently tegulated after brain injury. Howewer, we bave no plausible explanation for this finding, besides that it reflects the dyramios of GFA assembly, and it, therefore, urges the need for further studies to resolve the question of the functional role of GFAP upregulation during reactive gliosis Ikf. 491.

\section{STREP model}

Initially, STREP has been and is still being used with systemic administration to experimentally induce diabetes melditus in rats [e.g. 6.433]. It has been assumed that i.c.v. injected STREP would have the same effects in the central nervous system as in the periphery, i.e. inhibition of a brain insulin producing system and/or a decreased insulin receptor function $[46,47]$. Normally, insulin is thought to regulate glucose transport into the cell by stimulating the synthesis of glucose transporters and their translocation to, and activation at the plasma membrane [y2]. The autophosphorylation of the insulin receptor tyrosine kinase may be the initial event in insulin action [31]. Hence, botll an inhibited insulin synthesis/secretion and a decreased insulin receptor signalling in the target cells may impair the transport of glucose, and lead to a decrease in glucose utilization. More recently, if has been suggested that the central effects of i.c.v. injected STREP are mainly mediated via the insulin receptor, i.e. inhibition of its attophosphorylation and thus resulting in noninsulin-dependent diabetes confined to the brain $[13.50]$. However, neurodegenerative effects due to oxidative damage cannot be excluded yet, since a probable mechanism of action of STREP could be that it induces primarily axidative stress. (e.g. generation of $\mathrm{H}_{2} \mathrm{O}_{2}$ ), which would result in for instance DNA damage [ef. 5. 14, 60]. Accordingly, we have recently found indirect evidence that i.c.v. injected STREP might cause oxidative damage in the septum which is likely to contribute to a hippocampal cholinergic deficit [6]].

As just mentioned, we have recently found that i.c.v. injected STREP caused septal damage, as was evident from a reduction in septum weight, which could be used to explain the decrease in hippocampal ChAT activity since the latter was conelated with the reduced sepium weight [61]. In the present study, however, no reduction in septum weight of STREP-treated rats was found. Moreover, the reduction in hippocampal ChAT activity could not be related to possible damage to the septum, since it did not correlate with the increased GFAP content in the septum. On the other hand, direct damage of the hippocampus it self may be related with its cholinergic deficit, since GFAP content and ChAT activity in the hippocampus of STREP-treated rats correlated negatively with each other. Furher evidence against an involwement of septal damage in causing hippocampal deficits is provided by the lack of a correlation between GFAP contents in septum and hippocampus of STREP-treated rat. In addition, the septal GFAP content of STREP-treated rats was not correlated with their cognitive performance, whereas the hippocampal GFAP content was. This discrepancy in effects of i.c.v. injected STREP on the septal-hippocampal system is probably due to differences in the strain of rats used, viz. Lewis (previous study) versus Wistar (this study). Another possibility is the difference in the age of the rats used, viz. 18 (previous study) versus 14 (this study) months, since we showed that young rats are not sensitive to STREP $[3]$.

The present finding that in particular the hippocampus is severely affected, does not exclude one of the mechanisms of action for STREP, i.e. inlibition of energy metabolism versus oxidative stress. But it adds more evidence to the metabolic deficit after STREP treatment, since the 
hippocampal formation is one of the brain structures which have the largest number of insulin receptors on neurons 129.53 and the highesit glucose need $[26]$. Therefore, the hippocampus might be one of the most sensitive brain structures to STREP treatment. In this respect it is noteworthy to mention that the density of insulin receptors in the striatum is lower than in the hippocampus 29. 631. This could explain why the striatum is affected since an increase in GFAP content was found. However, to a lesser extent than the hippocampus, since there was no effect on striatal ChAT activity after an I.C.W. injection of STREP. In addition, it is likely that besides insulin receptors also insulin production may be affected since some neurons in the hippocampal formation may synthesize and secrete insulin $[10]$. However, it is thought that most, if not all brain insulin originates from the periphery [63]. This would reduce the relevance of the putative effect of STREP on the insulin production in the brain.

A characteristic feature of STREP treatment was that not all rats were affected to the same extend. Some rats showed no changes in behavioral measures whereas other rats showred marked effects. This could already be assumed based on our previous findings $[3,4,51]$. This feature may appear to be a disadvantage of the STREP model because group differences are difficult to find since STREP induces a great variability in the data. On the other hand, however, it provides a manner to correlate both the behavioral and biochemical markers which is a powerful tool to study brain-behavior relationships [ef. 51 .

In the present study we made a subdivision of STREP-treated rats into two groups on basis of the performance in the DNMTP task (non-impaired wersus impaired) in order clarify the effect of i.c.v. injected STREP on cognition. We found that this subdivision of the STREP groups into a non-impaired and an impaired group as based on their performance in the DNMTP task also applied to their performance in the Morris water escape task, which was confirmed by correlation andysis (all $r_{p} s<-0.63, p<0.05$ ). This is striking because it has been demonstrated for several experimental groups of rats (e.g. aged or ischemic rats) that the performances in different cognitive tasks did not correlate with each other, even when the stimuli modalities were the same (e.g. spatial information $[2,481)$. This may be due to the small variation in data and the involvement of different non-cognitive factors $[2,23,48]$. Apparently. STREP induces a behavioral deficit that affects the performance in various tasks in a similar manner. This indicates the usefulness of i.c.v. injected STREP as a model suitable for studying individual cognitive decline across several behaviorid domains.

In the brain of $\mathrm{AD}$ patients a decrease in the utilization of glucose has been observed which has been suggested to be related to the cognitive impairments of $A D$ [30]. It has been found that i.c.w. injected STREP decreased glucose metabolism [13, 50] and inhibited energy metabol ism [46, 47] in the cortex and hippocampus of rats. In addition, i.c.v. injected STREP caused cognitive inpaiments $13,4,37,51]$. These similarities between changes in $A D$ and STREP-treated rats led to the suggestion that the STREP model might be an aninal model of the sporadic form of $A D$ 13.13, 46. 47, 501. However, indirect evidence has been found that oxidative stress might be involved [61]. An impared mitochondrial function and thus energy metabolism may be the result of an increased oxidative damage, and alternatively an increased formation of reactive compounds coxidative stress) may be the result of an mitochondrial dysfunction (metabolic deficit) [40. 58, 651. This may apply to $\mathrm{AD}$ and it has already been suggested that amyloid accumulation and neurofibrillary tangles formation are a result of, and contribution to a reduced energy availability and increased oxidative processes [36].

Despite the fact that hippocampal ChAT activity correlated well with cognitive behavior, it has been found that there is more than only a cholinergic deficit in STREP-treated rats [I.1, 5II. Accordingly, it has been found that in $\mathrm{AD}$, apart from a decrease in cholinergic 
neurotransmission, different monoaminergic neurotransmitter systens are changed [0.9. 24. 28]. The other marker of neurodegeneration we used. GFAP, showed an increase in its conten in the septum, hippocampus and striatum. Likewise, in the brain of AD patients the GFAP content has been found to be increased [21]. Reactive astrocytes have been found particularly in the hippocampus of $\mathrm{AD}$ brains, which is the site of intense amyloid plaque formation and neurofibrillary tangles [64]. However, it is still largely unknown whether astrogliosis is induced by the neuropathology of AD (e.g. amyloid plaques and neurofibrillary tangles) or that it has a contribution in causing the neuropathology [21,57]. Interestingly, in relation to the metabolic inhibition of STREP, it has been hypothesized that a compromised brain energy metabolism (assumed to be due to an impaired brain microcirculation) causes neurons to release astroglial mitogens signalling reactive astrocytes to proliferate and become hypertrophic. This astroglial reaction would ostensibly contribute to senile plaques and neurofibrillary tangles formation [62].

In conclusion, in the present study we have found that i.c.v. injection of STREP affected cognitive performance in the DNMTP task and Morris water escape task and these cognitive impairments were related to an increased GFAP content and decreased ChAT activity in the hippocampus. GFAP content appeared to be a sensitive marker for neurodegeneration, but our findings indicated that it is important to take into account which protein fraction has been used for GFAP measurements. We suggest that the GFAP content in a soluble or cytoskeletal protein fraction are the most representative markers. Although the biochemical and behavioral changes after STREP treatment have great similarities with those in $A D$, it has to be noted that the changes after STREP treatment do not fully mimic the biochemical and behavioral features of $A D$ [see $11,42$. 55.611. This limits the usefulness of i.c.v. injected STREP as a model of sporadic AD. Nevertheless, it may provide a relevant model of neurodegenerative changes due to a metabolic insufficiency.

\section{ACKNOWLEDGEMENTS}

The authors would like to thank Dr. Joln Bothmer for his expert assistance with the dissections. Furthermone, we thank Bayer $\mathrm{AG}$ (Cologne, Germany) for the financial support.

\section{REFERENCES}

1. Aggleton J.P., Keith A.B. Rawlins J.N., Hunt P.R. and Suhgal A. (1992) Removal of the hippocanpus and tramsetion of the formix produre comparable deficits on delayed non-matching lo position by ratsis. Behav, Bian Res. 52, 61.71 .

2. Blokland A. (1993) Age-related changes in correlation between behavioral and biochemical parameters in Lewis rats. Behav. Neural Biol.60,52-61.

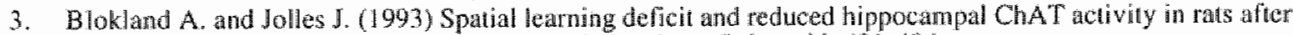
an jev injection of streptozotacin. Pharracol. Biochem. Behov. 44, 49l-494.

4. Blokland $A$. and Jolles J. (1994) Behavioral and biocherntcal effects of an icv injection of streptozotocin in old Lewis tats. Phamarol Bhochem. Behav. 47, 833-837.

5. Bolatf J.L... Nagamatsu S., Harris J. and Grodsky G.M. (1987) Protecton by thymidine, an inhibitor of polyadenosine diphosphata ribosylation, of streptozolocin inilabition of insulin secretion. Endocrinology $120,2117-2122$

6. Burvin R. Armoni M. and Karnieli E. (1994) In vivo insulin action in momal and streptozorocin-indunod diabetic rats. Physhol Belav. 56, I-6.

7. Decker M.W, and McGaugh J L. (1991) The role of interactions betwen the cholnergic system and other neuromodulatory systems in learning and memory. Synapse 7, 151-168.

8. Desjardins S. Mayo W. Vallec M. Hancock D., Le Moall M., Simon H. and Abrous D.N. (1997) Enfect of aging on the bus d expression of 6 -fos, c-jun, and erg-I proteins itn the lippocampus. Neurobiol. Agung 18. $37-44$

9. Devan B.D. Goad E.H. and Petri H.L. (1996) Dissociation of hippocampal and striatal contributions to 
spatal ravigation in the watter maze. Neurobiol. Learn. Ment 66, 305-323.

10. Devaskat SU., Gideings S.I., Rajakumar P.A. Camaghi J.R. Menon R.K. and Zahn D.S. (1994) lasulin gene expression and insulin synthesis in mammalian neuronal cells. J. Biol. Chen 269, 8445-8454.

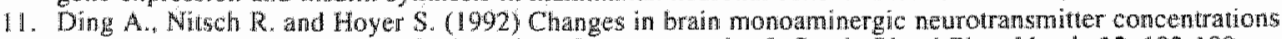
in rat afue intracerebroventricular injection of streptozotocin. J. Cereb. Blood Flow Mefab. 12, 103-109.

12. Dobrossy M.D., Svendser C.N. and Dunnet S.B. (1996) Bilateral striatal lesions impait retention of an operant test of short-rern memory. Brain Res. Bull. 41, 159-165.

13. Duell R. Sebrock H. Kuschnsky W. and Hoyer s. (1994) Intracerebroventricular injection of streptozotocin induces discrete local changes in cerebral glucose witization in rats. Int. D. Devl Neurosci. $12,737-743$

14. Dulin W.E. and Wyse B.M. (1969) Studies on the ability of compounds to block diabetogenic activity of wreptozotocin. Orabeles 18,459

15. Dunnet S.B. (1992) Aging, menory, and cholinergic systems: Sudies using delayed-matching and delayednonmatiching lasks in rats. In Neuropsychology of memory (ed. Squire L. R.). pp. 357-377. The Guilford Press, New York.

16. Dumnett S.B. (1993) The role and repair of forebrin cholinergic systems in short-term memory. Studies using the detayed matching-lo-position lask in rats. Ad\%. Neurol. 59, 53-65.

17. Dunnell $\$$.B., Everitt B.J. and Robbins T.W. (1991) The basal forebrain-contical cholinergic system: Interpreting the functional consequences of exciotoxic lesions. Trends Neurosci. 14, 494-501.

18. Fahrig T. (1992) Homologies between rat and rabbil Ca ${ }^{2}-$ channels: An immunobiochemical study. Neurosei. Res. Commun. 10, $115-123$.

19. Fahrig T. (1994) Changes in solubility of glat fubrillary actulic proten after ischemic brain damage in the mousw. Neurochem. 63, 1796.1801.

20. Fonnum $\mathbb{E}$. (1975) A rapid radiochemical method for the determination of choline acetyltransterase. J. Netrowent. 24, 407-409.

21. Furederickson R.C.A. (1992) Astroglia in Alzheimen's disease. Neurobiol. Aging 13, 239-253.

22. Gallagher M. Burwell R. and Burchinal M. (1993) Severily of spatial learning impairment in aging: Development of a learning undex for performance in the Mortis water maze. Behav. Neurosct. 107, 618 . 626.

23. Gallagher M. and Burwell R.D. (1989) Relationship of agerelated decline across several behavioral domains. Neurobiol. Aging 10, 691-708.

24. Gotries C.G. Andolisson R., Acquilomius S.M., Carlsson A., Eckernas S.A., Nordberg A., Oreland L., Svennerholm $L_{n}$. Wherg A. and Wintad B. (1983) Biochemeal changes in dementia disorders of the Alzheimer type (AD/SDAT). Newrobiol, Aging 4, 261-271.

25. Hartington C.R. (1990) Lowry protein assay containing sodium dodecyl sulfate in microtiter plates for protein determinations on fractions from bran lissue. Anal. Blochen. 186, 285-287.

26. Hawkins R.A., Mans A.M. and Davis D.W. (1983) Glucose availability to individual cerebral struetures is corrulated to glucose metabolism. Neurochem. 40, 1013-1018.

27. Hellweg R., Nitsely R., Hock $\mathbb{C}_{\text {. }}$ Jaksch $M$. and Hoyer $\mathbf{S}$. (1992) Nerwe growth lactor and choline acetyltransferase activity levels in the rat brain following experimental impairment of cetebral glucose and energy metabolism. J. Neurosed, Res, 31, 479-486.

28. Heregodts P. Brilyland M., Dekeyser J., Sollheid C., Michotte $Y$. and Ebingen G. (1989) Monoaminergic neurotrasmitter in Alzhemer"s disease. A. Newrol. Sci. 92, $101-116$.

29. Hill J.M. Lesniak M.A. Pert C.B. and Roth J. (1986) Autoradiographic localization of insulin receptors in rith brain: Promine ince in olfactory and limbic aneas. Neuroscience $17.1127-1138$.

30. Moyer S. (1992) Oxidative energy metabolism in Alzheimer brun. Mol Chem. Newopahol. 16, $207-224$.

31. Kahn C.R. (1985) The molecular mechanism of insulin action. Amon. Rev. Med. 36, 429-451.

12. Karnali p. and Armoni M. (1990) Regulation of glucose transporters in diabetes. Horm. Res. 33.99.104.

33. Katzman R. (1986) Alzheimer's disease. N. Eng. J. Med. 314, 664-973.

34. Kunklar P.E. and Kraig R.P. (1997) Reaclive astrocytosis from excitotoxic injury in hippocampal organ culture parallels that seen in vivo. J. Cereb. Blood Flow Merab. 17, 26-43.

35. Linneman D. and Skatslel T. (1994) Ragional changes in expression of NCAM, GFAP, and SIOO an aging rat brain. Neurobiol. Aging 15, 651-655.

36. Mattson M.P. (1995) Degenerative and protective signalling mechanisms in the neurofibrillary pathology of AD. Netrobiol. Aging 16, 447-463.

37. Mayer G., Nitsch R. and Hoyer S. (1990) Effets of changes in peripheral and cerebral ghucose netabolism on locomotor activity, learning and memory in adult male rats. Bran Res. $532,95-100$.

38. MeDonald R.J. and White N.M. (1994) Paralel informarion processing in the Water Maze: Evidence for independent memory systems involving dorsal stratum and hippocampus. Behav. Neural Biol. 61,260 270.

39. McNamara R.K. and Skellon R.W. (1993) The neuropharmacological and neurochemical basis of place lew aning in the Morris water maze. Brain Res. Rev. 18, 33-49.

40. Mecoce $\mathrm{P}$., MacGarvey U. and Beal M.F. (1994) Oxidative damage ro mitochondrial DNA is increased in Alzheiner's disease. Amm. Nernol. 36, 747.751.

41. Meibr-Ruge W. Bertoni-Freddari C. and Iwangoff P. (1994) Changes in brain glucose metabolismas a key 
10 the pathogenesis of Altheimer's disease. Geromology 40, $246-252$.

42. Money E.A., Kitk R.C. and McNaughton N (1992) Altheimers dernentia proluces a loss of alisctimination but no increase in rate of memory decay in delayed matching to sample. Netwopsyolologka 30, 133-14\%.

43. Mordes J.P. and Rossimi R.A. (1981) Animal models of abiabics. Am J. Med. 70, 35,360 .

44. Morris R.G.M. Garrand P. Rawlins J.N.P. and OKeefe J. (1982) Place wavigution is lmpained in rats with hippocampal. lesions. Nature $297,681.683$.

45. Nichols N.R., Finch C.E. and Nelson J.F. (1995) Food restricion detays the age-related increase in GFAP in $\mathrm{mRNA}$ in rat hypocampus. Neurobiol Aging 16. $105-110$.

46. Nisch $\mathbb{R}$. and Hoyer S. (1991) Local action of the diabetogenic drug, steptozotocin, on glucose and energy metabolism in the brain contex. Neurosic. Lett $128,199-202$.

47. Misch R., Mayer $\mathrm{G}$. and Hoyer S. (1989) The intracerebroventriculary streptozotocin-treated rat: Imparnent of cerebral glucose metabolism resembles the alterations of cabohydrate metabolism of the bran in Alzheimer's disease. J. Netural Trans. 1, $109-110$.

48. Pappas B.A. De la Torte J.C., Davidson C.M., Keyes M.T. and Fortin T. (1996) Chronic reduction of cerebral blood flow in the adult at: Late-emetging CAI cell loss and nemony dysfunction. Broin Res. 708 , $50-58$.

49. Paxinos $\mathrm{C}$. and Watson C. (1986) The rat brain in stereotaxic condinates. 2nd edn., Academic Press, San Diego.

50. Plaschke $K$ and Hoyer $S$. (1993) Action of the diabotonic drug streplozotocin on glycolytic and glycogenolytic metabolism in adult rat bran cortex and hippocampus. Wh. J. Dew Nerrosci. 11, 477-483.

51. Prickaerts J Blokland A.. Honig W. Meng F. and Jolles J. (1995) Spatial discrimination learning and choline acetyltransferase activity in streptozotocin-treated rats: Eftects of chronic treatment with acedyl Lcamitine. Bran Res. 674, 142.146.

52. Rolls E.T. (1994) Neuroptrysiology and cognitive functions of the striatum. Rev. Newrol. Partis 150 . 648. 660 .

53. Rosengren L.E., Lycke I. and Anderson O. (1995) Ghal fibrillary acidic protain in CSF of multiple sclerosis patients: Relation 10 neurological deficit. J. Netrol. Sci. 133, $61-65$.

54. Salngal A. (1987) Some limitations of indices derived from signal detection theory: exaluation of an alternative index for measuring bias in memory tasks. Psychophamacology $91,517.520$.

55. Sahgal A., Galloway P.H. McKeith I.G., Lloyd S., Cook J.H., Ferrier N. and Edwardson J.A. (1992) Matching-to-sample defichs in patients with senile dementias of the Alzhemer and Lewy body types. Arch. Nenrol. $49,1043-1046$

56. Samuel W. Masliah E., Brush D.E., Garcia-Munoz M., Patino P., Young S.J. and Groves P.M. (1997) Lesions in the dentate hilum and $\mathrm{CA} 2 / \mathrm{CA} 3$ regions of the rat hippocampus procilace cognitive deficits that correllate with site-specific glial activation. Newrobiol. Learn. Ment 68, 103-116.

57. Schipper H.M. (1996) Astrocytes, brain aging, and neurodegeneration. Neurobiol. Agimg $117,467-480$.

58. Shigenaga M.K. Hagen T.M. and Ames B.N. (1994) Oxidative damage and mitochondrial decay in aging. Proc. Natl Acad. Sci. U.S.A. 91, 10771-10778.

59. Sugaya K., Chouinard M., Greene R., Robbins M., Personet D., Kent C., Gallagher M. and Mckinney M. (1996) Motecular indices of neuromal and glial plasticity in the hippocampal formation in a rodent model of age-induced spatial leaming impaiment. W. Newrosci. 16, 3427-3443.

60. Takasu N. Komiya I. Takayuki A. Nagasawa Y. and Yamada T. (1991) Streptozotociti- and alloxath:

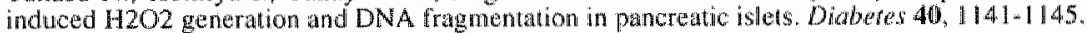

61. Terwel D., Prickaters J., Meng F. and Jolles J. (1995) Brain enzyme activilies afler intracemebroventricular Hijection of streptozotocin in rats receiving acetyl-L-camitine. Eur. J. Phannacol. $287,65-71$.

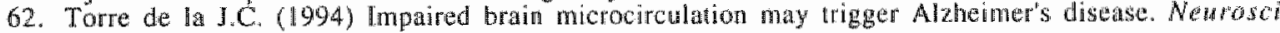
Biobehav. Rek. 18, 397-401.

63. Unger J.W Livingston J.N. and Moss A.M. (1991) Insulin receptors in the central nervous system: Localization, signalling mechanisms and functional aspects. Prog. Neurobiol. 36, 343-262.

64. Vijayan V.K. Geddes J., Anderson K. Chang-Chui H., Ellis W. and Cotman C.W. (1991) Astrocyte hypertrophy in Alzhemer's disease hippocampal formation. Exp. Merrol, 112, 72-78.

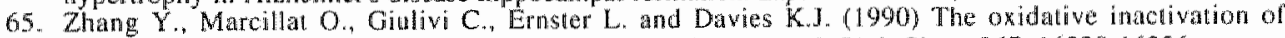
mitochondral electron transport chain components and A.TPase. J. Biol. Chen. 265, $16330 \times 16336$. 


\section{Nitric oxide synthase does not mediate neurotoxicity after an i.c.v. injection of streptozotocin in the rat*}

Jos Prickaerts1, Jan de Vente!, Wiel Honig』, Hellen Steinbuschl, Marjanne Markerink-van Ittersum 1 , Arjan Blokland2 and Harry W.M. Steinbusch 1

European Graduate School of Neuroscience 'EURON", IDepartment of Psychiary and Newropsychology, and 2 Department of Psychology, Maastricht University. The Netherlands

\section{ABSTRACT}

In the present study we evaluated the possible role of nitric oxide (NO) in mediating neuronal damage in middle-aged rats after an intracerebroventricular injection of streptozotocin. An intracerebroventricular injection of streptozotocin has been reported to decrease the central metabolism of glucose. This inhibition of the energy metabolism after streptozotocin treatment might induce an excitotoxic mechanism, which may lead to the stimulation of NO synthase and, consequently to the synthesis of NO. On the other band, streptozotocin might induce oxidative stress directly by liberation of NO from äts nitroso moiety. To investigate whether NO synthase is involved in a possible excitotoxic mechanism after streptozotocin treatment, some of the rats treated with streptozotocin $\left(1.25 \mathrm{mg} / \mathrm{kg}\right.$ in $4 \mu \mathrm{l}_{v}$ billaterally $2 \mu 1 /$ injection silte) were also treated with the NO synthase inhibitor $N$-nitro-L-arginine methyl ester (L-NAME, $20 \mathrm{mg} / \mathrm{kg}$ 1.p. $10 \mathrm{~min}, 6,24$ and $96 \mathrm{~h}$ after streptozotocin injection). To investigate whether NO liberated from streptozotocin may be responsible for neurotoxic effects, one additional group of control rats received an i.c.v. injection of the NO donor sodium nitroprusside (SNP, $10 \mu g$ in $4 \mu \mathrm{l}$ ). We found that streptozotocin affected the behavioral performances in the open field and two-way active avoidance task. In addition, glial fibrillary acidic protein immunostaining showed that neuronal damage was mainly located in peri- and paraventricular regions of the third and lateral ventricles, like for instance in the septum, caudate putamen and hippocampus. L-NAME treatment had no protective effect on the behawioral impairments and neuronal damage of streptozotocin-treated rats. This suggests the neuronal damage of streptozotocin may still be a result of the decrease in the central energy metabolism, but without the involvement of NO synthase. This was supported by measuring, using immunostaining, the $\mathrm{NO}$-mediated cyclic GMP production by the enzyme soluble guanylyl cyclase in contical slices, i.e. L-NAME did not prevent NO production after streptozotocin administration in vitro. In addition, it was found that SNP liberated NO in viro, whereas in wivo SNP administration did not lead to any behavioral and neuronal deficits at all However, the present study cannot exclude the involvement of NO liberated from streptozotocin in neuronal damage.

\footnotetext{
* "This study has been submitted to Neurosici. Res.
} 


\section{INTRODUCTION}

Treatment of middle-aged rats with a single intracerebroventricular (i.c.v.) injection of streptozotocin (STREP) may provide a relevant model of neurodegeneration [47, 55] which has been suggested to show great similarities with Alzheimer's disease (A.D) $[4,15,36,37,46]$. Substantial evidence has accumulated that i.c. $v$. injected STREP reduces the central metabolism of glucose. Although some questions regarding the precise mechanism of action of STREP are still unanswered, the inhibition of the energy metabolism may be caused by destruction of the insulin-secreting cells and/or interference with the insulin receptor system [1.5, 36. 37. 46].

Inhibition of the energy metabolisn by STREP could start an excitotoxic mechanism, which results in neurodegenerative damage eventually $[1,57]$. Nitric oxide (NO) might be involved in this excitotoxic mechanism or also referred to as $N$-methyl-D-aspartate (NMDA)-mediated neurotoxicity, because the increase in NMDA-dependent $\mathrm{Ca}^{2+}$ influx stimulates the enzyme NO synthase [c. 191. This has been corroborated by several studies showing that $\mathrm{NO}$ is involved in a NMDA-mediated neurotoxicity. For instance, in vitro studies showed that inhibition of NO synthase by $N$-nitro-L-arginine (L-NA) blocked neurotoxicity after NMDA agonist administration [11.26] This is in line with thein vivo finding that NO synthase inhibition with L-NA [22] or its methyl ester, L-NAME [51], can be neuroprotective after NMDA agonist administration into the brain. The contribution of NO to the neurodegenerative processes might be the oxidative damage caused by the free radical itself, which is corrobarated by both in vitro [11, 27] and in vivo [21, 30.1 studies demonstrating that sodium nitroprasside (SNP), a NO donor, was neurotoxic. However, the exact chemical species involved in this neurotoxic mechanism are still unknown.

It is known that STREP upon decomposition gives rise to $\mathrm{NO}[29,54,56]$ and $\mathrm{H}_{2} \mathrm{O}_{2}$ [53]. Thus apart from an oxidative stress due to inhibition of the energy metabolism, STREP may cause oxidative stress by itself which leads to neurodegenerative damage [of. 6, 16].

To evaluate the possible role of NO synthase activity in STREP-induced neurodegeneration, rats treated with STREP in combination with L-NAME (i.p.) were tested in the open field, Morris water escape task and shuttle box, and compared with the results of a control group. To investigate whether NO liberated from STREP may be responsible for the neurotoxic effect, we compared the results of the STREP group with a group of rats which received an i.c.v. injection with SNP. AS an index for the tissue reaction to neurotoxic damage we studied glial fibrillary acidic protein (GFAP) expression in brains of all the animals of all four groups. In an additional in vitro experiment we studied the effects of SNP or STREP together with L-NAME on NO production by measuring the NO-mediated cyclic GMP (CGMP) production by the enzyme soluble guanylyl cyclase $(\mathrm{sGC})$.

\section{MATERIALS AND METHODS}

\section{Animals}

All experimenal procedures were approved by the local ethical committee of the Mastricht University for animal experiments and me governmental guidelines. Forty-two 20-month-old male Lewis rats were tsed. One week before surgery the rats were housed individually in standard Makrolon cages on sawdust bedding in an air-conditioned room (about $20^{\circ} \mathrm{C}$ ). The rats had ad-lib food and water, and were kept under a 12//2-h light/dark cycle (lights on from 6.00 to $18.00 \mathrm{~h}$ ). For the additional in vitro experiment three 2 -month-old Lewis rats were used. 


\section{Stageral procedure aud treatrnent}

Two weeks before behavioral testing the animals were matched for body weights and assigned to a control group (CON; $n=10)$, a STREP-treated group $(n=10)$, a STREP/L-NAME-treated group $(n=12)$, or a SNP-treated group $(n=10)$. STREP was dissolved in a citrate buffer ( $5 \mathrm{mM}, \mathrm{pH} 4.5)$ and L-NAME and SNP were dissolved in $0.9 \%$ saline. All solutions were freshly prepared each day. Rats were anestherized with a combination of Rompun (2 mg/kg. s.c.) and hydrochloride ketamine $(50 \mathrm{mg} / \mathrm{kg}, \mathrm{i} . \mathrm{m}$.) and placed in a slereotaxic frame. The stereotaxic coordinates for i.c. $v$. injections were $-0.8 \mathrm{~mm}$ anterior, $1.55 \mathrm{~mm}$ lateral and $-3.8 \mathrm{~mm}$ ventral from the bregma [45]. An i.c.v. injection $(2 \mu$ l/site) took about $4 \mathrm{~min}$ for each injection site and the needle was left in place for $1 \mathrm{~min}$ after completion of the injection. The STREP and STREP/L-NAME group were given a bilateral i.c.v. injection of STREP $(1.25 \mathrm{mg} / \mathrm{kg}$ body weight dissolved in $4 \mu$ citrate buffer, $2 \mu$ il/site). In addition, the rats of the STREP/L.NAME growp were given an i.p. injection of L-NAME (20 $\mathrm{mg} / \mathrm{kg}) 10 \mathrm{~min}, 6,24$, and $96 \mathrm{ht}$ after STREP administration. The CON group was given citrate buffer $(2 \mu 1 /$ site) instead of STREP. The SNP group was given a bilateral i.c.w. injection of SNP (10 $\mu \mathrm{g}$ dissolved in $4 \mu 1$ salline, $2 \mu \mathrm{i} / \mathrm{site}$ ). One rat of the STREP/L-NAME group had a bleeding during surgery and died one day later and one rat of the SNP-group died during anesthesia before surgery. Further, one rat of the STREP/L-NAME group and one rat of the SNP-group. died within one day after surgery for unknown reasons. Before behavioral testing starled one STREP/L. NAME-treated rat was excluded from the experiment because its physical condition only worsened after surgery. During the complete experiment body weights and water consumption were monitored.

\section{Behavioral procedures}

Open field. The animals were allowed to recover for 11-15 days between surgery and the start of behavioral testing in the open field. The open field consisted of a square base $(100 \times 100 \times 30 \mathrm{~cm})$ subdivided into 36 equal squares by black lines (for details see [58]). One red fluorescent tube provided very dim illumination (about 2 (x) on the floor of the apparatus. Inmediately after a rat was placed in the center of the open field, the movements of the rat were scored. A crossing was scored as soon as the rat crossed a line with both hind legs. The total time spent in the corner, wall and center squares, as well as the total number of lines crossed and rearings were recorded manually with a personal computer. Testing was carried out on four consecutive days in 5 -min sessions.

Shock sensitivity. The day after the open field test, the shock sensitivity of rats was determined by the flinch reaction. A rat was placed in a box $(40 \times 25 \times 40 \mathrm{~cm})$ with a grid floor which was connected to a shock scrambler. The intensity of the shock was increased step wise, starting at $0.05 \mathrm{~mA}$, with increments of $0.05 \mathrm{~mA}$. Each shock lasted $0.5 \mathrm{~s}$. The shock intensity at which a rat first flinched was scored as the first shock sensitivity level. After the shock intensity was again increased by $0.05 \mathrm{~mA}$, the shock intensity was decreased in a similar manner as it was increased. The second shock sensitivity level was scored when a rat failed to flinch when a shock was given.

Morris waser escape task. Two days after the shock sensitiwity measurements, that is approximately three weeks anter surgery, the Morris water escape performance of the rats was assessed in a black water lank (diameter $1.22 \mathrm{~m}$ ). The escape platform (diameter $11 \mathrm{~cm}$ ) was submerged $(1.5 \mathrm{~cm}$ below the surface of the water. The water temperature was about $22^{\circ} \mathrm{C}$. The water tank was divided into four quadrants which were designated training, right-adjacent, left-adjacent and opposite relative to the position of the platform. Abundant spatial cuss were provided by the furniture in the room, including desks and computer equipment, and by the presence of the experimenter. A radio, which was playing sofily, provided background noise during testing in the water task.

The rats were started facing the wall of the tark from four different, randomly chosen, start positions, and tramed to find the invisible platform at a fixed invisible position in the water tank. A trial lasted until a rat had found the platform or until $60 \mathrm{~s}$ had elapsed. If a rat did not find the platform within $60 \mathrm{~s}$, it was placed on the platform for $3 \mathrm{~s}$ and then removed from the water tank. On the first day, the rats were given four trialls. On the three subsequent days the rats were given eight trials a day. The time berween subsequent trials was about 10 min. Escape latencies of each trial during the training were measured with a stopwatch. A probe trial lasting 60 $s$, during which the platform was removed from the water tank, was given after the last trial. In the probe trial all] rats were started, facing the wall of the tank, from the position opposite to the position of the removed platform. A video camera, mounted in the center above the circular pool, prowided a picture of the pool on a TV monitor and the movements of a rat during the probe trial were registered automatically (EthoVision, Noldus cquipment) and slored in a personal computer. 
Two-way acture avoldance rast. Three days after the Morris water escape task, acquisition of a twow way actue avoidance lask was assessed in an automated shutle box. The apparatus consisted of two equal compartments $(21 \times 27 \times 40 \mathrm{~cm})$, separated by a barrier (height $3 \mathrm{~cm}$ ). The floor consisted of a metal grid connected to a shock serambler. The animals were trained in one session of 50 trials. The session was preceded by an adaptation period of $5 \mathrm{~min}$. A trial started with the presentation of an auditory signal as the conditioned stimulus. The signal was terminated when a rat crossed the barrier within \& s, i.e. when the rat made an avoidance response; otherwise, a foo shock $(0.25 \mathrm{~mA})$ was giwen as the unconditioned stimulus. The foot shock and the auditory signal were teminated when the rat escaped to the other compartment or when 10 s had elapsed. The interinal interwal varied randomly between 35 and $60 \mathrm{~s}$.

\section{Morphological staining}

After behaviorat testing, that is at approximately five weeks after surgery, all rats were used for morphological staining studies. For GFAP immunostaining vibratome 50 un sections were used, which were cut from tissue that was fixated by an intracardiac perfusion and a 2 h postfixation at $4^{\circ} \mathrm{C}$ with somogyi fixative (final concentration $4 \%$ paraformaldehyde, $0.05 \%$ glutaraldehyde and $15 \%$ picric acid in $0.1 \mathrm{M}$ phosphate buffer, $\mathrm{pH} 7.4$ ) and was, thereafter, imbedded in $10 \%$ gelatin (porcine). Sections were successively rinsed three times 15 min in Tris-buffered saline ( $\mathrm{pH} 7.6$ ) containing $0.3 \%$ (v/v) Triton X-100 (TBS-Triton) "Tris-buffered saline (TB5; pH 7.6) and TBS-Triton. Subsequently the sections were incubated overnight at $4^{\circ} \mathrm{C}$ with rabbit amti-GFAP (1:500) in TBS-Triton. The polyclonal antibody to GFAP was kindly donated by Prof. Dr. F.C.S. Ramaekers (Department of Molecular Cell Biology and Genetics, Maastricht University). Thereafter the sections were again successively rinsed three times $15 \mathrm{~min}$ in TBS-Triton, TBS and TBS-Triton. The second antibody incubation was for 1 th at room temperature with goat anti-rabbit $(1: 60$, Jackson) in Tris-Triton, after which the sections were rinsed again three times 15 min in TBS-Triton, TBS and TBS-Triton. The last incubation was for I h a room temperature with rabbit peroxidase anti-peroxidase (Dako) at a 1:600 dilution in Tris-Triton. After the sections had been successively rinsed three tirnes $15 \mathrm{~min}$ in TBS-Triton, TBS and a Tris-HCl buffer (50 mM, $\mathrm{pH}$ 7.6), the peroxidase label was visualized by incubation in the Tris-HCl buffer containing $0.05 \%$ diaminobenzidine (Sigma) and $0.01 \% \mathrm{H}_{2} \mathrm{O}_{2}$ for about $10 \mathrm{~min}$ at room temperature. After rinsing three times 10 min with TBS, the sections were mounted on ehrome alum/gelatin coated slides. Finally, the slide mounted sections were delaydrated and coverslipped. GFAP was used a marker of reactive gliosis, which results from neuronal damage and, probably, neuronal cell death. Hence, the extent of neuronal damage was judged qualitatively from the GFAP-stained brain sections by one experimenter.

\section{Immunocytochemical determination of cGMP}

NO is a powerlul activator of SGC which synthesizes cGMP [35]. To study NO liberation/formation after SNP or STREP treatment, also ifi combination wilh L-NAME treatment, we additionally measured oGMP formation in sticos using immunocytochenistry. The proedure for incubation of tissue slices has been described in detail in Otr previous studies [13.31. Briefly. After decapitation the brains of (naive) rats were removed and immediately placed in alerated Krebs-Ringer bicarbonate buffer $(\mathrm{pH} 7.4)$, containing $1 \mathrm{mM}$ isobutylmethylxanthine (IBMX; Sigma). Brains were cut in the midsagittal plane, the tissue was placed on a fitter paper on a precooled $\left(4^{\circ} \mathrm{C}\right)$ stainless steel table and transversal 300-pm slices were cut using a Mcllwain tissue chopper. We used slices of the fromal contex, which are known to have a thighly (re)active NO-cGMP system 112, 31, 591. Slices were separated while being submerged in ice-cold Krebs buffer (aerated with $95 \% \mathrm{O}_{2}$ and $5 \% \mathrm{CO}_{2}$ ) and partitioned in incubation wells. Incubation time before adding the NO donor sodium nitroprusside (SNP; Fluka) was 30 min. The NO synthase inthibitor L-NAME (Fluka; $0.1 \mathrm{mM}$ ) was included from the start of the incubation. SNP $(0.1$ $\mathrm{mM})$ or STREP $(10 \mathrm{mM})$ was present for another, final, 10 min. The incubations were terminated by adding ice-cold fixative solution (final concentration $4 \%$ paraformaldehyde, $10 \%$ sucrose, $\mathrm{pH} 7.4$ ). Fixation was continued for $2 \mathrm{~h}$, followed by a 30-min wash in ice-cold $0.1 \mathrm{M}$ phosphate (pH 7.4) containing $10 \%$ sucrose. Subsequently, cryostat sections $(10 \mu \mathrm{m})$ were cut and thawed on 10 chrome alum/gelatin-coated slides and processed for inmunocy tochemistry. Sections were incubated overnigh at $4^{\circ} \mathrm{C}$ with rabbit anti-cGMP $(1: 300)$ in Tris-buffered saline (TBS; $p H 7.6$ ) containing $0.3 \%$ (w/v) Triton X-100. cGMP immunoreactivity was visualized using a fluorescein isothiocyanate-conjugated goat anti-rabbit antiserum (Jackson) at a 1:150 dilution. Specificity of the cGMP immunostaining has been described in detail before $[13,3 \mid]$, 
It is important to note that the in vitro slice incubations were performed in the presence of I MM MBMX inhibit phosphodiesterase activity. This was done because in st previous study we have found that in the absence of IBMX no or almosi no CGMP immunostaining can be obserwed in the rat brain [31). Therefore, cGMP determinations under so-called basal conditions do not reflect actual basal cGMP lewels, but rellect ongoing COMP synthesis in the absence of added stimulators or inhibitors of either $\mathrm{SCC}$ or NO synthase.

\section{Siaristical analysis}

Body weights and water consumption were weekly measured. Treatment effects on both measures during the complete study were evaluated with a two-factorial (Treatment and Week) ANOVA with repeated meatsures over Week.

The data of the open field test were aggregated in order to enhance reliability [41]. "The mean time spent in the squares of the comers, walls and center, respectively, was transformed to the raturall logarithm $\left(\mathrm{Ln}\left(\mathrm{t}_{1}+\mathrm{I}_{2}+\right.\right.$ $13+4$ )/4]; subscripts refer to day 1 through 4 of testing) to remove inhomogeneity [58]. Treatment effects on the open field measures were analyzed with a one-factorial ANOVA (Treatment).

During the acquisitions of the Morris water escape tasks, the mean escape latency was calculated per session of four trials. In the probe trials, the time spent in each quadrant was measured. Treatment effects during the acquisitions were evaluated with a two-factorial (Treatment and Session) ANOVA with repeated meastres over Session. For the probe trial, preferences for quadrants were evaluated per group by anallyzing the times spent in each guadrant with a repeated measures ANOVA over Quadrant, and additional $t-$ statistics which analyzed whether the cime spent in a quadrant differed from the chance level $(15 \mathrm{~s})$. Treatment effects on the time spent in the training guadrant, as well as the measures swimming speed and mean distance to platform $[18\}$, were evaluated with one-factorial ANOVA (Treatment).

The sensitivity to shock was calculated by averaging the two shock sensitivity levels. The shock sensitivity data were ordinal and, therefore, rank scores were calculated, which were used for parametric analysils with a one-factorial ANOVA (Treatment). The mean number of avoidance responses in the two-way active avoidance task was calculated per block of 10 trials and analyzed by a twomfactorial (Treatment and Trial block) ANOVA with repeated measures over Trial block.

Changes in body weights and water consumption during the experiment were further analyzed by a one-factorial ANOVA (Treatment) on orthogonal trend coefficients calculated over the successive weeks [60]. Likewise, orthogonal trend coefficients were calculated over the successive sessions of the Morris water escape task and the trial blocks of the two-way active avoidance task, respectively, and analyzed to assess changes in the course of learning these behavioral tasks. All Treatment effects were analyzed in more detail by Duncan's post hoc multiple range test.

\section{RESULTS}

\section{Body weights and wher consumption}

The body weights and water consumption during the experiment are depicted in Figure 1. The data of week 5 after surgery were not used for statistical analyses, since compared to week 4 no more changes in body weight and water consumption appeared (see Fig. 1). There was no overall difference in body weight between the four experimental groups during the complete experiment (general mean Treatment: $F_{3.33}=0.93$, n.s.). All groups showed a decrease in body weight during the experiment (Week effect: $F_{3,33}=73.86, p<0.01$ ), which was characterized by a linear and cubic trend. Ninety-three percent of variation in the decrease in body weight over the successive weeks can be predicted from a linear regression equation [60], and the remaining proportion of the variation may be explained almost completely by a cubic equation, viz. $6 \%$. The four treatment groups did not differ on any of the two trend components (Treatment effect: both Fs $<0.93$, n.s.). This indicates that they all showed a similar decrease in body weight during the experiment.

No difference between the groups was found for the overall amount of water consumed 

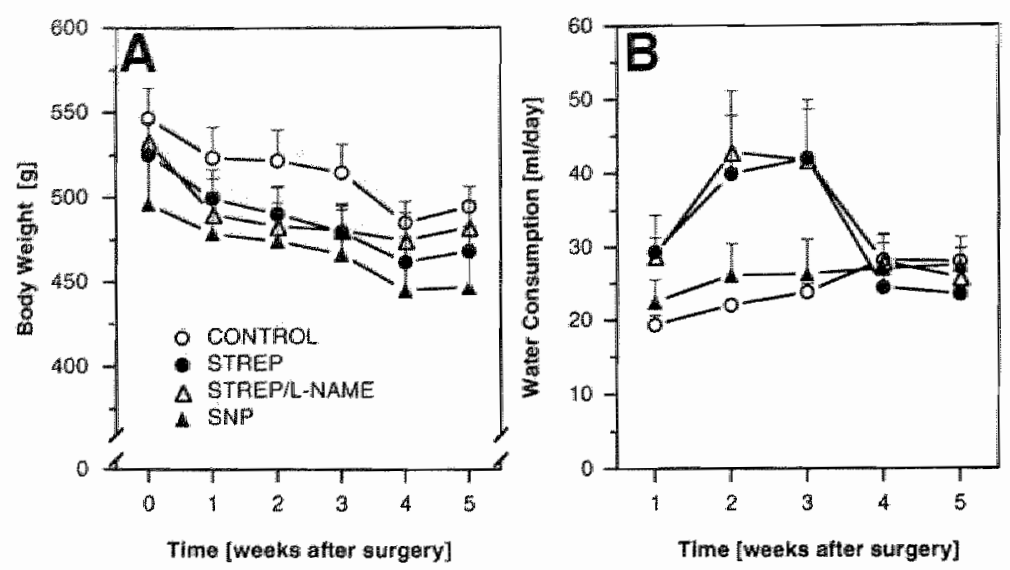

FIG. 1. Body weights (A) and water consumption (B) during the experiment. Behavioral testing was started two weeks after surgery, i.e. treatment. Rats were successively tested in the open field, Morris water escape task and shuttle box at two, three and fowr weeks, respectively, after surgery. Values are given as means with S.E.M.

during the experiment (general mean Treatment: $F_{3,33}=2.13$, n.s.), although inspection of Figure 1B would predict otherwise. A change in water consumption in the course of the experiment was found (Week effect: $F_{3,33}=8.11, p<0.01$ ), of which the variation may be explained for $96 \%$ by a quadratic trend component. A treatment effect was found on this trend component (Treatment effect: $F_{3,33}=4.36, p<0.05$ ). Post hoc analysis showed that the quadratic trend component was the same for the control and SNP group. In addition, the quadratic trend component was also not different between the STREP and STREP/L-NAME group, but it was more pronounced (negative) in the latter two groups when compared to the control and SNP group. This indicates that during the second and third week after surgery, all STREP-treated rats consumed more water than the control and SNP-treated rats.

\section{Open field}

The results of the open field test are summarized in Figure 2. There was a difference in the times spent in the center squares between the four experimental groups of rats (Treatment effect: $F_{3,33}=$ $5.45, p<0.01$; see Fig. 2A). Post hoc analysis showed that the SNP group spent the same amount of time in the center squares as the control group. Further, compared with these two groups of rats, all STREP-treated rats spent more time in the center squares, while there was no difference between the STREP group itself and the STREP group treated with L-NAME. Although inspection of Figure $2 \mathrm{~A}$ suggested a similar effect on the limes spent in the comer squares, there was only weak statistical evidence for this (Treatment effect: $F_{3.33}=2.71,0.05<p<0.10$ ). Post hoc it was only found that the STREP group spent less time in the corner squares than the control group. All rats spent the same amount of time in the wall squares (Treatment effect: $F_{3,33}=1.73$, n.s.) and had the same activity as measured by the total number of line crossings and rearing (Treatment eflect: both Fs $<0.69$, n.s.; see Fig. 2B).

\section{Shock sensitritity}

The shock sensitivities (MA) for the control, STREP, STREP/L-NAME and SNP group were, 

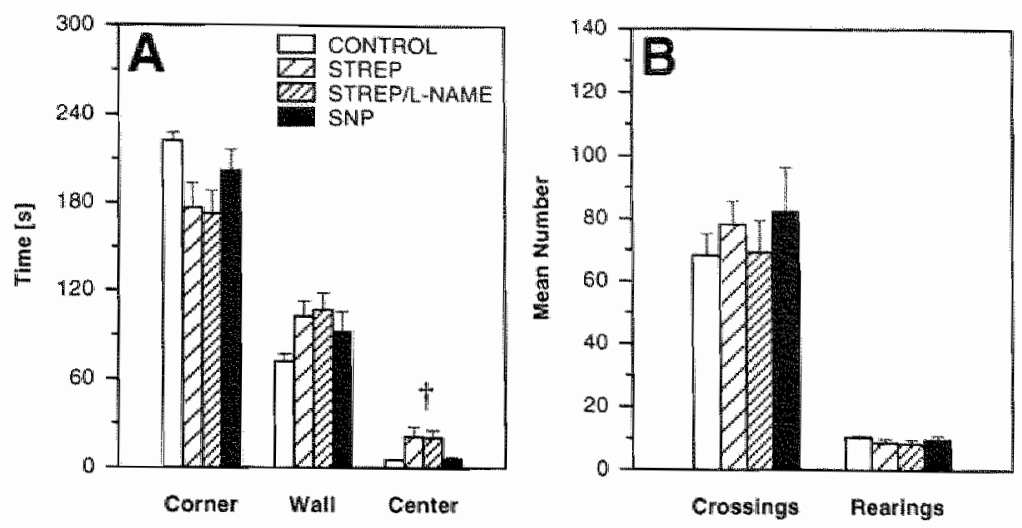

FIG. 2. Mean total time (+S.E.M) spent in the different areas (A) and the mean total number $(+S . E . M$.) of line crossings and rearings (B) in the open field (aggregated data of four 5 -min sessions, one session per day). th $\beta$ 0.01 Treatment effect.

median (range), $0.21(0.18-0.28), 0.20(0.13-0.30), 0.18(0.13-0.35)$ and $0.19(0.15-0.25)$, respectively. These shock sensitivities were not different between the four experimental groups $\left(F_{3,33}=1.86\right.$, n.s. $)$.

\section{Morris water escape lask}

The results of the acquisition of the water escape task and the probe trial are summarized in Figure 3. There was no difference in the overall escape latency between the four experimental groups (general mean Treatment: $F_{3,33}=0.72$, n.s.; see Fig. $3 A$ ). All groups reduced their escape latencies during acquisition of the spatial discrimination task (Session effect: $F_{6,198}=60.67, p<$
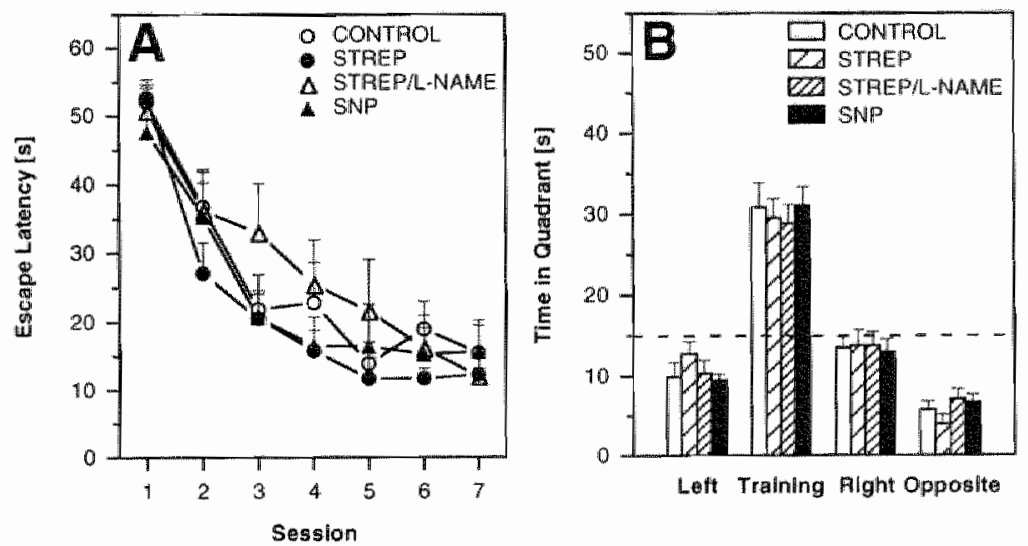

FIG. 3. Mean escape latencies (+S.E.M.) for each session during spatial discrimination learning in the Morris water escape rask (A). Mean times (+S.E.M.) spent in the four quadrants in the probe trial (B). The quadrants are organized with respect to the training quadrant, i.e. left-adjacent, right-adjacent and opposite. 
0.01). This improvement of performance over sessions was characterized by a linear, quadratic and cubic trend component. Eighty-one percent of the variation of the decrease in escape latency may be explained by a linear regression equation, sewenteen percent by a quadratic equation and the remaining iwo percent by a cubic equation. There was no difference between the groups on any of the trend component (Treatment effect: $F ;<1.77, \mathrm{~ns}$. ), indicating that the learning curves of the groups were simillar.

In the probe trial all groups showed a quadrant preference (Quadrant effect: $F_{\mathrm{s}}>21.70, p<$ $0.01)$. In addition, all groups spent most of their time in the training quadrant $(t s>5.33, p<0.01$; see Fig. 3B) and there was no difference between the groups for this measure (Treatment effect: $F_{3,33}=0.17$, n.s.). Netther was there a difference in the mean distance to the former location of the platform (Treatment effect: $F_{3,33}=0.36$, n.s.; data not shown) nor in the swimming speed (Treatment effect: $F_{3,3,3}=1.51$, n.s." data not shown). The latter indicates that the acquisition performance as measured with escape latencies, was probably not influenced by speed.

\section{Two-way active avoidance learning}

The results of the two-way active avoidance task in a shuttle box are summarized in Figure 4. There was no difference in the overall number of avoidance responses (general mean Treatment: $F_{3,33}=1.58$, n.s. $y$ and all rats increased the number of avoidances during acquisition of the avoidance task (Trial Block effect: $F_{3,33}=71.13, p<0.01$ ). This increase was characterized by a linear and quadratic trend, which may explain $87 \%$ and the remaining $13 \%$, respectively, of the variation in the increase of the number of avoidances. Only for the quadratic trend component there was a difference between the four experimental groups of rats (Treatment effect: $F_{3,33}=$ $3.95, p<0.05)$. Post hoc analysis revealed that this trend component was more pronounced in the STREP group compared to the control and SNP group, which both had the same quadratic trend component. This indicates that the speed of attaining its maximal number of avoidance responses was higher in the STREP group when compared with the control and SNP group.

\section{Morphology}

GFAP immunocytochemistry showed that after STREP treatment there was an increase in both the intensity of staining and the number of stained astrocytes in the rat brain. This is illustrated in

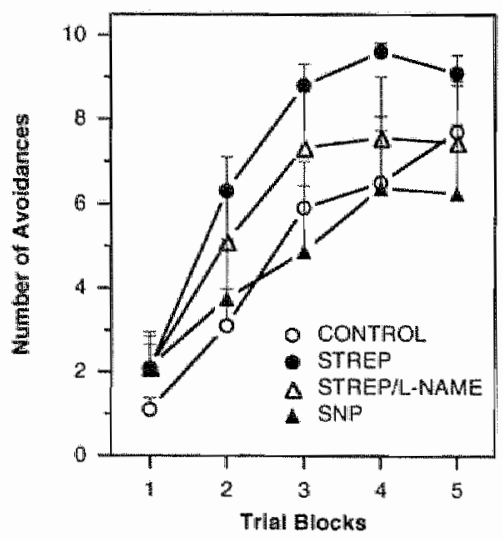

FIC. 4. Menn total number of avoidance responses (+S.E.M.) during acquisition of the active avoidance task in a shutule box. 

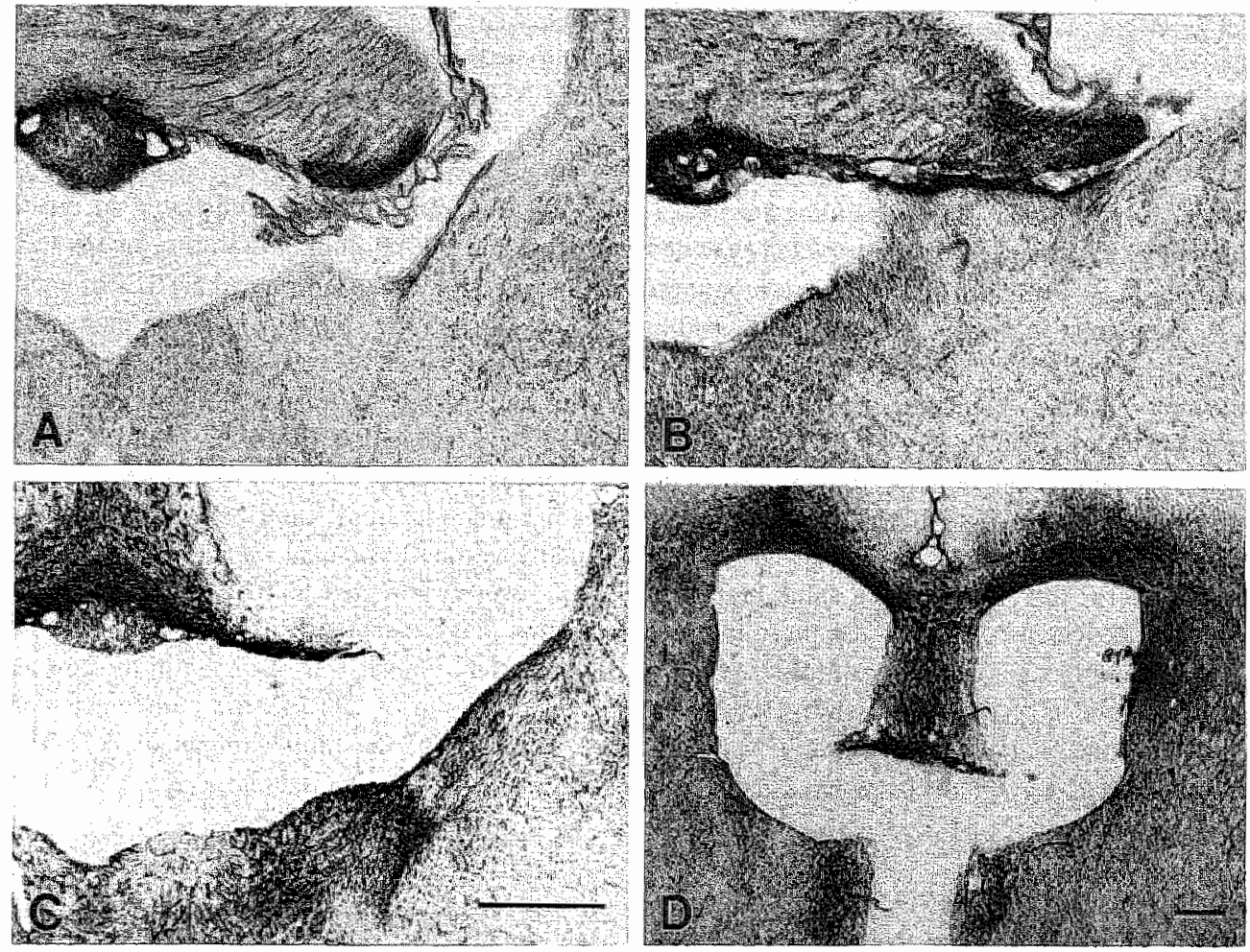

FIG 5. GFAP immunostaining in brain sections of a control rat (A) and STREP-treated rats, showing the sepual region where the lateral ventricles pass into the third ventricle at approximately $.0 .8 \mathrm{~mm}$ anterior from the bregma, that is the injection site. To illustrate few neuronal damage after STREP treatment, brain sections of the STREP-treated animal with least newronal damage was used (B). As example of severe STREP-induced neuronal danage, that is extensive cell loss and gliosis, the animal of the STREP/L-NAME group with most nearonal damage was used $(\mathrm{C})$. A minor magnification of a similar brain section of the same animal illistates the increase in the volume of the ventricular system and the extent of glosis in its adjacent regions (D). Scale bars are 100 $\mu \mathrm{m}$.

Figure 5 showing GFAP immunoreactivity near the site of injection in a control and wo STREP-treated rat. Figure $5 B$ shows the STREP-treated animal with the least neuronal damage. The results of the GFAP staining of the STREP/L-NAME group were not different from that of the STREP group. The animal from the STREP/L-NAME group with most STREP-induced damage is 
depicted in Figure 5C and SD to illustrate severe neuronal damage. The GFAP staining of brain sections showed that there was no difference in (low) GFAP immunoreactivity and brain morphology between the SNP and control group (data not shown).

Neuronal damage was reflected by gliosis and extensive celj loss, of which the latter was manlly indirectly evident from the increase in the volume of the ventricular system (see Fig. $5 \mathrm{C}$ and SDJ. Some rats appeared to be not so susceptible to STREP treatment, since the volume of the ventricles were not or only slightly increased, although their walls with the septum and striatum showed signs of irritations (see Fig. 5B) and an increased gliosis was observed compared to control rats (compare Fig. $5 \mathrm{~B}$ with $5 \mathrm{~A}$ ). Hematoxylin-eosin staining did not reveal any clear differences if cell number between these minor affected STREP-treated rats and control rats (data not shown).

STREP-induced neuronal damage was mainly found near the lateral ventricles and third ventricle in regions of the lateral septal nuclei and candate putamen. This is illustrated in Fig 6 showing the extent of damage, which was deduced from the GFAP immunostaining, in several coronal brain sections of the least (left column) and most (right column) affected STREP-treated rat. Damage in minor affected STREP-treated rats was mainly reflected by gliosis, while there was no extensive loss of cells. Besides that damage near the lateral ventricles was located in the lateral septum and striatum, gliosis (and also extensive cell loss in severely affected STREP-treated rast) was also found in the dorsal fornix and bed nucleus of stria terminalis. The same brain sections also showed damage near the third ventricle, mainly in paraventricular nuclei of the hypothalamus, the periventricular hypothalamic nucleus and the medial preoptic area. Anterior to the injection site, the fornix, medial septum and nuclei of the (mainly vertical) diagonal band were damaged after STREP treatment. In the same brain sections of severely affected STREP-treated rats the lateral ventricles were sometimes fused together, indicating that the septum was greally reduced in size because of the extensive cell loss (see Fig. 6, right column). Gliosis was observed in the corpus callosum and anterior commissure. Posterior to the injection site of STREP, the paraventricular nuclet of the thalanus and fimbria were damaged. In addition, the hippocampus of severely affected STREP-treated rats showed damage (involving cell loss) in the dentate gyrus, CA3 and CA2.

\section{cGMP imwunocytochemistry}

cGMP immunocytochemistry showed that under basal conditions in only a few cell somata and fibers in slices of the frontal cortex there was cOMP immunoreactivity (Fig. 7A). L-NAME incubation had no visible effect on the (already low) basal intensity of cGMP immunostaining (Fig. 7B). Incubation with STREP or SNP greatly increased the intensity of basal cGMP immunostaining in slices of the frontal cortex as is reflected in the increased number of fibers stained after STREP incubation (Fig. 7C), while SNP incubation even resulted in an increase in the number of cell somata stained (Fig. 7D). After incubation of slices with STREP or SNP together with L-NAME, no change in the intensity in CGMP immunostaining could be observed (see Fig. $7 D$ and $F$ ).

\section{DISCUSSION}

Morphological examination of the brain of middle-aged rats that had received an i.c. injection of STREP, revealed, using GFAP inmmnostaining staining, gliosis and extensive cell loss near the lateral and third ventricle, especially in the septum and striatum. Another structure which was 
affected by STREP was the hippocampus. These morphological findings were in agrement with our previous abservations $[47,55]$ and biochemical measurements 448 . The behavior in the open field and shuttie box was affected by STREP treatment, however to a lesser extent than expected [ct. 5]. In contrast with our previous studies we found no effect of STREP treament on spatial discrimination learning in the Morris water escape task [4, 47). This can be attributed to the lower dose of STREP used in the present study $(1.25$ versus $1.5 \mathrm{mg} / \mathrm{kg})$. L-NAME treatment was not protective against STREP which suggests that a possible excitotoxic mechanism as a result of a metabolic inhibition, does not involve NO synthase. Athough SNP administration had no behawioral and morphological effects at all, the involvement of NO liberated from STREP in neuronal damage cannot be excluded.

\section{Body werghts and water consumption}

STREP treatment did not affect the body weights of rats. This has also been found in a previous study in which we used Wistar rats instead of Lewis rats [48]. However, in that study neither an effect was found on the water consumption after STREP treatment, whereas in the present study STREP-treated rats had an increased water intake. L-NAME had no effeet on the water consumption of STREP-treated rats. Administration of SNP did not change the water consumption of control rats.

The integrity of the septal area, mainly its lateral part, is very important for the control of water intake and it has been found that septal lesions increased the water consumption of rats le.g. 251. The septum, especially its bateral nuclei, was affected in all the STREP-treated rats, which would explain the increase in their amount of water consumption. In addition, other brain regions like for instance the peri- and paraventricular preoptic-hypothalamic region and the subfornical organ, which are part of the circuitry subserving the control of water intake [0.g. 33], were damaged after STREP-treatment. Lesions in these regions would decrease the water intake and our data do not exclude the possibility that these regions influenced the water intake after STREP treatment. Damage in the latter regions and the septum could antagonize each others effects on drinking, which has indeed been demonstrated for lesions of the subfornical organ and septum [25]. It might be assumed that the water consumption of STREP-treated rats with most newronal damage, and therefore most likely involving all regions of water intake control, is not affected. However, inspection of the individual drinking data rewealed that just these rats had the highest water intake. This indicates that the effect of STREP on drinking can solely be explained by the damaged septum.

In the study with Wistar rats we found indications that the septum of these rats was less susceptible for STREP then the septum of Lewis rats. Therefore, the lack of a drinking effect in Wistar rats after STREP treatment might be due to a too small danaging effect on their septum.

\section{Behavior}

The open field provides occupancy mensures of which a decrease in the rotal time spent in the comer squares when aggregated over consecutive open field sessions, is considered as reflecting a lower level of anxiety [58]. The total number of line crossings in the open field provides a measure of horizontally directed locomotor activity (or locomotion [39l). An increase in the aggregated horizontal locomotor activity is assumed to reflect a lower levell of anxiety 158]. There was a (negative) correlation between the time spent in the comer squares and the number of line crossings as could be expected from previous studies, indicating that these measures are indices of the same trait of anxiety and, hence, do reflect anxiety-related behavior [49. 5\%). This implicates that the corners of the open field are the safest areas for the rats since they spert most of the time 
MINIMUM DAMAGE

MAXIMUM DAMAGE
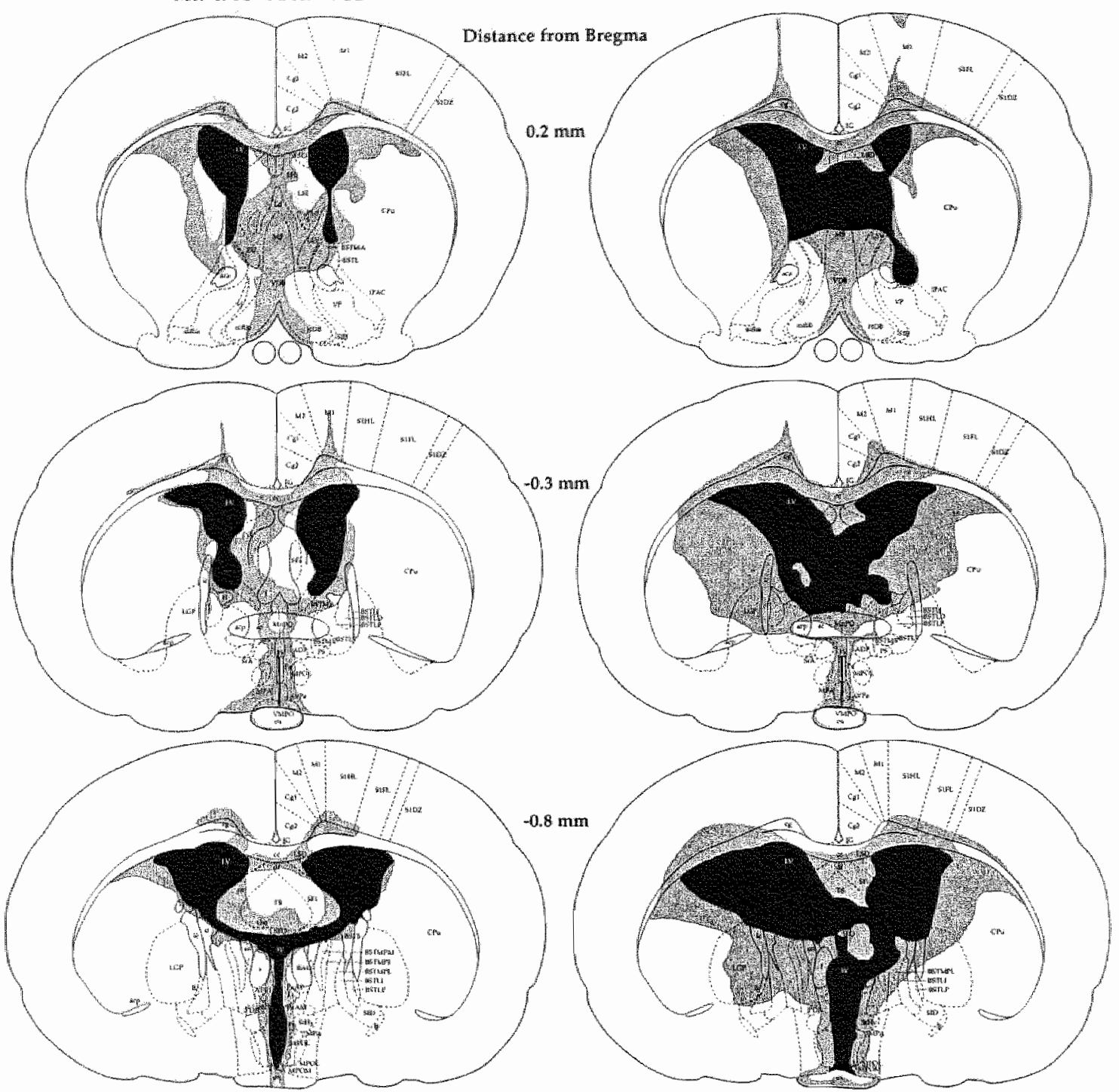

FIG. 6. Illustration of brain sections showing the animal with the minimum (left column) and the one with the maximum (right colurnn) extent of STREP-induced neuronal damage (gray area) as could be deduced from the GEAP immunostaining. Brain section are shown at 0.5 -mm intervals ranging from 0.2 to $-2.3 \mathrm{~mm}$ anterior from the bregna, that is $-1.0101 .5 \mathrm{~mm}$ from the site of injection $(-0.8 \mathrm{~m}$ manterior from bregma). Adapted from Paxinos and Warson [45].

there. We found statistical, though weak, evidence for a decrease in the time spent in the corner squares after STREP treatment, which was at the cost of the time spent in the center squares. This 


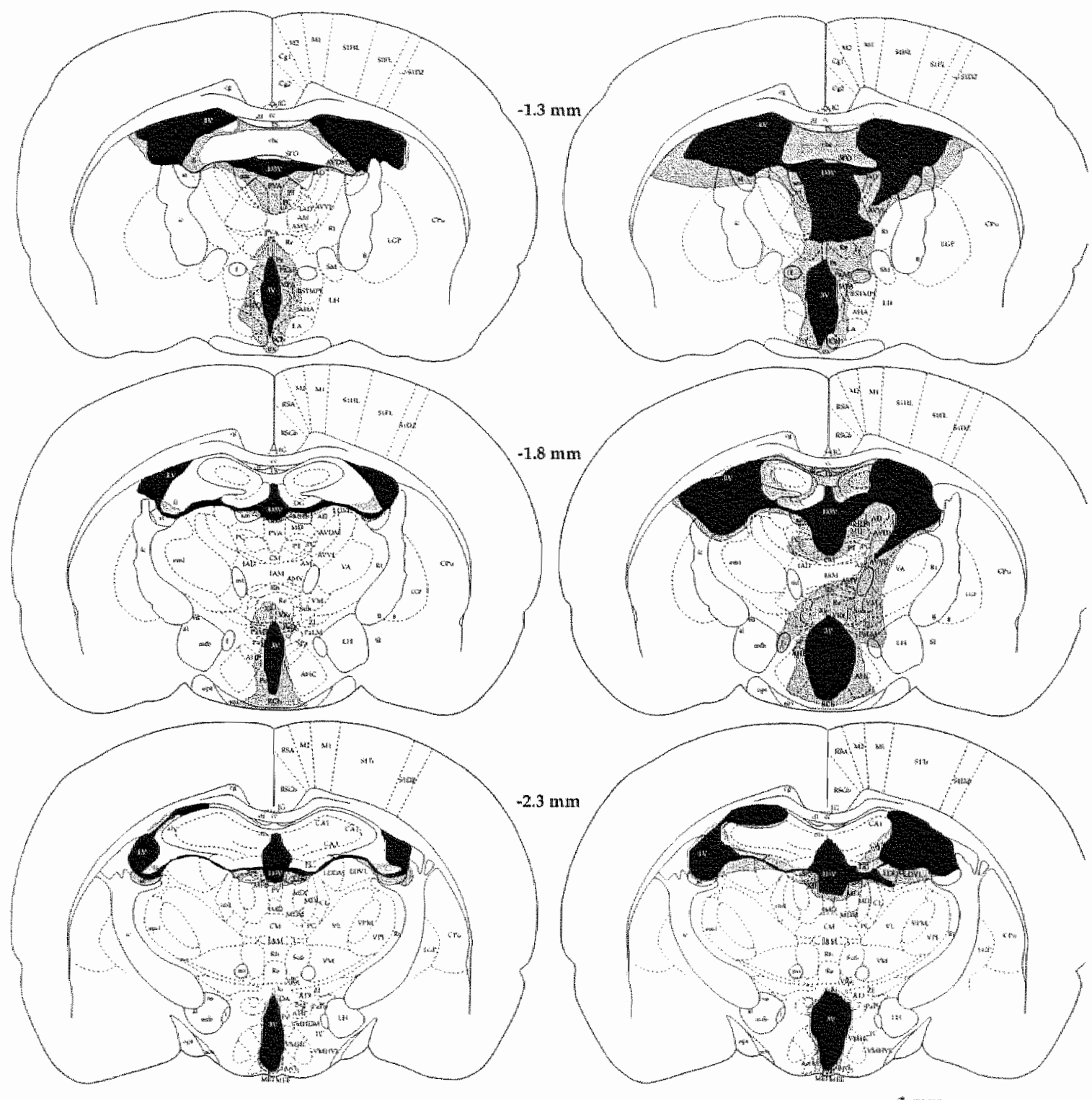

$1 \mathrm{~mm}$

FIG. 6. Continued.

finding is in agreement with our previous findings in the open field (for both middlewged [urpublished datal] and old (5) rats). However, the STREP effect was more prominent then, as was evident from the finding that the rats spent the same amount of time in all compartments, i.e. comers, walls, and center of the open field.

It has been reported that rats with hippocampal lesions have a decreased level of anxiety, i.te, a lack of knowledge of fear [39]. Our accupancy measures suggest that the hippocampal function 

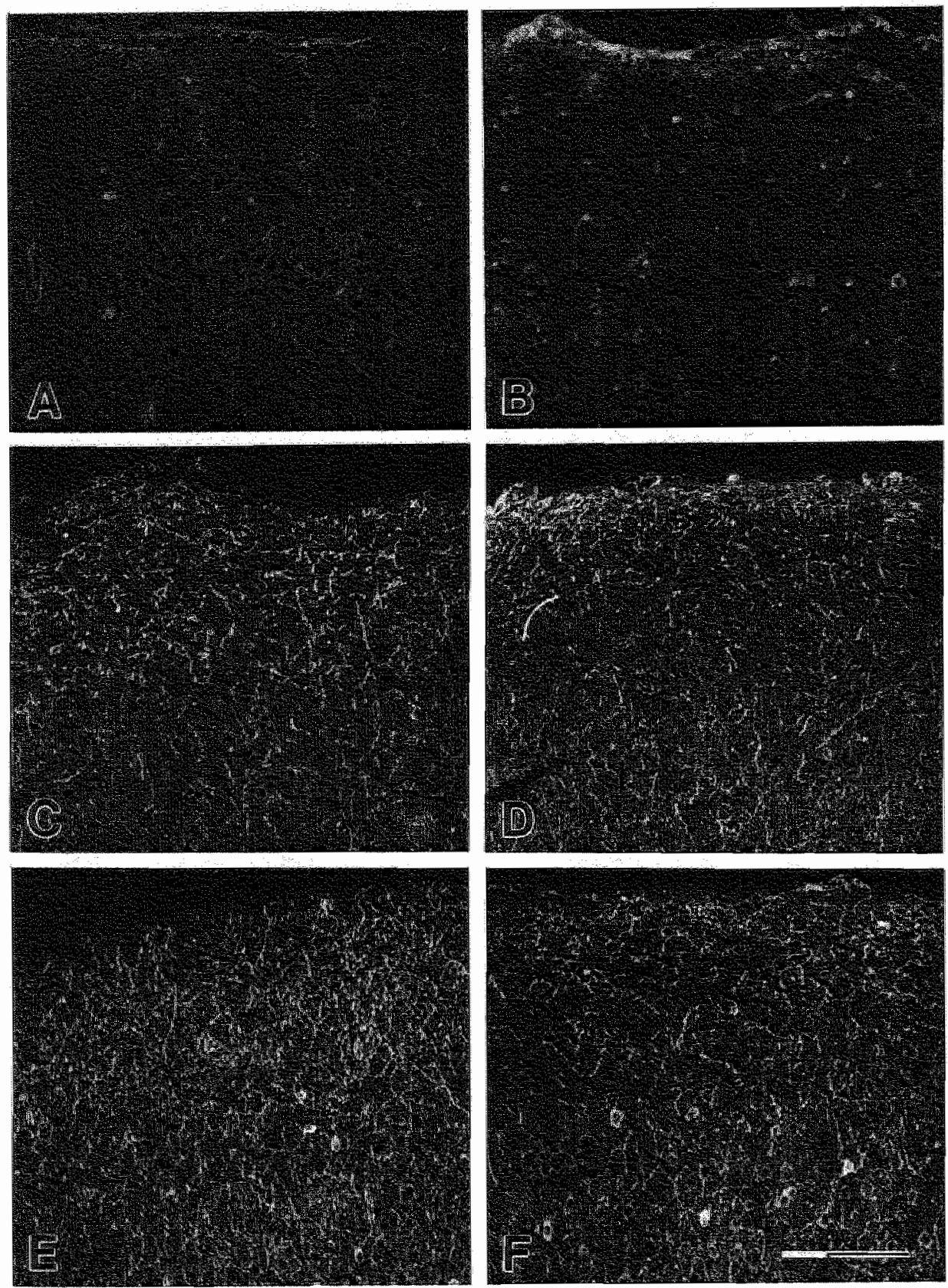

FIG. 7. Cyclic GMP immunostaining in the frontal cortex illustrating $\mathrm{NO}$-mediated cyclic GMP production by soluble guanylyl cyclase. Basal cyclic GMP immunostaining (A) and cyclic GMP immunostaining in the presence of $0.1 \mathrm{mM}$ L-NAME (B), $10 \mathrm{mM}$ STREP (C), $10 \mathrm{mM}$ STREP and $0.1 \mathrm{mM}$ L-NAME (D), $0.1 \mathrm{mM}$ SNP (E), or $0.1 \mathrm{mM}$ SNP and $0.1 \mathrm{mM}$ L-NAME (F). Scale bar is $50 \mu \mathrm{m}$. 
necessary for open field behavior, was affected by STREP. The other measure of anxiety, that is horizontal locomotor activity, was, howewer, not affected, but this is in accordance with our previous studies (for both middle-aged [unpublished data] and old [5] rats). The number of rearings provides a measure of vertically directed locomotor activity (or exploration [39]), although it did not reflect anxiety-pelated behavior since it did not correlate with the other measures of anxiely. The number of rearings was not affected after STREP treatment which is in contrast with its decrease as found in our previous studies (for both middle-aged [unpublished data] and old 6.5] rats).

STREP treatment did not affect the spatial discrimination learning in the Morris water escape task. However, in previous sudies spatial discrimination leaming in this task was impaired [4, 47]. Spatial discrimination learning, also referred to as place learning, is highly dependent on the integrity of the hippocampus [34]. Therefore our data suggest that the hippocampal function, as far as necessary for spatial discrimination in the Morris water task, of the STREP-treated rats was still intact in the present study.

The two-way active avoidance learning of STREP-treated rats was affected, although to a much lesser extent again as found in the previous studies (for both middle-aged funpublished data] and old [5] rats). Because there was no difference in shock sensitivity between the experimental groups, this effect on two-way active avoiclance performance in the shuttle box of STREP-ireated rats, was not influenced by an effect on the sensitivity to the electrical shock. The finding that STREP treatment seemed to improve two-way active avoidance learning has previously been explained with an impaired hippocampal function [see 3,5,40]. Briefly, rats with a normal hippocampal function can apply both place and cue strategies in avoidance learning. If a rat uses a place strategy int he two-way active avoidance task, it may experience a conflict situation because it is shocked in both parts of the apparatus. This conflict situation retards avoidance learning. If a rat does not use the maladaptive place strategy because of an impaired hippocampal function, it will use the cue strategy and will therefore not experience this conflict situation which, as a consequence, facilitates acquisition of the task. However, place learning as measured in the Morris water escape task of the same STREP-treated rats was not impaired. Perhaps because the damaging effect of STREP on the hippocampus was not large enough to affect the performance in the Morris water escape task. However, this remains highly speculative.

Taken together, the behavior of the STREP-treated middle-aged rats was affected to an much lesser extent when compared to our previous findings. This suggests that the hippocampal function was also much less affected by STREP. In addition, it was found that L-NAME treament had no effect on the behavior of STREP-treated rats in any of the tasks. SNP administration had no effect on the performance of rats in any of the behavioral tasks.

\section{Morphalogy}

The immunocytochenical GFAP staining revealed that neuronal damage was mainly located in peri- and paraventricular regions like the septum, striatum and hippocampus. These findings are in line with those of a previous study in which GFAP was measured semiquantitatively 448 . The cause of the damage to the hippocampus, or impairments in its functioning, may be related to an impaired innervation, since the dorsal fonix and fimbria were found to be damaged after STREP treatment. Moreover, the nost severely affected STREP-treated rats had a damaged dentate gyrus and $\mathrm{CA}$, through which information enters the hippocampus normally. Another cause of hippocanpal imparments may be related to an impaired septum, since the major innervation of the hippocampus, which goes via the fimbria and dorsal fornix, originates in the (medial) septum [44]. This is in accordance with the finding that the septum of all STREP-treated rats was damaged. 
In addition, the STREP-induced damage to the nuclei of the vertical diagonal band could have also contributed to a damaged/mpaired hippocampus, since it also innervates the (mainly ventral) hippocampus.

In a previous study with Lewis rats we have found that the hippocampal damage may be related to the septal damage after STREP-treatment [55]. However, in another study with Wistar rats we have found that probably only direct damage to the hippocampus itself is responsible [48]. This discrepancy in results was probably due to differences in the strain of rats used; Perthaps the septum of Wistar rats is less susceptible for STREP than the septum of Lewis. In the present study there is an indication that damage to the hippocampus was caused directly via the ventricles, since also the caudate putamen region located opposite from the hippocampus at the other site of their common ventricle, was affected. However, the contribution of a damaged septum cannot be ruled out either, although this might mainly apply to the worst affected STREP-treated animals in which besides septal damage, also severe hippocampal damage was found. Nevertheless, despite what the cause of hippocampal danage/impairment was, the damage to the hippocampus and more in particular its impaired functioning in STREP-treated rats appeared to be rather mild as could be gathered from the behavioral data in comparison with previous studies.

Although a hemotoxy-eosin staining did not show a gross effect on brain morphology in some STREP-treated rats, GFAP staining revealed that gliosis, which is indicative of neuronal damage, occurred. These rats can, therefore, be referred to as being less severely affected STREP-treated rats in contrast with more severely affected rats having not only gliosis, but also extensive cell loss. These morphological findings indicate that the susceptibility to STREP treatment was not the same for all rats. This has already been established for behavioral and biochernical markers in previous studies $[4,5,4,7,48$. . Therefore, the individual susceptibility appears to be a characteristic feature of STREP treatment. L-NAME treatment had no effect on the brain morphology of STREP-treated rats. Also no effects on the morphology of the brain were found after SNP administration.

\section{STREP}

As has been mentioned above, the effect of STREP on behavior was less pronounced than in our previons studies $\{4,5,47\}$. This might be due to the lower dose of STREP used (1.25 instead of 1.5 $\mathrm{n} \mathrm{g} / \mathrm{kg}$ ). Nevertheless, this lower dose of STREP caused substantial neuronal damage. However, behaviorally the effects were rather wak compared to the previous studies with a higher dose. This suggests that neuronal damage may have been larger in the previous studies using a thigher dose of STREP. A model of neurodegeneration in which the morphology of the brain structures remains grossly intact, has the most relevance, especially when one wants to measure cognitive decline. Based on the above findings it is more useful to inject the high dose of STREP since this resull in an more pronounced cognitive deficit. However, it is better to use middle-aged Wistar rats instead of Lewis rats. This because the neuronal damage in Wistar rats after STREP treatment seems to be less than in Lewis rats, since their septum weight was not affected, while their spatial discrimination leaning was affected still $[47,48,55]$.

It has been found that i.c.v. injected STREP reduced the central glucose metabolism which, assuming that STREP would have the same effects in the central nervous system as in the periphery, may be due to the inhibition of a brain insulin producing system and/or a decreased insulin receptor function 136.371 . More recently, it has been suggested that the central effects of STREP ate mainly mediated via the latter aspect as a result from a reduced phosphorylation, and thus activity, of the insulin receptor tyrosine kinase in the brain $[15.46$. However, besides that neurodegenerative effects may be due to an inhibition of the energy metabolism, oxidative 
danage may be involved (also), since a probable mechanism of action of STREP could be that it induces oxidative stress (e.g. $\mathrm{H}_{2} \mathrm{O}_{2}$ generation), which results in for instance DNA damage [ef. 6. 16. 53.

The neurodegenerative damage due to inhibition of the energy metabolism by STREP may be attributed to an excitotoxic metabolism [0t. 1.57]. Several in vitro studies have found indications of the involvement of NO produced by NO synthase, in an excitotoxic metabolism $[11,26,27]$. Therefore, it may be assumed that a metabolic/excitotoxic insult of STREP could involve NO. On the other hand, it is also possible that $\mathrm{NO}$ is directly involved in oxidative damage after STREP treatment, since it has been demonstrated in vitro that STREP may liberate NO [29,54.56], Oxidative damage (DNA damage or lipid peroxidation) can be caused by NO itself $[61]_{\text {, but also by } \mathrm{NO}}$ converted compounds like for instance ONOO- [2, 52].

Based on the above mentioned findings it may be assumed that NO plays a role in STREP-induced neurodegeneration. Thus, NO might be associated with the results of some former studies with STREP in which for instance oxidative DNA damage was found. However, it was found that DNA damage was caused by $\mathrm{H}_{2} \mathrm{O}_{2}$ of which the production was stimulated by STREP. whereas on the production of its $\mathrm{O}_{2}$ - intermediate no effect was found [53]. An increased $\mathrm{O}_{2}$ production might have been expected since a source of $\mathrm{O}_{2}$ - radicals is the electron transport chain, and especially an impaired one, which could result from an inhibition of complex IV of the electron transport chain by NO liberated from STREP [7. The latter might also explain a decrease in central energy metabolism. But these two possibilities are not relevant since no effect of STREP on $\mathrm{O}_{2}^{-}$production has been reported. Consequently, how $\mathrm{H}_{2} \mathrm{O}_{2}$ is generated or how STREP stimulates $\mathrm{H}_{2} \mathrm{O}_{2}$ generation remains unknown. Also, other explanations for neuronal damage with NO after STREP treatment may be possible. Very interesting in this context is the above mentioned suggestion that the metabolic defect after STREP treatment is due to an impaired insulin receptor via inhibition of its tyrosine kinase activity [15. 46]. This because it has recently been found that $N O$ inhibited several tyrosine kinase activities by $S$-nitrosylation of the receptor proteins $[17,50]$. All these findings urge the need to examine the possible involvement of NO in our STREP model, as has been done in the present study.

\section{NO synthase}

The neurodegenerative damage after STREP treatment could be caused by an excitotoxic mechanism or also referred to as NMDA-mediated neurotoxicity, which might involve NO produced by NO synthase [or. 19]. This has been corroborated by several in vitro and in vivo studies demonstrating the involvement of NO in NMDA-mediated neurotoxicity $111,22.26,27,30,511_{\text {, }}$ Moreover, it has been found that inhibition of NO synthase was neuroprotective on both behavior and brain morphology in in vivo models of ischemia, in which an excitotoxic mechanism is probably responsible for the neurotoxic insult $[9,10,38]$. Therefore, we administered L-NAME after STREP reatment, since this might have had a protective action. The dose and administration schedule of L-NAME were assumed to result in a continuous maximal inhibition of central NO synthase for at least 5 days after STREP administration [cr. 9, 24].

In the present study no effects of L-NAME were found on the behavior and brain morphology of STREP-treated rats. This indicates that L-NAME was not protective after STREP treatment. This might be due to the peripheral administration of L-NAME, while it might have been better to use a central one, which has been reported to be protective after central NMDA receptor stimulation in vivo [22, 51]. The lack of an effect of L-NAME could also be due to the possibility that NO synthase does not play a (major) role in NMDA-mediated neurotoxicity, or at 
least not in a STREP-induced excitotoxic mechanism. This is supported by our in vitro finding that L-NAME did not prevent the NO-mediated CGMP production after STREP administration which indicates that NO formation after STREP administration was independently of NO synthase. Furthermore, some in vitro experiments have provided evidence against a (primary) toxic action of NO in NMDA-mediated neurotoxicity because NO synthase inhibitors did not protect after NMDA receptor stimulation $[14,43]$. Furthermore, there is also an in wiwo study showing that NO is not likely to be involwed in NMDA-mediated neurotoxicity [23]. Finally, it might even be suggested that an excitotoxic mechanism does not play a major role in mediating neurotoxicity of STREP.

Systemic administration of STREP induces diabetes mellitus in rodents [e.g. 8. 32]. It has been found that NO synthase inhibition with L-NAME suppressed the development of diabetes in STREP-treated rodents as was, for instance, evident from the decreased glycemia levels in diabetic animals after treatment with L.NAME $[20,28]$. However, it has also been found that L-NAME treatment inhibition failed to protect (completely) against STREP-induced diabetes as glycemia values returned to diabetic levels two weeks after withdrawal of L-NAME [42]. Thus, assuming that STREP has centrally the same mechanism as peripherally, the L-NAME treatment might have been not long enough. But taken together, the present study does not favor a role of NO synthase in a possible STREP-induced excitotoxic insult. Hence, it is more likely that STREP was neurotoxic by liberation of the reactive $\mathrm{NO}$ (see below).

\section{NO}

Neurodegenerative damage after i.c.v. STREP treatment could also result from its liberated NO [cl. 29, 54, 56]. Therefore, we administered i.c.v. the NO donor SNP. The dose of SNP was based on an in vitro study in which STREP and SNP had the same quantitative effect on cGMP formation [56]. In addition, our dose of SNP $(10 \mu \mathrm{g})$ was almost the same as one $(8.6 \mu \mathrm{g})$ used in an in vivo study in which a neurodegenerative effect was found after intrahippocampal SNP administration [30]. The latter study also found that a three times higher dose of SNP was already lethal, which indicates that the application window of SNP doses is rather narrow. In the present study no effect at all on both behavior and brain morphology was found in SNP-treated rats. This might be stributed to the dose of SNP, which could have been too low still. Also the fact that we used an i.c.v. injection of SNP resulted in a further reduction of the concentration when compared to a local injection.

Until now there are only few experiments which studied behavior after a central administration of SNP, or another NO donor. This is in contrast with the large number of behavional studies after NMDA receptor stimulation. There are two studies in which SNP was injected centrally either into the nucleus basalis magnocellularis $[23]$ or i.e.v. $[21]$, and subsequently behavior was studied. In these two studies also no or only few behavioral and cytotoxic effects were found. The study which found no effects at all, might be explained by the low dose of SNP $(0.4 \mu \mathrm{g})$ injected into the nucleus basalis magnocellularis, which was 25 times lower than in our study. However, the study with i.c.v. injection of SNP used a dose more than 50 times higher (524 $\mu \mathrm{g})$ than in our study, but all rats died within $2-3 \mathrm{~h}$ which questions the physiological relevance of this dose. Hence, although our in vitro experiment showed that SNP increased cGMP formation which indicates that SNP donates NO, it remains largely unknown whether such NO after in wivo SNP injection is directly responsible for neurotoxicity resulting in neurodegeneration.

In conclusion, STREP may decrease the central metabolism of glucose or it may induce oxidative stress by liberation of nitric oxide. The impaired energy metabolism after STREP treatment may induce an excitotoxic mechanism, which may lead to the stimulation of NO 
synthase and, consequently to the synthesis of NO. The present sudy demonstrated that the neurodegenerative damage after STREP treatment rnay be still a result of the decrease in the central energy metabolism, but without NO synthase involvement. However, the inwolvement of NO liberated from STREP in neutodegenerative damage cannot be excluded.

\section{REFERENCES}

1. Beal M.F., Hyman B.T. and Koroshetz W. (1993) Do defects in mitochondrial energy metabolism underlue the pathology of neurodegenerative diseases? Trends Newrasci. 16, $125-131$.

2. Beckman J.S.. Ischiropoulos H., Zhu L., Van der Woerd M., Smith C. Chen J., Harrison J., Martin J.C. and Tsai M. (1992) Kinetics of superoxide dismutase-and iron-atalyzed nitration of phenolics by peroxymitite. Arch. Biochem. Brophys. 298, 438-445.

3. Black A.H., Nadel L. and OKeefe J. (1977) Hippocampal function in avoidance learning. Psychol. Bull. 84, $1107-1129$

4. Blokland A. and Jolles J. (1993) Spacial tearning deficit and reduced hippocampal ChA $\mathrm{T}$ activity in rats after an iow injection of streptozotocin. Pharmacol. Biochem. Behow. $44,491-494$.

5. Blokland A. and Jolles J. (1994) Behavioral and biochemical effects of an icwiection of streptozotocin in old Lewis rats. Pharmacal. Biochem. Behav. 47, 833-837.

6. Bolaff J.L., Nagamatsu S., Harris J. and Grodsky G.M. (1987) Protection by thymudine, an inhibitor of polyadenosine diphosphate ribosylation, of streptozotocin inhibition of insinin secretion. Emdocrinology? $120,2117-2122$.

7. Bolaños J.P., Peuchen S., Heales S.J.R., Land J.M. and Clark J.B. (1994) Nitric oxidemedianed inhibition of the mitochondrial respiratory chain in cultered anstrocyles. J. Mewroche

8. Burvin $\mathbb{R}_{\text {... }}$ Armoni M. and Karnieli E. (1994) In vivo insulin action in normal and streptozotocin-induced diabetic rats. Physiol. Behav. 56, 1.6 .

9. Caldwell M. O'Neill M., Earley B. and Leonard B. (1994) NG-nitro-Larginine protects against ischeniainduced increases in nitric oxide and hippocanpal neuro-degeneration in the gerbil. Ewr. J. Phamacol. 260 , 191-200.

10. Chalimoniuk M. and Strosznajder 1. (1995) Brain ischemia-teperfusion injury activates neuronal, constitutive form of nitric oxide synthase. Molecular action of nitric oxide. Eur, J. Newrosci. Suppl. 6, $26,15.28$.

11. Dawson V.L. Dawson T.M., London E.D., Brede D.S and Snyde S.H (1991) Nitric oxide mediates glutamate neurotoxicicy in primary cortical cultures. Proc. Nat Acad. Sci. U.S.A. 88, 6368m6371.

12. De Vente J. and Steinbusch H.W.M. (1992) On the stimulation of soluble and particulate guanylate cyclase in the rat brain and the involwement of nitric oxide as situdied by cCMP immunocytochemistry. Acra Histochem. 92, $13 \times 38$.

13. De Vente J., Young H.M. and Steinbusch H.W.M. (1996) Immunohistochemical visualization of cyclic nucleotides. In Methods in Nentoscience (ed. Manes M. D.), Vol. 3., pp. 68-79. Academic Pross, San Dirgo, CA.

14. Demerte-Pallardy C. Lonchampt M.-O., Chabrier P.-E and Braquet P. (1991) Absence of implication of L

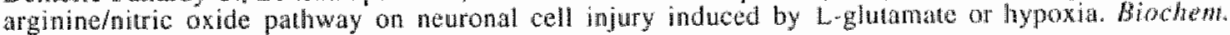
Biophys. Res. Comm. $\mathbb{1 8 1}$, 456-464.

15. Duell R. Schröck H., Kuschingky W, and Hoyer S. (1994) Intrucerebroventricular injection of streptozotocin induces discrete local changes in cerebral glucose ubitization in rats. Int. A. Devl Neurosci. 12, $737-743$.

16. Dulin W.E. and Wyse B.M. (1969) Sudies on the ability of compounds to block diabetogenic activily of streptozotocin. Diaberes 18,459 .

17. Estrada C., Gomez C. Martin-Nieto J. De Frutos T. Jimenez A. and Villalobo A. (1997) Nitric oxide rewersibly inhibits the epidernal growth factor receptor tyrosine kinase. Biochen. $J .326,369.376$.

18. Gallagher M. Burwell $R$. and Burchinal $M$. (1993) Severity of spatial learmimgangme in ang: Development of a learning index for performance in the Morris water maze. Behow. Neworki. 107.618626.

19. Garthwaite I. (1991) Glutamate, nitric oxide and cell-cell signalling in the nerwous system. Trends Newrosci. 14, 60-67.

20. Gonzalez E. Rosello-Catafau J., Xabs C., Jawerbaum A., Novano V., Gomez G. Golpi E. and Gimeno M.A. (1997) Influence of nitric oxide symthase and kinim antagonists on metwbolic paraneters in chronic streptozotocin-induced diabetes mellitus Prositaglandins $53,321-336$.

21. Cross P.M., Weaver D.F., Bowers R.J., Nag. S. Ho L.T., Pang J.J. and Espinosa F. I. (1994)

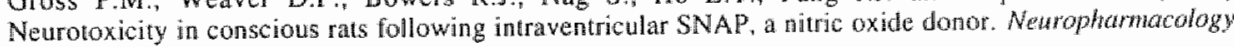
$33,915-927$.

22. Hammer B. Davis Parker W, Jr and Bennett $J . P .(1993)$ NMDA receptors increase OH radicals in wivo by 
using nitric oxide synthase and protein kinase C. NeuroReport 3, 72-74.

23. Harrington C.A. Mobley S.L. and Wenk G.L. (1994) Nitric oxide formation does not underlie the memory deficits produced by ibotenate injections into the nucleus basalis of rats. Behov. Neurosci. 108, 277-283.

24. Wadecola $C, X u X$. Zhang F. Hu J. and El-Fakahany E.E. (1994) Prolonged inhibition of brain nitric oxide synthase by short-1erm systemic atministration of nitro-L-arginine methyl ester. Neurochem. Res. 19, 501505.

25. Iovino $M$. and Steardo $L$. (1985) Thirst and vasopressin secretion following central administration of angiotensin II in rats with lesions of the septal area and subfornical organ. Neuroscience 15, 61-67.

26. Izumi Y., Benz A.M., Clfford D.B. and Zorumski C.F. (1992) Nitric oxide inhibitors attenuate N-methyl. D-aspariate excilotoxicity in rat hippocampal slices. Newrosci. Lett, 135, 227.230,

27. Izumi Y., Benz A.M. Clifford D.B. and Zorumski C.F. (1993) Neurotoxic effects of sodium nitroprusside in rat hippocampal slices. Exp. Neurol. 121, 14-23.

28. Kolb H., Kiesel U., Kroncke K.D. and Kolb-Bachofen V. (1991) Suppression of low dose streptozotocin induced diabetes in mice by administration of a mitric oxide synthase inhibitor. Life Sci. 49, PL213-217.

29. Kwon N.S. Lee S.H., Choi C.S., Kho T, and Lee H., S. (1994) Nitric oxide generation from streptozotocin. The FASEB Journal $8,529-533$.

30. Loiacono R.E. and Beart P.M. (1992) Hippocampal lesions induced by microinjection of nitric oxide donor nitroprusside. Eur. J. Phamacol. 216, 331-333.

31. Markerink-van Itersum M., Sicinbusch H.W.M. and De Vente J. (1997) Region-specific developmental patterns of artial natriuretic factor-and nitric oxide-activated guanylyl cyclases in the postnatal frontal rat brain. Neuroscience 78, $571-587$.

32. Mordes J.P. and Rossini R.A. (1981) Animal models of diabetics. Am. J. Med. 70, 353-360.

33. Mordes R., Garcia del Rio C., Bolanos J. and Mora F. (1986) Adjpsia-polydipsia induced by simultaneous lesion of the medioventral septurn and anteroventral third ventricle area in the rat. Brain res. Bull. 17, 823828

34. Morris R.G.M., Garrard P., Rawlins J.N.P. and O'Keefe J. (1982) Place navigation is impaired in rats with hippocampall Jesions. Noture 297,681.683.

35. Murad F., Mittal C.K., Arnold W.P., Katsuki S. and Kimura H. (1978) Guanylate cyclase: Activation by aride, nutro compounds, nitric oxide, and hydroxyl radical and inhibition by hemoglobin and myoglobin. Adv. Cyclic Nucl. Res. 9, 145-158.

36. Nitsch R. and Hoyer S. (1991) Locall action of the diabetogenic drug, streptozotocin, on glucose and energy metabolism in the brain contex. Newrosci. Lett. 128, 199-202.

37. Nitsch R., Mayer G. and Hoyer S. (1989) The intracerebroventriculary streptozotocin-treated rat: Impairment of cerebral glucose metabolism resembles the alterations of carbohydrate metabolism of the brain in Alzheimer's discase. J. Neurat Trans. 1, 109-110.

38. Nowicki J.P., Duval D., Poignet H. and Scatton B. (1991) Nitric oxide mediates neuronal death after focal cerebral ischemiat in the mouse. Eur. J. Pharmacol. 204, 339-340.

39. O'Keefe J. and Nadel L. (1978) The hippocampus as a cognitive map. Clarendon Press, Oxford.

40. Okaichi $Y$. and Okaichi $\mathbb{H}$. (1994) Effects of fimbria-fornix lesions on avoidance tasks with temporal elements in rats. Physiol. Behav. 56, 759-765.

41. Ossenkop K.-P. and Mazmanian D.S. (1985) The principle of aggregation in psychobiological correlational research: An example from the open-field test. Aniwn. Learn. Betav. 13, 339-344.

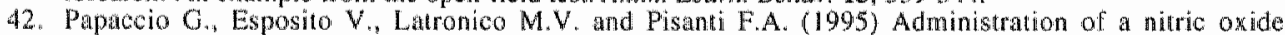
synthase inhibitor does not suppress low-dose streptozotocin-induced diabetes in mice. Int. J. Pancreatol. 17. $63-68$.

43. Pauwels P.J. and Leyson J.E. (1992) Blockade of aitric oxide formation does not prevent glutanate-induced neurotoxicity in neuronal cultures front rat hippocampus. Neurosci. Letr. 143, 27-30.

44. Paxinos G. (1994) The rat nerwous system. 2nd edn., Academic Press, San Diego.

45. Paxinos $\mathrm{G}$. and Watson $\mathrm{C}$. (1996) The rat brain in stereotaxic coordinates. compact 3nd edn. . Academic Press, San Diego.

46. Plaschke K and Hoyer S. (1993) Action of the diabetonic drug streptozotocin on glycolytic and glycogenolytic metabolism in adult rat brain contex and hippocampus. Int. J. Devl Neurosci. 11, 477-483.

47. Prickaerts J., Blokland A., Honig W., Meng F. and Jolles J. (1995) Spatial discrimination learning and choline acetyltransferase activity in streptozotocin-treated rats: Effects of chronic treatment with acetyl-L. carnitine. Brain Res. 674, 142-146.

48. Prickaerts J, Fahrig T. and Blokland A. (submitted) Cognitive performance and neurodegenerative markers in septum, hippocampus and striatum of rats after an i.c.v, injection with streptozotocin: A correlation analysis. Eur. J. Neurosci. .

49. Prickaeris J., Raaijmakers W. and Blokland A. (1996) Effects of myocardial infarction and captopril therapy on anxiety related behaviors in the rat. Physiol. Behav. 58, 43-50.

50. Simon D.I., Mullins M.E., Jia L., Gaston B. Singel D.J. and Stamler J.S. (1996) Polynitrosylated proteins: Characterization, bioactivity, and functional consequences. Proc. Natl Acad. Sci. U.S.A. 93, $4736-4741$ :

51. Strijbos P.J.L.M., Knowles R. and Rothwell N.J. (1993) Nitric oxide mediates glutamate neurotoxicity in the rat striatum. Brain Res. Assoc. Abstr. 10, 46, 8.6. 
52. Szabó C., Zingarell B., OConnor M. and Salzman A.L. (1996) DNA strand breakage activation of poly (ADP ribose) synthetase and cellular energy depletion are involved in the cytotoxicity in macrophages and smooth muscle cells exposed to peroxynitrite. Proc. Nat Acad. Sci. U.S.A. 93. 1753-1758.

53. Takasu N., Komiya I., Takayuki A., Nagasawa Y. and Yamada T. (1991) Streptozotocin-and alloxaninduced $\mathrm{H}_{2} \mathrm{O} 2$ generation and DNA fragmentation in pancreatic islets. Draberes $40,1141-1145$.

54. Tamaka Y., Shimizu H., Sato N., Mori M. and Shimomura X. (1995) Involvement of spontaneous nitric axide production in the diabetogenic action of streptozotocin. Pharmacology 50,69-73.

55. Terwel $\mathbb{D}$., Prickaerts J., Meng $F$, and Jolles J. (1995) Brain enzyme activities after intracerebroventricular injection of streptozotocin in rass receiving acetyl-L-carnitine. Eur. J. Phowmacol. 287,65-71.

56. Turk J., Corbett J.A., Ramanadham S., Bohrer A. and McDaniel M.L. (1993) Biochemical evidence for nitric oxide formation from streptozotocin in isolated pancreatic islets. Biochem. Biophys. Res. Comm. $197,1458-1464$.

57. Turski L. and Turski W.A. (1993) Towards an understanding of the role of glutamate in neurodegenerative disorders: Energy metabolism and newropathology. Experimentia 49, 1064-1072.

58. Wan der Staay F.J., Kerbusch S. and Raaijmakers W.G.M. (1990) Genetic correlations in validating emotionality. Behav. Genet. 20,51-62.

59. Wan Eden C.G., Steinbusch H.W.M., Rinkens A. and De Vente J. (1996) Developmental pattern of NADPH-diaphorase activity and nitric oxide-stimulated cGMP immunoreactivity in the frontal rat cortex and its role in functional recovery from aspiration lesions. $d$. Chern. Neuroanat. 10, 279-286.

60. Winer B.J. (1971) Statistical principles in experimental design. 2nd edn., McGraw-Hill. New York.

61. Zhang J., Dawson V.L., Dawson T.M. and Snyder S.H. (1994) Nitric oxide activation of poly(ADP-ribose) synthetase in neurotoxicity. Science $263,687-689$. 


\title{
Acute effects of acetyl-L-carnitine on sodium cyanide- induced behavioral and biochemical deficits*
}

\author{
Jos Prickaerts", Arjan Blokland2, John Bothmer-3, Wiel Hongl, Marjanne Markerink-van \\ Ittersum ${ }^{l}$ and Jelle Jolles 1
}

European Graduate School of Neurascience 'EURON', Department of Psychiatry and

Neuropsychology, and 2Department of Psychology, Maastrich University, The Nerherlands

3 Janssen Research Foundation, International Clinical R\&D, CNS, B-2340 Beerse. Belgiam

\section{ABSTRACT}

In the present study we investigated the effects of acute treatment with acetyl-L-carnitine $(50$ $\mathrm{mg} / \mathrm{kg}$, i.v. $90 \mathrm{~min}$ before the sodium cyanide injection) on a sodium cyanide-induced behavioral deficit in the Morris water escape task. In a first experiment the spatial discrimination performance of the rats was found to be dose-dependently impaired after an i.c.v. injection of sodium cyanide $(2.5$ and $5.0 \mu \mathrm{g})$. Acute treatment with acetyl-L-carnitine was found to increase the behavioral deficit after sodium cyanide. These findings were replicated in a second experiment. Based on these results it can be argued that an acute administration of acetyl-L-carnitine appears to potentiate a sodium cyanide-induced behavioral deficit. An additional in vitro experiment with rat brain synaptosomes showed clear effects of administered sodium cyanide on the energy-dependent incorporation of inositol into phosphoinositides and on the ATP concentration. In witro acetyl-L-carnitine administration had no effect on the sodium cyanide-induced energy depletion. The negative behavioral findings are in contrast with our previously found protective effect of chronic treatment with acetyl-L-carnitine (via drinking water) on the sodium cyanide-induced behavioral deficit. Since chronic acetyl-L-carnithe treatment has fio effect on the phosphoinositide metabolism it was suggested that acetyl-L-camitine may act via the formation of an ATP-independent reservoir of activated acyl groups. Thus, fatty acids as acylated derivatives can be used for reacylation processes during an acute period of energy depletion. However, we have no clear explanation for the discrepancy in behavioral results between the chronic versus acute treatment of acetyl-L-carnitine at present. Funther research is needed to characterize the mechanism of action of acetyl-L-carnitine in relation to sodium cyanide.

\section{INTRODUCTION}

In animal studies it has been found that chronic treatment with acetyH-L-carnitine (ALCAR) prevented an impaired cognitive performance in aged rats [5, 14-16], In human studies it has been Found that the progression of Alzheimer"s disease was significantly reduced in patients chronically treated with ALCAR [27, 30]. Several possible mechanisms of action of ALCAR in the

\footnotetext{
* This study is in press in Neurochem. Int.
} 
brain have been hypothesized 10 explain these neuroprotective effects on cognitive function. Many explanations are based on Lucarnitine of which ALCAR is the acetylated form. Both compounds occur naturally in the brain and play an important role in the transfer of acyl groups across the mitochondrial membrane for subsequent 8 -oxidation [7]. However, this role is of minor importance in the brain, since the brain almost exclusively depends on the oxidation of glucose to meat its energy denands.

ALCAR treatment is thought to be more effective than L-carnitine treatment because it is quite possible that the hydrophobic acetyl moiety of ALCAR improves the ability of the drug to cross the blood brain barrier and to be taken up by neuronal tissue [13]. ALCAR is regarded as a compound which may attenuate meurodegenerative processes by having a beneficial effect on the energy metabolism, viz. wia a stimulation of the use of alternative energy sources, such as lipid substrates or ketone bodies [3, 4, 6. 24, 32, 33].

In a previous study we have demonstrated that chronic treatment with ALCAR (via drinking water) had a protective effect on spatial memory performance in the Morris water escape task after acute sodium cyanide (NaCN) intoxication [81. Cyanide is an inhibitor of the electron chain enzyme complex IV and intravenous (i.w.) administration causes a period of 'histotoxic' hypoxia in the brain [21]. Cyanide does not cause neuronal damage per se [12], and low doses of cyanide are converted within minutes by mitochondrial sulphur transferase into non-toxic sulphur cyanide [17]. We injected $\mathrm{NaCN}$ intracerebroventricularly (i.c.w.) to avoid peripheral systems being affected and found that this treatment impaired spatial memory performance of rats [8]. In addition, the hippocampal phosphoinositide metabolism, which is ATP-dependent, was affected, indicating that energy metabolism was indeed impared after $\mathrm{NaCN}$ administration [8]. However, we also found that chronic ALCAR treatment had no effect on the affected hippocampal energy metabolism of $\mathbb{N a C N}$-treated rats as measured by the energy-dependent phosphoinositide metabolism [8]. This indicates that ALCAR did not enhance the utilization of alternative energy sources during the acute $\mathrm{NaCN}$-induced energy depletion. Consequently, other explanations have been suggested for the protective effect of chronic ALCAR treatment such as the formation of an ATP-independent reservoir of activated acyl groups [see 8 ].

Based on another study [28] we assumed that an acute administration of ALCAR could possibly be even more effective than a chronic ALCAR treatment. Hence, the effects of acute ALCAR administration (i. $v_{\text {. }}$ ) were evalluated on the spatial memory deficit. In addition, the effects of NaCN and ALCAR on the energy metabolism were in witro assessed with rat brain synaptosomes whose energy starus was determined by the measurement of energy-dependent inositol incorporation into phosphoinositides and by the measurement of synaptosomal ATP concentrations.

\section{MATEERIALS AND METHODS}

\section{Behwional procedires}

Animals. All experimental procedures were approved by the local ethical committee of the Maastricht University for animal experiments and met governmental guidelines. In a first experiment twenty-ëght 4-month-old male Lewis rats were used. After spatial discrimination learning (see below) the rats were randomly divided into two groups (control, $n=14 ; \mathrm{ALCAR}, n=14$ ). In a second experiment twenty-mine 3-month-old male Lewis rats were used which were also randomly divided into two groups after spatial discrimination learning (control, $n=14 ;$ ALCAR, $n=15$ ). All animals were housed individually in standard Makrolon cages on sawdusi bedding in an air-conditioned room (about $20^{\circ} \mathrm{C}$ ). They were kept under a $12 / 12 \mathrm{~h}$ light-dark cycle (lights on from $06.00-18.00 \mathrm{~h}$ ). 
Treatment. Two weaks before acquisition of the Morris water escape task, the rats were anesthetized with an intraperitoneal (i.p.) injection of pentobarbital $(60 \mathrm{mg} / \mathrm{kg}$ ) and placed in a stereotaxic frame. Bilateral stainless steel cannulas fouter and inner dianeter 0.65 and $0.30 \mathrm{~mm}$, respectively) were implanted above the lateral ventricles. The cannulas were fixed to the skull with acrylic dental cement (Paladur). The coordinates for injection of $\mathbb{N a C N}$ were: $-0.8 \mathrm{~mm}$ anterior, $1.55 \mathrm{~mm}$ lateral and $-3.8 \mathrm{~mm}$ from the bregma 1261 .

After acquisition of the Mornis water escape ask, the effects of NaCN injection were evaluated (see belowh. Ninely min before the $\mathrm{NaCN}$ injection the rats were lightly anesthetized with ether and an injection (volume 1 ng $/ \mathrm{kg}$ ) of ALCAR (50 mg/kg or $100 \mathrm{mg} / \mathrm{kg}$ ) or saline ( $9 \mathrm{~g} / \mathrm{NaCl}$ ) was given i.v. (peris wena). A pilot study revealed that after 90 min the ether anesthesia, also together with the ALCAR treatment, had no effect on the spatiall (baseline) performance, i.e. this was not different from the asymptotic performance at the end of acquisition. The ALCAR solution was neutralized ( $\mathrm{pH} 7.0$ ) with $0.25 \mathrm{M} \mathrm{NaOH}$. In the first experiment one rat of the control (saline) group died during ether anesthesia. In both experiments a lateral canrula of one rat of the ALCAR group clogged and became useless for $\mathrm{NaCN}$ injections, therefore these rats were excluded from the experiments.

Spatial discrimination leaming. Two weeks after surgery, the rats were trained on a standard spatiat Morris

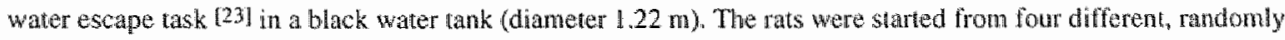
chosen, start positions and irained to find an invisible platform (diameter $11 \mathrm{~cm}$ ) at a fixed position in the water tank, $1 \mathrm{~cm}$ below the surtace of the water. The water temperature was about $22^{\circ} \mathrm{C}$. A rrial lasted until a rat had found the platform or until $60 \mathrm{~s}$ had elapsed. If a rat did not find the platiorm within $60 \mathrm{~s}$, it was placed on the platform for $3 \mathrm{~s}$ and then removed from the water tank. On the first day, the rats were given four trials. The rats were given eight trials on consecutive days until the rats had attained an asymptotic performance.

Efjects of $\mathrm{NaCN}$ injections. After the rats had attained an asymptotic periormance, the effects of $\mathrm{NaCN}$ injections were evaluated. ALCAR or saline was always injected 90 min before the NaCN injection. On day one, the rats received four trials (baseline performance), after which they were injected with NaCN (2.5 $\mu \mathrm{g}$ of salt weight in $2.5 \mu$ per injection site). The rats were given four additional trials, $1,5,10$ and 15 mim after the $\mathrm{NaCN}$ injection. The four possible stat positions were balanced per group during each post-injection trial. On day three, the rats underwent the same procedure as on day one, but now a dose of $5.0 \mu \mathrm{\mu g} / 2.5 \mu \mathrm{\mu l}$ per injection site was administered after the baseline measurement. The same procedure was repeated one week later for both doses. In the second experiment a dose of $100 \mathrm{mg} / \mathrm{kg}$ ALCAR (instead of $50 \mathrm{mg} / \mathrm{kg}$ ) was used in an additional third wheek and on both day one and day three a NaCN dose of $2.5 \mu \mathrm{g} / 2.5 \mu \mathrm{l}$ per injection site was administered.

\section{Biochemical procedures}

Preparation of synaptosomes. For the in vitro experiment we also used adult male Lewis rats (see above). After decapitation the head was immediately immersed in liquid nitrogen for $8 \mathrm{sec}$. All subsequent operations were performed at $0 \times 4^{\circ} \mathrm{C}$. The brain was taken out of the skull rapidly, and the bulbi olfactory, the cerebellum and brainstem were removed. The tissue was homogenized in $0.32 \mathrm{M}$ sucrose, $1 \mathrm{mM}$ EDTA, $10 \mathrm{mM}$ Tris- $\mathrm{HCl}$, pH 7.4, in a total volume 10 times the brain tissue volume by 10 up-and-down strokes of a Poter-Elivehjem Teflon-glass homogenizer (radial clearance $0.125 \mathrm{~mm}, 700 \mathrm{rpm}$ ). The homogenate was spun at $1,000 \mathrm{~g}$ for 10 minutes. After centrifugation of the supernatant at $10,000 \mathrm{~g}$ for 10 minutes, the resuluing crude mitochondrial-synaptosomal pelles was resuspended in homogenization bulfer and then applied to a discontinuous. Ficoll-sucrose gradient as described by Booth and Clark 1101 . The tubes were certrifuged at $100,000 \mathrm{~g}$ for 30 minutes. Myelin and synaptosones banded at the first and second interphases, respectively, with the free mitochondria being pelleted at the bottom. The synaptosomal layer was carefully removed and suspended in homogenization buffer. Affer centrifugation $\left(11,000 \mathrm{~g}, 15 \mathrm{mmin}, 4^{\circ} \mathrm{C}\right)$, the synaprosomal pellet is resuspended in Krebs-Ringer buffer containing $10 \mathrm{mM}$ glucose, $106 \mathrm{mM} \mathrm{NaCl} 4.7 \mathrm{mM} \mathrm{KCl}, 0.1 \mathrm{mM} \mathrm{CaCl}, 1 \mathrm{mM}$ $\mathrm{Na}_{2} \mathrm{HPO}_{4}, 24.9 \mathrm{mM} \mathrm{NaHCO}, 10 \mathrm{mM}$ Hepes, I mM Cynidine, $1.2 \mathrm{mM} \mathrm{MgCl}, \mathrm{pH} 7.4$, salurated with $\mathrm{O}_{2}: \mathrm{CO}_{2}$ $(95 \%: 5 \%)$, in which the synaptosomes were incubated at $37^{\circ} \mathrm{C}$ in a water bath. Protein determination was performed according to the method of Lowry [19].

The number/amount of intact synaptosomes during the incubation was determined by measuring the lotal laciale dehydrogenase ( $\mathrm{LDH}$, a cylosolically located enzyme) activily in the incubation medium after removing the intact synaptosomes by centrifugation. This total LDH activity was compared with the total LDH activity in an incubation mixture in which all synaptosomes were broken by sonication. The dala from inositol 
incorporation measurements and ATP concentration measurements were corrected for this leakage of synaplosomes which could be influenexd by the different treaments. LDH activity was measured by incubating a

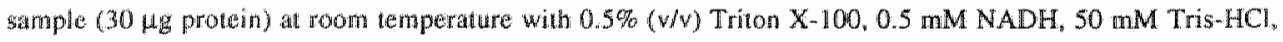
$1 \mathrm{mM}$ EDTA, and $0.25 \mathrm{mM}$ pyruwate, $\mathrm{pH} 7.4$. The rate of disappearance of NADH was measured at $340 \mathrm{~mm}$ against a blank containing no pyruwate. Single determinations for protein, inositol or ATP measurements were always done in triplicate.

$[3 H] m$ mositol incorporation. [3H]-inositol (Amersham) incorporation into phosphatidylinositol (PI), phosphatudylinositol monophosphate (PIP) and phosphatidylinositol bisphosphate (PIP $\mathrm{P}_{2}$ ) in synaptosomes of rat brain was assayed in the Krebs-Ringer buffer as oulined above. $95 \mu l$ of synaptosomes (approximately $250 \mu \mathrm{g}$ protein) were preincubated for $20 \mathrm{~min}$ in oxygenated buffer at $37^{\circ} \mathrm{C}$ in capped tubes. Thereafter, $5 \mu \mathrm{l}[3 \mathrm{H}]$-inositol ( $5 \mu \mathrm{Ci}$ ) was added, and $10 \mathrm{~min}$ later $25 \mu(0.2-20 \mathrm{mM}) \mathrm{NaCN}$ or buffer (control) was added. In case of ALCAR administration, synaptosomes were treated with $0,0.1,1$ or $10 \mathrm{mM}$ ALCAR after $10 \mathrm{~min}$ of preincubation, i.e. 20 min before the $\mathrm{NaCN}$ treatment. The incubation was terminated by the addition of $2 \mathrm{ml}$ ice-cold chloroform/methanol/13 $\mathrm{M} \mathrm{HCl}(200: 100: 0.75$, by vol.). Carrier lipids were added and a biphasic system was obtained by the addition of $0.375 \mathrm{mll} 0.6 \mathrm{M} \mathrm{HCl}$. The upper phase was renowed and the lower phase washed with $1 \mathrm{ml}$ chloroform $/$ methanol $/ 0.6 \mathrm{M} \mathrm{HCl}\left(3: 48: 47\right.$, by vol.). The resulting lower phase was dried under $\mathrm{N}_{2}$ and redissolved in chloroform/methanol/water $(75: 25: 2$, by vol.). Samples were taken for the determination of radioactivity incorporated into total phospholipids. Phospholipids were separated by high-performance thin-layer chromaiography (HPTLC; layer thickness $25 \mu \mathrm{m}$, Merck). Before use, the plates were impregnated with potassium oxalate and activated for $15 \mathrm{~min}$ at $110^{\circ} \mathrm{C}$. The extract was applied to the plate and the lipids were separated with chloroform/acetone/methanol/glacial acetic acid/water (40:15:13:12:8, by vol.). Lipids were visualized with iodine vapor. The spots were scraped from the plate and coumted for radioactivity in a liquid scintillation spectrometer.

ATP concentration measurement. $100 \mathrm{H}$ synaptosomes (approximately $250 \mu \mathrm{Hg}$ protein) were preincubated

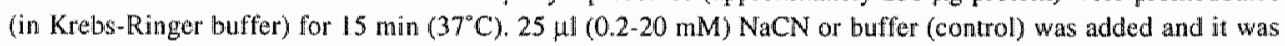
further incubated for another $15 \mathrm{~min}$ (or more). In case of ALCAR treatment, $0,0.1,1$ or $10 \mathrm{mM}$ ALCAR was administered just before the start of the preincubation, i.e. $15 \mathrm{~min}$ before the $\mathrm{NaCN}$ treatment. The incubation was stopped by the addition of ice cold concentrated perchloric acid $(33 \mu \mathrm{l})$. After centrifugation $(10,000 \mathrm{~g}, 5$ min) the supernatant was neutralized by addition of $95 \mu \mathrm{l} 4 \mathrm{M} \mathrm{KOH} / 1.1 \mathrm{M}$ imidazole/0.8 $\mathrm{M} \mathrm{KCl}$. After centrifugation $(10,000 \mathrm{~g}, 5 \mathrm{~min})$ the supernatant was taken off to measure ATP concentration. Assay conditions: for ATP measurement were $100 \mathrm{mM}$ Tris- $\mathrm{HCl}(\mathrm{pH} 7.4), 1 \mathrm{mM}$ glucose, $5 \mathrm{mM} \mathrm{MgCl}, 0.2 \mathrm{mM}$ DTT, 0.03 $\mathrm{mM}$ NADP, $0.5 \mu \mathrm{g} / \mathrm{ml}$ yeast glucose- $6 \%$ phosphate dehydrogenase, $1 \mu \mathrm{g} / \mathrm{ml}$ yeast hexokinase, for $30 \mathrm{~min}$ at $37^{\circ} \mathrm{C}$. The reaction was terminated by puncing the samples on ice. The appearance of NADPH (which is linearly pelated to the original amount of ATP) was measured fluorometrically (exctation at $355 \mathrm{~nm}$, emission at 460 (nm).

\section{Sratisnical analysis}

The mana escape latencies of the pre-injection trisls were averaged and taken as baseline performance for each rat. In order to evaluate whether $\mathrm{NaCN}$ treatment affected the performance of the rats, the escape latency during the trials after the animals had received $\mathrm{NaCN}$ was compared with the baseline tatency using a paired $t$ mlest. Troutment effects between groups were ewaluated using a $t$-test. For each dose of $\mathrm{NaCN}$, the effects of ALCAR treatment on escape latency 1,5 and 10 min after the NaCN injections were also analyzed in a two-factorial (Treatment and Minute) ANOVA with repeated measures over Minute. The biochenical data were analyzed with a Student-Newman-Keuls test.

\section{RESULTS}

\section{Effects of NaCN and ALCAR injections on spatial discrimination}

Spatial discrimination performance. Baseline performance before the $\mathrm{NaCN}$ injection did not differ between the ALCAR-treated rats and the control rats on any occasion in both experiments 
(is $<1.0$ and 1.3 , respectively, n.s.).

NaCN dose $2.5 \mu \mathrm{g}$ - ALCAR dose $50 \mathrm{mg} / \mathrm{kg}$. In the first experiment the pertomance of the control rats did not devate from baseline performance after injection of $2.5 \mu \mathrm{gaCN}(r s<1.6$. n.s.). The escape latency of the ALCAR-treated group deviated from its baseline performance during the first trial after injection, i.e. I. min after $\mathrm{NaCN}$ injection $\left(t_{12}=2.3, \mathrm{p}<0.05\right)$. During the second trial after $\mathrm{NaCN}$ injection, i.e. after $5 \mathrm{~min}$, the ALCAR-treated group only showed a tendency to deviate from baseline performance $\left(t_{12}=2.0,0.05<p<0.1\right)$. Further analysis showed that the escape latencies did decrease during the first three trials after the injection (Minute effect: $F_{2.48}=8.48, p<0.001$; see Fig. 1A). However, there were no differences in escape latency between the control and ALCAR group during the trials after injection of $\mathrm{NaCN}(t s<0.2, \mathrm{n} . \mathrm{s}$. $F$ s $<0.5$, n.s.).

In the second experiment the escape latencies of the control and ALCAR-trested rats deviated from baseline performance during the trials on $\mathbb{1}$ and 5 min after injection of $2.5 \mu \mathrm{Hg} \mathrm{NaCN}$ ( $t \mathrm{~s}>$ 3.3, $\mathrm{p}<0.01$; see Fig. 1C). During the first trial after injection the escape latency of the ALCAR-treated tended to be higher than that of the control group $\left(t_{26}=1.99,0.05<p<0.1\right)$. The decrease in escape latency was the same for both experimental groups during the first three trials after the NaCN injection (Treatment $\times$ Minute effect: $F_{2,52}=1.63, \mathrm{n.s.}$ ).

NaCN dose $5.0 \mu \mathrm{g}$ - ALCAR dose $50 \mathrm{mg} / \mathrm{kg}$. After $5.0 \mu \mathrm{g} \mathrm{NaCN}$ in the first experiment, the escape latencies of the control and ALCAR-treated rats deviated from baseline performance during the trials on 1 and $5 \mathrm{~min}$ after $\mathrm{NaCN}$ injection $(t s>4.5, p<0.001$; see Fig. 1B). During the first trial, 1 min after injection, the increase in escape latency of the ALCAR-treated rats was higher than that of the control rats $\left(t_{24}=-3.47, p<0.01\right)$. In addition, the decrease in escape latency of the ALCAR group during the first three trials after $\mathrm{NaCN}$ injection was higher than that of the control group (Treatment $\times$ Minute effect: $F_{2,48}=5.81, p<0.01$ ).

In the second experiment the escape latencies of the control and ALCAR-treated rats deviated from baseline performance during the trials on 1 and 5 min after injection of $5.0 \mu \mathrm{g} \mathrm{NaCN}\{t \mathrm{~s}>$ $6.6, p<0.001$; see Fig. 1D). During the second urial, 5 min after injection, the ALCAR-treated rats tended to have a higher escape latency than control rats $\left(i_{26}=2.0,0.05<p<0.1\right)$. In addition, the return of the escape latency to baseline performance of the ALCAR group after NaCN injection tended to be slower than that of the control group (Treatment $\times$ Minute effect: $F_{2.52}=2.54,0.05<$ $p<0.1$ ).

NaCN dose $2.5 \mu \mathrm{g}-\mathrm{ALCAR}$ dose $100 \mathrm{mg} / \mathrm{kg}$. In the second experiment a dose of $2.5 \mu \mathrm{g}$ $\mathrm{NaCN}$ appeared already to be sufficient to induce a behavioral deficit (see Fig. IC). Further, we assumed that after a dose of $5.0 \mathrm{Hg} \mathrm{NaCN}$, performance could be the result of a ceiling effect (see Fig. ID). Therefore, it was decided to test additionally a higher dose of ALCAR $(100 \mathrm{mg} / \mathrm{kg}) \mathrm{in}$ combination with the $\mathrm{NaCN}$ dose of $2.5 \mu \mathrm{g}$ in the rats of the second experiment.

Again it was found that the escape latencies of the control and ALCAR-treated rats deviated from baseline performance during the trials on 1 and 5 min after injection of $2.5 \mu \mathrm{g} \mathrm{NaCN}$ ( $\mathrm{ss}>$ $3,0, p<0.01$; see Fig. IE). However, there was no difference in performance between the control rats and ALCAR-treated rats $(t s<0.4$, n.s.; Fs $<1.1$, n.s. $)$

\section{Effects of NaCN and ALCAR on phosphomositide metabolism and ATP concentrations}

In a pilot study it was found that $\mathrm{NaCN}$ decreased the energy status of rat brain synaptosomes time- and concentration-dependently. A dose of approximately $0.7 \mathrm{mM} \mathrm{NaCN}$ had $50 \%$ of the maximal effect on [3H]-inositol incorporation into PI, PIP and $\mathrm{PIP}_{2}$ for an incubation period of 60 min. For $50 \%$ of the maximal effect on the ATP concentration. the dose of NaCN was approximately $0.3 \mathrm{mM}$ for an incubation period of $15 \mathrm{~min}$. With these results, an in vitro 


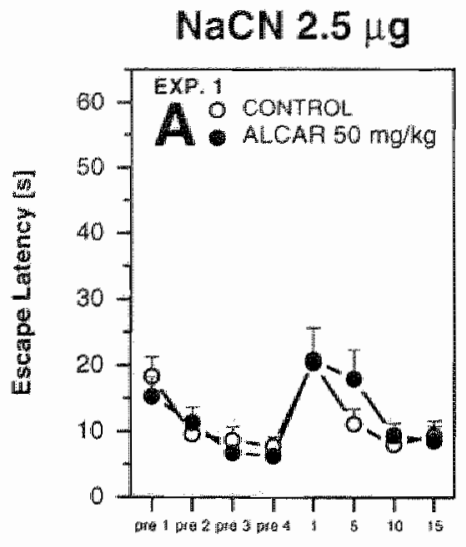

Trialls

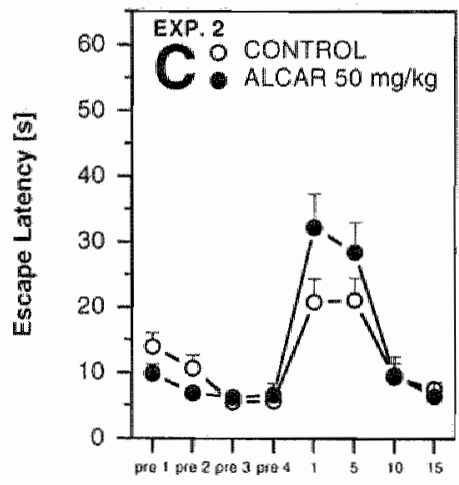

Trials

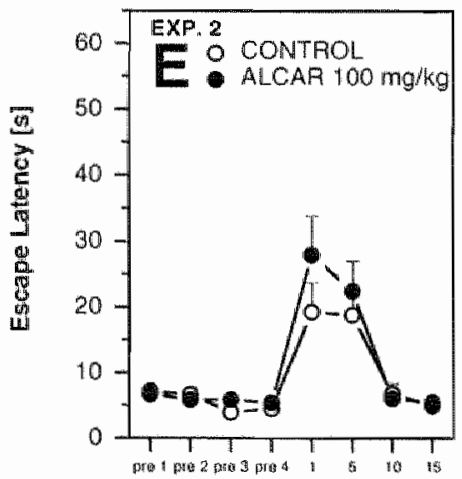

Trials
$\mathrm{NaCN} 5.0 \mu \mathrm{g}$

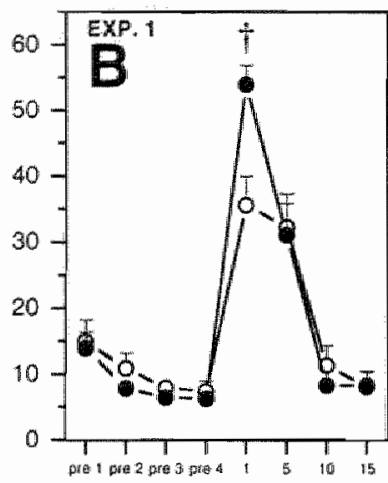

Trials

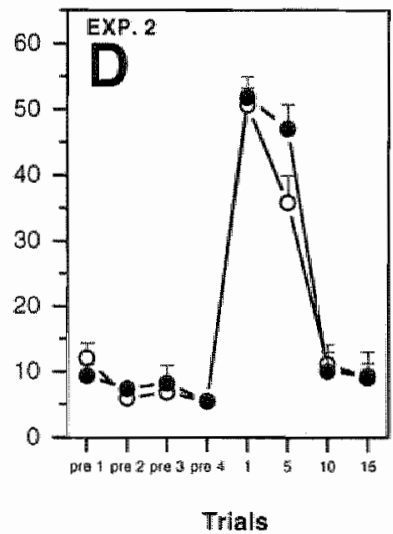

FIG. 1. Meam escape latency (+S.E.M.) of control and ALCAR-treated rats during the fout pre- and post-injection (NaCN 2.5 on $5.0 \mu \mathrm{g}, \mathrm{i}, \mathrm{c}, \mathrm{v})$ urials $(1.5,10$ and 15 min) in the Morris water ascape task. A dose of $50 \mathrm{mg} / \mathrm{kg}$ ALCAR (i.w) was tested with 2.5 and $5.0 \mu \mathrm{g} N \mathrm{NaCN}$, respectively, in both the first (A, B) and second experiment (C, D). An additional dose of $100 \mathrm{mg} / \mathrm{kg}$ ALCAR (i. was tested with $2.5 \mu \mathrm{g}$ aCN in the second experiment (E). Treatment effect: $\neq \mathrm{p} \times 0.01$

energy-deficiency model was established: $0.7 \mathrm{mM} \mathrm{NaCN}$ addition to rat brain synaptosomes for a 15 min incubation period (ATP measurements) or $60 \mathrm{~min}$ incubation period (inositol incorporation assay), which was used to evaluate properties of ALCAR. However, it is noteworthy 


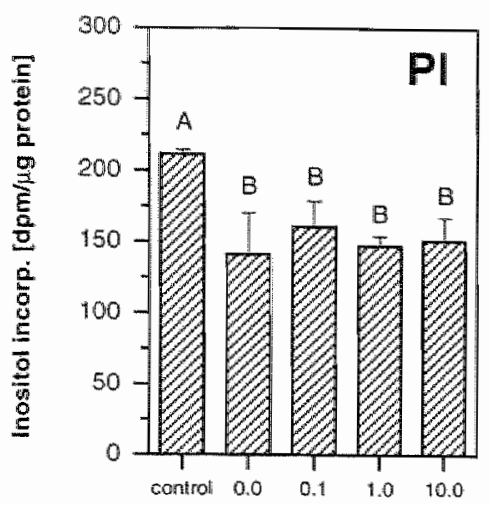

ALCAR conc. [mM]

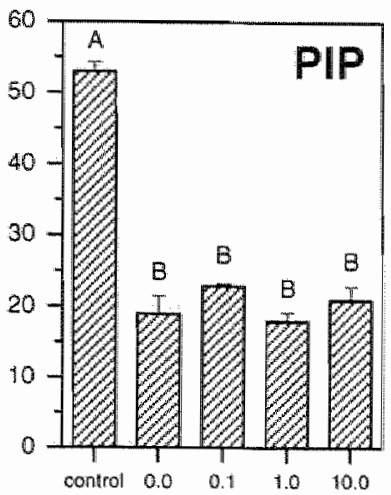

ALCAR conc. [min]

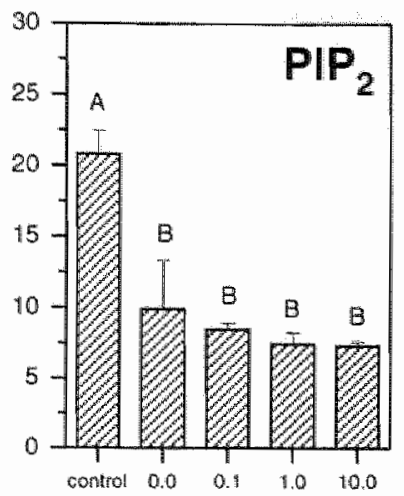

ALCAR conc. [nM]

FIG. 2. The effect of ALCAR administered in witro $(0,0.1,1$ and $10 \mathrm{mM})$ on the NaCN-(0.7 $\mathrm{mM})$-induced inhibition of the incorporation of $[3 \mathrm{H}]$-inositol $(5 \mu \mathrm{Ci} / \mathrm{incubation})$ into phosphatidylinositol (PI), phosphatidylinositol monophosphate (PIP) and phosphatidylinositol bisphosphate ( $\mathrm{PIP}_{2}$ ) of syraptosomes (approximately $250 \mu \mathrm{g}$ protein/inculation) prepared from rat forebrain, during a $60 \mathrm{~min}$ incubation al $37^{\circ} \mathrm{C}$ after 30 min preincubation without $\mathrm{NaCN}$. ALCAR was administered after $10 \mathrm{~min}$ of preincubation. Control is untreated synaptosomes, i.e. no ALCAR and no NaCN treatment. Each walue is the mean (+S.E.M.) of three deterninations. Values which were different (Student-Newman-Keuls test; $p<0.05$ ) were marked with different characters.

to mention that since the inositol incorporation showed no recovery after $60 \mathrm{~min}$ of $\mathrm{NaCN}$ incubation, which was also observed for the measured ATP concentrations, it appears that the synaptosomes could not recoven from the $\mathrm{NaCN}$-induced energy depletion.

The incorporation of [3H]-inositiol into ALCAR-treated $(0,0.1,1.0$ or $10 \mathrm{mM})$ synaptosomes was measured and compared with a sample of untreated synaptosomes, i.e. no ALCAR and no $\mathrm{NaCN}$ treatment. Figure 2 shows a clear effect of $\mathrm{NaCN}$ on the incorporation of [3H]-inositol into Pli, PIP and PIP2, as expected. However, the in vitro ALCAR-tleated synaptosomes were not different from the $\mathrm{NaCN}$-treated synaptosomes incubated without ALCAR, indicating that ALCAR administered in vitro has no effect on the lowered energy status of synaptosomes.

The ATP concentration of ALCAR-treated $(0,0.1,1.0$ or $10 \mathrm{mM})$ synaptosomes was measured and also compared with a sample of untreated synaptosomes. Figure 3 shows a clear effect of NaCN on the concentration of ATP in synaptosomes, as expected. The in witro ALCAR-treated synaptosomes, however, did not differ from the NaCN-treated synaptosomes incubated without ALCAR. This indicates again that ALCAR administered in witro has no effect on the lowered energy status of synaptosomes.

\section{DISCUSSION}

After a dose of $2.5 \mu \mathrm{g} N \mathrm{NaN}$ the spatial memory performance of the rats in the first experiment was not consistently affected. However, the data did suggest that at this dose of $\mathrm{NaCN}$, spatial discrimination behavior in the ALCAR-treated rats is yet somewhat affected. A dose of $5.0 \mu \mathrm{g}$ 


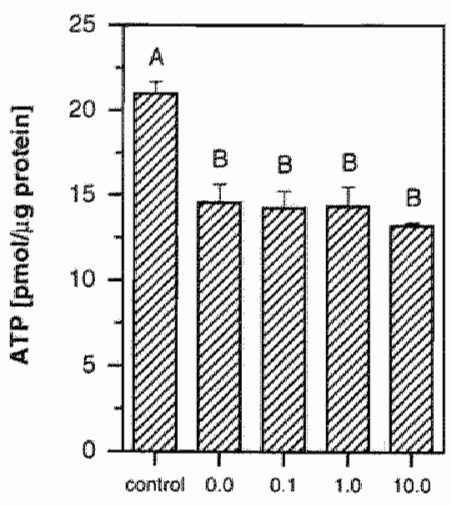

ALCAR conc. [mM]

FIIG. 3. The effect of ALCAR administered in vitro $(0,0.1,1$ and $10 \mathrm{mM})$ on the NaCN-(0.7 $\mathrm{mM})$-indeced decrease of the ATP conceniration of synaptosomes (approximately $250 \mu \mathrm{g}$ protein/incubation) prepared from rat forebrain, during a $15 \mathrm{~min}$ incubation at $37^{\circ} \mathrm{C}$ after 15 min preincubation without $\mathrm{NaCN}$. ALCAR was administered just before the start of the preincubation. Control is untreated synaptosomes, i.e. no ALCAR and no NaCN treatment. Each value is the mean $(+S$ S.E.M.) of three determinations. Vallues which were different (Student-Nowman-Keuls test; $p<0.05$ ) were marked with different characters.

$\mathrm{NaCN}$ was sufficient to disrupt the performance of the rats 1 and $5 \mathrm{~min}$ after injection. Acute treatment with ALCAR was found to increase the behavioral deficit after this dose of $\mathrm{NaCN}$.

The composition of neuronal membranes undergo age-associated changes $[29]$ and concomitantly it has been found that in aged rat brain there is a decrease in actiwity of the enzyme lysophospholipid acyltransferase which is necessary for the reacylation of plasma membrane phospholipids out of lysophospholipids and acyl-CoAs [4, 22]. These acyl-CoAs are produced out of free fatty acids (originating from deacylation of membrane phospholipids) by the enzyme acyl-CoA synthetase which requires energy (ATP). Arduini et al [2] proposed a working medhanism for L-carnitine which suggests that $\mathrm{L}$-carnitine is involved in brain phospholipid metabolism via the enzyme carnitine acyltransferase (probably a plasma membrane associated palmitoyltransferase). According 10 Arduini"s mechanism, L-carnitine provides an ATPindependent reservoir of acyl groups for reacylation, by shifting the equilibrium for carnitine acyltransferase toward acyl-L-carnitines. This could be of main importance when the ATP. dependent reacylation route via acyl-CoA synthetase is inhibited by a low energy metabolism in the brain, like during hypoxia or ischemia (see below). In addition, this might also explain the obserwed increase in acyl-L-carnitines during aging [20], i.e. a removal of a possible excess of acyl-COAs, which might arise because reacyllation is reduced $[4,22]$, takes place.

The above mentioned mechanism is probably not per se of importance in normal healthy adult rats, unless membranes have been damaged due to for instance hypoxia, ischemia or hypoglycemia and thus reacylation/deacylation processes become involved. When energy metabolism is impaired due to ischemia [18], hypoxia or hypoglycemia [11] there is an increase in deacylation of membrane phospholipids and a decrease in B-oxidation. This results in an increase in free fatty acids and acyl-CoAs, respectively, of which the latter are toxic for the mitochondrion 
both wia an inhibition of the electron chain enzyme complexes 241 and wia mitochondral membrane damage [25], and thus can contribute to an impaired neuronal function of even lead to neuronal damage. It seems likelly that ALCAR via L-carnitine diminishes the accumulation of toxic acyl-CoAs by shifting the equilibrium for carnitine acyltransferase towards acyl-L-carnitines [c. 24]. However, this may be of minor relevance in the brain because of the low carnitine acyltransferase activity in the brain due to its preference for glucose. But a detoxification function (and also the formation of a ATP-independent acyl reservoir) may still play a role, since it appears that next to an increase in acyl-COAs, there is an increase in acyl-L-carnitines in the brain during ischemia [sec 9. Taken together, it is likely that an increase in ALCAR and/or L-carnitine levels prevents, by formation of acyl-L-carnitines, a too high level of acyl-CoAs both inside and outside the mitochondrion. A mitochondrion membrane associated camitine acylransferase may be responsible for this inside the mitochondrion, while at its outside a mitochondrion and/or plasma membrane associated enzyme may be involved [of. 2l.

The increased NaCN-induced behavioral deficit by acute ALCAR treatment might be explained by an inhibition of the glycolytic flow, because it has been found that ALCAR is not only involved in brain energy metabolism by stimulating the use of alternative energy sources, but also by inhibiting the glycolytic flow $[3,4,6,32,33]$. Consequently, acute ALCAR treatment would further increase the energy deficit due to $\mathrm{NaCN}$. With respect to a possible inhibition of the glycolysis it has been found in an aging study that acute administration of ALCAR $(75 \mathrm{mg} / \mathrm{kg}$, i.p.) inhibited the glycolytic flow in the brain, next to the increased use of alternative energy sources, in both young and old rats [4. In addition, an indication was found that this dose of ALCAR had an effect on reacylation/dencylation processes. However, this only applied to young rats. Only a very high dose of ALCAR (500 $\mathrm{mg} / \mathrm{kg}$ ) apperared to have an effect on the reacylation/deacylation processes in the brain of old rats, while at the same time the glycolytic inhibition stayed the same as with the low dose. Hence, one may assume that a higher dose of acutely administered ALCAR might be protective against NaCN, since its ATP-independent acyl reservoir (or detoxification) function may then overcome its inhibition of the glycolytic flow. Therefore, the experiment was replicated and additionally a higher dose of ALCAR was tested.

In the second experiment a dose of $2.5 \mu \mathrm{g} \mathrm{NaCN}$ was already sufficient to produce it spatial memory deficit in all the rats. In addition, the spatial memory performance of the ALCAR-treated (same dose as in first experiment) rats tended to be even more affected than that of the control rats after both $2.5 \mu \mathrm{g}$ and $5.0 \mu \mathrm{g} \mathrm{NaCN}$ administration. This finding is in agreement with the first experiment with acute ALCAR and $\mathrm{NaCN}$. Therefore, it can be argued that an acute (i.v.) administration of ALCAR potentiates a $\mathrm{NaCN}$-induced behavioral deficit.

In contrast to acute administration, we thave found that chronic treatment with ALCAR (via drinking water) attenuated the $\mathrm{NaCN}$-induced behavioral deficit, although it had no effect on the energy-dependent phosphoinositide metabolism as was measured by the incorporation of inositol into PIP and PIP in a hippocampal lysed $P_{2}$ fraction. [8]. Additionally, we have found that in synaptosomes of chronically ALCAR-treated rats, the ALCAR treatment had no effect on the energy dependent inositol incorporation into PI, PIP and PIP2, and ATP concentrations after $\mathrm{NaCN}$ administration (unpublished data). Based on these findings the protective effect of chronic treatment with ALCAR against $\mathrm{NaCN}$ administration might be explained with the formation of the ATP-independent reservoir of actiwated acyl groups. Thus fatty acids as acylated derivatives are available and reacylation processes can continue during acute $\mathrm{NaCN}$ intoxication lor. 21. On the other hand, or maybe concomitantly, ALCAR may exert its protective action by preventing a possible accumulation of toxic acyl-CoAs [cf. 24],

As already discussed above, the potentiation of the $\mathrm{NaCN}$-induced behavioral deficit by acute 
administration of ALCAR might be explained by an inhibition of the glycolytic flow $[3,4,6,32,33$. Effects of ALCAR, after acute administration, on the possible formation of an activated acyl-Lcarnitues reservotr may be 100 small or short lasting in order to have a protective effect. However, when increasing the dose of ALCAR, which was tested with the smallest behawioral deficit, i.e. after $2.5 \mu \mathrm{gaCN}$, the lack of a behavional difference between the ALCAR and control rats after $\mathrm{NaCN}$ administration suggest that a possible inhibition of the glycolytic flow might be overcome (compare Fig. IE with $1 \mathrm{C}$ ).

Except for the assumed inhibition of the glycolytic flow, we have no clear explanation for the potentiating effect of acutely administered ALCAR on a NaCN-induced behavioral deficit. However, our in vitro data are not in complete agreement with the behavioral data since no potentiating effect was observed. But at least the lack of a protective effect of acute ALCAR treatment against $\mathrm{NaCN}$ is in agreement with our in vitro findings in synaptosomes that acutely administered ALCAR had no protective effect on the energy-dependent inositol incorporation into phosphoinositides and ATP concentrations after $\mathrm{NaCN}$ administration. In addition, these data are corroborated by another study which showed that acute L-carnitine administration did not prevent cyanide-induced death of cultured cells [25]. The explanation for the latter was that, although the toxic Acyl-CoAs were scavenged by L-carnitine, the increased phospholipase activity due to the energy deficiency was already sufficient, or such to cause enough membrane damage to result in cell death.

It has been found that the time of administration of a protective drug is best immediately after an ischemic insult, since otherwise the possibility exists that the drug treatment has no effects or even potentiates detrimental effects of the insult [e.g. 11]. Therefore, administration of ALCAR after the $\mathrm{NaCN}$ injection might have had resulted in a protective effect. However, $\mathrm{NaCN}$ causes hypoxia and it has been found that with respect to hypoxia the time of administration of a protective drug, i.e. before or after the insult, is not so important [e.g. 31]. Moreover, chronic ALCAR treatment that started long before the insult, did have a protective effect against NaCN treatment. Perhaps it is more useful to administer ALCAR or L-carnitine centrally. Especially L-camitine is interesting because it may have less or no inhibiting effect on the glycolytic flow as ALCAR has. Thus, the effective component in the brain can be assessed, viz. ALCAR or L-carnitine, or perhaps both. Concomitrantly, with respect to the ATP-independent reservoir of activated acyl groups, ALCAR has to be first converted to L-carnitine, in order to shift the equilibrium of the enzyme carnitine acyliransferase towards acy/-L-carnitines. Therefore, additional (mainly biochemical Icf. 2]) research is needed to investigate this possible action of ALCAR after chronic administration in relation to $\mathrm{NaCN}$.

In conclusion, chronic administration (via drinking water) of ALCAR may probably have exerted its positive action on the NaCN-induced spatial memory deficit viat the formation of an energy-independent acylcamines reservoir and/or detoxification of toxic acyl-CoAs. However, acute administration (i. $x$ ) of ALCAR appeared to potentiate the $\mathrm{NaCN}$-induced cognitive deficit, for which no addequate explanation exists at present. These findings indicate that the nootropic effect of acetyl-L-carnitine has more to do with neuroprotection than with cognition enhancement. Therefore, it is best to administer ALCAR chronically, and preferably as early as possible, to prevent/attenuate cognitive dysfunction such as occurs in for instance Alzheimer's disease. 
prevent the siroxic death of culured hepatocytes by inkibiting the mitochondrial pemeability transition. $J$. Brol. Biochem. $268,13791-13798$.

26. Paxinos $\mathrm{O}$ and Wason $\mathrm{C}$. (1986) The rat brain in stereoraxic coordinates. 2 nd edn. Academic Press, San Ditso.

27. Putegrew J.W., Klunk W.E. Panchalingam K., Kanfer J.N. and McClure R.I. (1995) Clinical and metrochemical effects of acetyl-L-carnitime in Alzheimer's disease. Netrobiol. Aging 16, 1 -4.

29. Rosenthal R.E. Willams R. Bogaer Y.E. Getson P.R. and Fiskum G. (1992) Prevention of postischemic rewroliogic injury through potentiation of brain energy metabolism by acetyl-L-carnitine. Sroke $23,1312-1317$

29. Soboeder F. (1984) Role of membrane lipid asymmetty in aging. Nesurabiol. Aging 5, 323-333.

30. Spagnoli A. Lucca U. Menasce G., Bandera L. Cizza G. Forloni G., Tetamanti M., Frattura L., Throboschil P, Comelli M., Senin U., Longo A., Petrini A., Brambilla G., Belloni A., Negri C. Cawazuti F. Salsi A. Calogero P. Parma E. Stramba-Badiale M. Vitali S., Andreoni G. Inzoli M.R., Santus G., Caregnatlo R., Peruzza M., Favaretio M., Bozeglav C., Aberoni M. De Leo D., Serraiotto L., Baiocehi A. Scoccia S., Culota P. and learacitamo D. (1991) Long-tem acetyl-L-carnitine treatment in Alzheimer's. disease. Neurology $41,1726-1732$.

31. Speiser Z., Uziel $f_{\text {, }}$, Defrith-Assa R., Giter S. and Urca G. (1991) Different behavioral deficils are induced by aroxiahypoxia in neonatal and senescent rats: Blockade by MK-801. Brain Res, 42, 181-186.

32. Villa R.F. and Gorini A. (1991) Action of L-acetylcarnitine on different cerebral mitochondrial poptaltions from hippocampus and striatum during aging. Neurochem. Res. $16,1125-1132$.

33. Villa R.F., Gormi A. Zanada $F$ and Benzi G. (1986) Action of L-acetylcamitine on different cerebral mitochondral populations from hippocampus. Arch. Int. Pharmacodyn. 279, 195-211. 


\title{
Possible role of nitric oxide-cyclic GMP pathway in object recognition memory: Effects of 7-nitroindazole and zaprinast*
}

\author{
Jos Prickaerts1, Harry W.M. Steinbusch1, Jos F.M. Smits² and Jan de Ventel \\ "European Graduate School of Neuroscience 'EURON', Department of Psychiatry and \\ Neuropsychology and 2Department of Pharmacology, Maastricht University, The Netherlands
}

\section{ABSTRACT}

The effects of 7-nitroindazole, a putative selective inhibitor of neuronal NO synthase, and zaprinast, a cGMP-selective phosphodiesterase inhibitor, were evaluated on recognition memory of rats in the object recognition test. This test is based on the differential exploration of a new and a familiar object. Two doses of 7 -nitroindazole $(10$ and $30 \mathrm{mg} / \mathrm{kg}$ ) and zaprinast ( 3 and $10 \mathrm{mg} / \mathrm{kg}$ ) were used. The substances were administered i.p. immediately after the exposure to two identical objects, i.e. at the start of the delay interwal. After a delay interwal of $1 \mathrm{~h}$, control rats spent more time exploring the new object which demonstrates that they recognized the familiar one. Both doses of 7-nitroindazole impaired the discrimination between the two objects after the 1 -h interwal. After a 4-h interval, control rats did not discriminate between the objects. The highest dose of zaprinast facilitated object recognition after the 4-h interval. In addition, this dose of zaprinast $(10 \mathrm{mg} / \mathrm{kg})$ reversed the recognition memory deficit induced by 7 -nitro indazol (10 $\mathrm{mg} / \mathrm{kg}$ ) at the $1 \mathrm{~h}$ interval. Interference with the NO-cGMP pathway can also induce cardiovascular effects which may influence memory performance. The highest dose of 7-nitroindazole slightly increased mean arterial blood pressure $1 \mathrm{~h}$ after its administration. Four h after administration of zaprinast $(10 \mathrm{mg} / \mathrm{kg}$ ), mean arteriall blood pressure was also slightly increased, but not after 1 h after zaprinast administration. However, these effects on blood pressure are apparently not related to, or explain the differential effects on object recognilion memory. These results therefore suggest that NO-cGMP signal transduction is inwolved in object recognition memory independently of its cardiovascular role. Finally, since 7-nitroindazole affected mean arterial blood pressute it can not be regarded as a sellective inhibitor of neuronal NO synthase.

\section{INTRODUCTION}

Long-term potentiation can be defined as a stable and long-lasting increase in the efficiency of synaptic transmission. This form of synaptic plasticity is believed to be the physiological substrate for leaming and memory [10]. It is likely that this increase in synaptic transmission is maintained, at least in part, by an increase in presynaptic neurotransmitter (glutamate) release. The latter implicates presynaptic changes of long-lasting nature.. For presynaptic changes to occur after

\footnotetext{
* The main part of this study has been published in Eur. J. Pharmacol. 337, 1251136 (1997).
} 
postsynaptic activation, it is necessary to hypothesize a retrograde messenger which is released posisynaprically 101. The molecule nitric oxide (NO) has many of the properties which are imposed on a retrograde messenger: it is synthesized postsynaptically by a calcium/calmodulin-dependent enzyme, NO synthase, is short-lived and freelly diffusible [10. 30 ]. Moreover, administration of NO synthase inhibitors can impair spatial learning $[15,27,40,62]$ and can block induction of long-term potentiation in hippocampal slices [11. 12.45].

NO is a powerful activator of the cyclic GMP (cGMP)-synthesizing enzyme soluble guanylyl cyclase [43]. It has been suggested that activation of soluble guanylyl cyclase may be a major pathway for the NO messenger function in the brain $118,29,561$. This is corroborated by studies showing that long-term potentiation induction in hippocampal slices can be blocked with soluble guanylyl cyclase inhibitors $[1,14,65]$.

The present study exanines whether the NO-cGMP transduction pathway is involved in memory processes. The effects of 7-nitroindazole, a NO synthase inhibitor, and zaprinast, a highly selective inhibitor of the CGMP-selective phosphodiesterase (phosphodiesterase type V) [6], were evaluated on recognition memory of rats. The used abject recognition test is based on spontaneous exploratory activity and the differential exploration of a new and a familiar object. The recognition of the familiar object reflects object recognition memory [26]. Zaprinast was used because we had found that NO-mediated CGMP accumulation in hippocampal slices in the presence of zaprinast was selectively enhanced in the CA2/CA3 region [19].

There are two isoforms of NO synthase that are activated by calcium: neuronal NO synthase and endothelial NO synthase. Consequently, interference with the NO-cGMP pathway can also induce cardiovascular effects [24, 25]. As there are few data available on the cardiovascular effects in wivo of zaprinast and especially 7-nitroindazole, we studied the effects of both compounds on mean arterial blood pressure in conscious animals. We used 7 -nitroindazole because it is assumed to be a selective inhibitor of neuronal NO synthase [41], and therefore its vascular effects should be minimal. An abstract of this work has been published previously $[51]$.

\section{MATERIALS AND METHODS}

\section{Object recognition menoy measurements}

Animals. In the object recognition memory test we used thirty male random-bred Tryon-Maze-Bright rats, which were supplied by our own animal facility. Two weeks before behavioral testing, the animals were housed indiwidually in standard Makrolon cages on sawdust bedding in an abr-conditioned room $\left(20^{\circ} \mathrm{C}\right)$. They had free access to food and water, and were kept under a reversed $12 / 12$-h lighlat/dark cycle (lights on from 19.001007 .00 h). At the start of behanvioral testing, the rats were three months old and weighing $342 \pm 4 \mathrm{~g}$ (mean \pm S.E.M.). At the last behavioral test, the rats were six months old and weighing $465 \pm 5 \mathrm{~g}$ (mean \pm S.E.M.).

Procedures. The object recognition test was performed as described elsewhere [26]. The apparatus consisted of a circulor arenn, $83 \mathrm{~cm}$ in dimmeter. Half of the $40 \mathrm{~cm}$ high wall was made of grey polyvinyl chloride, the other half of transparent polywinyl chloride. The light intensity was equal in the different parts of the apparatus. Two objects were placed in a symmetrical position about $10 \mathrm{~cm}$ away from the grey wall. Each object was available in triplicate. We used three different sets of objects. The different objects were: 1) a cone consisted of a grey polyvingl chloride base (maximal diameter $18 \mathrm{~cm}$ ) with a collar on top made of brass (total height $16 \mathrm{~cm}$ ), 2) a standard 11 brown glass bottle (diameter $10 \mathrm{~cm}$, height $22 \mathrm{~cm}$ ) filled with water, and 3) a massive netal cube $(10.0 \times 5.0 \times 7.5 \mathrm{~cm})$ with two holes (diameter $1.9 \mathrm{~cm})$. The objects could not be displaced by a rat.

In the week preceding testing, the animals were handled daily and were finally adapted to the procedure in two days, i.e. they were allowed to explore the apparatus (without any objects) twice for 5 min each day. Also the rats were then adapred to an i.p. injection by a puncture of the injection needle after the first exploration period. Two days after the last adaptation session, testing began. Testing took place in the same room as where 
the animals were housed. A radio, which was playing softly, provided buckground noise in the roon. Fillurescent red rubes and a light bulb, which was swiched on daring testing only, provided a constant illumination of about $20 \mathrm{~lx}$ on the flloor of the apparatus. All testing was done between 10.00 and $17.00 \mathrm{~h}$.

A lesting session comprised two trials. The duration of each trial was 3 min. During the first trial (T) the apparatus contained two identical objects (samples). A rat was always placed in the apparatus facing the wall at the middle of the front (transparent) segment. After the first exploration period the rat was put lack in its home cage. Subsequently, after a predetermined delay interval, the rat was put back in the apparatus for the second trial (T2), but now with two dissimilar objects, a familiar one (the sample) and a thew one. The times spent in exploring each object during $\mathrm{T} 1$ and $\mathrm{T} 2$ were recorded manually with a personal compurer.

Exploration was defined as follows: directing the nose to the object at a distance of mo more than $2 \mathrm{~cm}$ and/or touching the object with the nose. Sitting on the object was not considered as exploratory behavior. In order to avoid the presence of olfactory trails the objects were always thoroughly cleaned. All combinations and locations of abjects were used in a balanced manner to reduce potentia? biases due to preferences for particular locations or objects.

In a pilot study we had found that after a deflay interval of $1 h_{\text {, }}$ rats discriminated between the two objects in T2, i.e. they spent more time in exploring the new object than the familiar one. However, they did not discriminate between the two objects after a 4-ln interval. Therefore, 7-nitroindazole, which was expected to impair memory performance, was tested at a delay interval of $I \mathrm{th}$. On the other hand, zaprinast was expected to improve memory performance and was therefore tested at the 4-th interwal. The effects of a combined treatment, that is zaptinast and 7-nitroindazole, were tested at a $1-\mathrm{h}$ interval with doses based on the results of the first experiments of this study.

Treatment. 7-nitroindazole (Research Biochenicals International) was successively suspended in dimethyl sulfoxide and arachis oil ( 1 and $3 \%$ dimethyl sulfoxide for doses of 10 and $30 \mathrm{mg} / \mathrm{kg}$ respectively) and taken into solution with shaking and warming. Zaprinast (Sigma) was dissolved in $0.05 \mathrm{M} \mathrm{NaOH}$ (in $0.9 \mathrm{cog}$ NaCl). This solution was slowly titrated with $1 \mathrm{M} \mathrm{HCl}$ to bring a neutral $\mathrm{pH}$ (about 7.5). All solution were made just before use. Injections of 7-mitroindazole (injection volume $4 \mathrm{ml} / \mathrm{kg}$ ), zaprinast (injection volume $1 \mathrm{ml} / \mathrm{kg}$ ) and their wehicles, arachis oil and salline $(0.9 \% \mathrm{NaCl})$, respectively, were given i.p. immediately after $\mathrm{T} 1$ of each session.

The experimental protocol is summarized in Table 1 . The rats were matched for body weighis and assigned to two experimental groups of 15 animals each. In each group one dose of 7-nitroindazole and zaprinast was tested. The rationale for the doses used were based on data from the literature. For instance, $30 \mathrm{mg} / \mathrm{kg}$ 7-nitroindazole was used because this dose can already inhibit NO synthase activity maximally within $0.5 \mathrm{~h}$ [e.g. 381 . A dose of $10 \mathrm{mg} / \mathrm{kg}$ zaprinast was chosen to start with because this dose apparently has no or onlly a minintal effect on blood pressure [e.g. 23]. One group was first treated with 7 -nitroindazole $(30 \mathrm{mg} / \mathrm{kg})$ and four weeks later with zaprinast $(3 \mathrm{mg} / \mathrm{kg}$ ). The other group was first treated with zaprinast (10 mg/kg) and three weeks later with $7 \cdot$ nitroindazole $(10 \mathrm{mg} / \mathrm{kg}$ ). Finally, that is after a wash-out period of at least seven weeks after their

TABLE 1. Experimental protocol

\begin{tabular}{llllll} 
Group & $n$ & Week & Treatment & Dose (mg/kg) & Dellay (1) \\
\hline Group 1 & 15 & 1 & $7-N 1$ & 30 & 1 \\
Group 2 & 14 & 3 & ZAP & 10 & 4 \\
Group 1 & 15 & 5 & ZAP & 3 & 1 \\
Group 2 & 14 & 6 & $7-N I$ & 10 & 4 \\
Group 1 +2 & 28 & 13 & ZAP / 7-NI & $10 / 10$ & 1 \\
\hline
\end{tabular}

Rats were assigned to two groups of 15 rats each and were submitted to the object recognition test. If each group, one dose of 7 -nitroindazole (7-NI) and zaprinast (ZAP) wass tested at an delay interval of $1 \mathrm{~h}$ and $4 \mathrm{~h}$, respectively. The conbined treament was lested in all rats at $1-h$ delay. Except for the combined treatment experiment, each treatment experiment was done twice in a week. One rat was excluded because of a bias in its exploratory behavior and another rat died in the period before the combined treatment experiment. 
last teatment, all rats were used for a combined zaprinast $(10 \mathrm{mg} / \mathrm{kg})$ and 7 -nitroindazole $(10 \mathrm{mg} / \mathrm{kg})$ treatment. During this wash-out period one rat died for unknown reasons. Another rat was excluded from the entire experime necatise of its extreme bias toward one location of the objects within each trial.

Each rat served as its own control. Therefore, for each treatment experiment, rats were first submitted to a control session (vehicle) and $24 \mathrm{~h}$ later to a drug session (7-nitroindazole or zaprinast). This procedure, that is a control session followed $24 \mathrm{~h}$ later by a drug session, was repeated $48 \mathrm{~h}$ later. Thus each treatment experiment wass performed iwice in a ral. The combined treatment experiment, to which all rats were subjected, was. performed only once in a rat. In the control session, borh injections of wehicle (saline and arachis oil) were giwen. In the drugs session, $24 \mathrm{~h}$ laler; the rats received an injection of zaprinast and immediately thereafter an injection of 7 - nitroindazole.

Statistical analysis. The basic measures were the limes spent by rats in exploring an object during $\mathrm{T} 1$ and T2. The time spent in exploring the wo identical samples will be represented by ' $a 1$ ' and " $a 2$ '. The time spent in T2 in exploring the sample and new object will be represented by " $a$ " and " $b$ ", respectively. The following variables were calculated: $e l=a 1+a 2, e 2=a+b, h 1=e 1-e 2, d 1=b-a$ and $d 2=d 1 / e 2$ (see Table 2). $e$ l and $e 2$ are measures of the total exploration time of both objects during $\mathrm{T} 1$ and $\mathrm{T} 2$ respectively. $h 1$ was considered as an index. measure of global habituation of exploratory behavior from $\mathrm{T} 1$ to $\mathrm{T} 2, d 1$ and $d 2$ were considered as index measures of discrimination between the new and the familiar objects. In fact, $d 2$ is an relative measure of discrimination which corrects $d 1$ for exploration activity $(e 2)$. Thus, there should be no differences in $d 2$ indices between experiments with similar treatments at similar interwals. However, this needs not to be the case for $d 1$ because of differences in exploration activity.

Except for the combined treatment experiment, each treatment experiment was comprised of two control and two drug sessions. The results of both the two control sessions and the two drug sessions were averaged.

Comparisons within at session were based on a paired-comparisons t-test (SAS, $t$-test procedure) which tests the significance of a variable for a given session by comparing the mean value of this wariable with zero. This test, which is essentially the same as a paired $i$-test, was applied to the $h$ and $d$ variables. Because each rat served as its own control, comparisons of variables between control and drug sessions were based on a paired $t$-test.

Object preferences were tested within sessions by comparing el according to the nature of the objects (one-factorial analysis of variance) and, if apparent, evaluated in more detail with Duncan's post hoc multiple range test. Moreover, location preferences were tested by comparing $a 1$ and $a 2$ for each object (paired $t$-test).

TABLE 2. Measures involved in the object recognition test

\begin{tabular}{|c|c|c|}
\hline Exploration & Habituation & Discrimination \\
\hline$e^{\prime}=a 1+a^{2}$ & $h l=e 1-e 2$ & $d \mathbf{l}=b-a$ \\
\hline$a 2=a+b$ & & $d 2=d 1 / e^{2}$ \\
\hline
\end{tabular}

\section{Mecan arterial blood pressure measurements}

Amimals. For the blood pressure measurements we used ten male random-bred Tryon-Maze-Bright rats. One week before testing, the animals were housed individually. Housing conditions were the same as in the behavioral study as mentioned above, except that now the rats were kept under a normal $12 / 12$-h light/dark cycle (lights on from 07,00 to $19.00 \mathrm{~h}$ ). At the start of testing, the rats were three months old and weighing $340 \pm 7 \mathrm{~g}$ (meantS.E.M.).

Procedures and treatment, In the week preceding testing, the animals were handled daily and were finally adapted to an i.p. injection in two days by a puncture of the injection needle on each day. Two days later, animals were anesthetized with sodium pentobarbital $(60 \mathrm{mg} / \mathrm{kg}$, i.p.) and a catheter (PE-10) was inserted into the abdominal aorta through a femoral artery for measurement of mean arterial blood pressure. The catheter was 
exteriorized in the neck, filled with heparinized saline $(50 \mathrm{IU} / \mathrm{ml})$, and closed with a metal plug. After the operation, animals were allowed one day for recovery.

Mean arterial blood pressure measurements were performed at three subsequent days. On each day the catheters were connected to low-volume displacement pressure transducers (CP 01: Century Technology. Inglewood, CA). Signals were fed into a computer for on-line derivation of mean arterial balood pressure.

After an adaptation period of about $30 \mathrm{~min}$, four measurements of mean arterial blood pressure were taken at 5 min intervals. The average of these four measurements represented the baseline condition. On the first day, rats received an i.p. injection of saline $(0.9 \% \mathrm{NaCl})$. On the second and third day, zaprinasi (10 $\mathrm{mg} / \mathrm{kg}$. i.p.) and 7 -nitroindazole (10 or $30 \mathrm{mg} / \mathrm{kg}$, i.p.) were administered, respectively. Solutions and injection volumes were the same as mentioned in the behavioral experiments. After administration of a solution, the mean arterial blood pressure response was taken at 5 -min intervals for a maximun period of five hours (after salline and zaprinast administration), or for at least a three-hour period (after 7-nitroindazole administration). After the daily mean arterial blood pressure measurements, the catheters were flushed with heparinized salline and closed again with a metal plug.

Not all measurement were or could be done in all rats. One rat was excluded from the experiment because its catheter let loose. The mean arterial blood pressure responses after zaprinast administration were measured in all rats for a period of $4 \mathrm{~h}$. Moreover, in three rats the measurements were continued till 5 h had ellapsed. After 7 nitroindazole administration, the mean arterial blood pressure measurements of three rats could not be used due to technical problems. Hence, of both 7-nitroindazole treatments there were only three mean arterial blood pressure recordings used.

Statistical analysis. During the interval after administration of a solution, each mean arterial blood pressure response was expressed as the change in mean arterial blood pressure (in $\mathrm{mm} \mathrm{Hg}$ ) from pretreatmen baseline mean arterial blood pressure value. In addition, the mean arterial blood pressure response over an emtire interval was expressed as the average change in thean arterial blood pressure response from the baseline. Treatment effects on mean arterial blood pressure responses between the control (saline) and orug (zaprinast or 7 -nitroindazole) measurements were analyzed with a paired $t$-test.

\section{RESULTS}

\section{Effects of 7-nitroindazale in the object recognirion rest}

Rats were submitted to a 7 -nitroindazole experiment with a dose of either $10 \mathrm{mg} / \mathrm{kg}$ or $30 \mathrm{mg} / \mathrm{kg}$ 7-nitroindazole. The results of the 7-nitroindazole experiments are summarized in Table 3 . When comparing the total exploration time according to the nature of the objects, no object preferences

TABLE 3. Results of treatment with 7-nitroindazole (7-NI) on the measures of the object recognition test

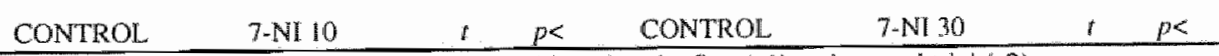

\begin{tabular}{|c|c|c|c|c|c|c|c|c|}
\hline \multicolumn{9}{|c|}{ A. Mean values ( \pm S. E.M.) of total exploration lime (s) during the first $(e 1)$ and second trial $(e 2)$} \\
\hline$E \mathbb{I}$ & & & 0.88 & & $21.36(1.22)$ & $17.35(1.49)$ & -1.85 & n.s. \\
\hline$e 2$ & $14.66(1.82)$ & $15.09(1.58)$ & 0.34 & m.s. & $21.68(1.23)$ & $23.12(1.67)$ & 0.79 & n.s. \\
\hline
\end{tabular}

B. Mean values ( \pm S.E.M.) of the index of global habituation (h.l) from the first to the second trial
h. 1 - $1.66(1.08)$
$-0.99(0.73)$
$0.60 \mathrm{~ns}$.
$-0.32(1.55)$
$-5.77(2.43)^{*}$
-1.75 nis.

C. Mean values ( \pm S.E.M.) of the index of discrimination $(d 1)$ between the new and familiar objects

\begin{tabular}{lllllllll}
$d 1$ & $4.38(0.70)^{* * 2}$ & $1.29(0.95)$ & -2.23 & 0.05 & $8.57(1.65)^{* *}$ & $2.76(1.58)$ & -2.59 & 0.05 \\
\hline
\end{tabular}

Rats received an i.p injection of 7-nitroindazole at a dose of $10(n=1 \mathrm{~A})$ or $30(n=15) \mathrm{mg} / \mathrm{kg}$ after the first trial. Each rat served as its own control (arachis oil treatment). For both the control and 7 -nitro indazol treatment the averaged data of two sessions are presented. The delay interval between the first and second trial was $1 \mathrm{~h}$. Between sessions effects are depicted with corresponding $l$ and $p$ values. Within session effects on the $h$ and $d$ measures are depicted with asterisks (*p $<0.05 ; * *_{p}<0.01$ ). 
were found in both 7-nitroindazole experiments. In addition, when comparing the exploration time according to the nature of the objects and their location in the apparatus, it was found that rats had a preference for the location of one of the objects in the 7 nitroindazolle 10 session.

In both 7-nitroindazole experiments, the total the spent in exploring the abjects in TI (el) as well as in $\mathrm{T} 2(e 2)$ did not differ between the control and 7-nitroindazole sessions (see Table $3 \mathrm{~A}$ ). The index measure of habituation of exploratory behavior $(h 1)$ showed that the exploration time did not change from $\mathrm{T} 1$ to $\mathrm{T} 2$ in both the control and 7-nitroindazole session of the 7-nitroindazole 10 experiment, i.e. in both sessions no within session effect was found (see: Table

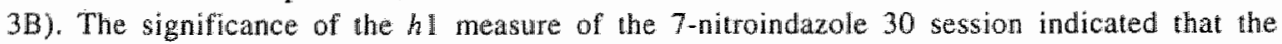
exploration time was increased from $\mathrm{T} 1$ to $\mathrm{T} 2$. However, the level of this $h 1$ measure was not different from its control level, i.e. no between session effect was found (see Table 3B).

The index measure of discrimination (dl) between the new and familiar objects of both 7-nitroindazole experiments showed that in the control sessions the familiar object was less explored thath the new object in T2 after the 1-h delay interval (within session effect on $d 1$; see Table 3C). In contrast, the $d 1$ measures of the 7-nitroindazole sessions indicated that after administration of 7 -nitroindazole at a dose of both 10 and $30 \mathrm{mg} / \mathrm{kg}$, the rats did not discriminate anymore between the objects.

\section{Effects of zaprinast in the object recognition test}

Rats were submitted to a zaprinast experiment with a dose of either $3 \mathrm{mg} / \mathrm{kg}$ or $10 \mathrm{mg} / \mathrm{kg}$ zaprinast. The results of the zaprinast experiments are summarized in Table 4 . When comparing the exploration time according to the nature of the objects and their location in the apparatus, two object preferences (one in the control session of the zaprinast 3 experiment and one in the zaprinast 10 session) and a location preference of one object (in the zaprinast 3 session) were found.

In the zaprinast 3 experiment, the lewel of exploration in $\mathrm{T} 1(e 1)$ of the zaprinast session was higher than that of the control session (see Table $4 \mathrm{~A})$. The level of exploration in $\mathrm{T} 2(e 2)$ was not different between the zaprinast 3 and the control session. In the control session, the within session effect on $h l$ indicated that the exploration time was increased from T1 to T2. However, there was no difference in $h 1$ between the control and zaprinast 3 session (see Table $4 \mathrm{~B}$ ).

The level of exploration in $T 1(e)$ was similar in the zaprinast and the control session of the zaprinast 10 experiment. After treatment with $10 \mathrm{mg} / \mathrm{kg}$ zaprinast, the level of exploration in

TABLE 4. Results of treament with zaprintast (ZAP) on the measures of the object recognition test

\section{CONTROL}

ZAP 3

$1 \quad p<$

CONTROL

ZAP 10

A. Mean values ( \pm S.E.M.) of tolal exploration time (s) during the first (el) and second trial ( $e 2)$

el $14.45(1.20)$

$19.77(1.56)$

$2.90 \quad 0.05$

$23.48(2.42)$

$23.78(2.02)$

$20.23(2.18)$

$24.53(2.31)$

0.13 n.s.

a $19.05(1.37)$

$20.68(1.16)$

0.96 m.s.

$3.25(1.68)$
$($ ) from the

tho the second trial
h. $-4.61(1.99)^{\text {* }}$
$.0 .92(1.29)$
1.47 n.s.
$-0.75(1.92)$
-1.62 n.s.

C. Mean values ( $(S . E . M$.) of the index of discrimination ( $d$ ) between the new and familiar objects

\begin{tabular}{lllllllll} 
d1 & $0.40(0.70)$ & $0.85(0.89)$ & 0.31 & n.s. & $1.04(1.22)$ & $6.33(0.69)^{* *}$ & 4.21 & 0.01 \\
\hline
\end{tabular}

Rals received an i.p injection of zaprinast at a dose of $3(n=15)$ or $10(n=14) \mathrm{mg} / \mathrm{kg}$ after the first triall. Each rat served as its own control (saline treatment). For both the control and zaprinast treatment the averaged data of two sessions are presented. The delay interval between the first and second trial was $4 \mathrm{~h}$. Between sessions effects are depicted with corresponding $t$ and $p$ values. Within session effects on the $h$ and $d$ measures are depicted with asterisks $(* p<0.05 ; * *<<0.01)$. 


\section{ACKNOWLEDGEMENTS}

We thank Sigma-Tau Company (Pomezia-Rome, Italy) for the financial support and for prowiding us with acetyl-L-ciarmitine.

\section{REFERENCES}

1. Ando S., Waki H. and Kon K. (1993) Differential fatly acid release from CAI and CA3 regrons of rat hippocampal slices under hypoxia and hypoglycemia. Neurosci. Letr. 151.48-50.

2. Arduini A., Denisova N. Virmani A. Avrowa N. Federici G. and Arigoni-Martelli E. (1994) Evidence for the involvement of carnitine-dependent long-chain acyltransferases in mearonal inglyceride and phospholipid acid turnover. J. Newrachem. 62, $1530-1538$.

3. Aureli T. Miccheli. A. Di Cocco M.E.L., Ghirardi O., Giuliani A., Ramacei M.T. and Conti Fi. (1994) Effect of acetyl-L-carnitine on recovery of brain phosphorus metabolites and lactic acid level duning reperfusion after cerebral ischemia in the rat - study by $31 \mathrm{P}$ - and 1 H-NMR spectroscopy. Brain Res. 643. 92-99.

4. Aureli T., Miccheli A., Ricciolini R., Di Cocco M.-A., Ranacci M.T., Angelucci L., Ghirardi O. and Cont F. (1990) Aging brain: Effect of acetyl-L-carnitine treatment on rat brain energy and phospholiphid metabolism. A study by $31 \mathrm{P}$ and IH NMR spectroscopy. Brain Res. 526, 108-1 $\ 2$.

5. Barnes C.A., Markowske A.L.. Ingram D.K., Kametan H., Spangrler E.L., Lemken V.J. ard Olton D.S. (1990) Acetyl-L-carnitine 2: Effects on learning and memory performance of aged rats in simple and complex mazes. Newrobiol. Aging 11, 499-506.

6. Benzi G., Villa R.F., Dossena M. Vercesi J Gorini A. and Pastoris O. (1984) Cerebral endogenous substrate utilization during the recovery period after profound thypoglycemia. I. Neurosci. Res. 11, 437. 450 .

7. Bieber L.L. (1988) Carmitine. Ann. Rew. Biochem. 57, $261-283$.

8. Blokland A, Bothmer J., Honig W. and Joltes J. (1993) Behavioral and biochemical effects of acute cemural metabolic inhibition: Effects of acetyl-L-carnitine. Eur. J. Pharmacol. 235 $275-281$.

9. Bohan T.P. (1992) Editoriall comment. Siroke 23, 1317-1318.

10. Booth R.F.G. and Clark J.B. (1978) A rapid melhod for the preparation of relatively pure metabolically competent synaptosomes. Biochem. $J, 176,365-370$.

11. Borlongan C.V., Cahill D.W. and Sanberg P.R. (1995) Loconotor and passive avoidance deficits following occlusion of the middle cerebral artery. Physiol Behav. 58, 909-917.

12. Brierly J.B., Brown A.W. and Calwerley J. (1976) Cyanide intoxication in the rat: Physiological and neuropathological aspects. J. Neurol. Neurosurg. Psychictry 39, $129-140$.

13. Burlina A.P., Sershen H., Debler E.A. and Lajtha A. (1989) Uptake of acetyl-L-carnitine in the brain. Neurochem. Res. 14, 489-493.

14. Caprioli A., Ghirardi O., Ranacei M.T. and Angelucci L. (1990) Age-dependent deficits in radial maze performance in the rat: Effect of chronic treatment with acetyl-L-Carnitine. Prog. Neuropsychopharmateol. Biol. Psychiat. 14, 359-369.

15. Ghirardi O., Caprioli A. Milano $S_{*}$, Giuliani A., Ramacci M.7. and Angehcci L. (1992) Achive awoidance learning in old rats chronically treated with levocarnitue aceiyl. Physiol. Behaw. 52, 185-187.

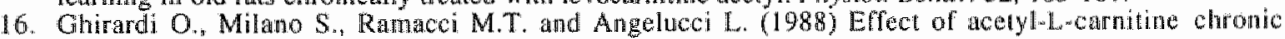
treament on discrimination models in aged rats. Physiol. Behov 44, $769-773$.

17. Hathway D.E. (1984) Molecular aspects of loxicology. pp. 84. "The Royal Society of Chemistry. London.

18. Ikeda M. Yoshida S., Busto R. Santiso M. and Oinsberg M.D. (1986) Polyphosphoinositides as a probable sotrce of brain free fatty acids accumulated at the inset of ischemia. $t$. Nemrochem. 47, $123-132$.

19. Lowry O.L., Rosebrough N.J. Farr A.L. and Randall R.J. (1951) Protein measurements witlithe Folin Phenol reagent. J. Biol. Chem, 193, 265-275.

20. Maccari F., Arseni A., Chiodi P.., Ramacei T. and Angelucci L. (1990) Levels of carnitines in brain and other tissues of rats of different ages: Effect of acelyl-L-carmitine administration. Exp. Gerontol. $25,127$. 13.4.

21. Macmillan V.H. (1989) Cerebral energy metabolism in cyanide encephalopaby. J. Cereb. Blood Flow Merab. 9, $156-162$.

22. Miccheli A. Delfini M., Aureli T., Di Cocco M.E., Tomasini A., Calderini G. and Cont F. (1988) Aging brain: H-1 and P. 31 NMP spectroscopy study on energy and phospholipid metabolism. Magn. Res. Med. Biol. $1,157-168$

23. Morris R.G.M. (1981) Spatial localization does not require the presence of local cues. Learn. Motiv. 12 . 239-261.

24. Ohtsuka $Y$. and Griffith O.W. (1991) L-carnitine protection in amonia intoxication. Biochexs. Pharwachl. 12. $1957-1961$.

25. Pastorino J.G., Snyder J.W., Serroni A., Hoek J.B. and Farber J.L. (1993) Cyclosporin and carnitine 


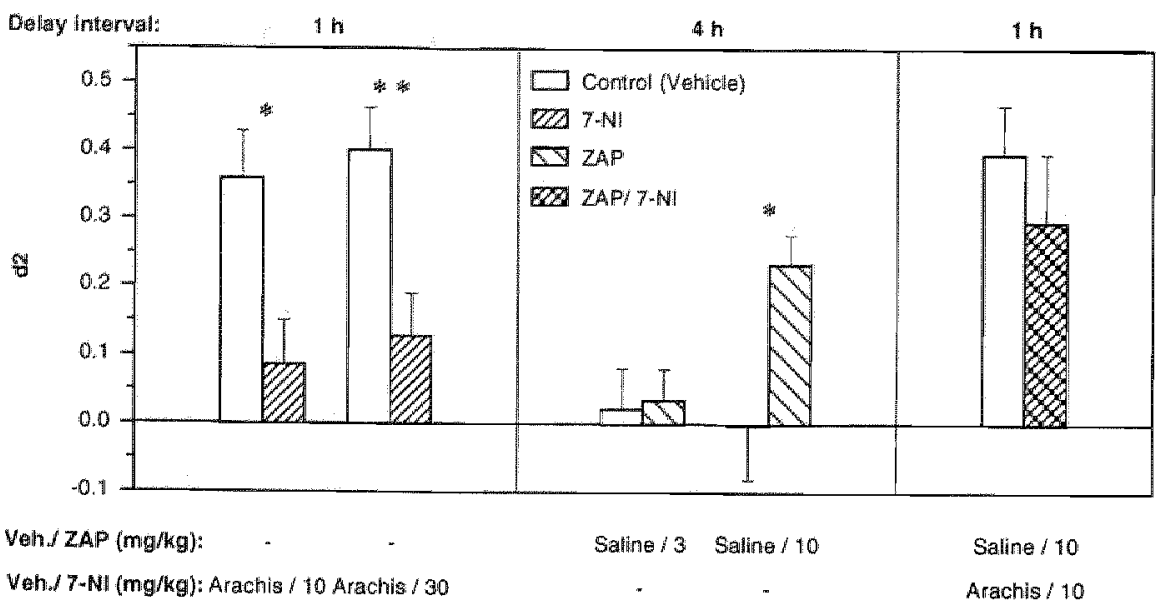

FIG. I. Effects of the differemt treatments on the index of discrimination $(d 2)$ in the object recognition test (mean values $\pm S . E . M$.). In the control sessions, wats were treated with vehicle (saline and/or arachis oil). In the drug sessions, rats were treated with zaprinast (ZAP) and/or 7 nitroindazole (7-NI). Bietween sessions effect: $p<$ $0.05 ; *^{*} p<0.0 .1$

discrimination index $d 2$, which is constituted by the index of discrimination $d 1$ divided by the exploration activity $(e 2)$, are presented in Figure 1.

Compared within sessions, $d 2$ was statistically significant in both control sessions of the 7 -nitroindazole experiment (both $t>5.10, p<0.01$ ), which indicated that rats discriminated between the new and familiar objects after a $1-\mathrm{h}$ interval. After treatment with both doses of 7 m nitroindazole, no more discrimination within each session was found (both $t<2.08$, n.5.). This finding is supported by the between session effect in both 7-nitroindazole experiments (see Fig. 1).

After a 4 -h interval, d2 revealed that there was no discrimination in the control sessions and the zaprinast 3 session (all $t<0.75$, n.s.). However, after treatment with the highest dose of zaprinust $(10 \mathrm{mg} / \mathrm{kg})$, rats were able to discriminate between the objects. This was indicated by the within session effect $(t=5.35, p<0.01)$ and between session effect (see Fig. 1).

When 7 -nitroindazole $(10 \mathrm{mg} / \mathrm{kg}$ ) was administered together with zaprinast $(10 \mathrm{mg} / \mathrm{kg}$ ), it was found that the discrimination index $d 2$ was not affected after the $1-h$ interval (see Fig. 1). In both the control and zaprinast/7-nitroindazole session, the rats discriminated between the objects (both $t$ $>2.94, p<0.01)$ and there was no difference in the level of discrimination between the control and zaprinast/7-nitroindazole session $(t=-0.96, n .5$.$) .$

Therefore, the results of the index of discrimination $d 2$ were not different from that of $d 1$. As expected, it was found that the level of discrimination, as defined by $d 2$, was not different between the control sessions of the 7-nitroindazole experiments and the combined treatment experiment at the $\mathbb{1}$-h interval (both $t<-0.09$, n.s.). Neither did $d 2$ differ between the control sessions of the zaprinast experiments at the 4 -h delay interval $(t=0.24$, n.s.; see also Fig. 1).

Effects of zaprinast and 7-mitroindazole on mean arterial blood pressure

Mean arterial blood pressure responses were measured at three subsequent days to assess 

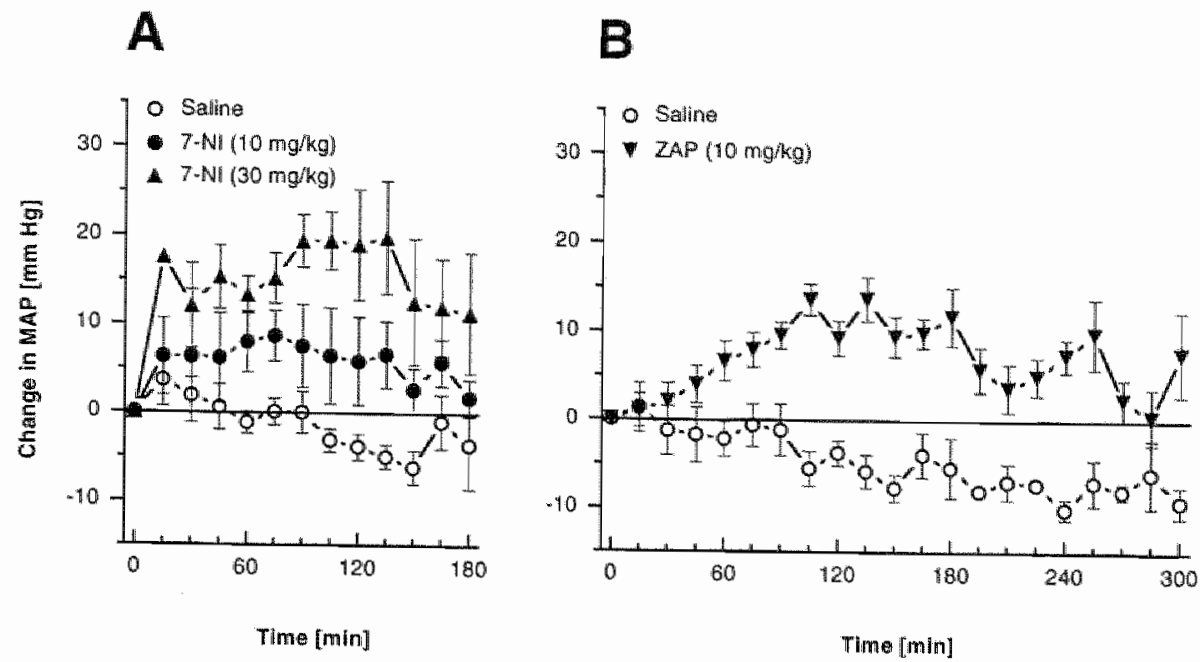

FIG. 2. Treatment effects on the mean arterial blond pressure (MAP), On day 1 the rats received an i.p. injection of saline and on day 2 they received an i.p. injection of zaprinast (ZAP) at a dose of $10 \mathrm{mg} / \mathrm{kg}$. On day 3 the rats received an i.p. injection of 7-nitroindlazole (7-ND) at a dose of either 10 or $30 \mathrm{mg} / \mathrm{kg}$. Data are presented ass changes in mean arterial blood pressure (mean \pm S.E.M.) from pretreatment baseline values. (A) Effects of the two 7-ritroindazole treatments and the corresponding saline control treatments, which have been averaged for clearness sake, on mean arterial blood pressure (each dose of 7 -nitroindazole, $n=3$ ). (B) Effects of the zaprinast treatment and the corresponding controls on mean arterial blood pressure $(0-240 \mathrm{~min}, n=9 ; 240-300 \mathrm{~min}$, remaining $n=\mathfrak{3}$ ).

respectively the effects of saline control, zaprinast $(10 \mathrm{mg} / \mathrm{kg})$ and 7 -nitroindazole $(10$ or 30 $\mathrm{mg} / \mathrm{kg}$ ) treatment. The profile of mean arterial blood pressure responses to the different treatments is presented in Figure 2. There were no differences in baseline mean arterial blood pressure values between the days, which had an average value of $123 \pm 1 \mathrm{~mm} \mathrm{Hg}$ (mean \pm S.E.M.).

Administration of 7-nitroindazole at a dose of $10 \mathrm{mg} / \mathrm{kg}$ had only incidentally as statistical effect on the individual mean arterial blood pressures compared with the saline treatment. At the higher dose of $30 \mathrm{mg} / \mathrm{kg}$ an elevation in mean arterial blood pressures was gemerally observed from 40 min to more tham 2 h after administration of 7-nitroindazole (see Fig. 2A). After administration of zaprinast, mean arterial blood pressures were generally increased from $\|$ to more than $4 \mathrm{~h}$ compared with the saline treatment (see Fig. 2B). For further statistical analysis, the average change in mean arterial blood pressure response over an interval (of whole hours) was calculated. After administration of 7-nitroindazole at the low dose, the average change in mean arterial blood pressure over the entire $3-\mathrm{h}$ interval was, even then, not different from that of the saline treatment $(t=0.66$, n.s.). For the high dose of 7 -nitroindazole it was found that over the interval of the first hour the average mean arterial blood pressure was not different from the saline treatment $(t=2.75, \mathrm{n} . \mathrm{s}$.). But from the $2-\mathrm{h}$ interval on, this dose of 7 -nitroindazole treatment produced an increase in the average mean arterial blood pressure (both $t>4.67, p<0.05$ ). Further, it was found that the average change in mean arterial blood pressure over the interval of the first hour was not different between the zaprinast and saline treatment $(t=1.17$, n.s). From the 2-h interval on, zaprinast treatment resulted in an elevation in the average mean arterial blood 
pressure at each interval $(a) 1>2.91, p<0.05)$.

\section{DISCUSSION}

\section{The NO-CGMP transduction pathway and memory formation.}

The results of the present study showed that both doses of 7 -nitroindazole (10 and $30 \mathrm{mg} / \mathrm{kg}$ ) impained the discrimination between the two objects after the $1-h$ interval. On the other hand the highest dose of xaprinast facilitated object recognition after the $4 \mathrm{~h}$ interval. In addition, this dose of zaprinast (10 mg/kg) reversed the recognition deficit induced by 7 -nitroindazole $(10 \mathrm{mg} / \mathrm{kg}$ ) at the $\mathbb{t}-\mathrm{h}$ interval.

The inhibition of NO synthase in the brain after systemic administration of $30 \mathrm{mg} / \mathrm{kg} 7-$ nitroindazole is probably already maximal 30 min after injection [16, 38]. Administration of 10 $\mathrm{mg} / \mathrm{kg}$ 7-nitroindazole probably only results in a partial inhibition of NO synthase activity in the brain [2]. The inhibition of NO synthase is transient with complete recovery after 24 hours [38]. The decline in memory performance after systemic administration of both doses of 7 -nitroindazole in our study is in agreement with the generally found impaired acquisition, as found in spatial memory tasks, after systemic administration of several nonselective NO synthase inhibitors $[15,27,40,62]$. Moreover, it has recently been reported that i.p. administration of 7-nitroindazole (25.65 mg/kg) impaired spatial acquisition as well [31, 39]. Likewise, in studies using local administration of NO synthase inhibitors it was abserved that NO synthase blockade in the hippocampus affected working memory, as found in the three-panel runway [47], and processes generated during or shortly after training, as found in passive avoidance learning [28]. At the same time it has been reported that NO levels in the hippocampus increased irnmediately after passive avoidance training [7]. Thus, it has been argued that $\mathrm{NO}$ affects mechanisms in the hippocampus that are important for memory consolidation [7, 28].

Biochemical and immunocytochemical studies have provided evidence that activation of soluble guanylyl cyclase and consequently cGMP formation, in the brain may be one of the major effects of NO [18,29.56]. cGMP degradation in the brain is very fast and appears to involve several isoforms of phosphodiesterase. Zaprinast is an inhibitor of the cGMP-selective phosphodiesterase (phosphodiesterase type $V$ ) and has been reported to increase cGMP levels in hippocampal slices [1.3. Recentlly, we have demonstrated an increase in NO-stimulated cGMP content in axonal fibers in the $\mathrm{CA} 2 / \mathrm{CA} 3$ area of the hippocampus of the rat in the presence of zaprinast using hippocampal slices 1191 . So far the presence of phosphodiesterase- $V$ in the brain has not been reported. Our results showed that there is a very restricted localization of the effects of zaprinast on NO-mediated cGMP accumulation, i.e, no effects of zaprinast were found in the cortex, the caudate putamen and the cerebellum (unpublished data). I.p. administration of zaprinast improved memory performance in our present study and completely attenuated the memory deficits of the additionally administered NO synthase inhibitor 7-nitroindazole. Based on these results, an effect of zaprinast on objeet recognition memory is likely to involve NO mediated cGMP synthesis in the CA2/CA3 area of the hippocampus. Although zaprinast is regarded to be well able of penetration into the brain [c.f. 57], our assumption needs further and more direct verification in future research by measuring cGMP levels or phosphodiesterase activity in several brain areas after zaprinast treatment.

Recently studies have appeared in the literature evaluating the role of cGMP in memory processes. In one study using the passive avoidance task with rats, it was found that the level of cGMP in the hippocampus increased immediately after training, and that administration of an 
analogue of CGMP into the hippocampus immediately after the training entanced memory performance $18 \%$. In addition, it was found that infusion of a soluble guanylyl cyclase inhibitor immediately after training caused full amnesia 191 . Furthermore, next to an increase in hippocampal cGMP levels also cGMP-dependent protein kinase activity increased immediately after training [9]. Thus, it was argued that cGMP is involved in memory consolidation [81, 1997). In another study using mice there was an indication that NO synthase inhibition due to systemic 7 . nitroindazole administration decreased cGMP levels in the hippocampus, and with a spontaneous alternation task it was demonstrated that an analogue of CGMP (i.c. $w$, administered) attenuated the effects of another systemically administered (nonselective) NO synthase inhibitor on spatial working memory [61]. Conceptually our results are in agreement with these recent findings. Because in our studies 7-nitroindazole and/or zaprinast were administered immediately after training, a strong argument has been found to advocate a role of NO-CGMP transduction in the consolidation of information. Taken together it can be argued that the NO-cGMP transduction pathway is involved in processes of memory formation.

\section{Behavioral side effects}

Two object preferences and two location preferences for an object were found. Due to the incidental character of these biases and the difference in objects between these biases, it may be assumed that these biases did not influence the behavional data. Normally, locomotor activity decreases after systemic administration of a NO synthase inhibitor [e.g. 52] and based on an earlier study it could be assumed that after administration of $30 \mathrm{mg} / \mathrm{kg} 7$-nitroindazole there is a decrease in locomotor activity [16]. In our study the index measure of thabituation of exploratory behavior (hl) was not different between the 7-nitroindazole sessions and its control sessions, that is there appears to be no difference in exploratory activity $\mathbb{t}$ h after control or 7 -nitroindazole treatments. Hence, it may be assumed that 7-nitroindazole does not affect behavioral activity. The same accounts for zaprinast as $4 \mathrm{~h}$ after its treatment $h 1$ is not different between the zaprinast sessions and its control sessions. However, $h \mathbb{l}$ showed that $I$ th after combined administration of zaprinast and 7-nitroindazole exploratory behavior decreased. There is no adequate explanation for this decrease in exploration. One possible explanation is that the combined treatment of zaprinast and 7-nitroindazole have synergistic effects on blood pressure. Moreover, the index measures of discrimination $d 1$ and $d 2$, which is $d /$ corrected for exploratory activity, show both the same effects and indicate that zaprinast attenuated the memory deficit after 7 -nitroindazole treatment. Thus, this memory performance is apparently not influenced by a decreased exploratory behturion induced by the combined treatment of zaprinast and 7-nitroindazole.

\section{Cardiovascular effects}

It is well established that NO plays a pivotal role in cardiovascular control l33. In fract the first known function of NO was endothelial cell dependent vasodilatation, explaining its earlier names endothelium-derived relaxing factor [48]. There are two constitutive isoforms of NO synthase wat are activated by calcium: neuronal NO synthase and endothelial NO synthase, although the father can also be found in neurons 201 . The produced $\mathrm{NO}$ can exert its vasodilator action both through a peripheral mechanism via its action on the vasculature, or through a central mechanism wat perivascular nerves $1.7,331$. It has been found that systemic administration of nonseltective No synthase inhibitors [e.g. 52] increased blood pressure. In the present study we that fourd that systemic administration of $30 \mathrm{mg} / \mathrm{kg} 7$-nitroindazole increased mean arterial blood pressare. Normally there is a lack of a pressor response in rodents after i.p. admuntration be 7-nitroindazole, which led to the general assumption that 7-nitroindazole is selective for newrenal 
NO symthase $116,36,41\}$, although there is some in vitro evidence that 7-nitroindazole may inhibit endothelaal WO synthase [a. However, closer examination of the in vivo data from rodents suggests that 7-nitroindazole is not totally devoid of cardiovascular effects. For example, in conscious rats i.p. administration of 7 -nitroindazole $(25-50 \mathrm{mg} / \mathrm{kg})$ had no effect on mean arterial blood pressure, although heart rate decreased immediately [36]. In addition, despite the apparent lack of peripheral vasoconstriction, a decrease in cerebral blood flow was measured, indicating a cerebrovascular effect. Thus, based on the putative selectivity of 7-nitroindazole for neuronal $\mathrm{NO}$ synthase, it was initially speculated that NO, synthesized and released from neuronal NO synthase (rather than endothelial NO synthase) in the brain, might play an important role in central cardiovascular control by perivascular nerves [36]. However, the same results can also be used to argue in the first place that 7-nitroindazole is not totally devoid of vascular effects because, for instance, the decrease in heart rate may be due to the baroreceptor reflex mechanism. Furthermore, the decrease in cerebral blood flow may indicate a direct effect upon endothelial NO synthase 10 induce vasoconstriction [c.5.35]. In another study it was reported that 7 -nitroindazole ( $80 \mathrm{mg} / \mathrm{kg}$, i.p.) did not increase mean arterial blood pressure over a $48 \mathrm{~h}$ period in conscious rats, although also in this study closer inspection of the data shows that there are at least indications that 7-nitroindazole influences blood pressure transiently [16]. Moreover, in a recent study it has been demonstrated that 7 -nitroindazole ( $50 \mathrm{mg} / \mathrm{kg}, 1 . \mathrm{p}$.) immediatelly increased mean arterial blood pressure and decreased heart rate in conscious rats, thus further adding evidence to the assumption that 7-nitroindazole affects directly endotheliall NO formation in vivo [63]. The latter study also suggests that the influence of anesthetics should be taken into consideration, because this might explain the lack of cardiovascular responses.

It has been found that systemic administration of cGMP-selective phosphodiesterase inhibitors like zaprinast (and also other phosphodiesterase inhibitors) decreased the blood pressure probably by lowering the total peripheral resistance in conscious rats, while other hemodynamic effects are only adventitious, e.g. an increase in heart rate is due to a reflexive mechanism. [22, 23]. Surprisingly, a slight increase in mean arterial blood pressure after administration of $10 \mathrm{mg} / \mathrm{kg}$ zaprimast has been found in the present study. Normally, a depressior response after systemic administration of zaprinast is found, but at doses that are above $10 \mathrm{mg} / \mathrm{kg}$ zaprinast $[22,23,25]$. Closer examination of the available data revealed that a slight pressor response after administration of low doses of zaprinast ( $510 \mathrm{mg} / \mathrm{kg}$ ) has also been observed $[22,23,25]$. The mechanism by which zaprinast elevates mean arterial blood pressure is not clear, but it might be due to ancillary pharmacological effects of zaprinast itself rather than reflexive mechanisms opposing an inutial vasodilatation $[22,23,25]$.

It could be assumed that the vascular effects may have influenced behavioral and memory performance [0.f. 52]. Inhibition of NO synthase after systemic 7-nitroindazole treatment is transient with complete recovery after 24 hours [38], Each behavioral experiment, which consisted of a control and a 24 h later treatment session, was performed twice. To exclude the possibility of inhibition of NO synthase during the second experiment, the second control session was performed $48 \mathrm{~h}$ after the first 7-nitroindazole session. Furthermore, there were no behavioral differences between the first and second control session. The mean arterial blood pressure measurements showed that the mean arterial blood pressure baseline right before 7 -nitroindazole administration, that is $24 \mathrm{~h}$ after zaprinast administration, had returned to control values. Thus, the mean arterial blood pressure measurements after 7-nitroindazole treatment were probably not influenced by the earlier zaprinast treatment. But more importantly, during the behavioral zaprinast experiments there was no more blood pressure influence in the second control sessions. Furthermore, there were no behavioral differences between the first and second control sessions of 
the zaprinast experiments. Whether blood pressure effects influenced the second control session of the 7-nitroindazole experiments is not likely, also because of the long time (48 h) between the second control sessions and the first 7-nitroindazole sessions. Administration of $10 \mathrm{mg} / \mathrm{kg} 7$. nirroindazole had no real effect on mean arterial blood pressure, while $30 \mathrm{mg} / \mathrm{kg}$. 7-nitroindazole increased mean arterial blood pressure. However, both doses of 7-nitroindazole affected memory performance similarly. Administration of $10 \mathrm{mg} / \mathrm{kg}$ zaprinast induced an increase in mean arterial blood pressure, while this dose of zaprinast improved memory performance. On basis of these data it is unlikely that effects on blood pressure after zaprinast treatment contributed to the memory improvement of zaprinast both alone and in the combination experiment with 7 -nitroindazole.

\section{Conclusions:}

Recent studies have shown that 7-nitroindazole impaired the acquisition of spatial tasks [31. 39]. Also, 7-nitroindazole prevented the induction of hippocampal long-term potentiation in vivo [21], Assuming that the synaptic plasticity of the hippocampus underlies memory formation it was, therefore, suggested that neuronal NO synthase plays a role in memory processes. However, because of the increase in mean arterial blood pressure after 7-nitroindazole administration in the present study, the selectivity of 7-nitroindazole for neuronal NO synthase can be questioned. In a study using mutant mice lacking neuronal NO synthase, it was found that hippocampal long-term potentiation could still be induced [46]. In addition, it has been found that a functional (membrane-targeted) endothelial NO synthase is required for long-term potentiation 1341. These findings suggested that endothelial NO synthase, rather than neutonal NO synthase, generates NO within the postsynaptic cell during long-term potentiation. Moreover, using (immuno) histochemical methods it has been demonstrated that endothetial NO synthase is highly concentrated in hippocampal neurons in contrast to neuronal NO synthase which was only sporadically found in interneurons [20]. Hence, it can be argued that endothel tal No synthase is involved in memory processes. However, one has to realize that the maximum inhibition of NO synthase in brain structures including the hippocampus, is never fully complete after systemic administration of 7-nitroindazole (maximally about $80 \%$ inhibition: $12,16,38,581$ ). Possibly the remaining NO synthase activity could still be sufficient for long-term potentiation induction and memory formation. The question which constitutive isoform of NO synthase, neuronal or endothelial, is involved in memory processes should be addressed in future research using nore specific types of NO synthase inhibitors. Recently, it has been reported in a study using knock-out mice for endothelial NO synthase and/or neuronal NO symthase that only long-term potentiation was reduced in the double knock-out mice [55]. This indicates that both isoforms of constitutive NO synthase can compensate for each other in the single knock-outs. Moreover, it suggests that both isoforms can be involved in memory processes, though it has very recently been argued that the contribution of neuronal NO synthase to long-term potentiation would be minimal $|60|$.

Our results suggest that cGMP is also involved in processes of memory fortiation. However, it is not clear how cGMP exents its action in this respect. Several mechanism of action of cGMP have been suggested to explain the role of cGMP in synaptic plasticity [30, 54]. For example, cGMP is thought to act through regulation of cGMP-gated ion channels, regulation of CAMP-phosphodiesterases or activation of cGMP-dependent protein kinases $[1,53,65,66]$. The latter has recently been corroborated by the finding that CGMP levels and cGMP-dependent protein kinsase activity were increased in the hippocampus after passive avoidance training 191 . Whether and how the possible actions of cCMP conld result in synthesis and/or release of neurotransmitter " and thereby explain the maintenance of long-term potentiation, is still not clear. 
To have some idea of processes of syuthesis we measured c-fos activity in a pillor experiment, which is a marker of early gene expression, in the rat brain after peripheral zaprinast administration, which should indirectly increase the cGMP levels. The experimental design was the same as in the present study, that is rats performed the same behavioral test and the substance administration was the same. Compared to the control (saline) treatment c-fos activity after the zaprinast treatment $(10 \mathrm{mg} / \mathrm{kg}$ ) was only found in brain areas which are involved in transient blood pressure regulation (e.g. paraventricular nucleus, central amygdala, area postrema, nucleus of the solitary tract), especially via the baroceptor input from the periphery [49]. No (increased) $c$-fos activity was found in the hippocampus. These findings suggest that the effects of zaprinast on memory formation in relation to the hippocampus can probably be explained with cGMP gated channels, rather than with protein phosphorylations by cGMP-dependent protein kinases or cAMP-dependent protein kinases resulting in gene expression and protein synthesis. Recently, it has been demonstrated that for rats which were shown novel objects, there is an increase in $\mathrm{c}$-fos activity in the perirhinal corte $x_{4}$ area TE of temporal cortex, occipital cortex and anterior cingulate cortex 164\%. Especially the relevance of the perirhinal cortex in object recognition can be argued, as was demonstrated in a study using a T-maze [42], though we neither measured an increased c-fos activity in this structure. Likewise, the hippocampus alone appears to be of minor importance for object recognition memory as no increased c-fos activity was found [64t] and its lesion had only minor effects [42] on object recognition. This is in agreement with our lack oll a $c$-fos effect in the hippocampus. Finally, in another study, which evaluated brain c-fos activity after peripheral zaprinast administration (also $10 \mathrm{mg} / \mathrm{kg}$ ), only an increased c-fos activity in the cerebral cortex was found [57]. However, in our study zaprinast did not increase cortical cufos activity. This could be due to differences in methods, e.g. our rats were first exposed to the apparatus. Moreover, this suggest that it is difficult to draw general conclusions about c-fos data in relation with behavior. At least the c-fos measurement indicate that zaprinast has central effects, whether this is a direct or indirect effect remains subject of further study.

Our behavioral results are in agreement with the generally found results on memory performance after systemic administration of NO synthase inhibitors. However, the possible role of NO and cGMP in memory formation has also been questioned, because it has sometines been found that systemic administration of NO synthase inhibitors did not affect acquisition in spatial memory tasks 14.5\%1. It was concluded that the behivioral effects after NO synthase inhibition may be a result of or be affected by nonspecific (physiological) effects [4, 58]. Nonspecific effects could be related to a decrease in locomotor activity and/or an increase in blood pressure. However, in the present study the behavioral data appeared not to be influenced by these effects. Nevertheless, in future behavioral studies focussed on learning and memory processes, it is better to use local instend of systemic administration to minimize effects on for instance locomotor and/or blood pressure fer. 521. But even data obtained after administration of NO synthase inhibitors into the brain data can be controversial as also no or only a moderate effect was found, when comparing several studies with each other, even with relation to the same behavioral task (e.g. passive aroidance task $[28,32,59]$ ). This could be due to differences in animal (rat versus mouse), differences in location of administration, type (selectivity) or dose of NO synthase inhibitor. In addition, it has been found that hippocampal long-term potentiation could still be induced after inhibition of NO synthase [3,5] or soluble guanylyl cyclase [14], although long-term potentiation was not maxinal anymore. This can be interpreted as long-term potentiation has a component that involves neither NO nor cGMP [cf. 14.30]. Based on these findings it can be argued that the function of the NO-cGMP transduction pathway in synaptic plasticity is probably only a modulating one.

The possible involvement of CGMP in memory formation could offer new possibilities for 
treatment of memory disorders [c..37. A first step in this direction might be searching for a way to increase cGMP levels in the brain. An indired way to increase cGMP levels is via CGMP-selectixe phosphodiesterase inhibitors like zaprinast or an alternative like dipyridamole. In addition, it is known that substances that have a nonselecrive phosphodiesterase inhibitor action, like the well-known substances caffeine and theophylline, can as well have positive effects on memory (for a review see $[44]$ ). Dipyridamole, caffeine and theophylline can also have effects on adenosine receptors and this occurs already at lower doses as compared to the phosphodiesterase inhibitor effect. Dipyridamole is an adenosine uptake inhibitor, while caffeine and theophylline are adenosine antagonists. These properties may also explain the effects on memory or cause the appearance of contradictory effect. Hence, it is not clear what mechanism of action of these substances explain the effects on memory. Furthermore, it is known that ginsenosides (found in ginseng extracts) can improve memory (e.g. 50]. This could be explained by the phosphodiesterase inhibiting effects (though calcium/calmodulin-dependent) of ginsenosides. All these findings and assumptions are interesting enough to encourage further study on the possible effects on memory by influencing the cGMP levels in the brain. However, the development and application of new drugs based on cGMP will still be a long way because one probably has to circumvent appearing (cardiovascular) side effects of these drugs that influence cGMP levels.

In conclusion, NO synthase and CGMP are possibly involved in recognition memory processes independently of its cardiovascular effects. This may have implications for possible treatments of memory disorders. The present study also demonstrated that 7 -nitroindazole is not a selective inhibitor of neuronal NO synthase. Future research is needed to demonstrate which isoform of constitutive NO synthase, neuronal or endathelial, is involved in memory formation, and how cGMP is involved.

\section{ACKNOWLEDGEMENTS}

The authors would like to thank A.M. Teuben and W. Honig for carrying out a part of the experimental work. and C.M. Eerdmans from the Deparment of Phamacology for helping with the blood pressure measurements. Furthermore, we thank Dr. W. Raaijmakers for his contribution to the initial experimental set up. Finally, in particular we thank Dr. A. Blokland for his helpful and inspiring commenis.

\section{REFERENCES}

1. Arancio O., Kandel E.R. and Hawkins R.D. (1995) Activity-dependent long-term enlinancenent of Iransmitter release by presynaptic $3^{\prime}, 5^{4}-\mathrm{cyclic}$ GMP in cullured hippocampal neurons. Nanke 376, 74-80,

2. Babbedge R.C., Bland Ward P.A. Hart S.L. and Moore P.K. (1993) Inhibition of rat cerebellar nitric oxide synthase by 7-nitro indazole and related substituted indazoles. Br. J. Pharmacol. 110, 225-228.

3. Bannerman D.M., Butcher S.P. and Morris R.G. (1995) Intracerebroventricular injection of nitric oxide synthase inhibitor does not affect long-term slope potentiation in vivo. Newropharmacology 33, 1387-1397

4. Bannerman D.M., Chapman P.F., Kelly P.A.T., Butcher S.P. and Morris R.G.M. (1994) Inthibition of nitric oxide synthase does not impair spatial learning. J. Nenrosci. 14, 7404.7414.

5. Bamnerman D.M., Chapman P.F., Kelly P.A.T., Butcher S.P. and Morris R.G.M. (1994) Inhibition of nitric oxide synthase does not prevent the induction of long-term potentiation in wiw. J. Neurosci. 14 , $7415-7425$.

6. Beavo J.A and Reifsnyder D.H. (1990) Primary sequence of cyclic nucleotide phosphodiesterase isoenzymes and the design of selective inhibitors. Trends Pharmacal. Sci. 11, 150-155.

7. Bernabeu R., de Stein M.L., Fin C., Izquierdo I. and Medina J.H. (1995) Role of hippocampal NO in the acquisition and consolidation of inhibitory avoidance learning. NewroReporn 6, 1498-1500.

8. Bernabeu R., Schmitz P., Faillace M.P., Izquierdo II, and Medina I.H. (1996) Hippocampal cGMP and CAMP are differentially involved in menory processing of inhibitory avoidance learning. Neurokeport 7 , 585-588. 
9. Bermabet R. Schroder N. Quevedo J., Cammarola M. Izquierdo L. and Medina J.H. (1997) Furher ewidence for the inwolvement of a hippocampal COMP/CGMPadependent protein kimase cascade in memory consolidation. NearoRepor \& 2221-2224.

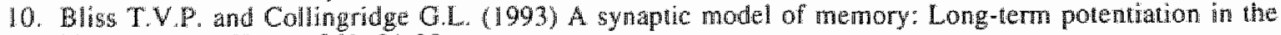
hippocampus Warte $361,31 \times 39$.

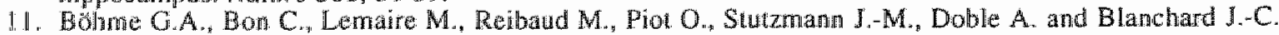
(1993) Allered syraptic plasticity and memory formation in nitric oxide synthase inhibitor-lieated rats. Proc. Nall Acad. Sci. U.S.A. 90, $9191-9194$.

12. Bon C., Böhne G.A., Doble A. Stuzmann J,-M. and Blanchard J.-C. (1992) A role for nitric oxide in long-term potrentiation. Rur. d. Phamacol. 4,420 424.

13. Boulton C.I. Irving AJ., Sotham E., Pother B., Garthwate J. and Collingridge G.L. (1994) The nimic oxide-cyclic GMP pathway and synaptic depression in rat hippocampal slices. Eur. J. Newasci. 6. 15281535

14. Boulnon C.L., Southam E. and Garthwite J. (1995) Nitric oxide-dependent long-term potentiation is. blocked by a spectific inhibitor of soluble guanylyl cyclase. Neuroscience $69,699-703$.

15. Chapman P.F. Atkins C., Alen M.T., Haley J.E. and Steinmetz J.E. (1992) Inhibition of nitric oxide synthesis impairs two different forms of learning NeuroReport $3,567-570$.

16. Connop B.P. Rolle N.G. Joegman R.J., Jhamandas K. and Beninger R.J. (1994) Potentiation of NMDAmediated toxicity on nigrostratal neurons by a low dose of 7-niro indazole. Newrophamacology 33,1439 1445.

17. Dawson T.M., Dason V.L. and Snyder S.H. (1992) A novel neuronal messenger molecule in brain: The free radicill, nitric oside. Ann. Mearol $32,297,311$.

18. De Vente J., Bol J.G.J.M., Berkelmans H.S., Schipper J. and Steinbuseh H.W.M. (1990) Immunocytochemistry of CGMP in the cerebellum of the immature, adult, and aged rat: The involwement of nitric onide. A micropharmacologicall study. Eur. N. Neurosici $2,845-862$.

19. De Vente J., Hopkims D.A., Markerink wan Ittersum M. and Stenbusch H.W.M. (1996) Effects of the 3'5phosphodiesterse inhibitors isobutylmethylxanthine and zaprinast on NO-mediated cGMP accumulation in the lippocampus stice preparation: An immunocylochemical study. J. Chem. Newrocmat. 16, $241-248$.

20. Dinerman J.L., Dawson T.M. Schell M.J., Snowman A. and Snyder S.H. (1994) Endothelial nitric ox ide synthase localized to hippocampal pyramidal cells: Implications for synaptic plasticity. Proc. Nati Acad. Sci. U.S.4. 91, 421.4.4218.

21. Doylle C. Holscher C., Rownian M.J. and Anwyl R. (1996) The selective neuronal nitric oxide symathase antagonist 7-nitro indazole blocks both long-tem potentiation and depotentiation of field excitatory postsymaptic potentials in rat bippocampal CAI in vivo. J. Neurasci. 16, 418-424.

22. Dundore R.L. Clas D.M... Whe ter L.T., Habeb P.G., Bode D.C., Buchholz R.A., Silver P.J. and Pagani E. D. (1993) Zaprinast increases cyclic GMP levels in plasma and in aortic tissue of rats. Eur. A. Phamocol. 249, $293-297$.

23. Dundone R.L. Habeeb P.G., Prat P.F., Becker L.T., Clas D.M. and Buchholz R.A. (1992) Differential henodynamic responses to selective inhibitors of cyclic nucleotide phosphodiesterases in conscious rats. At. Cardiovasic, Phamacol 19,937-944.

24. Dundore R.L.. Pratu P.F. Hallenbeck W.D., Wassey M.L., Silver P.J. and Buchholz R.A. (1990) Sodiurn nitroprusside potentiates the depressor response to the phosphodiesterase inhibitor zaprinast in rats. Eur. $J$. Phumacol. 185, 91-97.

25. Dundore R.L., Prut P.F., O'Connor B., Buchtholz R.A. and Pagani E.D. (1991) N omega-nitro-L-argining atenuates the accumulation of aontic cyche GMP and the hypotension produced by zaprinast. Eur. It. Phandacol. $200,33.87$.

26. Enmacer A and Delacour J. (1988) A new one tral test for neurobiological suditus of memory in rats. 1 : Buthioral data. Benav, Braw Res. 31, 47-59.

27. Estall L. B.. Grant S.J. and Cicala G.A. (1993) Inhibition of nitric oxide (NO) production selectively impairs leatring and memory in the rat. Pharmacol. Biochem. Behov. 46, 959-962.

28. Fin C., Cunha C.A., Bromberg E., Schmitz P.K., Bianchin M. Medina J.H. and Izquierdo I. (1995) Experiments suggesting a role for nitric oxide in the hippocampus in memory processes. Neurobial Learn. Men, 63, $113-115$

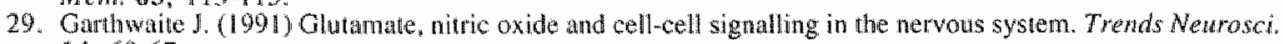
$14,60-67$.

30. Gathwaina d. and Boulton C.I. (1995) Nitric oxide signaling in the central nervous system. Anmu. Rew. Physiol. $57,683,706$.

31. Holscher C. MoCilinchey L., Anvyl R. and Rowan M.J. (1996) 7-Nitro indazole, a selective nearonal nitric oxtde synhase inhibitor in vivo, impairs spatial learning in the rat. Leam. Mem. 2, 267-278.

32. Huang A.M. and Lee E.H. (1995) Role of hippocampal nitric oxide in memory retention in rats. Pharmacol. Brochem. Bethow, 50, 327-372.

33. Iadecola C. Pelligrino D.A., Moskovitz M.A. and Lassen N.A. (1994) Nitric oxide synathase inhibition and cerebrovascular regulation. J. Cereb. Blood Flow Metab. 14, 175-192.

34. Kantor D.B., Lanzrein M.., Stary J. Sandoval G.M., Smith B., Sullivan B.M., Davidson N. and Schuman E.M. (1906) A role for endothelial NO synthase in LTP revealed by adenovirus-mediated inhibition and 
rescue Science $274,1744-1748$

35. Kelly P.A.T. Ritchie I.M. and Arbuthnot G.W. (1994) 7-nitro indazol uncouples cerebral blood fow hom metabolic demand in the consious rat. Br. $\$$, Phanacol 112, $348 \mathrm{P}$.

36. Kelly P.A.T., Ritchie I.M. and Arbuthnutt G. (1995) Inhibition of neuronal nitric oxide synthase by 7 . nitroindazol: Effects upon local cerebral blood flow and glucose use in the rat. J. Cereb. Blood Flow Metab. 15, 766-773.

37. Kim J I. (1996) Cyclic nucleotides and memory. NuroRepont 7, 385.

38. Mackenzie G.M., Rose S., Bland Ward P.A., Moore P. K., Jennet P. and Marsden C.D. (1994) Thime course of inhibition of brain nitric oxide synthase by 7-nitro indazole. NewroRepor 5, 1993-1996.

39. Mever R.C. Spangler E.L., Patel N. and Ingram D.K. (1996) Inthibition of neuronal nitric axide synthase impairs learning of rats in a 14-unit T-maze. Soc. Neurosei. 22, 139.62.3.

40. Mogensen J. Wörtwein G., Gustafsan B. and Ermens P. (1995) L-Nitroargmine reduces hippocampal mediation of place learning in the rat. Neurobiol. Learn. Mem. 64, 17-24.

41. Moore P.K. Babbedge R.C., Wallace P., Gaffer Z.A. and Hart S.L. (1993) 7-Niwo indazole, an intribitor of nitric oxide synthase, exhibits anti-nociceptive activity in the mouse without increasing blood pressure. Br: J. Phamacol 108, 296-297.

42. Mumby D.G. and Pinel J.P.I. (1994) Rhinall cortex lesions and object recognition in rats. Behav. Nevrosici. $108,11-18$.

43. Murad F. Mittal C.K... Arnold W.P., Katsuki S. and Kimura H. (1978) Guanylate cyclase Activation by azide, nitro compounds, nitric oxide, and hydroxyl radical and inhibilion by hemoglobin wand myoglobin. Adv. Cyclic Nuct Res. 9, 145-158.

44. Nehlig A., Daval J.L and Debry G. (1992) Caffeine and the central nervous system: Mechanisms of action. biochemicall, metabolic and psychostinulant effects. Brain. Res. Brain. Res. Rev 17, 139.170.

45. ODell T.I. Hawkins R.D. Kandel E.R. and Arancio O. (1991) Tests of the roles of two diffusible substances in long-term potentiation: Evidence for nitric oxide as a possible early retrograde messenger Proc. Nat Acad. Sci. U.S.A. 88. $11285-11289$.

46. ODell T.J., Huang P.L.., Dawson T.M., Dinerman J.L., Snyder S.H., Kandel E R. and Fishman M.C. (1994) Endotheliai NOS and the blockade of LTP by NOS inhibitors in mice lacking netronal NOS. Sicrence $265,542-546$

47. Ohno M., Yamamoto $T$. and Watanabe S. (1993) Deticits in working memory following inhibition of hippocampal nitric oxide synthesis th the rat. Brian Res.632, $36,40$.

48. Palmer R.M.J. Ferrige A.G. and Mancada S. (1987) Nitric oxide release accounts for the biological anctivity of endothelium-derived relaxing factor. Nature $327,524-526$.

49. Paxinos G. (1994) The rat newous system. 2nd edn., Academic Press, San Diego.

50. Petkov V.D., Cao Y., Todorov I., Lazarova M., Getova D., Stancheva S. and Alova L. (1992) Behavioral effects of stem-leaves axtract from panax ginseng C.A. Meyer. Acta Physiol. Pharmacol. Bulg 18, $41-48$.

51. Prickaers J. De Vente J., Raaijmakers W. and Steinbusch H.W.M. (1996) Involvement of the nitric oxide/cyclic GMP system in object recognition memory of the rat. Soc. Neurosci. 22, 139, 62.2

52. Sandi C., Venero $C$ and Guaza C. (1995) Decreased spontaneous motor activity and startle response in nitric oxide synthase inhibitor-treated rats. Eur. J. Pharmacol. 277, 89-97.

53. Schmid H.H.H.W., Lohmann S.M. and Walter U. (1993) The mitric oxide and cGMP signal transduction system: Regulation and mechanism of action. Biochin. Brophys". Acta 1178, 153-175.

54. Schuman E.M. and Madison D.V. (1994) Nitric oxide and syaptic function. Anmu. Rev. Nearosei. 17, 153.183

55. Son H. Hawkins R.D. Martin K., Kiebler M. Huang P.L. Fisliman M.C. and Kandell E R. (1996) Long term potentiation is reduced in mice that are doubly mutant in endothelial and neuronal nitric oxide symuase, Cell $87,1015-1023$.

56. Southam E. and Garthwate J. (1993) The nitric oxde-cyclic GMP signalling pathway in rat brain. Neuropharmicology $32,1267-1277$

57. Svenningsson P." Johansson B. and Fredholm B.B. (1995) Effect of different Ranhines and phosphodiesterase inhibitors on c-fos expression in rat stratum. Acta. Physiol. Scand. $154,17-24$.

58. Tobin J.R., Gorman L.K., Baxter M.G. and Traystman $\mathbb{R}$.J. (1995) Nitric oxide synthase inhibition does nol impair visual or spatial discrimination learning. Brain Res. 694, 177-182.

59. Toyoda M. Saito H. and Matsuki N. (1996) Nitric oxide but nol carbon monoxide is involved in spatial learning of mice. Jpn. J. Pharmacol. 71, 205-211.

60. Wilson R.I., Yanowsky J, Godecke A., Stewens D.R., Schrader J. and Hass H.L. (1997) Endothelial nitric oxide synthase and LTP. Nature $386,338$.

61. Yamada K., Hiramatsi M., Noda Y. Mamia T., Murai M., Kameyama T. Konori Y., Nikai T., Sugihara H. and Nabeshima T. (1996) Role of nitric oxide and COMP in the dizocilpine-induced impaiment of spontaneous alternation behavior in mice. Neuroscience $74,365-374$.

62. Yamada K. Noda Y., Naknyama S., Komori Y., Sugihara H., Hasegawa T. and Nabeshima T. (1995) Role of nitric oxide in learning and memory and in monoamime metabolism in the rat bratn. Br. J. Pharmacol. $115,852-858$

63. Zagvazdin Y., Sancesario G., Wang Y.-X., Share L. Fitzgerald M.E.C. and Reiner A. (1996) Evidence from its cardiovascular effects that 7 -nitroindazol may inhibit endotheliall nitric oxide synthase in $v^{n} w^{\prime}$. Eur 
1. Phamacol, $303,61-69$

64. Zhu X.O. Brow M.W. McCabe B.J. and Aggleton J.P. (1995) Effects of the nowelty or familiarity of wisual stimul on the expression of the immediate early gene c-fos in rat brain. Neuroscience 69, $821-829$.

65. Thuo M. Hu X., Schultz C., Kandel E.R. and Hawkins R.D. (1994) Role of guanylyl cyclase and cGMPdependent protetn kinase in long-term potentation. Nature 368, 635-639.

66. Zufall $\mathrm{F}$. (1995) Cyclic nucleotide gated channels, nitric axide; and neural function. Neuroscientist $2,24-32$. 


\section{Behavioral, neurochemical and neuroanatomical effects of chronic postnatal L-NAME treatment in neonatal and adult rats*}

Jos Prickaerts, Jan de Vente, Marjanne Markerink-van Ittersum and Harry W.M. Steinbusch

European Graduate School of Neuroscience 'EURON', Deparment of Psychiatry and Neuropsychology, Maastrich University, The Netherlands

\section{ABSTRACT}

In the present study we evaluated the consequences of interference wilh nimic oxide synthesis during development on brain function and behavior in later life. Rat pups received daily injection of the nitric oxide synthase inhibitor $N$-nitro-L-arginine methyl ester (L-NAME, 25 mg/kg s.c.) from postnatal day 0 to 24 . At postnatal day $8 \mathrm{~L}$-NAME-lreated rats had enlarged and heavier stomachs, while body weights appeared to be reduced. The stomachs were not affected in size and weight anymore at postnatal day 24 , whereas the body weights were still reduced by the L-NAME treatment, althongh they soon recovered after termination of the treatment. At 4 months-of-age, rats were tested in non-cognitive (open field) and cognitive (Morris water escape, two-way active avoidance) tasks. Open field behavior of adult rats postnatally treated with $L$ NAME was not affected. In the water escape task there were no differences between the saline and L-NAME-treated rats in spatial discrimination learning and spatial reversal learning. Furthermore, postnatal L-NAME treatment did not have an effect on the acquisition of the two-way active avoidance task. Subsequently, we tested rat pups during the L-NAME treatment at postnatal day 19 through 24 in the open field and the two-way active avoidance lask. L-NAME treatment appeared to increase the behavioral activity in the open field. There was no difference in behavior in the active aroidance task between saline and L-NAME-treated rats. Biochemical and immunocytochemical studies showed that at postnatal day 8 the basal cyclic GMP level was reduced, while the cyclic GMP formation due to incubation with the nitric oxide donor sodium nitroprusside appeared to be increased in the hippocampus, striatum and frontal cortex of LNAME-treated rats. Hence, nitric oxide synthase was inhibited whereas the soluble guanylyl. cyclase activity may be increased in sensitivity. At postnatal day 24 basal cyclic GMP levels and nitric oxide-medialed cyclic GMP formation in the brain structures of L-NAME-treated rats had nomal values again. Taken together, the findings of this study suggest that postnatal inhibition of nitric oxide synthase has profound neurochemical effects during development and may have short-lasting effects on non-cognitive behavior, but it does not affect behavior and brain function in later life.

\footnotetext{
* The main part of this study has been published in Neuroscience 87. 181-195 (1998).
} 


\section{INTRODUCTION}

It has been suggested that NO may play a role in the development of the nervous system $[12]$. Because $N O$ is a powerful activator of soluble guanyly) cyclase (sGC) which synthesizes cyclic GMP (cGMP) [33], cGMP may also be involved during development [cr. 8. 14, 46]. The expression of NO synthase and ${ }^{\circ} \mathrm{GC}$ in the rodent hippocampus has been found to increase during the early development of the brain with a maximum level at 1-2 weeks after birth after which there is a decrease to adult levels $115,45$. Moreover, it thas been found that the NO-mediated \$GC activity in the hippocampus, frontal cortex and striatum of the developing rat brain is high immediately after birth and starts declining between 1-2 weeks, until at 3 weeks after birth adult levels are reached 19. 28, 521. Furthermore, a number of studies, using different experimental animals and looking at different neuronal systems, have revealed that NO andfor cGMP are important factors in development and synaptogenesis by stimulating outgrowth and maturation of neurons le.g. $21,26,40$, 49. 57]. Hence, it may be argued that the NO-cGMP signal transduction is involved in the development of the nervous system. Therefore, we investigated whether interference with NO synthesis during development could impair brain function and behavior during later life.

In the first experiment of the present study we evaluated, using CGMP immunocytochemistry, whether treatment with the NO synthase inhibitor $N$-nitro-L-arginine methyl ester (L-NAME) for 23 days after birth, affected NO-mediated $\mathrm{sGC}$ activity in the brain of neonatal rats. The remaining rats were tested as adults (4 months old) in cognitive (Morris water escape task, shuttle box task) and non-cognitive (open field) tasks. In a second experiment with a similar L-NAME treatment, cGMP formation was assessed, now using a cGMP radioimmunoassay procedure, in the hippocampus, frontal cortex and striatum of both neonatal and adult rats. The neonatal rats were also used for behavioral studies.

\section{MATERIALS AND METHODS}

\section{Animals and treatment}

All experimental procedures were approved by the local ethical committee of the Maastricht University for animal experiments and met governmental guidelines. We used male and female random-bred Tryon-Maze-Bright rats which were supplied by our own animal facility. The day of birth was taken as postnatal day 0 (PO), On PO both males and fentes of a litter were randomly assigned to either the control group or the L-NAME-treated group. Maxinally ten neonatal rats were allowed to live per litter. The treated animals received subcutaneous (s.c.) injections of $25 \mathrm{mg} / \mathrm{kg}$ L-NAME once daily at 18.00 h. The last injection was given on P23. The injection volume was $2 \mu / \mathrm{g}$ urtill $\mathrm{PB}$, thus facilitating the administration of the correct dose of $L-N A M E$ at the very low body weights. Alter $\mathbb{P} 8$, injection volume was $1 \mu \mathrm{l} / \mathrm{g}$. The controls were injected with an equal volume of the saline velhicle $(0.9 \% \mathrm{NaCl})$. All animals stayed with their mother until they were weaned and housed in groups of two to fluree sunimals of the same sex and treatment. The rats were housed in standard Makrolon cages on sanwlust bedding in an air-conditioned room $\left(20^{\circ} \mathrm{C}\right)$. They had free access to food and water, and were kept under a 12/12-houl ligh/dark cycle (lights on between 06.00 to $18.00 \mathrm{~h}$ ). From approximately P90 on wards rats were housed individually.

The experimental protocol is summarized in Table 1. In the first experiment neonatal rats were used at P8 and P24 for cGMP immunocytochemishry or olherwise weaned at P24 to asses their behavior at later life. The behavioral experiments started at approximately P130. In the second experiment rats were used for cGMP biochemistry at $P 8, P 24$ and approximately P130. Also rats were used for histochemical NADPH diaphorase staining at P24. Rats were weaned at P19 and behavioral testing was performed between P20 and P24.

Only the male rats of the litters were used for all the behavioral experiments and cGMP determinations (both immunochernistry and biochemistry) at P24 and approximately P130. This was done to be able to compare our 
TABLE 1. Experimental protocol

\begin{tabular}{|c|c|c|c|c|}
\hline & Experiment 1 & & Experiment 2 & \\
\hline Measurements & Postnatal day & $n$ & Posinatal day & $n$ \\
\hline Immunocytochemistry cGMP & 8,24 & 8,8 & - & $\bar{\sigma}$ \\
\hline Biochemistry cGMP & - & - & $8,24,130$ & $84,8,8$ \\
\hline Hislochemistry NADPH & - & - & 24 & 8 \\
\hline Behavior: & & 20 & & 15 \\
\hline Open field & $130-133$ & & $20-23$ & \\
\hline Morris water task & $136-145$ & & - & \\
\hline "Two-way avoidance & 151 & & 24 & \\
\hline
\end{tabular}

Ai the day of birth (postnatal day 0) rats were vandomly assigned to either a control (saline) or L-NAME (daily 25 mg/kg s.c.)-treated group. Treatmemt continued from postnatal day 0 until 24 . Even groups were used for the several experimental procedures, except for the behavioral studies of the second experiment (control $n=7, \mathrm{~L}$ NAME $n=8$ ). In addition, only male rats were used, uniess when stated otherwise: fonly females, except for one male; sonlly females.

data with relevant data of the literature which was obtained using male rats exclusively. Hence, almost only female rats could be used for cGMP deterninations at P8 and histochemical staining at P24. The behavior of neonatal rats in the second experiment was studied in cohorts of animals born on the sane day after which the averaged performance of all animals was computed. Adult rats were subsequenly tested in the open field, Morris water escape task and two-way active avoidance learning in the shuttle box. Neonatal rats were not tested in the Morris water escape task because the time after weaning until they were killed for cGMP biochemistry (5 days) allowed ws to perform the open field test and the two-way active avoidance task only. Due to tecthical problems the two-way active avoidance data of one rat of the neonatal L-NAME group was excluded. A radio, which was playing softly, provided background noise during testing in the water task arid open field. All testing was carried out berween 10.00 and $15.00 \mathrm{~h}$.

\section{Weights and mortality}

During postnatal development all rats were daily weighed until P24. Mortality was also registered. Neonatal rats that were used for CGMP biochemistry were also used for measuring stomach weight at P8 or P24. The body/stomach weight ratio was calculated to correct for differences in stomach weight that were due to differences in body weight. Adult rats that were used for behawioral testing were also used for mensuring body weigh at P60, P90, P130 and P160.

\section{Operifield}

The open field consisted of a square base $(100 \times 100 \times 30 \mathrm{~cm})$ subdivided into 36 equal squares by black lines (for details see [5II). Two red fluorescent tubes provided very dim illumination (about $21 \mathrm{~lx}$ ) on the floor of the apparatus. Immediately after a rat was piaced in the center of the open field, the movements of the rat were scored. A crossing was scored as soon as the rat crossed a line with both hind legs. The total time spent in the conter, wall and center squares, as well as the total number of lines crossed and rearings were recorded mantally with a personal computer. Testing was carried out on four consecutive days in 5 -min sessions.

\section{Morris water escape task}

The Morris water escape performance of the aduit rats was assessed in a black water tank (diameter $1.22 \mathrm{~m}$ ). Neonatal rats were not tested in the Morris water escape task because the time after weaning until they were killed ( 5 days) allowed us to perform the open field test and the two-way actiwe avoidance task only. The water lank was divided ino four quadrants, norh-east, south-east, soulh-west, and north-west, or which were also designated training, right-adjacent, left-adjacent and opposite relative to the position of the platform. The rats were started facing the wall of the tank from four different, randomly chosen, start positions: north-cast, south-east, south-west, and north-west, and trained to find a platform (diameter $11 \mathrm{~cm}$ ) at a fixed invisible or 
variable visible position in the water tank, depending on the type of rask. The water temperature was about $22^{\circ} \mathrm{C}$. A trial lasted unil a rat had found the plation or unil $120 \mathrm{~s}$ thad elapsed. If a rat did not find the platorm within 120 s. it was placed on the platform for $3 \mathrm{~s}$ and then removed from the water tank. The time between subsequent trials was about $10 \mathrm{~min}$. Probe trials lasting $60 \mathrm{~s}$, during which the platform was removed from the water tank were given 24 hours after the last session of the water escape tasks. Ir a probe trial all rats were started facing the wall of the tank from the same position. Abundant spatial cues were provided by the furniture in the room, including desks, computer equipment the carrying cage, and by the presence of the experimenter. Escape latencies of each urial during the different acquisitions were measured with a stopwatch. In the probe trials the time spent in each quadrant was recorded manually with a personal computer.

Three days after the open field test, the water escape asks were started with a standard Morris spatial discrimination task [30]. During the acquisition of this spatial discrimination task (day 1-3) rats received wo dally sessions of four trials each. The rats were trained to find the invisible platform at a fixed position (south-east) in the water tank, $1 \mathrm{~cm}$ below the surface of the water. In this also called place version of the water escape task (and also the following ones), the water was not made opaque, because a black escape platform is virtually invisible in a black tank. In the probe trial (day 4) all rats were started firom the position opposite to the former position of the removed platform. After the probe trial, four spatial trials were given to reinstate the memory of the platform position.

The day after the probe rial of the spatial discrimination task, a spatial rewersal task was performed (day 5). Rats received two sessions of four trials egch. The rats were again trained to tind the invisible platform which had now been moved to a position opposite to its position in the earlier spatial discrimination task. The starting position in the probe trial (day 6) was again the same for all rats and opposite to the position of the removed platform.

One day after the probe trial of the reversal task, a visual discrimination task was performed. During the acquisition of this also called cue version of the water escape task (day 8-9) rats received two daily sessions of four urials each. The rats were trained to find a visible platform. The platform emerged $1 \mathrm{~cm}$ above the water and a white cloth was fixed on it as a visual cue; the surroundings were stild visible to the rats. The position of the platform in the water fank was varied at a random order. In the probe trial (day 10) all rats were started from the same position (north-east).

\section{Shock sensitivity}

Two days after the Morris water escape tasks, that was four days before the adult rats were subjected to acquisition of the two-way active avoidance task, their shock sensitivity was determined by the flinch reaction (for methods see [5]). Neonatal rats were subjected to the shock sensitivity measurements a few hours after the open ficld test, that was one day before the two way active avoidance task. A rat was placed in a box $(40 \times 25 \times$ $40 \mathrm{~cm}$ ) with a grid floor which was connected to a shock scrambler. The intemsity of the shock was increased step wise, starting at $0.05 \mathrm{~mA}$, with increments of $0.05 \mathrm{~mA}$. Each shock lasted $0.5 \mathrm{~s}$. The shock intensity at which a rat first flinched was scored as the first shock sensitivity level. After the shock intensity was again increased by $0.05 \mathrm{~mA}$, the shock intensity was decreased in a similar manner as it was increased. The second shock sensitivity level was scored when a rat failed to flinch when a shock was given.

\section{Tho-way active anoidance task}

Acquisition of a lwo-way active avoidance task was assessed in an automated shutule box. The apparatus consisted of two equal comparments $(21 \times 27 \times 40 \mathrm{~cm}$ ), separated by a barrier (height $3 \mathrm{~cm}$ ). The floor consisted of a metal grid connected to a shock scrambler. The animals were trained in one session of 50 triats. The session was preceded by an adaptation period of $5 \mathrm{~min}$. A triall started with the presentation of an auditory signal as the conditioned stimulus. The signal was terminated when a rat crossed the barrier within $8 \mathrm{~s}$, i.e. when the rat made an avoidance response; otherwise, a foot shock $(0.25 \mathrm{~mA})$ was given as the unconditioned stimulus. The foot shock and the auditory signal were terminated when the rat escaped to the other compartment or when $10 \mathrm{~s}$ had elapsed. The intertial interval varied randomly between 35 and $60 \mathrm{~s}$.

\section{Mnnumocytochemical detemination of CGMP}

The procedure for incubation of tissue slices has been described in detail in our previous studies [10. 28]. Briefly, 
rats were decapitated at P8 and P24, brains were removed and immediately placed in aerated Krebs-Ringer bicarbonate buffer $(\mathrm{PH} 7.4)$, contaning $1 \mathrm{mM}$ isobutylmethylxanthine (IBMX; Sigma). Brains were cut in the midsagittal plane, the tissue was placed on a filter paper on a precooled $\left(4^{\circ} \mathrm{C}\right.$ ) stainless steel table and transversal $300-1 \mathrm{~m}$ slices were cut using a Mcliwain tissue chopper. Our first objective was to assess whether postnatal LNAME treament could affect NO-mediated cGMP formation. Hence, we used slices of the frontal contex and striatum, which are known to have a highly active NO-CGMP system during development $[9,28,52]$, Slices were separated while being submerged in ice-cold $\mathrm{Krebs}$ buffer (aerated with $95 \% \mathrm{O}_{2}$ and $5 \% \mathrm{CO}_{2}$ ) and paritioned in incubation wells. Incubation time beliore adding the No donor sodium nitroprusside (SNP: Fluka) was 30 mins. The NO synthase inhibitor L-NAME (Fluka; $0.1 \mathrm{mM}$ ) was included from the start of the incubation. SNP (0.1. maM) was present for another, final, $10 \mathrm{~min}$. The incubations were terminated by adding ice-cold fixaive solution (final concentration $4 \%$ paraformaldehyde, $10 \%$ sterose, $\mathrm{pH} 7.4$ ). Fixation was continued for $2 \mathrm{~h}$, followed by a 30 -min wash in ice-cold $0.1 \mathrm{M}$ phosphate (pH 7.4) containing 10\% sucrose. Subsequently, cryostat sections (10 fum) were cut and thawed on to chrome alun/gelatin-coated slides and processed for immunocylochemistry. Sections were incubated overnight at $4^{\circ} \mathrm{C}$ with rabbit anti-cGMP $(1.300)$ in Tris-buffered saline (TBS; pH 7.6) containing $0.3 \%(\mathrm{v} / \mathrm{v})$ Triton $\mathrm{X}-100$. cGMP immunoreactivity was visualized using in fluorescein isothiocyanate-conjugated goat anti-rabbit antiserum (Jackson) at a 1:150 diturion. Specificity of the cGMP immunostaining has been described in detail before $[10,28]$.

\section{Biochemical determination of cGMP}

Biochemical measurement of cGMP levels was performed as described in our previous studies $[9,28]$. Briefly, the hippocampus, frontal cortex and striatum of rats at P8, P24 and P130 were chopped crosswise at $300 \mu \mathrm{m}$ and washed three times at 10 -min intervals with aerated Krebs buffer at $37^{\circ} \mathrm{C}$. The tissues were dispensed in Eppendorf tubes in a final volume of $250 \mu \mathrm{l}$, containing $25-500 \mu \mathrm{g}$ of tissue protein. L-NAME $(0.1 \mathrm{mM})$ wass present the whole incubation time $(30 \mathrm{~min})$. SNP $(0,1 \mathrm{mM})$ was added the final $10 \mathrm{~min}$ before terminating the incubation by adding $30 \%$ ice-cold trichloroacetic acid solution (final concentration $5 \%$ ). Each sumple was assayed in duplicate and, subsequently, the samples were sonicated and centrifuged at $10,000 \mathrm{~g}$ for 10 min a $4^{\circ} \mathrm{C}$. The supernatant was extracted four times with $1 \mathrm{ml}$ of water-saturated diethyl ether. To remove residual ether, samples were placed for 30 min at $50^{\circ} \mathrm{C}$. cGMP content was measured using a radioimmunoassay procedurel4] as described elsewhere $[48]$. Protein content was measured according to the method of Lowry [27].

It is important to note that all in virro slice incubations in this study were performed in the presence of 1 MM IBMX to inhibit phosphodiesterase actiwity. This was done because in a previous study we have found that in the absence of IBMX no or almost no CGMP immunostaining can be observed in the rat brain 1281. "Therefore, cGMP determinations with both the immunostaining and radioimmunoassay procedure under so-called basall conditions do not refleet actual basal cGMP levels, but reflect ongoing cGMP synthesis in the absence of added stimulators or inhibitors of either $5 \mathrm{GC}$ or NO synthase.

\section{Histochemical NADPH diaphorase staining}

For the NADPH diaphorase staining vibratome 50-4m sections were used, which were cut from tisste fixed by an intracardiac perfusion and a 2 -h postixation at $4^{\circ} \mathrm{C}$ will somogyi fixative (final concentration $4 \%$ paraformaldehyde, $0.05 \%$ glutaraldehyde and $15 \%$ picric acid in $0.1 \mathrm{M}$ phosphate buffer, pH 7.4 . Sections were rinsed two times $15 \mathrm{~min}$ in TBS $(\mathrm{pH} 7.6)$, followed by a 30 min rinse in a Tris $\mathrm{HCl}$ buffer $(50 \mathrm{mM}, \mathrm{pH} 8.0)$ containing $0.2 \%$ Triton $X-100$. Subsequently the sections were incubated for $30-60 \mathrm{~min}$ in the dark at $37{ }^{\circ} \mathrm{C}$ in a Tris-HCl buffer $(50 \mathrm{mM}, \mathrm{pH} 8.0$ ) containing $0.84 \mathrm{mg} / \mathrm{ml}$ NADPH (Bowhinger), $0.4 \mathrm{mg} / \mathrm{m}$ nitroblue telrazolium (Boehringer), and $0.2 \%$ Triton X-100. Thereater the sections were tinsed two times 15 min in TBS (pH 7.6) and mounted on chrome alum/gelatin-coated slides. Finally, the slide mouncel sections were dehydrated and coverslipped.

\section{Siaristical analysis}

Untess stated otherwise below, differences between the postnatally L-NAME-trealed and saline control group were analyzed using a t-test. Mortality data were analyzed with a Fisher's exact test (righi-tailed) for $a 2 \times 2$ lable (Gender and Death). To evaluate in more detail whether treatment affected survival, the Cochran-Mantel-Haenszel general association between death and treatment was calculated. In addition, Mantel-Hatenszel estimates of the 
common relative risk for death after treatment were calculated. The body weight during a defined period was analyzed with th three-factorial (Treatment, Gender and Day) analysis of wariance (ANOVA), with repeated measures over Day. When only one gender was analyzed, a two-way (Treatment and Day) ANOVA was used.

The data of the open field test were aggregated in order to enhance reliability [35]. The mean time spent in the squares of the comers, watls and center, respectively, was transformed to the natural logarithm ( $\mathrm{Ln}\left[\left(\mathrm{t}_{1}+t_{2}+\right.\right.$ $3+(4) / 4$ ] subscripts refer to day 1 through 4 of testing) to remove inhomogeneity [5i]. Treatment effects on the open field measunes were analyzed with a t-est.

During the acquisitions of the Morris water escape tasks, the mean escape latency was calculated per session of four trials. In the probe trials, the time spent in each quadirant was measured. Treatment effects during the acquisitions were ewaluated with a two-factorial (Treatment and Session) ANOVA with repeated measures over Session. For the probe trial, preferences for quadrants were evaluated per group by analyzing the times spent in exch quadram with a repeated measures. ANOVA over Quadrant, and additional istatistics which analyzed whether the lime spent in a quadrant differed from the chance level $(15 \mathrm{~s})$. Treatment effects on the time spent in the training quadran were evaluated with a t-test.

The gensitivity to shock was calculated by averaging the two shock sensitivity levels. The shock sensitivity data were ordinal and, therefore, analyzed nonparametrically with a Kruskal-Wallis one-factorial aralysis by ranks $\left(\chi^{2}\right.$-approximations). The mearn number of avoidance responses in the two-way active avoidance task was calculated per block of 10 trials and analyzed by a two-factorial (Treatment and Trial block) ANOVA with repeated measures over Trial bilock.

The biochenically measured CGMP lewels were transformed to the natural logarithm to remove inhomogeneity, because the CGMP dati showed high wariance at high values and low variance at low values. Thus, the log transformation corrected for differences in variance between values. Incubation and treatment effects on the cGMP levels were analyzed with a one-factorial (Incubation or Treatment) ANOVA, and were evaluated in more detail by Duncan's post froc multiple range test.

\section{RESULTS}

\section{Mortality}

In both experiments mortality appeared mainly between P1 and P3, and was rather incidental until P8. In the first experiment one control and four L-NAME-treated rat pups died. In the second experiment six. L-NAME-treated rat pups died. A Fisher"s exact test (right-tajled) showed that in none of the two experiments the gender of rats had any effect on mortality until P8. When andyzing whether the L-NAME treatment affected the survival until P8, it was found, while controlling for gender, that there was no relationship between treatment and mortality in the first experiment (Cochran-Mantel-Haenszel association, n.s.). But in the second experiment there was an association between treatment and mortality $(p<0.05)$ and subjects with L-NAME treatment were about 1.3 times (Mantel-Haenszel estimate) more likely to die before P8 than control-treated subjects.

\section{Weights}

When weaned at P24, the L-NAME-treated rats of the first experiment had a lower body weight compared to their control treated rats $\left(\sigma_{33}=2.29, p<0.05\right.$; see Fig. 1 A). However, analyzed over the total developmental period there was no difference in body weight between the two experimental groups (genenal mean Treatment: $F_{3,34}=0.94$, n.s.). Likewise, the L-NAME treatment appeared not to affect the course of the growth curve during development (Treatment $\times$ Day eflect: $F_{34,74}=1.50$, n.s.). Gender had no influence on these findings ( $F \mathrm{~s}<0.37$, n.s.). Only the male ruts were used for behavioral studies at later life and hence weighed during 

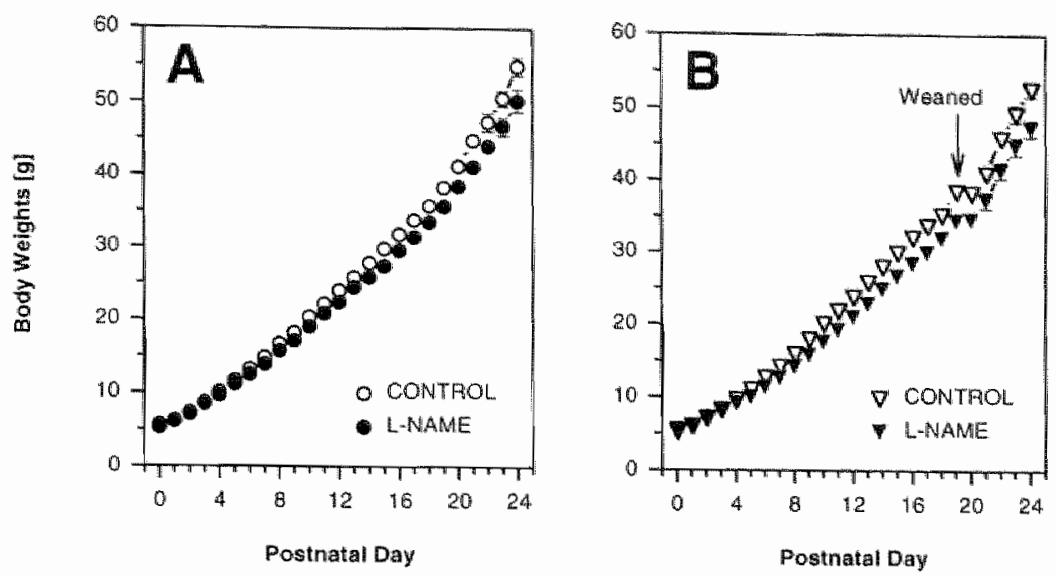

FIG. 1. Development of body weights of L-NAME (25 $\mathrm{mg} / \mathrm{kg}$ s.c. daily from post natal days 0 through 23 ) and CONTROL (saline)-injected rat pups that were used for experiments (A) in adulthood or (B) right after weaning. Values are given as means with S.E.M.

adulthood. It was found that at P60, P90, P130 and P160 the body weights were not different between the control and postnatally L-NAME-treated rats (all $t \mathrm{~s}<1.63$ n.s.). For instance, control and postnatally $L-N A M E-t r e a t e d$ rats were weighing (g) at $P 60$, respectively, $220 \pm 6$ and $225 \pm 6$ (mean \pm S.E.M.), while their body weights were, respectively, $395 \pm 7$ and $399 \pm 7$ at P130, that was just prior to behavioral testing.

L-NAME-treated rats of the second experiment had at the day of weaning, that is P19, a lower body weight than their controls $\left(t_{32}=3.23, p<0.01\right)$. At P24 the body weights of the L-NAME treated rats were still reduced $\left(\mathrm{t}_{31}=3.38, p<0.01\right.$; see Fig. $1 \mathrm{~B}$ ). It was found that $\mathrm{L}-\mathrm{N}$ AME treated rats had in general a lower body weight during development until P19 (general mean Treatment: $F_{3,30}=11.21, p<0.05$ ). In addition, L-NAME freatment affected the increase in body weight during this period (Treatment $x$ Day effect: $F_{8.336}=17.90, p<0.01$ ). Gender did not influence any of these findings (Fs $<1.08$, n.s.). When analyzing the body weights of the rats of the first experiment until P19, the same effects, except on the general mean, of L. NAME treatment were found, whereas there were initially no effects found when analyzed until P24.

The body and stomach weights of the rats of the second experiment that were used for the cGMP biochemistry at P8 (females, except for one male) and P24 (only males) were also measured, after which body/stomach weight ratios were calculated, as is summarized in Table 2. At P8 there was no difference in body weight between LN NAME and control-treated rats (see Table 2). Likewise, there was no general effect on body weight during this early development (general mean Treatment: $F_{1.6}=3.27, n, s_{.}$), although $L-N A M E$ appeared to influence the course of the growth curve (Treatment $\times$ Day effect: $F_{8,48}=3.51, p<0.01$ ). Further, L-NAME-reated rats had a lower body weight than control rats at P19 $\left(\mathbb{t}_{6}=2.86, p<0.05\right)$ and $\mathrm{P} 24\left(\mathrm{t}_{6}=3.60, p<0.05\right.$; see Table 2). However, until being weaned at P19 only the course of the growth curve was affected by L-NAME treatment (Treatment $\times$ Day effect: $F_{19,14}=32.51, p<0.01$ ), while the treatment had no effect on the body weights in general during this developmental period (general mean 
TABLE 2. Body and somach werghts (g) at postratal day (P) and 24 of rats which had been postmatally trated with adaly s.c. injection of saline (CONTROL) or L-NAME (25 mg/kg).

\begin{tabular}{|c|c|c|c|c|c|c|}
\hline & \multicolumn{3}{|c|}{ CONTROL } & \multicolumn{3}{|c|}{ L-NAME } \\
\hline & Body & Siomach & Ratio & Body & Stomach & Ratio \\
\hline$\overline{\text { P\& }}$ & $15.8 \pm 0.6$ & $0.48 \pm 0.06$ & $0.031 \pm 0.004$ & $13.8 \pm 0.9$ & $1.87 \pm 0.16^{* * *}$ & $0.137 \pm 0.011$ \\
\hline P24 & $58.3 \pm 0.9$ & $1.76 \pm 0.16$ & $0.030 \pm 0.002$ & $50.2 \pm 2.1 \%$ & $1.75 \pm 0.29$ & $0.034+0.005$ \\
\hline
\end{tabular}

Values are shown ass mean \pm S.E.M. of four rats per group. For statistical analysis a $r$-test was used. ${ }^{\text {ac }} \mathrm{p}<0.05$ and wh $^{2} \mathrm{p}<0.01$ compared 10 CONTROL value at the same postnatal day.

Treatment: $\left.F_{1,6}=4.46, n . s.\right)$. L NAME treatment increased the stomach weights at P8 as is also clear from the decrease in body weight/stomach weight ratio (see Table 2). This increase in stomach weights is illustrated by the enlargement of the stomachs as is represented in Figure 2. At P24 L-NAME treatment did not influence the stomach weight anymore, although there was a decrease in body weights (Table 2 ).

To have a complete view on the effects of postnatal L-NAME treatment on the growth curve, the body weight data of the rats in the first and second experiment were also analyzed until P8. In the first experiment the body weights at P8 of L-NAME-treated rats were not different from those of the control rats $\left(t_{37}=1.57\right.$, n.s.), which was also the case when seen over this whole early developmental period (general. mean Treatment: $F_{3,35}=0.94$, n.s.). However, the increase in body weight of L-NAME-treated rats appeared to be slower than that of the control rats (Treatment $x$

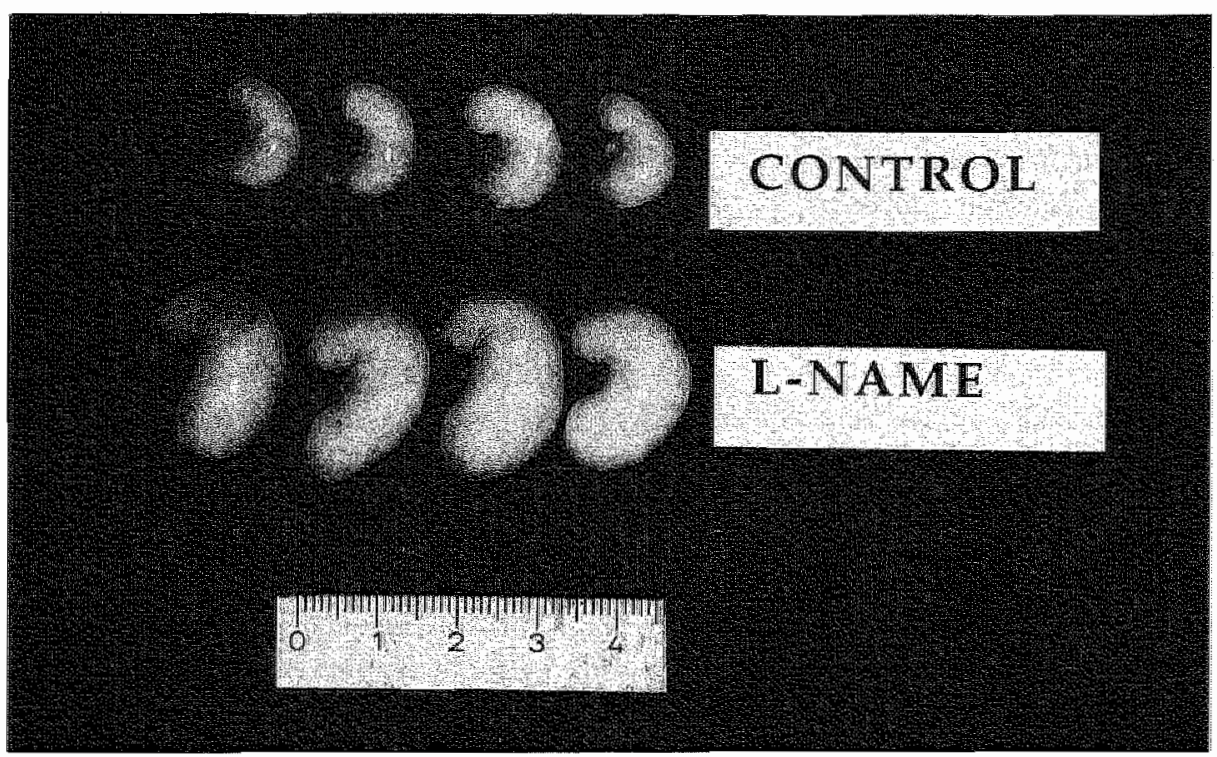

FG. 2. Stomach hypertrophy at postnatal day $8 \mathrm{in} \mathrm{L-NAME-hreated} \mathrm{rat} \mathrm{pups.} \mathrm{The} \mathrm{upper} \mathrm{four} \mathrm{stonachs} \mathrm{on} \mathrm{the}$ photograph are notnal stomachs of CONTROL (saline) injected rat pups, and the lower four stomachs are of $L$ NAME Ereated rat pups. The scale bar is in $\mathrm{cm}$. 
Day effect: $F_{8.280}=32.51, p<0.01$ ). In the second experiment L-NAME treatment already had a clear effect on the growth curve at P8 ws was evident from the group effect an this day $(44=3.29$, $p<0.01$ ), general treatment effect and treatment by day interaction effect (both $F \mathrm{~s}>4,20, p<$ 0.05 ). All these findings were not influenced by gender (all $F_{5}<1.09$, n.s.)

\section{cGMP immunocytochenistry}

CGMP immunocytochemistry showed that in slices of control rats of both the frontal cortex and striatum at P8, there was intense cGMP immunoreactivity in cell somate and dendrites, and in fibers containing numerous varicosities. This is illustrated in Figure $3 \mathrm{~A}$ which shows cGMP immunostaining in the frontal cortex at P8 under basal conditions. The results of the cGMP immunostaining of the striatum are not represented because they were comparable with those of
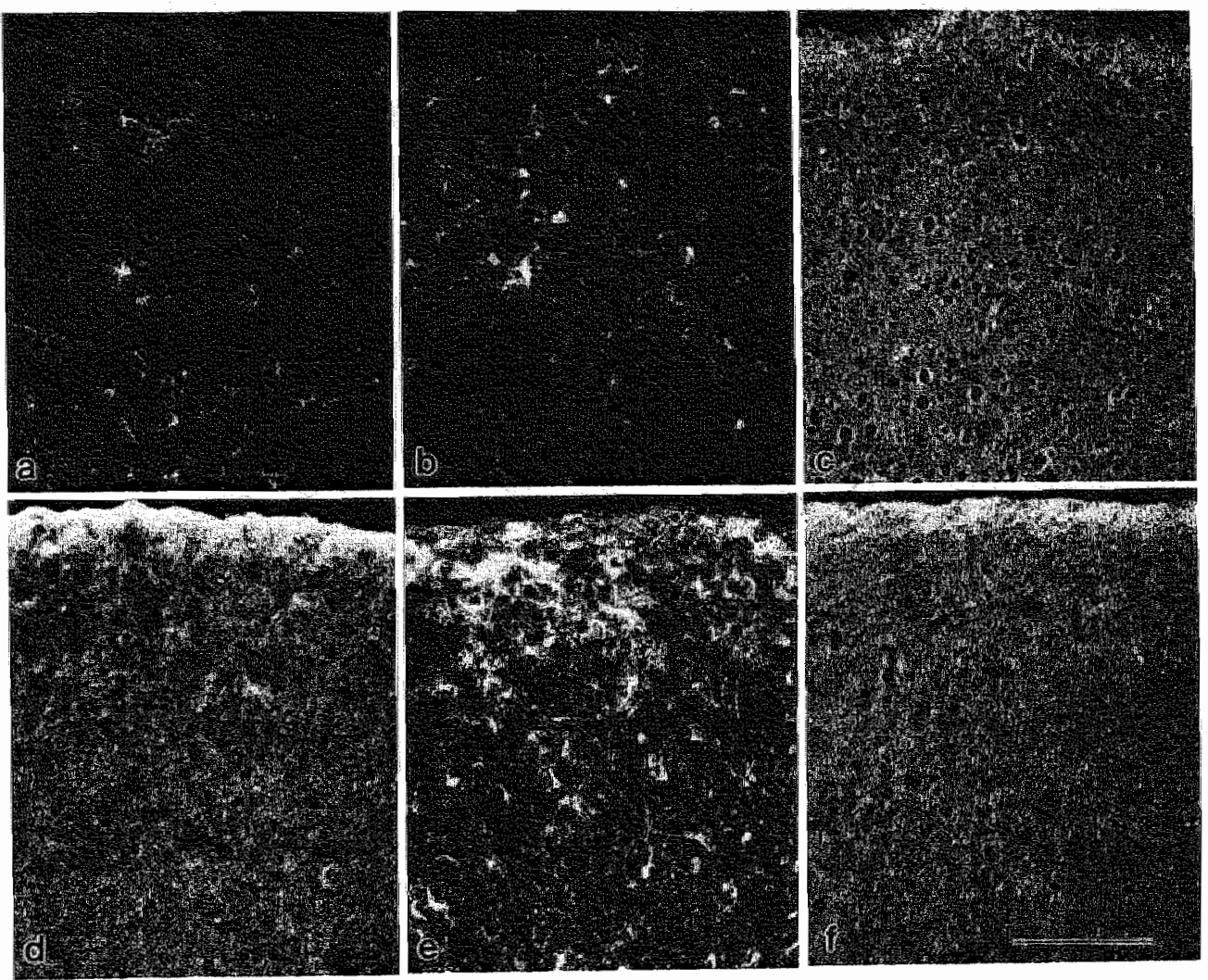

FIG. 3. Cyclic GMP immunostaining in the frontal contex of saline atreated (CONTROL; A, B, C) rat pups and L-NAME-treated (D, E, F) rat pups at postnatal day 8. Basal cyclic GMP immunostaining (A, D) and cyclic GMP immunostaining in the presence of $0.1 \mathrm{mM} \mathrm{SNP}(B, E)$ or $0.1 \mathrm{mM}$ L-NAME $(C$, F). The exposure times of the different photographs were not standardized. Scale bar is $100 \mu \mathrm{m}$. 
Whe frontal conex. Incubation with SNP greatly increased the intensity of basal cGMP immunostaining in 8-day-old control slices of the frontal cortex (see Fig. 3B). After incubation of control slices of the two brain structures of the same age with L-NAME, the cGMP immunoreactivily was allmost completely absent (see Fig 3C). Basal cGMP immunostaining in cell somata and fibers in frontal cortex of L-NAME-treated 8-day-old rat pups was almost completely abolished (see Fig. 3D), just as was found after in vitro L-NAME incubation (see Fig. 3F). However, after incubation with $\mathrm{SNP}$ again a great intensity in cGMP immunostaining could be found in the frontal cortex slices at P8 of L-NAME-treated neonates (see Fig. 3E).

At 124 , both frontal cortex and striatal slices of control animals showed almost no cGMP immunoreactivity (data not shown). Furthermore, at P24 no differences could be observed in cGMP immunostaining of frontal cortex and striatal slices between L-NAME-treated and control rats for all incubation conditions (data not shown).
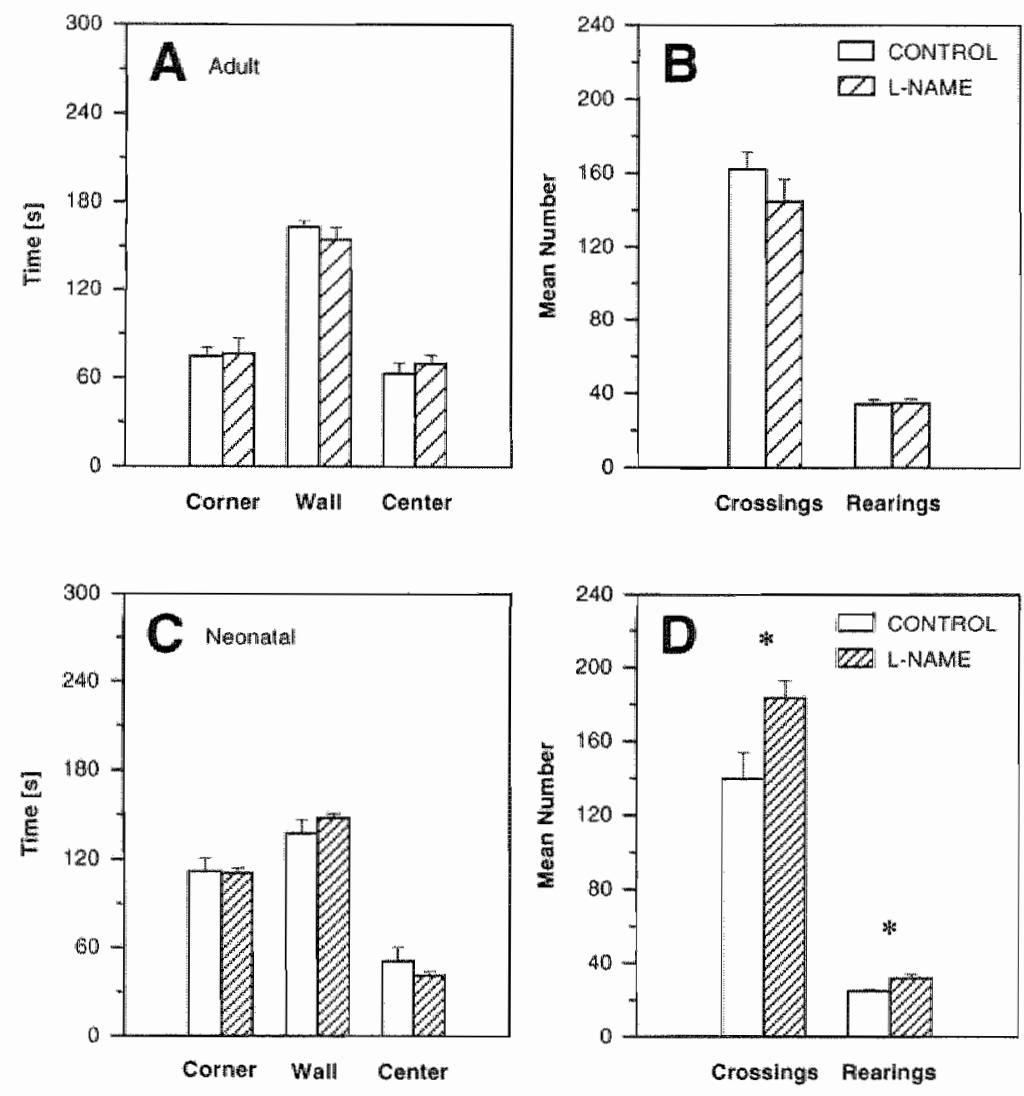

FIG. 4. Performance in the open field test of $(A, B)$ adult rats (4-month-old) that had been postnatally treated with L-NAME or saline (CONTROL), and of (C, D) neonatal rats (postnatal day 20 ) that were treated with $L$ NAME or saline. Mean total time (+S.E.M.) spent in the different areas (A, C) and the mean total number ( + S.E.M.) of line crossings and rearings (B, D) in the open field (aggregated data of four 5 min sessions, one session per day). * $p<0.05$ Treatment effect. 


\section{Open field}

The results of the open field test are summarized in Figure 4. Postnatal treatment with L-NAME did not affect the behavior of the rats in the open field in adulthood (all is < 1.16, n.s.; Fig. 4A and $B$ ). When tested at $\mathrm{P} 20-23$, rats that were being treated with L-NAME spent ulso the same amounts of time in the different areas of the open field as the control rats (all $t s<0.56, n, 5$.; Fig. 4C). The L-NAME-treated neonates were, however, more active than their controls, as measured by the total number of line crossings and rearings $\left(t_{13}=-2.60\right.$ and -2.44 , respectively, $p<0.05$; Fig. 4D).

\section{Morris water escape rask}

The acquisitions of the different water escape tasks are summarized in Fignre $5 \mathrm{~A}, \mathrm{~B}$ and $\mathrm{C}$. The corresponding probe trials are summarized in Figure 5D, E and F. All adult rats reduced their escape latencies during acquisition of the spatial discrimination task (session effect: $F_{5,90}=66.13$, $p<0.01$; Fig. 5A). There was no difference in the overall session performance (general nean Treatment: $F_{1,18}=1.48$, n.s.) and the rate of improvement over sessions (Treatment $\times$ Session effect: $F_{5,90}=0.41$, n.s.) between the postnatally L-NAME-treated rats and their control group. In the probe trial both groups of rats showed a quadrant preference (Quadrant effect: both $F$ $14.29, p<0.01 \%$. In addition, it was found that both groups spent nost of their time in the training quadrant (both $s s>4.43, p<0.01$; Fig. 5D). The time spent in the training quadrant was the same for the L-NAME-treated group and the control group $\left(t_{i}=0.04, n . s.\right)$. During the reinstatement session there was no difference between the two groups in escape latency (s) (mean \pm S.E.M.: CONTROL $=4.5 \pm 0.4$ and $L-N A M E=4.7 \pm 0.7 ; t_{18}=-0.27$, n.s. $)$.

During the spatial discrimination reversal task both groups improved their performance (Session effect $F_{1.18}=26.03, p<0.01$; Fig. 5B). Again, no difference was found in overall reversal performance and rate of improvement over sessions (both $F_{s}<0.43$, n.s.) between the L-NAME-treated rats and their control group. In the probe trial it was found that both groups had a quadrant preference (Quadrant effect: both $F s>9.09, p<0.01$ ). Further analysis showed that for both groups only the time spent in the training quadrant was above chance level (both $4 s>$ 2.66, $p<0.05$; Fig. 5E), i.e. again most of the time was spent in the training quadrant. Likewise, again there was no difference in the time spent in the training quadrant between the $L_{w}$ NAME-treated group and the control group $\left(t_{18}=0.30, \mathrm{n} . \mathrm{s}_{\mathrm{s}}\right.$ ).

During the visual discrimination task all rats reduced their escape latencies (Session effect $F_{3,18}=27.01, p<0.01$; Fig. $5 \mathrm{C}$ ). while there was no difference in overall performance and rate of improvement (both $F \mathrm{~s}<2.17$, n.s.) between the L-NAME-treated rats and the control rats. In the probe trial after the visual task, both groups of rats showed a preference for a quadrant (Quadrant effect: both $F_{5}>7.20, p<0.01$ ), which appeared to be the northmeast quadrant (both $t s>3.83, p<$ 0.01 ; Fig. $5 \mathrm{~F}$ ). The time spent in this quadrant was the same for both groups $\left(\mathrm{t}_{18}=0.05\right.$, n.s. $)$.

\section{Shock sensirivity}

The shock sensitivities ( $\mathrm{mA}$ ) for the control adult rats and the L-NAME-treated adult rats were, median (range), $0.19(0.15-0.33)$ and $0.15(0.08-0.23)$, respectively. These shock sensitivities were not different from each other $\left(\chi^{2}=2,69, \mathrm{n} . \mathrm{s}\right.$ ). The shock sensitiwities (mA) measured at P23 for the control and L-NAME-treated rats were, median (range), $0.10(0.05-0.15)$ and 0.13 $(0.08-0.18)$, respectively. These shock sensitivities were not different between the two groups of $\operatorname{rats}\left(\chi^{2}=1.14\right.$, n.s. $)$. 

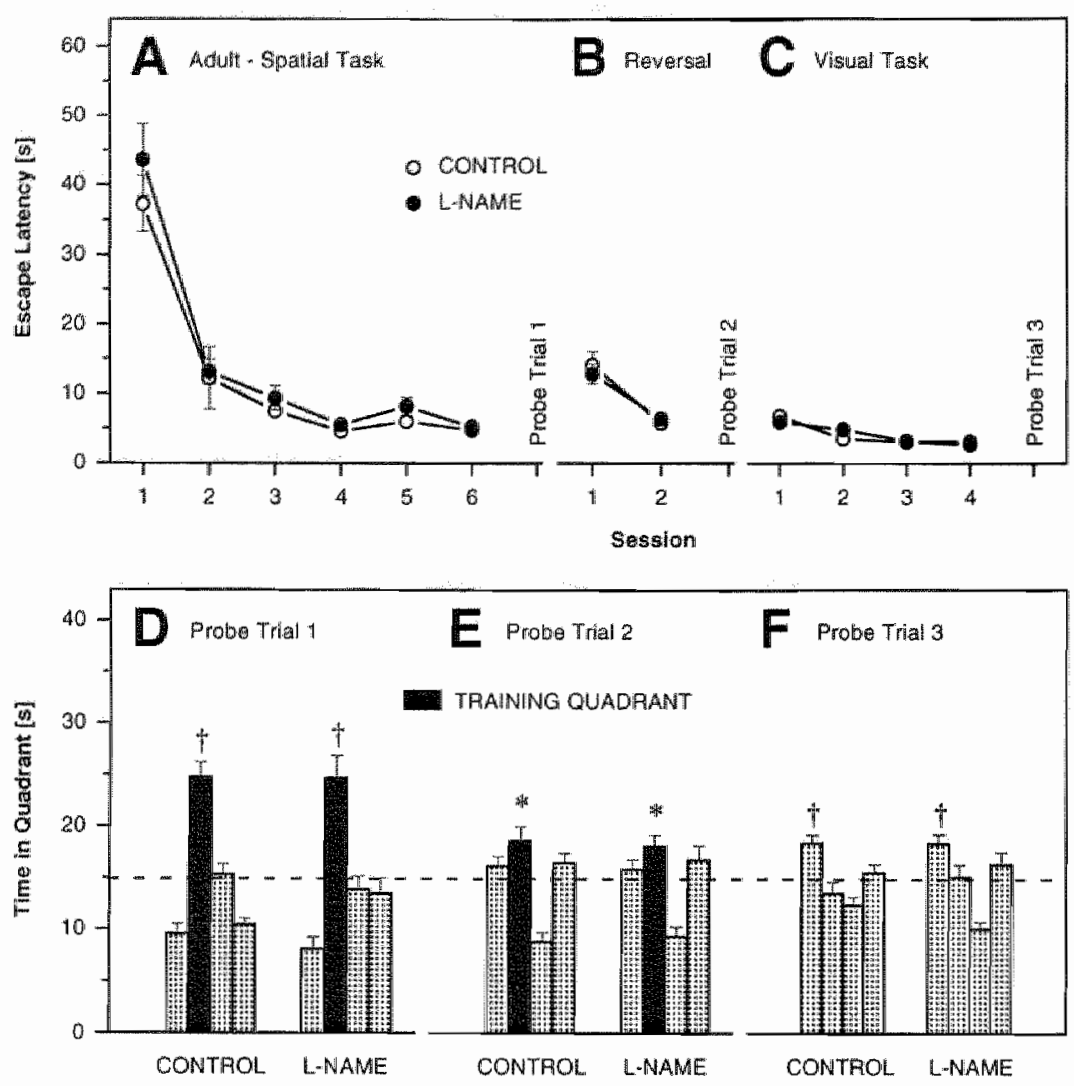

FIC. 5. Performance of adult rats (4-monthold) that had been postnatally treated with L-NAME or saline (CONTROL) in the Morris water escape iank. Mean escape latencies (HS.E.M.) for each session during acquisition of (A) the spatial discrimination task, (B) the reversal task, and $C$ ) the visual discrimination task (four triats por session, (wo sessions per day). Mean times (+S.E.M.) spent in the four quadrants in (D) the first probe trial after the spatial task, (E) the second probe trial after the reversal task, and (F) the thro probe trial after the visual task (probe trials always 24 hafier the last session). The four quadrants are organized with respect ro the

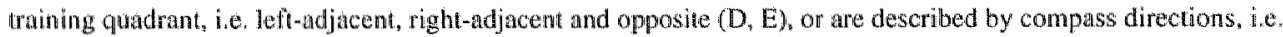
north-east, south-east, solith-west and north-west (F). $p<0.05$ and $p<0.01$ for time spent in quadrant above chance lowel $(15 \mathrm{~s})$.

\section{Two-way active avoldance leaming}

The results of the two-way active avoidance task in a shuttle box are summarized in Figure 6. During acquisition of the avoidance task of the adult rats, both the control group and the LNAME-treated group increased the number of avoidances. (Trial block effect: $F_{4,72}=27.16, p<$ 0.01; Fig. 6A). There was no difference in the mean performance (general mean: $F_{1,18}=0.00$, n.s.) and the rate of improvement (Treatment $\times$ Trial block effect: $F_{4,72}=1.09$, n.s.) over the trial blocks between the two groups of adult rats. None of the neonatal rats showed a change in the number of avoidances over the trial blocks (Trial block effect: $F_{4.48}=0.76$, n.s.; Fig. $6 \mathrm{~B}$ ). In 

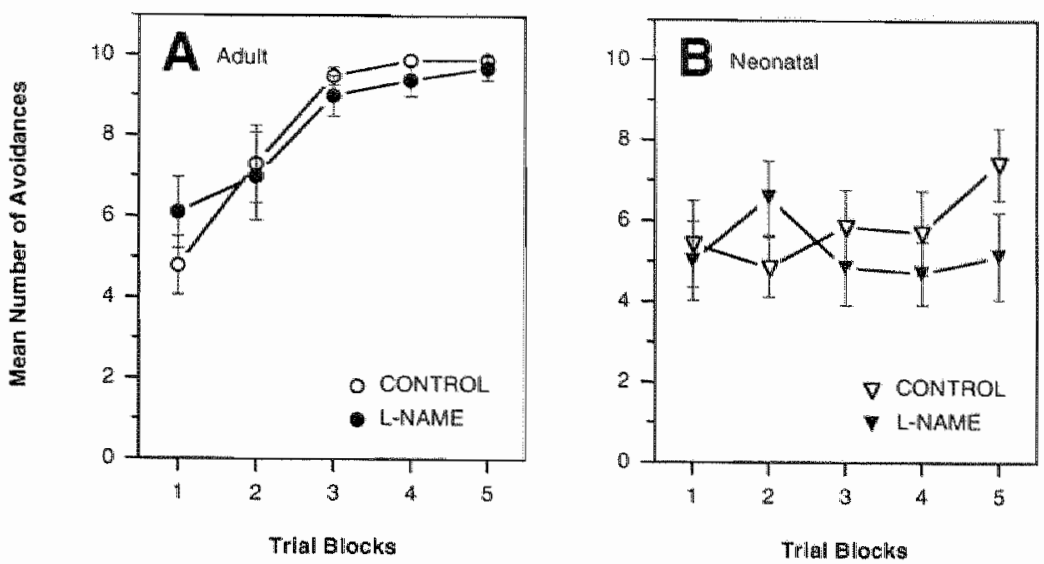

FIG. 6. Mean total number of avoidance responses ( $t$ S.E.M.) during acquisition of an active swoidance rask in a shuttle box of (A) adult rats (4-month-old) that had been postnatally treated with L-NAME or saline (CONTROL), and of (B) neonatal rats (posinatal day 25) that were treated with L-NAME or salino.

addition, the mean number of avoidances made by the neonatal rats over the trial blocks was not different between the L-NAME-treated group and the control group (general mean: $F_{1,12}=0.40$, n.s.).

\section{cGMP biochemistry}

The results of the radioimmunoassay determination of cGMP levels in the hippocampus, frontal cortex and striatum are summarized in Table 3. The slices of control rats showed that basal cGMP levels in the hippocampus, frontal cortex and striatum declined with advancing age. In vitro incubation of control slices of these brain structures with SNP or L-NAME stimulated and inhibited, respectively, cGMP formation at all ages.

At P8 the L-NAME treatment led to reduced basal cGMP levels in the hippocampus, frontal cortex and striatum as compared with the control treatment, whereas at P24 and P130 basal cGMP levels were not affected anymore in these brain stnctures. Incubation with SNP at P8 increased basal cGMP levels in the hippocampus, frontal contex and striatum of the L-NAME-treated rats. Incubation of hippocampal and frontal cortex slices of L-NAME treated rats with L-NAME at P8 did not further decrease the basal cGMP levels, while the basal cGMP level of the L-NAME-treated rats' striatum at P8 could still be decreased by L-NAME incubation. At P24 and PI30 incubation of slices of the three brain structures with SNP or L-NAME had the same effect on CGMP formation as in their corresponding slices of control rats.

\section{NADPH histochemistry}

NADPH histochemistry of hippocampus, frontal cortex and striatum of rats at P24 revealed several types of non-pyramidal interneurons. This is illustrated in Figure $7 \mathrm{~A}, \mathrm{~B}$ and $\mathrm{C}$ showing NADPH-positive cells in the cortex, striatum and hilus of the dentate gyrus, respectively. NADPH staining was also observed in blood vessel walls and thin, varicose fibers throughout all three brain 
TABLE 3. Cyclic GMP levels (pmol/mg protein) in slices of different brain regions ar posinatal day (P) \&, 24 and 130 of rats which had been postnatally treated with a daily s.C. injecrion of saline (CONTROL) or L-NAME $(25 \mathrm{mg} / \mathrm{kg})$.

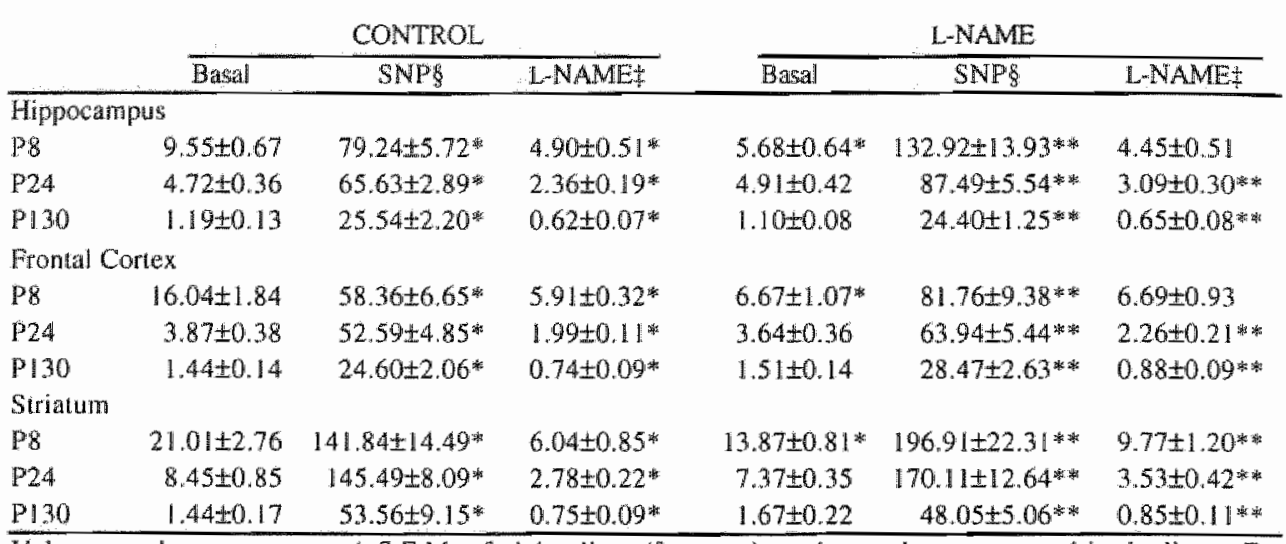

Values are shown as means \pm S.E.M. of eight slices (four rats); each sample was assayed in duplicate. For statistical analysis values were transformed to the natural logarithm to remove inhomogeneity. A one-factorial ANOVA, followed by a Duncan's multiple range test was used.

FL-NAME (0.1 mM) was present the whole incubation ( $30 \mathrm{~min}$ ).

$\$ S N P(0.1 \mathrm{mM})$ was added the final $10 \mathrm{~min}$ before terminating the incubation.

" $\mathrm{p}<0.05$ compared 10 CONTROL basal levels; "the $<0.05$ compared to L-NAME basal levels.

structures (e.g. Fig 7A). In the hippocampus, NADPH staining was mainly concentrated in neurons located against the inner sides of the pyramidal cell layer (mainly the CAI field) and the granule cell layer of the dentate gyrus (see Fig. 7E). There were no differences in NADPH staining in the cortex, striatum and hippocampus between L-NAME-treated (see Fig. 7B, D and F) and control rats (see Fig. 7A, B and C) at P24.

\section{DISCUSSION}

\section{Mortality and weights}

For the behavioral studies and most of the biochemical studies only male rats were used. The mortality and body weights data offered the possibility to check whether gender affected mortality and weights differently. With respect to mortality it was found that in both experiments almost exclusively L-NAME-treated animals died and that mortality occurred mainly within the first three days after beginning of the treatment. In the second experiment even a statistically reliable relationship between treatment and mortality was found, that is rat pups treated with L-NAME were about 1.3 times more likely to die than control-treated subjects. Additionally, we found that gender appeared not to influence the mortality due to the postnatal L-NAME treatment.

In both experiments it was found that L-NAME treatment, which started at P0, affected the increase in body weights of the neonates. This effect appeared to be largest in the second experiment because an additional effect was found on the overall growth curve (general mean) and the body weights were already affected at an earier postnatal day. In a very recent study in 

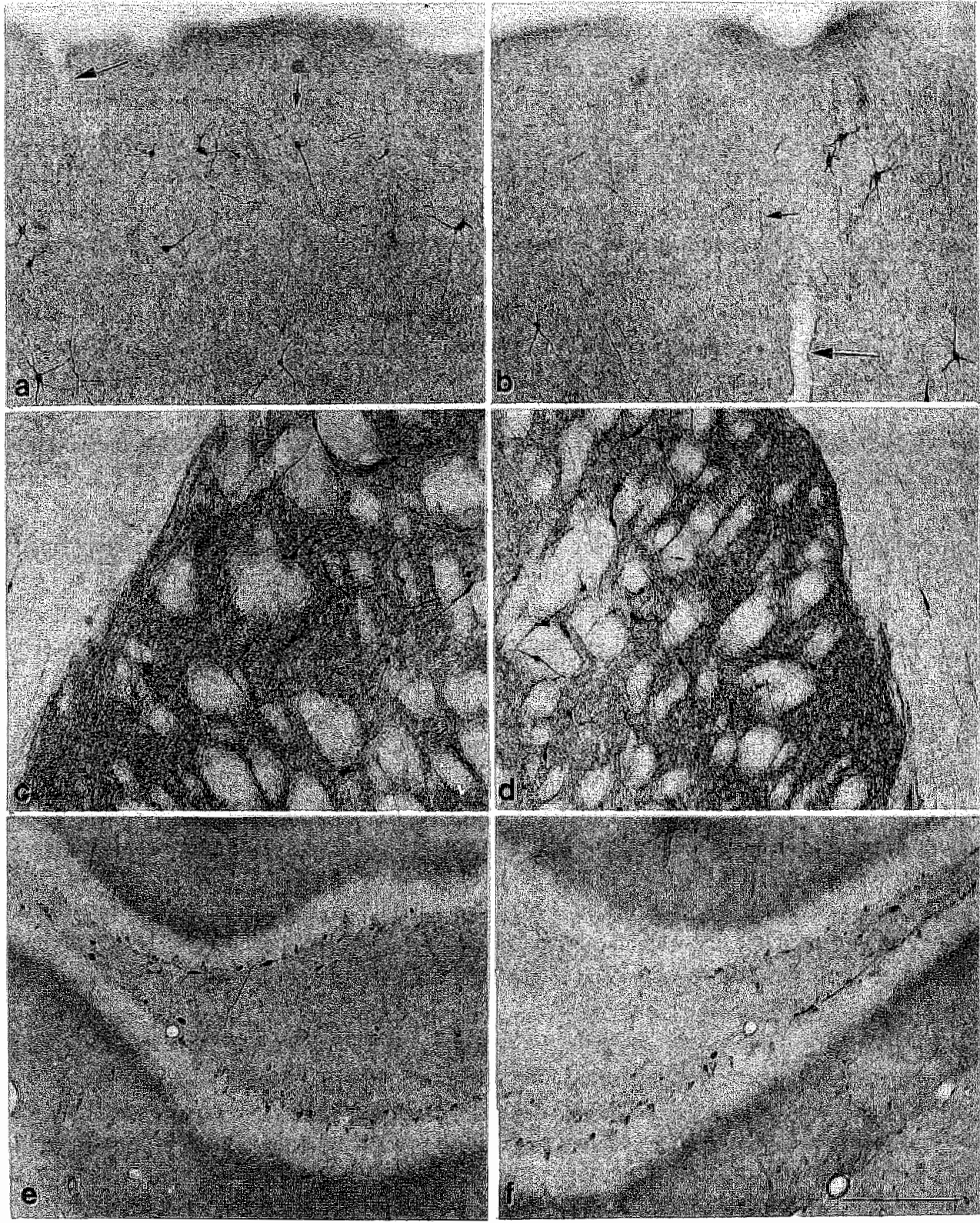

FIG. 7. NADPH staining in brain sections of saline (CONTROL; $A, C, E$ ) and L-NAME-treated $(B, D$, F) rat pups at posinatal day 24. NADPH-posiniwe neurons and fibers in the cortex $(A, B)$, striatum $(C, D)$, and hilus of dentate gyrus (E, F). Small arrows indicate thin, varicose fibers and large arrows indicate blood versel walls. Scale bar is $100 \mu \mathrm{m}$. 
which rat pups received an injection of the NO synthase inhibitor $N$-nitro-L-arginine (L-NA, rwice daily $50 \mathrm{mg} / \mathrm{kg}$ i.p.) from $\mathrm{P} 810 \mathrm{P22}$, the growth curve was reported not to be affected [58]. This finding is in contrast with the reduction we found in the course of the growth curve of neonates treated with L-NAME from PO to P23. This contradiction is surprising because L-NA was administered i.p. (instead of s.c.), twice daily (instead of once), and at a high dose $(50 \mathrm{mg} / \mathrm{kg} \mathrm{L}$ NA versus $25 \mathrm{mg} / \mathrm{kg}$ L-NAME). L-NA is thought to be responsible for inhibition of NO synthase after its methyl ester L-NAME is in vivo demethylated [36]. Furthermore, peripheral administration (i.p. and I.w.) of L-NA appears to be more potent than L-NAME, that is there is an even quicker onset and a lower final degree of NO synthase inhibition in the brain compared to L-NAME 141], For instance, compared to an i.p. administrated dose of L-NAME, a ten times lower dose of L-NA was found to have the same degree of NO synthase inhibition in the cerebellum. Therefore, a dose of $50 \mathrm{mg} / \mathrm{kg}$ L.NA, twice daily administered $\mathrm{i} . \mathrm{P}$. should be sufficient to continuously inhibit central $N O$ synthase maximally. Although we used only one daily administration of L-NAME ar a dose of $25 \mathrm{mg} / \mathrm{kg}$, it can be assumed that NO synthase activity was quickly maximally inhibited within 2 h after its administration, which lasted at least $24 \mathrm{~h}$, so central NO synthase activity was also continuously maximally inhibited [s. 24]. The major difference between the Lu-NA study and the present one is that treatment in the former study yet started at P8. Note that the central effect in our study as described by the decrease in basal cGMP levels due to the L-NAME treatment, appeared within the first 8 days after birth (see below). This could explain the lack of an effect on body weights because the L-NA treatment started after this early period as NO synthase apparantly needs to be inhibited immediately after birth to affect the growth rate of newborn rats. In addition, another explanation could be that it was mentioned, although the growth curves were reported to be similar between the L-NA-treated rats and control rats, that subjects receiving L-NA injections were slightly heavier at the beginning of the experiment and remained so. Hence, this could have occluded a possible treatment effect of L-NA on the growth curve.

The rats of our first experiment were weaned within 1 day after ending of the L-NAME treatment, that is P24. The rats of the second experiment were weaned at P19, while their treatment was also continued until P24. When analyzed until PI9 the effect of L-NAME treatment on the course of the growth curve was found in both experiments. However, in the first experiment, body weights were not affected anymore at $\mathrm{P} 24$ while in the second experiment the weights were still reduced conpared to the controls at P24. This finding suggests that at the end of the developmental period body weights already started recowering from the $L-N A M E$ treatment, unless rats were weaned before the end of the developmental period. In addition, we found that gender did not influence the growth curves.

It is possible that the reduced body weights and increased mortality of L-NAME-treated neonates are due to the enlarged stomachs as were found at P8. Although L-NAME is a non-selective inhibitor of NO synthase and thus inthibits both the neuronal and endothelial isoform of the enzyme, enlarged stomachs and increased mortality have also been observed in mice lacking the neuronal NO synthase gene [22]. The most evident effect of disrupting the neuronal NO synthase gene is the development of grossly enlarged stomachs with accompanying hypertrophy of the circular muscle layer of the stomach and the pyloric sphincter [22]. These effects may be seen as adaptive responses to primary effects due to the lack of NO production, like a deficiency of pyloric relaxation. The resulting gastric outlet obstruction and thas disrupted digestion, might be responsible for the reduction in the course of the growth curve or even for the 
increase in mortality of L-NAME-treated neonates.

Finally, postmatally undernourished rats have been found to show long-lasting decreases of body weight and/or brain weight [e.g. 1, 44]. In addition, undernourished rats showed also other effects which persisted into adulthood, like an affected monoaminergic metabolism [44] and an impaired cognitive performance in a passive awoidance task [1]. On the other hand effects like a decrease in locomotor activity and an increased shock sensitivity appeared to be of a more short-lasting nature [1]. Hence, it should be taken into account that possible behavioral and biochemical effects may (at least particularly) be a result of undernutrition of treated-rats and not of a developmental defect. However, it can be argued that in the present study undernutrition did not explain the observed results. Firstly, because no locomotor effect was found in the first 5 -min open field session of neonatal rats, while had they been undernourished a decrease in locomotor activity could be expected 11. Secondly, because of the low and transient decrease in body weights after the L-NAME treatment.

\section{Open field}

The open field thest is widely used to assess emotional reactivity/anxiety. It provides measures of locomotor activity: horizontally directed activity (or locomotion [34]) is measured by the number of line crossings, and vertically directed activity (or exploration [34]) is measured by the frequency of rearings. An increase in both activity measures when aggregated over consecutive open field sessions, is assumed to reflect a lower level of anxiety [50]. The open field test also provides occupancy measures of which an increase in the aggregated time spent in the corner squares is considered as reflecting a higher level of anxiety [51]. This occupancy measure of LNAME-treated neonates was not affected, whereas our activity measures indicated that inhibition of NO synthase had an effect on the locomotor activity of neonatal rats. This effect may be due to an affected neuronall development as a result of the chronic L-NAME treatment and/or to an acute effect of the L-NAME treatment, of which the latter has been reported to thave an acute anxiolytic effect 154 . Howewer, the increase in locomotor activity of L-NAME-treated neonates can not be interpreted in tems of anxiety-related behaviors, because there appeared to be no correlation between their activity measures and the occupancy measure. Based on earlier studies, it was expected that the activity meastres and the occupancy measure of the open field test would be (negatively) correlated, as they are indices of the same trait of anxiety 137. 51]. This discrepancy can be attributed to differences in treatment, strain and (young) age of rats. For the adult rats it was found that neither the activity measures nor the occupancy measure in the open field were affected by postnatal L-NAME treatment, suggesting recovery of behavioral function after termination of the treatment.

Open field-like tasks are often used to assess only activity by measuring the number of line crossings and/or rearings during one session. Recently, it has been found that inlitbition of NO synthase with L-NA during development from P8 to P29, had no effect in adulthood (5month-old rats) on the number of rearings, while the number of line crossings decreased during a 60-min exposure in an open fielld [58\%. However, closer inspection of this open field data of this study showed that during the first 5 min the number of line crossings and rearings were not different between the L-NA-treated group and the control group. This is in agreement with our findings of the first open field session, which lasted also 5 min, in which no effects of the $L$ NAME treatment on the activity measures were found in adulthood.

Whether chronic postnatal inhibition of NO synthase affects locomotor activity in neonatal rats is not known, although acute NO synthase inhibition with L-NAME has been found to 
decrease locomotor activity in adult rats [e $3: 42]$. In our study the locomotor activity of the neonates treated with L-NAME was not affected in the first session of the open field test. This suggests that the increase in behavioral activity of the L-NAME-treated neonates as measured over four consecutive sessions, was not due to acute effects of the treatment, but can be attributed to a chronic effect, although the influence of the very young age of the rats cannot be ruled out either.

Some of the neonatal and adult rats of the present study (all females) were used for another experiment which included blood pressure measurements (unpublished data). At P24, that is 24 hours after the last L-NAME injection, a small but statistical ellevation in blood pressure of the LNAME-treated neonatal rats was observed (about $15 \mathrm{~mm} \mathrm{Hg}$ ), indicating that during behavioral testing blood pressure had been increased. In adulthood there was no difference in blood pressure between L-NAME-treated and control rats. Whether the increase in blood pressure at P24 was due to NO synthase inhibition or another effect which chronically changed blood pressure, is not clear, but it is in agreenent with the increase in blood pressure found after acute NO synthase inhibition with L-NAME [42]. However, the latter also resulted, as mentioned above, in a decrease in locomotor activity which was not in agreement with our increase in behavioral activity [42]. In spite of this divergence in behavioral effects the possibility still exists that an increase in blood pressure of the L-NAME-treated neonates might have influenced their behavior in the open field.

\section{Morris water escape task}

Spatial discrimination learning not only involves place leaming, which is learning a position in space which is in this case the position of the hidden platform in the Morris water escape task, but also involves non-spatial components like procedural learning (such as learning to search for an escape plation) and visual or other sensorimotor processes together with possible motivational/emotional processes necessary for executing the task [31, 56]. After acquisition of the spatial Morris water escape task, a probe trial can reveal whether the rats have actually learned the position of the platform. Furthermore, a spatial discrimination reversal task after the acquisition of the spatial task, measures mainly place leaming because the rats are already familiar with the procedural component. It was found that in both the spatial and its reversal task, the performance of L-NAME-treated rats during the acquisition and in the probe trial did not differ from that of control rats. This indicates that place learning was intact in adult rats that had been treated postmatally with L-NAME. In addition, this implicates that other processes like visual or other sensorimotor processes were also not affected. This was supported by the data of the visual for cue) version of the Morris water escape task, in which postnatal L.NAME treatment had no effect on visual discrimination learning. However, in the probe trial after the visual task, both groups had a bias towards the north-east quadrant, while no preference for any quadrant was expected because the visible platiorm had been positioned randomly during acquisition of the visible task. The north-east quadrant had even never been used as a training quadrant in the spatial tasks as is illustrated by Figure 5 in which the description of the quadrants for the third probe trial coincide with the organization of the quadrants in the second probe trial (see Fig. $5 E$ and F): the south-east quadrant in the third probe trial served during the second probe trial as the training quadrant, and the north-west quadrant corresponded with the opposite quadrant in the second probe trial which had also been the training quadrant during the first probe trial. The bias toward the north-east quadrant cannot be explained by the experiences of the animals with the previous spatial discrimination learning. It is more likely that the bias corresponds towards the location of the carrying cage which was standing nearby the north-east quadrant. In spite of this finding, there was no difference in the quadrant preference between L-NAME-treated rats and control rats. 
Hence, there is still no indication that sensorimotor processes were influenced by the L-NAME treatment.

Recently, it has been reported that the inhibition of NO synthase with L-NA during development of the rat from P8 to P29, had no effect on the atcquisition of the spatial Morris water escape task in adulthood (5-month-old rats) [58]. Hence, it can be assumed that spatial discrimination in adult life is not affected by postnatal inhibition of NO synthase. This is supported by our finding that chronic postnatal L-NAME treatment from P0 to P24 did not affect the acquisition and probe trial performance of adult rats (about 4 months old) in the spatial Morris water task. The support for the assumption is further extended by the lack of an effect on the performances in the spatial reversal task. Hence, it can be concluded that inhibition of NO synthase during development does not affect spatial behavior in later life.

\section{Shock sensitivity and wo-way active avoidance task}

The effects of postnatal NO synthase inhibition on shock sensitivity and behavior in the two-way active avoidance task have not been evaluated yet in neonatal and/or adult rats. The shock sensitivity allows one to monitor possible differences in responsiveness to electrical foot shock. For both neonatal and adult rats there was no difference found in sensitivity to the electrical shock between L-NAME-treated rats and control rats, indicating that chronic postnatal inhibition of NO synthase had neither long-lasting nor short-lasting effects on shock sensitivity of rats. This implicates that possible differences in two-way active avoidance performance between control and L-NAME treated rats, are not influenced by differences in shock sensitivity.

None of the neonatal rats learned the two-way active avoidance task in the shuttle box. This lack of a generall learning effect could be due to a shock intensity that might have been too high for the neonates. However, the neonates were not behaviorally inhibited. They started just as the adult rats, which received also the same shock intensity, at a sort of chance level where about $50 \%$ of the responses were correct, i.e. avoidances, while the remaining ones were incorrect escapes responses. However, in contrast with the adult animals, the neonates did not improve their performance during the subsequent trials. Another possible explanation for the lack of a learning effect in neonates could be that the ability for learning this task was not yet fully developed at P24. All adult rats learned the task and there was no difference in the rate of acquisition between the postnatally L-NAME-treated rats and control rats. Hence, it may be argued that chronic inhibition of NO synthase during development does not affect cognitive behavior in the two-way active avolidance task in later life.

\section{cGMP determinations and anatomy}

The quantitative (Table 3) and qualitative (Fig. 3) data revealed that at P8 the L-NAME treatment had resulted in a decline in basal cGMP levels in the frontal rat brain. Further inhibition of NO synthase with L-NAME in vitro was still somewhat effective in the striatum, but not in the hippocampus and frontal cortex. The NO donor SNP increased cGMP levels in all three brain structures of controls and L-NAME-treated neonates. Closer examination of the data showed that at P8 the cGMP response to SNP appeared to be increased in comparison with the one in controltreated neonates. This suggests that the NO-mediated SGC activity may have even been increased in sensitivity. All these data show that the L-NAME treatment was effective in inhibiting NOS activity and demonstrate the plasticity of the NO-cGMP signal transduction during development.

It has been found that the NO-mediated $\mathrm{SGC}$ activity in the frontal rat brain was maximal within the first two weeks after birth and declined thereafter $19,28,52]$. This is supported by our 
findings that basal cGMP levels and, to a lesser extent, the cGMP response to SNP declined during development. However, the decline was not so fast as was expected, because basal cGMP levels had yet not reached the adult levels at the end of the developmental period as had previously been found 19.28, 521. Likewise, de NO-mediated cGMP response started declining only after the developmental period. However, it should be noted that the cGMP measured in L-NAME-treated rats is certainly partly caused by the activity of the particulate isoform of guanylyl cyclase (pGC). pGC is insensitive to NO but instead activated by natriuretic peptides. Furthermore, we have prewiously shown that $\mathrm{pGC}$ is prominently present in the frontal rat brain during the first two weeks after birth [28],

The radioirnmunoassay data demonstrated only a partly reduction in basal cGMP by $L$ NAME treatment. However, the immunocytochemical data showed an almost complete disappearance of the intensely fluorescent cells in frontal cortex and striatum. It thas to be realized that cGMP will be bound to several target proteins like phophodiesterases and cGMP-dependent protein kinases [43]. This pool of bound cGMP is probably not visualized by the cGMP antisera because of shielding by the target protein. The cGMP antisera used in the present immunocytochemical study are directed against cGMP which is free in the cytosol before it is conjugated to tissue proteins by the fixative [10].

There are two constitutive isaforms of NO synthase that are activated by calcium: neuronal NO synthase and endothelial NO synthase, although the latter can also be found in neurons. In the present study we had no main interest which isoform of NO synthase could be involved in brain development. Also note that L-NAME is a non-selective inhibitor of NO synthase. However, independently of the isoform of $\mathrm{NO}$ synthase, its localization and that of $\mathrm{SGC}$ do not fully overlap. For instance, $\mathrm{NO}$ in the hippocampus is produced by NO synthase-containing cells which can be pyramidal cells, granule cells or interneurons 411 . More in detail, immunocytochemical staining revealed that endothelial NO symthase was highly concentrated in both pyramidal cells and granule cells of the hippocampus, whereas neuronal NO synthase was only found in hippocampal interneturon [11]. On the other hand, cGMP in the hippocampus can be produced by astrocytes and some interneuron [9]. However, NO synthase and $\$$ GC do not necessarily have to be co-localized in the same cells because NO is freely diffusible. This is in line with the finding that these enzymes apear to thave a complementary distribution, e.g. postsynaptic (NO synthase) versus presynaptic $\left(\mathrm{SGC}^{3}\right)[47]$.

NADPH diaphorase staining after somogyi fixation, in which mainly paraformaldehyde is used, stains both endothelial and neuronal NO synthase, as is also evident from the finding that blood vessel walls, which have a high concentration of endothelial NO synthase [23], were stained. Normally, the NO synthase content of a neuron fully accounts for its NADPH diaphorase staining [7]. Therefore, it can be argued that all NO synthase-containing cells were stained in the present study, independently of the NO synthase isoform. NADPH-positive cells and NADPH-stained varicose fibers were found throughout the hippocampus, cortex and striatum at $\mathrm{P} 24$. Furthermore, NADPH-positive cells in the hippocampus were mainly concentrated in neurons located against the inner sides of the pyramidal cell layer (mainly the CAI field) and the granule cell layer of the dentave gyrus, although pyramidal and granule cells itself apparently did not stained. There was no difference in NADPH diaphorase staining between the L-NAME-treated rats and control rats. These findings suggest that NO synthase or its containing cells were not morphologically affected at the end of development after the chronic treatment with L-NAME. The lack of an effect on cells containing NO synthase can be generalized over all cells in the hippocampus, cortex and striaturn, because hematoxylin-eosin staining neither revealed differences in in tissue structure or cellular composition at P24 (data not shown). Therefore, it is further suggested that chronic postnatal 
treatment with L-NAME has no effect on the maturation of the central nervous system at the pure morphollogical level.

\section{NMDA and development}

$N$-methyl-Di-aspartate (NMDA) receptor stimulation may lead to the production of NO by stimulation of the enzyme NO synthase by the NMDA-dependent Ca2+-influx [14]. NMDA receptors have a pronounced role in the development of the nervous system [e.g. 2, 3, 201, and, recently, the effects of chronic postnatal NMDA receptor blockade on brain function and behavior in adulthood have extensively been studied $[13.16-191$. If NO mediates neuronal responses to NMDA receptor stimulation in the brain, then interference with NO synthesis should induce changes similar to those of NMDA receptor manipulation.

In the present study, mortality was rather high during postnatal L-NAME treatment, In a study about the effects of postnatal NMDA receptor blockade using the NMDA antagonist MK- 801 , it was reported that mortality was also rather high, although depending on the dose used [13]. Further, it has been found in studies using MK-801 that body weights were very reduced (minimally about $35 \%$ ) and recovered only partially after postnatal treatment $[13,16,18,19,53]$. This is in contrast with our data showing a quick and complete recovery of an only modest decrease in body weight (about $10 \%$ ), probably already soon after termination of the L-NAME treatment. In open field-like lasks it has been reported that the postnatal MK-80I treatment increased locomotor activity [13, 16, 18]. This effect could be of long-lasting nature [13]. All these findings were done in only one session. However, in our first session of the open field test, no effect of LNAME treatment was found on locomotor activity for both the neonatal and adult rats.

In the Morris water escape task it was found that spatial discrimination learning was impaired in adulthood (about 4-month-old) of postnatally MK-801-treated (from P8-P19) rats, although eventually these rats learned the task as well as control rats as was evident from the probe trial performance in which both groups of rats spent the same time in the training quadrant $[18]$, Furthermore, learning in the spatial reversal task was impaired in the MK-801-treated rats, while the treatment did not affect the performance in the visual discrimination task. Also the hippocampal NMDA receptor function was affected in adult rats similarly postnatally treated with MK-801, as changes in affinity and sensitivity were found 117. 191. However, it should be mentioned that some studies reported no effect on spatial discrimination learning 1291 and hippocampal NMDA receptor function [93] shortly after termination of the chronic postnatal MK- 801 treatment. although it is likely that this was due to the early termination (already at Pl5) of the treatment or the low dose (twice as low) of MK-801 used. Place leaming is highly dependent on the integrity of the hippocampus as hippocampal lesions impair the performance in the spatial Morris water escape task during its acquisition and reversal learning [321. On the other hand, at hippocampal dysfunction will not affect the performance in a visual Morris water escape task. Likewise, it has been found that place learning is also dependent on functional NMDA receptors, whereas visual discrimination learning is not $[31,39$, but set 61 . It has, therefore, been argued that the deficits in place learning as found in the studies with postnatally MK-80I-treated adult rats might be due, at leasi in part, to long-lasting disturbances in hippocampal function and NMDA receptor function [16, 18 !. However, in the present study no place learning deficits were found in adult rats after chronic treatment with L.NAME during development, and, therefore, it can be suggested that the hippocampus and NMDA receptor function is normal in adult rats treated postnatally with L. NAME.

Taken together, the findings of the present study indicate that chronic postnatal inhibition of 
NO synthase with L-NAME had no effect on behavior and brain function in adulthood. This is in contrast with the findings after chronic postnatal NMDA receptor blockade with $M K-801$. In addition, it has recenty been found that chronic postnatal NMDA receptor blockade with HA-966, which acts at the glycine site of the NMDA receptor instead of the PCP site like MK-801 does, also impaired behavior (in a cogritive novelty test) in adulthood [5s]. Hence, chronic postnatal NMDA receptor blockade can impair behavior in adult life which conld be a result of a disturbed neuronal development. It is important to note that probably other glutamate receptor subtypes like amino-3-hydroxy-5-methyl-4-isoazolepropionate (AMPA) and kainate receptors are also involwed in neuronal development (e.g. affinity changes of the different glutamate receptor subtypes may reflect processes of synaptogenesis in the human hippocampus and adjacent cortex (251). However, it should be mentioned that the effects on the several variables (e.g. NMDA receptor affinity, locomotor activity or weights) were not universally found in all studies. This was likely to be due to the differences between the several studies in the site of the NMDA receptor blockade, in doses of substances used and durations of treatment. Therefore, caution should be exercised when interpreting the results of several treatment studies, especially when comparing studies which different type and doses of drugs, e.g. MK-801 versus L-NAME. Nevertheless, the lack of similarity in the effects of postnatal NO synthase inhibition and NMDA receptor blockade in adult rats, may suggest that a role of $\mathrm{NO}$ does not function necessarily to mediate neuronal responses after NMDA receptor stimulation in the developing brain.

\section{Conclusions}

In the present study we observed that cognitive behavior (spatial learning in water escape task, active avoidance learning) and non-cognitive behavior (open field test) were not affected in adult rats that had postnatally been treated with L-NAME. The former finding is in agreement with an earlier study in which cognitive behavior (spatiall learning in water escape task) of adult rats was not impaired after chronic postnatal NO synthase inhibition with L-NA [58]. Biochenical and immunocytochemical cGMP determinations in hippocampus, frontal cortex, and striatum revealed that during early development (first week) the NO-cGMP signal transduction was affected as basal cGMP levels decreased, while the NO-mediated SGC response appeared to be increased in LNAME-treated neonates. This indicates a form of neuronal plasticity ats a reaction to the inhibition of NO synthase. At the end of the developmentall period (after three weeks) the NO-cGMP signal transduction in the different brain structures of $\mathrm{L}$-NAME-treated rats appeared to function as normal again. In addition, L-NAME treatment appeared to have no gross effects on brain morphology. However, because an increase in behavioral activity was found in neonatal rats, it appeared that non-cognitive processes were influenced by the chronic postnatal L-NAME treatment, which may be indicative of an affected brain function. Short-lasting effects on cognition were, as far as measured (active avoidance learning), not detected. To summarize, inhibition of NO synthase during development may have short-lasting effects on non-cognitive behavior, but it apparently has no long-lasting effects on behavior and brain function in later life.

\section{ACKNOWLEDGEMENT'S}

The authors would like to thank Hellen Steinbusch for her expert technical assistance. Especially, we would like to thank Dr. Arjan Blokland for his helpful comments. 


\section{REFERENCES}

1. Ameida S.S., Soares E.G., Bichuete M.Z, Graeff F.O. and de Ohveira L.M. (1992) Ethects of early

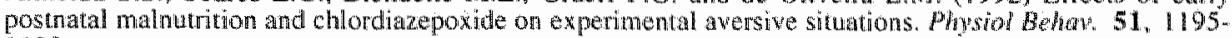
1199.

2. Brady R.J, Gorter J.A., Monroe M.T. and Swann I.W. (1994) Dewelopmental altertitons in the sensitwity of hippocampal NMDA receptors to A PS. Brain Res. Dev. Broin Res. 83, 190-196.

3. Brewer G.J. and Coman C.W. (1989) NMDA receptor regulation of neuromal morphology in cultured hippocampall neurons. Netrosci. Lef. 99, 268-273.

4. Brooker G., Harper J.F., Terasaki W.L. and Moylan L.D. (1979) Radioimmunoassay of cyclue AMP and cyclic GMP. Aar. Cyclic Nucleotide Res, $10,1-26$.

5. Cahill L. and MoGaugh J.L. (1900) Amygdaloid complex lesions differentinlly affect retention of lasks using appetive and aversive reinforcement. Behaw. Netrosct. 104, 532, 543 .

6. Cain D.P., Saucier D. and Boon F. (1997) "Testing hypotheses of spatial larning: The role of NMDA receptors and NMDA-mediated long-term potentation. Behan. Brain Res. 84, 179-193.

7. Dawson T.M. Bredi D.S., Fotuhi M., Hwang P.M. and Suyder S.H. (1991) Nitric oxide synthase and neuronal NADPH diaphorase are identical in brin and perpheral lissues Proc. Nan Acad. Sci. U.S.A. 88, $7797-780$.

8. De Vente J., Bol J.J.M. Berkelmans H.S. Schipper J. and Steinbusch H.W.M. (1990) Immunoytochemistry of CGM in the cerebellum of the immature, adult, and aged rat" The involvement of nitric oxide: A micropharmacological study Eur. No Neurose. 2, 845-862.

9. De Vente J. and Steinbusch H.W.M. (1992) On the stimulation of soluble and particulate guanglate cyclase in the rat brain and the involvement of nitatic oxide as studied by coMP immunocytoetwemistry. Acra Histochem. 92, 13-38.

10. De Vente J., Young H.M. and Steinbusch H.W.M. (1996) Immunohistochemical vissulization of cyclic nucleotides. In Methods in Nenroscience (ed. Maines M. D.), Vol. 31. pp. 68-79. Academie Press. San Diego, C.A.

11. Dinerman J.L., Dawson T.M. Schell M.j., Snowman A. and Snyder S.H. (1994) Endohelial nitric oxide synthase localized to hippocampal pyramidal cells: Implications for synaptic plastacity, Proc. Narl Acad. Sici. U.S.A. 91, 4214.4218.

12. Edelman G.M. and Gally J.A. (1992) Nitric oxide: Linking space and time in the brain. Proc. Nat Acad. Sci. U.S.A. 89, $11651-1.1652$.

13. Facchineti F. Ciani E. Dallolio R. Virgili M, Contestabile A. and Fonnum F. (1993) Suructural, neurochemical and behavoural consequences of neonatal blockade of NMDA receptor throwgh chronic treatment with CGP 3955 I or MK-801. Der. Bran Res. 74, 219.224.

14. Garthwaite I. (1991) Gilutamate, nitric oxide and cell-cell signalling in the nervous system. Trends Neurosci. $14,60-67$.

15. Giuli G., Luzi A., Poyard M. and Guellaen G. (1994) Expression of mouse brain soluble guanylyl cyclase and NO synthase during ontogeny, Dew. Broin Res. 81, 269-283.

16. Gorter J.A."Boterblom M.H., Feenstra M.G. and Boer G.J. (1992) Chronle neonatal NMDA receptor blockade with MK-80! atters monoamine metabolism in the adult rat. Nevrosci. Lest. 137, 97-100.

17. Gorter J.A. and Brady R.J. (1994) NMDA receptor responses in adult hippocampal CAI region alter neonatal treatment with MK-801: Compatison with NMDA receptom responses in tho immoture nut. Brain Res. 665, 63-68.

18. Gorter J.A. and de Bruin J.P. (1992) Chronic neonatal MK-80 I teatnent results in an impaiment of spatal learning in the adule rat. Broth Res. $580,12-17$.

19. Gorter J.A., Veeman M. and Mirmiran M. (1992) Hippocampat tueuronal responsiveness to NMDA agonists and antagonists in the adult tat neonalally treated with MK.801. Brain Re.5.52, 176-181.

20. Hattor $\mathrm{H}$ and Wasterlair $\mathrm{C} G$. (1990) Excinatory amino acids in the developing bram "Ontogerny plasticity, and excitotoxicily. Pediarr. Netrol, 6,219-228.

21. Hindley S., Jumrlink B.H.J., Gysbers J W., Middlemiss P.J., Herman M.A.R. and Rahbone M.P. (I997) Nitric oxide donors enhance neurotrophin induced reurite outgrowh through a cGMP dependent mechanism. J. Nerrosici. Res. $47,427-439$.

22. Huang P.L. Dawson T.M. Bredt D.S. Snyder S.H. and Fishman M.C. (1993) Targeted distuption of the neuronal nitric oxide synthase gene. Cell 75, $1273-1286$.

23. Iadecola C. Pelligrimo D.A.. Moskovitz M.A. and Lassen N.A. (1994) Nitric oxide synthase inhibition and cerebrovascular regulation, J. Cereb. Blood Flow Metab. 14, 175-192.

24. Iadecola C., Xu X., Zhang $E_{.,}$Hu J and El-Fakahany E. E. (1994) Prolonged inhibition of brain nitric oxide synthase by short-tiem systemic administration of nitro-L-arginite nedbyl ester. Newrocfem. Rex. 19, 501505 .

25. Johnson M., Perry R.H., Piggon M.A., Cour J.A., Spunden D. Lloyd S., Ince P.G. and Perry F.K. (1996) Glutamate receptor binding in the humart hippocampus and adjacent contex dung developmemt cund aging. Neurobiol. Aging 17, 639-651.

26. Kalb R.G. and Agostin J (1993) Molecular evidence for nitric oxide-mediated motor neuron development. Neurosci 57, $1,-3$. 
27. Lowmy O.L., Rosebrough N.J. Farr A.L. and Randall R.J. (1951) Protein mensurements with the Folin Phenol reagent. J. Biol Chem. $193,265,275$.

26. Markerink-wan Itarsum M. Steinbusch H.W.M. and De Vente J. (1997) Region-specific developmental

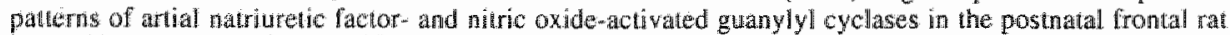
brein. Neuroscience $78,571-587$.

29. Melamb R.L. Williams L.R. Nangy K.P. Wison W.A. and Tison H.A. (1990) MK-801 impedes the acoustition of apatial merory task in rats. Pharnacal. Biochem. Behav. 37, 41-45.

30. Morris R.G.M. (1981) Spatial localization does not require the presence of local cues. Learm. Moriv, 12 . 239.261

31. Morts R.G.M. (1989) Synaplic plasticity and learning: Selective impaiment of leaming in rats and blockade of long-tem potentiation in viwo by the NMDA receptor antagonist APS. J. Nenuosic 9.30493057.

32. Morris R.G.M., Garard P., Rawlins J. N.P. and OReefe J. (1982) Place nawigation is impaired in rats with hippocampal tesions. Nature 207,681-683.

33. Murad F. Miltal C.K., Arnold W.P. Katsuki S. and Kimura H. (1978) Guanylate cyclase: Activation by axide, nitro compounds, fitric oxide, and hydroxyl radical and inhibition by hemoglobin and myogiobin. Adv. Cyclic Nucl Res. 9, 145-158.

34. OKeefeJ and Nadel Le (1978) The hippocampus as a cogniwhe map. Clarendon Press, Ox ford.

35. Ossenkop K. P. and Mramunian D.S. (1985) The principle of aggregation in psychobiological correlational research: An example from the open field test. Animb. Learn. Behav. 13, 339-344.

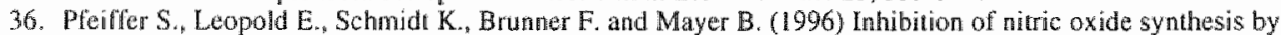
MG-nitro-L-arginine methyl ester (L-NAME). Requiremen for bioacuvation to free acid NG-aitro-L-arginine. Br. A. Pharmicol. 118, 1433-1440.

37. Prickaerts J., Raaimakers W. and Blokland A. (1.996) Effects of myocandial infarction and captopril therapy on anxiety related behaviors in the rat. Physiol. Bethas. 58, 43-50.

38. Pudiak C.M. and Bozanth M.A. (1993) L-NAME and MK-801 atternate senstization to the locomotorstimuluting effect of cocaine. Life Sici, $53,1517-1524$.

39. Robinson G.S. Crooks G., Shinkman P.G. and Gatlagher M. (1989) Behavioral effects of MK.801 mimive delicits associated with hippocampal damage. Psychobiology 17, 156-164.

40. Roskams A.J. Bredit D.S., Dawson T.M. and Ronnett G.V. (1994) Nitric oxide mediates the formation of synaptic comnections in developing and regenerating olfactory receptor newrons. Newnon 13, 2894299.

41. Salter M. Duffy C. and Hazelwood R. (1995) Determination of brain nitric oxide synthase inthibition in viwo: Ex viwo assays of nitric oxide synthase can give incorrect resulls. Newrophamacology $34,327-34$.

42. Sandi C., Venero C. and Guaza C. (1995) Decreased spontaneous motor activily and startle response in mitric oxide synthase inhibiton-treated Iats. Eur. J. Pharmacol. 277, 89-97.

43. Schmid H.H.H.W. Lolimann S.M. and Walter U. (1993) The nitric oxide and cGMP signal transduction system: Regulation and mechanism of action. Biochim. Biophys. Acta 1178, 153-175.

44. Seidler F.J. Bel J.M. and Slotkin T.A. (1990) Undernutrition and owernutrition in the neonatal rat: Longterm effects on noradrenergic pathways in brain regions. Pediatr. Res. 27, 191.197.

45. Smigrodzki R. and Levitt P. (1996) The alpha 1 subunit of soluble guanylyl cyclase is expressed prenatally in tho rat brain. Dev. Brain Res. 97, 226-234.

46. Southam E., East S.I. and Garthwaite J. (1991) Exchatory amino acid receptors coupled to the nitric oxide/cyclic GMP pathwy in at cerebellum during developmen. d. Neurochem, 56, 2072-2081.

47. Southam Lu and Gathwait I. (1993) The nitric oxide-cyclic GMP signalling pathway in rat brain. Neuropharmacology 32, 1267-1277

48. Steiner A.J., Parke C.W. and Kipnis D.M. (1972) Radiommunoassay for cyclic mucleotides. 1. Preparation of antiboties and iodinated oydic nucleotides. J. Biol. Chem 274, 1106 . 1113.

49. Truman J.W. De Vente J. and Ball E.E. (1996) Nitric oxide-sensitive guanylate cyclase activity is associated with the maturational phase of neuronal development in insects. Development $122,3949 * 3958$.

50. Wan der Stany F.J and Blokland A. (1996) Behavioral difference between outbred Wistar, inbred Fischer 344, Brown Norwity, and hybrid Fischer 344 x Brown Norway rats. Physiol. Behaw, 60, 97-109.

51. Wan der Staby F.J., Kerbusch $\mathrm{S}$, and Ratimakers W.G.M. (1990) Gentic correlations in walidating motionality. Behav. Gentet, 20, $51-62$.

52. Wan Eden C.G., Steinbusch H.W.M. Rinkens A. and De Vente J. (1996) Dewelopmental pattern of NADPH diaphorsse activity and nitio oxide-stimulated cGMP immunoreactivity in the frontal rat cortex and ins role in lunetional recovery from aspiration lesions. $J$. Chem. Neuroanat 10, 279-286.

53. Vư Lookeren Campagne M., Vermeuelen J.P. Boer G.j and Balázs R. (1995) Treatment wilh NMDA receptor antagonists does not affect developnental changes in NMDA teceptor properties in wivo. Neurochen. Int, 27, 355-366.

54. Volke V., Koks S., Vasar E., Bourin M., Bradwajn J. and Mannisto P.T. (1995) Inhibition of nitric oxide synthase canses anxiolytic like behaviour in an elewated plus-maze. Neuroreport 6, 1413-1416.

55. Wangen K., Myhrer T.. Moldstad J.N., Iversen E.G. and Fonnum F. (1997) Modulatony treatment of NMDA receptors in neonatal rats affects cognitive behavior in adult age, Dev. Brain Res. 99, 126-130.

56. Whishaw I.Q. and Tomie J.A. (1987) Cholinergic receptor blockade produces imparments inu a sensonimotor subsysten for place navigation in the rat: Evidence from sensory, notor, and acquistion tests 
In a swimming pool. Behav. Neunoset. 101, 603-616.

57. Willams C.V., Nordquist D. and Meloon S.C. (1994) Correlation of nitric oxide synthise expecsion with changing pattens of axonal projections in the developing visual system. J. Newosci. $14.1746-1755$

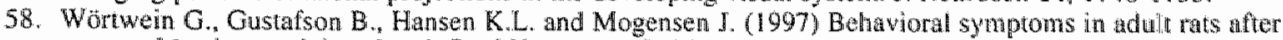
postnatal L-nitro-arginine Im. Jew Newrosci. 15, 147-154. 


\section{Chapter 9}

\section{Concluding Remarks}

\section{Animal models based on metabolic deficits}

In the present thesis two animal models were used for evaluating consequences of, or influences on energy depletion. One model, i.c.V. injected streptozotocin (STREP), is bused on a chronic inhibition of the energy metabolism, while the other one, i.c.r. injected sodium cyatide ( $\mathrm{NaCN}$ ), is based on a short period of acute energy depletion.

\section{THE STREPTOZOTOCIN MODEL}

Several studies have provided evidence that a single i.c.w. injection of STREP reduced the central metabolism of glucose in middle-aged rats $[32,73,74,83]$. Concomitantly, a decrease in (spatiall) cognitive function $[18,19,67]$ and a decrease in several neurotransmitters $[18,19,31,50]$ were observed in STREP-treated rats. It has been suggested that the middle-aged STREP-treated rat could be regarded as a relevant animal model of sporadic Alzheimer"s disease [18, 32, 73, 74, 83], since it has been hypothesized that the cognitive dysfunction [55) (and also the amyloid accumulation in the brain (69)) of Alzheimer"s disease (AD) patients is caused by a major decrease in central glucose metabolism.

Substantial evidence has accumulated that i.c.v. injected STREP reduces the central metabolism of glucose by destruction of the insulin-secreting cells and/or interference with the insulin receptor system $132,73,74,83$. However, some questions are still unanswered regarding the precise mechanism of action of STREP in causing neurodegenerative damage. For instance, oxidative damage may be inwolved (also) in causing neuronal damage, since a probable mechanism of action of STREP could be that it induces oxidative stress (e.g. generation of $\mathrm{H}_{2} \mathrm{O}_{2}$ (102)). In a study described in this thesis (Chapter 3), it has been found that STREP affected enzyme activities in the septum rather indiscriminately whereas it affected only ChAT activity in the hippocampus. The hippocampal ChA $T$ activity was strongly correlated with that of the seprum, and, therefore, the reduced ChAT activity in the hippocampus can be explained by an effect of STREP at the level of the septum. The fact that enzyme activities were affected rather indiscriminately in the septum, together with a reduced weight of the septum suggests that STREP acts as a non-selective neurotoxin near the site of injection. This wonld favor the possibility that STREP causes (non-selective) oxidative damage in stead of (only) decreasing the central metabolism of glucose.

In a following study the effects of STREP were investigated on (spatial) cognitive behavior and two biochemical markers of neurodegeneration, choline acetyltransferase (ChAT) activity and glial fibrillary acidic protein (GFAP) content (Chapter 4). The GFAP conterst in the septum, hippocampus and striatum of STREP-treated rats was increased, while (again) ChAT activity wats decreased in their hippocampus only. The cognitive deficits in the behavioral tasks were correlated with the increased GFAP conterts and the decreased ChAT activity in the hippocampus. The GFAP content and ChAT' activity in the hippocampus of STREP-treated rats correlated negatively with each other, thus suggesting that direct damage of the hippocampus it self may be related with its cholinergic defucit. Moreover, the reduction in hippocampall ChAT activity was not 
related to possible damage (increased GFAP) of the septum and there was no reduction in septum weight of STREP-treated rats. However, in the previous study, i.c.v. injected STREP caused septal damage, as was evident from ex reduction in septum weight, which could be used to explain the decrease in hippocampal ChAT activity (Chapter 3). This discrepancy in effects of i.c.v. injected STREP on the septohippocampal system is probably due to differences in the strain of rats used, viz. Lewis (Chapter 3) versus Wistar (Chapter 4). It may be better to use middle-aged Wistar rats instead of Lewis rats for the STREP model, since the neuronal damage in Wistar rats after STREP treatment seems to be less than in Lewis rats, and a model of neurodegeneration in which the morphology of the brain structures remains grossly inact, has the most relewance when one wants to measure cognitive decline.

A characteristic feature of STREP treatment was that not all rats were affected to the same extend. Some rais showed no changes in behavioral measures whereas other rats showed marked effects. This could already be assumed based on findings in another study described in this thesis (Chapter 2 [5te allso 18,191 ). This feature may appear to be a disadvantage of the STREP model, but on the other hand it provides a manner to correlate boib the behavioral an biochemical markers. Furthermore, an arbitrary subdivision of STREP-treated rats into two groups on basis of their cognitive performance applied to all behavional tasks that measure different aspects of cognitive behavior. This is striking because it has been demonstrated for several experimental groups of rats (e.g. aged or ischemic rats) that the performances in different cognitive tasks did not correlate with each other, which may be due to the small spread in data and the involvement of different non-cognitive factors $116,37,79]$. Apparently, STREP induces a behavioral deficit that affects various aspects of learning and memory.

As mentioned above, indirect evidence was found, asing Lewis rats, that i.c.w. injected STREP might cause oxidative damage, particularly to the septum which would thus result in a hippocampal dysfunction (Chapter 3). The finding, using Wistar rats, that in particular the hippocampus is directly severely affected, does not exclude one of the mechanisms of action for STREP, i.e. inhibition of energy metabolism versus oxidative stress. But now it adds more evidence to the metabolic deficit after STREP treatment, since the hippocampal formation is one of the brain structures which have the largest number of insulin receptors on neurons [52, 108] and the highest glucose need [49]. Therefore, the hippocampus might be one of the most vulnerable brain structures to STREP treament. A metabolic deficit (or mitochondrial dysfunction) may be the result of an increased oxidative damage, and allematively an increased radical formation (oxidalive stress) may be the result of an impaired energy metabolism 168, 93, 6191. This may apply to our STREP model, but also to AD of which it has already been suggested that amyloid accumulation and tangles formation are a result of, and contribution to a reduced energy availability and increased oxidative processes [66]. Furthermore, despite the fact that hippocampal ChAT activity correlated well with cognitive behavior, it was found that there is more than only a cholinergic deficit in STREP-treated rats (Chapter 2 (see also 31). Accordingly, it has been found that in $A D$, apart from a decrease in cholinergic neurotransmission, several monoaminergic neurotransmitter systems are changed 144.51\%. The other marker of neurodegeneration, GFAP, showed an increase in its content in the septum, hippocampus and striatum. Likewise, in the brain of AD patients, especially the hippocampus, the GFAP content has been found to be increased [36]. Although these biochemical and behavioral changes after STREP treatment have great similarities with those in $A D$, it has to be noted that the changes after STREP treatment do not fully mimic the biochemical and behavioral features of AD 1see 31.31 .891 and Chapter 3 in which the enzymatic changes did not closely resemble the more specific changes in enzyme activities as abserved in AD). Although this limits the usefulness of i.c.v. injected STREP as a model of sparadic AD, it 
may provide a relevant model of neuradegeneration.

A further sudy was conducted to investigate the rolle of nitric oxide (NO) in mediating neuronal damage after STREP treatment (Chapter 5). The inhibition of the energy metabolism after STREP treament might induce an excitotoxic mechanism, also referred to as $N$-methyl-Daspartate (NMDA)-mediated neurotoxicify [11. 107], which may involve the synthesis of the reactive NO by stimulation of the enzyme NO synthase [of. 38]. On the other hand, STREP might induce oxidative stress directly by liberation of $\mathrm{NO}$ from its mitroso moiety 161.103, 106. STREP caused neuronal damage (as assessed by GFAP expression) which was mainly located in peri- and paraventricular regions of the third and lateral ventricles, like for instance in the septum, caudate putamen and hippocampus. Treatment (i.p.) with the NO synthase inhibitor $N$-nitro-L-arginine methyl ester (L-NAME) had no protective effect on the neuronal damage and the behavional impairments of STREP-treated rats. This suggests the that the STREP-induced neuronal danage may still be a result of the decrease in the central energy metabolism, but without the involvement of NO synthase. In addition, L-NAME did not prevent NO production after STREP administration in vitro which suggests the involvement of NO liberated from STREP in neuronal damage. However, it remains unknown how STREP stimulates the in previous studies reported $\mathrm{H}_{2} \mathrm{O}_{2}$ generation $[102\}$, i.e. whether $\mathrm{NO}$ is involved hereby. Hence, other explanations for neuronal damage with NO after STREP treatment may be offered. Very interesting in this context is the abowe mentioned suggestion that the metabolic defect after STREP treatment is due to an impaired insulin receptor via inhibition of its tyrosine kinase activity [32.83], This because it has recently been found that NO inhibited several tyrosine kinase activities by $S$ mitrosylation of the receptor proteins $[34,94]$. All these findings urge the need to further examine the possible involvement of NO in our STREP model.

\section{THE SODIUM CYANIDE MODEL}

In a previous study the NaCN nodel has already been characterized, both behaviorally and biochemically [17]. It was found that an .c.w. injection of $\mathrm{NaCN}$ impaired spatial memory performance of rats whose hippocampal phosphoinositide metabolism, which is ATP dependent, was affected. The latter indicates that the energy metabolism was indeed impaired after injection of $\mathrm{NaCN}$, which is an inhibitor of the electron chain enzyme complex IV 6.51 .

\section{Acetyl-L-carniline}

In animal studies was found that chronic treatment with acetyl-L-carnitine (ALCAR) prevented an impaired cognitive performance in aged rats $[10,25,40,41]$, In human studies it was found that the progression of Alzheimer's disease (AD) was significantly reduced in patients chronically treated with ALCAR [81, 95]. Several possible mechanisms of action of ALCAR in the brain have been offered to explain these neuroprotective effects on cognitive function. For instance, ALCAR is regarded to attenuate neurodegenerative processes by having a beneficial effect on the energy metabolism, viz. via a stimulation of the use of alternative energy sources, such as lipid substrates or ketone bodies $[4,5,12,77,109,110]$. Hence, in the present thesis the putative nootropic (cognitive enhancing/meuroprotective) effect of ALCAR was tested in both animal models of energy depletion. 


\section{ACETYL-L-CARNITINE AND STREPTOZOTOCIN}

It was found that chrontc treatment (via drinking water) with ALCAR attenuated both the STREP-induced (spatial) cognitive impaiment and the decrease in hippocampal ChAT activity (Chapter 2). The latter suggests that ALCAR had a neuroprotective effect on cholinergic neurons after a STREP.induced decrease in central energy metabolism. This neuroprotectiwe action could be mediated by the supposed energy enhancing mechanism of action of ALCAR, i.e. the stimulation of the use of altemative energy sources 14, 5, 12]. In that case ALCAR should spare neurons non-selectivity. However, ALCAR did not spare all the enzyme activities in the septum (although ChAT seemed to be preserved), but preserved the cholinergic function in the hippocampus to some extent (which was also the only enzyme affected there; Chapter 3). Therefore, other explanations may be offered and especially one suggesting that ALCAR has a positive effect on the neurotrophic action of nerve growth factor (NGF) on the septohippocampal cholinergic system [2, 9\%" 100]. Thus, a positive effect of ALCAR on NGF could also explain the increase in ChAT activity in the hippocampus in the STREP group treated with ALCAR isee also 82. 101]. Furthermore, the fact that NGF plays a role in oxidant homeostasis [78] further strengthens the possibility that NGF is irvolved in the neuroprotective effect of ALCAR against possible oxidative damaging effects of STREP.

\section{ACETYL-L-CARNITINE AND SODIUM CYANIDE}

In a previous study is has been found that chronic treatment with ALCAR had a protective effect on spatial memory performance after NaCN intoxication, although it had no effect on the affected hippocampal energy metabolism. This indicates that ALCAR did not enhance the utillization of alternative energy sources during the acute $\mathrm{NaCN}$-induced energy depletion. Another possibility for a protective effect of ALCAR offers the finding that when the energy metabolism is impared (e.g. ischemia) there is an increase in deacylation of membrane phospholipids and a decrease in B-oxidation $[1,56,801$. This results in an increase in free fatty acids and acylCoAs, respectively, of which the latter are toxic $[77,80]$, and thus can contribute to an impaired neuronal function or even lead to neuronal damage. In addition, the ATPudependent reacylation roate via acylCoA synthetase (which uses the free fatty acids) is inhibited by a low energy metabolism in the brain. The protective effect of chronic treatment with ALCAR against acute $\mathrm{NaCN}$ administration might be explained via L-carnitine with the formation of an ATP-independent reservoir of activated acyl groups, i.e. acetyl-L-carnitines, by the enzyme carnitine acyltransferase. Thus fatty acids as acylated derivatives are avalable and reacylation processes can continue during $\mathrm{NaCN}$ intoxication [ef. 3], On the other hand, or maybe concomitantly. ALCAR may exert its protective action by preventing a possible accumulation of toxic acylCoAs [ch. 77].

This thesis describes the study investigating the effect of acute ALCAR treatment (i. .) on spatial discrimination performance of NaCN-treated rats (Chapter 6), since we assumed based on another study [B7], that an acute administration of ALCAR could possibly be even more effective than a chronic treatment. Howewer, it was found that acute administration of ALCAR appears to potentiate the $\mathrm{NaCN}$-induced behavioral deficit. This anexpected finding may be explained by an inhibition of the glycolytic flow, because it has been reported that ALCAR not only stimulates the use of alternative energy sources, but also inhibits the glycolytic flow $[4,5,12,109,110]$. As a consequence, acute ALCAR treatment would further increase the energy deficit due to $\mathrm{NaCN}$, although this was not confirmed by an additional in witro study as ALCAR had no effect on a 
$\mathrm{NaCN}$-induced energy deficiency. Effects of the in wivo ALCAR administration on the possible formation of an activated acyl-L-carnitines reservoir may be too small or short lasting to have a protective effect. Perhaps it is more useful to administer ALCAR or L-carnitine centrally. Especially the determination of the effective component, i.e. ALCAR or L-carnitine, is needed. For instance, with respect to the reserwoit of activated acyl groups, ALCAR has to be first converted to L-carnitine, in order to shift the equilibrium of the enzyme carnitine acyltransferase towards acyl-L-carnitines. Moreover, L-carnitine is interesting because it may have no inhibiting effect on the glycolytic flow as ALCAR has.

\section{Nitric oxide and plasticity}

Two functions of $\mathrm{MO}$ in neural plasticity have been examined in this thesis: its role together with cyclic GMP (cGMP), in memory formation and its role in the early development of the brain. With regard to memory formation it was found that treatment (i.p.) with the NO synthase inhibitor 7-nitroindazole impaired object recognition memory (Chapter 7). Treatment (i.p.) with the phosphodiesterase inhibitor type $V$ zaprinast, which indirectly increases cGMP levels, improved memory performance, also after 7-nitroindazole treatment. It was concluded that, like in other previous behavioral studies, that NO [13,35,92] and CGMP [14, 15] play a role in the consolidation of information rather than in its retrieval. Thus, it can be argued that the NO-cGMP signal transduction is involved in processes of memory fomation.

However, there are some questions with respect to the relationship between NO and cognitive performance. This also accounts for the relationship between NO and long-term potentiation (LTP) which is a form of (synaptic) plasticity which is believed to be the physiological substrate of memory formation. For instance, inhibition of NO does not always impair spatial discrimination learning $[7,104]$. In addition, no effect on spatial reversal learning was found $[7]$, Moreover, it has also been found that even when NO synthase inhibitors initially affected spatial learning, at the end of training still normal performance was achieved as was evident from the probe trial performance after spatial discrimination learning in the Morris water escape task $133,70$. 841. Recently, it has been found in our laboratory that hippocampal NO synthase inhibition with $N$-nitro-L-arginine had no effect on probe trial performance and reversal learning, although the acquisition of spatial discrimination was little affected (unpublished data). This is in agreement with the just mentioned studies using peripheral administration of NO synthase inhibitors. With respect to the inwolvement of NO in synaptic plasticity, LTP can still be induced, though not maximally, after blockade of NO [6.8] or CGMP [20]. This suggests that LTP has a component that involves neither NO nor cGMP [c. 20. 39].

Nevertheless, the finding that an (indirect) increase of cGMP levels improved the memory formation in normal healthy rats (Chapter 7) urges the need for further investigations into the cognitive effect of cGMP manipulations which may offer more favorable possibilities for treatment of cognitive dysfunctions, than interfering with NO itself or NO synthase. Moreover, because a latter approach may even have detrimental effects on cognition because of the toxic potential of a too high NO level, and also because the relationship between NO and cognition is more and more questioned (see above).

A growing number of studies has provided evidence that NO and CGMP is an important factor in the development of neurons $153,59,88,105,112]$. Hence, it may be argued that the NO-cGMP signal transduction is involved in the development of the nervous system. Therefore, it was investigated 
whether interference with NO symthesis during development could impair brain function and behavior during later life (Chapter 8). It was found that chronic postnatal inhibition of NO synthase with L-NAME had no effect on cognitive behavior [see also 113] and brain function in adulthood.

Particularly NMDA receptors have been linked to specific neuronal functions in synaptic plasticity, development and also neurodegeneration (and may provide a venue for therapeutic intervention [er. 581). It can be argued that there should be some connection between NMDA receptor stimulation and NO formation by the enzyme NO synthase [ef. 38]. Therefore it may be assumed that if $\mathrm{NO}$ mediates (behavioral) responses to MMDA receptor stimulation in the brain, then interference with the NO synthesis should induce (behavioral) changes similar to those of NMDA receptor manipulations.

Studies using NMDA antagonist have shown that NMDA receptors have a relationship with both LTP and (spatial) cognitive behavior $[30,72,86]$. Since it appears that NO is not essential for (spatial) cognitive performance and both NO and cGMP are not essential for LTP induction, the NO-cGMP transduction system has probably only a modulating function in synaptic plasticity (see above). Thus, there is a discrepancy between the effects of NO synthase inhibitors and NMDA antagonists on both LTP and memory formation. However, recently the relationship between NMDA and (spatial) cognitive function is also more and more questioned $19,23,60,917$, although it is still certain that LTP is NMDA-receptor-dependent [28]. Newertheless, a clear discrepancy between NO and NMDA with respect to LTP, i.e. synaptic plasticity, remains. Furthermore, NMDA receptors have a pronounced role in the development of the nervous system [e.g. 21, 22, 48]. Concomitantly, it has been found that chronic postnatal NMDA blockade affects cognitive behavior in adult life $[42,43,111]$. Again, there is a lack in similarity in the effects of $N O$ synthase inhibition (see Chapter 8 [see also 113]) and NMDA receptor blockade. This is further corroborated by comparing the studies which investigated the effects of NMDA and NO manipulations on several other behaviors such as locomotor activity which was increased by NMDA antagonist, $154,63,64,85,96]$, whereas NOS inhibition decreased locomotor activity $[29,85,90,97$. [14]. Therefore, it is suggested that $\mathrm{NO}$ in the brain is involved in many processes concerning cognition, locomotion, or development, but not necessarily to mediate neuronal responses after NMDA receptor stimulation.

Finally, there is one possible important role left for $\mathrm{NO}$, viz. its involvement in neurodegenerative processes or in more detail: NMDA-mediated neurotoxicity [11, 107]. This line of research is relatively new and thus far the recent studies and also the one described in this study (Chapter 5) with an in vivo administration of a NO donor did not have any clear results, i.e. rnainly it is not clear whether NO mediates NMDA neurotoxicity $[45,47,62]$. However, there is some in vivo evidence that NO may still mediate NMDA neurotoxicity as indications were found of a neuroprotective action of NO blockade against administration of a NMDA agonist [46, 98] Furthermore, in vivo models of central ischemia, which are particularly thought to result in a NMDA-mediated neurotoxicity, revealed that NO synthase inhibition, just as NMDA antagonism, had a neuroprotective effect $[24,26,27,57,75,76]$. Therefore, NO inhibition may be a powerful tool for the treatment of neuronal damage after a stroke, although its application for treatment of neurodegenerative disorders appears to be limited. 


\section{Conclusions}

Treatment of middle-aged rats with a single i.c.v. injection of STREP maty provide a relewant model of neurodegeneration which has several similarities with AD. STREP reduces the central metabolism of glhcose most likely by interference with the insulin receptor system. However, besides that neurodegenerative effects may be due to an inbibition of the energy metabolism, oxidative damage may be involved (directly), since a probable mechanism of action of STREP could be that it induces oxidative stress (e.g. $\mathrm{NO}$ and $\mathrm{H}_{2} \mathrm{O}_{2}$ generation). Ir contrast to the chronic STREP model, there is only a short period of acute energy depletion after i.c.. NaCN administration. Hence, the NaCN model can be used test the acute effects of compounds that are presumed to preserve neuronal function during low energy states.

Chronic ALCAR treatment (via drinking water) prevented/attenuated cognitive decline in both the STREP and NaCN model. The positiwe effect of ALCAR in the STREP model may be explained with a beneficial effect of ALCAR on the energy metabolism, i.e. stimulation of the use of alternative energy sources. However, a possible neurotropic effect of ALCAR on NGF cannot be ruled out either. In the NaCN model chronically administered ALCAR may probably exert its positive action via the formation of an energy-independent acyl(carnitines) reservoir and/or detoxification of toxic acylCoA. However, acute administration (i.v.) of ALCAR appeared to potentiate the NaCN-induced cognitive deficit, for which no adequate explanation exists. These findings indicate that the nootropic effect of ALCAR has more to do with neuroprotection than with cognition enhancement. Therefore, it is best to administer ALCAR chronically, and preferably as early as possible, to preventattenuate cognitive dysfunction such as occurs in for instance $\mathrm{AD}$.

Chronic postnatal inhibition of NO formation had no effect on cognitive performance in later life, which questions a role of NO in early brain dewelopment. On the other hand, NO and cGMP were involved in processes of memory formation. However, other studies indicated that NO only plays a moderate, or even no role at all, in this respect. Hence, further investigations into the cognitive enhancing effects of increasing cGMP levels may offer favorable possibilities for treatment of cognitive dysfunctions.

\section{REFERENCES}

1. Ando S. Waki H. and Kon K. (1993) Differential falty acid release from CAI and CA3 regions oft" ratt hopocampal slices under hypoxia and hypoglycemia. Neurosci. Lett. 151, 48-50.

2. Angelucci L., Ramatci M.T. "Taglialatela G., Hulsebosh C. Morgan B., Wertbach-Perez Kond Pere\% Polo J.R. (1988) Nerve growth factor binding in aged rat centril nerwous system: Effect of acetyl-L-carniline. $d$. Newrosci. Res. $20,491-496$.

3. Arduini A., Denisowa N., Virmani A., Avrova M. Federici G. and Arrigoni-Martelli E. (1994) Evidance for the involvement of carnitine-dependent long-chain acyltransferases in neuronal triglyceride and phospholipid acid turnover. N. Nesurochent. $62,1530-1538$.

4. Aureli T., Miccheli A., Di Cocco M.E.L., Ghirardi O. Giuliani A., Ramacci M.T. and Conti (1994) Effect of acetyl- Larnitire on recovery of brain phosphorus metabolites and lactic acid level during reperfusion after cerebral ischemia in the rat - studly by $31 \mathrm{P}$ - and IH-NMR spectroscopy. Bran Res. 643, 92.99.

5. Aureli T., Miccheli A. Ricciolini R. Di Cocco M.A., Ramacei M.T., Angelucei L., Ghürardi O. and Conti F. (1990) Aging brain: Effect of acetyl-L-carnitine treatment on rat brain energy and phospholipid melabolism. A study by $31 \mathrm{P}$ and $1 \mathrm{H}$ NMR spectroscopy. Brain Res. 526, 108-1 12.

6. Bannerman D.M. Butcher S.P. and Morris R.G. (1995) Intracerebroventricular injection of mitric os inde symthase inhibitor does not affect long-term slope potentiation in vivo. Neurophamacology $33,4387$. 1397 .

7. Bamerman D.M., Chapman P.F., Kelly P.A.T', Butcher S.P. and Morris R.G.M. (1994) Inhibition of 
nitric oxide synthuse does not inpar spatial learning, J. Neurosci. 14, 7404-7414.

8. Banturman D.M., Chapman P.H. Ketly P.A.T. Bucher S.P. and Morris R.G.M. (1994) Inhibiton of vitric oxide synthase does not prekent the induction of long-term potentiation in vivo. J. Nethose. 14 , $7415-7425$

9. Barneman D.M. Good M.A., Butcher S.P., Ransay M. and Mortis R. G.M. (1995) Distinct components of spatial letming revaled by prior training and NMD receptor blockade. Nanure 378, 182-186.

10. Barneg C.A. Markowska A.L. Ingram D.K., Kametani H.y Spangler E.L. Lemken V.J. and Olton D.S. (1990) Acetyl-L-carnitine 2: Effects on learning and memory perfornance of aged rats in simpte and complen mazes. Newrobiol. Aging 11, 499-506.

11. Beal M.F., Hyman B.T. and Koroshetz W. (1993) Do defects in mitochondrial anergy metabolism underlie the pathology of neurodegeneratiwe diseases? Trends Netrosci. 16, 125-131

12. Benat G., Villa R. D., Dossena M., Vercesi L., Gonini A. and Pastorts O. (1984) Cerebral endogenous

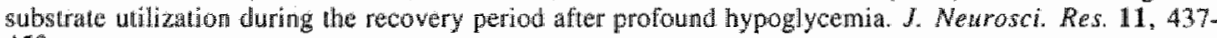
4.50

13. Bermabeu R., de Stein M.L., Fin C., lzquierdo I. and Medina J.H. (1995) Role of hippocampal NO in the acquisition and consolidation of inhibitory avoidance leaming. Nevrokeport 6, $1498-1500$.

14. Bernabeu R., Selmirz P. Fetillace M.P., Izquierdo I. and Medina I.H. (1996) Hippocampal cGMP and cAMP are differentially inwolved in memory processing of inhibitury avoidance learming. NeuraReport 7 585. 588 .

15. Barnabeu R., Schroder N., Quevedo J., Cammarota M. Lzquierdo 1. and Medina J.H. (1997) Further evide ace for the in volverrent of a hippocampal cGMP/cGMP-dependent protein kinase cascade in memory consolidation. NewroReport 8, 2221-2224.

16. Blokland A. (1993) Age-related changes in correlation between bethavional and buochemical parameters in Lewis rats. Behav. Netral Biol. 60,52-61.

17. Blokland A., Bothmer J., Honig W. and Jolles J. (1993) Behavioral and biochemical effects of acute central metabolic inhibition: Effects of acelyl-Lacamitine. Eur. $J$, Pharmacol, 235, 275-281.

18. Blokland A and Jolles J. (1993) Spatial learning deficit and reduced hippocampal ChAT activity in rats after an dow injection of streptozotocin. Pharmacol. Brochem. Behav, 44, 491-494.

19. Blokland A. and Jolles J. (1994) Behawioral and biochemical effects of an icv injection of streptozotocin in old Lewis rats. Pharmacol. Biochem. Behav. 47,833+837.

20. Boulton C.L., Southam E. and Garthwate J. (1995) Nitric oxide-dependent long-term potentiation is blocked by a specific inhibitor of solubie gutnylyl cyclase. Neuroscience 69, 699-703.

21. Brady R.J., Gorter J.A. Monroe M.T, and Swann J.W. (1994) Developmental alterations in the sensitivity of hippocampal NMDA receptors to AP5. Brain Res. Dev. Brain Res. 83, 190-196.

22. Brewer G.J. and Cotman C.W. (1989) NMDA receptor regulation of nemonal morphology un cultured hippocampal neurons. Neurosci. Len. 99, 268-273.

23. Cain D.P. Saucier D. and Boon F. (1997) Testing hypotheses of spatial learning: The role of NMDA receptors and NMDA-mediated long-term potentiation. Behav. Brain Res. 84, 179-193.

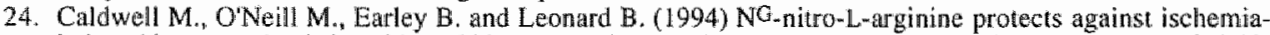
induced increases in nitric oxide and hippocampal neuro-degeneration in the gerbil. Eur. J. Phamacol. 260 , 191-200.

25. Caprioli A. Chirandi O. Ramace $M . T$ and Angelucei L. (1990) Age-dependert deficits in radial maze perfounance in the rat: Effuct of elwonic treatment with acely-L-carnitine. Prog. Neuropsychopfarmacol. Biol. Psychiat. 14,359.369.

26. Chalimoniuk M. and Stroszajder J. (1995) Brain ischemia-reperfusion injury activates neuronal, constitutive form of nitric oxide symthase. Molecular action of nitric oxide. Eur. J. Newrosci. Sappl. 6 , $26,15,28$

27. Choi D.W. (1995) Calcium: Still center-stage in hypoxic-ischemic neuronal death. Tyends Newrosci. 18 , $58-60$

28. Collingridge G.L. and Bliss T.V.P. (1995) Memories of NMDA receptors and LTP. Trends Nenrosic. 18, 54.56 .

29. Connop B.P. Ronfe N.G., Boegnn R.J., Jhamandas K. and Beninger R. J. (1994) Potentiation of NMDAmediated toxicity on nigrosinatal neurons by a low dose of 7-nitro indazole. Newropharmacology 33, 1439 . 1445 .

30. Dnvís S., Butcher S.P. and Morris R.G.M. (1992) The NMDA receptor antagonist D-2-amino-5 phosphopentanoate (D-APV) impairs spatial learning and LTP in vivo at indracerebral concentations compurable to those that block LTP in vitro. N. Newrosci. 12, 21-34, 1992 .

31. Ding A. Nitsch R. and Hoyer S. (1992) Changes in bran monoaminergic neurotransmitter concentrations in rat after intracerebroventricular injection of streptozotocin. J. Cereb. Blood Flow Merab. 12, 103-109.

32. Duelli R., Schrock H., Kuscthinsky W. and Hoyer S. (1994) Intracerebroventricular injection of strepiozotocin induces discrete local changes in cerebral glucose utilization in rats. Im. J. Dew/ Newrosci. $12.737-743$

33. Estall U.B.. Grats S.J, and Cicala G.A. (1993) Inbibition of nitric oxide (No) production selectively impairs learning and memory in the rat. Phamacol. Biochem. Bikhan, 46, 959-962.

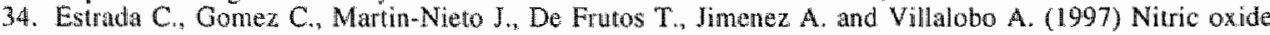


reversibly inhibits the epidemal growth factor receptor tyrosine kinase. Biochem. $1.326,369-376$.

35. Fin C. Cunha C.A., Bromberg E. Schmitz P.K. Biznchin M. Medina J.H. fand Izquerdo I. (1995) Experiments suggestimg a nole for nutric oxte in the hippocampus in menory processes. Neumbiol team. Mem. 63, 113-115.

36. Frederickson R.C.A. (1992) Astroglia in Alzheimer's disease. Neurobiot Aging 13, 239.253.

37. Gallagher M. and Burwell R.D. (1989) Rellationship of agemelated decline across sevoral behavioral domains. Neurabiol. Aging 10,691-708.

38. Garthwaite J. (1991) Glutamate, nitric oxide and cell-cell signalling in the merwous system. Thends Neturosic: 14, 60-67

39. Garthwaite J, and Boulton C.L. (1995) Nitric oxide signaling in the central nervous system. Anmu. Rew. Physiol. 57, 683-706.

40. Ghirardi O., Caprioli A., Milano S. Giulieni A. Ramacci M.T. and Angelucei L. (1992) Active Avoudance Learnirg in old rats chronically treated with levocamitine acotyl. Physiof. Behav. 52. 185-187

41. Ghirardi O., Milano S., Ramacei M.T. and Angelucci L. (1988) Effect of acetyl-L-carnitine elmonic treatment on discrimination models in aged rats. Physiol. Behow.44,769-773.

42. Gonter J.A., Botterblom M.H. Feenstra M. G. and Boer G.J. (1992) Clitronic neonatal NMDA neceptor blockade with MK-801 aliers monoamine metabolism in the adult rat. Newrosch. Lert. 137. 97-100.

43. Gorter J.A. and de Bruin J.P. (1992) Chronic neonatal MK-801 treament results in an impairment of spatial learning in the adult rat. Brain Res. $580,12-17$.

44. Cottries C.G., Andolfsson R., Acquilonius S.M., Carlsson A., Eckernäs S.A., Nordberg A.. Oreland L., Swennerholm L., Wiberg A. and Wüblad B. (1983) Biochemical changes in dementia disorders of the Alzheimer type (AD/SDAT). Newrobiol. Aging 4, 261-271.

45. Gross P.M. Weaver D.F., Bowers R.J., Nag S., Ho L.T., Pang J.J. and Espinosa F.J. (1904) Neurotoxicity in conscious rats following intraventricula SNAP, a nitric oxide donor. Newopharmacology $33,915 \div 927$.

46. Hammer B., Davis Parker W.J. and Benmen J.P. (1993) NMDA receptors increase OH radicals in viwo by using nitric oxide synthase and protein kinase C. NeuroReport 5, 72-74.

47. Hanrington C.A. Mobley S.L. and Wenk G.L. (1994) Nitric oxide formation does non underlie the memory deficits produced by ibotenate injections into the nucleus basalis of rats. Behav. Neurosci. 108, 277-283.

48. Hattori $H_{\text {. }}$ and Wasterlain C.G. (1990) Excitatory amino acids in the developing bran: Orutogeny. plasticity, and excitotoxicity. Pediatr. Newrol. $6,219.228$.

49. Hawkins R.A., Mans A.M. and Davis D.W. (1983) Glucose availability to individual cerabral structures is correlated to glucose metabolism. J. Newrochem. 40, 1013-1018.

50. Hellweg R. Nitsch R. Hock C. Jaksch M. and Hoyer S. (1992) Nerve growth factor and cholline acelyltansferase activity levels in the rat brain following experimemal impaiment of cerebral glucose and energy metabolism. I. Neurosci. Res, 31, 479-486.

51. Hernegodts P., Bruyland M., DeKeyser J., Solheid C., Michotte Y. and Ebinger G. (1989) Monoaninergic neurotransmitter in Alzheimer's disease. J. Newrol. Sci, 92, 101-116.

52. Hill J.M., Lesniak M.A., Pert C.B. and Roth J. (1986) Autoradiographic localization of insulim receptors in rat brain: Prominence in olfactory and limbic areas. Newoscience 17, 1127-1138.

53. Hindley S., Jurlink B.H.J., Gysbers J.W., Middlemiss P.J., Herman M.A.R. and Rathbone M.P. (1997) Nitric oxide donors enhance neurotrophin induced neurite outgrowth through a $\mathrm{COMP}$ dependen mechanism. J. Nerrosici. Res. 47,427.439.

54. Hirmatsu M. Cho A.K. and Nabeshina T. (1989) Comparison of the behavional and biochemical effects of the NMDA receptor antagonists, MK-801 and phencyclidine. Eur. J. Pharmacol. 166, 359-366.

55. Hoyer S. (1992) Oxidative energy metabolism in Alahemer brain. Mol. Chem. Newropathol. 16, 207-224.

56. Ikeda M. Yoshida S., Busto R. Santiso M. and Ginsberg M.D. (1986) Polyphosphoinosinides as a

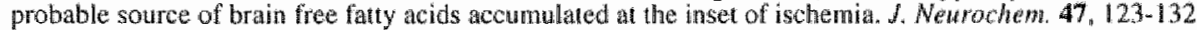

57. Kahn R.A., Panah M. and Weinberger J. (1997) Modulaton of ischemic excitatory newrotransmitter and ganma aminobutyric acid release during global temporary cerebral ischemia by selective neuronal nitric axide synthase inhibition. Anesthesia Analgesia 84, 997-1003.

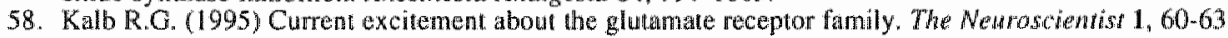

59. Kalb R.G. and Agostini J. (1993) Molecular evidence for nitric oxide-mediated motor neuron developmernt. Neurasci. 57, 1.8.

60. Kenth J.R. and Rudy J.W. (1990) Why NMDA-receptor-dependent long-rem potentiation may not be a mechamism of learning and memory. Reappraisal of the NMDA-recepor blockade strategy. Psychobiology 18, $251-257$

61. Kwon N.S. Lee S.H. Choi C.S., Kho T. and Lee H., S. (1994) Nitric oxide generation from streptozotocin. The FASEB Journal 8, 529-533.

62. Loiacono R.E. and Bear P.M. (1992) Hippocampal lesions induced by microinjection of nitric oxide donor nitroprusside. Ear. J. Pharmacol. 216, 331-333.

63. Loscher W. Amnies R. and Honack D. (1993) Comparison of competitive and uncompetitive MMDA receptor antagonists with regard to monoaminergic newronal actiwity and behavioural defets int rats. Lup. J. Pharmacol, 242, 263 m. 274 .

64. Loscher W. and Honack D. (1992) The behaviourd effects of MK-801 in rats: Involvement of 
Wopam nergic, serotonatgic and noradrenergic systems. Eus, f. Pharmacol. 215, 199-208.

65. Machllan V.H. (1989) Cerebral energy metabolism in cyanide encephalopathy. J. Cereb. Rlowd Flow Metor. 9. 156.162.

66. Mentsom M.P. (1995) Degenerative and protective signalling mechanisms in the nemrohbrillary pathology of AD. Neurobiol. Aging $16,447.463$

67. Mayer $\mathrm{G}$. Nitsch $\mathrm{R}$ did Hoyer $\mathrm{S}$. (1990) Effects of changes in peripheral and cerebral glicose metabolism on locomolor activity, learning and memory in adult malle rats. Brain Res. 532, 95-100.

68. Mecocei P. Maccarvey U. and Beal M.F. (1994) Ox lidative damage to mitochondrial DNA is increased in Axheimer's disease. Ann. Neurol. 36, 747-751.

69. Meier- Ruge W. Bertoni-Freddari C. and Iwangoff P. (1994) Changes in brain glucose metabolism as a key to the pathogenes of Alzheinen's disease. Gerontology $40,246-252$.

70. Mogensen $J_{\text {, Wortwein }} G_{\text {. }}$, Gustafson B. and Ermeins $P$. (1995) L-Nitroarginine reduces hippocampal mediation of place learning in the rat. Neurobial. Learn. Mern. 64, 17-24.

71. Money E.A. Kirk R.C. and McNaughton N. (1992) Alzheimer's dementia produces a loss of discriminalion but no increase in rate of memory decay in delayed matching to sample. Newropsychologia 30, 133-143.

72. Morris R.G.M. (1989) Synaptic plasticity and learming: Selective imparment of learning in rats and blockade of long-lem polertiation in vivo by the MMDA receptor antagonist A.PS. J. Neurosci. 9, 30493057 .

73. Nitsch $\mathbb{R}$, and Hoger $\mathbf{S}$. (1991) Local action of the diabetogenic drug, streptozotocin, on glucose and energy metabolistin the brain cortex. Neurose. Lett. 128, 199-202.

74. Nisch R. Mayer G. and Hoyer S. (1989) The intracerebroventriculary streptozotocin-treated rat: Impairnent of cerebral glucose metabolism resembles the alterations of carbohydrate metabolism of the brain in Alzheimer's disease. J. Neural Trans" 1, 109.110.

75. Nowicki J.P., Duwal D. Poignet H. and Scaton B. (1991) Nitric oxide mediates neuronal death after focal cerebril ischemia in the mouse. Eur. J. Pharmacol. 204, 339-340.

76. Ohno M. Yamamoto T. and Watanabe S. (1994) Intrahippocampal administration of the NO synthase inhibitor L-NAME prevents working memory deficits in rals exposed to transient cerebral ischemia. Brain Res. 634, 173-177.

77. Ohtsuka Y. and Griffuth O.W. (1991) L-carnitine protection in amona intoxication. Biochem. Phamacol. 12. $1957-1961$.

78. Pan Z and Perez-Polo R. (1993) Role of nerve growth factor in oxidant homeostasis: glutathione metabolism. f. Newrachem. 61, 1713-1712.

79. Pappas B.A., De Ia Torre J.C., Dawidson C.M., Keyes M.T. and Fortin T. (1996) Chronic reduction of cerebral blood flow in the adult rat: Latememerging CA 1 cell loss and memory dysfunction. Brain Res. 70\$, 50.58 .

80. Pastorino J.G., Snyder J.W., Serroni A., Hoek J.B. and Farber J.L. (1993) Cyclosporin and carnitine prevent the anoxic death of cultured hepatocytes by inlibiting the mitochondrial permeability transition. $l$. Biol. Biochem. 268, 13791-13798.

81. Pettegrew J.W, Klunk W.E., Panchalingan K., Kanfer J.N. and McClure R.J. (1995) Clinical and neurochemical effects of acety]-L-carnitine in Alzheimer"s discase. Neurobiol. Aging 16, 1-4.

82. Piovesan P., Pacifici L., Taglialaela G., Ramaci M.T. and Angelicci L. (1994) Acetyl-Leamitine treatment increases choline acelyltransterase activity and NGF levels in the CNS of adult rats following total fimbiritfornix transection. Brain Res. 633,77.82.

83. Plaschke $\mathrm{K}$. and Hoyer $\mathrm{S}$. (1993) Action of the diabetonic drug streptozotocin on glycolytic and

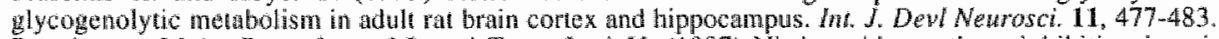

84. Prendergast M.A. Buccafusco J.J. and Terry J. A.V. (1997) Nitric oxide synthase inhibition impairs spatial navigation learning and induces conditioned taste aversion. Pharmacol. Biochem. Behav, 57, 347352 .

85. Pudiak C.M. and Bozarth M.A. (1993) L-NAME and MK-801 attenuare sensitization to the loconotorstimulating effect of cocaine. Life Sci. 53, 1517-1524.

86. Robinson G.S., Crooks G., Shinkman P.G. and Gallagher M. (1989) Behavioral effects of MK-801 mimic delicits associated with hippocampal damage. Psychobiology 17, 1.56-164.

87. Wosemal R.E. Williams R.,Bogater Y.E. Getson P.R. wnd Fuskum G. (1992) Prevention of postischemic misologio injury through potentiation of brain energy metabolism by acetyl Licarnitine. Srroke 23. $1312 \times 1317$.

88. Roskans A.J. Bred: D.S. Dawson T.M. and Ronneti G.V. (1994) Nitric oxide mediates the formation of synaptic connections in developing and regenerating olfactory meceptor meurons. Newron 13, $289-299$.

89. Sahgall A., Galloway P.H., Mckein 1.G. Lloyd S. Cook J.H., Ferrier N. and Edwardson J.A. (1992) Matching-10-sample deficits in patients with senile dementigs of the Alzheimer and Lewy body types. Arch. Neurol. 49, 1043-1046.

90. Sandi C. Venero C. and Guaza C. (1995) Decreased spontaneous motor activity and startle response in nitric oxide synthase inhibitor-treated nats. Eur. J. Pharmacol, 277, 89.97.

91. Samcier" D. and Cain D.P. (1995) Spatial learning without NMDA receptor-dependent long-term potentiation. Natwe $378,186 \mathrm{~m} 189$.

92. Schuman E.M. and Madison D.V. (1994) Nitric oxide and symaptic function. Annu. Rev. Neurosci. 17 , 
$153-183$.

93. Shigenaga M.K., Hagen T.M. and Anes B.N. (1994) Oxidative damage and milochondrial decay in aging. Proc Narl Acod. Sci, U.S.A. 91. 10771-10778.

94. Simon D.I., Mullins M.E., Jia L.. Gaston B., Singel D.J. and Stamler J.S. (1996) Polynitrosylant proteins: Characterization, bioactivity, and functional consequences. Proc. Narl Acad. Sci. U.S.A. 93. 4736-4741.

95. Spagnoli A., Lucca U., Menasce G., Bandera L., Cizza G., Forloni G., Tenamanti M., Fratturir L., Tiraboschi $\mathbb{P}$., Comelli M. Senin U., Longo A., Petrini A., Brambilla G. Belloni A., Negri C., Cavazzuti F., Salsi A., Calogero P., Parma E., Stramba-Badiale M., Vitali S., Andreoni G. Inzoli M.R., Sanus G., Caregnato R., Peruzza M., Favaretto M. Bozeglav C., Alberoni M., De Leo D. Serraioto L., Baiocehi A., Scoccia S., Culotta P. and Jeracitano D. (1991) Long-term acetyl-L.carnitine treament in Alzheimer"s disease. Neurology 41, 1726-1732.

96. Start M.S. and Starr B.S. (1994) Comparison of the effects of NMDA and AMPA antagonists on the locomotor activity induced by selective D1 and D2 dopamine agonists in reserpine-treated mice. Psychophamacology (Berlin) 114, $469-476$.

97. Start M.S. and Starr B.S. (1995) Do NMDA receptor-mediated changes in motor behaviour inwolve nitric oxide? Eur. J. Pharmacol. 272, 211-217.

98. Strijbos P.J.L.M., Knowles R. and Rothwell N.J. (1993) Nitric oxide mediates ghntamate neurotoxicity in the rat striatum. Brain Res. Assoc. Abstr. 10, 46,8.6.

99. Tagliallatela G., Angelucci L., Ramacci M.T., Werbach-Perez K., Jackson G.R. and Perez-Polo J.R. (1991) Acetyl-L-carnitine enhances the response of $\mathrm{PCl} 2$ cells to nerve growt factor. Dew. Brain. Res. 59, 221230 .

100. Taglialatela G., Argelucci L., Ramacei M.T., Wernbach.Perez K., Jackson G.R. and Perez.Polo J.R. (1992) Stimulation of nerve growth factor receptors in PC12 by acelyl-L-carnitine. Biochew. Pharmacol. 44, 57758.5 .

101. Taglialatela G., Navarra D., Cruciani R., Ramacci M.T. Alema G.S. and Angeluce L. (1994) Aceiyl-Lcarnitine tratment increases nerve growth factor levels and choline acetyltransferase activily in the central mervous system of aged rats. Exp. Gerontol. 29, 55-66.

102. Takasu N., Komiya I., Takayuki A., Nagasawa Y. and Yamada T. (1991) Streptozotocin- and alloxaninduced $\mathrm{H}_{2} \mathrm{O} 2$ generation and DNA fragmentation in pancreatic islets. Diabetes $40,1141-1145$.

103. Tanaka $Y_{\text {, }}$ Shimizu H., Sato $N_{\text {., Mori }} M_{\text {. and Shimomura }} Y_{\text {. (1995) Involvement of sponancous mitric }}$ axide production in the diabetogenic action of streptozotocin. Phamacology 50,69-73.

104. Tobin J.R., Gorman L.K., Baxter M.G. and Traystman R.J. (1995) Nitric oxide symathase imhibition does not impair visual or spatial discrimination learning. Brain Res. 694, 177-182.

105. Truman J.W., De Vente J. and Ball E.E. (1996) Nitric oxide-sensitive guanylate cyclase activity is associated with the maturational phase of neuronal development in insects. Development 122, 3949-3958.

106. Turk J., Corbett J.A., Ramanadham S, Bohrer A. and McDaniel M.L. (1993) Biochemical evidence for nitric oxide formation from streptozotocin in isolated pancreatic islets. Biochent. Biophys, Res. Connn. $197,1458-1464$.

107. Turski L. and Turski W.A. (1993) Towards an understanding of the role of glutannte in neurodegenerative disorders: Energy metabolism and neuropathology. Experimentia 49, 1064-1072.

108. Unger I.W., Livingston J.N. and Mass A.M. (1991) Insulin receptors in the central nervous system: Localization, signalling mechanisms and functional aspects. Prog. Newrobiol. 36, 343-262.

109. Villa R.F, and Gorini A. (1991) Action of L-acetylcarnitine on differeme cerebral mitoctiondrial populations from hippocampus and striatum during aging. Newrochem. Res. 16, $1125-1132$.

110. Villa R.F., Gorini A., Zanada F, and Benzi G. (1986). Action of L-ascetylcarniene on different cerebral mitochondrial populations from hippocampus. Arch. Im. Pharmacodyn. 279, 195-211.

111. Wangen K., Myhrer T., Moldsid J.N., Iversen E.G. and Fonnum F. (1997) Modulatory treatmenn of NMDA receptors in neonatal rats affects cognitive behavior in adult age. Dev. Brain Res. 99, 126-130.

112. Williams C.V., Nordquist D. and Mcloon S.C. (1994) Correlation of mitric oxide synthase expression with changing patterns of axonal projections in the developing wisual system. J. Newrosci. 14, 1746-1755.

113. Wörtwein G., Gustafson B., Hansen K.L. and Mogensen J. (1997) Behavioral symptoms in adult rats after posinatal Lu"nitro-arginine. Int. J. Dev/ Nesurosci. 15, 147-154.

114. Yamada K., Noda Y., Nakayama S., Komori Y., Sugihara H., Hasegawa T. and Nabeshima T. (1995) Role of nitric oxide in learning and memory and in monoamine metabolism in the rat brain. Br. J. Pharmacol. $115,852-858$.

115. Zhang Y., Marcillat O., Giulivi C., Enster L. and Davies K.J. (1990) The oxidative inactivation of mitochondrial electron transport chain components and ATPase. J. Biol. Chem. 265. 16330-16336. 


\section{Summary}

This thesis describes studies investigating newrodegeneration and plasticity at a behaviorit, neurochemical and neuroanatomical level. In the first chapter (ntroduction) of this thesis the terms neurodegeneration (decline in function and dying of neuronal tissue) and plasticity (ability of neuronal tissue to change or adapt) are explained and an overview is given of (possible) processes of neurodegeneration and plasticity (the later only in relation with memory). The last part of the first chapter explains the aims of this thesis in more detail.

With regard to neurodegeneration this thesis" angle of view is that energy depletion may be a cause of neurodegeneration, especially since it has been suggested that the cognitive/cholinergic dysfunction of Alzheimer's disease, which is one of the most common neurodegenerative disorders, is related with a reduction in central glucose metabolism. Two animal models were used for the evaluation of consequences of, or influences on energy depletion. One model, an intracerebroventricular (i.c.v.) injection of streptozotocin, is based on a chronic inthibition of the glucose energy metabolism by destruction of the insulin-secreting cells and/or interference with the insulin receptor system, which ultimately results in neuronal damage. However, oxidative stress may be involved (also) in causing neuronal damage, since streptozotocin can generate the reactive compounds $\mathrm{H}_{2} \mathrm{O}_{2}$ and nitric oxide (NO). In the present thesis the neurodegenerative mechanism of streptozotocin was further investigated and based on the obtained data, streptozotocin's relevance was evaluated as a model of neurodegeneration in general, or sporadic Alzheimer's disease in particular (Chapter 2-5). The other model used in this thesis, i.c.v. injected sodium cyanide (NaCN), is based on a short period of acute energy depletion, without causing lasting neuronal damage (Chapter 6). The $\mathrm{NaCN}$ model can be used to test the acute effects of drugs that are presumed to preserve neuronal function during low energy states. The putative nootropic (cognitive enhancing/neuroprotective) effect of acetyl-L-carnitine was tested in both models of energy depletion (Chapter 2, 3 and 6 ). Acetyl-L-carnitine has been reported to have a beneficial effect on the energy metabolism, wiz. stimulation the use of alternative energy sources, such as lipid substrates or ketone bodies. However, it is noteworthy to mention that besides an influence on the energy metabolism, also other actions of acetyl-L-carnitine have been reported such as, for instance, a positive effect on nerve growth factor receptors.

With regard to plasticity this thesis describes two studies. One sudy investigated the role of the NO-cyclic GMP (cGMP) signal transduction in memory formation (Chapter 7). The other one evaluated the consequences of interference with nitric oxide synthesis during development on brain function and behavior in later life (Chapter 8 ). In the final chapter (Concluding remarks) the main findings of the above mentioned studies and their implication are discussed.

In Chapter 1, an overview is given of plasticity, neurodegeneration, and aims of the thesis. respectively. With regard to plasticity, operational definitions of, and behavioral tasks meaturing learning and memory were presented. Thereafter, brain structures and neurotransmitter/neuromodulator systerns involved in memory formation were summarized. Neurodegenerative processes were mainly discussed by taking Alzheimer's disease as an example. In addition, models of neurodegeneration and therapies for neurodegenerative disorders (mannly based on the discussed neurodegenerative processes) were presented. Finally, the ams of the thesis were discussed in more detail to give additional information, especially in order to gain more insight into the several possible mechanisms of action of the chemical compounds used (e.g. streptozotocin, aceryl-L-carnitine).

In Chapter 2, the effects of chronic treatment with acetyl L-carnitine $75 \mathrm{mg} / \mathrm{kg}$ dailly, via drinking water) were evaluated on spatial discrimination learning in the Morris water escape task 
and choline acetyltransferase activity of i.c.v. streptozotocin-treated $(1.5 \mathrm{mg} / \mathrm{kg}$ in $4 \mu \mathrm{l}$, bilaterally $2 \mu$ /injection site) middle-aged rats. I.c.W. injected streptozotocin may provide a relevant model of neurodegeneration, in particular Alzheimer's disease, that is induced by a decrease in the central metabolism of glucose. Chronic treatment with acelyl-L-carnitine attenuated both the streptozotocin-induced impairment in spatial bias and the decrease in hippocampal choline acetyltransferase activity. Whether acetyl-L-carnitine enhanced the utilization of alternative energy sources and thus antagonized the effects of streptozotocin treatment was not investigated. Other explanations are also possible, such as a positive effect of acetyl-Li-carnitine on nerve growth factor. To conclude, the findings indicate that acetyl-L-carnitine treatment has a neuroprotective effect, but further studies are needed to characterize the mechanism of action of acetyl-L-carnitine in this model.

In Chapter 3, enzyme activities previously investigated in Alzheimer's disease (peptidases, dehydrogenases and acetyltransferases) were measured in the septum and hippocampus of control and i.c.v. streptozotocin-treated ( $1.5 \mathrm{mg} / \mathrm{kg}$ in $4 \mu \mathrm{i}_{\text {, }}$ bilaterally $2 \mu 1 /$ injection site) rats. It may be assumed that i.c.v. streptozotocin treatment of rats might be of relevance to investigate pathogenetic aspects of Alzheimer's disease, since it likewise inhibits the central glucose metabolism, and induces changes in neurotransmitter systems and behavior. Streptozotocintreated rats receiving acetyl-L-camitine $(75 \mathrm{mg} / \mathrm{kg}$ daily, via drinking water) were also included in this study in order to further assess possible nearoprotective effects of this compound. All enzyme activities in the septum were affected by streptozotocin, with the exception of choline acetyltransferase activity. By contrast, choline acetyltransferase activity was the only enzyme activity affected in the hippocampus. The weight of the septum was reduced in streptozotocintreated animals. The hippocampal choline acetyltransferase activity was correlated with that of the septum, and, therefore, the reduced choline acetyltransferase activity in the hippocampus can be explained with an effect of streptozotocin at the level of the septum. The fact that enzyme activities were affected rather indiscriminately in the septum, together with a reduced weight of the septum suggests that streptozotocin acts as a non-selective neurotoxin. This would favor the possibility that streptozotocin causes (nos-selective) oxidative damage in stead of (only) decreasing the central metabolism of glucose. Furthermore, these enzymatic changes do not closely resemble the nore specific changes in enzyme activities as obserwed in Alzheimer's disease. Acetyl-L-camitine partly prevented the streptozotocin-induced damage, as reflected by an attenuation of the streptozotocin-induced decrease in hippocampal choline acetyltransferase activity. This neuroprotective action of acetyl-L-carnitine could not be mediated by an energy enituncement of acetyl-L-camitime, since in that case all neurons/enzyme activities should have been spared non-selectively in the septum. Another explanation could be that acetyl-L-carnitine has a positive effect on the neurotrophic action of nerve growth factor on the cholinergic system. In conclusion, although it has to be considered that there are some limitations to streptozotocin as. a mordel of Alzheimer's disease, it may be valuable to test neuroprotective effects of compounds.

In Chapter 4 , the effects of an i.c.w. injection of streptozotocin ( $1.5 \mathrm{mg} / \mathrm{kg}$ in $4 \mu \mathrm{l}$, bilaterally 2 ulfinjection site) were evaluated on cognitive behavior and biochenical markers in the brain of middle aged Wistar rats. We found that streptozotocin-treated rats showed an impaired cognitive performance in the delayed non-matching to position task and the Morris water escape task. The streptozotocin-treated rats could be subdivided into a non-impaired and an impaired group on the basis of the performance in the delayed non-matching to position task. This subdivision also applied to the perfornance in the Morris water escape task which indicates that streptozotocin induced an individual cognitive decline across various behavional domains. Glial fibrillary acidic 
protein, an indicator of reactive astroglial changes and indirect marker of neuronal damage, was measured in three different (soluble, Triton X-100 soluble and crude cytoskeletal) protein fractions and its content in the fractions of the septum, hippocampus and striatum of streptozotocin-treated rats was increased. Furthemore, the glial fibrillary acidic protein response of each protein fraction to streptozotocin treatment appeared to be differently regulated. In streptozotocin-treated rats the choline acetyltransferase activity was decreased in the hippocampus only, which was correlated with the hippocampal glial fibrillary acidic protein contents of all three hippocampal proten fractions, thus suggesting that the cholinergic deficit is a consequence of direct damage to the hippocampus. This is supported by the finding that there was no reduction in septum weight of streptozotocin-treated rats and the decrease in hippocampal choline acetyltransferase activity was not related to possible damage (increased glial fibrillary acidic protein content) of the septum. This indication of direct damage to the hippocampus of streptozotocin-treated Wistar rats, adds more support to the metabolic deficit after streptozotocin treatment, since the hippocampus is one of the brain structures with the highest number of insulin receptors and highest glucose need, and, therefore, probably most vulnerable. The cognitive deficits in both tasks were related to the increased glial fibrillary acidic protein contents, especially of the soluble and cytoskeletal fraction, and the decreased choline acetyltransferase activity in the hippocampus. All findings regarding glial fibrillary acidic protein indicate that it is important to take into account which protein fraction thas been used for measuring the glial fibrillary acidic protein response to a stressor. Furthermore, intracerebroventricular injected streptozotocin may provide a relevant model for studying neurodegenerative changes due to a metabolic insufficiency and testing newroprotective effects of substances.

In Chapter 5 , the possible role of NO was evaluated in mediating neuronal damage in middle-aged rats after an i.c.v injection of streptozotocin. An i.c.v injection of streptozotocin has been reported to decrease the central metabolism of glucose. This intuibition of the energy metabolism after streptozotocin treatment might induce an excitotoxic mechanism, which may lead to the stimulation of NO synthase and, consequently to the synthesis of NO. On the other hand, streptozotocin might induce oxidative stress directly by liberation of nitric oxide from its nitroso moiety. To investigate whether NO synthase is involved in a possible excitotoxic mechanism after streptozotocin treatment, rats treated with streptozotocin $(1.25 \mathrm{mg} / \mathrm{kg}$ in $4 \mu \mathrm{l}$, bilaterally 2 ul/injection site) were also treated with the NO synhase inhibitor $N$-nitro-L-arginine methyl ester (L-NAME, $20 \mathrm{mg} / \mathrm{kg}$ intraperitoneal (i.p) $10 \mathrm{~min}, 6.24$ and 96 h after sureptozotocin injection). To investigate whether $\mathrm{NO}$ liberated from streptozotocin may be responsible for neurotoxic effects, one additional group of control rats received an i.c.w. injection of the NO donor sodium nitroprusside $(10 \mu \mathrm{g}$ in $4 \mu 1)$. It was found that streptozotocin affected the behavioral performances in the open field and iwo-way active avoidance task. In addition. glial fibrillary acidic protein immunostaining showed that neuronall damage was mainly located in periand paraventricular regions of the third and lateral ventricles, like for instance in the septum; caudate putamen and hippocampus. L-NAME treatment had mo protective effect on the behavioral impairments and neuronall damage of streptozotocin-treated rats. This suggests that the neuronal damage of streptozotocin may still be a result of the decrease in the central energy metabolism, but without the involvement of NO synthase. This was supported by measuring, using immunostaining, the $\mathrm{NO}$-mediated cGMP production by the enzyme soluble guanylyl cyclase in cortical sllices, i.e. L-NAME did not prevent NO production after streptozotocin administration in vitro. In addition, it was found that sodium nitroprusside liberated NO in wiro, whereas in vivo sodium nitroprusside administration did. not lead to any behavioral and neuronal deficits at all. However, the involvement of NO liberated from streptozotocim in neuronal damage cannot be 
excluded yet.

In Chapter 6 , the effects of acute treament with acetyl-L-camitine $(50 \mathrm{mg} / \mathrm{kg}$. i.w. $90 \mathrm{~min}$ before the $\mathrm{NaCN}$ injection) were investigated on a NaCN-induced behavioral deficit in the Morris water escape task. In a first experiment the spatial discrimination performance of the rats was found to be dose-dependently impaired after an i.c.y. injection of $\mathrm{NaCN}(2.5$ and $5.0 \mu \mathrm{g})$. Acute treatment with acetyl-L-camithe was found to increase the behavional deficit after NaCN. These findings were replicated in a second experiment. Based on these results it can be argued that an acute administration of acetyl-L-carnitine appears to potentiate a $\mathrm{NaCN}$-induced behawioral deficit. An additional in vitro experiment with rat brain synaptosomes showed clear effects of administered $\mathrm{NaCN}$ on the energy-dependent incorporation of inositol into phosphoinositides and on the ATP concentration. In witro acetyl-L-carntine administration had no effect on the $\mathrm{NaCN}$ induced energy depletion. The negative behavioral findings are in contrast with our previously found protective effect of chronic treatment with acetyl-L-carnitine (via drinking water) on the $\mathrm{NaCN}$-induced behavioral deficit. Since chronic acetyl-L-carnitine treatment has no effect on the phosphoinositide metabolism it was suggested that aceryl-L-carnitine may act via the formation of an ATP-independent reservoir of activated acyl groups. Thus, fatty acids as acylated derivatives can be used for reacylation processes during an acute period of energy depletion. However, we have no clear explanation for the discrepancy in behavioral results between the chronic versus acute treatment of acetyl-L-carnitine at present. Further research is needed to characterize the mechanism of action of acetyl-L-carnitine in relation to $\mathrm{NaCN}$.

In Chapter 7, the effects of 7-nitroindazole, a putative selective inhibitor of neuronal NO synthase, and zaprinast, a cGMP-selective phosphodiesterase inhibitor, were evaluated on recognition memory of rats in the object recognition test. This test is based on the differential exploration of a new and a familiar object. Two doses of 7 -nitroindazole (10 and $30 \mathrm{mg} / \mathrm{kg}$ ) and zaprinast ( 3 and $10 \mathrm{mg} / \mathrm{kg}$ ) were used. The substances were administered i.p. immediately after the exposure to two identical objects, i.e. at the start of the delay interval. After a delay interwal of $1 \mathrm{~h}$, control rats spent more time exploring the new object which demonstrates that they recognized the familiar one. Both doses of 7 -nitroindazole impaired the discrimination between the wo objects after the 1-h interval. After a 4 -lin interval, control rats did not discriminate between the objects. The highest dose of zaprinast racilitated object recognition after the 4-h interwal. In addition, this dose of zaprinast ( $10 \mathrm{mg} / \mathrm{kg}$ ) reversed the recognition memory deficit induced by 7 . nitroindazole (10 $\mathrm{mg} / \mathrm{kg}$ ) at the 1 -h interval. Interference with the NO-cGMP signal transduction can also induce cardiovascular effects which may influence memory performance. The highest dose of 7-nitroindazole slightly increased mean arterial blood pressure $1 \mathrm{~h}$ after its administration. Four h after administration of zaprinast ( $10 \mathrm{mg} / \mathrm{kg}$ ), mean arterial blood pressure was also slighty increased, but not after $\|$ h after zaprinast administration. However, these effects on blood pressure are apparently not related to, or explain the differential effects on object recognition memory. These results therefore suggest that NO-cGMP signal transduction is involved in object recognition memory independently of its cardiovascular role. In addition, since 7-nitroindazole affected mean arterial blood pressure it cannot be regarded as a selective imhibitor of neuronal NO symthase.

In Chapter 8, the consequences of interference with NO synthesis during development were evaluated on brain function and behavior in later life. Rat pups received a daily injection of the NO synthase intibitor L-NAME (25 mg/kg subcutaneous) from postnatal day 0 to 24 . At postnatal day 8 L-NAME-treated rats had enlarged and heavier stomachs, while body weights appeared to be reduced. The stomachs were not affected in size and weight anymore at postnatal 
day 24, whereas the body weights were still reduced by the L-NAME treatment, although they soon recowered after termination of the treatment. At 4 months of age, rats were tested in noncognitive (open field) and cognitive (Mortis water escape, two way active avoidance) tasks. Open field behavior of adult rats postnatally treated with LNAME was not affected. In the water escape task there were no differences between the saline and L.NAME-treated rats in spatial discrimination learning and spatial reversal learning. Furthermore, postnatal L-NAME treatment did not have an effect on the acquisition of the wo-way active avoidance task. Subsequently, we tested rat pups during the $\mathbb{L}$-NAME treatment at postratal day 19 throwgh 24 in the open field and the two-way active avoidance task. L-NAME treatment appeared to increase the behavioral activity in the open field. There was no difference in behavior in the active avoidance task between saline and L-NAME-treated rats. Biochemical and immunocytochemical studies showed that at postnatal day 8 the basal cGMP level was reduced, while the CGMP fomation due to incubation with the NO donor sodium nitroprusside appeared to be increased in the hippocampus, striatum and frontal cortex of L-NAME-treated rats. Hence, NO synthase was inhibited whereas the soluble guany!yl cyclase activity may be increased in sensitivity. At postnatal day 24 basall cGMP levels and NO-mediated CGMP formation in the brain structures of L-NAME-treated rats had normal values again. Taken together, the findings of this study suggest that postnatal inhibition of No synthase has profound neurochemical effects during development and may have short-lasting effects on non-cognitive behavior, but it does not affect behavior and brain function in later life.

In Chapter 9, the various findings and conclusions of this thesis are discussed. The main conclusions were that treatment of middle-aged rats with a single i.c.v. injection of streptozotocin may provide a relevant model of neurodegeneration which has several similarities with Alzheimer's disease. Streptozotocin reduces the central metabolism of glucose most likely by interference with the insulin receptor system. However, besides that neurodegenerative effects may be due to an inhibition of the energy metabolism, oxidative damage may be involved (directly), since a probable mechanism of action of streptozotocin could be that it induces oxidative stress (e.g. NO and $\mathrm{H}_{2} \mathrm{O}_{2}$ generation). In contrast to the chronic streptozotocin model, there is only at short period of acute energy depletion after i.c.v. NaCN administration. Hence, the $\mathrm{NaCN}$ model can be used test the acute effects of drugs that are presumed to preserve neuronal function during low energy states.

Chronic acetyl-L-carnitine treatment (via drinking water) prevented/attenuatted cognitive decline in both the streptozotocin and $\mathrm{NaCN}$ model. The positive effect of acetyl-L-cantine in the streptozotocin model may be explained with a beneficial effect of acetyl-L.-carruitine on the energy metabolism, i.e. stimulation of the use of alternative energy sources. However, a possible neurotropic effect of acetyl-L-carnitine on nerve growth factor cannot be ruled out either. In the $\mathrm{NaCN}$ model chronically administered acetyl-L-carnitine may probably exert its positive action via the formation of an energy-independent acyl(carnitines) reservoir and/or detoxification of toxic acylCoAs. However, acute administration (i.v.) of acetyl-L-carnitine appeared to potentiate the $\mathrm{NaCN}$-induced cognitive deficit, for which no adequate explanation exists. These findings indicate that the nootropic effect of acetyl-L-carnitine has more to do with neuroprotection than with cognition enhancement. Therefore, it is best to administer acetyl-L-carnitine chronically, and preferably as early as possible, to preventattenuate cognitive dysfunction such as occurs in for instance Alzheimer's disease.

Chronic postmatal inhibition of $\mathrm{NO}$ formation had no effect on cognitive performance in later life, which questions a role of NO in early brain development. On the other hand, NO and cGMP 
were involved in processes of memory formation. However, other studies indicated that NO only piays a moderate, or even no role at all, in this respect. Hence, further investigations into the cognitive enhancing effects of increasing CGMP levels may offer favorable possibilities for treatment of cognitive dysfunctions. 


\section{Samenvatting}

Dit proefschrift beschrijf studies waarin neurodegeneratie en plasticitei onderzocht werden op gedrags-, neurochemisch-en neuroanatomisch niveau. In het eerste hoofdstuk (Introduction) worden de begrippen neurodegeneratie (afname in functie en doodgaan van neuronasl weefsel) en plasticiteit (eigenschap van neuronal weefsel on te veranderen of zich aan te passen) uitgelegd. Tevens wordt een overzicht gegewen van (mogelijke) processen van neurodegeneratie en plasticiteit (dit laatste alleen in relatie tor geheugen). Hei laatste deel van het eerste hoofdsmk behandelt de beoogde onderzoeksdoelen van dit proefschrift.

De invalshoek van dit proetschrift met betrekking tot neurodegeneratie is cat energie depletie de oorzaak kan zijn van neurodegeneratie. In het bijzonder omdat de cogritieve/cholinerge disfunctie bij de ziekte van Alzheimer, een van de meest voorkomende neurodegeneratieve aandoeningen, gerelateend is an een afname in het centrale glucose metabolisme. Twee diermodellen werden gebruikt om de consequenties van, of de invloeden op energie depletie te evalueren. Het ene model waarbij streptozotocine intracerebroventriculair (i.c.v.) wordt geinjecteerd, is gebaseerd op een chronische inhibitie van het glucose metabolisme door middel van destructie van de insuline-secrerende cellen en/of interferentie met het insuline receptor systeem, wat uiteindelijk resulteert in neuronale schade. Oxidatieve stress kan echter (ook) betrokken zijn bij het veroorzaken van neuronale schade ondat streptozotocina de reactieve verbindingen waterstofperoxide $\left(\mathrm{H}_{2} \mathrm{O}_{2}\right)$ en stikstofmonoxide (NO) kan genereren. In dit proefschrift werden de mogelijke neurodegeneratieve mechanismen van streptozotocine verder onderzacht. Gebaseerd op de verkregen data werd de relevantie van stteptozotocine als een model voor neurodegeneratie, of meer in het bijzonder de ziekte van Alzheimer, geëvalueerd (Hoofdstuk 2-5). Het andere model dat in dit proefschrift word beschreven makt gebruik van een i.c.w. injectie met natriumcyanide $(\mathrm{NaCN})$ en is gebaseerd op een korte periode van acute energie depletie, zonder dat blijvende schade wordt veroorzaakt (Hoofdstuk 6). Het NaCN model kan gebruikt worden om acute effecten te testen van substanties die verondersteld worden het neuronaal functioneren in stand te houden tijdens lage energie niveau's. Het veronderstelde noütropische (cognitief verbeterende/neuroprotectieve) effect van acetyl-L-carmitine werd getest in beide modellen wan energie depletie (Hoofdstuk 2, 3 en 5). Het is bekend dat acetyl-L-carnitine een gunstig effect heeft op het energie metabolisme, al. de stimulatie wan het gebruik van alternatieve energiebronnen zoals vetsubstraten en ketonlichanen. Maar het is van belang om op te merken dat behalve de beinvloeding van het energie metabolisme, eveneens andere werkingsmechanismen van acetyl-L-carnitine zijn gevonden zoals bijwoorbeeld eet positief effect op "nerve growth factor (NGF)" receptoren.

Met betrekking tot plasficiteit worden in dit proefschrilt fwee studies beschreven. Een studie anderzocht de rol van de NO-cyclisch GMP (cGMP) signaal transductie in de opslag van informatie in het geheugen (Hoofdstuk 7). De andere studie evalueerde de consequenties van interferentie met de synthese van NO gedurende de ontwikkeling op hersenfunctioneren en gedrag tijdens het Jatere leven (Hoofdstuk 8). In het laatste hoofdstuk (Concluding remarks) worden de belangrijkste bevindingen van bovengenoemde studies en hun implicaties bediscussieerd.

Hoofdstuk 1 geeft een overzicht van respectievelijk plasticiteit, neurodegeneratie en de doelstellingen van dit proefschrift. Met betrekking tot plasticiteit werden de operationalisaties en gedragstaken yoor het meten wan leren en geheugen gepresenteerd. Hiema werd een samenvatting gegeven van hersenstructuren en neurotransmitter/nearomodulator systemen die betrokken zijn bij de opslag van informatie in het geheugen. Neurodegeneratieve processen werden met name behandeld aan de hand vam de ziekte van Alzheimer. Verwolgens werden modellen voor 
neurodegeneratie en therapiesn voor neurodegeneratieve aandoeningen (wnl. gebaseerd op de ziekte van Alzheimer) gepresenteerd. Tenslotte, werden de beoogde doelstellingen van dit proefschrift meer in detail besproken om zodoende meer informatie te verschaffen over, en inzicht te geven in de verscheidene mogelijke mechanismen van de gebruikte chemische substanties (bijx. streptozotocine, acetyl-L-carnithe).

In Hooffstuk 2 werden de effecten geevalueerd van een chronische behandeling met acetylL-carnitine (75 mg/kg dagelijks, via het drinkwater) op spatieel leren in de Morris zwemtaak en choline acetyltransferase activiteit văn met i.c. $\psi$ streptozotocine $(1.5 \mathrm{mg} / \mathrm{kg}$ in $4 \mu \mathrm{l}$, bilateraal 2 $\mu$ /injectie) behandelde ratten van middelbare leeftijd. Een i.c.w. injectie met streptozotocine kan een relevant model voor neurodegeneratie, in het bijzonder de ziekte van Alzheimer, opleveren dat geinduceerd wordt door een afname in het centrale glucose metabolisme. Chronische behandeling met acetyl-L-carnitine verminderde zowel de door streptozotocine geïnduceerde verstoring in spatieel geheugen als afgenomen choline acetyltransferase activiteit in de hippocampus. Of acetyl-L-carnitine het gebruik van altematieve energiebronnen stimuleerde en aldus de effectan van streptozotocine behandeling antagoniseerde, was niet onderzocht. Derhalve zijn andere verklaringen ook mogelijk, zoals een positief effect van acetyl-L-carnitine op NGF. Geconchdeerd kan worden dat chronische behandeling met acetyl-L-carnitine een neuroprotectief effect heeft, maar dat meer onderzoek vereist is om het werkingsmechanisme van acetyl-L-carnitine in dit model te karakteriseren.

Hooldstuk 3 beschrift de metingen wan enzym activiteiten, die eerder al onderzocht waren bij de ziekte van Alzheimer (peptidasen, dehydrogenasen en acetyltransferasen), in het septum en de hippocampus van i.c.v. streptozotocine $(1.5 \mathrm{mg} / \mathrm{kg}$ in $4 \mu \mathrm{l}$, bilateraal $2 \mu l /$ injectie) behandelde ratten. Het wordt aangenomen dat i.c.v. behandeling met streptozotocine van belang kan zijn om pathogenetische aspecten van de ziekte van Alzheimer te onderzoeken. Dit omdat streptozotocine eveneens het centrale glucose metabolisme remt en veranderingen in neurotransmitter systemen en gedrag induceert. Streptozotocine behandelde ratten die acetyl-L-carnitine $(75 \mathrm{mg} / \mathrm{kg}$ dagelijks, via het drinkwater) kregen, werden ook onderzocht om mogelijke neuroprotectieve effecten van acetyl-L-carnitine vast te stellen. Alle enzym activiteiten in het septum waren aangedan doof streptozotocine, met wizondering van de choline acetyltransferase activiteir. Daarentegen was in de hippocampus de choline acetyltransferase activiteit de enige enzym activiteit die aangedaan was. Verder bleek het gewicht van het septum te zijn afgenomen in streptozotocine behandelde dieren. De choline acetyltransferase activiteit in de hippocampus correleerde met die van het septum. Derhalve kan de afigenomen hippocampale choline acetyltransferase activiteit verklaard worden door een effect van streptozotocine op het septum. Het feit dat de enzym activiteiten in het septum nogal aspecifiek waren aangedaan, naast de gewichtsafname van het septum, suggereert dat streptozotocine als een aselectief neurotoxine werkt. Dit ondersteunt de mogelijkheid dat streptozotocine (aselectieve) oxidatieve schade veroorzarkt in plats van (alleen) een afname in het centrale glucose metabolisme. Bovendien lijken de veranderingen in enzym activiteiten niet op de meer specifieke veranderingen zoals gevonden bij de ziekte van Alzheimer. Acetyl-L-carnitine voorkwam ten dele de door streptozotocine veroorzakte schade zoals bleek wit de vermindering van de door streptozotocine geinduceerde afname in hippocampale choline acetyltransferase activiteit. Deze neuroprotectieve werking van acetyl-L-carnitine kan niet gemedieerd zijn via een verbetering van het energie metabolisme omdat in dat geval alle neuronen/enzym activiteiten aselectief gespaard zouden zijn gebleven. Een andere verklaring kan zijn dat acetyl-L-carnitine een positief effect heeft op de 
neurotrofe werking van NGF op het cholinerge systeem. Samengevat kan gesteld worden dat streptozotocine waardevol is om neuroprotectieve effecten van substamties te testen, alhoewel er rekening mee gehouden moet worden dat streptozotocine als model voor de ziekte van Alzheimer enkele tekortkomingen kent.

In Hoofdstuk 4 werden de effecten van een i.c.v. injectie met streptozotocine $(1.5 \mathrm{mg} / \mathrm{kg}$ in 4 $\mu 1$, bilateraal $2 \mu 1 /$ injectie) geevalueerd op cognitief gedrag en biochemische "markers" in het brein van Wistar ratten van middelbare leeftijd. De streptozotocine behandelde tatten hadden een verstoorde cognitieve prestatie in de "delayed mon-matching to position (DNMTP)" taak en de Morris zwemtak. Op basis wan de prestatie in de DNMTP taak konden de sireptozotocine behandelde ratten ingedeeld worden in een "non-impaired (geen effect)" en "impaired (wel effect)" groep. Deze indeling bleek ook van toepassing op de prestatie in de Morris zwemtak wat aangeeft dat streptozotocine een afname in verschillende typen van cognitie-gerelateerd gedrag induceert. "Glial fibrillary acidic protein (GFAP)", een indicator voor reachieve astrogliosis en indirecte "marker" voor neuronale schade, werd gemeten in drie verschillende eiwit fracties: een water oplosbare, Triton X-100 oplosbare en ruwe cytoskelet fractie. De GFAP hoeveelheden in de fracties van septum, hippocampus en striatum van streptozotocine behandelde ratten waren toegenomen, alhoewel de GFAP respons van elke eiwit fractie op streptozotocine anders gereguleerd bleek te worden. Choline acetyltransferase activiteit was alleen in de hippocampus van streptozotocine ratten afgenomen en correleerde met de GFAP hoeweelheid van alle drie de hippocampale eiwit fracties. Dit suggereert dat het cholinerge gebrek in de hippocampus een gevolg is van directe beschadiging van de hippocampus. Deze suggestie wordt ondersteund door de bevinding dat het septum gewicht in streptozotocine behandedde ratten niet was afgenomen en dat de afname in hippocampale choline acetyltransferase activiteit niet gerelateerd was aan een septum beschadiging, d.w.z. toegenomen GFAP hoeveelheid. Directe beschadiging van de hippocampus duidt op een energie deficiëntie als gevolg van streptozotocine behandeling omdat de hippocampus éen van de hersenstructuren is met de meeste insuline receptoren en hoogste glucose behoefte, en die derhalve waarschijnlijk het meest kwetsbaar is. De cognitieve gebreken in beide taken waren gerelateerd aan de toegenomen GFAP hoeveelheid, met name de oplosbare en cytoskelet fractie, en de afgenomen choline acetyltransferase activiteit in de hippocampus. Alle bevindingen met betrekking tot GFAP duiden erop dat het van belang is om er rekening mee te houden welke eiwit fractie gebrutht wordt om de GFAP respons op een "stressor" te meten.

In Hoofdstuk 5 werd de mogelijke rol wan NO geevalueerd in het medietren wan neuronale schade in ratten van middelbare leeftijd ta een i.c.v. injectie met streptozotocine. Het is bekend dat een i.c.w. injectie met streptozotocine leidr tot cen afname in het centrale glucose metabolisme. Deze remming wan het energie metabolisme na sitreptozotocine behandeling zou een excitotoxisch mechanisme in gang kunnen zetten dat kan leiden tot de stimulatie van NO synthase en dientengevolge de productie van NO. Aan de andere kant zou streptozotocine oxidatieve stress veroorzaken door afsplitsing van zijn $N O$ groep. Om te onderzoeken of NO synthatse betrokken is bij een mogelijk excitotoxisch mechanisme na streptozotocine behandeling, werden ratten die met streptozotocine ( $1.25 \mathrm{mg} / \mathrm{kg}$ in $4 \mu \mathrm{l}$, bilateral $2 \mu \mathrm{l} / \mathrm{injectie)} \mathrm{behandeld} \mathrm{waren,} \mathrm{eweneens} \mathrm{belliandeld}$ met de NO synthase remmer N-nitro-L-arginine methyl ester (L-NAME, $20 \mathrm{mg} / \mathrm{kg}$. intraperitoneaal (i.p.) $10 \mathrm{~min}, 6,24$ en 96 ur na streptozotocine injectie). Om na te gan of NO afkomstig van streptozotocine verantwoordelijk kan zijn voor neurotoxische effecten, kreeg een additionele groep wan controle ratten een i.c.v. injectie met de NO donor natriumnitroprusside (10 $\mu \mathrm{g}$ in $4 \mu \mathrm{l}$ ). Streptozotocine bleek een negatief effect te hebben op de gedragsprestaties in het open veld en op het actief vermijdingsleren. Aanvullendle immunokleuring voor GFAP liet zien 
dat neuronale schade woomamelijk gelokaliseerd was in peri-en paraventriculaire gebieden van de derde en laterale ventrikels, zoals bijy. in hef septum, caudate putamen en de hippocampus. LNAME behandeling had geen protectief effect op de gedragsstoomissen en neuronale schade van streptozotocine behandelde ratien. Dit suggereert dat neuronale schade als gevolg van streptozolocine toch een gevolg kan zijn van een afname in het centrale energie metabolisme, maar wel zonder de betrokkentheid van NO symthase. Dit wordt ondersteund door in witro experimenten, waarbij door middel van immunokleuring de NO-gemedièerde cGMP productie door de oplosbare vorm van het enzym guanylyl cyclase werd gemeten, en waaruit bleek dat LNAME niet kon woorkomem dat NO werd geproduceerd na streptozotocine toediening. Tevens werd gevonden dat natriumnitroprusside NO afsplitst in vitro, terwijl in wivo natriumnitroprusside loediening niet leidde tot gedrags- en neuronale gebreken. Toch kan de betrokkenheid van NO afgesplitst van streptozotocine in vivo niet uitgesloten worden.

Hoofdstmk 6 beschrijf de resultaten van acute behandeling met acetyl-L-carnitine $(50 \mathrm{mg} / \mathrm{kg}$, intraveneus 90 min vóor natriumcyanide ( $\mathrm{NaCN}$ ) injectie) op een door $\mathrm{NaCN}$ veroorzaakte verstoring wan het gedrag in de Morris zwemtalk. In een eerste experiment werd gevonden dat de spatiële prestaties van fle ratten dosis-afhankelijk verstoord werd door een i.c.v. injectie met $\mathrm{NaCN}$ (2.5. en 5.0 $\mu \mathrm{g}$ ). Acute behandeling met acetyl-L-carnitine bleek de gedragsstoornis te verergeren. Deze bevinding werden gerepliceerd in een tweede experiment. Er zijn derhalve argumentem om te stellen dat acute toediening van acetyl-L-camitine de door $\mathrm{NaCN}$ veroorzaakte verstoring in gedrag potentieent. Een aanvullend in vitro experiment met synaptosomen uit het uattenbrein liet duidelijke effecten zien van $\mathrm{NaCN}$ toediening op de energie-afhankelijke incorporatie van inositol in fosfoinositiden en op ATP concentraties. In vitro acetyl-L-carnitine toediening had geen effect op de door $\mathrm{NaCN}$ veroorzalakte energie depletie. De negatieve gedragseffecten zijn in tegenstelling met de eerder gevonden protectieve effecten van chronische behandeling net acetyl-L-carnitine $(75 \mathrm{mg} / \mathrm{kg}$ dagelijks, wia het drinkwater) op de door $\mathrm{NaCN}$ veroorzaakte gedragsstoornis. Ondat chronische acetyl-L-carnitine behandeling geen effect heeft op het fosfoinositiden metabolisme, is gesuggereerd dat acetyl-L-carnitine een effect kan hebben door middel van de vorming van een ATP-onathankelijk reservoir van geactiveerde acyl groepen. Aldus zijn vrije vetzuren als geacyleerde derivaten beschikbar voor reacyleringsprocessen gedurende een acute periode van energie gebrek. Op het moment is er echter geen eenduidige verklaring voorhanden woor de discrepantie in de gedragsdata tussen de chronische en acute behandeling met acetyl-L-carnitine. Meer onderzoek is derhalve vereist om het werkingsmechanisme wan acetyl-L-carnitine te karakteriseren in relatie met $\mathrm{NaCN}$.

In Hoofdstuk 7 werden de effecten van 7 -nitroindazol, een mogelijke selectieve remmer van neuronal NO synthase, en zaprinast, een cGMP-selectieve fosfodiesterase remmer, geëvalueerd op het herkenningsgeheugen van ratten in de voorwerp herkenningstaak. Deze taak is gebaseerd op het verschil in exploratie tussen een nieuw en bekend woorwerp. Twee doseringen van 7nitroindazol (10 en $30 \mathrm{mg} / \mathrm{kg}$ ) en zaprinast ( $3 \mathrm{en} 10 \mathrm{mg} / \mathrm{kg}$ ) werden gebruikt. De substanties werden i.p. toegediend meteen na blootstelling aan twee identieke voorwerpen, d.w.z. aan de start van het tijdsinterval. Na een tijdsinterval wan 1 uur, spendeerden de controle ratten meer tijd aan het nieuwe voorwerp wat aangeeft dat ze het bekende voorwerp herkenden. Beide doseringen van 7 -nitroindazol verstoorden de discriminatie tussen de twee woorwerpen na het 1 uur interval. Na een 4 uur interval discrimineerden de controle ratten niet meer tussen de voorwerpen. Behandeling met de hoogste dosis van zaprinast leidde wel tof woorwerp herkenning na het 4 uur interval. Dezelfde zaprinast dosis $(10 \mathrm{mg} / \mathrm{kg}$ ) hief de door 7 -nitroindazol $(10 \mathrm{mg} / \mathrm{kg}$ ) verstoorde voorwerp herkenning bij het 1 ur interval op. Interferentie met de NO-cGMP signaal transductie 
kan ook cardiovasculaire effecten tot gevolg hebben die geheugen prestaties kunnen beinvloeden. De hoogste dosis wan 7-nitroindazol verhoogde de gemiddelde arterièle bloeddruk I wur ha toediening. Vier uur na zaprinast toediening $(10 \mathrm{mg} / \mathrm{kg})$ was ook de geniddelde arteriele bloeddruk lichtelijk verhoogd. Maar deze effecten op bloeddruk konden niet de verschillende effecten op het voorwerp herkenningsgeheugen verklaren. Derhalve kan aangenomen worden dat de NO-cGMP signaal transductie betrokken is bij het voorwerp herkenningsgeheugen onafhankelijk van de cardiovasculaire functie. Daarnaast kan 7-nitroindazol niet beschouwd worden als een selectieve remmer van neuronaal NO synthase gezien de effecten op de gemiddelde arteriele bloeddruk.

In Hoofdstuk 8 werden de consequenties geëvalueerd wan interferentie met NO synthase gedurende de ontwikkeling op hersenfunctie en gedrag tijdens het latere leven. Rattenpups kregen dagelijks van postnatale dag 1 tot 24 eén injectie met de NO synthase remmer $\mathbb{L}-\mathrm{NAME}$ (25 mg/kg, subcutaan). Op postmatale dag 8 hadden de L-NAME behandelde ratten vergrote en zwaardere magen, terwijl hun lichaarnsgewichten waren afgenomen. De magen waren niet meer aangedaan in maat en gewicht op postnatale dag 24, terwijl de لlichaamsgewichten nog steeds afgenomen waren, alhoewel ze wel snel herstelden na beëindiging van de L-NAME behandeling. Op een leeftijd van 4 maanden werden de ratten in niei-cognitieve (open weld) en cognitieve (Morris zwemtaak, actief vermijdingsleren) taken getest. Het gedrag in het open veld was bij de volwassen ratten niet aangedaan. In de Mornis zwemtakk waren er geen verschillen tussen de saline en L-NAME behandelde ratten. De postnatale L-NAME behandeling had ook geen effect op het actief vermijdingsleren. Verwolgens werden pups tijdens de L-NAME behandeling op postnatale dag 19 tot en met 24 getest in het open veld en op actief vemijdingsleren. L-NAME behandeling leidde tot een toename in de gedragsactiviteit in het open veld. Er was geen verschil tussen de saline en L-NAME behandelde pups in het gedrag in de actief vermijdingslerentaak. Biochemische en immunocytochemische studies lieten zien dat op postnatale dag 8 het basale cGMP niveau verlaagd was, terwijl de cGMP productüe als gevolg van incubatie met de NO donor natriumnitroprusside was toegenomen in de hippocampus, frontale cortex en het striatum van I.. NAME behandelde ratten. Hieruit kan afgeleid worden dat NO synthase geremd was, terwijl de activiteit van het oplosbare guanylyl cyclase toegenomen was. Op postnatale dag 24 hadden de basale cGMP niveau's en de NO-gemedięerde cGMP productie weer normale warden. Samengevat duiden de resultaten van deze studie erop dat postnatale remming van NO synthase grondige neurochemische effecten heeft tijdens de ontwikkeling en tot korte-termijn effecten op niet-cognitief gedrag leidt, maar het heeft verder geen effect op gedrag en hersenfunctie tijdens het latere leven.

In Hoofdstuk 9 zijn de bevindingen en conclusies van dit proefschrift bediscussieerd. De belangrijkste conclusies waren dat behandeling van ratten wan middelbare leeftijd met cen i.c.v. injectie met streptozotocine een relevant model voor neurodegeneratie kan opleveren dat verscheidene overeenkomsten vertoont met de ziekte van Alzheimer. Streptozotocine verlaght het centrale metabolisme van glucose meest watarschijnlijk door interferentie met het insuline receptor systeem. Maar behalwe dat neurodegeneratieve effecten een gewolg kunnen zijn van een geremd energie metabolisme, kan een mogelijke werkingsmechanisme wan streptozotocine het veroorzaken van oxidatieve stress zijn (bijw. he genereren wan $\mathrm{NO}$ en $\mathrm{H}_{2} \mathrm{O}_{2}$ ). In tegenstelling tot het chronische streptozotocine model is er na i.c.v. toediening van $\mathrm{NaCN}$ alleen een korte periode van acuut energie gebrek. Derhalve is het $\mathrm{NaCN}$ model geschikt om acute effecten van substanties te testen die verondersteld worden neuronal functioneren in stand te houden tijdens een toestand van lage energie niveau's. 
Chronische acetyl-L-carnitine behandeling (wian het drinkwater) voorkomt/vermindert de cognitheve achteruitgang in zowel het streptozotocine als NaCN model. Het positieve effect van acetyl-L-canitine in het streptoxotocine model kan verkladard worden met een gunstig effect van acetyl-L-carnitine op het energie metabolisme, $\mathbb{d}$.w.z. stimulatie van het getruik van alternatieve energiebronnen. Een mogelijk pasitief effect van acetyl-L-carnitine op NGF kan echter ook niet uitgesloten worden. In het $\mathrm{NaCN}$ model kan het positieve effect van chronische acetyl-L-carnitine behandaling werklaard worden met de vorming van een energie-onafhankelijk acylcarnitines reservoir. Acute toediening van acetyl-L-carnitine bleek echter de door $\mathrm{NaCN}$ geinduceerde cognitiewe stoomis te potentieren, waarvoor echter geen afdoende verklaring gegeven kan worden. Deze bevindingen geven aan dat het noötropische effect van de substantie acetyl-L-carnitine meer met neuroprotectie dan met cognitie verbetering te maken heeft. Derhalve kan acetyl-L-carnitine het beste chronisch, liefst zo vroeg mogelijk, toegediend worden ter voorkoming/vermindering van cognitieve disfuncties zoals die woorkomen bij bijw. de ziekte van Alzheimer.

Chronische postnatale remming van de NO productie had geen effect op de cognitieve prestaties tijdens het latere leven. Dit zet vraagtekens bij de betrokkenheid van NO bij de vroege ontwikkeling van het brein. Aan de andere kant bleek NO wel, en ook cGMP, betrokken te zijn bij processen voor de opslag van informatie in het geheugen. Maar andere studies hebben aangegeven dat NO slechts een bescheiden, of zelfs geen rol speelt in deze context. Derhalve kan toekomstig onderzoek naar mogelijkheden om cognitieve disfuncties te behandelen, zich het beste richten op de cognitie verbeterende effecten van verhoogde cGMP niveau's. 


\section{Dankwoord}

Zonder een aantal mensen zon dit proefschrift er nooit gekomen zijn. Vandaar ook dat ik het proefschrift heb opgedragen aan iedereen die (ooit) heeft bijgedragen, in welke hoedanigheid dan ook. Maar tot enkele mensen wil ik hier zelfs nog een persoonlijk woord van dank richten. Nu is dit begin van het dankwoord natuurijk een cliché begin. En als je al die reeds geschreven dankwoorden leest. denk je 'jongens is dat nou echt nodig...dat valt toch well mee, zon proefschrift". Niet dus, en daar is een AIO opleiding net lang (of kort?) genoeg voor om achter te komen. Dus zoals alle cliche's is dit slecht weer eens een bevestiging van de reads bekende waarheid.

Allereerst wil ik mijn promotor Jelle Jolles bedanken. Beste Jelle, bedankt dat je me de mogelijkheid bood on te promoveren op een onderzoeksgebied dat mijn grootste interesse heeft, nl. hersenen en gedrag. Harry Steinbusch, waarde co-promotor en uiteindelijk ook promotor, jou wil ik bedanken omdat jouw komst destijds mij de gelegenheid bood om het onderzoek met anatomie te kunnen uitbreiden. Beste Jan de Vente, jou heb ik leren kennen als een goede wetenschapper en fijne collega. Omdat jouw bijdrage ann het onderzoek essentieel was, zowel inhoudelijk als ondersteunend, beschoww ik je als de derde officieuze co-promotor. Dit brengt ons automatisch bij de tweede officiele co-promotor en uiteindelijk enige co-promotor. Arjan Blokland, zonder jou had dït proefschrift er heel anders (niet?) thitgezien. Om te begimen ben je de grondlegger van mijn promotieonderzoek. Daarnaast werkte jouw pragmatische -KISS*aanpak van onderzoek altijd zeer inspirerend, alhoewel die eerste " $S$ " zeker niet op dit proefsclarift betrekking heeft. Tevens wil ik je niet alleen bedanken voor de grote wetenschappelijke, maar zeker voor de vriendschappelijke steun. Letzendlich, Thomas Fahrig und. besonders Franz Josef varn der Staray, euch möchte ich geme danken für die gute und angenehme Zusammenarbeit während und auch mach meinem Aufenthalt in Köln.

Naast al dit theoretisch geweld moet het praktische zeker niet vergeten worden. Voor de grote ondersteuning bij het gedragsonderzoek wil ik Wiel Honig bedanken. 'Korter of langer', dat is de vraag waar jij het antwoord op weet. Wiel zoals jou is er maar eentje (gelukkig), bedanki voor de plezierige samenwerking. Marjanne Markerink-van Ittersum, jij bent zonder twijfel de collega met de meeste goedheid en zonder jou waren de biochemische bepalingen nooit van de grond gekomen of mogelijk geweest. Biedankt. Voor het anatomisch werk moet ik Hellen Steinbusch bedanken, omdiat ik zonder haar simpelweg nooit al die data had kunnen verkrijgen. Verder wil ik Huab Hamers bedanken woor de prima soft- en hardware ondersteuning bij de gedragsopstellingen. Tenslotte bedank ik al die (ex-)studenten die hebben meegewerkt bij de experimenten. leders bijdrage, zonder dat ik nu al jullie namen ga noemen, varièrend van een eiwitbepaling tot complete gedragsexperimenten, is een grote hulp geweest.

Er is ook een aantal collega's, van wie sommige alweer elders werkzam zijn, die ik will bedanken omdat ze op én of andere manier hebben bijgedragen, zelfs al in de oude tijden (dus nog voor aanvang van dit $\mathrm{A}$ TO schap). Allereerst Wijnand Raaijmakers, jij bent tenslotte de man via wie het voor mij allemaal begon in Maastricht. Daamaast wil ik (s)John(ny) B., Martin K., Matthijs H., Wim R., Rudolf $P$. en Jeroen (may the blues be with you) ten $H$., van het fameuze maar reeds ter zielle gegane zaalvoetbaiteam Brain Wave, bedanken voor de prettige samenwerking op het velid en zeker ook daurbuiten, zoals aan de tap.

De vorige alinea is al een opmaat voor de zaken naast het werk. Bij mij zijn dat mijn vrienden en de liefde voor muziek. Allereerst mijn goede vrienden: Folkert, Gert-Jan, Armand, HP en Wilmar, ook beter bekend als de Heeren (nee, dit is geen typefout). Mannen, jullie vriendschap en

\footnotetext{
* Keep it Short and Simple
} 
Steun was ook bij het tot standkomen van dit proefschrift van grote waarde. Er is nog een aantal mensen geweest die ik zeker wil bedanken voor de vaak zeer uiteenlopende manieren waarop zij mij er door sleepten als ik er weer eens niet meer in geloofde. Beste Frank, als bet nodig was, bleek je er te zijn, bedankt. Guido, goede broer van me, het werken in de tuinen en vertoeven tussen jouw veestapel (en ook die van de 'boerderij") waren woor mij van onschatbare warde om de teller af en toe op nul te zetten, allhoewel jezelf dat misschien niet zo in de gaten had. Beste Pa en lieve Ma, ik ben dankbaar dat jullie mij altijd zijn blijven steunen. Zeker niet vergeten will ik mijn paranimfen: Folkert (van de Heeren) en mijn lieve zus Astrid, wiens beide bijdragen zich niet tot het proefschrift beperken, en op wiens steun ik eigenlijk mijn hele leven al kan rekenen. Tenslotte kom ik uit bij de muziek en in het bijzonder mijn drumstel; het ideale instrument om je op af te reageren. Als ik geen wetenschapper was geworden, dan misschien wel muzikant. Helaas of gelukkig mocht het niet zo zijn. Maar muziek maken is altijd een geweldige uitlaatklep en vandaar ook dat ik de (ex-)leden van het Ronald Rondas Combo, ex-Litte Wing, Night Mission en Recycle (met name Erik en Y'vonne) wil bedanken voor het vele plezier dat samen muziek maken oplevert.

Maar het allerbeste heb ik woor het laatste bewaard. Jeanette, mooie vrouw wan me, bedankt dat je altijd in me bent blijven geloven met een liefde die onbeperkt is. 


\section{Curriculum vitae}

Jos Prickaerts werd op 31 maart 1966 geboren te Berg en Terblijt. Na het behalen wan het Wwobeta diploma aan het Jeanne d"Are College te Maastrich, ging hij in 1986 biologie studeren aan de Universiteit Urecht. Na het 'cum laude' behalen van het propedense diploma, koos hij voor de afstudeerrichting medisch gerichte biologie. De eerste stage werd gedaan in Utrech bij de wakgroep Vergelijkende Fysiologie en betrof electrofysiologisch onderzoek naar informatieverwerking in horizontale cellen wan het kattennetvlies. De tweede stage werd gedan in Maastricht bij de vakgroep Neuropsychologie en Psychobiologie van de Universiteit aldaar. Het onderwerp betrof de effecten van chronisch alcohol gebruik op cognitie bij de rat. Na een studentassistentschap met als onderwerp hartinfarct en angst, was hij de laatste 7 maanden van zijn studie werkzaam als onderzoeksassistent in opdracht van het famaceutische bedrijf SERVIER (Parijs, Frankrijk). Na het behalen van het doctoral diploma in 1992 bleef hij werkzaam in Maastricht, maar dan als toegevoegd onderzoeker op een project van 12 maanden voor het bedrijf SIGMA-TAU (Ponezia-Rome, Italie). Beide 'farmaceutische' projecten betroften onderzoek naar de werkzaamheid van potentiele noötropica van de desbetreffende firma's. Eind 1993 werd hij assistent in opleiding bij de divisie Neurodegeneratie en Plasticiteit van wat dan de sectio Neuropsychologie (Vakgroep Psychiatrie) komt te heten, en warvan dit proefschrift hut resultaat is. Het promotieonderzoek werd uitgevoerd binnen EURON (European Graduate School of Neuroscience) dat onderdeel is van het Hersenen en Gedrag Instituut. Daarnaast werd een gedeelte van een onderzoek uitgevoerd in samenwerking met, en bij de CNS-Research afdeling van het farmaceutische bedrijf BAYER in Duitsland (Keulen). Voor laatstgenoemde firma is hij sinds begin 1998 werkzaam als toegewoegd onderzoeker te Maastricht op een project met betrekking tot potentiele noötropica. In zijn wrije tijd is hij een fanatiek liefhebber van muziek, zowel passief als actief. Hij is dan ook regelmatig achter zijn drumstel te vinden. 


\section{Publications}

\section{Papers}

First awhor

Prickaerts J., Blokland A., Bothmer J.. Honig W., Markerink-Wan Ittersum M. and Jolles J. (In Press) Acute effects of acetyl-L-carnitine on sodium cyanide-induced behaviorall and biochemical deficts. Neurochemistry International.

Prickaerts J., Blokland A., Honig W. Meng F. and Jolles J. (1995) Spatial discrimination learning and choline acetyliransferase activity in streptozotocin-treated rats: Effects of chronic treament with acetyl-Lcarniline Braln Research 674, 142-146.

Prickterts J., De Vente J., Honig W. Steinbusch H., Markerink-Van Ittersum M., Blokland A. and Steinbusch H.W.M. (Submitted) Nitric oxide synthase does not mediate neurotoxicity after an i.c.v. injection of streptozotocin in the rat. Neuroscience Research.

Prickaerts J., De Vente J., Markerink-Van Ittersum M. and Steinbusch H.W.M. (1998) Behavioural, neurochemical and neuroanatomical effects of chronic postnatal L-NAME treatment in neonatal and adult ralls. Neuroscience $87,181-195$.

Prickaerts J., Fahrig T. and Blokland A. (Submitted) Cognitive performance and neurodegenerative markers in hippocampus, septum and striatum after an i.c.r. injection of streptozotocin: $A$ correlation analysis. Behawiourat Brain Research.

Prickaerts J., Raaijmakers W. and Blokland A. (1996) Effects of myocardial infarction and captopril therapy on anxiely-related betaviors in the rat. Physiology and Behovior 60, 43-50.

Prickaerts J., Steinbusch H.W.M., Smils J.F.M. and De Vente J. (1997) Possible role of nitric oxide-cyclic GMP pathway in object recognition: Effects of 7-nitroindazole and zaprinast. Europeam Joumal of Pharmacology 337, 125-136.

\section{Co-author}

Blokland A. De Vente J., Prickaerts J., Honig W., Markerink-Van Ittersum M. and Steinbuseh H.W.M. (In Press) Local inhibition of hippocarnpal nitric oxide synthase does not impair place learning in the Morris water escape task in rats. European Jownal of Neuroscience.

Blokland A., Honig W. and Prickaerts J. (In Press) Effects of haloperidol and $d$-ampheramine on working and

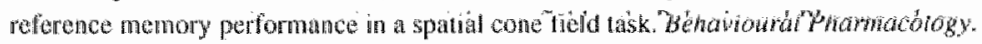

Blokland A., Prickaerts J., Honig W. and De Vente J. (Subnitted) State-dependent impairment in object recognition sifter bippocampal NOS indibition. NetroReporn.

Blokland A., Prickarts J. and Ruaijmakers W. (1992) Reduced level of anxieny in adult Lewis rats after chronic ethanol consumption. Physiology and Behovior 51, 245-248.

Blokland A. Prickaers J. and Ranimakers W. (1993) Absence of impairments in spatial and temporal discrimination leaning in Lewis rats after chronic ethanol consumption. Pharmacology Biochemistry and Behavior 46, 27-34,

Lankheed M.J.M., Prickatrs J.H.H.J. and Van de Grind W.A. (1992) Responses of cat horizontal cells to sinusoidal gritings. Vision Resetarch 32, $997-1008$.

Lankheet M.J.M., Van Wexel R.J.A., Prickaerts J.H.H.J, and Van de Grind, W.A. (1992) The dynamics of ligh adaptation in cat thorizontal cell responses. Vision Research 33, $1153-1171$.

"Terwel D. Prickachs $\mathrm{J}_{\text {. }}$ Meng F. and Jolles J. (1995). Brain enzy me activities after intracerebroventriculat injection of streptozotocin in rats receiving acetyl-L-carnitine. European Joumal of Pharmacology 287 , $65-71$. 


\section{Abstracts (published)}

Prickaerts J., Blokland. A., De Vante J, Jolles J. and Steinbusch H.W.M. (1995) Behavioral, neuroehemical and morphological observations in streptozotocin-treated rats. Europech Joural of Neuroscience $\$ 8.171$, 68.03 .

Prickaerts J., Blokland A., Honig W., Steinbusch H.W.M. and Jolles J. (1995) Behavhoral and neurochenical observations in streptozolocin-treated rats: Effects of acelyl-L-carninine. Neuroscience Research Communicarions 17,56 , poster 37 .

Prickaerts J., De Vente J. Raaijmakers W. and Steinbusch H.W.M. (1996) Involvement of the nitric oxide/cyclic GMP system in object recognition memory of the rat. Society for Newroscience Abstracts 22.139. 62.2.

Prickaerts J. and Ralijmakers W. (1993) Different models for the assessment of anxiety in rats: A correlative study. Behaviourd Processes 29, 140-141.

Rataimakers W., Prickzerts J., Schoffelmeer A. and Jolles J. (1994) The novel cognition enhancing agent $S$ 12024-2 facilitates object and social recognition memory after acute and clronic treatment in rats. Society for Nerroscience Abstracts 20, 1765, 721.17.

\section{Research reports}

Raaijmakers W., Prickaerts J, and Terwel D. (1993) S 12024-2: Behavioral, cognitiwe and neurochenical effects. An investigation in rats into the effects of acute or chronic treatment with $S 12024-2$ on social and object recognition memory and on vasopressin in brain and blood plasma. Report to SERVIER.

Jolles J., Prickaerts J., Blokland A., Terwel D., Bothmer I. and Steinbusch H. (1994) The effects of acelyl-L. carnitine and congeners on brain energy metabolism and cognition in the rat: Progress repor 1092-1994 to SIGMA TAU.

Prickaerts J. and Blokland A. (1998) The effects of two putative cognition enhancers in three modiels of animal cognition. Report to BAYER AG. 